


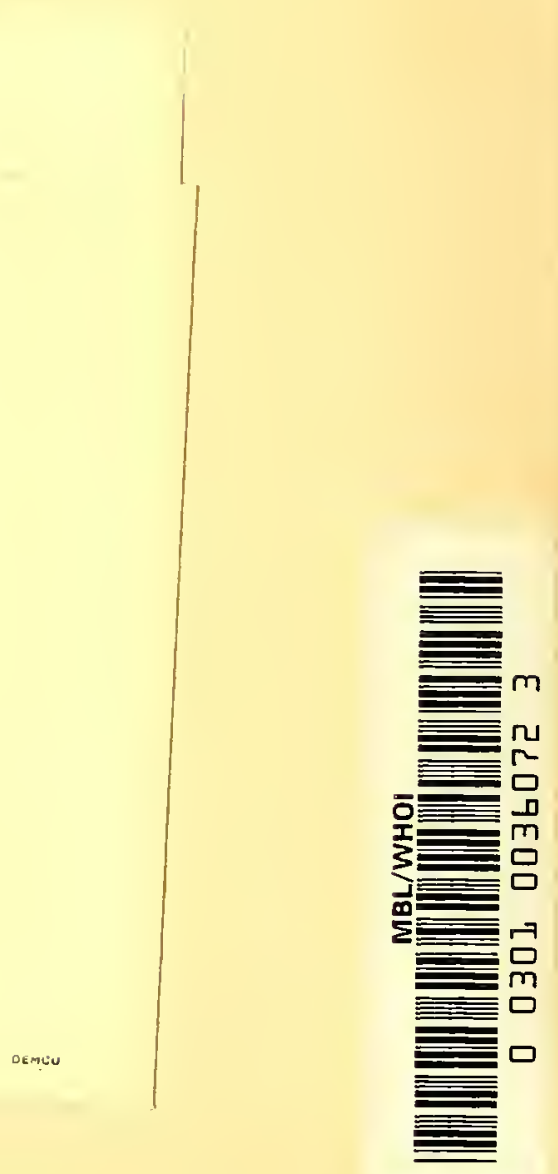



SUMMARY 'TECHNICAL REPORT

OF THE

NATIONAL DEFENSE RESEARCH COMMITTEE 
Manuscript and illustrations for this volume were prepared for publication by the Summary Reports Group of the Columbia University Division of War Research under eontraet OEMsr-1131 with the Office of Scientifie Research and Development. This rolume was printed and bound by the Columbia University Press.

Distribution of the Summary Technical Report of NDRC has been made by the War and Navy Departments. Inquiries concerning the availability and distribution of the Summary Technical Report volumes and microfilmed and other reference material should be addressed to the War Department Library, Room 1A-522, The Pentagon, Washington 25, D. C., or to the Office of Naral Research, Navy Department, Attention: Reports and Doeuments Section, Washington 25, D. C.

\section{Copy No.}

\section{4}

This volume, like the seventy others of the Summary Technical Report of NDRC, has been written, edited, and printed under great pressure. Inevitably there are error's which have slipped past Division readers and proofreaders. There may be errors of fact not known at time of printing. The author has not been able to follow through his writing to the final page proof.

Plase report errors to:

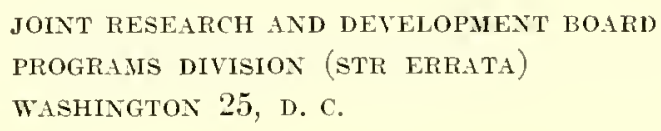

A master errata sheet will be compiled from these reports and sent to recipients of the rohme. Your help will make this book more useful to other readers and will be of great value in preparing any revisions. 
SUMIIARY TECHNICAL REPORT OF TIIE

COMMITTEE ON PROPAGATION, NDRC

VOLUME 3

\title{
THE PROPAGATION
}

\section{OF RADIO WAVES THROUGH \\ THE STANDARD ATMOSPHERE}

\author{
OFFICE OF SCIENTIFIC RESEARCH AND DEVELOPMENT \\ VANNEVAR BUSH, DIRECTOR \\ NATIONAL DEFENSE RESEARCH COMMITTEE \\ JAMES B , CONANT, CHAIRMAN \\ COMMITTEE ON PROPAGATION \\ CHAS. R. BURROWS, CHAIRMAN
}

DAPA LBRARY

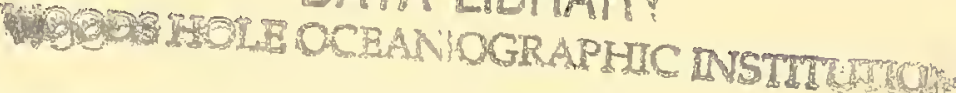

WASHINGTON, D.C., 1946 


\title{
NATIONAL DEFENSE RESEARCH COMMITTEE
}

\author{
James B. Conant, Chairman \\ Richard C. Tolman, Vice Chairman \\ Roger Adams \\ Army Representative ${ }^{1}$ \\ Frank B. Jewett \\ Navy Representative 2 \\ Karl T. Compton \\ Commissioner of Patents ${ }^{3}$ \\ Irvin Stewart, Executive Secretary
}

1 Army representatives in order of service:

$\begin{array}{ll}\text { Maj. Gen. G. W. Strong } & \text { Col. L. A. Denson } \\ \text { Maj. Gen. R. C. Moore } & \text { Col. P. R. Faymonville } \\ \text { Maj. Gen. C. C. Williams } & \text { Brig. Gen. E. A. Regnier } \\ \text { Brig. Gen. W. A. Wood, Jr. } & \text { Col. M. M. Irvine }\end{array}$

Col. E. A. Routheau
${ }^{2}$ Navy representatives in order of service:

Rear Adm. H. G. Bowen Rear Adm. J. A. Furer

Capt. Lybrand P. Smith Rear Adm. A. H. Van Keuren

Commodore H. A. Schade

${ }^{3}$ Commissioners of Patents in order of serice:

Conway P. Coe Casper W. Ooms

\section{NOTES ON THE ORGANIZATION OF NDRC}

The duties of the National Defense Research Committee were (1) to recommend to the Director of OSRD suitable projects and research programs on the instrumentalities of warfare, together with contract facilities for carrying out these projects and programs, and (2) to administer the technical and scientific work of the contracts. More specifically, NDRC functioned by initiating research projects on requests from the Army or the Navy, or on requests from an allied government transmitted through the Liaison Office of OSRD, or on its own considered initiative as a result of the experience of its members. Proposals prepared by the Division, Panel, or Comnittee for research contracts for performance of the work involved in such projects were first reviewed by NDRC, and if approved, recommended to the Director of OSRD. Upon approval of a proposal by the Director, a contract permitting maximum flexibility of scientific effort was arranged. The business aspects of the contract, including such matters as materials, clearances, vouchers, patents, priorities, legal matters, and administration of patent matters were handled by the Executive Secretary of OSPD.

Originally NDRC administered its work through five divisions, each headed by one of the NDRC members. These were:

Division A - Armor and Ordnance

Division B - Bombs, Fuels, Gases \& Chemical Problems

Division C - Communication and Transportation

Division D -- Detection, Controls, and Instruments

Division $\mathrm{E}$ - Patents and Inventions
In a reorganization in the fall of 1942 , twenty-tbree administrative divisions, panels, or committees were created, each with a chief selected on the basis of his outstanding work in the particular field. The NDRC members then became a reviewing and advisory group to the Director of OSRD. The final organization was as follows:

Division 1-Ballistic Research

Division 2-Effects of Impact and Explosion

Division 3 - Rocket Ordnance

Division 4 -Ordnance Accessories

Division 5-New Missiles

Division 6 - Sub-Surface Warfare

Division 7 - Fire Control

Division 8 - Explosives

Division 9 -Chemistry

Division $10-$ Absorbents and Aerosols

Division 11 - Chemical Engineering

Division 12 - Transportation

Division 13 - Electrical Communication

Division 14 - Radar

Division 15 - Radio Coordination

Division 16-Opties and Camouflage

Division 17 - Physics

Division 18 - War Metallurgy

Division 19 - Miscellaneous

Applied Mathematies Panel

Applied Psychology Pane]

Committee on Propagation

Tropical Deterioration Administrative Committee 


\section{NDRC FOREWORD}

$\mathrm{A}^{\mathrm{s}}$ EVENTS of the years preceding 1940 revealed more and more clearly the seriousness of the world situation, many scientists in this country came to realize the need of organizing scientific research for service in a national emergency. Recommendations which they made to the White House were given careful and sympathetic attention, and as a result the National Defense Research Committee [NDRC] was formed by Executive Order of the President in the summer of 1940 . The members of NDRC, appointed by the President, were instructed to supplement the work of the Army and the Navy in the development of the instrumentalities of war. A year later upon the establishment of the Office of Scientific Research and Development [OSRD], NDRC became one of its units.

The Summary Technical Report of NDRC is a conscientious effort on the part of NDRC to summarize and evaluate its work and to present it in a useful and permanent form. It comprises some seventy volumes broken into groups corresponding to the NDRC Divisions, Panels, and Committees.

The Summary Technical Report of each Division, Panel, or Committee is an integral survey of the work of that group. The first volume of each group's report contains a summary of the report, stating the problems presented and the philosophy of attacking them, and summarizing the results of the research, development, and training activities undertaken. Some volumes may be "state of the art" treatises covering subjects to which various research groups have contributed information. Other's may contain descriptions of devices developed in the laboratories. A master index of all these divisional, panel, and committee reports which together constitute the Summary Technical Report of NDRC is contained in a separate volume, which also includes the index of a microfilm record of pertinent technical laboratory reports and reference material.

Some of the NDRC-sponsored researches which had been declassified by the end of 1945 were of sufficient popular interest that it was found desirable to report them in the form of monographs, such as the series on radar by Division 14 and the monograph on sampling inspection by the Applied Mathematics
Panel. Since the material treated in them is not duplicated in the Summary Technical Report of NDRC, the monographs are an important part of the story of these aspects of NDRC research.

In contrast to the information on radar, which is of widespread interest and much of which is released to the public, the research on subsurface warfare is largely classified and is of general interest to a more restricted group. As a consequence, the report of Division 6 is found almost entirely in its Summary Technical Report, which runs to over twenty volumes. The extent of the work of a division cannot therefore be judged solely by the number of volumes devoted to it in the Summary Technical Report of NDRC: account must be taken of the monographs and available reports published elsewhere.

Though the Committee on Propagation had a comparatively short existence, being organized rather late in the war program, its accomplishments were definitely effective. That so many individuals and organizations worked together so harmoniously and contributed so willingly to the Committee's effor'ts is a tribute to the leadership of the Chairman, Charles R. Burrows. The latest information in this field was gathered from the four corners of the earth, organized, and dispatched to the points where it would aid most in the prosecution of the war.

Much credit must be given, not only to the members of the Committee and its contractors, but also to the many other individuals who gave so generously of their time and effort. This group included a number of our Canadian and British allies. In addition to the assistance given the war effort, a considerable contribution has been made to the knowledge of short-wave transmission and especially to the interrelation of this phenomenon with meteorological conditions. Such information will be most valuable in weather forecasting and in furthering the usefulness of the whole radio field.

Vannevar Bush, Director

Office of Scientific Research and Development

J. B. Conant, Chairman

National Defense Research Committee 



\section{FOREWORI)}

$\mathrm{T}$

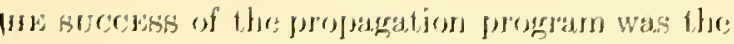

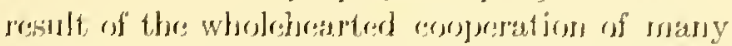

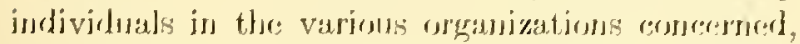

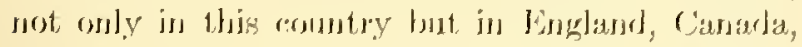

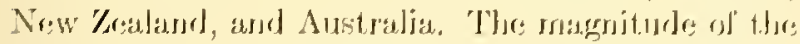

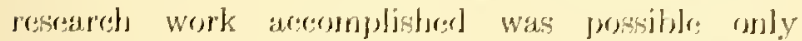
breasuse of the willingreses of the workens in sodny organizations to nordrentakes these pasts of ther overall

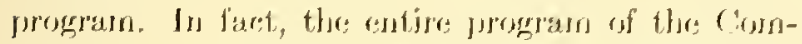

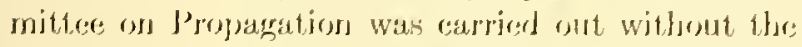

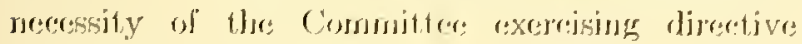
anthurity oves any jorojocet.

J). Jukbent Hoykins of the Netjonal Physical

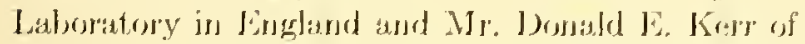
the Radiation Jaboratory at the Maskachusetts

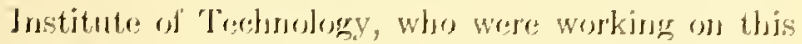
phase of the: war elfort when the J'ropsagation Cornsnitere was fromed, werre justromarestal in giving de

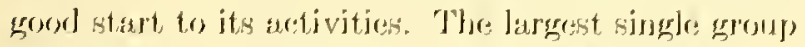

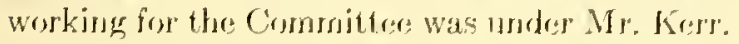

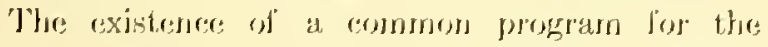
United Nations ju sadios-weve propegetion resulted

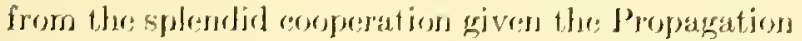
Missions an England by Sir lidward Appleton ared

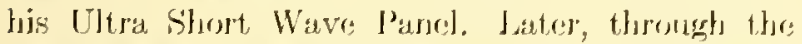

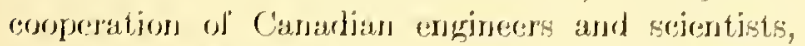
Dr. W, Ji. Mekindry of the National Jiescarets Comucil of' Camarka and I)r. Andsew 'Themenon of the

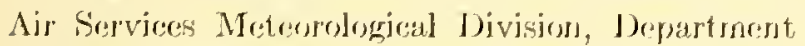
of 'Jransport, Toponto, Canarla, undortook to rarry on a part of the porogram origriolly assigned to the United States. The joregrass was funther gromerled out by the willinguess of the Now Zealand Government to undertake an experingent for whicels their siluation was particalarly favorabls. J)r. F. J. S. Alexander of New /oaldusd and J)r. Paul A. Anderson of the State College of Washingtors initiated this work. Needless to sidy, the latoror of the Cosnmittee on J'opagation comld bardly have becon alfective without the cosoperation of the Amry and Navy. Maj. Gien. IJ. M. MreClelland jersonally established

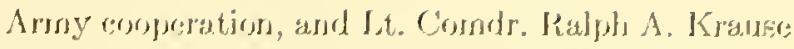

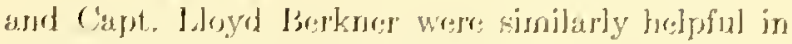
orgardizing Navy liaison and hedp.

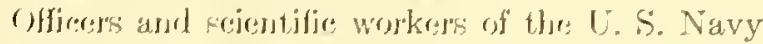

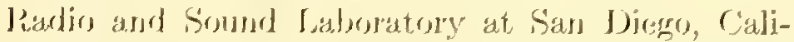
formia, altorod their program ors goropagation to fit

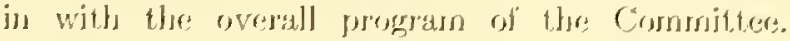

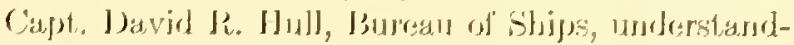

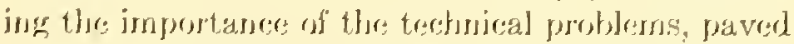
the way fore reffecotive ceoreperation by this laboratory.

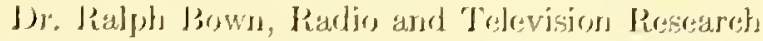

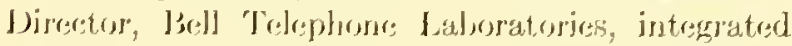

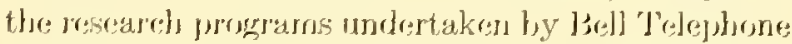

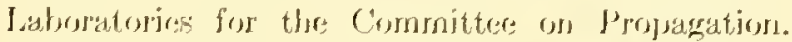

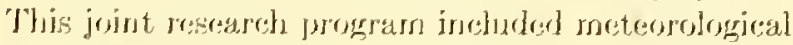

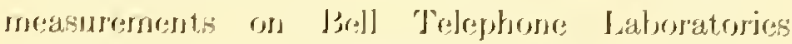
property by inoteorologists of the Arrsy Air Foresess working with (ol. J). X. Yates, Jireretor, and L.t. Col. Jarry Wrexler of the Wrather Wirng, Arrny

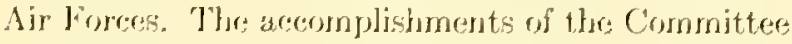
on l'oppagation ars a gorod rexample of the efforetivenoss of eoroporation - all parts were essential and uenas Juones thase the rost.

I want to thask IOr. Karl T. Corngetor, J'essident of Masiachusetis fnstitute of 'Teceluologry, who was always willing to disedess problerns of the Comnitese and who hrofped ros to solves many of the more diffecult ones, and alsos, Prof. S. S. Attwrond, University of Michigan, whoss: continuad counsol thronghout my term of offices was in no sunall way responsible for the sucoess of our activity.

Creedit is also due Jedl Telephose Lalsoratories, which marle my services avalable to the Coversment and paid my salary from August 1949 to September 1945, and to Cornell University, which has allowed me timo off with pay to complete the work of the Committec on Propagation since Septernter 1945.

Chas. R. Jurpows

Chairman, Committee on Propagation 


\section{PREFACE}

$\mathrm{T}$ HE Materlal presented in this book was prepared by the Columbia University Wave Propagation Group at the request of the Committec on Propagation of the National Defense Research Committee. The International Radio Propagation Conference, meeting at Washington in Mar 1944, recommended that a book be prepared dealing with problems of radio wave propagation in the standard atmosphere at frequencies above 30 megacycles. The importance of these higher frequencies is apparent when it is recalled that most radars operate in this range and that an increasing number of communication circuits are being equipped for operation above this frequency.

A certain amount of evidence from operational theaters indicates that lack of familiarity with the underlying theory of propagation and calculations based thereon not infrequently has resulted in ineffective installation and operation of radar and communication sets. This is ascribable, in part at least, to the lack of publications which give a clear picture of the problems of propagation or show how the important factors may be evaluated.

A considerable volume of basic material on propagation had appcared in technical journats before World War II, and during it a great quantity of classified material has come from laboratories and operational theaters illustrating new applications of old principles, giving valuable information on propagation problems as well as on characteristics of radar and communication sets, antennas, targets, siting problems, etc. But this information has not been coordinated under one cover for practical use by signal personnel in the field. The Columbia University Wave Propagation Group was asked to undertake this task and it is hoped that this book will, in some measure, answer the need.

Our effort, then, has been to provide a book de- signed for men with college training in radio, physics, or electrical engineering, which states the basic laws of propagation, that is, shows how the characteristics of the earth and the atmosphere control the propagation of radio waves; gives the fundamental properties of the basic types of antenna systems, particularly their directivity and gain; gives the reflecting properties of targets such as airplanes for use in detection by radar; teaches the reader how to calculate field strength or obtain the coverage diagrams given a particular set, power, and site; gives tho fundamental information required in the above calculations for application to the radar and communication sets used in operational theaters; and provides illustrative material and sample calculations which show how the laws of propagation may advantageously be used in the location and operation of radar systems, communication sets, and countermeasure equipment designed to deceive the enemy and to prevent jamming of equipment by enemy action or by mutual interaction of our own sets.

The members of the group chiefly responsible for the preparation of the manuscript were Drs. S. Rosseland, W. M. Elsasser, P. Newman, and Prof. S. Fich. Others who contributed special sections were Messrs. E. R. Wicher, M. Ettenberg, M. Siegel, and Capt. E. J. Emmerling, on special assignment from the Signal Corps.

The editor wishes to acknowledge also the courtesy of the Radiation Laboratory Wave Propagation Group, under Mr. Donald E. Ferr, in supplying the universal coverage charts given in Chapter 6, and the steady interest and assistance rendered by Dr. Chas. R. Burrows, Chairman, NDRC Committee on Propagation.

S'TEPHEN \$. ATTwOOD

Editor 


\section{CONTENTS}

1 Propagation of Radio Waves: Introduction and Objectives . . . . . . . . 1

2 Fundamental Relations . . . . . . . . 12

3 Antennas . . . . . . . . . . 22

4 Factors Influencing Transmission . . . 45

5 Calculation of Radio Gain . . . . . . 60

6 Coverage Diagrams . . . . . . . 129

7 Propagation Aspects of Equipment Operation $\quad 160$

\& Diffraction by Terrain . . . . . . 170

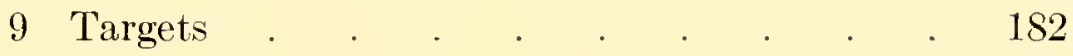

10 Siting . . . . . . . . . . . 187

Glossary . . . . . . . . . . 197

Bibliography $^{\mathrm{a}}$

OSRD Appointees .

Contract Numbers . . . . . . . 200

Service Project Numbers _ . . . . 201

Index . . . . . . . . . . 203

${ }^{2}$ Refer to Bibliography of C. P. Summary Technical Report, Volume 1. 



\section{Chapter 1 \\ PROPAGATION OF RADIO WAVES: INTRODUCTION AND OBJECTIVES}

\subsection{FACTORS INFLUENCING PROPAGATION}

$\mathrm{T}$ HE PROPAgation of radio waves through the atmosphere and around the curve of the earth, at frequencies above $30 \mathrm{mc}$, is influenced by so many factors that it is desirable to give first an overall survey of the problem. This chapter presents the problem of propagation in broad perspective in contrast with many of the later chapters which are devoted to detailed consideration of special phases and methods of calculation.

1.1 .1

\section{Assignment}

The International Radio Wave Propagation Conference recommended that a book be prepared dealing with problems of radio wave propagation in the standard atmosphere at frequencies above $30 \mathrm{mc}$. The importance of these higher frequencies is apparent when it is recalled that most radars operate in this range and that an increasing number of communication circuits are being equipped for operation above this frequency.

A certain amount of evidence from operational theaters indicates that lack of familiarity with the underlying theory of propagation and calculations based thereon not infrequently has resulted in ineffective installation and operation of radar and communication sets. This is ascribable, in part at least, to the lack of publications which give a clear picture of the problems of propagation or show how the important factors may be evaluated.

A considerable volume of basic material on propagation had appeared in technical journals before World War II, and during the war a great quantity of classified material came from laboratories and operational theaters illustrating new applications of old principles, giving valuable information on propagation problems as well as on characteristics of radar and communication sets, antennas, targets, siting problems, etc. But this information has not been coordinated under one cover for practical use by signal personnel in the field. The Columbia University Wave Propagation Group was asked to undertake this task, and it is hoped that this volume will, in some measure, answer the need.

\section{Pirpose}

Our effort then has been to provide a book, designed for men with college training in radio, physics, or electrical engineering, which:

1. States the basic laws of propagation, that is, shows how the characteristics of the earth and the atmosphere control the propagation of radio waves;

2. Gives the fundamental properties of the basic types of antenna systems, particularly their directivity and gain;

3. Gives the reflecting properties of targets such as airplanes for use in detection by radar;

4. Teaches the reader how to calculate field strength or obtain the coverage diagrams, given a particular set, power, and site;

5 . Gives the fundamental information required in the above calculations for application to the radar and communication sets used in operational theaters;

6. Provides illustrative material and sample calculations which show how the laws of propagation may advantageously be used in the location and operation of radar systems, communication sets, and countermeasure equipment designed to deceive the enemy and to prevent jamming of equipment by enemy action or by mutual interaction of our own sets.

\subsection{FUNDAMENTAL PROBLEMS AND LIMITATIONS}

\subsubsection{Meaning of Propagation}

By propagation is meant the movement of radio waves through the atmosphere, and the transfer, by a wave mechanism, of radiant energy from a transmitting antenna to a receiving antenna. The problem of propagation requires an understanding of the manner in which the wave energy is emitted and received as well as of the manner in which it flows through the atmosphere. The radio engineer must 
understand this general mechanism, be able to evaluate the factors which play contributory roles, and, for a given amount of power emitted from a given transmitter, be able to compute the strength of the radiation field at any point in space or to locate all the points in space where a given field strength occurs.

The problem divides naturally into two parts, (1) the one-way transmission or communication problem, and (2) the two-way transmission or radar problem. In the former the prime requisite is to calculate the amount by which the wave and its field strength are attenuated in passing from the transmitter to a receiver and yet permit a ficld at the receiver suffi-
1.2 .2

\section{Atmospheric Layers}

The atmosphere from one point of view is composed of two layers, the troposphere and the stratosphere. The former is a layer adjacent to the earth which extends upward approximately $10 \mathrm{~km}$, in which the temperature decreases about $6.5 \mathrm{C}$ per kilometer with increasing altitude to a value, at the upper boundary, of about $-50 \mathrm{C}$. Above this is the stratosphere in which the temperature remains approximately constant at $-50 \mathrm{C}$.

The ionosphere, as its name implies, is a layer (or series of layers) composed of ions and free electrons lying at an elevation of approximately $100 \mathrm{~km}$. See

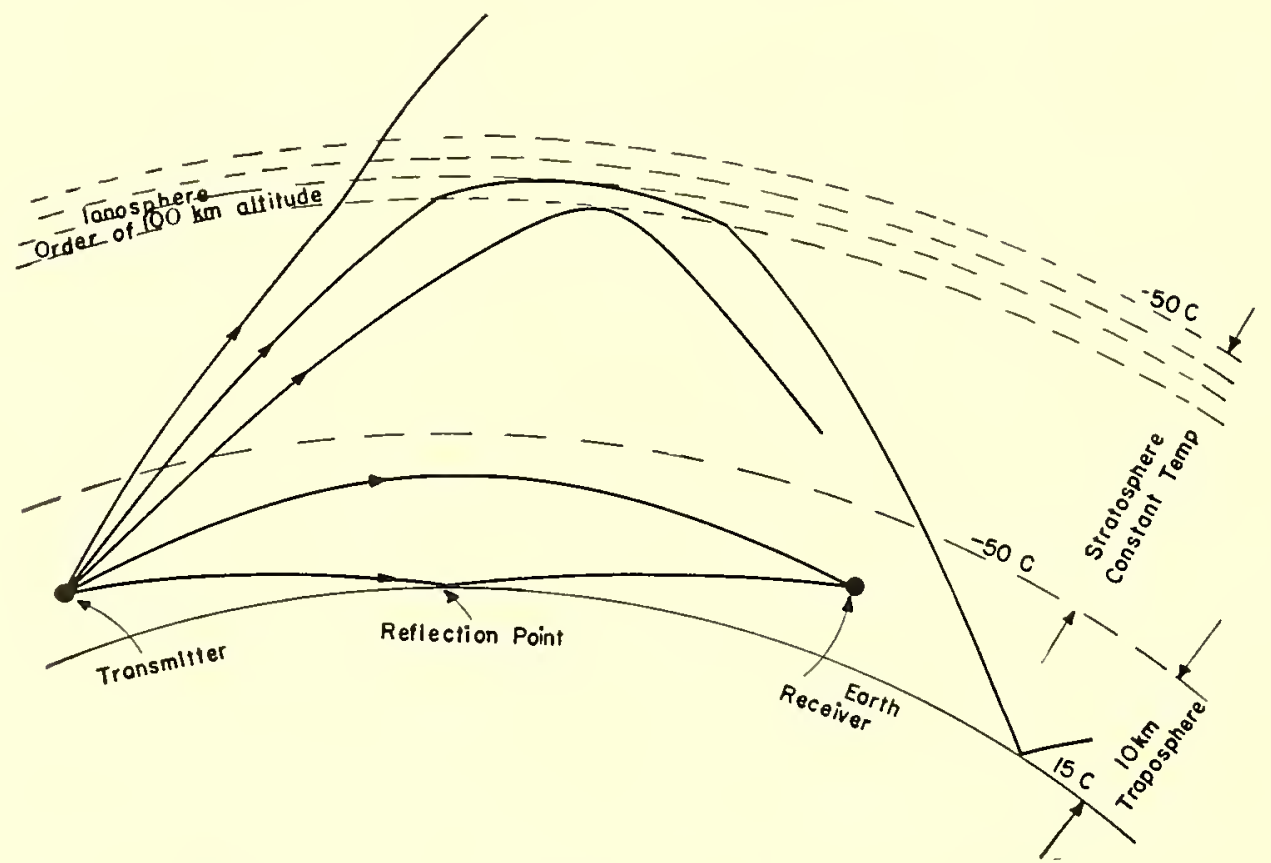

Figure 1. Transmission along and reflection from the ionosphere oceurs primarily with frequencies below $30 \mathrm{me}$. At higher frequencies useful transmission is primarily concerned with the nearly horizontal rays in the troposphere; higher angle radiation passes through the ionosphere and is lost.

cient at least to produce the minimum detectable signal. In the latter problem the attemuation must be calculable for the two-way journey from transmitter to the target and back to the receiver, which frequently uses the same antenna as the transmitter. In this type of problem, due account must also be taken of the reflecting and reradiating properties of the target.

Knowledge of these factors is indispensable for the design, installation, and successful operation of both communication and radar systems.
Figure 1. These layers play an important role in the transmission of waves at frequencies below $30 \mathrm{mc}$ and are responsible for transmission over very long distances. At the higher frequencies, which are the concern of this volume, the portion of the waves which penetrate the ionosphere is not useful for transmission.

From this it follows that propagation at the higher frequencies (above $30 \mathrm{mc}$ ), to be useful, must occur entirely in the troposphere. This volume therefore is concerned only with tropospheric propagation. 
1.2 .3

\section{Standard Atmosphere}

Propagation of radio waves in the troposphere is materially influenced by the distributions of temperature, pressure, and water vapor. The condition most nearly approximated in the Temperate Zone has been accepted as the so-called standard atmosphere, and propagation under this condition has been studied and calculations made thereon.

In the standard atmosphere specified by the National Advisory Committee on Aeronautics [NACA] the temperature is assumed to decrease with altitude at the rate of $6.5 \mathrm{C}$ per kilometer, starting from $15 \mathrm{C}$ at sea level, with a sea level dry air pressure of 1013.2 millibars, which is equivalent to $760 \mathrm{~mm} \mathrm{Hg}$ pressure (see Table 1). the total pressure and moisture vapor pressure, respectively, in millibars, at height $h$ above sea level. In the moist standard atmosphere, $n$ decreases linearly with height $h$ at the approximate rate of $0.039 \times 10^{-6}$ units per meter.

There are several reasons why this book concerns propagation in the moist standard atmosphere.

1. The atmosphere in certain places (particularly the temperate zoncs) and over considerable periods of time is substantially standard in character.

2. Calculations based on the standard atmosphere serve as a standard against which propagation in nonstandard atmospheres may be compared.

3. A great deal of propagation information now available in the field is based on propagation calculated for standard conditions.

TABLE 1. Properties of the atmosphere.

\begin{tabular}{|c|c|c|c|c|c|c|c|c|}
\hline \multicolumn{5}{|c|}{ NACA standard dry atmosphere } & \multicolumn{4}{|c|}{ Moist standard atmosphere } \\
\hline Feet & Meters & $\underset{C}{t} \underset{\text { Temp }}{t}$ & $\begin{array}{c}p-e \\
\text { Dry air } \\
\text { pressure } \\
\text { millibars }\end{array}$ & $\begin{array}{l}\text { Index of } \\
\text { refraction } \\
(n-1) 10^{6}\end{array}$ & $\begin{array}{c}e \\
\text { Water vapor } \\
\text { pressure } \\
\text { millibars }\end{array}$ & $\begin{array}{c}\text { Saturated } \\
\text { water vapor } \\
\text { pressure } \\
\text { millibars }\end{array}$ & $\begin{array}{l}\text { Per cent } \\
\text { relative } \\
\text { humidity }\end{array}$ & $\begin{array}{l}\text { Index of } \\
\text { refraction } \\
(n-1) 10^{\circ}\end{array}$ \\
\hline 500 & 152 & 14.0 & 995 & 274 & 9.5 & 16 & 59.4 & 312.4 \\
\hline 1,000 & 305 & 13.0 & 977.1 & 270 & 9 & 15 & 60.0 & 309 \\
\hline 1,500 & 457 & 12.0 & 960 & 266 & 8.5 & 14 & 60.7 & 304 \\
\hline 2,000 & 610 & 11.0 & 942.1 & 262 & 8 & 13.1 & 61 & 295.6 \\
\hline 3,000 & 915 & 9.1 & 908.1 & 254 & 7 & 11.6 & 60.3 & 284 \\
\hline 4,000 & 1,220 & 7.1 & 875.1 & 247 & 6 & 10.1 & 59.4 & 273 \\
\hline
\end{tabular}

To simulate the actual atmosphere of the temperate zones it is necessary further to specify a water vapor pressure. The value chosen is 10 millibars at sea level, decreasing with altitude at the rate of 1 millibar per $1,000 \mathrm{ft}$ up to $10,000 \mathrm{ft}$. With this addition the conditions for a moist standard atmosphere are specified in Table 1 . This value of water vapor pressure yields a valtue of relative humidity approximating 60 per cent.

Listed also in Table 1 is the index of refraction $n$. The gradient of this quantity, $d n / d h$, controls the curvature of the rays for a wave moving in the approximately horizontal direction; $n$ is given by the formula

$$
(n-1) 10^{6}=\frac{79}{T}\left(p-e+\frac{4800 e}{T}\right),
$$

where $T$ is the absolute temperature, $p$ and $e$ are

\subsubsection{Propagation in the Moist Standard Atmosphere}

The radiation energy emitted by a transmitter is a wave spreading out in three dimensions, which may be represented by a series of concentric spherical wave fronts or by a system of lines called rays. The velocity at any point on the wave ir ront is given by

$$
v=\frac{c}{n}=\frac{3 \times 10^{3}}{n} \text { meters per second. }
$$

Since $n$ decreases with height, the upper portions of the wave front move with higher velocities than the lower portions, and the wave paths as represented by the rays are curved slightly downward, as shown in Figure 2.

The radius of curvature of the rays $\rho$ is given by 


$$
\frac{1}{\rho} \cong-\frac{d n}{d h}=+0.039 \times 10^{-6} \text { units per meter }
$$

and $\rho$, therefore, is equal to $25.5 \times 10^{6}$ meters, which is approximately four times the radius of the earth $(\rho=4 a)$. As a result the distance to the radio horizon is some 15 per cent greater than the geometrical lineof-sight distance from the transmitter to the horizon. This curvature of the rays by the atmosphere is called refraction.

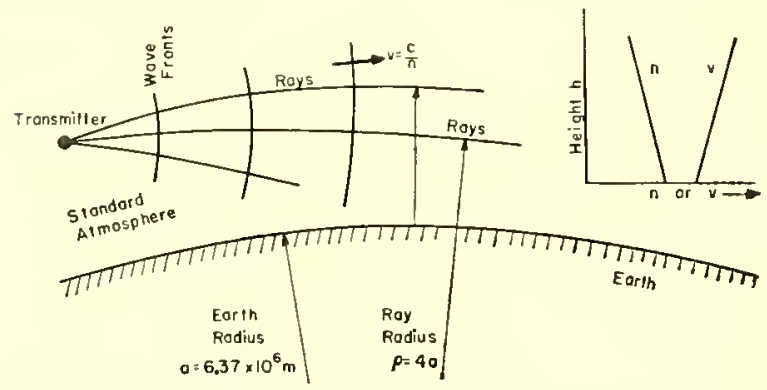

Figure 2. Curvature of rays in the standard atmosphere.

For the purpose of calculating wave propagation, only relative curvature of the earth and the rays is of interest. We can be compensated for the effects of refraction by replacing the actual earth with a radius $a$ by an equivalent earth with a radius $k a$ and replacing the actual atmosphere (in which the index of refraction $n$ decreases with height) by a homogeneous atmosphere (constant $n$ ) in which the rays are straight lines. Since $1 / a$ is the curvature of the earth and $1 / \rho$ the curvature of the rays, we may set their difference equal to $1 / 7 a$, the curvature of the equivalent earth. Thus

$$
\frac{1}{a}-\frac{1}{\rho}=\frac{1}{l_{i} a}
$$

and

$$
k=\frac{1}{1-(a / \rho)}=\frac{1}{1+a \frac{d n}{d h}}
$$

Since $\rho=4 a, k=4 / 3$, and $k a$, the radius of the equivalent earth, equals $8.49 \times 10^{6}$ meters. See Figures 4 and 5 in Chapter 4.

\subsubsection{Propagation in Nonstandard Atmospheres}

Though this subject is beyond the seope of this volume it is desirable to present a brief discussion of the salient features.
Not infrequently the lower atmosphere is stratified in horizontal layers in which the variations with height of the temperature and moisture content are nonstandard. Of particular interest is a sharp rise in temperature with iucreasing height (temperature inversion), or a sharp decrease in water vapor content, or a combination of the two. If these variations from the standard distribution are sufficiently great, horizontal radio ducts may be formed in the atmosphere. In this event an appreciable fraction of the wave energy (only that fraction moving in the nearly horizontal direction) may be constrained to propagate along the duct to distances far beyond the horizon and the ficld strength may be large compared with that obtainable under standard conditions. This phenomenon produces a marked bending of the wave paths or rays and is known as super-refraction. To take fullest advantage of this phenomenon the antennas should be located in the duct.

Ducts are of various types:

1. Overland. These are surface ducts formed at night by the radiation cooling of the earth.

2. Oversea. In the trade-wind belt there appears to be a continuous duct of the order of $50 \mathrm{ft}$ thick starting at sea level.

3. Land to sea. Warm dry air flowing from land out over cooler water often yields surface ducts 100 or more feet thick.

4. Elevated. Caused by subsidence of large air masses, these ducts may be found at elevations of perhaps 1,000 to $5,000 \mathrm{ft}$ and may vary in thickness from a few feet to $1,000 \mathrm{ft}$. They are common in Southern California and certain areas in the Pacific.

Depending upon the strength and the thickness of the duct, there is a limiting frequency below which the duct cannot trap the wave energy. Though trapping does at times occur at $200 \mathrm{mc}$, it is more likely to occur at the bigher frequencies such as $3,000 \mathrm{me}$.

Ability to calculate performance under standard conditions is necessar'y if performance under nonstandard conditions is to be evaluated.

\subsubsection{Radio Gain}

The basic problem to be solved is that of computing the radio gain of a transmitting-receiving system.

The radio gain of a transmitting-receiving system 
is defined as the ratio of received porrer $P_{2}$, delivered to a load matched to the receiving antenna, to power $P_{1}$, supplied to the transmitting antemu, with both antennas adjusted for maximum power transier. Thus

$$
\text { Radio gain }=\frac{P_{2}}{P_{1}},
$$

which is equal, in the decibel scale, to

$$
10 \log _{10} \frac{P_{2}}{P_{1}}=\text { radio gain in dccibels. }
$$

The attenuation is the reciprocal of the gain. Since $P_{2} / P_{1}<1$, the gain in decibels is necessarily a negative quantity. The attenuation in decibels is a positive quantity equal in magnitude to the gain in recibels.

The radio gain can be taken as the product of physically significant factors. Among these are the gains $G_{1}$ and $G_{2}$ of the transmitting and receiving antennas respectively; and $A^{2}$ which accounts for all other influences modifying the transmission of power. $A$ is called the gain factor.

$$
\text { Radio gain }=\frac{P_{2}}{P_{1}}=C_{1} C_{2} A^{2} \text {. }
$$

The radar problem involves double transmission over the path as well as the reradiating properties of the target, $16 \pi \sigma / 9 \lambda^{2}$.

$$
\text { Radar gain }=\frac{P_{2}}{P_{1}}=G_{1} G_{2} A^{4}\left(\frac{16 \pi \sigma}{9 \lambda^{2}}\right),
$$

where $\sigma$ is the radar eross section of the target and $\lambda$ is the wavelength. adjusted for maximum power transfer. $\Lambda_{0}=3 \lambda / 8 \pi d$ where $d$ is the distance between doublets. $A_{p}$ is the path gain factor which includes all additional influences modifying the transmission of porrer.

These factors may also be related to the field strength, $E$, at any point in space by

$$
E=E_{0} \sqrt{G_{1}} A_{p}
$$

and

$$
\frac{A}{A_{0}}=\frac{E}{E_{0} \sqrt{G_{1}}}
$$

Here $E_{0}$ is the free-space field at a point in space set up by a doublet transmitter and $E_{0} \sqrt{\sigma_{1}}$ is the free-space field of a transmitter with antenna gain $C_{1}$.

The primary function of this book is to show how the factors $A$ and $A_{p}$ may be calculated, taking into account all contributory influcnces which modify their magnitudes.

\subsubsection{Radio Gain of Doublet Antennas in Free Spaee}

This is the fundamental and simplest case of transmission of radiant energy, against which other transmitting combinations may be compared. Two doublet antennas (for which the gains, by definition, are unity) are sct up in free space in a manner which insures the maximum transfer of power to the receiver eircuit, i.e., the doublets are parallel to each other, have a common equatorial plane, and the

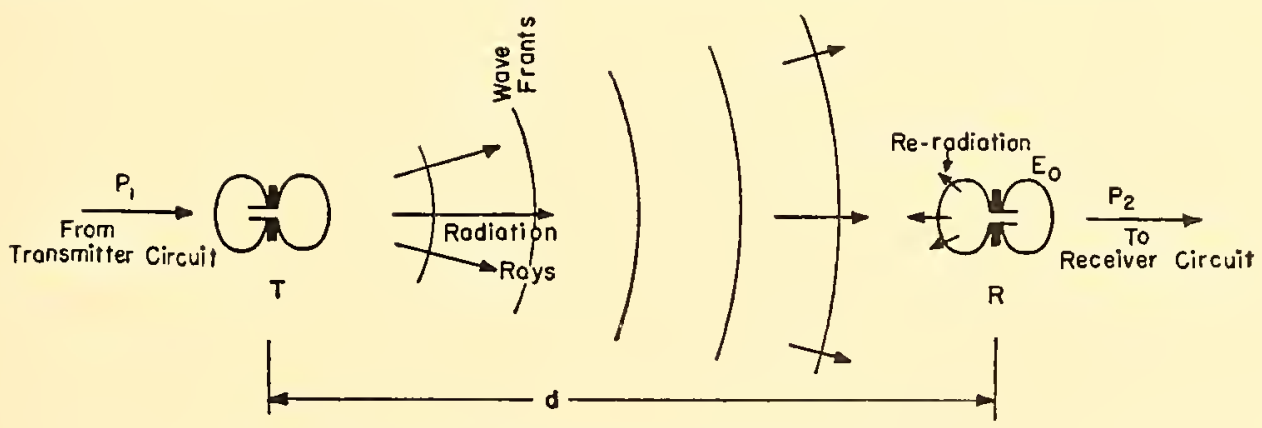

Figure 3. Doublet antennas in free space.

The gain factor, $A$, may also be split into two factors, so that

$$
A=A_{0} A_{p}
$$

Here $A_{0}$ is the frec-space gain factor for doublet antennas (see Sections 1.2.7, 2.1.3, 2.2.2, and 5.1.2) receiver circuit impedance is matched to that of the receiving antenna (see Figure 3). Then for free space,

$$
\text { Free-space gain }=\frac{P_{2}}{P_{1}}=A_{0}^{2}=\left(\frac{3 \lambda}{8 \pi d}\right)^{2},
$$


and the free-space field strength at distance $d$ from the transmitter is

$$
E_{0}=\frac{3 \sqrt{5} \sqrt{P_{1}}}{d}
$$

$P_{1}$ is the power radiated by the doublet transmitting antema (see Section 2.1.1).

\subsection{SURVEY OF PROPAGATION \\ 1.3.1 Outline}

We will first consider those factors which are instrumental in modifying the transmission or the attenuation that arises from the presence of the earth, then give typical eurves of both vertical and horizontal variation of field strength, and lastly, consider the problem of coverage.

\subsubsection{Factors Modifying Transmission}

The important factors which affect the distribution of ficld strength are the following:

1. Antenna characteristics. For many applications the most important feature is the gain which is a measure of the directivity of the antemna. From a pattern gives the relative amount of power per unit area radiated in that direction.
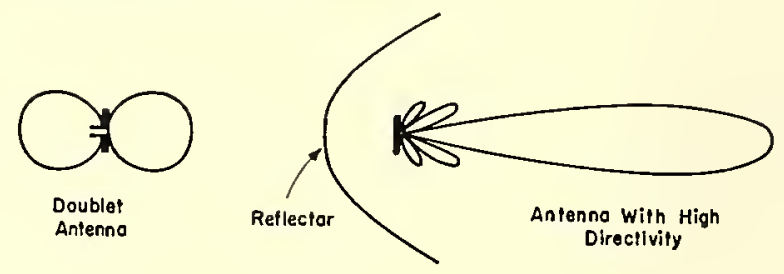

Figure 4. Antenna radiation patterns.

2. Polarization. The wave is said to be polarized horizontally or vertically according to whether the electric vector $E$ is parallel to the earth's surface or is in a plane perpendicular thereto. A horizontal electric doublet (axis parallel to the earth's surface) radiates horizontally polarized waves, whereas a vertical doublet radiates vertically polarized waves.

Too many factors are involved to make it possible to state in general which type of polarization should be used in a particular case.

3. Refraction. As explained in Section 1.2.4, refraction in the standard atmosphere can be taken into account by using an equivalent earth with a radius equal to $4 / 3$ that of the actual earth and a homogeneous atmosphere in which the rays traverse straight line paths.

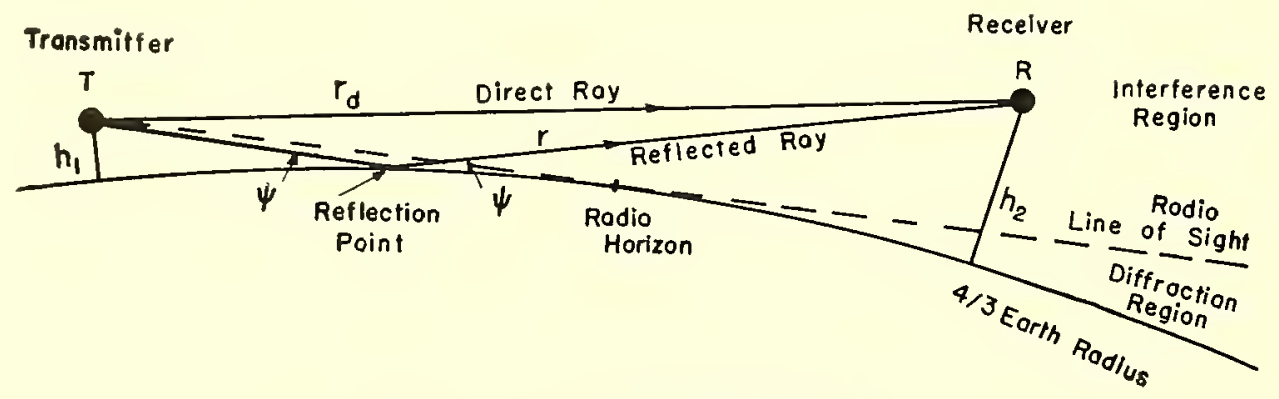

Figure 5. Geometrical relations for rays.

value of 1.09 for the half-rave dipole, the gain may increase to several thousand for the highly directive parabolic antennas used in the microwave range.

Antennas with high gains which concentrate the radiated energy into beams of small angles require less power to produce a detectable signal. This is particularly important in larlar where the attenuation of the two-way path is pronounced.

Qualitative radiation patterns for the doublet antenna and an antenna with high directivity are illustrated in Figure 4 . The radial distance to the
4. Reflction. Well above the line of sight (see Figure 5) the field at the receiver $R$ is the vector sum of the fields radiated along the paths of the direct and reflected rays. The contribution from the reflected ray path depends primarily on the manner in which the earth (or sea) acts as a reflecting body.

Upon reflection, the angle of incidence $\left(90^{\circ}-\psi\right)$ is equal to the angle of reflection, irrespective of the polarization of the wave, but the strength of the ficld in the reflected ray relative to that in the incident ray depends upon (a) the grazing angle $\psi$, 
(b) the type of polarization, (c) the reflecting properties of the earth or sea, and (d) the divergence factor.

The incident beam or bundle of rays, in general, is partially absorbed by the earth, while the reflected portion is reduced in strength and suffers a phase shift relative to the incident beam. (In the case of sea water with horizontal polarization, the earth acts substantially as a perfect reflector, for which the reflection is 100 per cent complete and the phase shift is 180 degrees for all grazing angles. This is true for vertical polarization only at zero grazing angle.)

The divergence factor is introduced to account for the fact that an incident bundle of rays striking a spherical surface diverges upon reflection and produces a further decrease in strength of the reflected beam.

Reflection from hills, trees, and other obstacles must frequently be taken into accolmt, particularly in the siting of very high frequency $[\mathrm{VHF}]$ communication sets.

5. Diffraction. The mechanism by which radio waves curve around edges and penetrate into the shadow region behind an opaque obstacle is called diffraction. The explanation usually given is based on Huyghens' principle. This, in effect, states that every elementary area on a wavefront (see $P$ in Figure 6 ) is a center which radiates in all directions

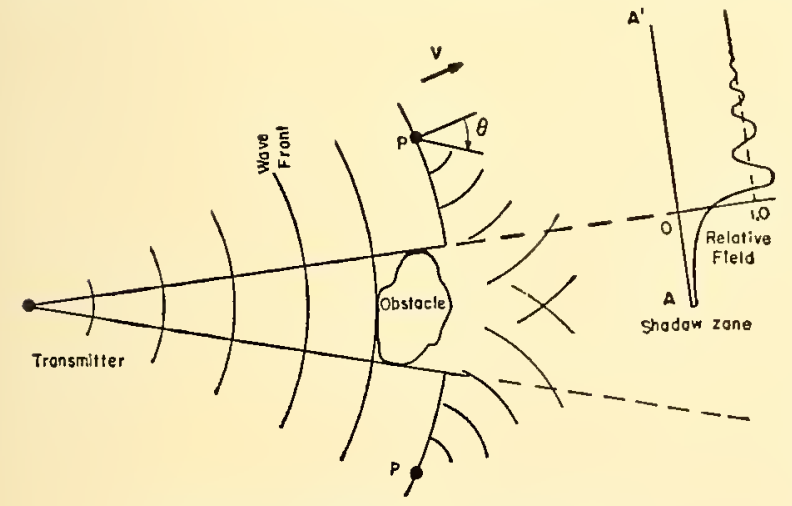

Figlre 6. Diffraction around an obstacle.

on the forward side of the wavefront; the intensity of radiation is a maximum in the direction perpendicular to the wavefront and depends on angle $\theta$ according to the function $(1+\cos \theta)$. The field at any point, either inside or outside the shadow zone, is obtained by summing the contributions from all the elementary areas comprising the wavefront.

As a result of these calculations, the field along the line $A A^{\prime}$ in Figure 6 varies approximately as indicated in the curve. Unity represents the field value if the obstacle were removed. It is seen that the field strength rises from a minimum at point $A$ to 0.5 at the edge of the shadow zone and thereafter oscillates about unity. The field outside the shadow zone, therefore, at certain points is stronger and at other points is weaker than it would be if there were no obstacle. The curve, of conrse, varics with the position of the line $A A^{\prime}$, the size and shape of the obstacle, the wavelength of the radiation, and the type of polarization. The diffraction of radiant energy into the shadow zone increases with increasing wavelength.

Of prime importance for propagation of radio waves is the diffraction of these waves into the diffraction region below the line of sight (see Figure 5). But it should be noted that the influence of diffraction is not confined to this region but extends well above the line of sight. [In general, the influence extends upward far enough to affect the shape of the lower part of the first lobe in a coverage diagram (see Figures 25 and 26 of Chapter 5). In this region the cliffraction contribution must be added to the contributions of the direet and reflected rays to give the correct value of the field strength at $R$ in Figure 5.]

Of importance in communication problems is diffraction of waves around obstacles such as hills, trees, houses, etc. This is illustrated in Figure 6 . Again diffraction is important in problems involving propagation above two different earth conditions. An especially important case is that of a radar set well inland and searching far out over the sea. Here the shore line is treated as a diffracting edge for the radiation from the image antenna.

6. Absorption and scattering. No account is taken in this book of the absorption and scattering of radio waves by the various constituents of the atmosphere. Oxygen, water vapor, water droplets, and rain all contribute to absorption. Their influence, however, is important only in the microware range and in general tends to increase with frequency.

1.3 .3

\section{General Nature of the Radiation Field}

In Section 1.3.2, reference has been made to the role of reflection by the earth. The resultant of the direct and indirect rays at points in the region above the line of sight gives rise to the lobes of an inter- 
ference pattern (see Figures 9 to 12). The maximum number of lobes is the largest integral number of times that the quarter wavelength is contained in the transmitter height.

In the case of horizontal polarization over a smooth surface, e.g., a calm sea, the reflected and direct rays are comparable in strength, so that at certain points (on lines for which the points correspond to a path difference of a half wavelength) where the reinforcement is a maximum, the field may be as much as twice the free-space field. More exactly, the free-space field is multiplied at points of maxima by $(1+F D)$, where $D$ is the value of the divergence factor for the point and $F$ gives the relative strength of the reflected and direct rays attributable to the antenna beam pattern. At points of minima (the nulls) the field is $(1-F D)$ times the free-space field.

In general the magnitude of the reflected wave is reduced both by the increased divergence resulting from reflection from the convex surface of the earth but also because the electrical properties of the earth are such that only part of the incident energy is reflected. The magnitude of the reflection coefficient is then $\rho D$ instead of the $D$ used in the preceding paragraph, where $\rho$ is the magnitude of the reflection coefficient for plane waves impinging on a plane surface. The field strength, then, lies between $(1+\rho F D)$ and $(1-\rho F D)$. As a result of the smaller value of $\rho F D$ for vertical polarization the maxima of the interference pattern are reduced and the nulls strengthened.

At low heights (see Figure 3 of Chapter 5) the effect of diffraction is important, so that when reference is made to the optical interference region, it should be understood that the portion of the optical region near the earth is not included. It must be considered instead as part of the diffraction region.

The diffraction region, accordingly, designates a layer in the optical region as well as the region below the line of sight (see Figure 3 in Chapter 5). Below the line of sight the fietd falls off exponentially. Within the diffraction region, fields are strengthened by raising the receiver or transmitter antennas.

\subsubsection{Typical Radio Gain Curves}

Three types of graphical representation of radio gain in a vertical plane through the transmitter antenna are possible, namety, (1) at a specified distance, radio gain against height; (2) at a specificd height, radio gain against distance; and (3) a set of contour lines representing constant radio gain.

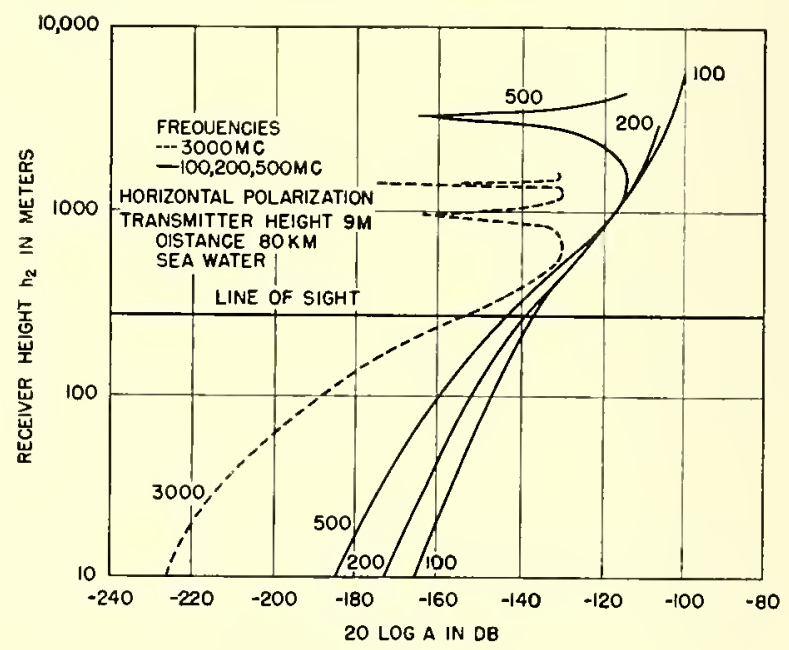

Figure 7. Radio gain vs receiver height for horizontal polarization.

In Figure 7 , curves of type (1) are exhibited for various frequencies. The transmission is over sea water with horizontally polarized waves. It may be observed that the higher the frequency the lower the first maximum and the narrower the lobe. Figure 8 gives similar information for rertically polarized

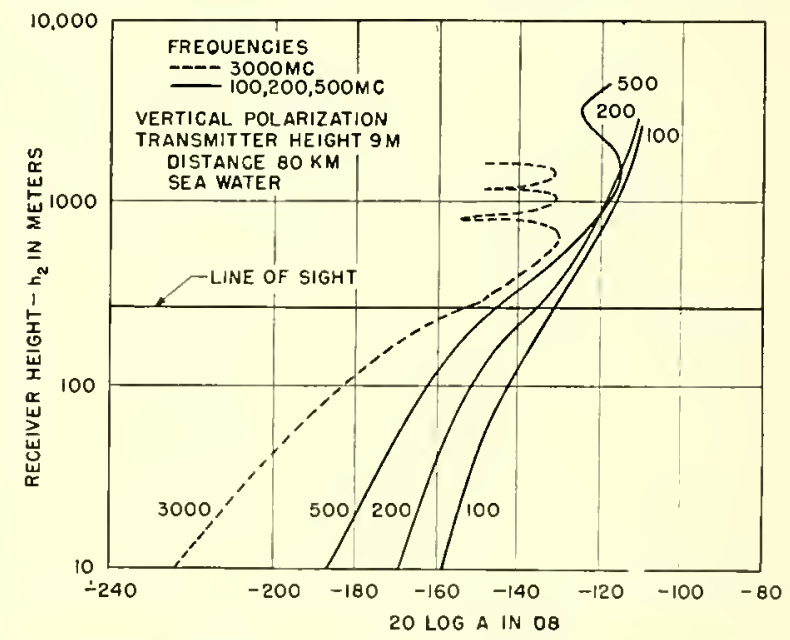

Figure 8. Raclio gain vs receiver height for vertical polarization.

waves. Note that the minima are not so deep with vertical polarization. Curves of type (2) exhibit similar characteristics (see Figure 6 of Chapter 5).

In Figures 9 to $12^{a}$, rertical coverage diagrams of

a Figures 7 to 12 have been adapted from Radiation Laboratory Report C-6. 
type (3) are given. These illustrate the effects of frequency, polarization, and transmitter height.

A comparison of Figures 9 and 10 shows the effect of frequency. As the frequency increases, the lobes become more numerous, narrower, and lower. Another effect is exhibited along the surface. For the higher frequency the corresponding decibel lines come in closer to the transmitter. This illustrates the fact that for the higher frequency the shadow effect is more pronounced along the surface of the earth.

A comparison of Figures 10 and 11 shows that for horizontal polarization the nulls are deeper but the lobes extend out farther. Along the line of sight, rertical polarization gives the higher field strength, while well within the diffraction region the field strength is about the same. The last observation liolds for all frequencies greater than $300 \mathrm{mc}$, the greater the frequency the less difference in the diffraction region between the two polarizations.

A comparison of Figures 9 and 12 shows the effect of the height of the transmitting antenna. As the antenna height is increased, the lobes are narrower and depressed toward the horizon. The range is improved. However, there are broad nulls for the higher antenua in which detection will fail. Below the horizon, the corresponding decibel contours are pushed to the right so that point-to-point communication is improved. It should be observed that the effect of height upon the lobe structure is similar to that of frequency.

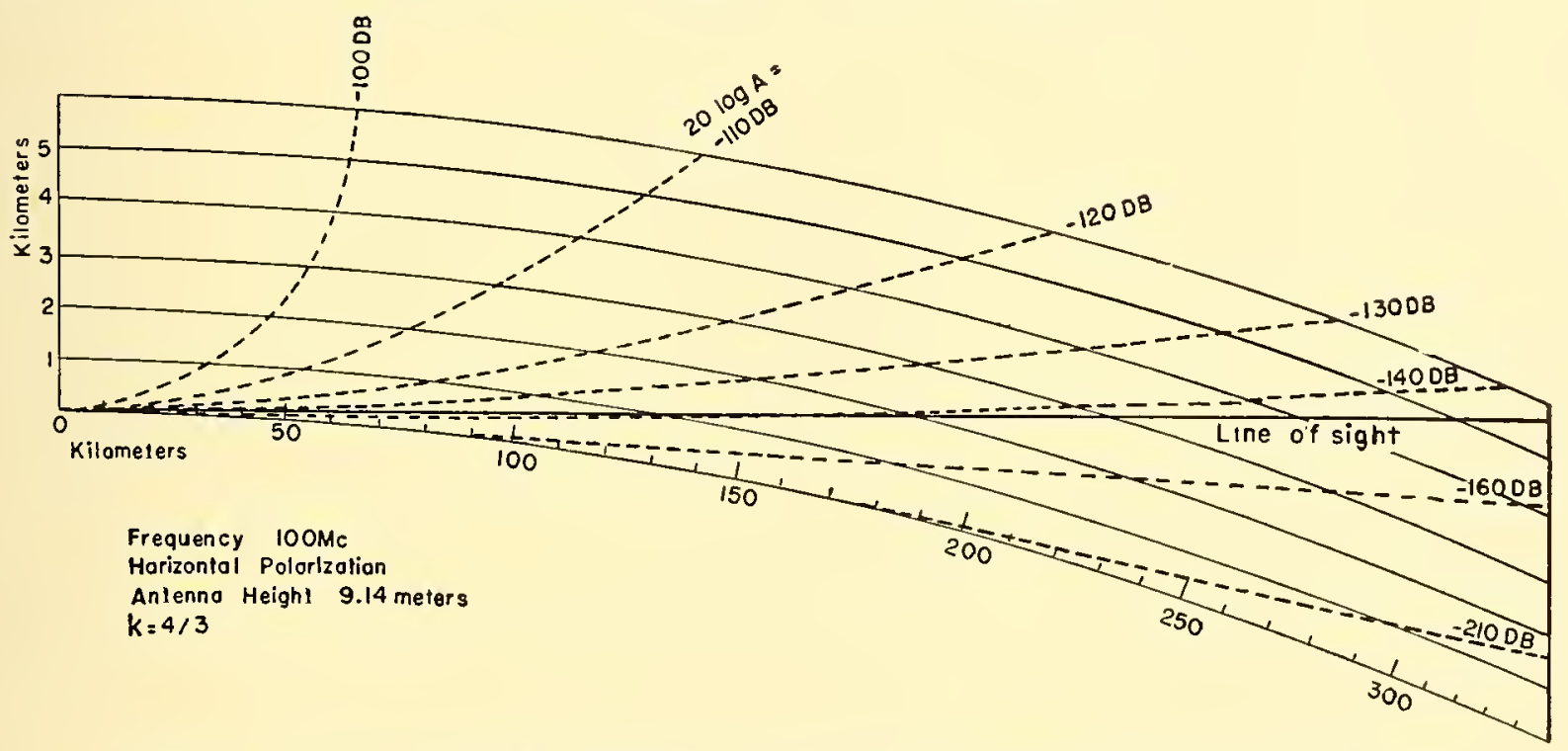

Figure 9. Contours of constant radio gain factor for horizontal polarization on 100 me over sea water.

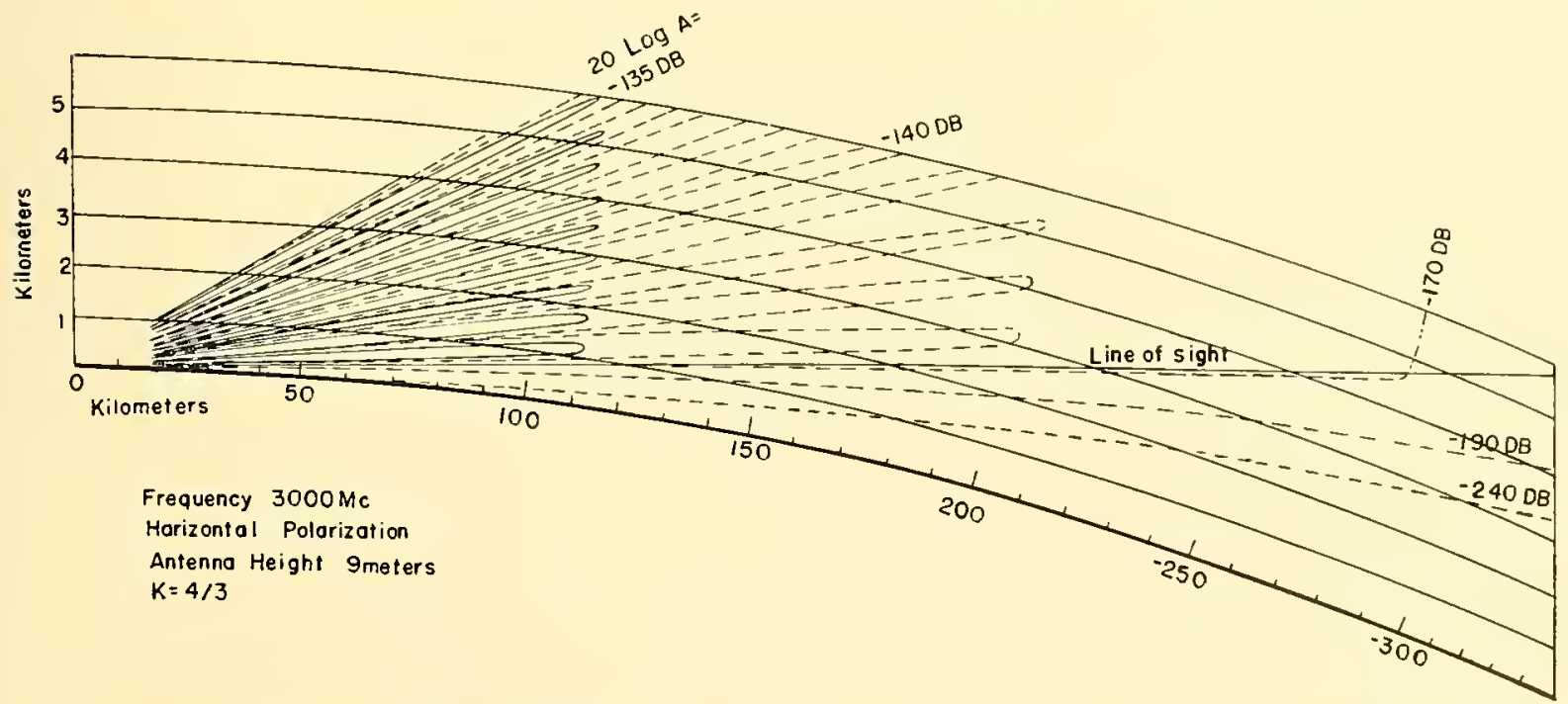

FIgURE 10. Contours of constant radio gain factor for horizontal polarization on 3000 me over sea water. 
Which contour actually represents the limit of detection for a given radar depends on the power output of the transmitter, the minimum power detectable by the receiver, the antenna gains, and the radar cross section of the target. For eommunication sets, the same quantities, except the target cross section, apply.

\subsection{ORGANIZATION OF THIS VOLUME}

\subsubsection{Arrangement of Material}

This rolume is composed of two classes of material. One class, comprising Chapters 2, 5, and 6, is devoted primarily to the major problem of calculating the field strength, while the other class discusses collateral problems of importance if these caleulations are to be utilized for obtaining the most effective use of radar and communication sets.

Chapter 2 is devoted to a presentation of basic relationships such as the definition of radio gain, the transfer of power between doublet antennas in free space, antenna gain, receiver sensitivity and noise, and the definitions of radar gain and cross section.

The problem of computing the field strength or radio gain at any point in the atmosphere is given at length in Chapter 5, and Chapter 6 extends this material to the calculation of coverage diagrams. In Chapter 7 these calculations are related to the

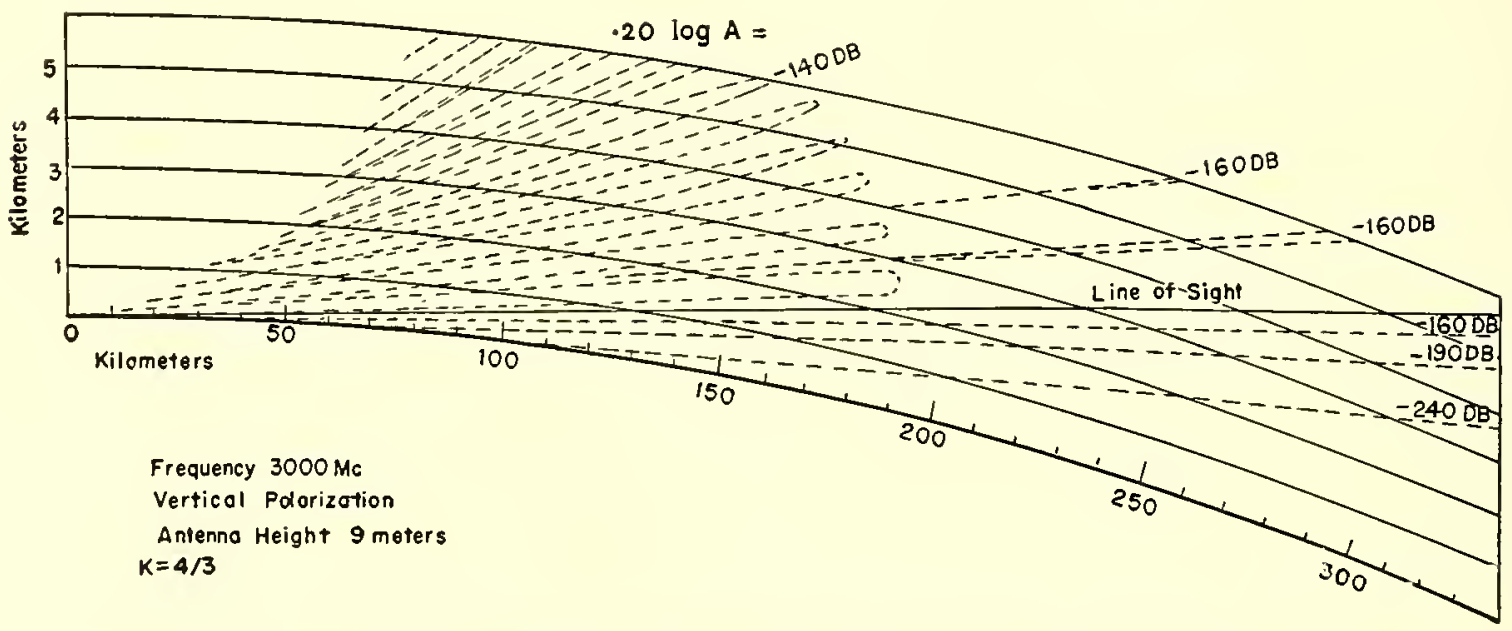

FigURE 11. Contours of constant radio gain factor for vertical polarization on 3000 me over sea water.

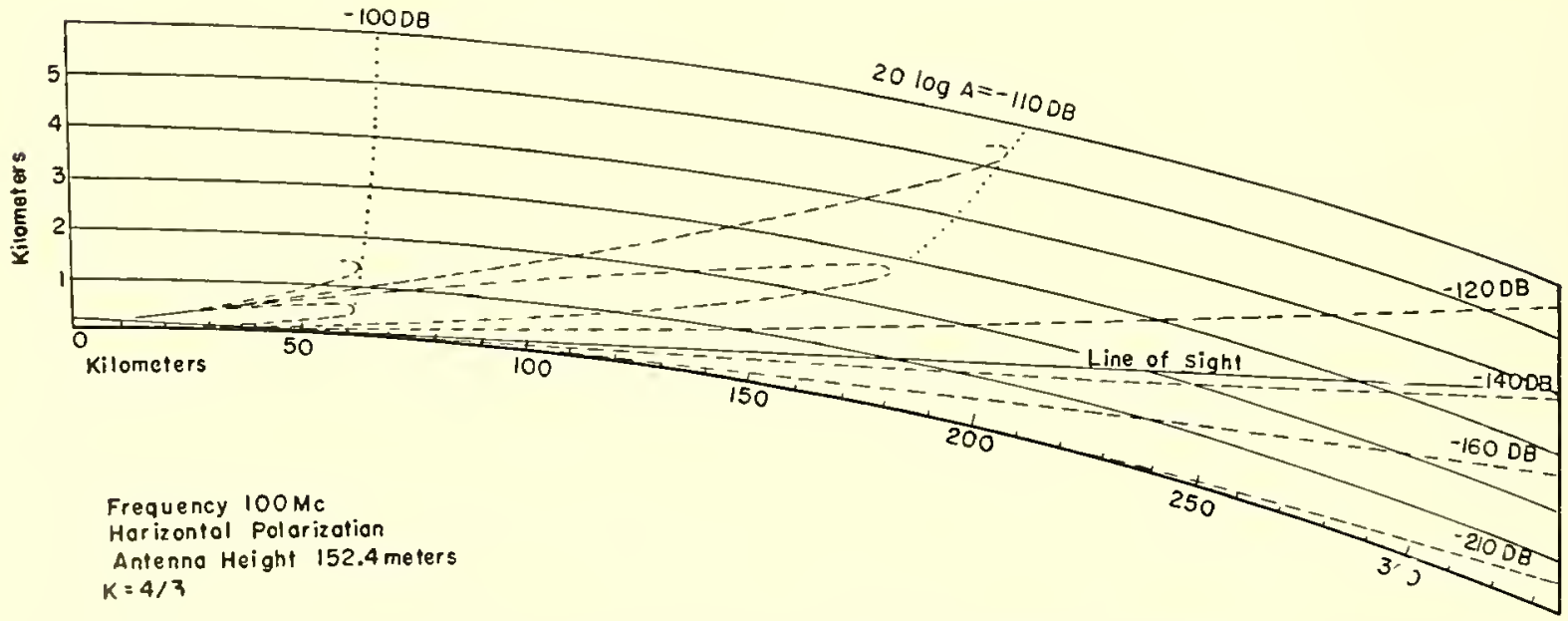

Frgure 12. Contours of constant radio gain factor for horizontal polarization on 100 me over sea water. 
performance characteristics of particular radar and communication sets.

In Chapter 3 will be found a discussion of the radiating properties of a wide variety of antenuas. Particular attention is devoted to a consideration of the shape of the radiation patterns, methods for improving directivity of antennas, and computation of the gains.

A general discussion of the factors which modify the manner in which radio waves are transmitted through the atmosphere is given in Chapter 4. Here also is given the reflecting properties of sea water and various types of soil.

An important practical problem is that of diffraction of waves around obstacles such as hills and trees. A simplified treatment of this problem is given in Chapter 8.

Chapter 9 is devoted to a presentation of the reflecting problems of targets and their bearing on the operation of radar sets. Successful operation of these sets is dependent, in no small measure, on the proper choice of siting. Factors bearing on the siting problem are evaluated in Chapter 10.

\subsection{UNITS AND FREQUENCY RANGES}

\subsection{1 \\ Units}

In this book the units used are those of the mks rationalized system, in which distances are expressed in meters, masses in kilograms, and time in seconds; and the formulas have been rationalized so that the factor $4 \pi$ appears in equations involving point sources, $2 \pi$ in equations involving line sources, and is generally absent from equations for uniform or unidirectional fields.

The Coulomb formula, for illustration, for point sources in classical electrostatic units,

$$
f=\frac{q_{1} q_{2}}{\epsilon r^{2}}
$$

with $f$ in dynes, $q_{1}$ and $q_{2}$ in statcoulombs, $r$ in centimeters and $\epsilon$ referred to unity in free space, is transformed to

$$
f=\frac{q_{1} q_{2}}{4 \pi \epsilon_{0} \epsilon_{r} r^{2}}
$$

Here force $f$ is given in newtons ( 1 newton $=10^{5}$ dynes), $q_{1}$ and $q_{2}$ in coulombs, $r$ in meters, $\epsilon_{r}$ is the dielectric constant relative to that of free space $\epsilon_{0}$.
Similarly, the Coulomb formula for magnetic poles,

$$
f=\frac{m_{1} m_{2}}{\mu r^{2}}
$$

with $m_{1}$ and $m_{2}$ in unit poles in the electromagnetic system and $\mu$ equal to the permeability, transforms to

$$
f=\frac{m_{\mathrm{a}} m_{2}}{4 \pi \mu_{0} \mu_{r} r^{2}},
$$

where $m_{1}$ and $m_{2}$ are now given in webers, $\mu_{r}$ is the permeability relative to that of free space $\mu_{0}$.

In the mks rationalized system, the free-space values of $\epsilon_{0}$ and $\mu_{0}$ must carry the burden of the change of units and the inclusion of $4 \pi$, and thus take on the values

$\epsilon_{0}=8.854 \cdot 10^{-12} \cong \frac{1}{36 \pi} 10^{-9}$ farads per meter,

$\mu_{0}=4 \pi \cdot 10^{-7} \cong 1.257 \cdot 10^{-6}$ henries per meter.

With these values, $c$, the velocity of light in free space, is equal to

$c=\frac{1}{\sqrt{\epsilon_{0} \mu_{0}}}=2.998 \cdot 10^{8} \cong 3 \cdot 10^{8}$ meters per second

and the impedance of free space is

$$
\sqrt{\frac{\mu_{0}}{\epsilon_{0}}}=376.7 \text { ohms. }
$$

This system of units has been chosen because it is unified, free from numerical factors required in equations using arbitrary choices of units, and has been adopted by the International Electrotechnical Commission. Since the various Armed Services use differing sets of units for their operational instructions, it would have been impossible to choose any one that would have becn satisfactory to all; hence the choice of using the only system which is generally recognized and scientifically sound.

\begin{tabular}{|c|c|c|c|}
\hline Symbol & $\begin{array}{c}\text { Frequency } \\
\text { name }\end{array}$ & $\begin{array}{l}\text { Frequency } \\
\text { me }\end{array}$ & $\begin{array}{c}\text { Wavelength } \\
\text { meters }\end{array}$ \\
\hline LF & Low & $0.03-0.3$ & $10,000-1,000$ \\
\hline $\mathrm{MIF}$ & Medium & $0.3-3$ & $1,000-100$ \\
\hline HF & High & $3-30$ & $100-10$ short waves \\
\hline VHF & Very high & $30-300$ & 10-1 ultra-short \\
\hline UHF & Ultra-high & $300-3,000$ & waves \\
\hline SHF & Super-high & $>3,000$ & $<.1$ microwaves \\
\hline
\end{tabular}

\subsubsection{Symbols for Frequency Ranges}

The following symbolism has been adopted for various ranges of frequency.

TabLe 2. Symbols for frequency ranges. 


\section{Chapter 2}

\section{FUNDAMENTAL RELATIONS}

\section{THE ELECTRIC DOUBLET \\ IN FREE SPACE}

\subsubsection{Radiation of an Electric Doublet}

$I^{T}$ is covrexiext to present the basic relationships of radiation and reeeption by antennas in their simplest form, that of the radiation and reception of electrie doublets in free space. The resulting formulas will later be generalized to include other types of antennas and their positions relative to the earth.

An electrie doublet is a rectilinear antenna, which is symmetrical about the point or points of conneetion thereto and is so short that its directive properties are independent of its length. The field of such an antenna does not depend on the distribution of current along the wire, beease the wire is so short

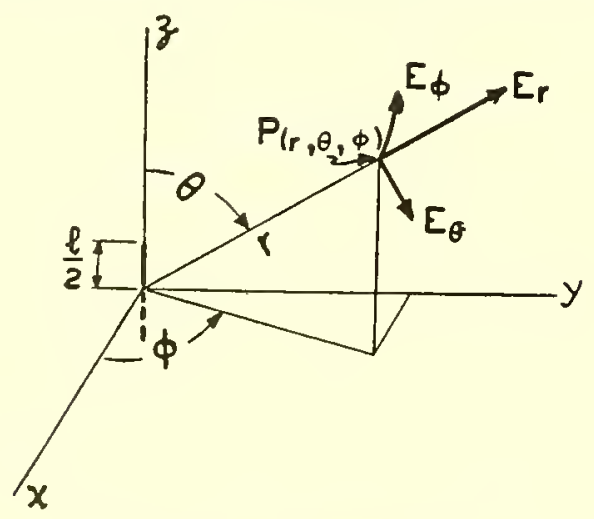

Figure 1. Polar condinate system.

that there is no phase difference between waves reaching a point in space from different portions of the wire. In symbols, $l<<\lambda$ where $l$ is the length of the antenna and $\lambda$ is the warelength of the radiation.

To facilitate the analysis of the field of the doublet. antenna, the spherical polar coordinate system shown in Figure 1 is introduced. The upper half of the doublet is shown in the figure. The distance from the center of the antenna to a point in space is here renoted by $r$. Elsewhere in this rohme this cuantity is written $d$.
Let $d l$ be an infinitesimal portion of $l$, the length of the doublet, and let the current in this portion be the real part of $I e^{j(2 \pi c t / \lambda)}$. Let $d E_{r}, d E_{\theta}, d E_{\phi}$ and $d H_{r}$, $d H_{\theta}, d H_{\phi}$ be the components of the electric and magnetic field strengths at any point $P(r, \theta, \phi)$ due to the current in $d l$. A straightforward solution of the fundamental equations of electromagnetic theory gives the following ralues for these components, ralid at distances large compared with the length of the doublet:

$$
\begin{aligned}
& d E_{r}=60 I\left[\frac{1}{r^{2}}-\frac{j \lambda}{2 \pi r^{3}}\right] d l \cos \theta e^{j(2 \pi \lambda)(c t-r)} \\
& d E_{\theta}=60 \pi I\left[\frac{j}{\lambda r}+\frac{1}{2 \pi r^{2}}-\frac{j \lambda}{-1 \pi^{2} r^{3}}\right] d l \sin \theta e^{j(2 \pi \lambda)(c t-r)} \\
& \text { rolts per meter, }
\end{aligned}
$$

$d E_{\phi}=0, d I I_{r}=0, d H_{\theta}=0$, $d H_{\phi}=\frac{I}{2}\left[\frac{j}{\lambda r}+\frac{1}{2 \pi r^{2}}\right] d l \sin \theta e^{j(2 \pi \lambda)(c t-r)}$ amperes per meter, where

$$
\begin{aligned}
& c=\text { velocity of light }=3 \times 10^{8} \text { meters per second } \\
& j=\sqrt{-1}
\end{aligned}
$$

and atl distances are measured in meters. Electric field strengths are in volts per meter and magnetic field strengths are in amperes per meter. Unless otherwise explicitly stated, the mks rationalized units are used throughout this rolume.

Equation (1) ean be simplified at once. Since the time variation of the field is assumed sinusoidal, $e^{j(2 \pi c t / \lambda)}$ may be omitted. The term $e^{-j(2 \pi r / \lambda)}$ gives the phase, and it too can be omitted when only the amplitude is required. From here on, unless otherwise stated, it is understood that root-mean-square (rms) values will be used for $d E_{r}, d E_{\theta}, d H_{\phi}$, and $I$.

The field in the neighborhood of the doublet is called the induction field and is given by the terms in equation (1) which include the highest powers of $r$ in the denominators. This field is important when mutual effects between elosely spaced antemnas, or antemnas and reflectors or directors, are involved.

The radiation field, of greater interest for most of the purposes of this rolume and the only important field at large distances $(r>>\lambda)$, is given by the 
terms in equation (1) containing $r^{-1}$. Thus the radiation field for the element $d l$ of the doublet may be written:

$$
\begin{aligned}
& d E_{\theta}=\frac{60 \pi I d l \sin \theta}{\lambda r} \text { rolts per meter, } \\
& d H_{\phi}=\frac{I d l \sin \theta}{2 \lambda r}=\frac{d E_{\theta}}{120 \pi} \text { amperes per meter. }
\end{aligned}
$$

The other components are relatively negligible except near the antenna or near ground for low antennas. The electrie field $d E_{\theta}$ is perpendicular to the radius vector $r$ and lies in the $r, z$ plane, and the magnetic field $d H_{\phi}$ is perpendicular to $r$ and to $d E_{\theta}$. It will be noted that $E / H=120 \pi \cong 376.7$ ohms. This is the impedance of free space in the mks rationalized system of units, the ohms of the electrical engineer.

Equation (2) describes the radiation field of a differential element of the doublet. To get the radiation field of the whole doublet, these equations must be integrated over the length $l$. This gives

$$
\begin{aligned}
& E_{\theta}=\frac{60 \pi \sin \theta \int_{l / 2}^{l / 2} I d l}{\lambda r} \text { volts per meter, } \\
& H_{\phi}=E_{\theta} / 120 \pi \text { amperes per meter. }
\end{aligned}
$$

Equation (3) may be written in exactly the form of equation (2) by introducing the effective length, $L$, of an antenna, which is defined as the length that a straight wire earrying current constant over its length would have if it produced the same field as the antenna in question. Calling the current measured at the input point $I_{i}$,

$$
L=\frac{\int_{-l / 2}^{l / 2} I d l}{I_{i}} \text { meters, }
$$

and hence

$$
\begin{aligned}
& E_{\theta}=\frac{60 \pi I_{i} L \sin \theta}{\lambda r} \text { volts per meter, } \\
& H_{\phi}=\frac{E_{\theta}}{120 \pi} \text { amperes per meter, }
\end{aligned}
$$

so that equations (5) are the same as equations (2) with $I_{i} L$ replacing $\int I d l$. For a short dipole or doublet the current varies linearly from $I_{i}$ at the midpoint to zero at each end so that from equation (4) $L=l / 2$ for a doublet.

The power per unit area, IV (that is, the power flowing through a unit area normal to the direction of propagation), is represented by Poynting's vector and is given by the product $E_{\theta} H_{\phi}$ times the sine of the angle between $E_{\theta}$ and $H_{\phi}$. This angle is 90 degrees. Consequently,

$$
\begin{aligned}
& W=E H \quad \text { watts per square meter, } \\
& W=\frac{E^{2}}{120 \pi} \text { watts per square meter, } \\
& E=\sqrt{120 \pi} \bar{W} \quad \text { volts per meter. }
\end{aligned}
$$

T'o find $P$, the power output of the doublet, $W$ is integrated over a large sphere concentric with the source. Using equations (5),

$$
P^{\prime}=\frac{E^{2} d^{2}}{45} \quad \text { watts }
$$

ind

$$
E=\frac{3 \sqrt{5} \sqrt{P}}{d}
$$

where $d$ is written in place of $r$. The subscripts $\theta$ and $\phi$ have been dropped at this point because the $E$ and $I I$ referred to in equations (7) are the fields in the equatorial plane, where $\sin \theta=1$.

As the antenna is part of a circuit, it is often convenient to think of the radiated power as being dissipated in a fictitious resistance called the radiation resistance, defined by

$$
R_{r}=\frac{P}{I_{i}^{2}} \text { ohms, }
$$

where $P$ is the radiated power and $I_{i}$ the rms input current. For the doublet,

$$
R_{r}=80 \pi^{2}\left(\frac{L}{\lambda}\right)^{2} \text { ohms, }
$$

where $L$ is the effective length given by equation (4).

\subsubsection{Reception by an Eleetrie Doublet}

When an electromagnetic wave falls upon an antenna, a current is induced in the antemma and power is abstracted from the wave. If the antenna is comnected to a load, the power abstracted is dissipated in two ways: (1) by absorption in the load (reception), and (2) by reradiation from the antenna (scattering).

In this classification, the power dissipated by the antenna itself (due to its ohmic resistance) is ignored because this loss is likely to be negligible compared with the power dissipated through reradiation. Hereafter, power absorbed by the load will be called received power and power reradiated by the antenna 
will be called scattered power. The sum of these is equal to the power abstracted from the wave.

The calculation of the received and scattered power may be carried out by means of the equivalent circuit of Figure 2. In this figure, $Z_{a}$ is the impedance

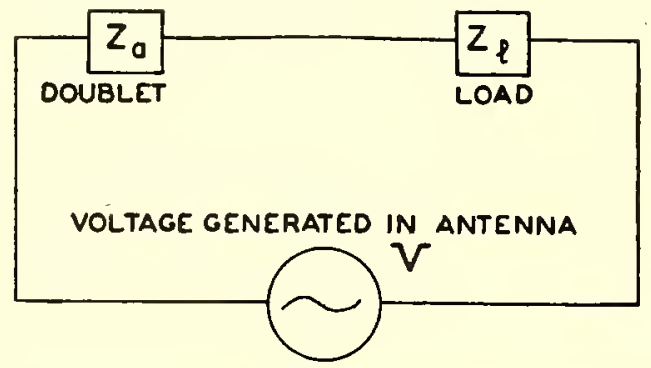

Figure 2. Equivalent cireuit of antenna and load.

of the doublet and $Z_{l}$ is the impedance of the load, that is, the impedance connected across the terminals of the antemna when it is acting as a receiver. $V$ is the voltage generated in the antenna.

The load is supposed to be tuned, which means that the reactance part of $Z_{l}$ is set equal and opposite to the reactance part of $Z_{a}$, so that $Z_{a}+Z_{l}=R_{a}$ $+R_{l}$, that is, the total impedance is simply the sum of the resistance parts of the impedances of the antenna and the load. Hence

$$
I=\frac{V}{R_{a}+R_{l}}
$$

gives the current. But $P,=R_{l} I^{2}$ is the power absorbed by the load and hence is equal to

$$
P_{r}=\frac{V^{2} R_{l}}{\left(R_{a}+R_{l}\right)^{2}}
$$

where $P_{r}$ is called the received power. In the same way

$$
P_{s}=\frac{V^{2} R_{a}}{\left(R_{a}+R_{l}\right)^{2}}
$$

is the power scattered by the doublet.

It is easy to show that the maximum power is delivered to the load if $R_{a}=R_{l}$. In this, the matched load case,

$$
P_{r}=P_{s}=\frac{V^{2}}{4 R_{a}}=\frac{T^{r_{2}}}{4 R_{l}}
$$

Now the resistance of the doublet, neglecting its low ohmic resistance, is only the radiation resistance [equation (9)] and the potential or voltage across the terminals is equal to $E_{0} L$, where $E_{0}$ is the field strength of the incident plane wave and $L$ is the effective length of the doublet. Inserting these quantities into equation (13),

$$
P_{r}=P_{s}=\frac{E_{0}{ }^{2}}{120 \pi} \cdot \frac{3 \lambda^{2}}{8 \pi} .
$$

In these equations it has been assumed that the line of the doublet has been oriented parallel to the electric vector of the incident wave in order to obtain maximum power absorption.

The factor $E_{0}^{2} / 120 \pi$ will be recognized from equation (6) as the power per unit area of the incident wave. The formula thus says that all the power crossing an area $3 \lambda^{2} / 8 \pi$ is received, and that all the power crossing an equal area is scattered. The area $3 \lambda^{2} / 8 \pi$ is therefore called the absorption cross section or scattering cross section of the matched doublet. Since the antenna has been placed parallel to the polarization of the incident wave, this is the maximum absorption cross section. Moreover, this formula holds only when the doublet las been matched to its loarl, and consequently $3 \lambda^{2} / 8 \pi$ is the maximum absorption cross section.

It will be noted, however, that $3 \lambda^{2} / 8 \pi$ is not the maximum scattering cross section. This maximum is achieved by shorting out the load, that is, setting $R_{l}=0$. In this case,

$$
P_{r}=0
$$

and

$$
P_{s}=\frac{E_{0}^{2}}{120 \pi} \cdot \frac{3 \lambda^{2}}{2 \pi} .
$$

Hence the scattering cross section of the shorted (dummy) doublet is four times the scattering cross section of the matched load doublet.

It should be noted in passing that the cross sections introduced here should not be confused with the radar cross section which is discussed in Section 2.4.

\subsubsection{Transmission between Doublets in Free Space}

Assume that two doublets, one to function as a transmitter and the other as a receiver, a distance $d>\lambda$ apart, are adjusted for maximum power transfer. This means that the axes of the doublets are parallel and he in their common equatorial plane and that each is matched to its comnected circuit. Then the power radiated by the transmitting doublet, from equation ( $\bar{\zeta})$, is equal to

$$
P_{1}=\frac{E_{0}^{2} d^{2}}{45} \text { watts, }
$$


The general relation between the input voltage at the receiver and the received power is $\tau_{t}=\sqrt{P_{2} R_{l}}$, where $R_{l}$ is the resistance of the receiver load circuit (which is equal to the radiation resistance for maximum power transfer) and $V_{t}$ is the input voltage. Hence, using equation (14),

$$
\begin{aligned}
V_{t} & =0.0178 E_{0} \lambda \sqrt{R_{l}} \text { volts } \\
& =\frac{1}{8 \pi \sqrt{5}} E_{0} \lambda \sqrt{R_{l}}=\frac{E_{0} L}{2}
\end{aligned}
$$

\subsubsection{Antenna Gain. Polarization}

The equations of Section 2.1 may be further generalized to apply to any type of antenna through the introduction of a quantity called the antenna gain. The term gain, as applied to an antenna, is a measure of the efficiency of the antenna as a radiator or receiver as compared with that of a doublet antenna, with all antennas located in free space.

Quantitatively, the gain, $G_{1}$, of a directive transmitting antenna is the ratio of the power $P_{1}{ }^{\prime}$ radiated ly a doublet antenna to the power $P_{1}$ radiated by the antenna in question to give the same response in a distant receiver, with both transmitting antennas adjusted for maximum transfer of power. Hence

$$
r_{1}=\frac{P_{1}^{\prime}}{P_{1}} .
$$

The gain ( $r_{2}$ of a directive receiving antenna is the ratio of the power $P_{\mathrm{I}}^{\prime \prime}$ radiated by a transmitting antenna, which produces a certain response in the matched load circuit of a distant doublet receiving antenna, to the power $P_{1}$ radiated by the same transmitting antenna to produce the same response in the matched load circuit of the receiving antenna in question, with both receiving antennas adjusted for maximum transfer of power. Hence

$$
C_{2}=\frac{P_{1}^{\prime \prime}}{P_{1}} \text {. }
$$

From the definitions given above it follows that for a transmitting and receiving antenna combination in free space, with gains $r_{1}$ and $r_{2}$ and adjusted for maxinum power transfer, the power ratio is equal to

$$
\frac{P_{2}}{P_{1}}=G_{1} G_{2}\left(\frac{3 \lambda}{8 \pi d}\right)^{2}=G_{1} G_{2} A_{0}^{2}
$$

where $P_{1}, C_{1}$ are the porrel output and gain of the transmitter and $P_{2}$ is the power delivered to the matched loud of a receiving antenna of gain $G_{2}$.
If the antennas are not in free space, equation (26) becomes

$$
\begin{aligned}
\frac{P_{2}}{P_{1}}=G_{1_{1}} C_{2}\left(\frac{3 \lambda}{8 \pi d}\right)^{2} A_{p}{ }^{2} & =G_{1} C_{2}\left(A_{0} A_{p}\right)^{2} \\
& =G_{1} C_{2} A^{2}
\end{aligned}
$$

where $A$ is the gain factor and $A_{p}$ is the path-gain factor. Note that for highly directive antennas $A_{p}$ may depend upon the directivity characteristic of the antennas, e.g. when the antenna discriminates between the direct and reflected waves.

Since power is proportional to the square of field strength, equation (20), for any transmitting antenna, becomes

$$
E=E_{0} \sqrt{\bar{r}_{1}} A_{p}
$$

In defining gain, the electric doublet is selected here as the comparison antemna in place of the isotropic radiator (that is, a hypothetical antenna which radiates equally in all directions) which is sometimes used in the literature. Since the gain of an isotropic radiator relative to a doublet is $2 / 3$, the gain of any antenna referred to an isotropic radiator is $3 / 2$ the value referred to a doublet antenna.

$$
C^{\prime}(\text { isotropic })=1.5 G \text { (doublet) } \text {. }
$$

The chiel objections to the isotropic radiator are that it does not occur in practice and cannot be prorluced experimentally, even approximately.

In experimentally measuring the gain of an antenna, a half-wave dipole is often used as a reference antenna. While the gain of a half-wave dipole relative to a doublet is approximately unity, being 1.09 for a very thin dipole, it depends somewhat on its actual dimensions so that it is better to express the experimental gain in terms of the doublet antenna even though a longer antenna is used as a reference antenna in making the measurements.

When antemnas are oriented so that the directions of polarization make an angle $\gamma$ with each other

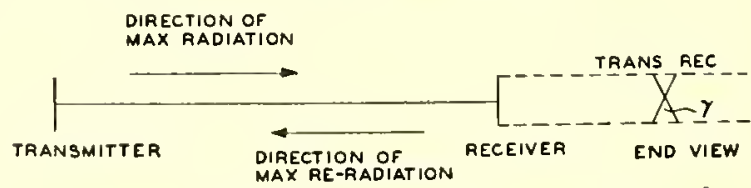

Figrne 4. Relation of antenna axes and wave polarization.

(while the maxima of their angular patterns still point toward each other), the formulas for power transfer, equations (18), (22), and (2 $\bar{\gamma})$, are multiplied by a factor $\cos ^{2} \gamma$ (see Figure 4 ). 


\section{2 .3}

\section{The Reciprocity Principle}

So far in this chapter the radiation and reception of power by antennas have been treated separately. Actually, nrany of the properties of an antenna are the same for either reception or radiation; in particular, the current distribution, the effective length, and the gain are unchanged. The reciprocity principle, from which these propositions may be proved, may be stated as follows: If an electromotive force $V$, inserter in antenna 1 at a point $x_{1}$, causes a current $I$ to flow at a point $x_{2}$ in antenna 2 , then the voltage $V$ applicd at $x_{2}$ ill produce the same current $I$ at $x_{1}$.

From this principle the statement of the equivalence of current distribution, effective length, and gain follow readily.

This theorem does not hold when the propagation of a wave takes place in an ionized medium in the presence of a magnetic field (the ionosphere), but it does hold for all cases of transmission discussed in this volume.

\section{RECEIVER SENSITIVITY}

The sensitivity of a radio receiver is that characteristic which determines the minimum strength of signal input capable of causing a desired value of signal output. In high-fiequency receivers the limiting factor for reception is usually set noise, that is, noise prorluced in the tubes or other clements, such as crystals, of the receiver itself. At frequencies below about $100 \mathrm{mc}$, atmospheric disturbances sometimes exceed the set noise in intensity, but at higher frequencies atmospheric static is negligible. Man-made noise (automobiles, ete.) may be a source of serious trouble, but such interference can often be eliminated by proper siting. Consecuently, lor high-frequency receivers, sensitivity may be expressed, at least approximately, in terms of set noise only.

Although set noise has an important bearing on sensitivity of radar receivers, there are other lactors which must be considered for this type of equipment.

There are several types of set noise. Though all noise sources in a well-designed receiver are mininized with the exception of the thermal noise whose magnitude is independent of equipment construction, the total set noise is usually several times the purely thermal noise.

\section{Thermal Noise}

Thermal noise is generated by the random (temperature) notion of electrons in a conductor; it is, therefore, a universal property of matter and independent of the design features of the receiver. The rms thermal-noise voltage that appears across the terminals of any circuit element is a function of the frequency interval (receiver bandwidth) over which the noise is averaged; it is given by

$$
r_{n}=\sqrt{4 k T \Delta f \cdot R}
$$

where $R$ is the resistance across which the noise voltage is measured. If the banckwidth in cycles per second, $T$ the absohite temperature, and $k$, the Boltzmann constant, is equal to $1.38 \times 10^{-23}$ watt-second per degree. The noise roltage is independent of the reactance components in the circuit.

Consider now, for the purpose of definition, a receiver without internal noise, that is, let all the noise be generated in the receiring antenna of resistance $R_{a}$. If $R_{l}$ designates the load resistanee (that is, the resistance of the receiver exchusive of its antenna), the average noise power delivered to the receiver will be

$$
P_{n}=\frac{\Gamma_{n}^{2} R_{l}}{\left(R_{a}+R_{l}\right)^{2}}
$$

where $V_{n}$ is the rms value of the noise generated in the antenna.

The noise powel is maximum when the receiver is matched to its input; this maximum is

$$
P_{n}=\frac{T_{n}^{2}}{4 R_{a}}=k T \Delta f \text { watts }
$$

by equation (30). Assuming equivalent temperature $T^{\prime}=290$ degrees absolute, and measuring $\Delta f$ in megacycles,

$$
P_{n}=4 \times 10^{-15} \Delta f \text { watt. }
$$

This result means that in an idealized receiver, noise is the thermal noise of an antenna of equivalent temperature $T=290$ degrees absolute, and the minimum detectable signal would be approximately equal to $4 \times 10^{-15} \Delta f$ watt.

\section{3 .2}

\section{Noise Figure}

The sensitivity of a set cannot be described in terms of the thermal noise alone, because the set noise is usually several times the purely thermal noise. For this purpose another quantity called the 
noise figure is used. The noise figure of a system (taken here to be a receiver, for definiteness) is defined as

$$
F_{n}-\frac{P_{n o} / P_{n i}}{P_{s o} / P_{s i}}
$$

where $P_{n i}=$ noise power $(k T \Delta f)$ from the antemma which is being delivered to the receiver.

$P_{n o}=$ noise power at the output of the receiver, that is, the noise after the amplifications and additions arising in the receiver circuit.

$P_{s i}=$ signal power from the antenna which is being delivered to the receiver.

$P_{s o}=$ signal power at the output of the receiver, that is, the signal power after detection and amplification have taken place.

The ratio $P_{s e} / P_{s i}$ is called the receiver gain. This quantity is called $g$ and must not be confused with antenna gain $G$. Using equation (32), equation (34) may be written

$$
F_{n}=\frac{P_{n o}}{k T \Delta f} \cdot \frac{1}{g} .
$$

The bandwidth $\Delta f$ is measured by finding the area under a curve of power-gain versus frequency and equating this area to the area of a rectangle whose width is interpreted as $\Delta f$ and whose height corresponds to the gain at the frequency at which the gain is a maximum.

\subsection{3}

\section{Receiver Sensitivity}

Frequently receiver sensitivity is defined by the assumption that a received signal can be discriminated when its output power is ecmal to the noise output power. 'This assumption, while true for' a large class of receivers, is too rough for radar receivers. The method given here will explain the procedure used for calculating the minimum discernible power of receivers for which the assumption is true. The sensitivity of radar receivers is considered in Section 2.3.5.

Refering to equation (34), the assumption that signal output power is equal to noise output power means that $P_{\text {so }}=P_{n o}$. Hence

$$
F_{n}=\frac{P_{s i}}{P_{n i}}
$$

But $P_{s i}$ is, on the assumption discussed above, just the minimum discernible signal power, $P_{\min }$, at the receiver input, that is, before amplification. Hence, using equations (32) and (33),

$$
P_{\min }=k T \Delta f \cdot F_{n} \cong 4 \times 10^{-15} \Delta f \cdot F_{n} \text { watt. }
$$

\subsubsection{Measurement of the Noise Figure}

Remembering that the cases under discussion are those for which the minimum discernible signal is equal to the noise output power, equation (37) gives an estimate of the minimum detectable power from a measurement of the noise figure $F_{n}$ which may be obtained as follows.

An antenna (or other signal generator) whose impedance is matched to the receiver is connected to the receiver. With the signal output reduced to zero (so that the antenna furnishes only noise power to the receiver), the receiver gain is increased until the noise gives a measurable output and the output noise power is measured with a power meter. Now a signal is impressed on the antenna and increased to a point where the receiver output power is doubled, and the input signal power is measured. Thus, referring to equation (34),

$$
P_{s o}=P_{n o} \quad \text { and } \quad F_{n}=\frac{P_{s i}}{P_{n i}}=\frac{P_{s i}}{k T \Delta f},
$$

so that the measurement of the impressed signal power indicated here gives $F_{n}$.

If the receiver consists of several elements in cascade, including attenuators, amplifiers, and converters, the overall noise figure can be compounded from the noise figures and gains of the individual componeuts by means of the following equation:

$$
F_{n}=F_{n 1}+\frac{F_{n 2}-1}{g_{1}}+\frac{F_{n 3}-1}{g_{1} g_{2}},
$$

where $F_{n}=$ overall noise figure,

$F_{n k}=$ noise figure of the $k$ th element,

$g_{k}=$ gain of the kth element.

In using this equation it is understood that the successive stages are matched.

It is clear from equation (38) that most of the noise comes from the early stages of reception; in high-frequency radar sets, it comes from the crystal mixer and the first intermediate-frequency (i-f) stage. This means of course that noise picked up at 
later stages is much less amplified by the system than the noise from the early stages.

In equipment specifications, the noise figure is usually expressed in the decibel scale as decibels above thermal noise. Actual noise figures vary from a few decibels above thermal noise in the very highfrequency [VHF] region (receivers built a few years ago often have appreciably higher noise figures) to larger values for microwave recciver's.

\subsubsection{Sensitivity of Radar Receivers}

It is by no means true for radar receivers that $P_{\min }=P_{\text {wo }}$; as a matter of fact, $P_{\min }>>P_{n o}$. That is, the minimum discernible power considerably exceeds the noise level.

The largest single additional loss in radar reception is scanning loss which is relate to the rotation of the antenna (one or several revolutions per minute). As an example, for one particular radar which has a bandwidth $\Delta f=2 \mathrm{mc}$, this loss is from $10 \mathrm{db}$ to $12 \mathrm{db}$.

In case the antenma does not rotate, there is no scanning loss. This fact would seem to be of limited operational importance, since it would usually be necessary to locate the target (a plane, for cxample) by scanning.

Another loss, closely connected with scanning loss, is sweep-speed loss. This loss is due to the fact that practical targets, such as airplanes, reflect rapidly varying amounts of power to the radar receiver, these amounts depending on the precise orientation of the target at the moment when the radar beam swceps over it. Conscquently, sweepspeed loss will depend on the speed of rotation of the antenna, on the distance of the target from the antenna, and, to some extent, on the beamwidth and the nature of the target. The overall figure for this loss on the same radar used to illustrate scanning loss is about $4 \mathrm{db}$ for targets 200 miles from the radar.

In addition to these losses, careful experiments with the radar used as an example above have indicated that there is an operator loss of about $4 \mathrm{db}$ for even experienced operators. This might be thought of as a loss due to the difference between laboratory and field conditions.

Statistical consideration about the extent of noise fluctuation and about the fact that a target need not be seen on every sweep lead to further small losses which total, for the radar under discussion, $2 \mathrm{db}$.

Summarizing for the case of the radar of the above example, the minimum detectable porer is about $34 \mathrm{db}$ above $k T \Delta f$ or about $8 \times 10^{-11.6}$ watt, not $12 \mathrm{db}$ above $k T \Delta f$ or $8 \times 10^{-13.8}$ watt, as would be indicated from the noise level alone. This amounts to $22 \mathrm{db}$ or a factor of 166 ; that is, the actual minimum discernible porer is 166 times that calculated from noise alone. It will be seen in the results of the next section that the maximum range of a radar set varies with the inverse fourth root of the minimum discernible power. Consequently, a calculation of the maximum range of the radar of the cxample, which assumed that the minimum discemible power was equal to the noise power, would give a range too great by a factor of $\sqrt[4]{166}=3.59$. Since this would be a serions error, it shows the importance of a very careful consideration of radar receiver sensitivity in calculations of this type.

\subsection{RADAR CROSS SECTION AND GAIN}

\subsubsection{Radar Cross Section}

The total scattering of a target may be described by the use of a parameter (having the dimensions of an area) called a scattering cross section. This concept has already been presented in the latter part of Section 2.1.2, where both scattering and absorption cross sections of doublets were discussed. The scattering cross section $S$ is defined by

$$
S=\frac{P_{s}}{W_{i}^{r}}
$$

where $P_{s}$ is the total porrer scattered by the target irrespective of its angular distribution and $W_{i}$ is the incident power per unit area.

The scattering cross section $S$, which gives information about the total scattcred energy, is not directly useful in radar work because in such applications one is interested only in that fraction of the total scattered power which is scattered in the direction of the radar; that is, one wants a parameter involving the scattered power per unit area at the receiver instead of the total scattered power. If the target is an isotropic scatterer,

$$
W_{r}=\frac{P_{s}}{4 \pi d^{2}},
$$


where $\mathrm{H}_{r}$ is the seattered power per unit area at the receiver, $d$ the distance from the target to the receiver, and $P_{s}$ the total scattered power. This gives, nsing equation (39),

$$
S=4 \pi i^{22^{2}} \frac{\mathrm{Ir}^{r}}{\mathrm{II}_{i}^{+}}
$$

as a formula for the scattering cross section of an isotropic scatterer which involves scattered power per mit area at the receiver $W_{r}$ instear of total scattered power $P_{s}$.

For targets other than isotropic scatterers, however, this procedure fails since one cannot say that $W_{r}=P_{s} / 4 \pi d^{2}$. Nerertheless, it is useful to define a parameter $\sigma$ which is called the radar cross section, by

$$
\sigma=4 \pi d^{2} \frac{\mathrm{H}^{r} r}{\mathrm{H}_{i}^{r}}
$$

in analogy with equation $(40)$. Here $W_{r}$ is the actual power per unit area at the receiver. From the preceding discussion it is apparent that $\sigma$ may be thought of as the seattering cross section which the target in question would have if it seattered as much energy in all directions as it actually does scatter in the direction of the radar receiver. For a target scattering isotropically, $\sigma=S$, but for any other type of target $\sigma$ does not, in general, equal $s$.

A radar gain formula analogous to the radio gain but applicable to two-way transmission can be developed from equation (41) by replacing $W_{r}$ and $W_{i}$ witb the directly measurable quantities $P_{1}$ (power output) and $P_{2}$ (received power). From equation (6), $W_{i}=E^{2} / 120 \pi$ in which $E$ is the field strength incident on the target. Substituting this value of $E$ into equation $(7)$ gives $\mathrm{I}_{i}^{r}=3 P_{1} / 8 \pi d^{2}$ for a doublet transmitter in free space. Inchuding the gain of any type of transmitting antenna, this takes the form

$$
\mathrm{W}_{i}=\frac{3 P_{\mathrm{I}}\left(i_{1}\right.}{8 \pi l^{2}}
$$

Further, the power received hy a doublet with a matched load, equation (17), may be written

$$
P_{2}=\frac{3 \lambda^{2}}{8 \pi} \mathrm{IJ}_{n}
$$

if $E^{2} / 120 \pi$ is replaced by $\mathrm{H}_{r}^{r}$, where here $E$ is the field at the receiver. If the receiver is not a doublet, equation (43) may be replaced by

$$
P_{2}=\frac{3 \lambda^{2}}{8 \pi} H_{r} r_{2}
$$

where $C_{2}$ is the gain of the recriver. Substituting the values for $\mathrm{H}_{i}$ and $\mathrm{H}_{r}$, given by equations (42) and (43), into equation (41) yields

$$
\frac{P_{2}}{P_{1}}=C_{1} G_{2} \frac{\sigma}{4 \pi d^{2}}\left(\frac{3 \lambda}{8 \pi d}\right)^{2} .
$$

This is the radar gain for two-way transmission in free space. By means of it, $\sigma$ may be measured, or if $\sigma$ and $P_{2} / P_{1}$ are known, it may be used to calculate ranges. Generalizing equation (45) we have

$$
\frac{P_{2}}{P_{1}}=G_{1} G_{2} \frac{\sigma}{4 \pi d^{2}}\left(\frac{3 \lambda}{8 \pi d}\right)^{2} A_{p}{ }^{4}
$$

where $A_{p}$ is the path gain factor (see Section 2.2.1).

It may be observed here that some writers call $\sigma A_{p}{ }^{4}$, not $\sigma$, the radal cross section. These writers call their $\sigma$, for the case $A_{p}=1$ (free space), the freespace radar cross section $\sigma_{0}$. Since, in this volume, the complicated terms appearing in $A_{p}$ are treated separately and not as part of the cross section, this distinction is not made here.

For some simple targets, $\sigma$ may be calculated. The following are a few of the values.

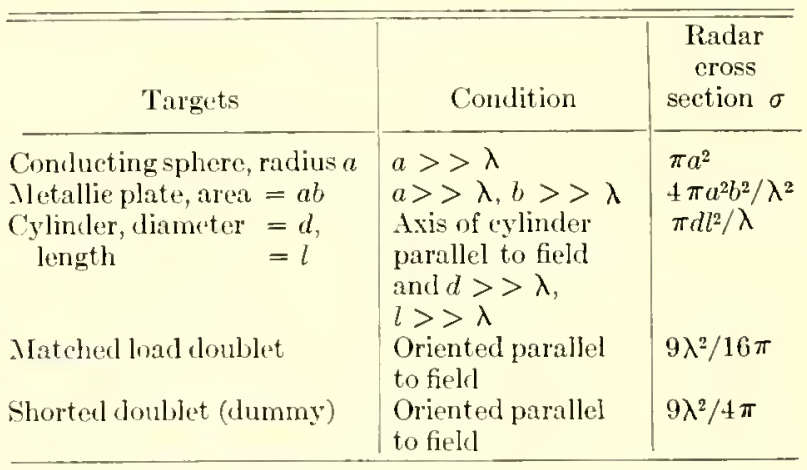

Objects of tactical interest (ships, airplanes) have very complicated radar eross sections. In particular, a strong dependence on the aspect of these unsymmetrical targets is observed. For ships the situation is still further complicated by the rariability of the inciclent field orer the target area.

Some writers on the subject of targets use a characteristic length $L$ (sometimes also called a scattering coefficient) which is related to $\sigma$ by

$$
\sigma=4 \pi L^{2}
$$

\subsubsection{Radar Gain}

It is possible to wite equations for two-way transmission which bear a formal resemblance to corresponding equations for one-way transmission by 
introducing a quantity $G_{R}$, called the gain of the target. $G_{R}$ is the gain of a target in the direction of the radar receiver relative to a shorted (dummy) doublet.

By writing formulas connecting the radar gain with the power per square meter incident on the target and the power per square meter seattered back to the receiver, it is possible to establish a connection between radar gain and the radar cross section defined in the last paragraph, and from this to calculate a gain formula involving $G_{R}$ instead of $\sigma$.

Applying equations (15) and (6),

$$
P_{s}=W_{i} \frac{3 \lambda^{2}}{2 \pi}
$$

for the case where the target is a shorted doublet. $P_{s}$ is the total scattered power and $W_{i}$ is the power per square meter incident on the target. For a target with a radar gain $G_{R}$ it follows that

$$
P_{s}=W_{i} \frac{3 \lambda^{2}}{2 \pi} G_{R} \text {. }
$$

In a similar way a formula for $W_{n}$, the scattered power per unit area at the receiver, can be developed. A target which scattered equally in all directions would scatter an amount

$$
P_{s}^{\prime}=4 \pi d^{2} W
$$

But

$$
P_{s}^{\prime}=\frac{3}{2} G_{R} P_{s}
$$

where $P_{s}$ is the amount seattered by an actual target with gain $G_{R}$. [The factor $3 / 2$ appears because the gain of the target relative to an isotropic radiator is $(3 / 2) G_{R}$.] Hence

$$
4 \pi d^{2} W_{r}=\frac{3}{2} G_{R} P_{s} .
$$

Eliminating $P_{s}$ from equations (49) and (52),

$$
\frac{\mathrm{H}_{r}}{\mathrm{H}_{i}}=\frac{9 \lambda^{2} G_{R}^{2}}{16 \pi^{2} d^{2}}
$$

Putting this value of $\mathrm{W}_{r} / W_{i}$ in equation (41),

$$
\sigma=\frac{9 \lambda^{2}}{4 \pi} G_{R}{ }^{2}
$$

which is the required general formula connecting target gain and radar cross section. It will be noted that the factor $9 \lambda^{2} / 4 \pi$ is just the radar cross section of the shorted doublet.

Inserting the value of $\sigma$ given by equation (54) into equation (45),

$$
\frac{P_{2}}{P_{1}}=4 G_{1} G_{2} C_{R}^{2}\left(\frac{3 \lambda}{8 \pi d}\right)^{4},
$$

which is the radar gain formula for free space in terms of the gain of the target relative to a dummy doublet.

The reasonableness of the factor 4 in the above equation may be marle apparent by the following analogy. Compare the doublet antenna with a generator whose internal resistance corresponds to the radiation resistance of the antenna. When the generator is shorted all the power is dissipated in the internal resistance. When the doublet is shorted all the power is reradiated. The maximum power that can be extracted from either the generator or the antenna occurs when the load resistance equals the internal generator, or antenna radiation, resistance. It is $1 / 4$ the above short-circuit power. This is the 4 that occurs in the above equation.

Equation (55), in the nonfree-space case, takes the form

$$
\frac{P_{2}}{P_{1}}=4 G_{1} G_{2} G_{R}^{2}\left(\frac{3 \lambda}{8 \pi d}\right)^{4} A_{p}^{4},
$$

where $A_{p}$ is the path gain factor defined by equation (20). 


\section{Chapter 3}

\section{ANTENNAS}

\section{Function of Antennas}

A TRANSIITTING ANTExNA converts the power delivered to it into electromagnetic radiation (neglecting losses); a receiving antenna abstracts power from an incident electromagnetic wave and delivers to the receiver that part which is not reradiated or lost in the antenna. In the short and microwave region the power conversion is effected with a very small loss so that for most practical purposes the porer loss inside the antenna may be disregarded. Apparent losses caused by reflection owing to mismatch between the antenna and its input circuit are of a different nature and are not included herein.

For many purposes it is desirable to concentrate the power radiated into a beam of comparatively small angle as in this way the field strength in the preferred direction is enhanced. The gain of a directional antenna is defined by means of a comparison of the given antenna radiation pattern with that of an eleetric doublet.

The gain of an antenna is the ratio of porrer that must be supplied to a doublet to the power that must be supplied to the antenna considered in order that, at a given large distanee, the electric field at the maximum of the antenna pattern is equal to the field at the same distance in the equatorial plane of the doublet. From the reciprocity prineiple it is found that the gain of a receiving antenna is equal to the gain of the same antenna used as a transmitter. A discussion of antenna gain and reciprocity is given in Chapter 2, Section 2.1.

\section{Directive Antennas}

Polar plots of antenna radiation patterns are of two kinds: either the relative magnitude of the Poynting rector (power per unit area) is plotted along the radius vector, or the relative magnitude of the radiation electric field strength is plotted in the same way. Usually the value of the radius vector at the maximum of the pattern is taken equal to unity. The Poynting vector plot is obtained from the field strength plot by squaring the radial distances (Figure 1).

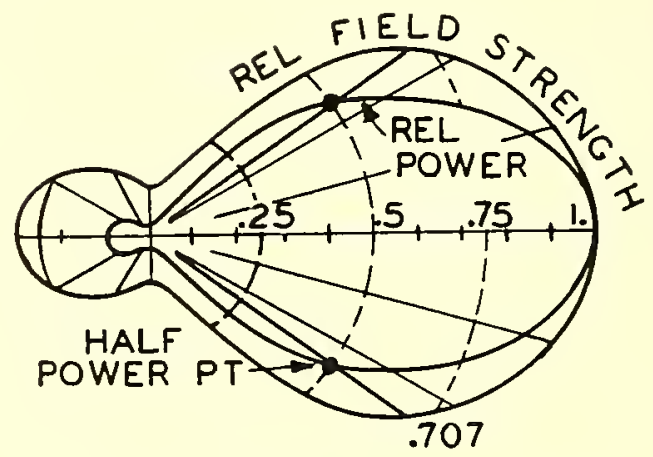

Figure 1. Antenna radiation patterns.

If an antemna system is designed so that most of its power is concentrated into a comparatively small cone, the corresponding part of the radiation pattern is called the main lobe. Commonly there are a number of secondary maxima (side lobes) much smaller than the main lobe. The width of the main lobe is measured by the angle hetween half-power points. Half-power points are those points in the polar diagram of the antenna pattern where the power per unit area is equal to one-half that at the maximum, the field strength being $1 / \sqrt{2}=0.707$ times that at the maximum. This angle is also referred to as the beam width. The beam width varies from a degree or less for some specialized radar antennas to very large angles such as 50 to 60 degrees, depending on the design and purpose of the antenna. The larger the beam width the smaller the gain.

It should be noted that an antenna radiation pattern may have high directivity with respect to one plane going through the antenna and little or no directivity in another plane. Thus a doublet antenna (for definition see Section 2.1.1) is directive in a plane which contains the antenna itself but is nondirective in the equatorial plane perpendicular to the antenna (see Figure 11). 
3.1 .3

\section{Antenna Pattern Factors in Ground Reflection}

With highly directive antennas the magnitude of the direet wave may differ appreciably from that of the ground-reflected wave owing to their difference in angle of emergence from the antenna (Figure 2). This must be taken into aecount by using the

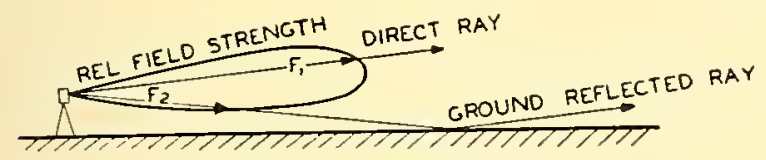

Figure 2. Antenna pattern factors.

antenna pattern factors $F_{1}$ and $F_{2}$ in computing the interference pattern above the line of sight. This subject is dealt with in Section 5.2.6.

\subsubsection{Standing-Wave Antennas}

An important class of antennas is that in which standing waves of the currents and the voltages are set up. In a transmitting antenna of this type, for instance, a progressive or traveling wave is supplied from the connected source of power. This is reflected from the end of the antenna and the interaction of the two sets of waves moving in opposite direetions results in a standing-wave system.

In this event the eurrent amplitude is zero at the ends of the antenna and assumes differing values at the other positions on the antenna. The distribution of eurrent amplitudes is usually assumed to vary sinusoidally with the distance from the end of the antenna. This is a good approximation where the diameter of the antenna wire is small compared with the length, but may be seriously in error for thick-wire antennas.

The simplest, and one of the most commonty used, standing-vave antennas is the half-wave dipole antenna, discussed in Section 3.2.3.

3.1 .5

\section{Resonant Antennas}

Many antennas are operated at or near resonance, which means that the reaetive eomponent of their impedanee vanishes or is very small.

Two types of resonant antenna may be distinguished: either (1) the radiating element as a whole is resonant, as in the ease of the half-wave dipole, shortened the right amount; or (2) the antenna system is made resonant by adding suitable reactive eomponents to the radiative elements. To illustrate, the center-fed half-wave dipole of exactly half-wavelength, assuming sine distribution of current, has an inductive reaetance; it may be made resonant by the addition in series of a capacitive reactance. This is known as antenna loading and is common at the longer wavelengths where half-wave dipoles would be too cumbersome. Another example is that of a dipole radiator shorter than the halfwave dipole and having the form of a metallie tube; this is combined with a tunable eavity resonator

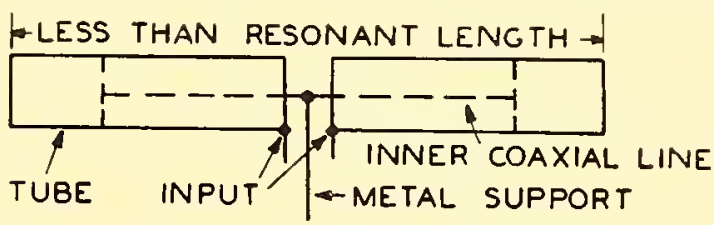

Figure 3. Antenna tuned to resonance by a shunt impedance.

inside the tube that acts as a shunt impedance, the whole system being tuned to resonance (Figure 3 ).

Although the actual antenna impedanee is made up in a complicated way of distributed capaeitances and induetances, the input impedance of the simpler types of antennas for a limited frequency band containing the resonance frequency is essentially that of an ordinary series lesonant eireuit [the resistance at resonance being essentially the radiation resistance of the antenna (see Seetion 3.1.7)]. The input impeclance of eertain other antennas is essentially that of parallel-resonant cireuits with very large shunt resistances at resonance (see Section 3.2.2). For illustration, see Figure 6.

\subsubsection{Traveling-Wave Antennas}

In this type of antemna there is no standing-wave system set up since the progressive or traveling wave of current fed into the antenna is absorbed, without refleetion, by a terminal resistanee placed at the end of the antenna, which is equal to the eharaeteristic impedanee of the antenna regarded as a transmission line. Such antennas are necessarily nonresonant.

The traveling-wave antenna radiates most strongly in the general direction of the wave motion. The major lobe makes an angle $\alpha<90$ degrees with this 
direction as indicated in Figure 20. Here we have a long-wire antemna with the input at the left and the characteristic imperlance (resistance) at the right.

A traveling-wave $V$ antema uses two of these clements (see Section 3.3.2) and a rhombic is composed of four elements (see Section 3.3.3), with the elements arranged at angles which produce maximum directivity of the combinations.

Antennas of the nonresonant or traveling-wave types are used both for longer and for very short waves. (However, there is an intermediate frequeney region extending from about 100 to $3,000 \mathrm{me}$ where the half-wave dipole is of such convenient size that standing-wave dipoles or dipole arrays are most frequently employed.)

In the microwne band where transmission is effected by wave guides it is possible to terminate a ware guide with a horn which "matches the impedance of the wave guide to that of free space" and acts as a directive antenna (Section 3.7). A slot or a series of slots in the sicle of a wave guide may also act as an antenna at these frequencies.

\subsubsection{Radiation Resistanee}

The radiation resistance $R_{r}$ of an antenna is the ratio $P$, of the total power radiated in all directions to the square of the current at the point of measurement. The power may be computed by integrating the radial component of the Poynting vector over a spherical surface surrounding the antenna. Then if $I_{i}$ is the cffective value of the input current,

$$
R_{r}=\frac{P}{I_{i}^{2}} .
$$

The radiation resistance of the doublet antenna is stated in equation (9) in Chapter 2 to be

$$
R_{r}=80 \pi^{2}\left(\frac{L}{\lambda}\right)^{2} \text { ohms. }
$$

\subsubsection{Influence of Near-by Condueting Bodies}

The impedance of an antema is affected by the presence of conductors in the vicinity and depends upon the mutual impedances between the conductors and the antenna. The mutual impedance decreases with increasing distance so that for conducting bodies of comparable size the effect is negligible for distances greater than, perhaps, 2 to 3 wavelengths.

But for conductors set less than a wavelength apart, such as an antenna and reflector (or director) combination or as antema arrays, the mutual effect plays an important role and modifies the input impedance of the antemna.

For an antenna set near a large conducting body, such as a large metallie sheet or the carth, the mutual effect is cared for in a different way. If the earth, for instance, is assumed plane and perfectly conducting, its effect is the same as that of the mirror image of the antemna in the ground. As shown in Figure 4,

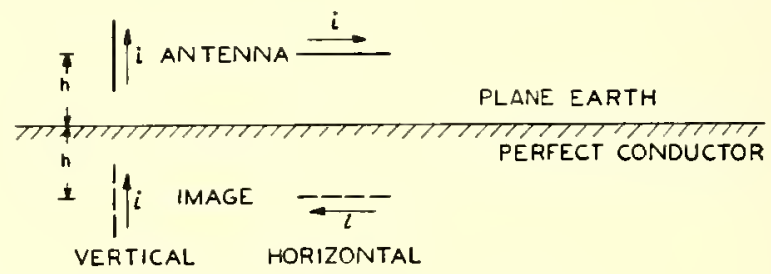

Figure 4. Method of images.

the image of a vertical antenna is a similar antenna with current in the same direction, while the current is reversed for a horizontal antenna. The radiation field at any point above ground is obtained by summing the radiation fields of antenna and image.

\subsection{STANDING-WAVE ANTENNAS}

\subsubsection{Linear Antennas}

A linear antema is a straight thin rod supplied with alternating current. According to whether the connection to the antenna is made at the niddle or
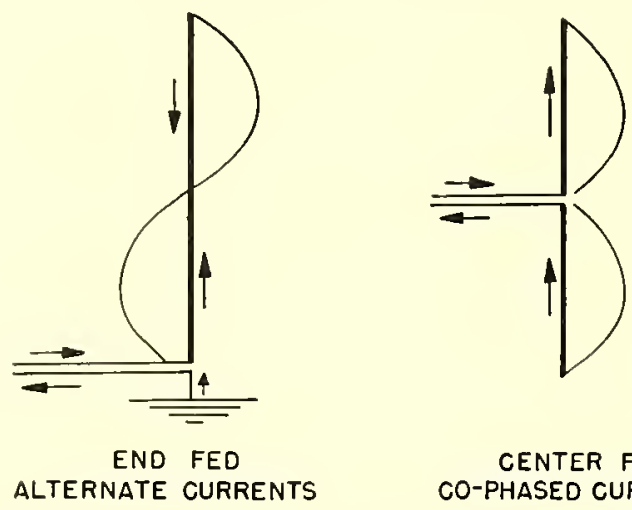

CENTER FED CO-PHASED CURRENTS

Figure 5. Distribution of current amplitudes with linear antennas. 
at the end, center-fed and end-fed antennas are distinguished. Center-fed linear antennas are also called dipole antennas.

Typical current amplitude distributions are illustrated in Figure 5. The amplitude is always zero at the open end while the amount at the input point depends on the position of the input connection. For thin wires, compared with the length, the distribution of amplitudes is approximately sinusoidal.

3.2 .2

\section{Ialf-Wave Antennas}

Figure 6 illustrates two types of half-wave dipole or center-fed antenmas and one encl-fed antenna, together with their lumped-circuit analogues. The
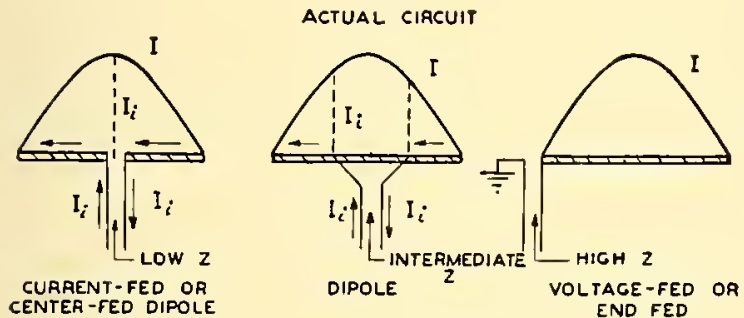

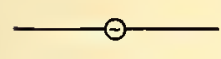

A

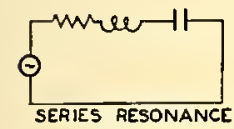

LUMPED-CIRCUIT ANALOGUE
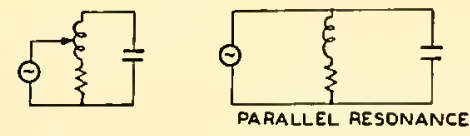

Figure 6. Three methods of exciting half-wave antennas and their analogues in lumped-constant resonant circuits.

input current required varies with the position of the input point. 'The voltage distribution in gencral has a maximum at the points of current zero and has a minimum where the current is maximum.

\subsubsection{Half-Wave Dipole}

The half-wave dipole, shown in $A$ and $B$ of Figure 6 and in Figure 7 , is the type most frequently used in the 100 to $3,000 \mathrm{mc}$ range. In this range the length $\lambda / 2$ lies between 1.5 and 0.05 meters. In this section it is assumed that the current distribution is sinusoidal.
1. Radiation field. The radiation ficld at point $P$, Figure 7 , where $d>>$, is obtained by dividing the half-wave current distribution into an infinite

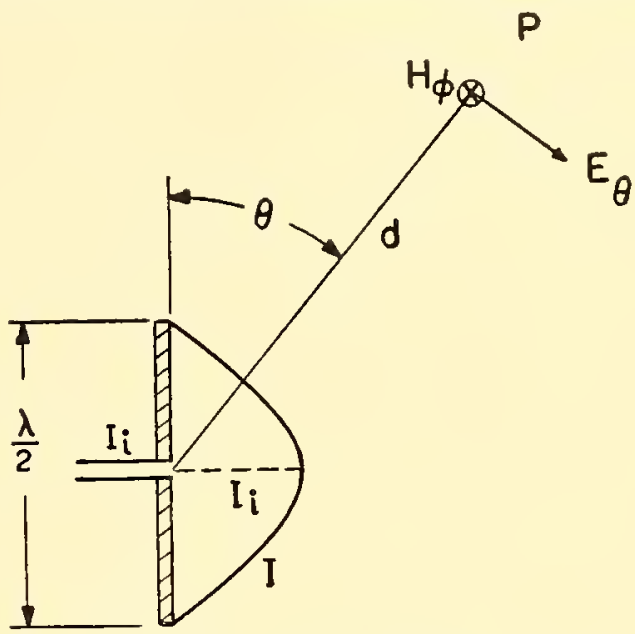

Figure 7. Half-wave dipole.

number of infinitesimal doublets, using equation (2) in Chapter 2 and taking into account the differences in phase at $P$ introduced by the differences in the distances which the radiation from the various doublets must travel. The net result, using $d$ in place of $r$, is

$$
\begin{gathered}
E_{\theta}=\frac{60 I_{i}}{d} \frac{\cos [(\pi / 2) \cdot \cos \theta)]}{\sin \theta} \text { volts per meter, } \\
H_{\phi}=\frac{E_{\theta}}{120 \pi} \text { amperes per meter. }
\end{gathered}
$$

The normal part of the field, $E_{\theta}$ (difference), preseribes the antenna pattern factor (measured in relative field strength) and is plotted in Figure 11. The corresponding pattern for a doublet is $E_{\theta} \sim \sin \theta$, which is a circle in polar coordinates. These patterns are circularly symmetric about the antenna axis. Squaring the radial lengths in the above patterns gives the pattern in terms of relative power per unit area in the same angular direction.

The radial component of radiated power per square meter (Poynting's vector) is given by

$$
W_{t}=E_{\theta} H_{\phi}=\frac{E^{2}}{120 \pi}=\frac{30 I_{i}{ }^{2}}{\pi d^{2}}\left[\frac{\cos \left(\frac{\pi}{2} \cos \theta\right)}{\sin \theta}\right]^{2}
$$

In the equatorial plane,

$$
E_{\theta}=\frac{60 I_{i}}{d} .
$$


2. Gain of half-urae dipole. The gain of the dipole relative to a doublet is the ratio of the power supplied to the doublet to the power supplied to the dipole to produce the same field strength at the same distance in the direction of maximum radiation (here the equatorial plane, $\theta=90$ degrees $)$.

For equal maximum fields, comparing equations (3) in Chapter 2 and (6) in this chapter,

$$
\int I d l=\frac{\lambda}{\pi} I_{\imath}
$$

The power per unit area for the doublet, using equation (3), in Chapter 2 , is

$$
W_{\text {doublet }}=\frac{E^{2}}{120 \pi}=\frac{30 I_{i}^{2} \sin ^{2} \theta}{\pi d^{2}},
$$

and for the dipole the pover per unit area is given by equation (5).

The dipole gain is then

$$
G=\frac{\int W_{\text {doublet }} d A}{\int W_{\text {dipole }} d A}=\frac{\text { Power radiated by doublet }}{\text { Power radiated by dipole }},
$$

where the integration is carried out over spheres surrounding the antennas. Carrying out this operation,

$$
\left.G_{\text {dipole }}=1.09 \quad \text { (or } 0.4 \mathrm{db}\right) .
$$

3. Radiation Resistance. The radiation resistance of the half-wave dipole is

$$
R_{r}=\frac{1}{I_{i}{ }^{2}} \int H_{\text {dipole }} d A=73.1 \text { ohms. }
$$

4. Impedance of an Infinitcly Thin Dipole. The formulas given here are valid only for a half-wave

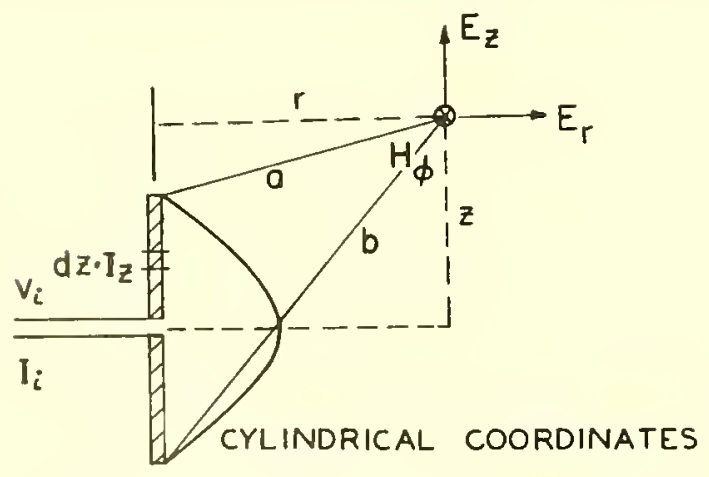

Figure 8. Half-wave dipole field components.

dipole composed of wire of vanishing thickness. For wire of finite dimensions, see Section 3.2.7.
Here (type $A$ in Figure 6 ) it is necessary to calculate the voltage $V_{i}$ required at the input to establish a current distribution $I_{i} \cos [(2 \pi / \lambda) z]$, as shown in Figure 8. To do this, the total field of the dipole must be known, including the induction field which is significantly large at short distances as well as the radiation field. In cylindrical coordinates, the total field is given by

$k_{r}=+j 30 I_{i}\left[\frac{z-\lambda / 4}{a r} e^{-j\left(\frac{2 \pi a}{\lambda}\right)}+\frac{z+\lambda / 4}{b r} e^{-j\left(\frac{2 \pi b}{\lambda}\right)}\right]$,

$E_{z}=-j 30 I_{i}\left[\frac{1}{a} e^{-j\left(\frac{2 \pi a}{\lambda}\right)}+\frac{1}{b} e^{-j\left(\frac{2 \pi b}{\lambda}\right)}\right]$,

$H_{\phi}=-j \frac{I_{i}}{4 \pi}\left[\frac{1}{r} e^{-j\left(\frac{2 \pi a}{\lambda}\right)}+\frac{1}{r} e^{-j\left(\frac{2 \pi b}{\lambda}\right)}\right]$.

By the reciprocity theorem a small current length $I_{z} d z=I_{i} \cos [(2 \pi / \lambda) z] \cdot d z$ induces a voltage $\left(-d V_{i}\right)$ at the input point which is equal to the voltage $d V_{z}=E_{z} d z$ induced in $d z$ by a small current length $I_{i} d z$ taken at the input point. Hence

$$
\frac{E_{z} d z}{I_{i} d z}=\frac{-d \Gamma_{i}}{I_{i} \cos \left(\frac{2 \pi}{\lambda} z\right) \cdot d z},
$$

and the total input voltage is

$$
V_{i}=2 \int_{z=0}^{z=\lambda}-E_{z} \cos \left(\frac{2 \pi}{\lambda} z\right) \cdot d z .
$$

Carrying out the operation indicated and dividing by $I_{i}$ gives the impedance of the half-wave dipole as

$$
Z=73.1+j+2.5 \text { ohms. }
$$

The dipole thus has an inductive reactance of 42.5 ohms if a sine distribution of current amplitudes is assumed.

The reactance can be altered by changing the length of the wire. Increasing the length increases the inductance; decreasing the length decreases the inductance, first to zero for resonance, and then for still shorter lengths to a capacitive reactance. Changes in length of only 4 to 5 per cent will produce large changes in the reactance.

\subsubsection{Modifications of the Half-Wave Dipole}

Two modifications will be given.

1. Quarter-wave dipole with artificial ground. I conrenient device for doubling the effective length 
of a dipole is to use an artificial ground plane. It usually takes the form of a number of grounded rods spreading radially from the base of the antenna

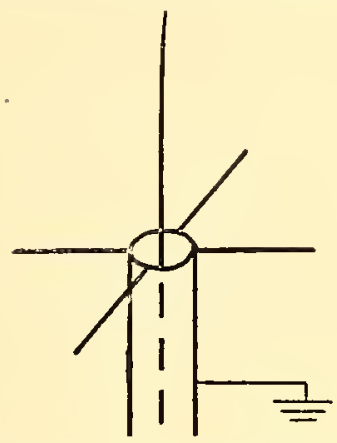

FIGtiRe 9. Quartel-wave dipole with artificial ground.

(Figure 9). If the antenna is a quarter-wave dipole the effect of the artificial ground is to produce an image quarter-rave dipole; the radiation resistance and the radiation pattern of the system are those of a half-wave dipole.

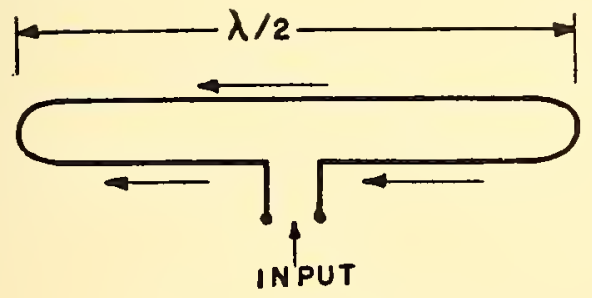

Figure 10. Folded dipole.

2. Folded dipole. Another variant of the dipole antenna is the folded dipole, shown in Figure 10.
It is essentially a center-fed half-rvave dipole with a parasitic counterpart "dummy" (see Section 3.5) in its immediate neighborhood and connected to the latter at the ends of the dipole. The induced current in the dummy has the same distribution as, and is in phase with, that of the primary dipole. Hence the radiation pattern is essentially that of a simple halfwave dipole. The radiation resistance is four times that of the ordinary dipole.

\subsubsection{Multiple Half-Wave Long Antennas}

For an antemna of length equal to an integral number, $n$, of half wavelengths, the radiation field is given by:

1. $n$ is odd:

$$
E_{\theta}=\frac{60 I_{2}}{d} \frac{\cos \left(\frac{n \pi}{2} \cos \theta\right)}{\sin \theta} .
$$

2. $n$ is even:

$$
E_{\theta}=\frac{60 I_{i}}{d} \frac{\sin \left(\frac{n \pi}{2} \cos \theta\right)}{\sin \theta},
$$

where $d$ is the radial distance to a field point and $l_{i}$ is the input current at the center of one of the half-wave elements.

The radiation patterns are illustrated in Figure 11 for the doublet, $n=1$ (the half-rave dipole), and $n=2,3,4$.
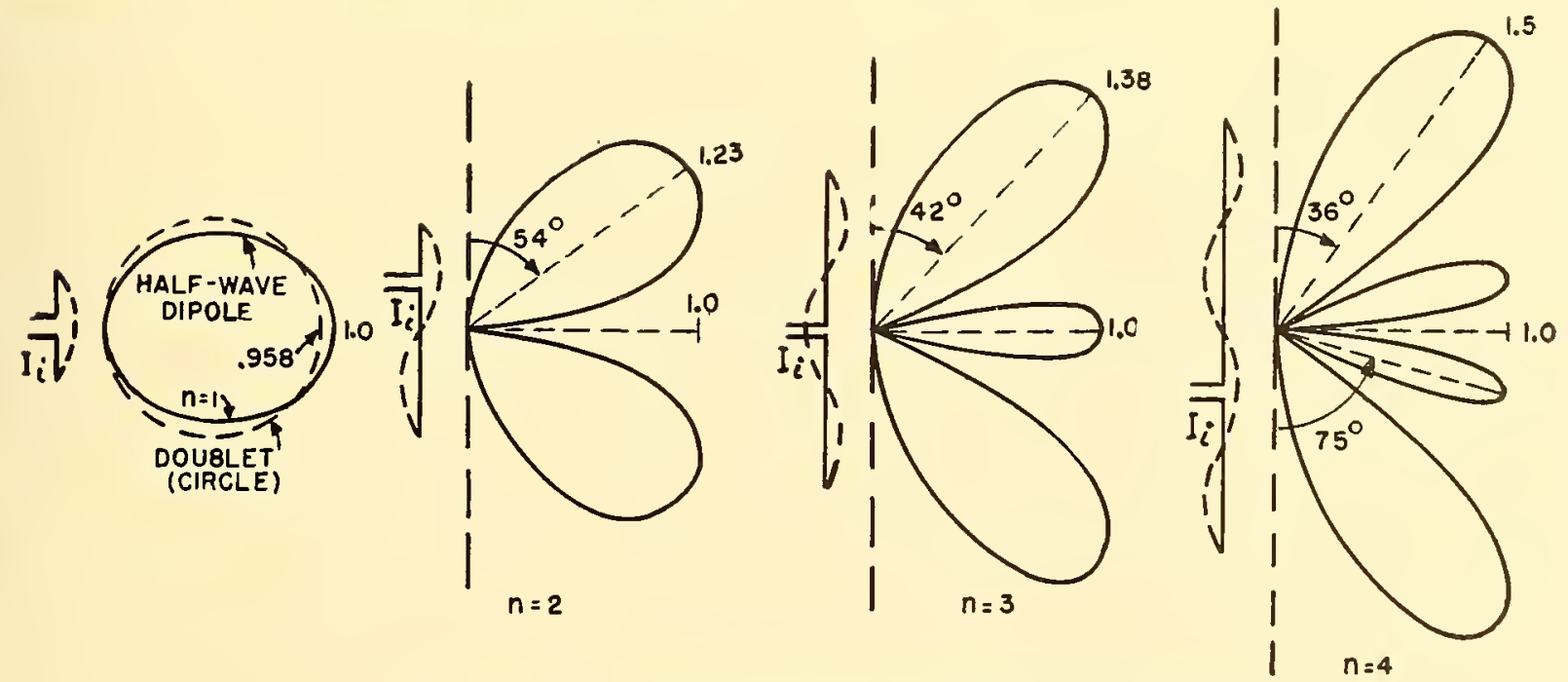

Figure 11. Antenna radiation patterns (relative field strength). 
The radiation resistance, both for integral and nonintegral numbers of half wavelengths, is plotted in Figure 12.

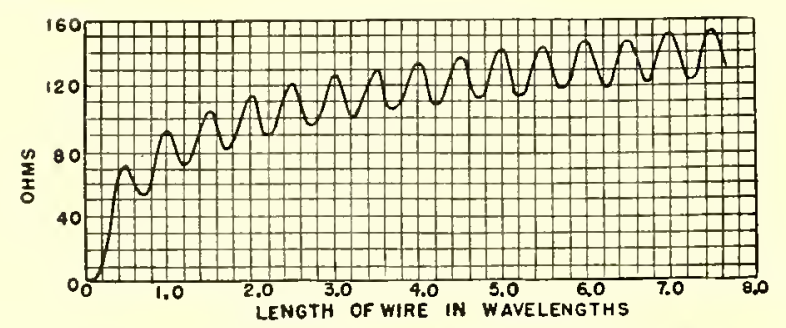

Figure 12. Radiation resistance for linear antennas.

In Table 1 the radiation resistances and the power gains for integral half-wavelength antennas are listed.

\subsubsection{Cophased Half-Wave Dipoles}

The directivity and gain of linear antennas may be increased considerably by the suppression of alternate current loops, leaving therefore only loops in which the eurrents are all eophased. The suppressed loops are contained in either (1) quarter-wave stubs or (2) short inductive elements, as indicated in Figure 13. The suppressed loops are practically nonradiative.

The radiation field at distance $d$ is the vector sum of the fields from the $n$ half-wave elements. The contribution from each element lags that of the next element above by an angle

$$
\alpha=\frac{\lambda}{2} \cos \theta \cdot \frac{2 \pi}{\lambda}=\pi \cos \theta \text { radians, }
$$

TABle 1. Comparison of alternate and cophased half-wave dipoles.

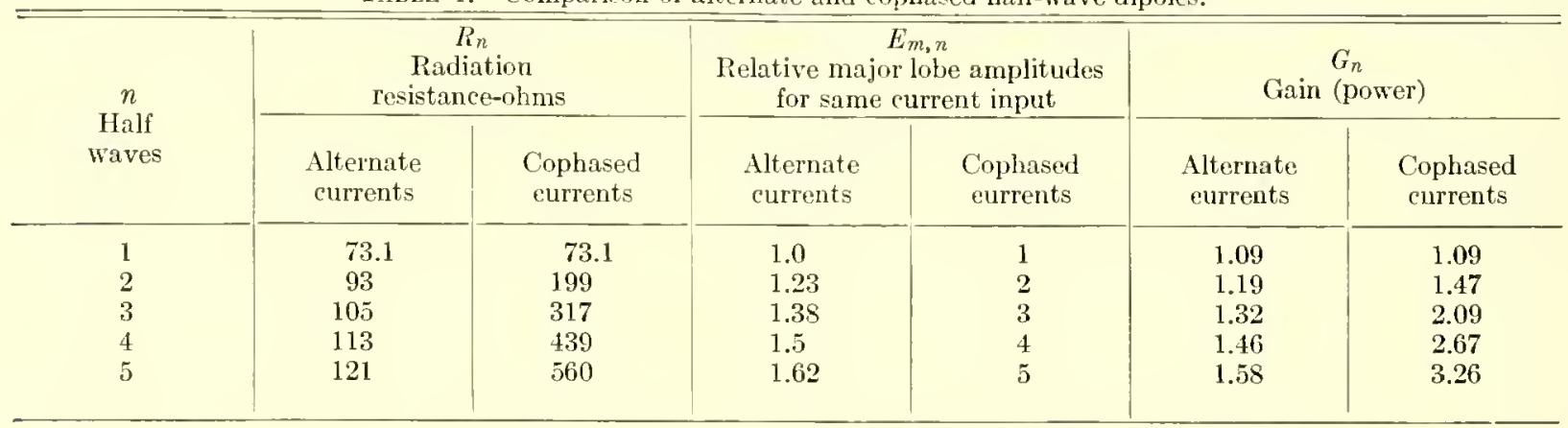

$$
G_{n}=\frac{R_{\text {doublet }}}{R_{n}}\left(\frac{I_{\text {doublet }}}{I_{n}}\right)^{2}=\frac{R_{\text {doublet }}}{R_{n}}\left(\frac{E_{m, n}}{E_{\text {doublet }}}\right)^{2} .
$$
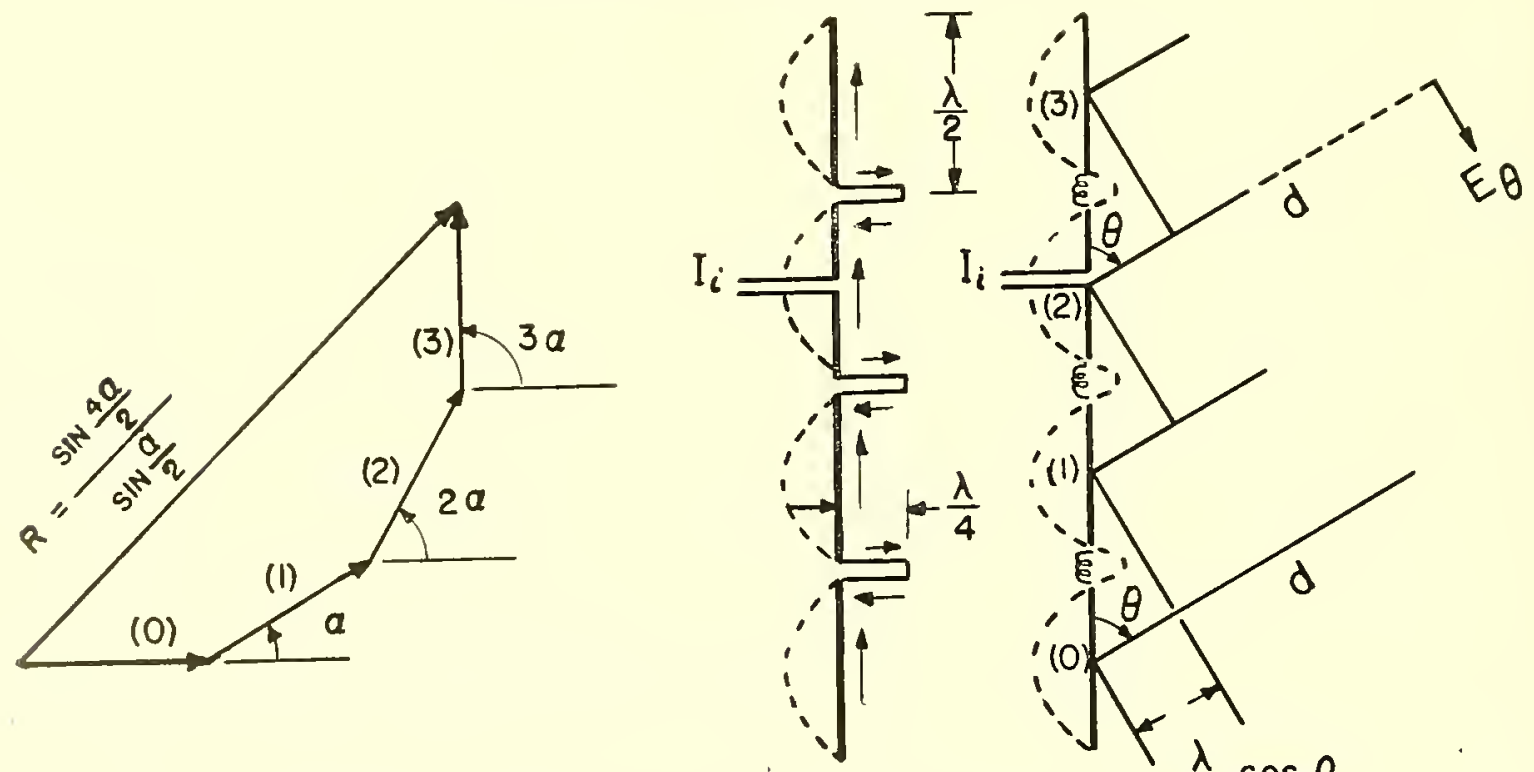

FIGURE 13. Cophased half-wave dipoles. 
determined by the extra distance $[(\lambda / 2) \cos \theta]$ which it must travel. The radiation field is then equal to the radiation ficld of one half-wave element (as a function of angle $\theta$ ) multiplied by the vector
The radiation patterns for various values of $n$ are plotted in Figure 14. Table 1 gives the radiation resistances, relative lengths of major lobes, and the gains, with comparative figures for the doublet and

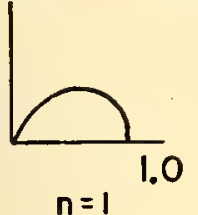

$n=1$

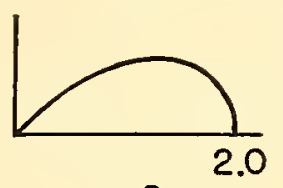

$n=2$

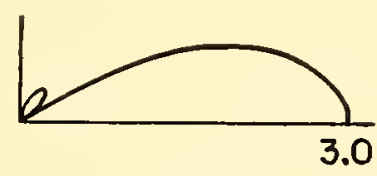

$n=3$

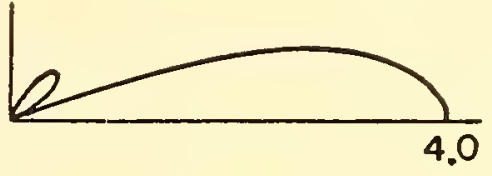

$n=4$

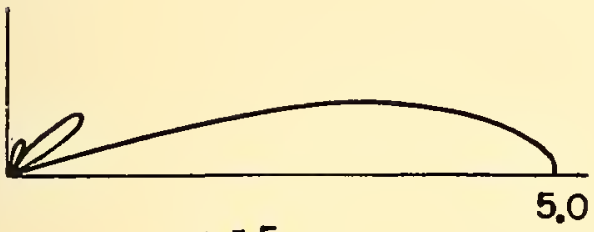

$n=5$

Figure 14. Cophased half-wave dipoles (relative fields).

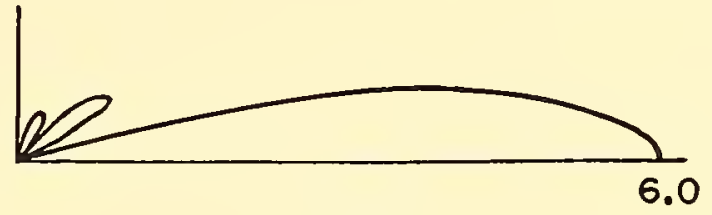

$n=6$

the multi-half-wave antennas of Section 3.2.5.

resultant for the $n$ elements. Thus

$$
\begin{aligned}
E_{\theta} & =\frac{60 I_{2}}{d}\left[\frac{\cos \left(\frac{\pi}{2} \cos \theta\right)}{\sin \theta}\right] \times \\
& =\frac{60 I_{2}}{d}\left[\frac{\cos \left(\frac{\pi}{2} \cos \theta\right)}{\sin \theta}\right]\left[\frac{\sin \frac{n \alpha}{2}}{\sin \frac{\alpha}{2}}\right] .
\end{aligned}
$$

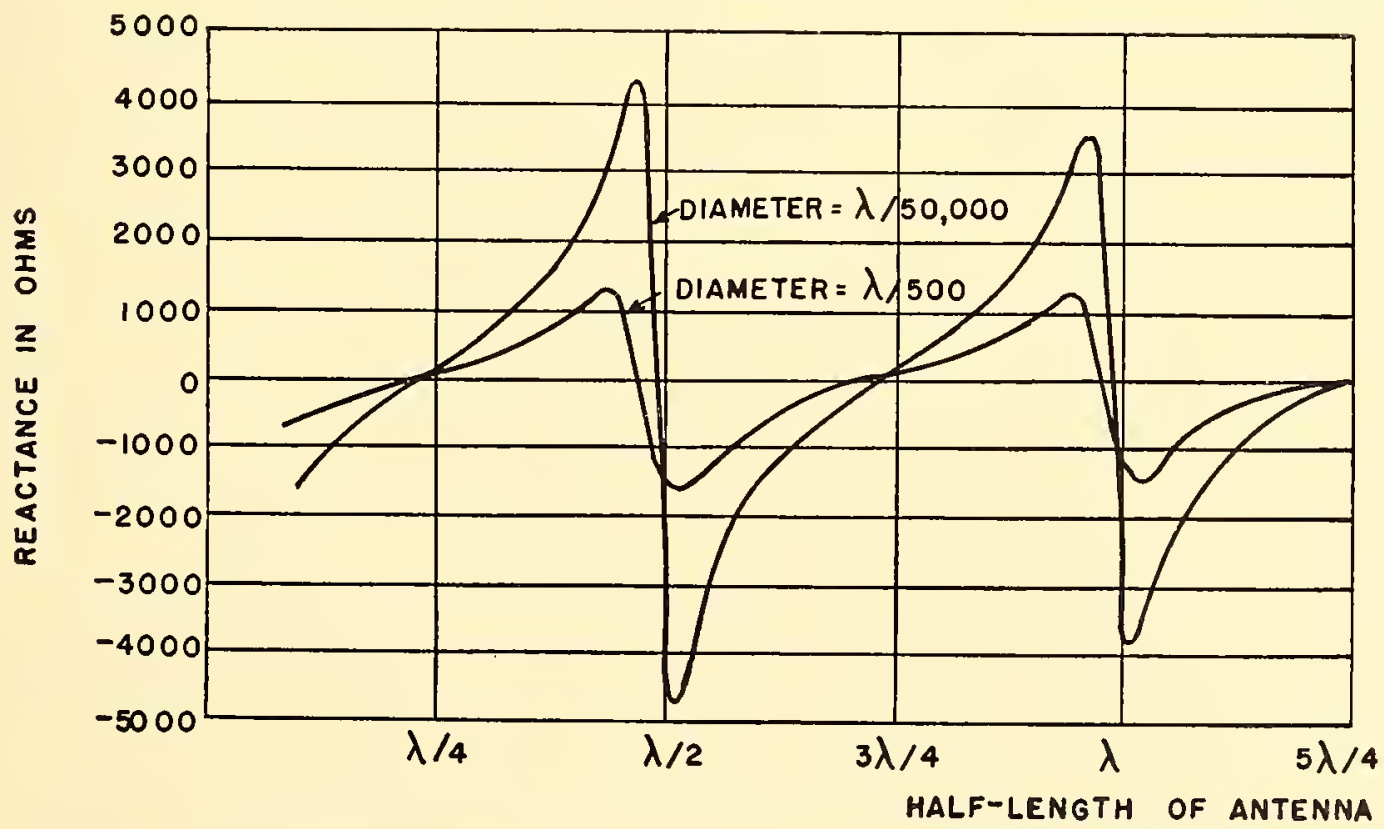

FigURE 15. Reactance at input of a center-fed antenna of arbitrary length.

\subsubsection{Effects of Finite Diameter on Center-Fed Linear Antennas}

Figure 15 shows the input reactance, and Figure 16 the input resistance of a center-fed antenna of arbitrary length. The input impedance is a series combination of the two components. The important 
regions of the curves correspond to antenna halflengths near $\lambda / 4$ and near $\lambda / 2$. The former represents a center-fed half-wave antenna, whereas the latter lepresents a pair of end-fed half-wave antennas exeited in phase. The half-length of the antenna was used in plotting, beeause in these terms the reactance curves resemble those for an open-ended transmission line.

In the regions of principal interest the reactance eurves are nearly straight lines whose slopes depend on the diameter of the antennas expressed in wavelengths. The slopes of the reactance curves decrease as the antenna diameter increases. This feature is important in radar antennas which need to be insensitive to small changes in frequency. The curves than when the half-length approximates $\lambda / 4$, as it does for a single center-fed antenna. The values for an antenna whose half-length is $\lambda / 4$ is not readable on the curve, but the component representing radiation ranges from 73 ohms for infinitely thin antennas, through $6 \pm$ ohms for a diameter of $0.0001 \lambda, 55 \mathrm{ohms}$ for a diameter of $0.01 \lambda$, to less than 50 ohms for certain large-diameter radar antennas. The change is mainly due to a decrease in the resonant length of the thicker antennas.

$A$ leature of Figure 15 which is not easily readable is that the lengths at which the reactance is zero are less than $\lambda / 2$ and $\lambda$. The amount by which an antenna with zero reactance is shorter than these lengths depends on the antenna diameter. For very

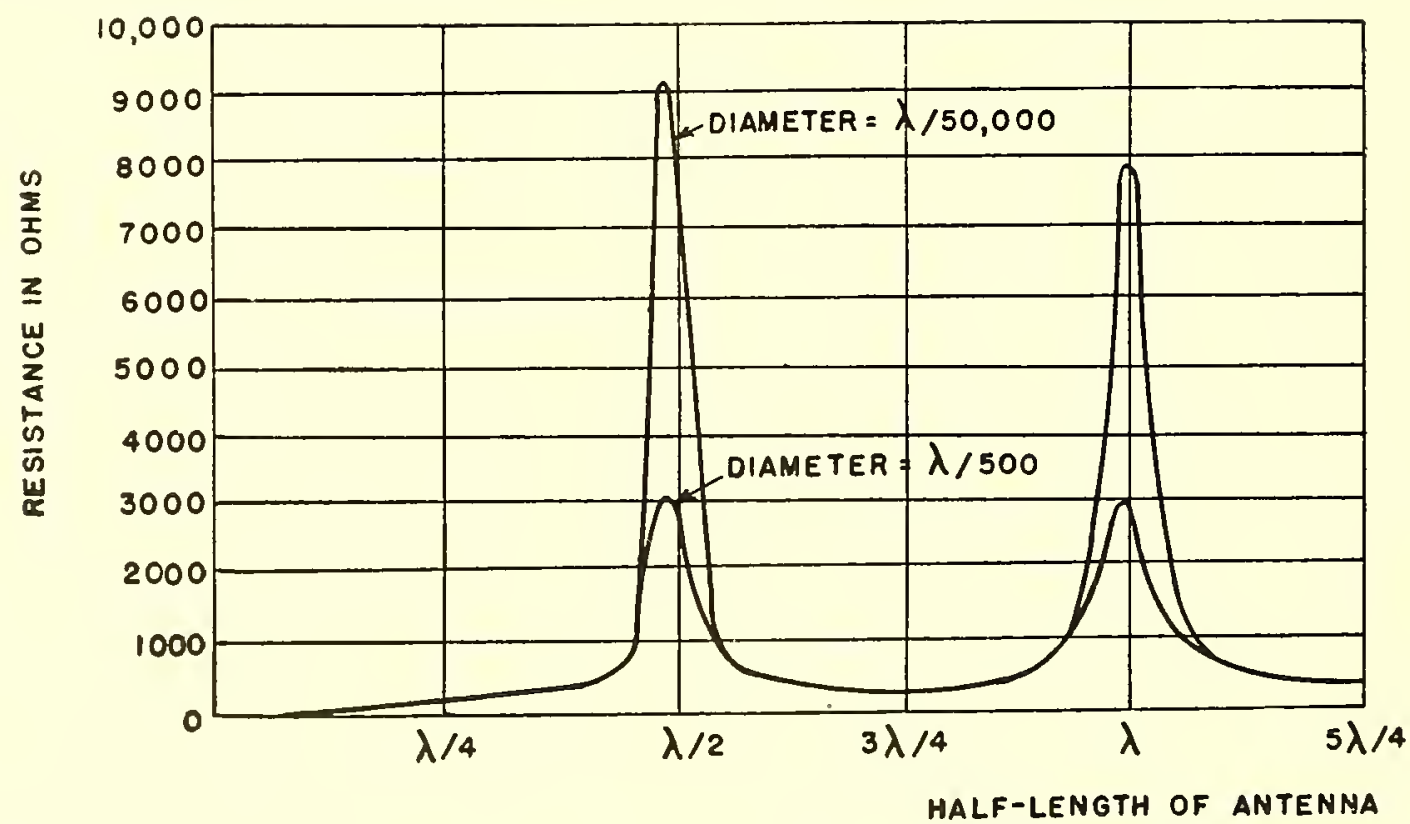

FigrRE 16. Resistance at input of a center-fed antenna of arbitrary length.

show that antennas of large diameter present less than a specified amount of reactance, say one ohm, over a greater range of antenna length than stender antennas do. In terms of frequency, this means that a given length of antenna has less than one-ohm reactance over a wider range of frequency when the antenna has a large diameter than when it has a small diameter. Radar antennas are commonly made of tubing and frequently have diameters in excess of $\lambda / 20$.

Figure 16 shows that the input resistance also depends on antenna diameter. This dependence is more pronounced when the half-length is about $\lambda / 2$ slender antennas the shortening is slight, but for large-diameter antennas or for special shapes as shown in Figure 17, a resonant length may be as

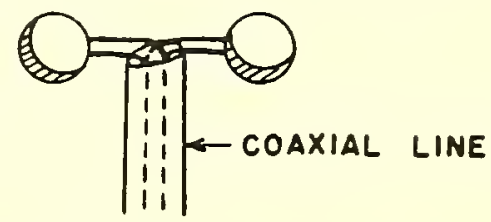

Figure: 17. Non-cylindrical half-wave antenna.

much as 20 per cent shorter than $\lambda / 2$. Special shapes, such as the one shown in Figure 17 , have the ad- 
vantage of being insensitive to small changes in frequency and at the same time are not so subject to corona (breakdown of the air because of large potential gradients) as slender antennas are.

\subsubsection{Standing-Wave $\mathbf{V}$ Antennas}

This type of antenna (Figure 18) utilizes the directive properties of the multi-half-wave antenni.
Two such elements are combined in a $\mathrm{V}$ arrangement so that the major lobe of each (at angle $\alpha$ with each element) is parallel to the axis of the $\mathrm{V}$. By feeding the two halves of the $\mathrm{V}$ with currents 180 degrees out of phase the lobe structure is reversed to produce maxima, forward and backward, along the axial direction, while the field in the plane perpendicular to the axis is greatly redneed. The

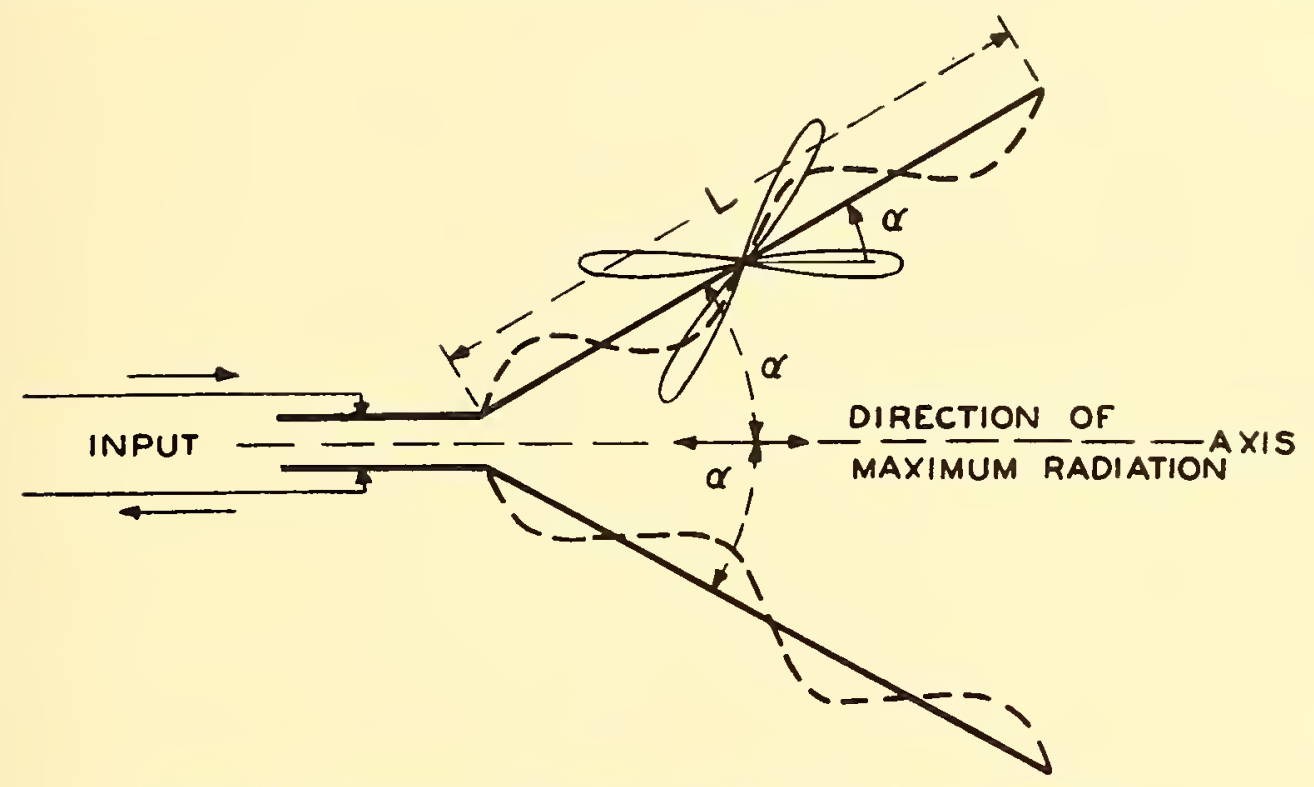

Figure 18. Standing-wave $V$ antema $\left(\alpha=36^{\circ}\right.$ for $n=4$ half-wavelengthis).

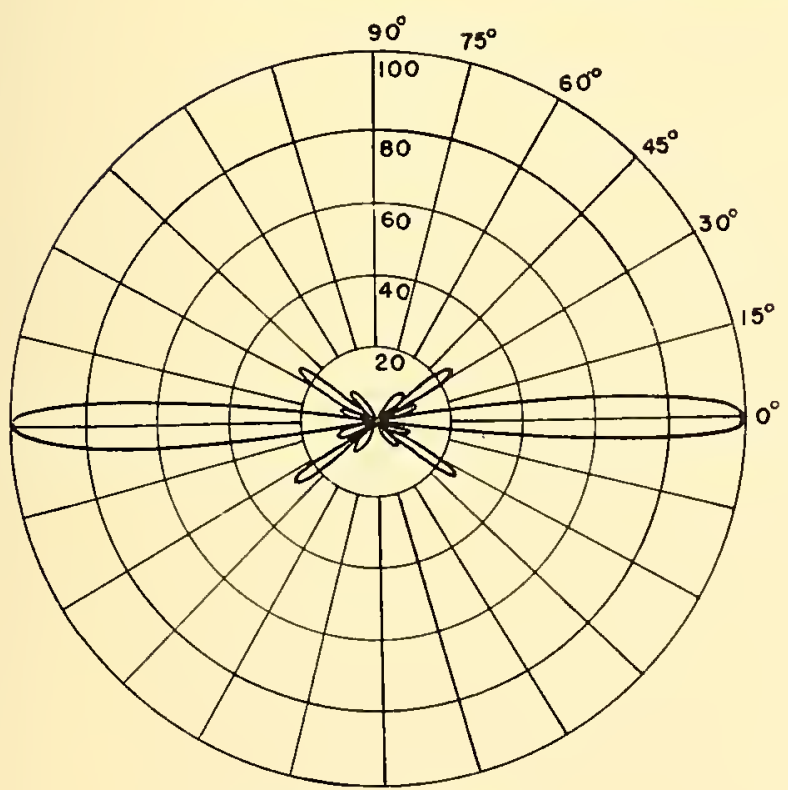

IN PLANE OF THE $\mathrm{V}$

$n=16, \alpha=17.5^{\circ}$

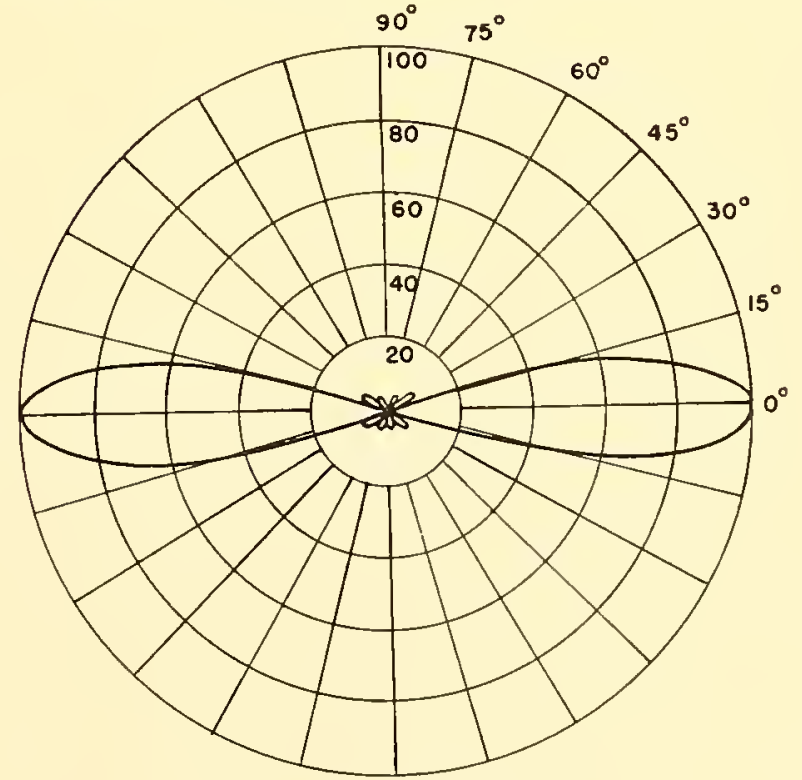

IN PLANE $\perp$ TO $\mathrm{V}$

$n=16, \alpha=17.5^{\circ}$

Figure 19. Power distribution for standing-wave $V$ antenna. (Courtesy of IRE) 
value for angle $\alpha$ is equal to the angle between each element and its maximum lobe (see Figure 11). Figure 19 gives the (porrer) radiation pattern for $n=16$ half-warelengths.

The directivity of this antenna system may be improved by adding one or more reflectors (see Section 3.4.6). The reflector is a $\mathrm{V}$ antemna of identical type. The legs of the reflector are placed parallel to those of the prinary $\mathrm{V}$ and lie in the same plane as the original $\mathrm{V}$. The reflector is set approximately $\lambda / 4$ behind the primary $\mathrm{V}$.

\subsection{TRAVELING-WAVE ANTENNAS \\ 3.3.1 Field and Pattern}

A traveling-wave antenna is one in which only progressive (or traveling) waves are allowed. Reflected waves are eliminated by terminating the end opposite the input point in the characteristic impedance. See Figure 20.
The major lobes given by this equation are plotted in Figure 21, and the major lobe angles with the wire $\theta_{m}$ are plotted in Figure 22. Angle $\theta_{m}$, it will be noted, decreases with increasing wire length.

\subsubsection{Traveling-Wave $V$ Antenna}

As in the case of the standing-wave antenna a pair of lines arranged at a suitable angle with each

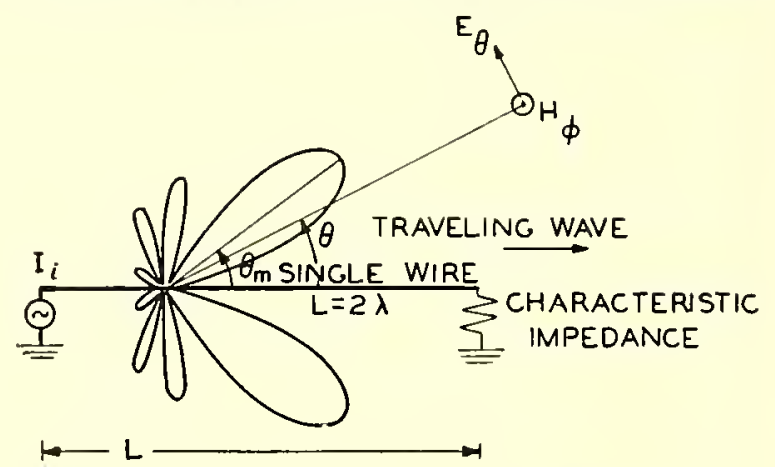

Figure 20. Lobe structure for $L=2 \lambda$ traveling-wave antenna in free space.

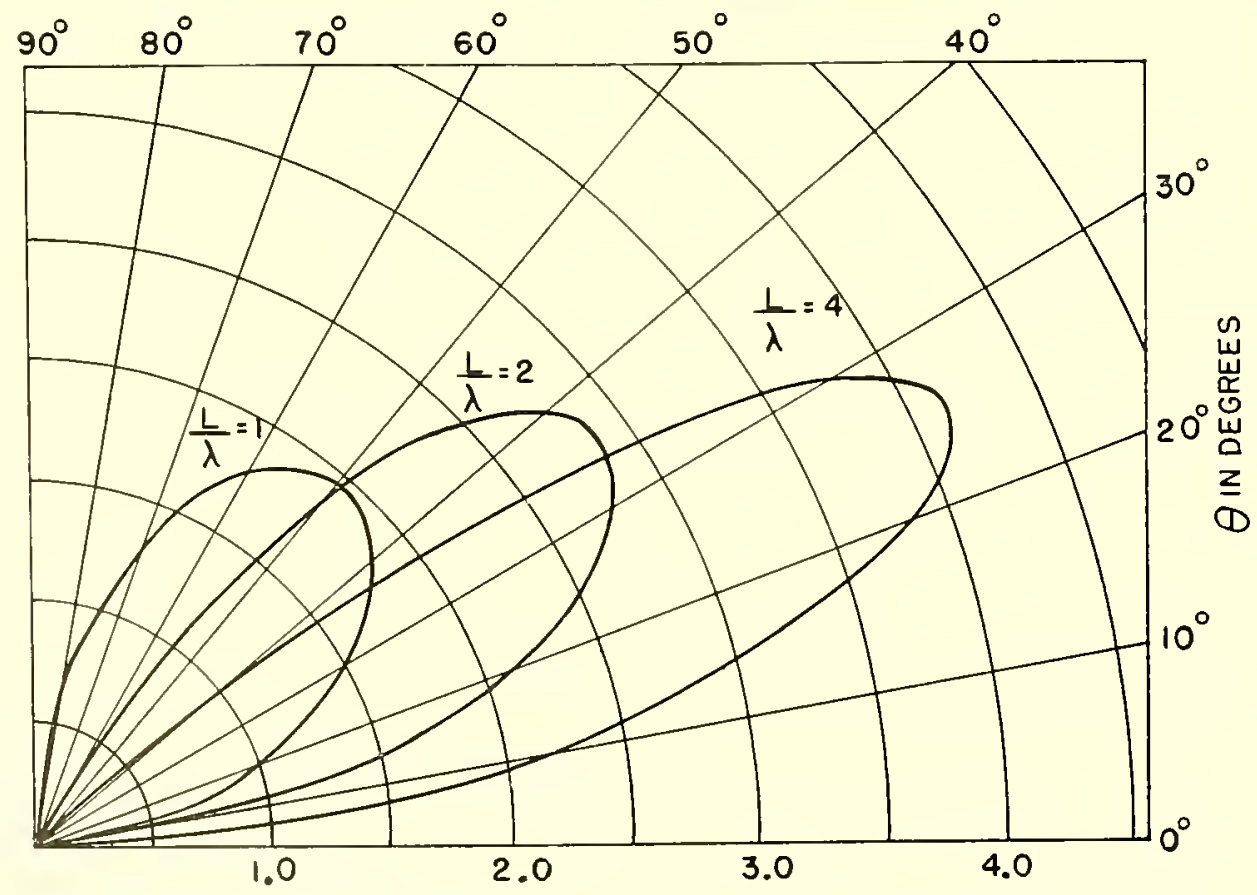

Figure 21. Major lobes (relative field strength) for traveling-wave antemna.

The equation of the radiation field, neglecting wire losses, is

$$
E_{\theta}=\frac{60 I_{i}}{d} \frac{\sin \theta}{1-\cos \theta} \sin \left[\frac{\pi L}{\lambda}(1-\cos \theta)\right] .
$$

other, and carrying traveling waves, can be made to produce a directional pattern with fairly high gain.

The traveling-ware $\mathrm{V}$ antenna can be designed so that the plane of the $V$ is horizontal and the maximum lies in the direction of the axis of sym- 
metry, as in Figure 18. In this case the radiation is horizontally polarized. It can also be used as an inverted $V$ in a vertical plane with the point of the $V$ directed upwards; the radiation is then vertically polarized. This antenna, also called a semi-rhombic, is represented by the upper half of Figure 20.

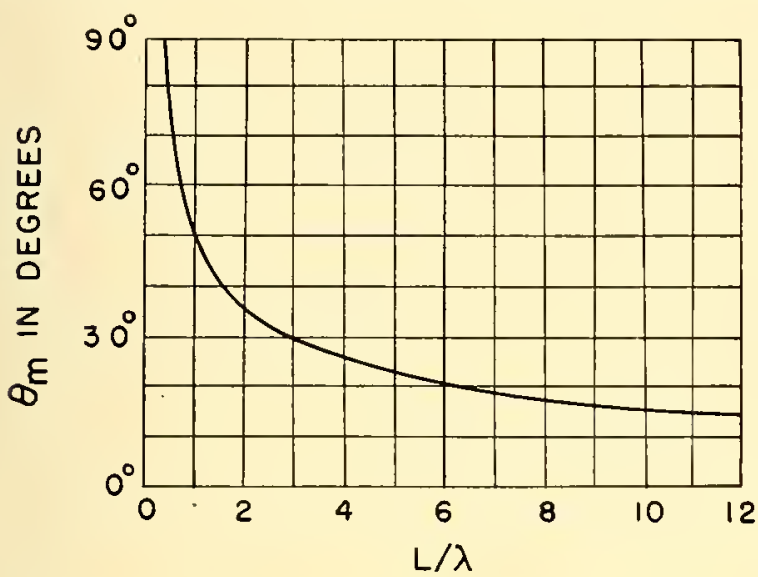

Figure 22. Angles for major lobes for traveling-wave antemna.

3.3 .3

\section{Rhombic Antenna}

This type of antenna is based on the same principle as the traveling-rrave $V$ antenna. The rhombic antenna consists of four wires arranged in the form of a rhomboid or diamond (Figure 23). The reflectionless termination of the wires is achieved by connecting the two wires at the end opposite the input to a resistance equal to their characteristic impedance.

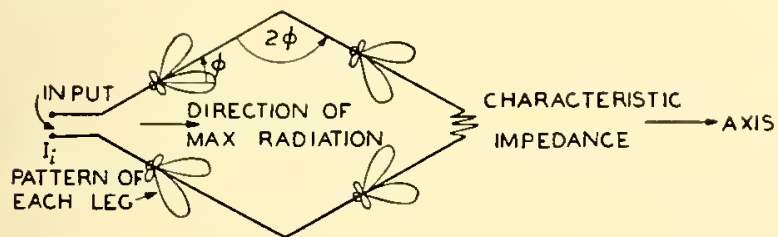

Figure 23. Rhombic antenna.

As in the case of the $\mathrm{V}$ antenua, the rhombic antenna can be used both horizontally and vertically; at the longer waves the horizontal arrangement is usually more practical. The optimum tilt angle of the rhombic (angle $\phi$ of Figure 23) is not very critical provided the legs are not less than two warelengths long. The radiation pattern is not very sensitive to frequency and the rhombic antenna can therefore be used over a fairly wide frequency range (of the order of 2 to 1 ). Rhombic antennas have appreciably higher gains than $\mathrm{V}$ antennas.
The field in the axial direction is equal to

$E_{\text {axial }}=\frac{2 t 0 J_{i}}{d} \frac{\cos \phi}{1-\sin \phi} \sin ^{2}\left[\frac{\pi L}{\lambda}(1-\sin \phi)\right]$.

Effect of Perfectly Conducting Ground. If the rhombic is placed in a horizontal plane, height $I$ above ground, the effect of the image rhombic must also be considered. The net result is that the direction of the resultant lobe maximum is tilted up by an angle $\epsilon$. It can be shown that, for a given angle $\epsilon$ and wavelength $\lambda$, to point the major lobe at vertical angle $\epsilon$ the following relations for $H$, $L$, and $\phi$ must hold :

$$
\begin{aligned}
& H=\frac{\lambda}{4 \sin \epsilon}, \\
& L=\frac{0.371 \lambda}{\sin ^{2} \epsilon}, \\
& \phi=90^{\circ}-\epsilon .
\end{aligned}
$$

Figure 24 illustrates the radiation pattern (relative field strength) for a particular case.

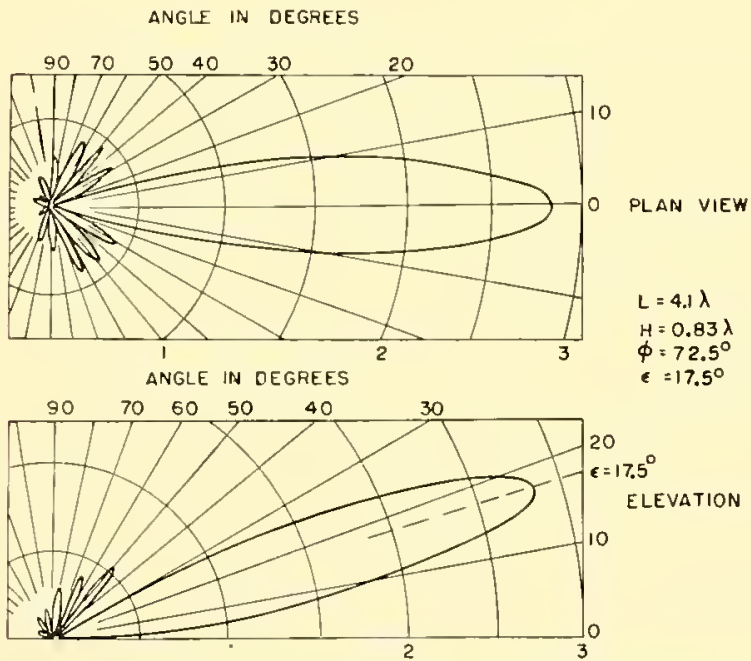

FIgURE 24. Rhombic antenna above ground (relative field strength). (Courtesy of Bell System Technical Journal.)

\section{ANTENVA ARRAYS}

3.4 .1

\section{Principle of Arrays}

An antenna array is a combination of several antennas, usually of equal strength and equally spaced in any one given direction. One-, two-, and three-dimensional arrays may be distinguished. The 
spacings in different directions may be different for two- or three-dimensional arrays. The use of arrays permits great increases in the amount of power radiated, in directivity, and gain.

Although the most eommon array element is a half-wave dipole, the elements of an array may be radiators of any type; in particular, the elements may themselves be arrays. In this way it is possible to interpret a two-dimensional array as an array of arrays. A vertical curtain may be eonsidered either as a horizontal array of elements which, themselves, are vertical, or it may be eonsidered a vertical array of elements which, themselves, are holizontal arrays; similarly for three-dimensional arrays.

In most arrays the elements radiate very nearly equal power, but in the binomial array the elements, although identieal in structure, differ in the amount of power radiated because of differing current distributions. In most arrays there is a eonstant phase hift (which might be zero) between adjacent elements. By suitable phasing a great variety of antenna patterns ean be produced.

\subsubsection{Basic Types of Dipole Arrays}

There are three basic types of dipole arrays.

1. Broadside array. The eenters of the elements are arranged in a line, with the axes of the elements parallel to each and perpendicular to the line. With the currents adjusted all in phase, the maximum radiation is broadside to the plane of the elements.

2. End-fire array. The geometrie arrangement is the same as in the broadside array, but through appropriate phasing of the currents in the elements the maximum radiation can be direeted primarily along the line joining the centers.

3. Colinear array. Here the axes of the antenna elements are arranged along the line of eenters with the currents all in phase. The radiation is a maximum in the equatorial plane perpendieular to the line of centers.

To illustrate the principles most simply, two halfwave dipole elements are eonsidered first, and later extension is made to arrays composed of a larger number of elements.

\subsubsection{Two-Dipole Side-by-Side Array}

T'wo half-wave dipoles are placed side by side with sparing $s$ and the currents $I_{1}$ and $I_{2}$ are equal but differ in phase by angle $\psi$ (see Figure 25). If $I_{2}$ lags
$I_{1}$ by time angle $\psi$, the field of the second element at. $P$ lags that of the first by angle $\alpha$ where $\alpha$ is

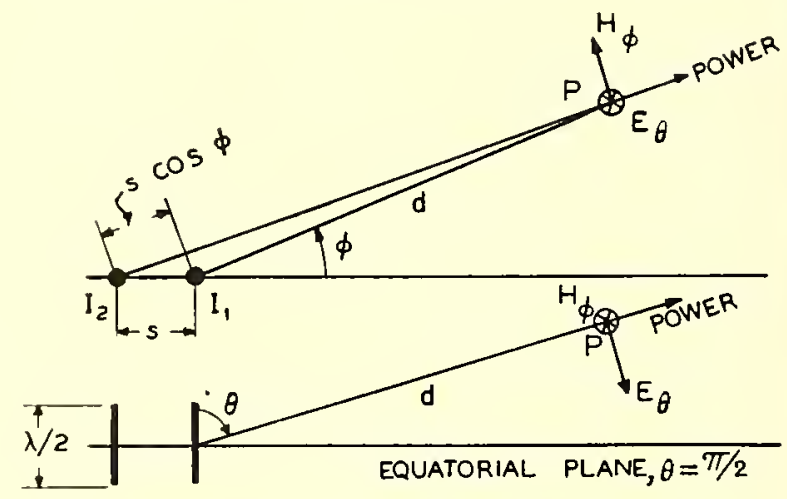

Frgure 25. Two dipole side-by-side array.

eomposed of $\psi$ and the time delay caused by the extra distance traveled, $(2 \pi / \lambda) s \cos \phi \sin \theta$,

$$
\alpha=\psi+(2 \pi / \lambda) s \cos \phi \sin \theta .
$$

For equal eurrents, $I_{1}=I_{2}=I$, the field is equal to

$$
\begin{aligned}
H_{H} & =\frac{60 I}{d}\left[e^{j 0}+e^{-j \alpha}\right]\left[\frac{\cos \left(\frac{\pi}{2} \cos \theta\right)}{\sin \theta}\right], \\
& =\frac{60 I}{d}\left[\frac{\sin \alpha}{\sin \frac{\alpha}{2}}\right]\left[\frac{\cos \left(\frac{\pi}{2} \cos \theta\right)}{\sin \theta}\right] .
\end{aligned}
$$

The first bracket gives the directional characteristie of an array of two elements, while the second bracket gives the direetional eharacteristic of the element itself.
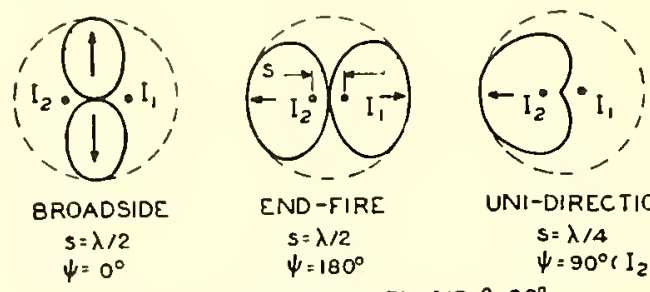

UNI-DIRECTIONAL

$s=\lambda / 4$ $\psi=90^{\circ}\left(I_{2}\right.$ LAGS I,

EQUATORIAL PLANE $\theta=90^{\circ}$

FIGURE 26. Radiation patterns (field strength) for two dipole side-by-side array.

Three special eases are particularly to be noted. The field patterns for the equatorial plane $\left(\theta=90^{\circ}\right)$ and $\left|I_{2}\right|=\left|I_{1}\right|$ are plotted in Figure 26 . 
1. Broadside. Here $s=\lambda / 2$, the currents are in phase $\left(\psi=0^{\circ}\right)$. The maximum field is broadside and twice that of each dipole.

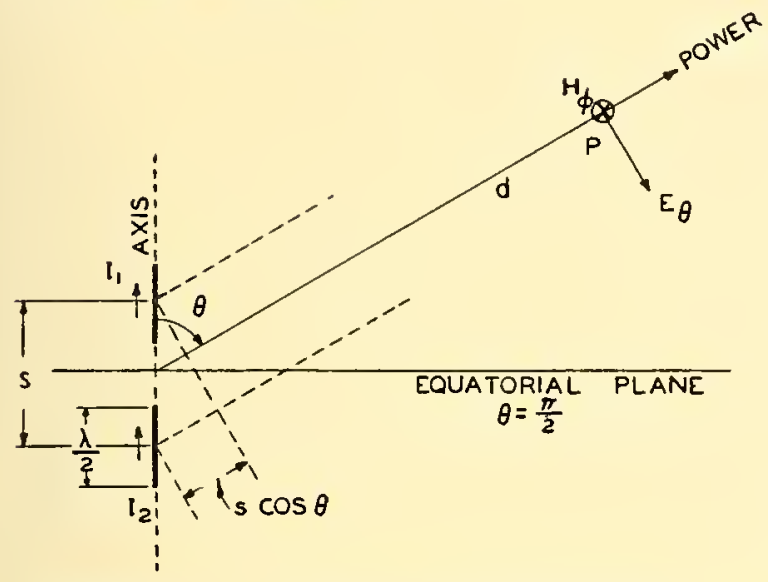

Figure 27. Two half-wave dipole colinear array.
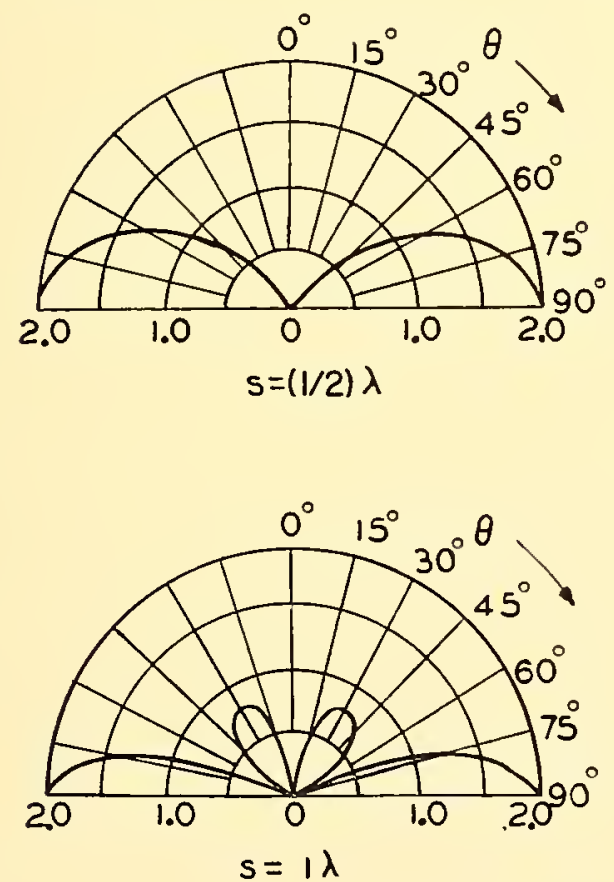

3.4 .4

\section{Two-Dipole Colinear Array}

For two equal eurrents in time phase (see Figure 27 ), the field is equal to

$$
E_{\theta}=-\frac{60 I}{d}\left[\frac{\cos \left(\frac{\pi}{2} \cos \theta\right)}{\sin \theta}\right]\left[\frac{\sin \alpha}{\sin \frac{\alpha}{2}}\right],
$$

where

$$
\alpha=\frac{2 \pi}{\lambda} s \cos \theta \text {. }
$$

The field is cireularly symmetrieal about the axis. Its variation with $\theta$ is plotted in Figure 28. This is, of course, equivalent to a rertical antenna with center leight at distance $s / 2$ above at perfeetly conducting flat earth.
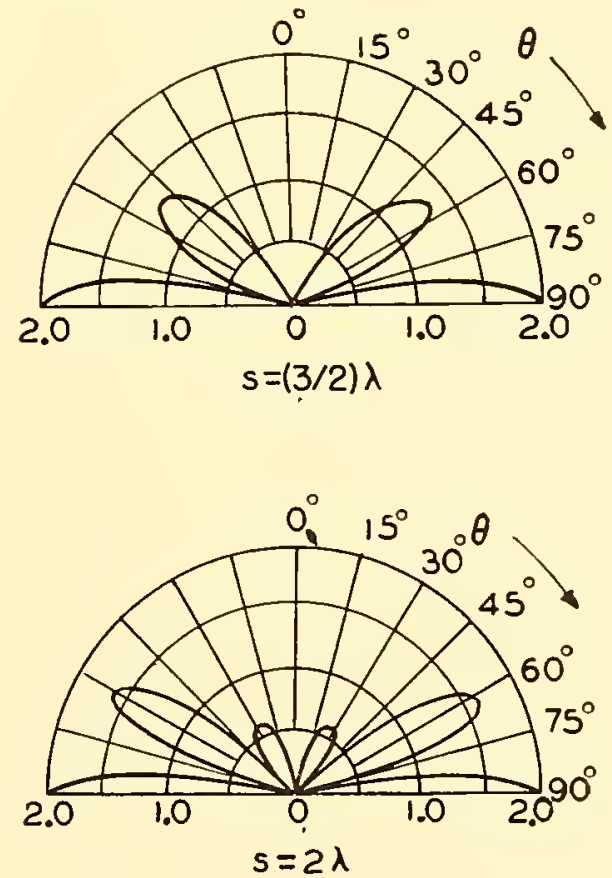

Figure 2S. Two half-wave dipole colinear array.

2. End-fire. Again $s=\lambda / 2$, but the currents are out of phase $\left(\psi=180^{\circ}\right)$. The maximum field is found in both directions along the line of centers.

3. Unidirectional couplet. Here $s=\lambda / 4$ and $I_{2}$ lags $I_{1}$ by $\psi=90^{\circ}$. The result of this combination is to produce a maximum field along the line of centers in the direetion looking from the leading to the lagging current and zero field in the reverse direction.

\subsubsection{One-Dimensional Array}

Two geometrical arrangenents will be considered.

1. Broadside. Consider $n$ elements with equal cophased currents equally spaced (see Figure 29).

$$
E_{\theta}=E_{e l}(\theta, \phi)-\frac{\sin \frac{n \alpha}{2}}{\sin \frac{\alpha}{2}},
$$




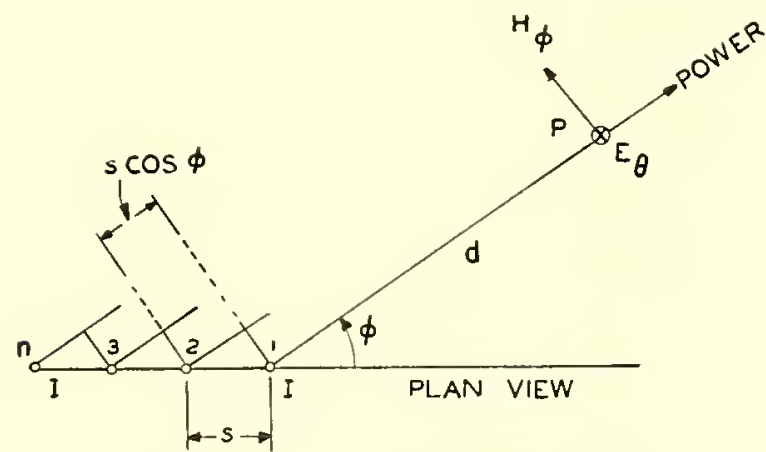

Figure 29. Broadside array, elements perpendicular to paper.

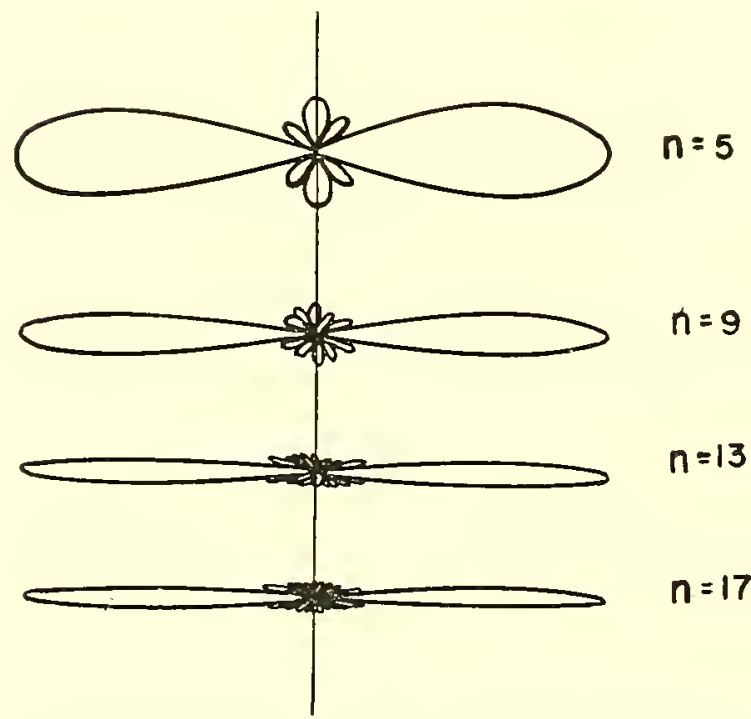

Figtre 30A. Effect of array length for broadside with element spacing $s=\lambda / 2$. (From Radio Engineers' Handbook by Terman.)

where

$$
\alpha=\frac{2 \pi}{\lambda} s \cos \phi .
$$

For center-fed half-wave dipoles, from equation (3)

$$
E_{e l}=\frac{60 I}{d} \frac{\cos \left(\frac{\pi}{2} \cos \theta\right)}{\sin \theta} .
$$

The patterns for the equatorial plane are illustrated in Figures $30 \mathrm{~A}$ and $30 \mathrm{~B}$. Figure $30 \mathrm{~A}$ shorrs the increase in directivity with increasing number of elements. Figure 30B illustrates the effect of element spacing on the production of side lobes. Figure 31 gires the gain for various spacings and array lengths. From this it appears that $s=5 \lambda / 8$ is approximately the optimum spacing.

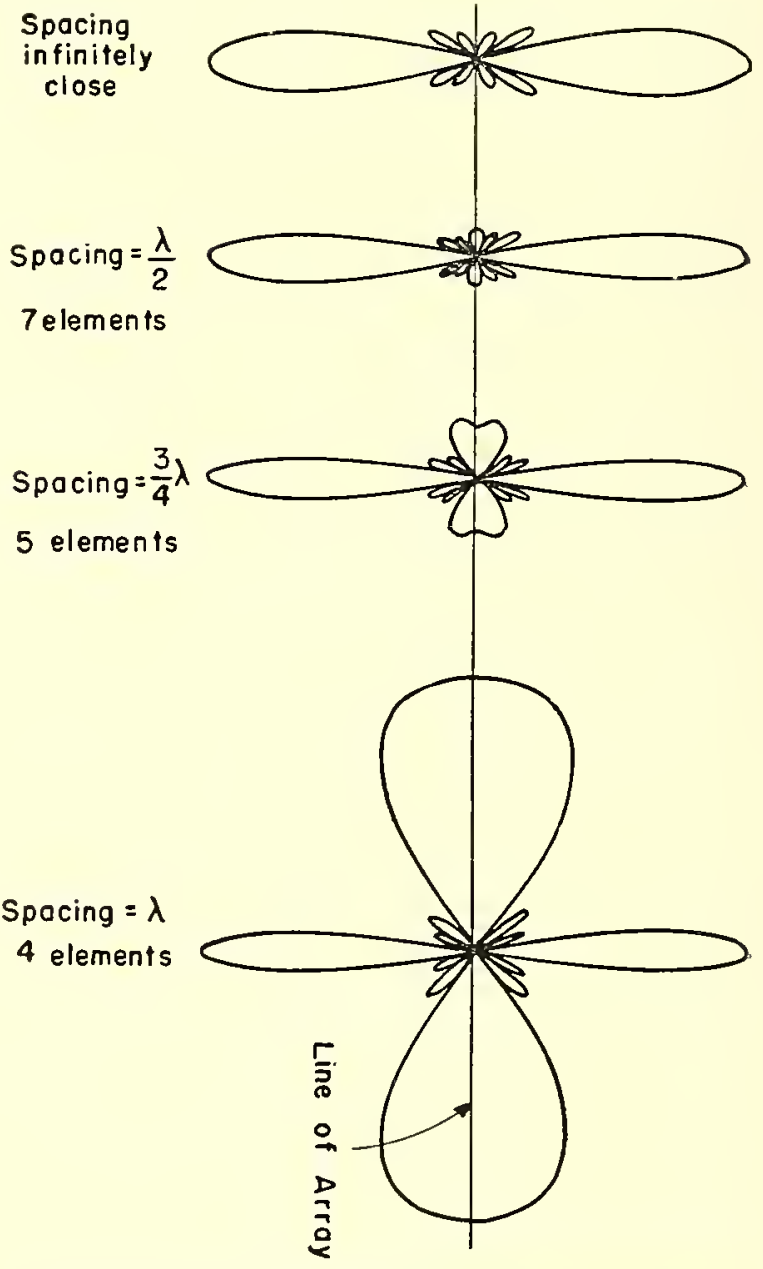

Figure 30B. Effect of element spacing for broadside for array length $L=3 \lambda$. (From Radio Engineers" Handbook by Terman.)

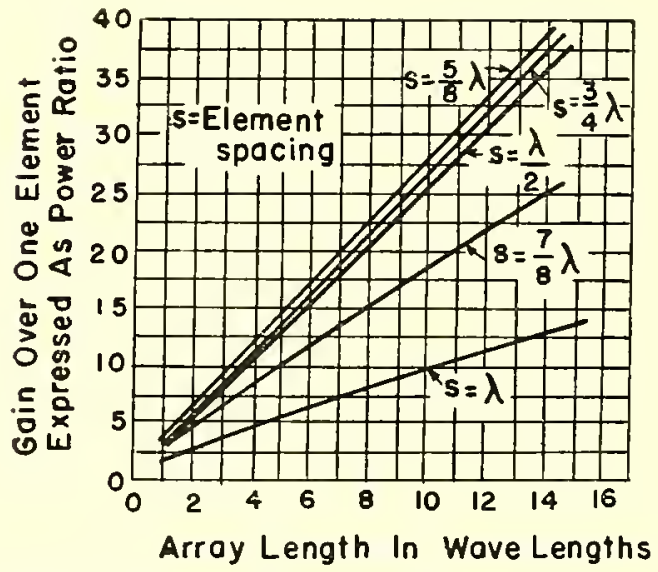

Figure 31. Gain for broadside array of doublets as a function of array length and spacing. (From Radio Engineers' Handbook by Terman.) 
2. Broadside: pattern factor and beam width. For illustration, suppose that the antenna consists of a vertical array of $m$ horizontal center-fed dipoles spaced $s$ apart with all fed in phase to give a broadside beam strongly direetive in the rertical plane. For this arrangement the field strength in the horizontal plane is given by $m$ times equation (27), that is,

$$
E_{\text {horizontal }}=m \frac{60 I_{i}}{d} \frac{\cos \left(\frac{\pi}{2} \cos \theta\right)}{\sin \theta}
$$

with angle $\theta$ measured from the dipole axis. See also equation (15) and Figure 11 (for $n=1$ ).

In the vertical plane, the beam is much narrower. If $\phi$ is the angle from the vertical and $\beta=90^{\circ}-\phi$ is the angle from the (horizontal) broadside direction, the field in the vertical plane $\left(\theta=90^{\circ}\right)$ is given liy

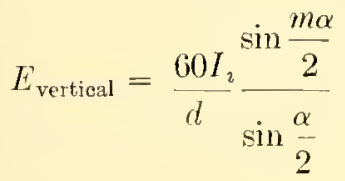

with

$$
\alpha=\frac{2 \pi}{\lambda} s \cos \phi=\frac{2 \pi}{\lambda} s \sin \beta
$$

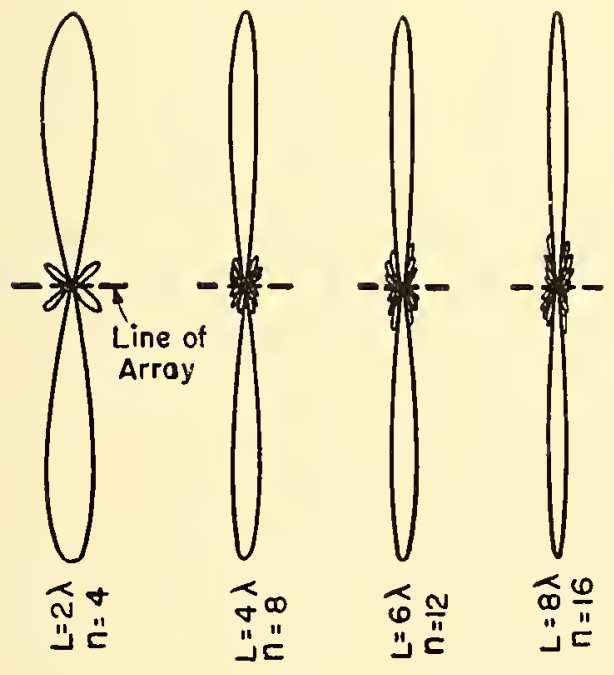

$$
\begin{aligned}
& \text { Midpoint Spocing } s=\lambda / 2 \\
& \text { Effect of Arroy Length }
\end{aligned}
$$
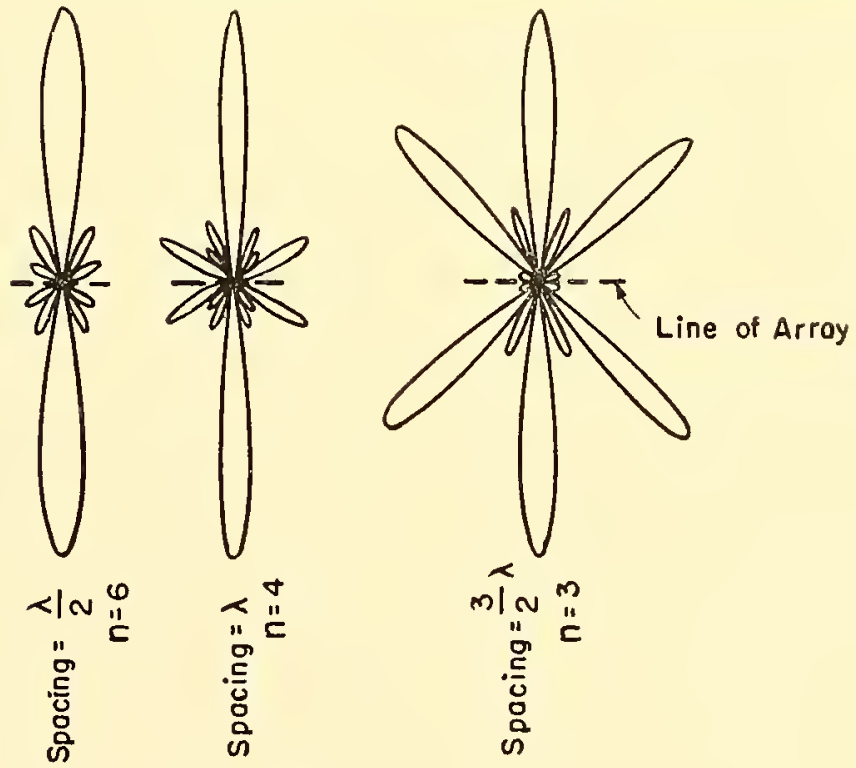

Array Length $=3 \lambda$

Effect of Element Spocing

Figure 33. Cophased colinear half-wave dipoles. (From Radio Engineers' Handbook by Termano)
Figure 32. Cophased colinear half-wave dipoles.

The bean width in the vertical plane is determined by the angle between the half-porrer points, or the angle between the points where the field strength is

$$
\frac{E_{\text {vertical }}}{E_{\max }}=\frac{\sin \frac{m \alpha}{2}}{m \sin \frac{\alpha}{2}} .
$$

The relative field strength is then

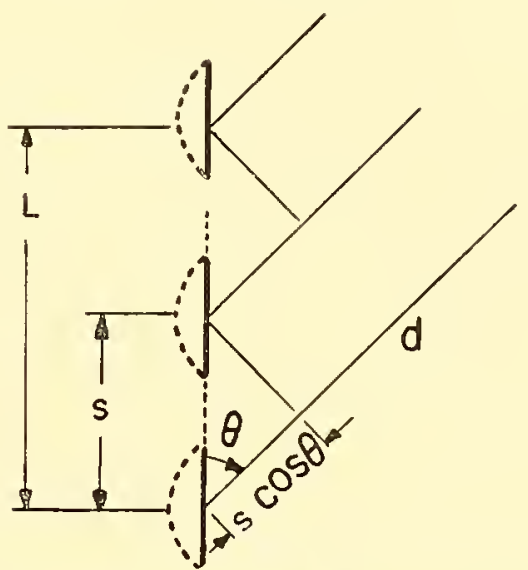

$$
\begin{aligned}
& \text { is } \\
& \text { ine }
\end{aligned}
$$

The maximum value of equation (29), corresponding

$$
E_{\max }=\frac{60 I_{i}}{d} m .
$$


0.707 of the maximum. Thus, equation (31) is set equal to 0.707 and $\beta$ determined. The beam width is equal to $2 \beta^{\mathrm{c}}$.

To illustrate, let $s=\lambda / 2$. For $m=2,3,4,8,12,16$ dipoles in the array, the corresponding beam widths are $60^{\circ}, 36.4^{\circ}, 26.4^{\circ}, 12.8^{\circ}, 8.5^{\circ}$ and $6.3^{\circ}$. A few field patterns are illustrated in Figure $30 \mathrm{~A}$ and gains are shown in Figure 31.

3. Colinear array (see Figure 32). For $n$ equal cophased currents, equally spaced,

$$
E_{\theta}=E_{e l}(\theta, \phi) \frac{\sin \frac{n \alpha}{2}}{\sin \frac{\alpha}{2}},
$$

where

$$
\alpha=\frac{2 \pi}{\lambda} s \cos \theta .
$$

For center-fed half-ware dipoles, from equation (3),

$$
E_{e l}=\frac{60 I_{i}}{d} \frac{\cos \left(\frac{\pi}{2} \cos \theta\right)}{\sin \theta} .
$$

The patterns for various array lengths and spacings are given in Figure 33.

If $s=\lambda / 2$, equation (32) for half-ware dipoles reduces to equation (18).

\subsubsection{Unidirectional Broadside and Colinear Arrays}

If an array is backed up with a similar array, the latter may serve to eoncentrate the radiation in one direction, provided the currents in the arrays are properly adjusted in magnitude and phase. Patterns for the unidirectional broadside and colinear arrays are given in Figure 34.

The broadside array is a collection of unidireetional couplets of the type illustrated in Figure 26. Increasing the number of couplets $n$ appreciably narrows the beam width.

A similar improrement is obtained in the colinear combination.

\subsubsection{Multidimensional Arrays}

Enough has already been given to show that it is not difficult to extend the principles of summation of fields from elements to cover two- and threedimensional arrays.
3.4 .8

\section{Binomial Arrays}

Most array patterns show, in addition to the main maximum, secondary maxima (side lobes) which are inconvenient in radar work. Side lobes are practically eliminated in the binomial array.

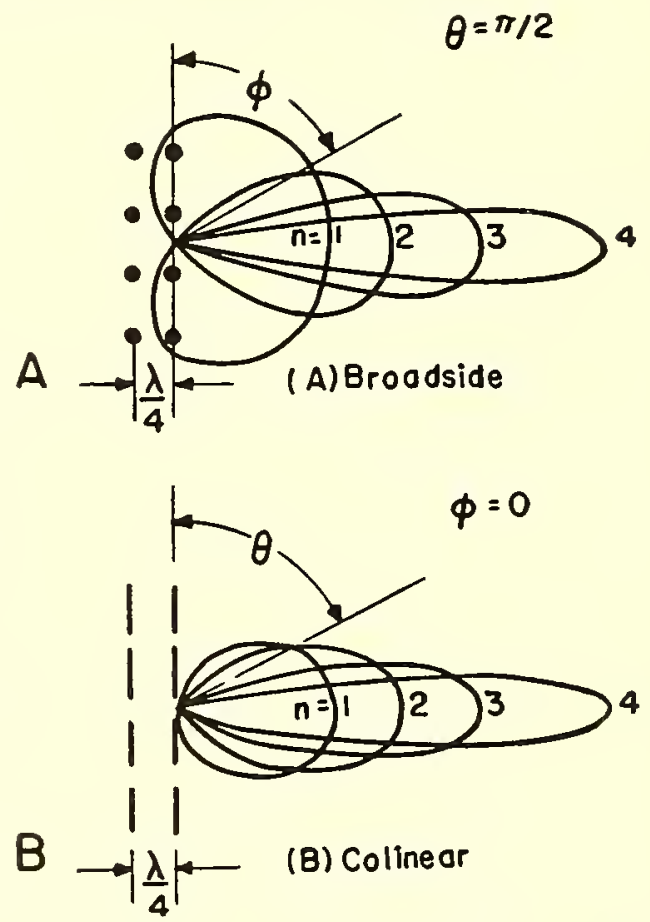

FIGURE 34. Unidirectional broadside and colinear array.

Consider first a two-element half-wave dipole broadside array with equal cophased currents and elements spaced a half wavelength apart. See equations (21) and (22). Then $\psi=0, s=\lambda / 2, \alpha=\pi \cos \phi$. Then with

$$
\begin{aligned}
E_{e^{\top}}(\theta) & =\frac{60 I}{d} \frac{\cos \left(\frac{\pi}{2} \cos \theta\right)}{\sin \theta}, \\
E & =E_{e l}\left(e^{j 0}+e^{-j \alpha}\right) .
\end{aligned}
$$

This gives the broadside field in Figure 26 which has only two equal major lobes. There are no side lobes.

Now consider the equation

$$
\begin{aligned}
E & =E_{e l}\left(e^{j 0}+e^{-j \alpha}\right)^{2}, \\
& =E_{e l}\left(1+2 e^{-j \alpha}+1 e^{-j 2 \alpha}\right) .
\end{aligned}
$$

This represents three half-rave dipoles in broadside; spacing $s=\lambda / 2$, currents in phase but with relative magnitudes $1: 2: 1$. The pattern has no side lobes. 
Again, for five similar dipoles,

$$
\begin{aligned}
E & =E_{e l}\left(e^{j 0}+e^{-j \alpha}\right)^{4} \\
& =E_{e l}\left(1+4 e^{-j \alpha}+6 e^{-j 2 \alpha}+4 e^{-j 3 \alpha}+1 e^{-j \downarrow \alpha}\right) .
\end{aligned}
$$

Here the cophased current magnitudes are $1: 4$ : $6: 4: 1$, and there are no side lobes.

The scheme is to follow the pattern of binomial coefficients in adjusting the relative current values.

$$
\begin{aligned}
& m=1 \\
& 2 \\
& 3 \\
& 4 \\
& 5 \\
& 11 \\
& \begin{array}{lll}
1 & 2 & 1
\end{array} \\
& \begin{array}{llll}
1 & 3 & 3 & 1
\end{array} \\
& \begin{array}{lllll}
1 & 4 & 6 & 4 & 1
\end{array} \\
& \begin{array}{llllll}
1 & 5 & 10 & 10 & 5 & 1
\end{array}
\end{aligned}
$$

Each number is the sum of the two immediately above.

3.4 .9

\section{Ring Arrays}

A set of radiating elements can be arranged on the perimeter of a circle with equal angular chistances and equal phase shift between the elements; the diameter of the ring must be properly chosen; the resulting radiation pattern can be made very nearly uniform, i.e., circular, in the plane of the ring, while the

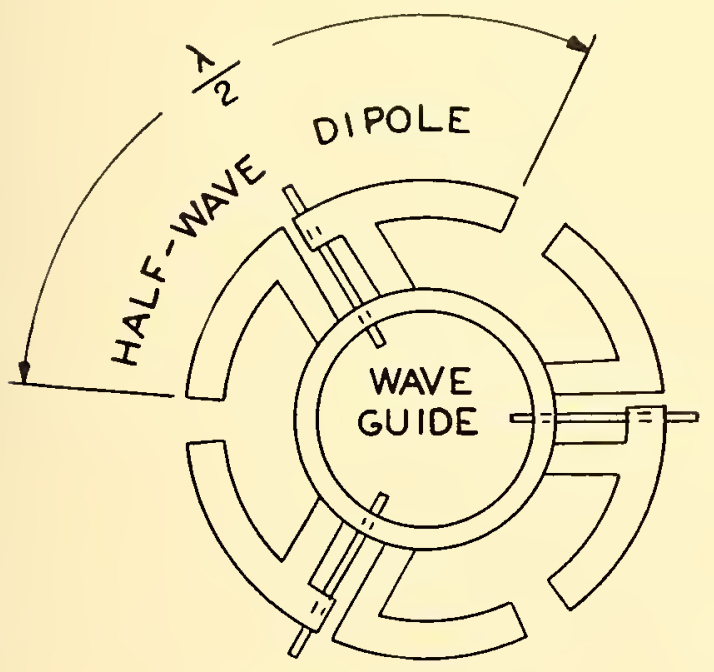

Figure 35. Ring array.

directivity of the pattern obtained in a plane perpendicular to that of the ring is increased compared to that of the single element.

If a number of such rings are stacked on top of each other with a common vertical axis, a linear array is formed whose elements are the rings. A radiation pattern is thus produced that has pronounced direc-

tivity in elevation while it is nearly uniform in azimuth. This device is frequently used in microwave beacons.

The most common form of the ring antenna is the triple dipole shown in Figure 35. The elements are threc half-wave dipoles spaced 120 degrees apart.

3.5

3.5.1

\section{PARASITIC REFLECTORS AND DIRECTORS}

\section{Parasitic Antennas}

A parasitic antenna or "dummy" is an antenna which is not connected to the antenna input terminals; if placed in the vicinity of a driven antenna a current is induced in the former which modifies the radiation field. Parasitic antennas provide a simple means of producing a moderate increase of directivity. Depending on the relative phase of the currents in the two antennas, the maximum of the radiation pattern either is found in the direction of the parasite and the latter is then called a director; or it is found in the direction of the primary element and the parasite is then called a reflector. In order to obtain good directive action the two dipoles must be close together, that is, a fraction of a wavelength.

\subsubsection{Half-Wave Dipole and Parasite}

The geometrical arrangement corresponding to the following discussion is illustrated in Figure 36. The radiation field at any point in the equatorial plane $\left(\theta=90^{\circ}\right)$ is equal to

$$
E_{\theta}=\frac{60 I_{0}}{d} e^{j 0}+\frac{60 I_{1}}{d} e^{j[\beta-(2 \pi / \lambda) s \cos \phi]},
$$

where $I_{0}$ is the center-fed input current to the antenna, $I_{1}$ is the center vahe of the current in the parasite, and $\beta$ is the angle by which $I_{1}$ leads $I_{0}$. The relation between $I_{1}$ and $I_{0}$ is given by

$$
I_{1}=I_{0} \frac{\left|Z_{01}\right|}{\left|Z_{11}\right|} e^{j \beta}
$$

where $Z_{01}$ is the vector mutual impedance of antenna and parasite, and $Z_{11}$, the vector self-impedance of the parasitic antenna, is equal to $R_{11}+j X_{11}=$ $\left|Z_{11}\right| e^{j \delta} . \delta$ is the phase angle of the parasite selfimpedance. 
The field pattern in the equatorial plane is dependent directly on the spacing and indirectly also, since the spacing controls the mutual impedance and thus the voltage induced in ${ }^{5}$ the parasite. The current ohms; if longer, the inductive reactance is increased; if shorter, it becomes at first less inductive, then resonant with $X_{11}=0$, and finally, capacitatively reactive. Field patterns for $s / \lambda=0.1$ and $s / \lambda=0.25$,

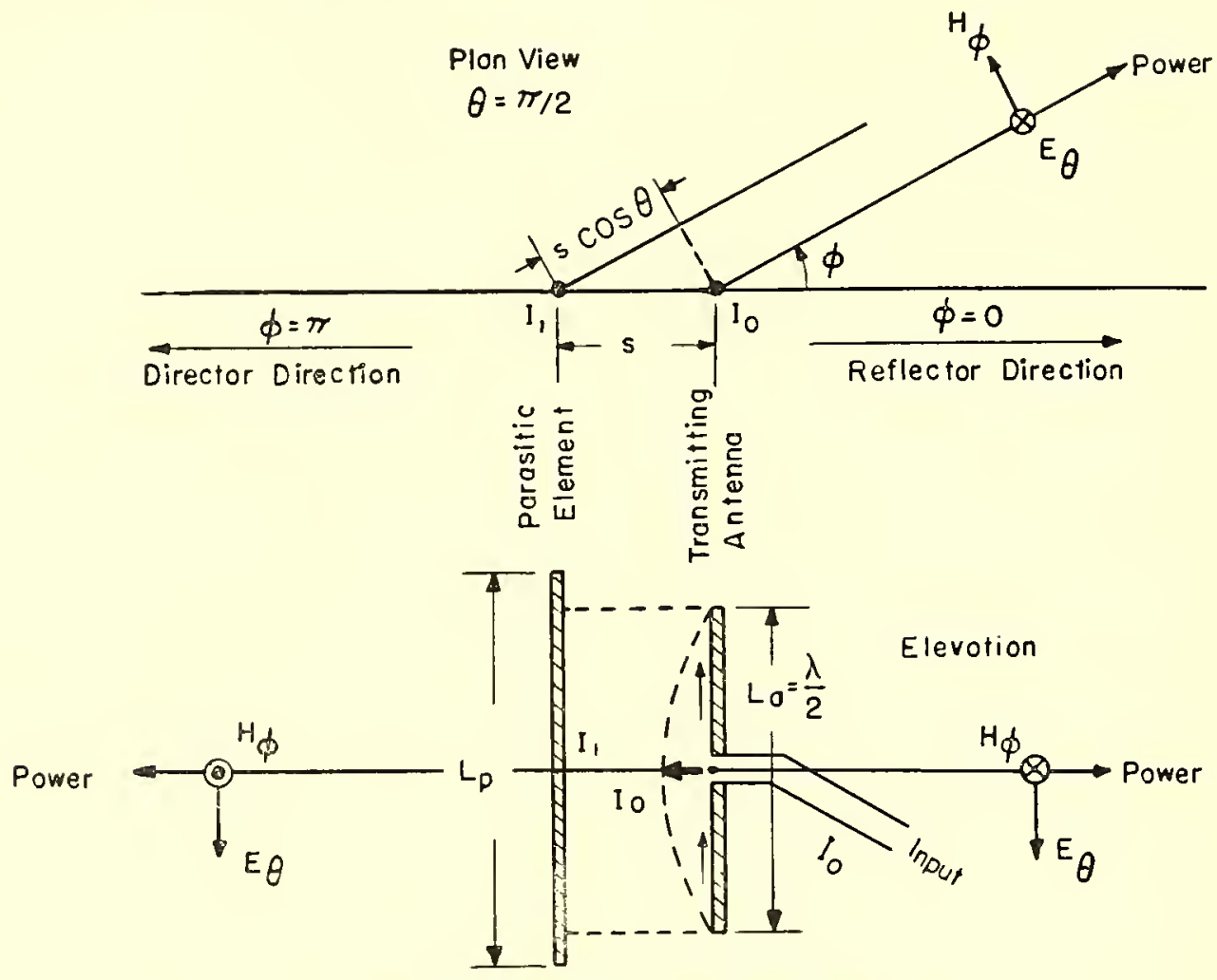

Flgure 36. Half-wave dipole and parasite.

in the parasite is further dependent on its selfimpedanec, which can be changed by altering the length of the parasite. Cut to a length of just $\lambda 2$, the self-impedance is inductive, $Z_{11}=73.1+j+2.5$

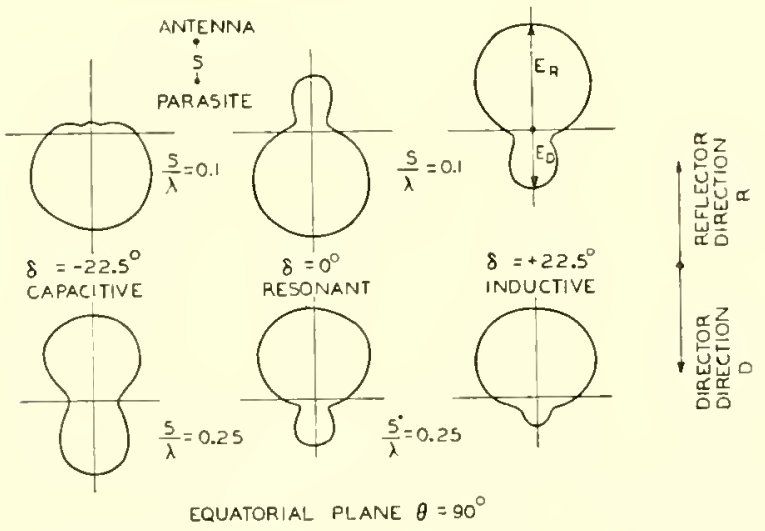

Figure 37. Relative field of half-wave antenna and parasite. (Courtesy of I. R. E.) and for $\delta=+22.5^{\circ}, 0^{\circ}$, and $-22.5^{\circ}$, are plotted in Figure $3 \pi$. These illustrate that, by controlling the spacing and length of parasite, it is possible to dircet the pattern maximum into either the $R$ or $D$ direction, so that the parasite acts primarily either as a reflector $\left(E_{R}>E_{D}\right)$ or as a director $\left(E_{D}>E_{R}\right)$.

1. Purasite as a reflector. For good reflector performance, the spacing $s / \lambda$ should lie between 0.15 and 0.25 with the parasitic element made slightly longer (perhaps 5 per cent) than $\lambda / 2$ in order to increase its inductive reactance. $A$ few of the equatorial field patterns are shown in the lower row of Firsure 37. To olstain the strongest field in the $R$ direction, it is necessary to lengthen the parasite to a particular length (obtained by trial). If this is done, Figure 38 indicates that the ficld $E_{R}$ is a maximum for $s / \lambda=0.15$ and that the ratio of $E_{R}$ to $E$ for the antenna atone is 1.83 ; for $s / \lambda=0.25$ it is 1.65 . This does not, however, give the best front-to-back ratio. 
2. Parasite as a director. Good director performance is obtained when $s / \lambda=0.1$ and the parasitic element is cut slightly shorter (perhaps 4 per cent) than $\lambda / 2$ to produce a capacitative reactance. Sec Figure 37, upper row, for the field patterns and

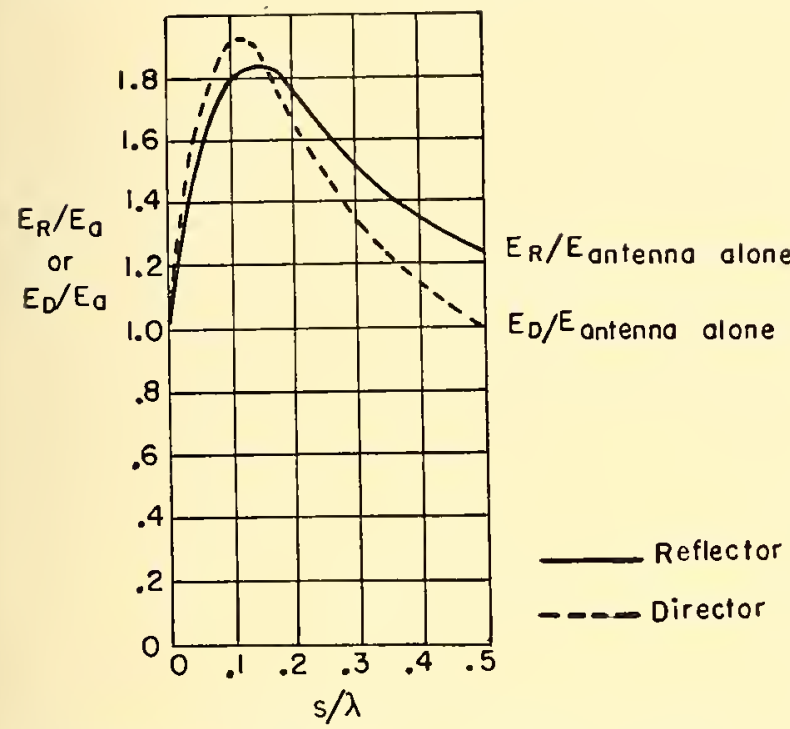

Figure 38. Adjustment of parasite for strongest fields $E_{R}$ and $E_{D}$. (Courtesy of I. R. E.)

Figure 38 for the best ratio of $E_{D}$ to $E$ for the antenna alone. The latter, again, does not give the best front-to-back ratio.

\section{Multiple Parasites. Yagi Antennas}

By using several parasites, rather pronounced directive effects can be achieved. Figure 39 shows a typical example. This antenna uses three parasitic

\section{PARASITES PATTERN}

$$
\begin{aligned}
& a=.248 \lambda \\
& b=.588 \lambda \\
& c=.535 \lambda
\end{aligned}
$$
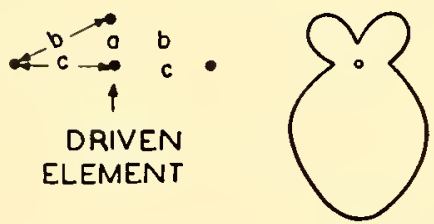

Figure 39. Antenna with three parasite elements. (From Radio Engineers' Handbook by Terman.)

dipoles arranged in a triangle or parabolic curtain. In order to obtain the most favorable pattern in such cases, careful tuning of the parasites is required.
The most commonly used of the multiparasitic arrays of half-wave dipoles is the Yagi antenna (Figure 40). It has one reflector and several (usually 2 to 5) directors. Since the voltage at the center of a dipole is always zero, it is possible to weld all the

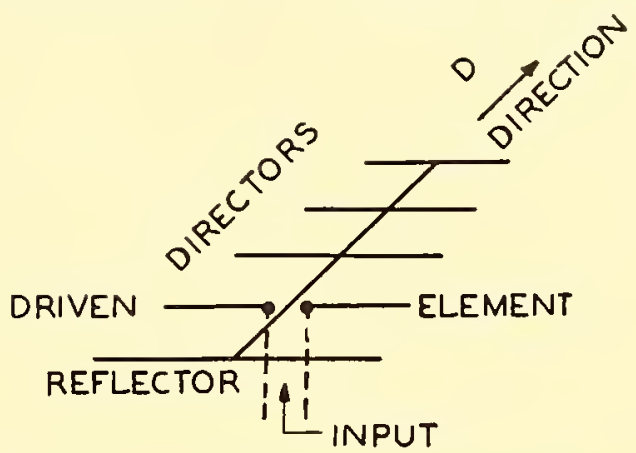

Figure 40. Tagi antema with three directors.

parasites to a central sustaining rod, as shown. By increasing the number of directors, it is possible to obtain highly directive patterns.

The spacings between the elements of a Yagi array are not uniform. They are determined so that the phase difference of the currents in adjacent elements is equal to their distance expressed in wavelengtis. If this condition is fulfilled, the elements are in phase with respect to radiation in the $D$ direction. In practice, the spacing is determined experimentally rather than by calculations, which become very cumbersome when several directors are employed.

3.5 .4

\section{Reflecting Screens}

A plane-conducting screen placed behind a radiating dipole has a similar effect in the forward direction as an inage dipole whose distance $s$ from the prinary dipole is twice that of the screen and which has a phase shift of $180^{\circ}$ from the primary dipole. Radiation in the backward direction is confined to the weak fields leaking around the edgos of the screen. The pattern in the forward direction is given by the array formula of equation (22), and end-fire array with $\psi=180^{\circ}$ and $s=\lambda / 2$. Good results are achieved when the distance from the screen to the dipole is small (less than $\lambda / 4$ ) but larger spacings are also used. The change in input impedance of the primary element caused by the presence of the screen is appreciable.

Reflecting screens are used primarily in connection with broadside arrays (curtains) to eliminate one 
of the two main lobes in opposite directions. An adequate screening effect is produced by a set of wires parallel to the direction of the radiating dipole with spacings somewhat less than a tenth of a wavelength.

\subsubsection{Corner-Reflector Antenna}

A simple directional derice that gives an appreciable power gain (of the order of 10 to 20 ) is a corner reflector, which is essentially a combination of two reflecting sheets and a dipole. In the case shown in Figure 41 where the angle subtended by
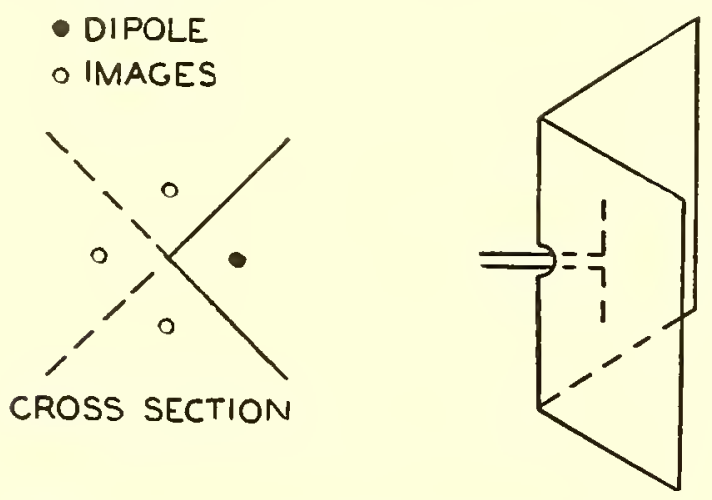

Figure 41. Corner reflector intenna.

the corner is $90^{\circ}$, the corner is equivalent to the combined radiation of three image antemnas. The reflector can also be made of wires parallel to the direction of the radiating dipole. The reflecting wires do not, however, act as parasitic antennas but are taken so long that they are practically equivalent to conductors of infinite length.

These should not be confused with corner reflectors which are extensively used as targets and consist then of three mutually perpendicular conducting planes (see Section 9.2.4).

\subsection{PARABOLIC ELEMENTS \\ 3.6.1 Parabolic Reflectors}

These reflectors are the derices most commonly used to produce highly directive rachation patterns in the microware region. The three main types are shown in Figure 42; they are the parabolic cylinder, the paraboloid of revolution, and the truncated paraboloid, the latter being a rectangular section cut from a paraboloid of revolution. If the parabolic cylinder is relatively short and provided with flat metallic covers at the top and bottom, its shape and

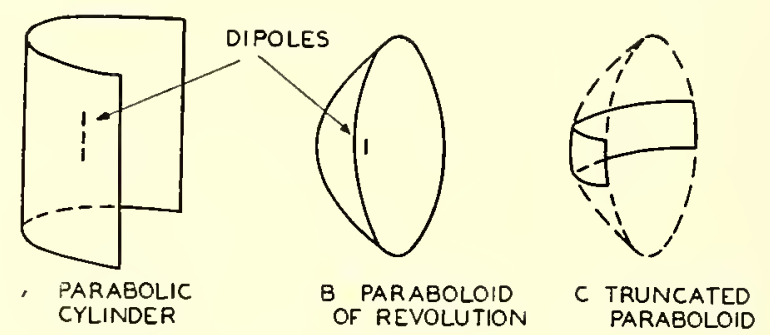

Figcre 42. Types of parabolic reflectors.

its electrical properties resemble those of a sectoral horn (see Section 3.7.2).

The directive action of the parabolic reflector depends on two geometrical properties of the parab-

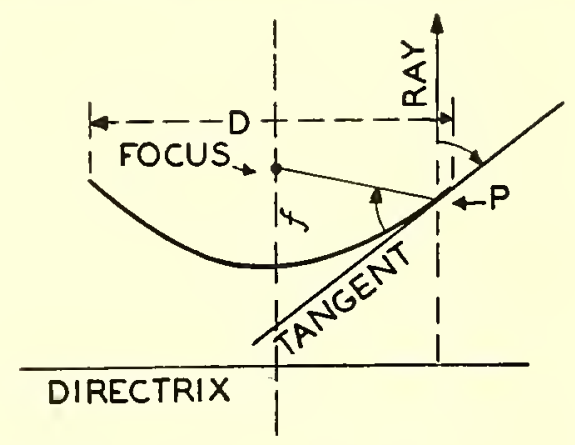

Figure 43. Properties of a parabola.

ola (Figure 43). A ray coming from the focus is reflected into a cirection parallel to the axis of the parabola, and the distance from any point $P$ on the parabola to the line called the directrix is equal to the distance from $P$ to the focus. Consequently, the effect of the parabola in the forward direction is equivalent to that of a distribution of sources in the directrix that all oscillate in phase (but usually have varying intensities over the directrix).

The parabolic eylinder produces a directive pattern only in a plane perpendicular to the generating line of the cylincler (horizontal plane in A of Figure 42). In order to concentrate the beam in a plane parallel to the generating line of the cylincter (rertical plane in Figure 42), an additional directive device must be employed. Usually this is a colinear array of dipoles, as shown; the direction of polarization is parallel to the focal axis. In microwave work this type of antenna offers advantages over the tro-dimensional curtain of dipoles employed in VHF directional antennas. 
For the paraboloid of revolution or the truncated paraboloid, a simple source of radiation at the focus is used. Often this is a half-wave dipole, sometimes combined with a parasitic dipole which acts as a reflector (Section 3.5.2).

In other types, the energy is brought to the focal point by a wave guide and is then reflected back onto the parabolic surface.

If the warelength is small compared with the dimensions of the parabolic reflector, the following approximate formula holds for the radiation pattern produced by a parabola:

$$
E=\mathrm{constan} t \frac{\sin \left[\frac{\pi D}{2} \sin \frac{\theta}{\lambda}\right]}{\pi D \sin \theta / \lambda}(1+\cos \theta),
$$

where $D$ is the aperture of the reflector and $\theta$ the angle from the axis. The half-power points correspond approximately to

$$
\sin \theta \cong \theta \cong \frac{0.52 \lambda}{D}
$$

These formulas correspond to the case of nearly uniform illumination of the reflector from the source at the focus. In practice a source that concentrates the field toward the center of the parabola is used in order to reduce the magnitude of the sicle lobes.

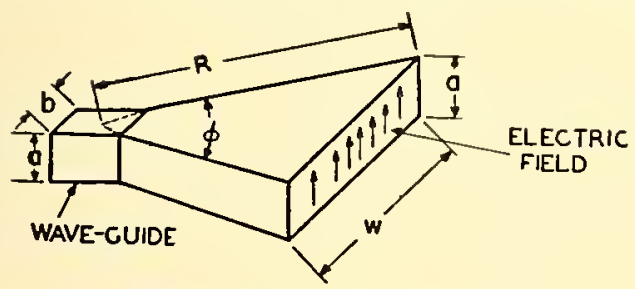

Figure 44. Sectoral horn.
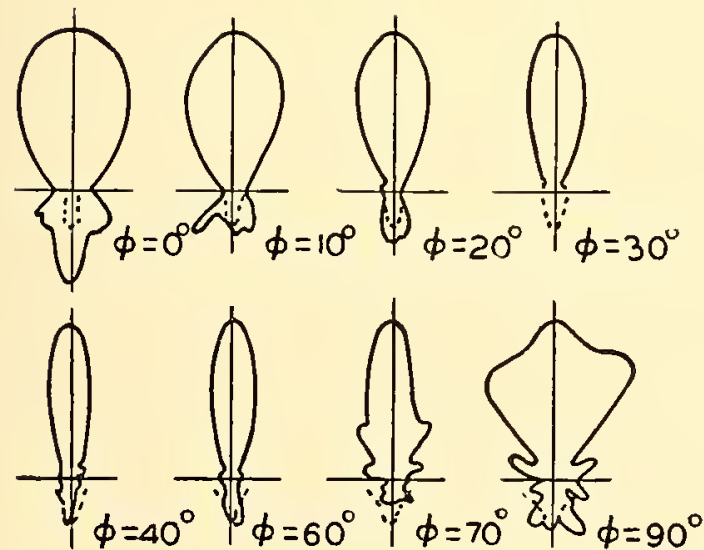

Figure 45. Radiation pattern for a sectoral horn having various flare angles.
The half-power angle is then more nearly equal to $\theta=0.6 \lambda / D$.

The maximum gain of a parabolic reflector is

$$
G=\left(\frac{\pi D}{\lambda}\right)^{2} \frac{1}{4}
$$

For $D=2$ meters and $\lambda=0.1$ meter, the gain is approximately 1,000 .
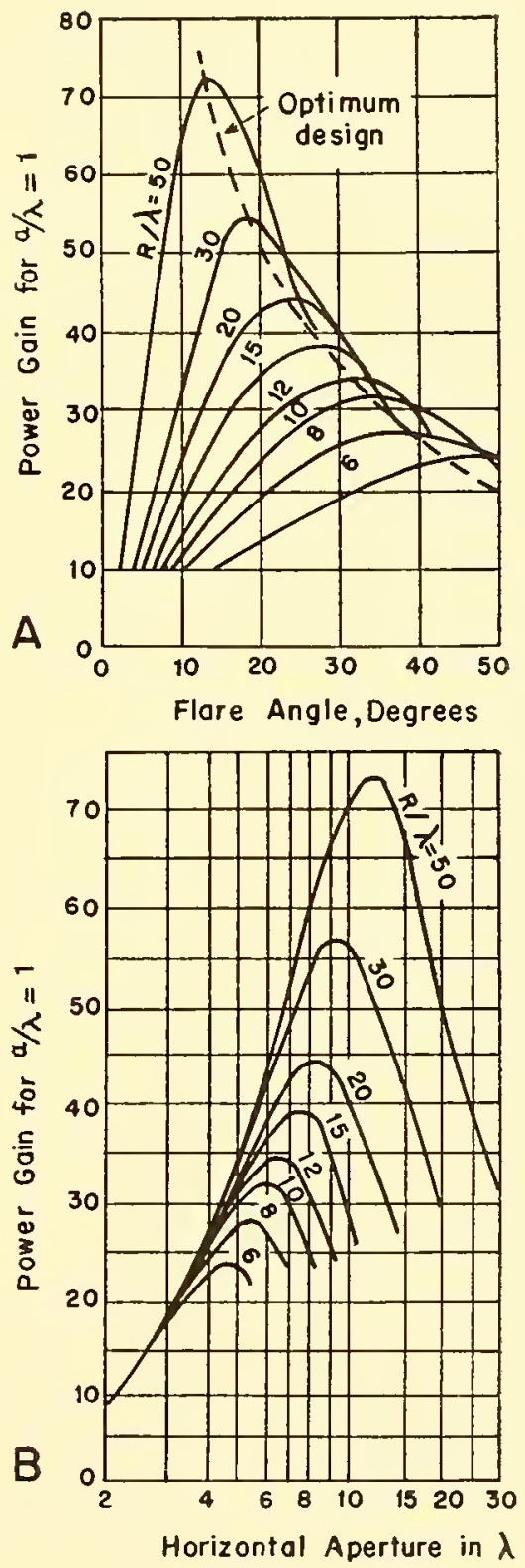

Figure 46. Gain of sectoral horn with $T E_{1}, 0$ wave. (These curves are for a vertical aperture ratio $a / \lambda=1$. For other ratios the gain given should be multiplied by $a / \lambda$.$) (From Radio Engineers' Handbook by Terman.)$ 
3.7

HORNS

3.7 .1

\section{Types of Horns}

Many of the antennas previously described are used in the high-frequency $[\mathrm{HF}]$ and very highfrequency [ $[\mathrm{THF}]$ bands of frequencies. Horns cannot readily be used at these frequencies because the sizes required would be excessive.

But at the microwave frequencies, the size of the horn is small and it is easy to feed energy to it through a ware guide. In this arrangement the horn acts as transition between the impedance of the wave guide and the 377 ohms impedance of free space and thus reduces to a minimum the reflection of energy backward into the guide (such as would occur if the wave guide ended in an open pipe).

Common types of horns are sectoral (discussed in Section 3.7.2), pyramidal, conical, biconical, ete. Only the first type is discussed in this section.

\subsubsection{Sectoral Horn with $T E_{1,0}$ Wave}

For this case the horn is flared only in width and is an extension of the wave guide of width $b$ and depth $a$. For the $T E_{1,0}$ wave the electric field is parallel to the dimension $a$ and varies in strength cosinusoidally across the wave guide and horn opening, as shown in Figure 4t. The length of the horn is $R$ and the flare angle is $\phi$.

Figure 45 illustrates the pattern shapes in the plane parallel to dimension $b$ for various flare angles.

For this type of wave, the cutoff frequency of the wave guide is

$$
f_{c}=\frac{3 \times 10^{8}}{2 b}
$$

with $b$ in meters. The operating frequency should be near but not greater than trice this value.

The gain depends upon the length $R$ and the flare angle $\phi$, and is plotted in Figure 16. 


\section{Chapter 4 \\ FACTORS INFLUENCING TRANSMISSION}

4.1

4.1.1

$\mathrm{R}^{\mathrm{n}}$

EFRACTION is caused by the variation of the dielectric constant (squiare of refractive index) of the atmosphere. Although the atmosphere is tenuous and the variations of refractive index are small, the effect of refraction upon the fieldstrength distribution of waves is considerable. As will be shown, refraction under average conditions may be taken into account by using an earth with a modified radius. A representative average value of modified earth radius commonly used is lia with $k=4 / 3$. Under certain conditions, especially in warmer climates, a slightly higher value of $k$ might be preferable.

The case where $a$ is replaced by $4 a / 3$ is referred to as standard refraction. It corresponds to a linear variation of refractive index with height in the atmosphere. In lecent years, more complicated variations of refractive index in the atmosphere have received considerable attention and have proved to be of great operational interest. This volume, however, is restricted to consideration of standard atmosphere propagation.

4.1 .2

\section{Snell's Law}

Let $n_{0}$ and $n_{1}$ denote the refractive indices of two media separated by a plane boundary. The ordinary law of refraction known as Snell's law is then usually stated (see Figurc 1), as

$$
n_{0} \sin \beta_{0}=n_{1} \sin \beta_{1},
$$

where $\beta_{0}$ and $\beta_{1}$ are the angles which the ray makes with the perpendicular to the boundary. It is convenient to use the complementary angle $\alpha$, so that

$$
n_{0} \cos \alpha_{0}=n_{1} \cos \alpha_{1} \text {. }
$$

For several plane-parallel boundaries, Snell's law generalizes to

$$
n_{0} \cos \alpha_{0}=n_{1} \cos \alpha_{1}=n_{2} \cos \alpha_{2}=\cdots .
$$

In the atmosphere, the refractive index is a continuous function of the height. Again, it is usually legitimate to consicler the atmosphere as horizontally stratified, so that the refractive index is a function of height only. The case of a continuously variable refractive inclex is readily obtained by

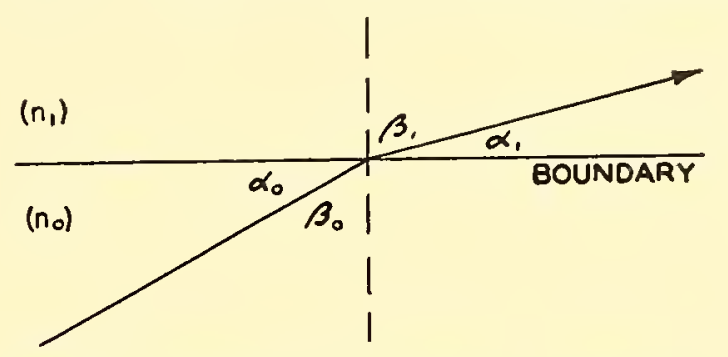

Figure 1. Refraction at bonndary between two media.

passing to the limit of an infinity of parallel boundaries infinitely close together, Sncll's law remaining the same; thus

$$
n(h) \cdot \cos \alpha=n_{0} \cos \alpha_{0}
$$

where now $n$ and $\alpha$ are continuous functions of the height. In place of a discontinuous change in direction, there will now occur a bending of the rays (Figure 2).

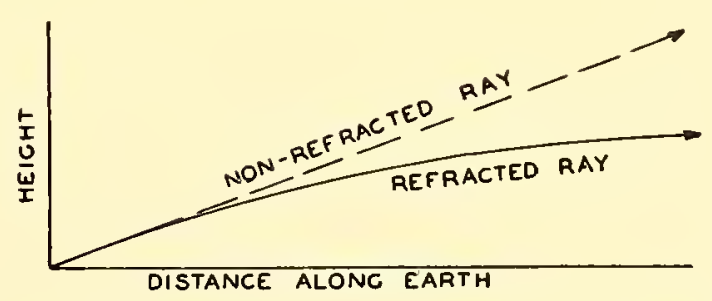

FIgURE 2. Refraction in the atmosphere with variable $n(h)$.

If the boundaries are not plane but spherical, Snell's law must be modified. Analysis shows that over a spherical earth surrounded by an atmosphere in which the refractive index $n$ is a function of the distance $r$ from the earth's center, the law of refraction becomes

$$
n(r) \cdot r \cos \alpha=n_{0} r_{0} \cos \alpha_{0}
$$

where $\alpha$ is the angle between a ray and the horizontal (see Figure 3). 
Refraction is of practical importance only when the angle between the rays and the horizontal is small. In the determination of gain as given in later

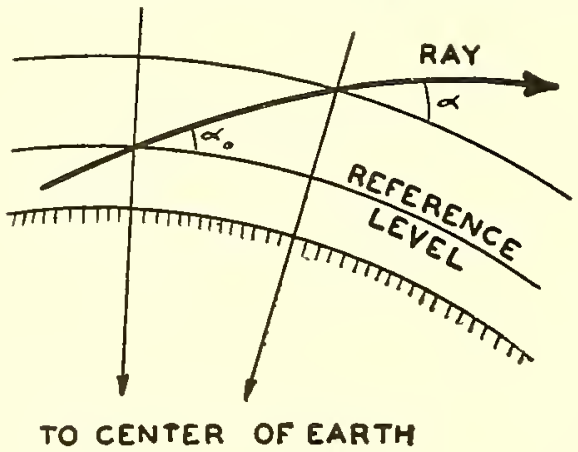

Figure 3. Refraction over curved earth.

chapters, the effect of refraction becomes completely negligible when $\alpha$ is more than a few degrees.

For small angles, $\cos \alpha$ may be replaced by $1-\alpha^{2} / 2$. In this case equation (1) is well approximated by

$$
\frac{1}{2}\left(\alpha^{2}-\alpha_{0}^{2}\right)=n-n_{0}+\frac{h}{a},
$$

where $h$ is the height above the ground, so that $r_{0}=a$ and $r=a+h$. This is the practical form of Snell's law for the atmosphere above a curved earth. The reference level (see Figure 3) is here taken at the surface of the earth where $n_{0}$ is the index of refraction.

\subsubsection{Modified Refractive Index}

In place of the sum $\left(n_{0}+h / a\right)$ that appears in equation (2), it is customary to define and use a quantity $M$ given by

$$
M=\left[(n-1)+\frac{h}{a}\right] 10^{6} .
$$

$M$ is called the modified refractive index. It gives a unit that is convenient for practical use. The modified index is then said to be expressed in $M$ units, values of which commonly lie in the range of 300 to 500 . Using this definition, equation (2) becomes

$$
\frac{1}{2}\left(\alpha^{2}-\alpha_{0}^{2}\right)=\left(M-M_{0}\right) \cdot 10^{-6} .
$$

An important special case is that in which the refractive index decreases linearly with height, $n-n_{0}=$ constant $\times h$. Then equation (2) may be written in the form

$$
\frac{1}{2}\left(\alpha^{2}-\alpha_{0}^{2}\right)=\frac{h}{l a},
$$

where $k$ is the factor mentioned in Section 4.1.1 which determines the modified earth's radius ka. Comparing the above expression with equation (2), and differentiating, it follows that

$$
\frac{1}{k a}=\frac{d n}{d h}+\frac{1}{a}
$$

or

$$
k=\frac{1}{1+a \frac{d n}{d h}}-\frac{1}{a} \cdot \frac{d h}{d M} \cdot 10^{-6} .
$$

Proof of the fact that refraction is negligible unless the angle is very small may readily be deduced from the preceding formulas. Thus, on differentiating equation (4),

$$
d \alpha=d M \cdot \frac{10^{-6}}{\alpha}
$$

and in the standard linear case, by equation (5),

$$
d \alpha=d h / k \alpha \alpha \sim 1.2 \cdot 10^{-7} \mathrm{dh} / \alpha
$$

for $k=43$. Taking $\alpha=0.05$ radians $\left(3^{\circ}\right)$ and $d h=100$ meters, one finds $d \alpha=0.00024$ radians (50 seconds of arc), a very small change in angle. This is the standard deflection which is accounted for by replacing $a$ by $k a$. The deviations from this value experienced with nonstandard refraction are even smaller. The larges the angle $\alpha$ with the horizontal at which a lay issues from the transmitter, the less the angular deviation. In communication work and for certain radar problems, however, angles of less than one degree are of importance, and $d \alpha$ may then become comparable to $\alpha$.

\section{4.i.4 Graplical Representation}

Figures 4 to 6 show three different ways of representing rays subject to refraction. Figure 4 gives a true picture apart from the exaggeration of heights. In the case of standard lefraction, the curvature of the rays is always concave downwards, the center of curvature being below the surface of the earth. The middle ray shown is the horizon ray and to the lower right is the diffraction region into which rays do not penctrate. Figure 5 shows a diagram with 
modified earth's radius, $k a$, in which the rays are straight lines. Figure 6 , finally, is a plane earth diagram; the rays are here curved upwards.

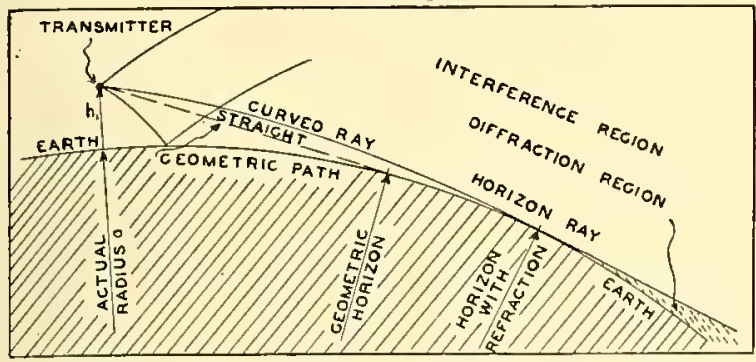

FIgURe 4. Ray curvature over earth with radius $a$.

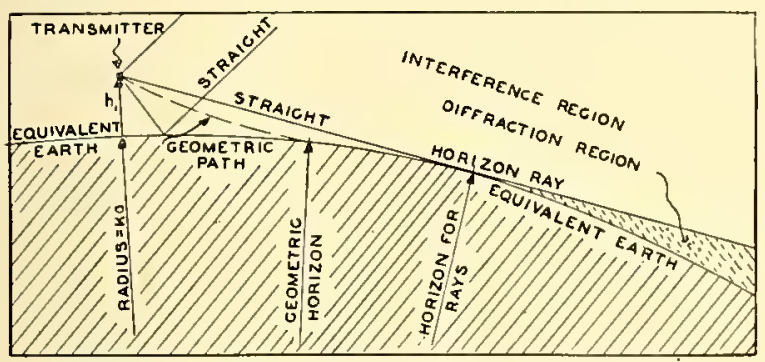

Figure 5. Rays in a homogeneous atmosphere (equivalent radius $k a$ ).

These diagrams may be considered as resulting from each other by changing the earth's curvature by an arbitrary factor. From this viewpoint Figure 6

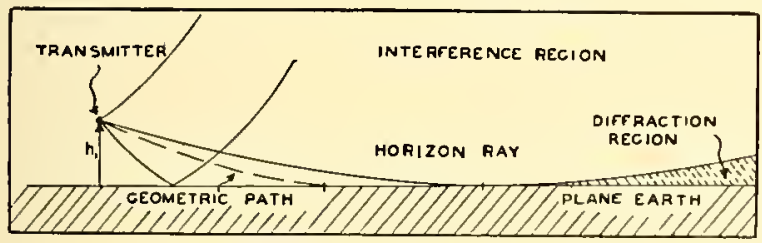

Figure 6. Rays in a plane earth diagram. (Radius of curvature of rays is $-k a$.)

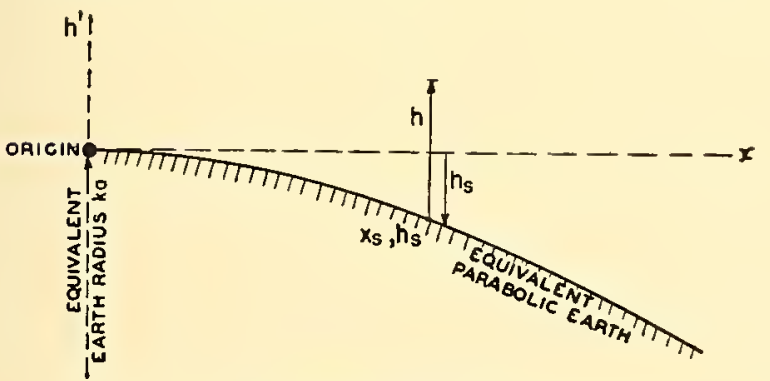

Figure 7. Equivalent parabolic earth diagram.

represents the limiting case of an infinite earth's radius. The plane earth diagram is widely used for problems of nonstandard propagation.
In drawing diagrams for a curved earth of equivalent radius $k a$, it is customary to replace the spherical earth outline by an equivalent parabola (see Figure 7). The equation for the surface reduces from the circular form,

$$
x_{s}^{2}+\left(h_{s}+k a\right)^{2}=(k a)^{2},
$$

to the parabolic form,

$$
h_{s}=-\frac{1}{2 k a} x_{s}{ }^{2},
$$

for $h_{s}<<x_{s}$. The height $h$ measured from the surface of the earth, instead of from the $x$ axis, is given by

$$
h=h_{s}+\frac{1}{2 l_{l} a} x^{2},
$$

in which $h$ is laid off perpendicular to the $x$ axis and not to the earth's surface. For elarity in drawing rays or field-strength diagrams, the vertical scale is expanded by an arbitrary factor $p$, whence

$$
h=p\left(h_{s}+\frac{1}{2 k a}-x^{2}\right) .
$$

This distortion of vertical distances, it can be shown, does not distort angles. The parabolic representation to be reasonably aceurate must be restricted to leights in the atmosphere small compared with the extent of the horizontal seale.

\subsubsection{Curvature Relationships}

The curvature of a ray is defined as the reciprocal of the radius of curvature $\rho$. Let $\psi$ be the angle between the ray and a nearly horizontal $x$ axis.

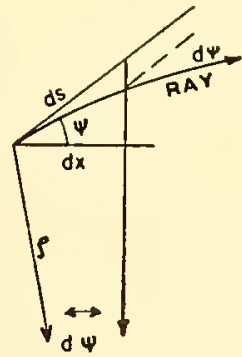

A

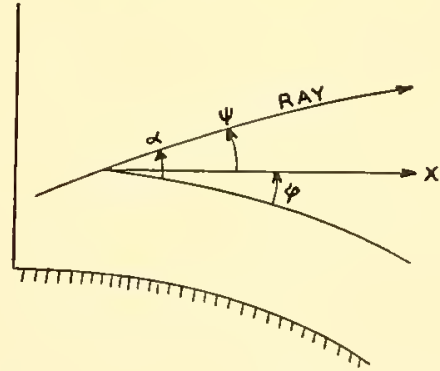

8
Figure 8. Angular relationships of rays.

By Figure $\delta, \rho=-d s / d \psi$, and since $\psi$ is a small angle we may, to a sufficient approximation, put $d s=d x$, so that

$$
\frac{1}{\rho}=-\frac{d \psi}{d x}
$$


Here the curvature has been defined so that it is positive when the ray curves in the same direction as the earth; with this system the curvature of the earth itself is positive. Referring to Figure SB,

$$
\frac{1}{\rho}=-\frac{d \psi}{d x}=-\frac{d \alpha}{d x}+\frac{d \phi}{d x} .
$$

But $d \phi, d x=1 / a$, and since $\alpha$ is a small angle

$$
\frac{d \alpha}{d x}=\frac{d \alpha}{d h} \cdot \frac{d h}{d x}=\alpha \frac{d \alpha}{d h}=\frac{1}{2} \frac{d\left(\alpha^{2}\right)}{d h} .
$$

Consequently, by equation (2)

$$
\frac{1}{\rho}=-\frac{1}{2} \frac{d\left(\alpha^{2}\right)}{d h}+\frac{1}{a}=-\frac{d n}{d h} .
$$

From this, the curvature of the ray is equal to the vertical rate of decrease of the refractive index. Notice that $d n / d h$ is msually negative, so that the true curvature of a ray is usnally concave downwards.

A simple relationship exists between $m=\rho^{\prime} a$, the ratio of the radius of curvature of a ray to the radius of the earth, and $k$. Combining equations (6) and $(9)$ gives

$$
\frac{1}{k}+\frac{1}{m}=1 .
$$

Consider again the special case where $d n / d h=$ constant, so that $n$ is a linear function of the height (standard refraction). Consider the plane earth diagram of Figure 6 . The angles between corresponding curres are the same as in the true diagram, Figure 4. Hence, for the plane earth diagram, equation (s) becomes $1 / \rho^{\prime}=-d \alpha / d x$, where $\rho^{\prime}$ is the raclius of curvature of the ray in the plane earth representation. It is readily found that

$$
\begin{aligned}
\frac{1}{\rho^{\prime}}=\frac{1}{\rho}-\frac{1}{a} & =-\frac{1}{2} \frac{d\left(\alpha^{2}\right)}{d h}=-\left(\frac{d n}{d h}+\frac{1}{a}\right) \\
& =-\frac{d M}{d h} \cdot 10^{-6}=-\frac{1}{k a} .
\end{aligned}
$$

Since II usually increases with height, the curvature of rars is concave upwards in this cliagram. Again, equation (11) shows that when the modified earth:s radius $k a$ is introduced (Figure 5), this amount of upward curvature is just canceled and the rays appear as straight lines.

\subsubsection{Alternate Method}

Instead of taking account of refraction by changing the earth's curvature, another method is sometimes more convenient. It may be shown that the ratio of the field $E$ to the free-space field $E_{0}$ transforms in the same way, whether (1) radius $a$ is replaced by $k a$, or (2) the horizontal distance $x$ is replaced by $x l^{-2,3}$ and at the same time all elevations $h$ are replaced by $h k^{-1 / 3}$. An angle $\alpha$ must then be replaced by $\alpha k^{-1}{ }^{3}$. Method (2) is usually less convenient than method (1) because it involves a change of horizontal distance which makes it necessary to transform the ratio $E / E_{0}$ rather than the field itself. In method (1) where only curvatures are changed, this difficulty does not appear as the distance $x$ and hence $E_{0}$ remains unaltered.

Method (2) may be used to advantage to account for deriations of $k$ from the standard value of $k=1 / 3$. Coverage diagrams are usually drawn for this ralue; the deviations oning to a change in f. may then be estimated by multiplying distances, heights, or angles with the appropriate powers of $k /(t / 3)$.

\subsubsection{Computation of Refractive Index}

The following equation gives the dependence of the refractive index on temperature, pressure, and humidity :

$$
(n-1) \cdot 10^{6}=\frac{79}{T}\left(p+\frac{4,500 e}{T}\right)
$$

where $T$ is the absolute temperature, $p$ is the total pressure, and $e$ the water-rapor pressure, both the latter in millibars.

Introducing $M$ from equation (3), the modified refractive index, for use on a plane earth diagram, is equal to

$$
M=\frac{79}{T}\left(p+\frac{4,800 e}{T}\right)+0.157 h,
$$

where the height $h$ is in meters. If $h$ is in feet, the last term is 0.04 h .

Tables have been prepared by means of which $M$ can be computed lapidly from meteorological data, nanely temperature, humidity, and pressure given as a function of height. For this purpose $M$ is the sum of three terms which are computed independently:

$$
M=M_{d}+U_{w}+M_{c} .
$$

The dry term $M_{d}$ is obtained from Table 1 as a function of temperature and height in meters above the ground. (If the pressure at the ground $p_{0}$ is substantially different from 1,000 millibars all values of $M_{d}$ should be multiplied by $p_{0} / 1,000$. In 
TABle 1 (Continued)

\begin{tabular}{|c|c|c|c|c|c|c|c|c|c|c|c|c|}
\hline & $t\left({ }^{\circ} \mathrm{C}\right)$ & & & & & $M_{d}$ & & & & & & \\
\hline$h(\mathrm{~m})$ & 20 & 22 & 24 & 26 & 28 & 30 & 32 & 34 & 36 & 38 & 40 & \\
\hline 0 & $2 c 9.6$ & 267.8 & 266.0 & 264.2 & 262.5 & 260.7 & 259.0 & 257.5 & 255.7 & 254.0 & 252.4 & 0.0 \\
\hline 10 & 269.3 & 267.5 & 265.7 & 263.9 & 262.2 & 260.4 & 258.7 & 257.2 & 255.4 & 253.7 & 252.1 & 32.8 \\
\hline 20 & 269.0 & 267.2 & 265.4 & 263.6 & 261.9 & 260.1 & 258.4 & 256.9 & 255.1 & 253.4 & 251.8 & 65.6 \\
\hline 30 & 268.7 & 266.9 & 265.1 & 263.3 & 261.6 & 259.9 & 258.2 & 256.5 & $25+.9$ & 253.2 & 251.6 & 98.4 \\
\hline 40 & 268.4 & 266.6 & 264.8 & 263.0 & 261.3 & 259.6 & 257.9 & 256.2 & $25 \pm .6$ & 252.9 & 251.3 & 131.2 \\
\hline 50 & 268.1 & 266.3 & 264.5 & 262.7 & 261.0 & 259.3 & 257.6 & 255.9 & 254.3 & 252.6 & 251.0 & 164.0 \\
\hline 75 & 267.3 & 265.5 & 263.8 & 262.0 & 260.3 & 258.6 & 256.9 & $25+.2$ & 253.6 & 251.9 & 250.3 & 248.1 \\
\hline 100 & 266.5 & 264.7 & 263.0 & 261.2 & 259.5 & 257.8 & 256.2 & 2.54 .5 & 252.9 & 251.3 & 249.7 & 328.1 \\
\hline 150 & 265.0 & 263.2 & 261.5 & 259.7 & 258.0 & 256.3 & 254.7 & 253.1 & 251.5 & 249.0 & 248.3 & 492.1 \\
\hline 200 & 263.4 & 261.7 & 260.0 & 258.3 & 256.6 & 254.9 & 253.3 & 251.7 & 250.1 & 248.5 & 246.9 & 656.2 \\
\hline 250 & 261.9 & 260.2 & 258.5 & 256.8 & 255.1 & 253.5 & 251.9 & 250.3 & 248.7 & 247.2 & 245.6 & 820.2 \\
\hline 300 & 260.3 & 258.6 & 256.9 & 255.3 & 253.6 & 252.0 & 250.4 & 248.9 & 247.3 & 245.8 & 244.3 & 984.3 \\
\hline 350 & 258.8 & 257.2 & 255.5 & 253.9 & 252.2 & 250.6 & 249.0 & 247.5 & 245.9 & 244.4 & 242.9 & $1,148.0$ \\
\hline 400 & 257.3 & 255.7 & 254.0 & 252.4 & 250.8 & 249.2 & 247.7 & 246.2 & 244.6 & 243.1 & 241.6 & $1,312.0$ \\
\hline 450 & 255.8 & 254.2 & 252.6 & 251.0 & 249.4 & 247.8 & 246.3 & 244.8 & 243.3 & 241.8 & 240.3 & $1,476.0$ \\
\hline 500 & 254.4 & 252.8 & 251.1 & 249.5 & 248.0 & 246.4 & 244.9 & 243.4 & 241.9 & 240.4 & 239.0 & $1,640.0$ \\
\hline 600 & 251.4 & 249.8 & 248.3 & 246.7 & 245.2 & 243.7 & 242.2 & 240.7 & 239.2 & 237.8 & 236.4 & $1,969.0$ \\
\hline 700 & 248.5 & 247.0 & 245.5 & 243.9 & 242.4 & 240.9 & 239.5 & 238.0 & 236.6 & 235.2 & 233.8 & $2,297.0$ \\
\hline 800 & 245.6 & 244.1 & 242.6 & 241.2 & 239.7 & 238.3 & 236.9 & 235.4 & 234.0 & 232.7 & 231.3 & $2,625.0$ \\
\hline 900 & 242.8 & 241.3 & 239.8 & 238.4 & 237.0 & 235.6 & 234.2 & 232.8 & 231.4 & 230.1 & 228.8 & $2,953.0$ \\
\hline 1,000 & 239.9 & 238.5 & 237.1 & 235.7 & 234.3 & 232.9 & 231.6 & 230.3 & 228.9 & 227.6 & 226.3 & $3,281.0$ \\
\hline 1,500 & 226.3 & 225.1 & 223.8 & 222.6 & 221.4 & 220.2 & 219.0 & 217.8 & 216.6 & 215.5 & 214.3 & $4,921.0$ \\
\hline 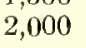 & 213.5 & 212.4 & 211.3 & 210.3 & 209.2 & 208.1 & 207.1 & 206.0 & 205.0 & 203.9 & 202.9 & $6,562.0$ \\
\hline & 86.0 & 89.6 & 93.2 & 96.8 & 100.4 & 104.0 & 89.6 & 93.2 & 96.8 & 100.4 & 104.0 & \\
\hline
\end{tabular}

general this correction may safely be neglected unless the difference between $p_{0}$ and 1,000 is quite large, corresponding to an elevation of the ground level of several thousand feet above sca level.)

The wet term $M_{w}$ is obtained from Table 2 as a function of temperature and relative humidity. Finally, $M_{c}=0.157 h$, if $h$ is in meters, or $M_{c}=0.048 h$, if $h$ is in feet, may readily be computed by means of a slide rule. $M$ is then obtained by addition.

\subsubsection{Atmospheric Stratification}

Ordinary weather data give comparatively little information about the atmospheric stratification near ground level. Radiosonde data are too widely spaced (vertical distances of the order of 100 meters between successive readings) for reliable determination of the variation of $M$ with height at low levels. Special instruments have therefore been dereloped in recent years for low-level soundings. Such instruments contain temperature and humidity measuring elements that are relatively free of lag; they are attached to airplanes or dirigibles or they are carried aloft by moans of captive balloons or kites. The above tables are for usc in connection with such measurements.
Two main cases must be distinguished. First, the refractive index or $\boldsymbol{M}$ is very nearly a linear function of the height in the lower layers (at heights above about 500 to 800 meters the rariation of refractive index with height will deviate from linearity only in very exceptional instances). This is the standard case where $d M / d h$ is independent of $h$, and by using equation (6), $k$ is conveniently obtained from the slope of the $M-h$ curve. It is found that the vertical temperature gradient has a comparatively small influence on $k$, while fairly small variations of the humidity gradient will affect $k$ appreciably.

The other case is that of nonstandard refraction. Here $M$ is not a linear function of the height. The most important special case is that of superrefraction, which occurs when, in certain height intervals, $M$ decreases with height instead of following the usual increase with elevation. Such a decrease of $M$ in certain layers of the atmosphere is caused by a steep negative moisture gradient or steep positive temperature gradient, or cven more by a combination of both influences.

With superrefraction, propagation conditions are greatly different from those encountered with stanclard refraction and the methods to be given later for the determination of the transmitted power 
Table 2

\begin{tabular}{|c|c|c|c|c|c|c|c|c|c|c|c|}
\hline \multirow{2}{*}{$t\left({ }^{\circ} \mathrm{C}\right)$} & \multicolumn{10}{|c|}{$M_{w}$} & \\
\hline & 10 & 20 & 30 & 40 & 50 & 60 & 70 & 80 & 90 & 100 & \\
\hline-20 & 0.6 & 1.2 & 1.8 & 2.5 & 3.1 & 3.7 & 4.3 & 4.9 & 5.5 & 6.130 & -4.0 \\
\hline-18 & 0.7 & 1.5 & 2.2 & 2.9 & 3.7 & 4.4 & 5.1 & 5.8 & 6.6 & 7.309 & -0.4 \\
\hline-16 & 0.9 & 1.7 & 2.6 & 3.5 & 4.3 & 5.2 & 6.1 & 6.9 & 7.8 & 8.678 & +3.2 \\
\hline-14 & 1.0 & 2.1 & 3.1 & 4.1 & 5.1 & 6.2 & 7.2 & 8.2 & 9.3 & 10.287 & +6.8 \\
\hline-12 & 1.2 & 2.4 & 3.6 & 4.8 & 6.1 & 7.3 & 8.5 & 9.7 & 10.9 & 12.121 & +10.4 \\
\hline-10 & 1.4 & 2.9 & 4.3 & 5.7 & 7.1 & 8.6 & 10.0 & 11.4 & 12.9 & 14.284 & +14.0 \\
\hline-8 & 1.7 & 3.4 & 5.0 & 6.7 & 8.4 & 10.1 & 11.7 & 13.4 & 15.1 & 16.754 & +17.6 \\
\hline-6 & 2.0 & 3.9 & 5.9 & 7.8 & 9.8 & 11.7 & 13.7 & 15.7 & 17.6 & 19.568 & 21.2 \\
\hline-4 & 2.3 & 4.6 & 6.9 & 9.1 & 11.4 & 13.7 & 16.0 & 18.3 & 20.6 & 22.871 & 24.8 \\
\hline-2 & 2.7 & 5.3 & 8.0 & 10.6 & 13.3 & 16.0 & 18.6 & 21.3 & 23.9 & 26.589 & 28.4 \\
\hline \pm 0 & 3.1 & 6.2 & 9.3 & 12.4 & 15.5 & 18.5 & 21.6 & 24.7 & 27.8 & 30.911 & 32.0 \\
\hline 2 & 3.5 & 7.0 & 10.5 & 14.1 & 17.6 & 21.1 & 24.6 & 28.1 & 31.6 & 35.139 & 35.6 \\
\hline 4 & 4.0 & 8.0 & 12.0 & 16.0 & 20.0 & 24.0 & 28.0 & 32.0 & 35.9 & 39.939 & 39.2 \\
\hline 5 & 4.3 & 8.5 & 12.8 & 17.0 & 21.3 & 25.5 & 29.8 & 34.0 & 38.3 & 42.533 & 41.0 \\
\hline 6 & 4.5 & 9.1 & 13.6 & 18.1 & 22.6 & 27.2 & 31.7 & 36.2 & 40.8 & 45.280 & 42.8 \\
\hline 7 & 4.8 & 9.6 & 14.5 & 19.3 & 24.1 & 28.9 & 33.7 & 38.5 & 43.3 & 48.175 & 44.6 \\
\hline 8 & 5.1 & 10.2 & 15.4 & 20.5 & 25.6 & 30.7 & 35.9 & 41.0 & 46.1 & 51.215 & 46.4 \\
\hline 9 & 5.4 & 10.9 & 16.3 & 21.8 & 27.2 & 32.6 & 38.1 & 43.5 & 49.0 & 54.394 & 48.2 \\
\hline 10 & 5.8 & 11.6 & 17.3 & 23.1 & 28.9 & $3 \pm .7$ & 40.5 & 46.2 & 52.0 & 57.793 & 50.0 \\
\hline 11 & 6.1 & 12.3 & 18.4 & 24.5 & 30.6 & 36.8 & 42.9 & 49.0 & 55.1 & 61.270 & 51.8 \\
\hline 12 & 6.5 & 13.0 & 19.5 & 26.0 & 32.5 & 39.1 & 45.6 & 52.1 & 58.6 & 65.094 & 53.6 \\
\hline 13 & 6.9 & 13.8 & 20.7 & 27.6 & 34.5 & 41.4 & 48.3 & 55.2 & 62.1 & 69.010 & 55.4 \\
\hline 14 & 7.3 & 14.6 & 22.0 & 29.3 & 36.6 & 43.9 & 51.2 & 58.5 & 65.9 & 73.169 & 57.2 \\
\hline 15 & 7.8 & 15.5 & 23.3 & 31.0 & 38.8 & 46.5 & 54.3 & 62.0 & 69.8 & 77.505 & 59.0 \\
\hline 16 & 8.2 & 16.4 & 24.6 & 32.8 & 41.0 & 49.2 & 57.4 & 65.6 & 73.9 & 82.060 & 60.8 \\
\hline
\end{tabular}

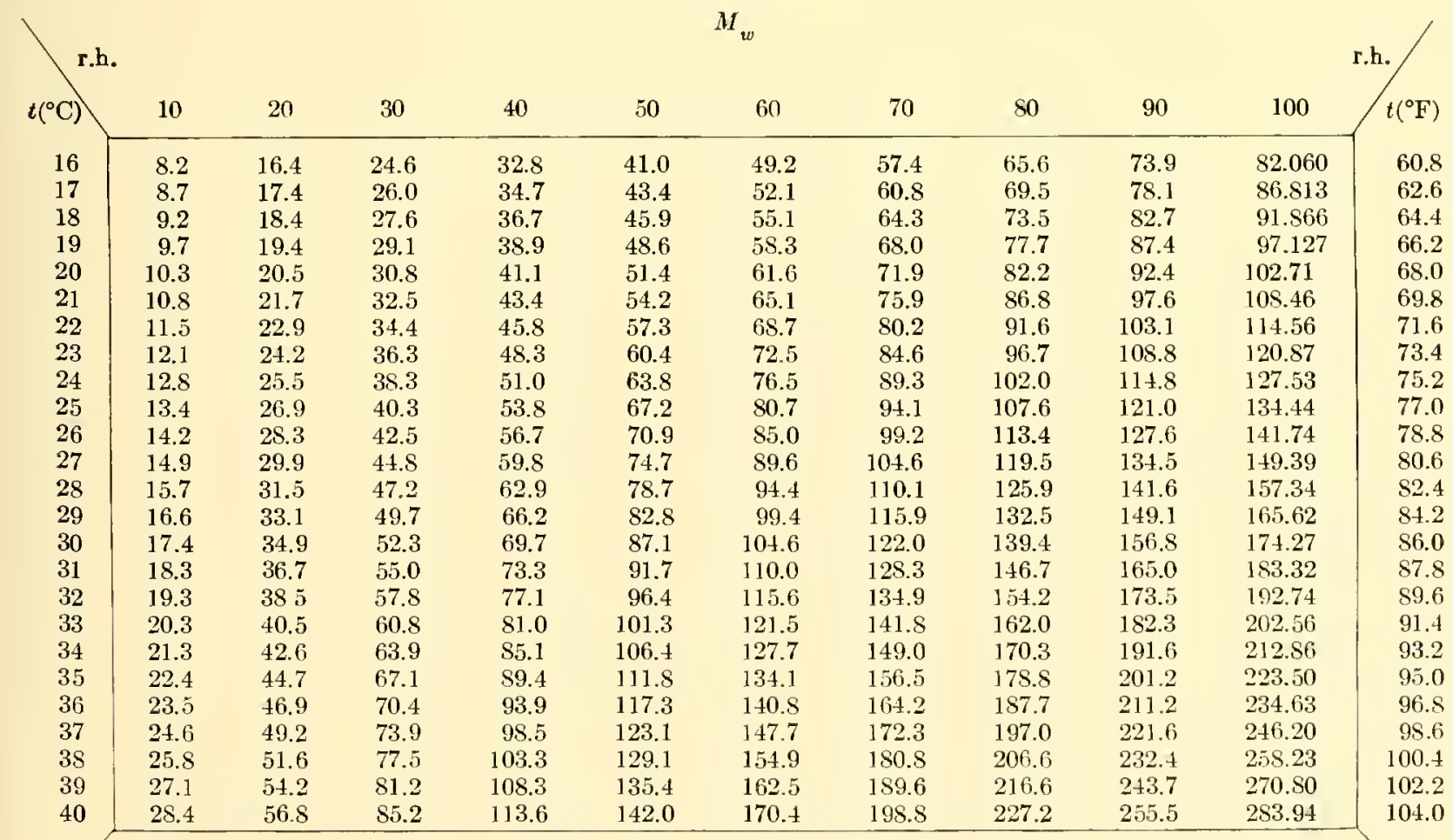


do not apply. This is especially true for the field near or below the optical horizon. The discussion of nonstandard propagation is beyond the scope of this volume.

High-angle corerage is generally independent of lefraction and is therefore also unaffected by the variations of $M$ in the lowest layers of the atmosphere.

\subsubsection{Direct Determination of $\boldsymbol{k}$}

In Figure 9 the reciprocal of $k$ is plotted as ordinate against the rertical gradient of relative humidity as abseissa. The values shown refer to a standard temperature gradient of -0.65 degrees Centigrade per 100 meters; unless the temperature gradient differs greatly from this value, the corresponding values of $k$ will not be much affected. The curves given refer to 100 per cent relative humidity at ground level, and an auxiliary table is provided
4.2

\subsubsection{Ground Reflection and Coverage}

The rcinforcement of the direct ray by the groundreflected ray is of great importance both in radar and in communication work. In favorable cases, the reflected ray may be of comparable intensity to the direct ray and thus the received intensity may be approximately doubled in places where the two rays have the same phase. This means, in many cases, the possibility of an appreciable increase in range relative to that in free space.

\subsubsection{Complexity of Reflection Problem}

In order to facilitate the discussion of the problems encountered in reflection, it is neccssary to analyze the complex phenomena into simpler constituents.

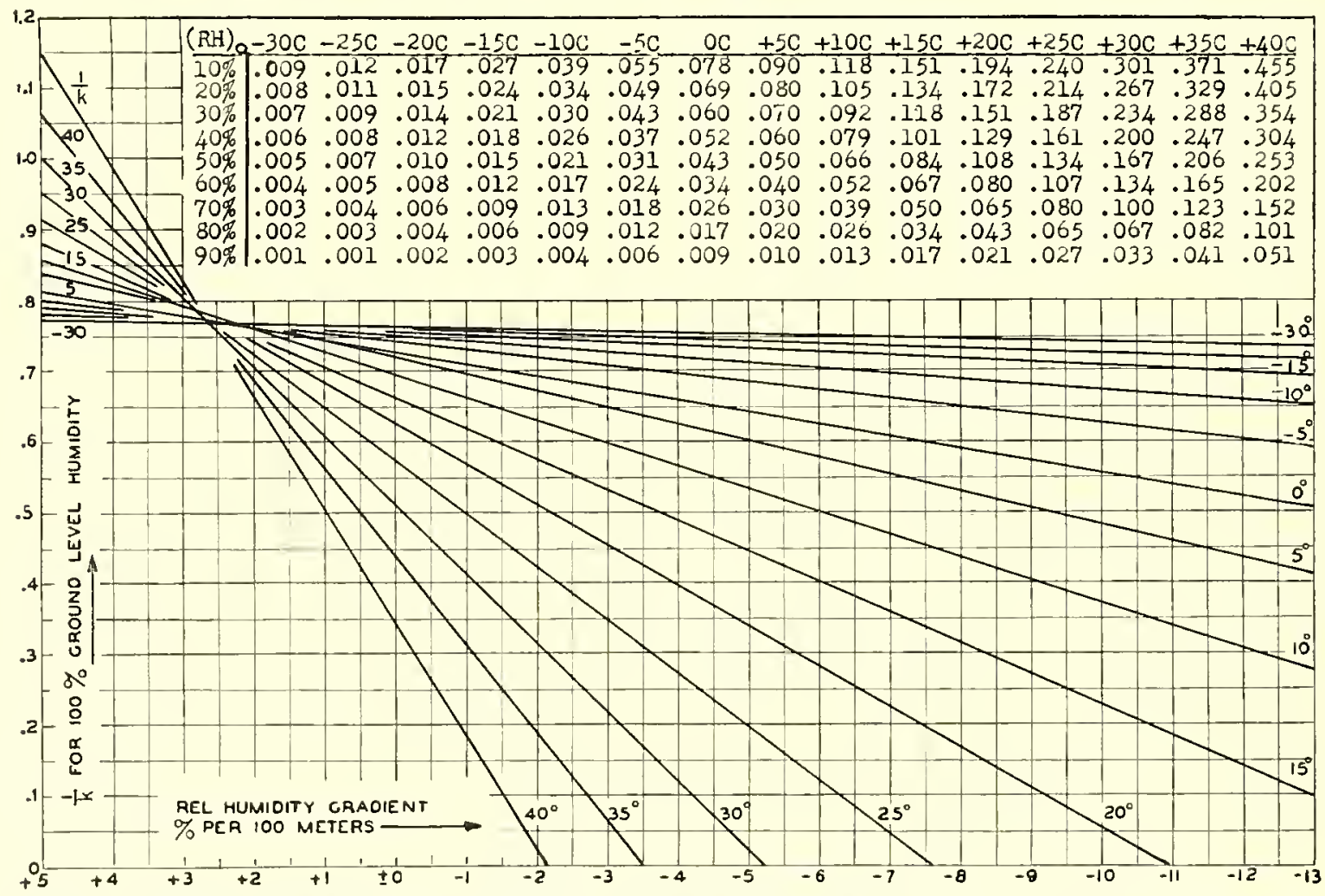

Figure 9. Craph $1 / k$ versus RH (relative humidity) gradient and temperature for 100 per cent RH at ground. Add correction tabulated to obtain $1 / k$ for RH at ground less than 100 per cent.

at the top of the graph which gives figures to be ardded for other values of the relative humiclity. The sensitivity of $k$ to moisture gradients in warm climates is obrious from these data.
First of all, the incident radiation field is resolved into nearly plane wave components, each forming a narrow pencil of rays striking the reflecting surface within a small area which we shall call the reflection 
point. There are two types of such rays depending on their state of polarization. If the electric vector is parallel to the reflecting plane, the rays are said to be horizontally polarized and if the electric vector is parallel to a vertical plane through the rays, they are said to be vertically polarized. When considering a very irregular surface, the reflected field may show extreme complexity even though the incident wave is linearly polarized. Inereasing roughness may result in diffuse reflection which is ineffective in reinforcing the direct wave. The existence of diffuse reflection depends primarily on the size of the irregularities of the surface in comparison with the wavelength of the incident radiation and on the grazing angle of the incident field. This problem will be discussed in more detail later.

\subsubsection{Plane Reffecting Surface}

Consider first the simplest case, when a plane wave strikes a plane surface such as that of an absolutely calm sea. The incident ray is then split into two parts. One is the reflected ray, which is returned to the atmosphere, and the other is the refracted ray, which is absorbed by the sea. At the point of reflection, the ratio of any scalar quantity in the reflected wave to the same quantity in the incident wave is defined as the reflection coefficient of the sea for plane waves of given frequency. Thus defined, the reflection coefficient can and will be different for the various components of the field.

For simplicity, let us assume the reflecting plane to be the $x y$ plane of a rectangular coordinate system, the $x z$ plane to coincide with the plane of incidence, and the reflection point to be the origin of the coordinate system.

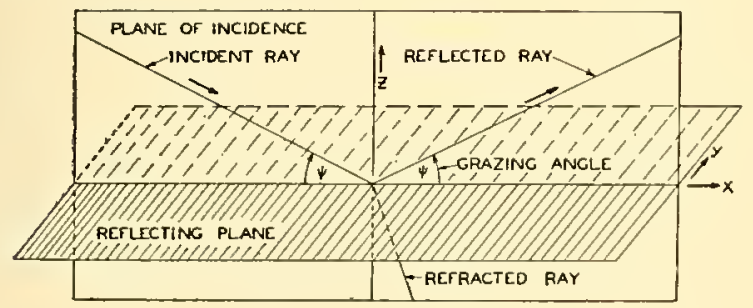

FiguRE 10. Geometry of reflection and refraction.

For horizontal polarization, the electric vector for the incident wave then is

$$
E_{i}=E_{0} e^{j 2 \pi f[t-(1 / c)(x \cos \psi-z \sin \psi)]}
$$

where $\psi$ is the grazing angle, $f$ the frequency of the radiation and $c$ the velocity of light in free space. The electric vector of the corresponding reflected field is given by the similar expression

$$
E_{r}=E_{0} \rho e^{-j \phi-j 2 \pi f[t-(1 / c)(x \cos \psi+z \sin \psi)]}
$$

where $\rho$ and $\phi$ are real constants. The ratio of the reflected to the incident field at the reflection point $(z=x=0)$ is seen to be

$$
R=\rho e^{-j \phi} .
$$

By definition this is the reflection coefficient for horizontally polarized waves. Thus the reflection coefficient is a complex quantity, the amplitude of which is the reflection coefficient of the wave amplitude and the phase is the lag in phase of the reflected wave with respect to the incident wave at the point of reflection.

The reflection coefficient for vertically polarized radiation is clefined in the same way. It is found, however, that the expression of $\rho$ and $\phi$ in terms of the grazing angle $\psi$ and the ground constants are quite different for the two types of polarization. For an arbitrary position of the plane of polarization, the wave must be separated into its vertically and horizontally polarized components, and the proper reflection coefficient applied to each component separately.

The quantities $\rho$ and $\phi$ are determined by the boundary conditions for the electric vector at the reflecting surface, namely, that the tangential components of the electric rector on the two sides of the boundary surface shall be equal. This brings in the ground constants, that is, the conductivity and dielectric constant of the reflecting body. How these boundary conditions are applied may be illustrated by the simple example of horizontally polarized rays reflected from a surface of infinite conductivity. In the surface itself, the sum of the incident and reflected field strength must always be such that the currents set up in the body just suffice to produce the reflected ficld. Within a reflecting body of infinite conductivity, an infinitely weak field is sufficient, and hence the boundary condition is such that the reflected field, at the reflection point, shall be equal in magnitude and opposite in phase to the incident field, so that the resultant field is zero. Hence for infinite conductivity and horizontal polarization

$$
R=-1, \quad \rho=1, \quad \phi=180^{\circ} .
$$


4.2 .4

\section{Fresnel's Formulas}

For finite conductivity, the reflection cocfficient may assume a variety of values. The gencral formulas, as derived from electromagnetic theory, are given in equations (19) and (20). For horizontal polarization

$$
R=\frac{\sin \psi-\sqrt{\epsilon_{c}-\cos ^{2} \psi}}{\sin \psi+\sqrt{\epsilon_{c}-\cos ^{2} \psi}},
$$

and for vertical polarization

$$
R=\frac{\epsilon_{c} \sin \psi-\sqrt{\epsilon_{c}-\cos ^{2} \psi}}{\epsilon_{c} \sin \psi+\sqrt{\epsilon_{c}-\cos ^{2} \psi}},
$$

where $\epsilon_{c}$ is the complex relative diclectric constant of the reflecting ground which is given by

$$
\epsilon_{c}=\epsilon_{r}-j \epsilon_{i} .
$$

A material that acts like a good conductor for ization the phase change is $180^{\circ}$ from $\psi=0$ up to an angle $\psi_{0}$ determined by $\tan \psi_{0}=1 / \sqrt{\epsilon_{r}}$. Here, the coefficient is zero. For larger angles the phase change is zero. As $\epsilon_{r}$ increases indefinitely, $\psi_{0}$ approaches zero and for infinite $\epsilon_{r}$ the phase shift is zero everywhere, except for $\psi=0$ where it is indeterminate. The angle $\psi_{0}$ is called the Brewster angle. For $\psi=0$ the amplitude is unity, and for $\psi=90^{\circ}$

$$
\rho=\frac{\sqrt{\epsilon_{r}}-1}{\sqrt{\epsilon_{r}}+1}
$$

for both cases of polarization. When $\epsilon_{i}$ is no longer zero, the amplitude $\rho$ will show a deep minimum for a ecrtain value of $\psi$ instead of the zero found for $\epsilon_{i}=0$. The angle corresponding to the minimum is called the pseudo-Brewster' angle. These various points are illustrated by examples in Figure 16.

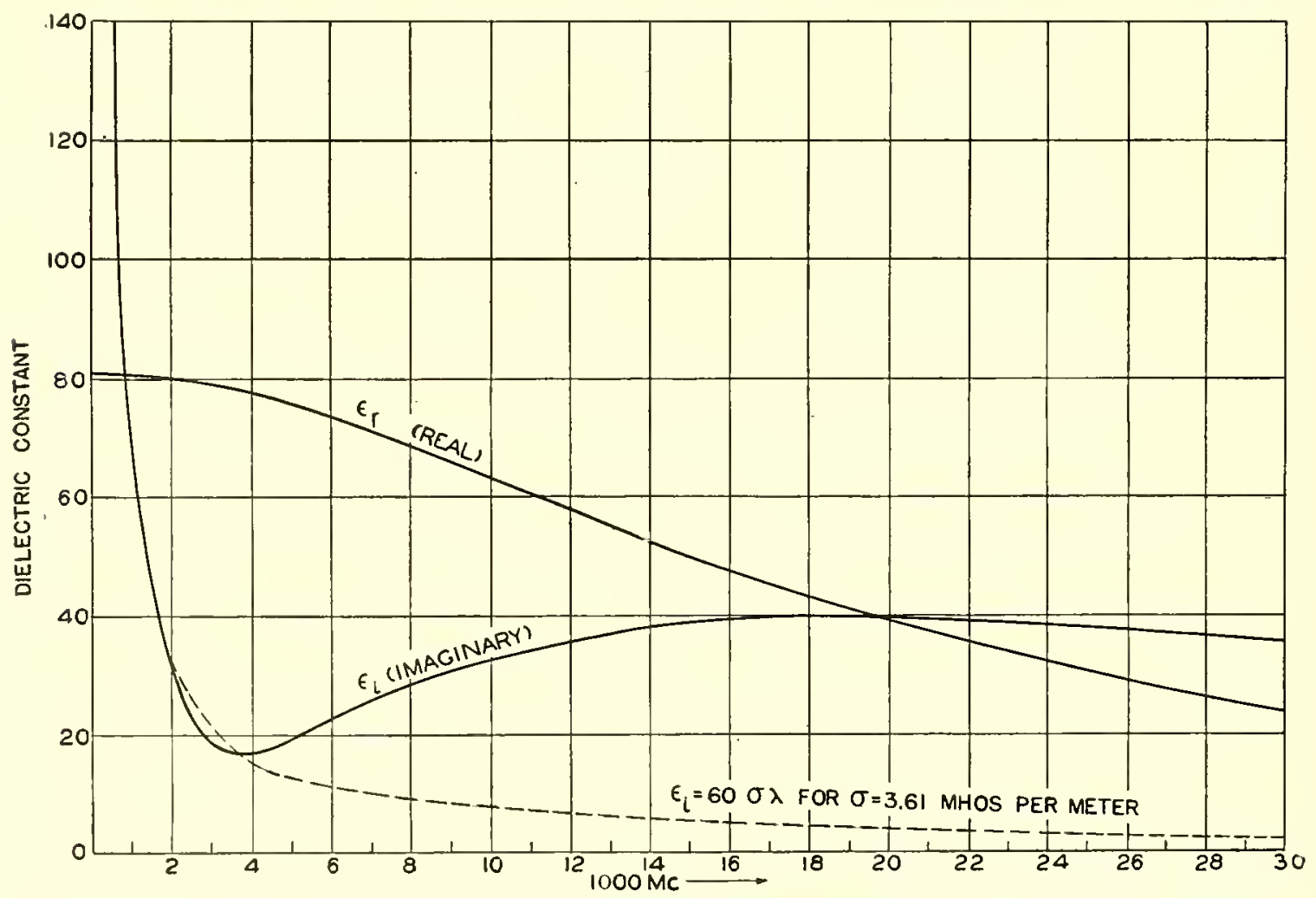

Figure 11. Dielectric constant of sea water at $17 \mathrm{C}$.

low-frequency waves may act as an approximately pure dielectric for microwaves. The case $\epsilon_{i}=0$ is therefore of considerable practical importance. When $\epsilon_{i}=0$, and $R$ consequently is real, the phase lag is $180^{\circ}$ for horizontal polarization. For vertical polar-

\subsubsection{The Complex Dielectric Constant of Water}

As much of the available radar and communication equipment is either shipborne or erected along the coast, reflection from sca water is one of the 
principal problems to be discussed here. For microwaves the salt content in sea water makes little difference, so that it may be assumed that the dielectric constant and conductivity are the same over all oceans at the same temperature. With increasing temperature, the real part of the dielectric constant diminishes roughly by one unit per $5^{\circ} \mathrm{C}$. Figure 11 gives the dielectric constant $\epsilon_{r}$ of ordinary sea water at $17 \mathrm{C}$ as a function of frequency.

The dielectric constant also diminishes with increasing salinity, but in the UHF-SHF region, normal variations of salinity have much smaller influence than changes in temperature and frequency. The imaginary part $\epsilon_{i}$ of the dielectric constant is, for frequencies less than say $1,000 \mathrm{mc}$, related to the conductivity $\sigma$ as follows:

$$
\epsilon_{i}=+60 \sigma \lambda
$$

( $\sigma$ in mhos per meter and $\lambda$ in meters). At $25 \mathrm{C}$ the average conductivity of sea water is usually given as

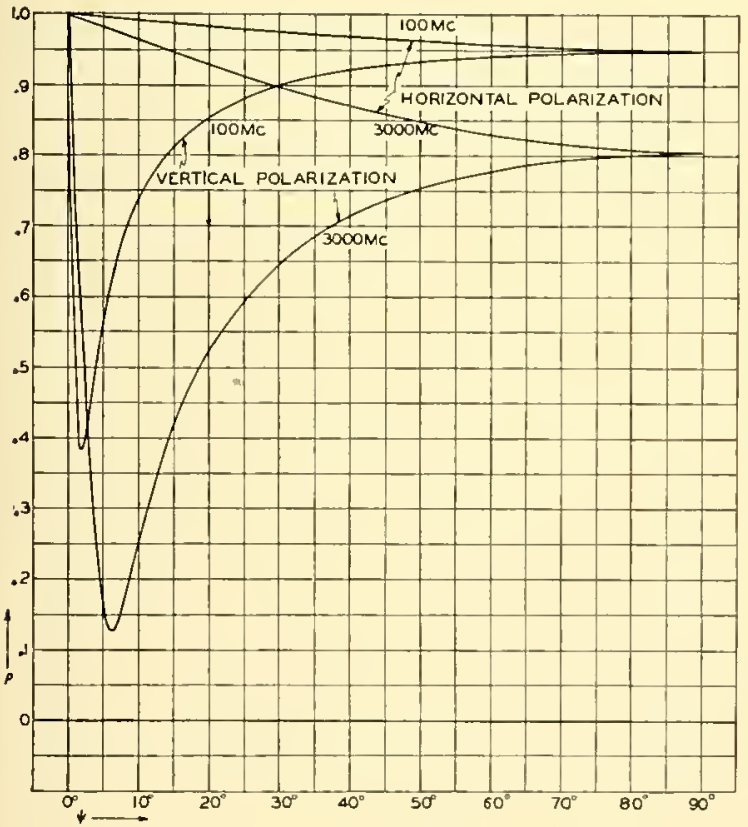

Figure 12. Amplitude of the reflection coefficient $\rho$ versus angle of reflection $\Psi$ for sea water. (From Radiation Iaboratory Report C-11.)

4.3 mhos per meter. The temperature dependence is given by

$$
\sigma=\sigma_{25}[1+0.02(t-25)]
$$

where $t$ is temperature in degrees centigrade.

The conductivity of fresh water is much smaller.
An average for inland lakes is $\sigma=10^{-3}$ mho per meter. For wavelengths shorter than about $10 \mathrm{~cm}$, the dielectric constant is influenced by the fact that water is built up of polar molecules. The maximum effect is found for wavelengths of the order of $1 \mathrm{~cm}$. For this region, there is no appreciable difference between salt and fresh water.

The probable run of $\epsilon_{i}$ for sea water is shown in Figure 11. The part of the $\epsilon_{i}$ curve extending from 6,000 me to $30,000 \mathrm{me}$ corresponds essentially to results obtained at Clarendon Laboratory, Oxford. Other investigators give results which are maikedly different. This curve is thus affected by considerable uncertainties and should be used with caution.

In Figures 12 to 15 are shown amplitude and phase of the reflection coefficient for a smooth sea for

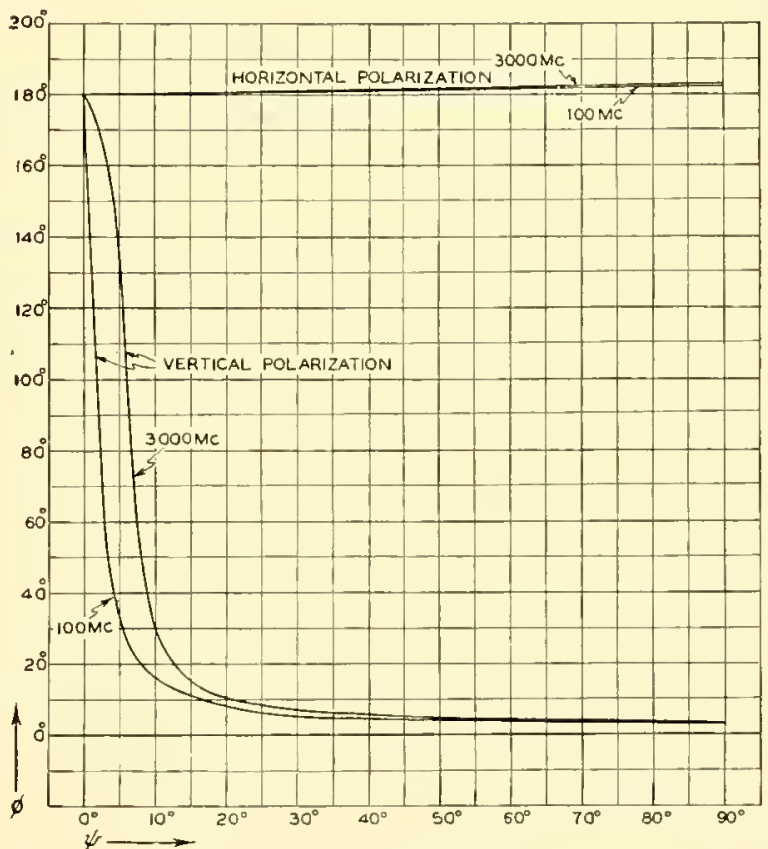

Figure 13. Phase of the reflection coefficient $\phi$ versus angle of reflection $\Psi$ for sea water. Reflected wave $\mathrm{E}_{r}$ lags incident wave $\mathbf{E}_{i}$ by $\phi$. (From Radiation Laboratory Report C-11.)

various frequencies. Figure 12 gives the amplitude $\rho$ of the reflection coefficient for both kinds of polarization, for reflection angles up to 90 degrees, and for 100 and 3,000 mc. Figure 13 gives the phase $\phi$ under the same conditions. The next two figures give $\rho$ and $\phi$ on a greatly enlarged scale of $\psi$, in the interval $\psi=0$ to $\psi=5.5^{\circ}$, which embraces all cases of practical interest. 


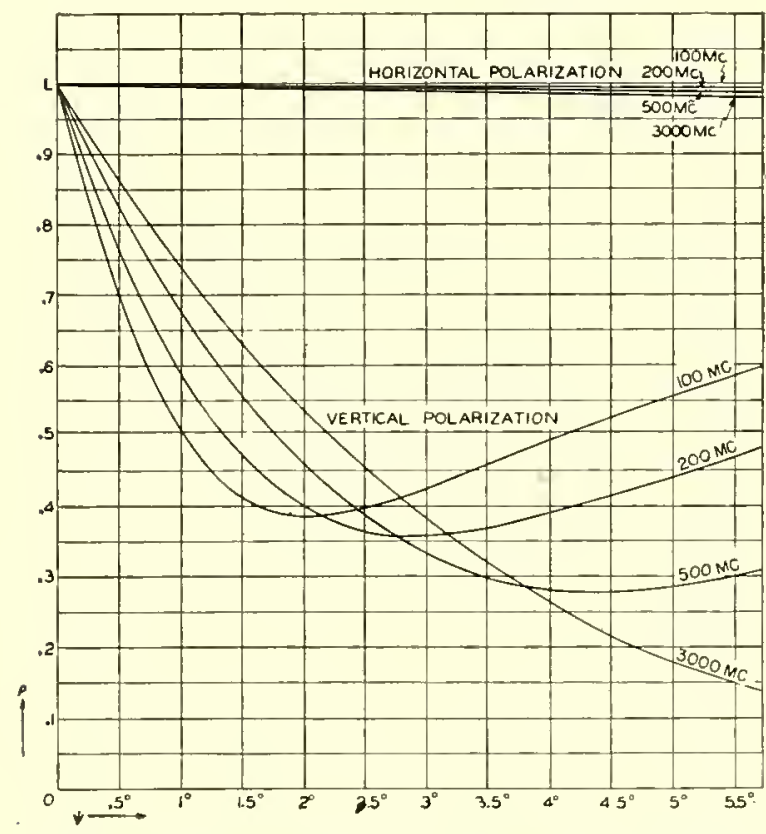

Figure 14. Amplitude $\rho$ of the reflection coefficient versus reflection angle $\Psi$ from $\Psi=0$ to $\Psi=5.5^{0}$ for sea water.

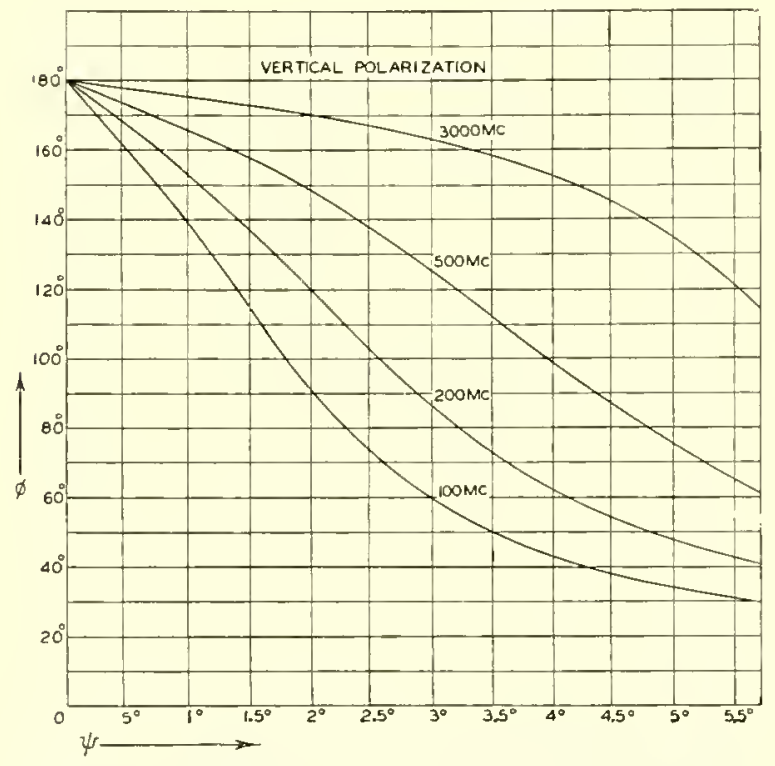

Figure 15. Phase $\phi$ of the reflection coefficient versus reflection angle $\Psi$ from $\Psi=0$ to $\Psi=5.5^{\circ}$ for sea water. $\mathrm{E}_{r}$ lags $\mathrm{E}_{i}$ by $\phi$. (From Radiation Laboratory Report C-11,)

\subsubsection{Overland Transmission}

Conditions over land are very different from those found over the sea. Land as a reflecting surface has larger irregularities and their effect is more pro- nonnced. Therefore in selecting a radar site, it is preferable to choose a location which is surrounded by relatively smooth ground. The electrical properties of the earth vary considerably for different localities, so that it is necessary to study the ground conditions for each particular case. Experimental data concerning reflection of very short waves from ice- or snow-covered ground seem to be lacking. Precise information might be of operational interest, particularly in Arctic regions. Laboratory experiments indicate that ground covered by ice or snow will influence the propagation of short waves somewhat in the same way as very dry ground.

\subsubsection{Conductivity of Soil}

Extensive investigations have been made on the conduetivity of different types of soil, particularly on low and medium frequencies. For $10 \mathrm{mc}$, the observed values range from $6 \cdot 10^{-3}$ mhos per meter for chalk to 0.13 mhos per meter for blue clay. The conductivity increases with increasing moisture

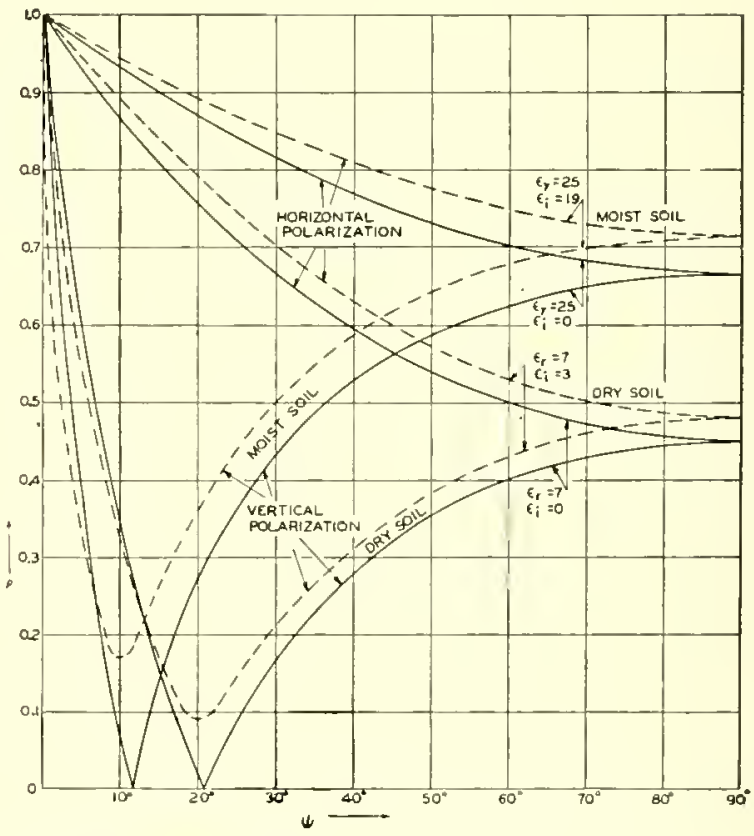

Figure 16. Amplitude of the reflection coefficient for moist and dry soil.

content, so that marked seasonal changes may be anticipated for a given locality. It also varies with frequency. Under field conditions it will not be possible to measure the conduetivity in individual cases and one will have to assume a value of about 
$10^{-2}$ mhos per meter for poorly conducting ground like chalk or very dry soil and take a value of about $10^{-1}$ for good conductors like blue clay or waterbogged marshy land. Fortunately, the amplitude of the reflection coefficient is not very sensitive to minor changes in conductivity when the frequency is sufficiently high, say $200 \mathrm{mc}$ or higher. Then the real part of the dielectric constant is the most important factor.

\subsubsection{Dielectric Constant of Soil}

It is not possible to give a standard table of dielectric constants of various types of soil, because the variation with the moisture content is considerable. For very dry ground $\epsilon_{r}$ is likely to be about 4 , but this value may rise to 25 when the ground is thoroughly soaked with water. The dielectric constant of ground will normally decrease with increasing frequency.

Above $200 \mathrm{mc}$, the dielectric constant will dominate the conductivity term, and for field conditions the ground may be assumed to be a pure dielectric. This is illustrated in Figure 16 for $\epsilon_{r}=7 ; \epsilon_{i}=3$

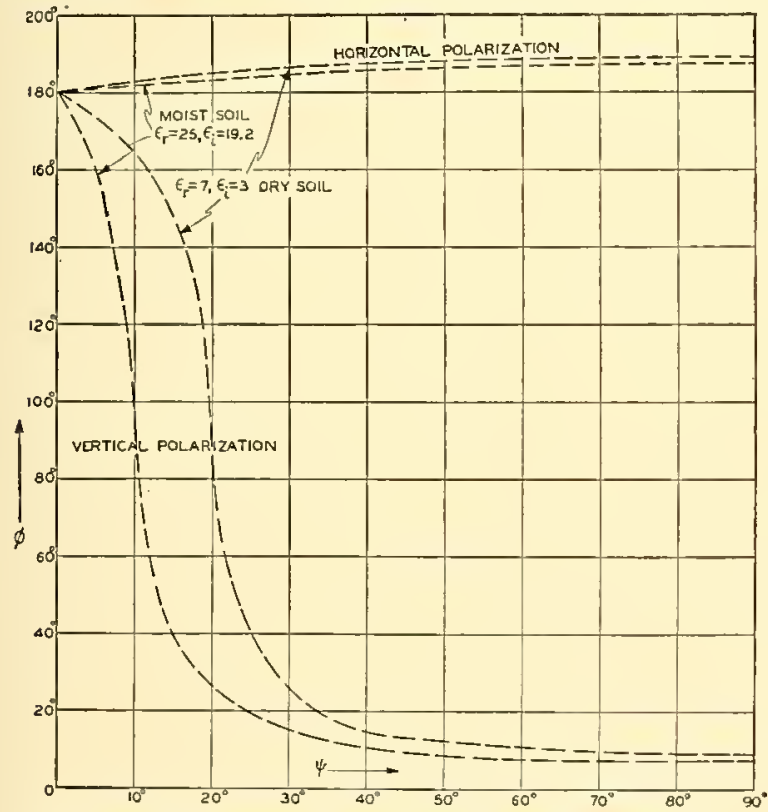

Figure 17. Phase of the reflection coefficient for moist and dry soil.

and $\epsilon_{i}=0$; and for $\epsilon_{r}=25, \epsilon_{i}=19$ and $\epsilon_{i}=0$. Except for values close to the Brerster angle, the zero conductivity curves give a usable approximation.
In Figure 17, the phase, $\phi$, of the reflection coefficient corresponding to the above values of $\epsilon_{r}$ and $\epsilon_{3}$ is also given.

\subsubsection{The Divergence Factor}

The preceding considerations apply only to reflection from plane surfaces. For reflection from a sphere like the earth, the divergence of a bundle of rays is increased when it suffers reflection, and the plane earth reflection coefficient, $R$, must be multiplied by a divergenes factor denoted by $D$, which accounts for the earth curvature. This factor ranges from unity at close range where the earth can be considered plane to zero at points just above the tangent line. [Note: When the divergence factor approaches zero at grazing angles less than the last minimum, other components of the wave must be

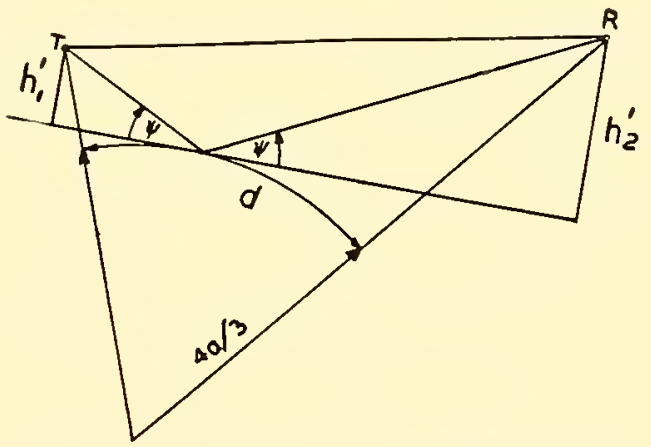

Figure 18. Geometry for divergence factor.

considered.] To a sufficient approximation $D$ is given by the expression

$$
D=\left[1+\frac{2 h_{1}{ }^{\prime} h_{2}{ }^{\prime}}{d k a \tan ^{3} \psi}\right]^{-\frac{1}{2}}
$$

where (see Figure 18),

$h_{1}{ }^{\prime}, h_{2}{ }^{\prime}=$ heights of transmitter and receiver above tangent plane at reflection point.

$d=$ distance between transmitter and receiver measured along the surface of the earth.

$\psi=$ grazing angle above tangent plane. $k a=$ equivalent earth radius.

\subsubsection{Irregularity of Ground}

The formulas for reflection from a plane or a spherical earth can only be applied with confidence granting a certain smoothness of the reflecting surface, depending on the wavelength. A rule of 
thumb for the applicability of the reflection formulas is that the rertical heiglit of the irregularities should not exeed $\lambda / 16 \psi$, where $\lambda$ is the wavelength and $\psi$ the grazing angle in radians. Suppose, for instance, that the wavelength is 1 meter and the grazing angle, 1 degree. The limit of tolerance is then $56 / 16=3.5$ meters. Hence, on this wavelength one may expect specular reflection over sea in most cases. For $\lambda=10 \mathrm{~cm}$, on the other hand, the limit is only $35 \mathrm{~cm}$, and for $\lambda=3 \mathrm{~cm}$ it is $11 \mathrm{~cm}$. For larger grazing angles, the limit of tolerance will be correspondingly smaller.

\subsection{DIFFRACTION (GENERAL SURVEY)}

4.3 .1

\section{Definition}

The term diffraction will be understood to apply to those modifications of the field produced by material bodies outside the transmitter that cannot be described by the ray methods of geometrical opties.

With this limitation of the term diffraction, there are three main topies to be considered:

1. Diffraction by the earth's curvature.

2. Diffraction by irregular features of the terrain, such as hills, houses, etc.

3. Diffraction by objects, primarily metallic (targets) in two-way transmission (radar echoes). Also scattering by raindrops.

\subsubsection{Diffraction by Earth's Curvature}

The diffraction field in this case is the field appearing below the line of sight determined by use of the equivalent value of the earth's radius $k a$. The case of an idcalized earth with smooth surface and given electrical properties can be treated mathematically, and the field obtained is often designated as the standard field (see Chapter 5). If one moves away from the transmitter horizontally, at a fixed height above the earth, the field strength decreases exponentially with distance once the line of sight is passed. Similarly the field strength decreases exponentially with height above the ground on going vertically downwards from the line of sight. In many instances, the rariation in the field strength, in the diffraction region is independent of the electric properties of the ground. The main exception oceurs in a comparatively shallow layer near the ground. Only for the important case of propagation over sea water and for frequencies below 100 megacycles does this layer become high enough to cover an appreciable part of the whole diffraction region.

\subsubsection{Diffraction by Terrain}

The problem of diffraction by terrain features requires special treatment. Frequently a ficld of appreciable magnitude is found behiud hills, houses, ete. Diffraction is also important when there is a sudden change in ground properties, as for instance in a transition from land to sea. In this case the shore line acts as a diffracting edge. Only a limited number of cases lend themselves to evaluation by simple formulas. The cases are those which can be treated by the Fresnel-Kirchhoff method of optics which leads to a somewhat intricate but straightforward mathematical formula for the diffracted fiek strength. In spite of its apparent limitations, the Fresnel-Inirchlioff formula is often applicable to short-wave propagation problems. It is treated in Chapter 8.

\subsubsection{Diffraction by Targets}

This problem can be dealt with theoretically by methods similar to those used in computing diffrac-

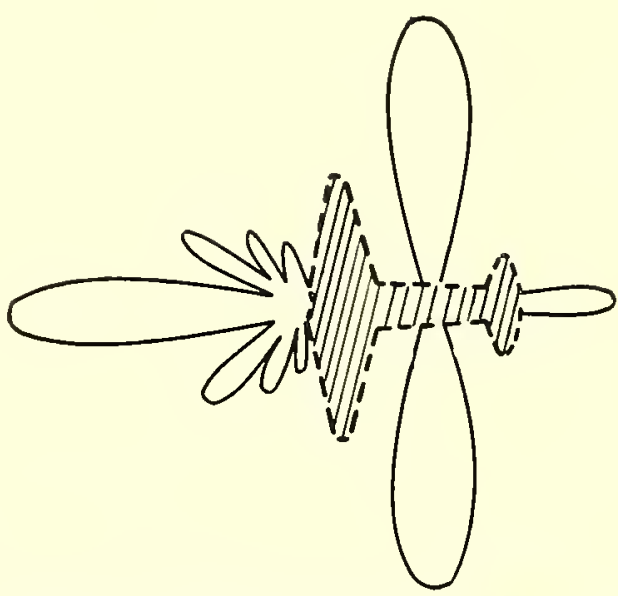

Figure 19. Reflecting pattern of an airplane.

tion by terrain features, the main difference being that the angle of seattering is nearly 180 degrees instead of approximately 0 degrees.

In the case of target diffraction, theory is less 
useful than in many other problems of wave propagation. This is due to the fact that radar targets such as airplanes or ships have an extremely complex structure; the seattered intensity will therefore often change by many decibels as a result of only a small tilt of the target. Figure 19 shows a typical reradiating or reflecting pattern for an airplane. Numerous measurements of the average rarlar cross section of planes and ships have been made.

A phenomenon of great importance in the microwave region is the scattering of radiation by water drops in the atmosphere. Small droplets such as are found in fogs and most types of clouds do not give reflection risible on the scopes. Only drops large enough to produce aetual precipitation give appreciable radar echoes. However, this does not necessarily mean that rain is falling at the locality indicated by the scope; frequently vertical updrafts of air will maintain drops afloat that in still air would fall to the ground; moreover, drops falling from a comparatively high cloud can evaporate before reaching the ground. Especially in tropieal regions, the last-named phenomenon is more common than is ordinarily thought. 


\section{Chapter 5}

\section{CALCULATION OF RADIO GAIN}

$\mathrm{T}$ 111s CHAPTER is devoted to the definition and calculation of the various factors which enter into a computation of the fietd strength of radio waves propagated in the standard atmosphere above the earth.

In Chapter 2, particularly in Sections 2.1 and 2.2, are given the basic definitions of path-gain factor and radio gain for transmission between doublets and other antenna types in free space. The present chapter shows how these quantities must be modified to account for the influences introduced by the curvature and electrical properties of the earth.

The methods of computation are presented in considerable detail to enable the interested reader to apply them to his particular problem, and sample calculations are given which should assist in reducing to a minimun the time required for obtaining the answers in a given case.

\subsubsection{Definitions Relative to Radio Gain}

The radio gain is defined as the ratio of received porrer $P_{2}$, delivered to a load matched to the receiver antenna, to transmitted power $P_{1}$, with both antennas adjusted for maximum power transfer. For doublet antennas in free space this ratio is given by $(3 \lambda / 8 \pi d)^{2}$ and is denoted by $A_{0}{ }^{2}$, that is,

$$
\frac{P_{2}}{P_{1}}=A_{0}{ }^{2}=\left(\frac{3 \lambda}{8 \pi d}\right)^{2}
$$

and the free-space ginin factor is given by

$$
A_{0}=\frac{3 \lambda}{S \pi d}
$$

in which $d$ denotes the distance from the transmitter to the receiver measured in the same units as the wavelength $\lambda$.

When the radiation is emitted and received by directive antennas and the propagation takes place through a refracting and absorbing atmosphere, and reflection and diffraction effects of the ground are taken into account, the cxpression for the radio gain becomes a very complicated affair.

For the general case of onc-way transmission, the radio gain is given by

$$
\frac{P_{2}}{P_{1}}=G_{1} G_{2} A^{2},
$$

where $G_{1}$ and $G_{2}$ are the gains of the transmitting and recciving antemnas, respectively, and $A$, the gain factor, is equal to

$$
A=A_{0} A_{p}
$$

with $A_{p}$ equal to the path-gain factor. [See equation (27) in Chapter 2.]

For radar or two-way transmission, the rackar gain is decreased because the energy traverses the path both ways and is influenced by the radiating properties of the target as given by the radar cross section $\sigma$. Combining equations (46) in Chapter 2 and (2) in this chapter, the radar gain equals

$$
\frac{P_{2}}{P_{1}}=G_{1} C_{2}\left(\frac{16 \pi \sigma}{9 \lambda^{2}}\right)\left[A_{0}^{4} A_{p}^{4}\right]=G_{1} C_{2}\left(\frac{16 \pi \sigma}{9 \lambda^{2}}\right) A^{4} .
$$

Comparing equations (3) and (5), it is seen that the gain factor $A$ may be used also for two-way transmission, provided the additional term $16 \pi \sigma / 9 \lambda^{2}$ is included in the formula.

Later on it will be shown how to split up $A$ and $A_{p}$ into a product of various factors, represented by graphs which make it possible to carry out computations in specific cases.

The gain factor $A$ may also be expressed in terms of the field strength $E$ and free-space field strength of a doublet transmitter $E_{0}$. From equation (28) in Chapter 2,

$$
E=E_{0} \sqrt{G_{1}} A_{p} .
$$

Combining this equation with equation $(t)$

$$
\frac{A}{A_{0}}=\frac{E}{E_{0}} \frac{1}{\sqrt{G_{1}}},
$$

where $E_{0} \sqrt{C_{1}}$ is the free-space field of the transmitting antenna with gain $G_{1}$. 
The free-space field, in terms of the transmitted power $P_{1}$, is given by

$$
\sqrt{G_{1}} E_{0}=\frac{3 \sqrt{5} \sqrt{P_{1}}}{d} \sqrt{G_{1}}
$$

for a point in the direction of maximum radiation. In terms of the power $P_{2}$ delivered to the load circuit of a receiving antenna, with matcher load and oriented for maximum pickup, the ficld at any point in space is equal, from equation (17) in Chapter 2, to

$$
E=\frac{8 \pi \sqrt{5}}{\lambda} \sqrt{\frac{P_{2}}{G_{2}}} .
$$

It is sometimes convenient to express $E$ in terms of the (radiation) field at one meter from the transmitter, whence $E_{0}=E_{1} / d$ and, from equation (6),

$$
E=\frac{E_{1}}{d} \sqrt{G_{1}} A_{p} .
$$

\subsubsection{Factors Affecting Attenuation and Gain}

The above definitions are quitc general. In the absence of the earth, there remains only the freespace attenuation which results from the spreading out of the radiated energy as it moves away from the transmitter. At a distance which is sereral times larger than the wavelength, the field strength varies inversely as the distance from the antenna.

The presence of the earth affects the field through two sets of quantities. One set is geometric and includes the heights of the antennas and their distance apart, the curvature of the earth, and shape of terrain features. The other set is electromagnetic and depends on the dielectric constant and conductivity of the earth and of its atmosphere, the polarization and the wavelength of the radiation.

\subsubsection{Simplifying Assumptions}

The present chapter is mainly concerned with the computation of the field-strength distribution of a transmitter for certain idealized standard conditions, so chosen as to give a fail average picture of propagation conditions for very high-frequency radiation. The reasons for this limitation are stated in Chapter 1. In substance, the limitations are imposed by the great complexity of the general problem, which makes it necessary to proceed in successive steps. The first step is to consider propa- gation under standard conditions, which will be defined farther on. Successive steps take into account diffraction by terrain, that is, by trees, hills, mountain ranges, or shore lines, or by nonstandard propagation effects in the atmosphere.

The fundamental importance of a knowledge of propagation under standard conditions is first of all due to the fact that in a large number of cases conditions do not differ significantly from standard. On the other hand, when they do deviate significantly, the standard solution sets up a criterion for the discovery of deviations and the evaluation of the influence of the nonstandard conditions upon propagation.

The basic assumptions which define what we have been calling standard propagation conditions will now be given.

1. Standard atmosphere. It is assumed that the iudex of refraction of the atmosplere has a uniform negative gradient with increasing elcvation. As has been pointed out in Chapter 4 , the influence of such an atmosphere upon propagation is equivalent to that of a homogeneous atmosphere over an earth of radius $k a$, where $k$ is a constant that usually is taken equal to $4 / 3$.

2. Smooth earth. The earth is assumed to be perfectly smooth. It can be considered sufficiently smooth if Rayleigh's criterion is satisfied, that is when the height of surface irregularities times the grazing angle (in radians) is less than $\lambda / 16$ (see Section 4.2.10).

3. Ground constants. The dielectric constant and conductivity of the earth are assumed uniform. For wavelengths less than one meter this assumption is particularly valid since in this case propagation is largely independent of the ground constants. In the VHF ( 1 to $10 \mathrm{~m}$ ) range, the same is true with the important exception of vertically polarized radiation over sea water. For the VHF range, the assumption of uniform earth constants is unsatisfactory for paths partly over land and partly over sea water, or over sea water with large land masses near-by (see Chapters 8 and 10).

4. Doublet antenna and antenna gain. For the formulas of this chapter, the radiating system is assumed to be a doublet antenna (i.e., a straight wire, short compared to the wavelength). Actual antennas have radiation patterns different from that of a doublet, usually having greater directivity. The antenna gain of a half-ware dipole is 1.09 times (or $0.4 \mathrm{db}$ greater than) that of a doublet, the field maximum being the same in the two cases. This 
gain is insignificant in practice. For other types of antenna systems and for microware frequencies, the gain may be many times larger.

The propagation problem, thus limited, has been solved mathematically; but the explicit mathematical formulas are far too complicated to be of much use to the practical computer. Much additional work has been done, however, to bring the solution into a form suitable for practical use. This involves reducing the computations to the use of graphs, nomograms, and tables, and it is this final stage of the problem which is the subject of subsequent parts of this chapter as well as of Chapter 6 .

\subsubsection{Curved-Earth Geometrieal Relationships}

Let $h_{1}$ and $h_{2}$ denote the heights of transmittex and receiver above the earth's surface, respectively, and let $d$ denote the distance from the base of the transmitter to the base of the receiver, measured along the earth's surface. For a number of eases concerned with high-frequency radiation orer the earth's surface, it is sufficient to identify the straightline distance from transmitter to receiver with the distance $d$ between the bases measured along the curved earth. But when path differences are of importance, as they are in interference problems of reflection and in diffraction, it is necessary to compute distances to a higher order of accuracy.

Throughout this chapter, the earth will be assumed to have the equivalent radius $k a$, and the atmosphere to be homogeneous, and radiation to travel along straight lines.

The straight line from the transmitting antenna and tangent to the earth's surface (the so-called line of sight) touches the earth along a circle which constitutes the radio horizon of the transmitter. The distance measured along the earth from the transmitter to the radio horizon will be denoted by $d_{T}$; and the horizon distance of the receiver by $d_{R}$. These geometrical relations are illustrated in Figure 1.

From this figure, it follows that

$$
k a+h_{1}=\frac{k a}{\cos \left(d_{T} / k a\right)} .
$$

Inasmuch as

$$
\frac{k a}{\cos \left(d_{T} / k a\right)} \cong \frac{k a}{1-\frac{1}{2}\left(d_{T} / k a\right)^{2}} \cong k a+\frac{1}{2} \frac{d_{T}{ }^{2}}{k a},
$$

equation (11) assumes the form

$$
h_{1}=\frac{1}{2} \frac{d_{T^{2}}}{k a},
$$

or

$$
d_{T}=\sqrt{2 k a h_{1}}
$$

Similarly, the hotizon distance of the receiver is

$$
d_{R}=\sqrt{2 \operatorname{liah}_{2}} .
$$

The sum of the two horizon distances is given by $d_{L}$, where

$$
d_{L}=d_{T}+d_{R}
$$

\subsection{Optical and Diffraction Regions}

The points visible from the transmitting antenna (on an earth of equivalent radius ka), i.e., the points above the line of sight, constitute the optical region (Figure 1). The rest of space lies beyond the transmitter horizon and below the line of sight and is called the shadow or diffraction region.

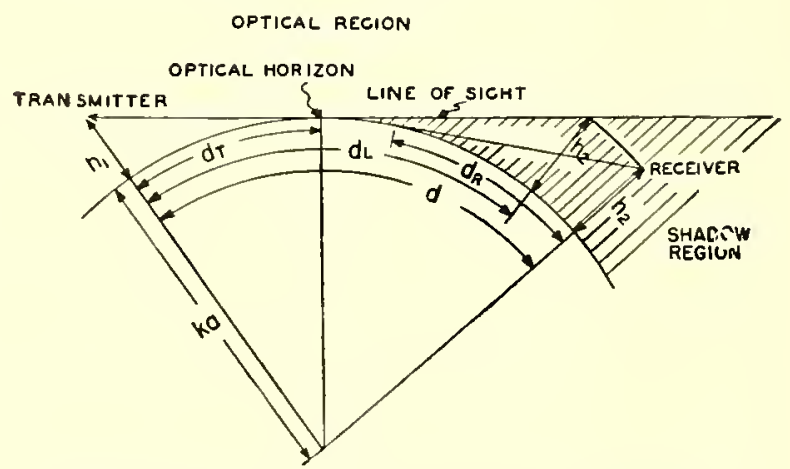

F1gure 1. Geometry for radio wave propagation over curved earth.

It is frequently necessary to know whether a receiving antenna lies in the optical region or the diffraction region of a given transmitter. This evidently is equivalent to knowing whether the distance $d$ of the receiver from the transmitter is smaller or larger than the combined horizon distance $d_{L}$. By equations (13), (14), and (15), it follows that in the optical region

$$
d<\sqrt{2 l_{i} a}\left(\sqrt{h_{1}}+\sqrt{h_{2}}\right)
$$

and in the shadow region

$$
d>\sqrt{2 k} \bar{a}\left(\sqrt{h_{1}}+\sqrt{h_{2}}\right) \text {. }
$$


A graphical representation of the equation

$$
d_{L}=\sqrt{2 k a}\left(\sqrt{h_{1}}+\sqrt{h_{2}}\right)
$$

is given in Figure 2. For $k=4 / 3, a=6.37 \times 10^{6} \mathrm{~m}$, this takes the form

$$
d_{L}=4120\left(\sqrt{h_{1}}+\sqrt{h_{2}}\right) \text { meters, }
$$

where $h_{1}, h_{2}$ are given in meters and $d_{L}$ in meters.
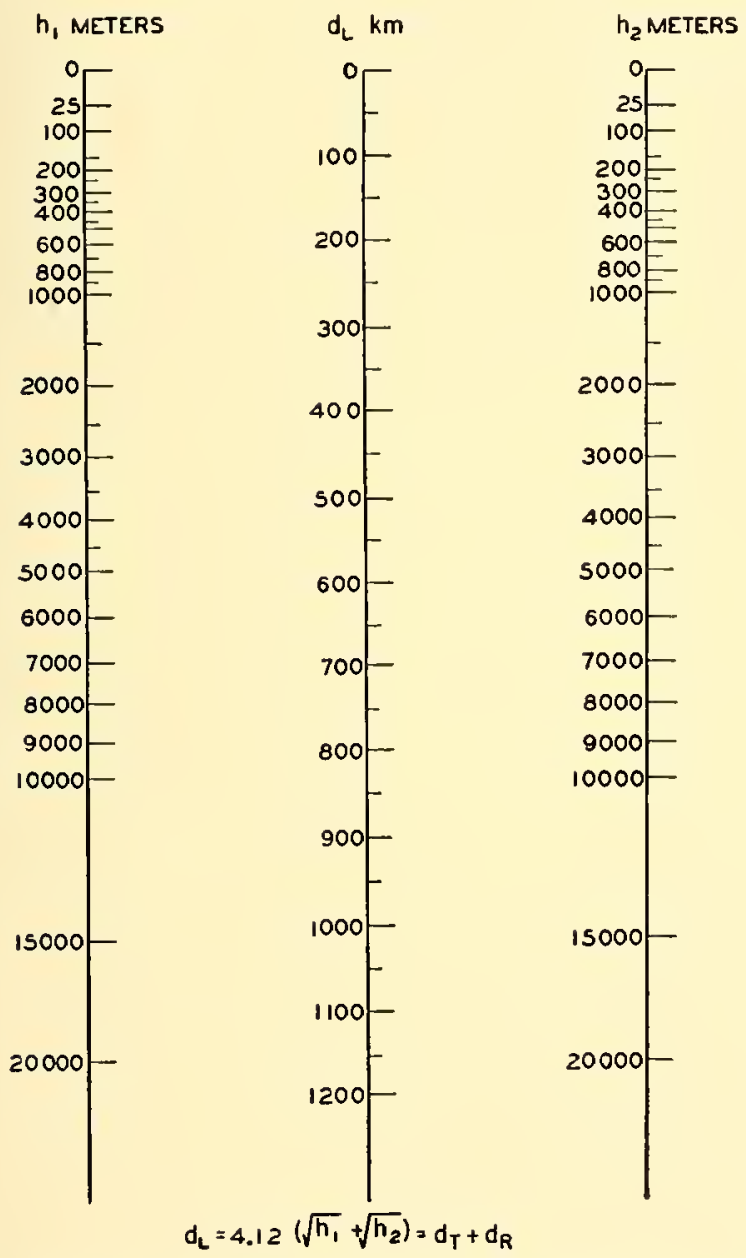

Figure 2. Sum of transmitter and receiver horizon distances for standard refraction. To change scale: Divide $h_{1}$ and $h_{2}$ by 100 , and divide $d_{L}$ by 10 .

\subsubsection{Nature of the Radiation Field in the Standard Atmosphere}

The mathematical solution for the radiation field takes various forms for particular cases. The treatment for low antennas, for instance, differs from that for high antennas, and similarly the equations must be handled differently for the two types of polarization. These and related problems are discussed in general in the following.

1. General form of field variation. The mathematical expression for the radio gain of the radiation field of a doublet under standard conditions is given as the sum of an infinite number of complex terms or modes. (See Section 5.7.6.) Disregarding the phase factor, a representative term (mode) of this series has the form

$$
F(d) \cdot f_{1}\left(h_{1}\right) \cdot f_{2}\left(h_{2}\right)
$$

These modes are attenuated unequally. Well within the diffraction region, the first mode contributes practically all of the field so that the effects of distance and height are separable. In this region, the problem of numerical computation is simplified, since it is possible to use separate graphs for the dependence on height and distance. As the receiver is moved toward the transmitter, the number of modes required for a good approximation increases. For low antennas, the addition of the modes is practicable and the graphical aids are useful for short distances. These conditions are illustrated in Figure 3 for horizontal polarization or ultra-short waves.

In the optical region, the methods of geometrical optics give a result equivalent to that of the rigorous solution at points which are not close to the line of sight. The field is then the sum of a direct and a reflected wave, resulting in an interference pattern.

The preceding discussion is illustrated by Figure 4 which shows the variation of field strength with distance for fixed antenna heights, for propagation. over dry soil with a wavelength of 0.7 meter on vertical polarization. The number's refer to the number of modes required for a better than 99 per cent approximation. The interference patteru is illustrated by the oscillatory nature of the curve. It will be observed that beyond the first maximum, the points found by geometric opties give a value of the field which is slightly too low. (See dots in Figure 4.) In faet, as the line of sight is approached, the optical formula approaches zero whereas the exact solution does not. The geometric-optical method breaks down in the optical region as the line of sight is approached. It may be noted that Figure 4 has been drawn for $k=1$ rather than for the customary value of $k=4 / 3$ corresponding to standard atmosphere conditions and is for a hypothetical isotropic radiator. 
TRANSMITTER LOW

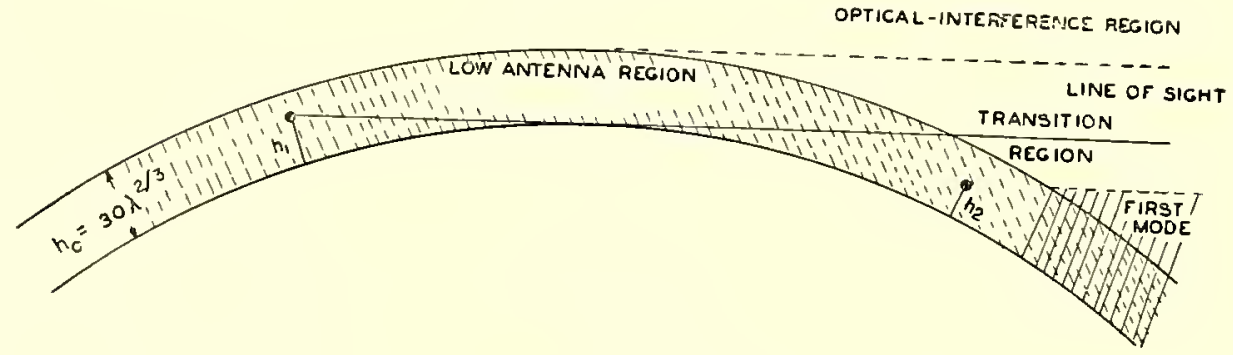

TRANSMITTER ELEVATEO

OPTICAL-INTERTERENCE REGION

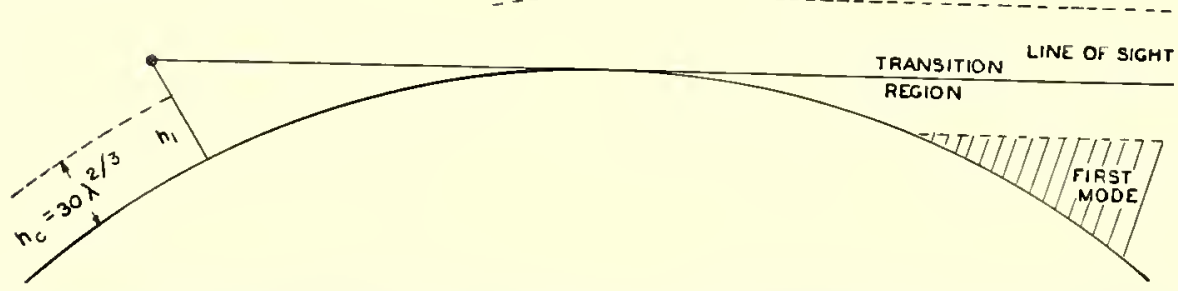

Figt RE 3. Field regions as related to transmitter heights for horizontal polarization or ultra-short waves. Iow antenna region $=\lambda_{l}>2 h_{1} h_{2} ; h_{1}, h_{2}<30 \lambda^{2 / 3}$.

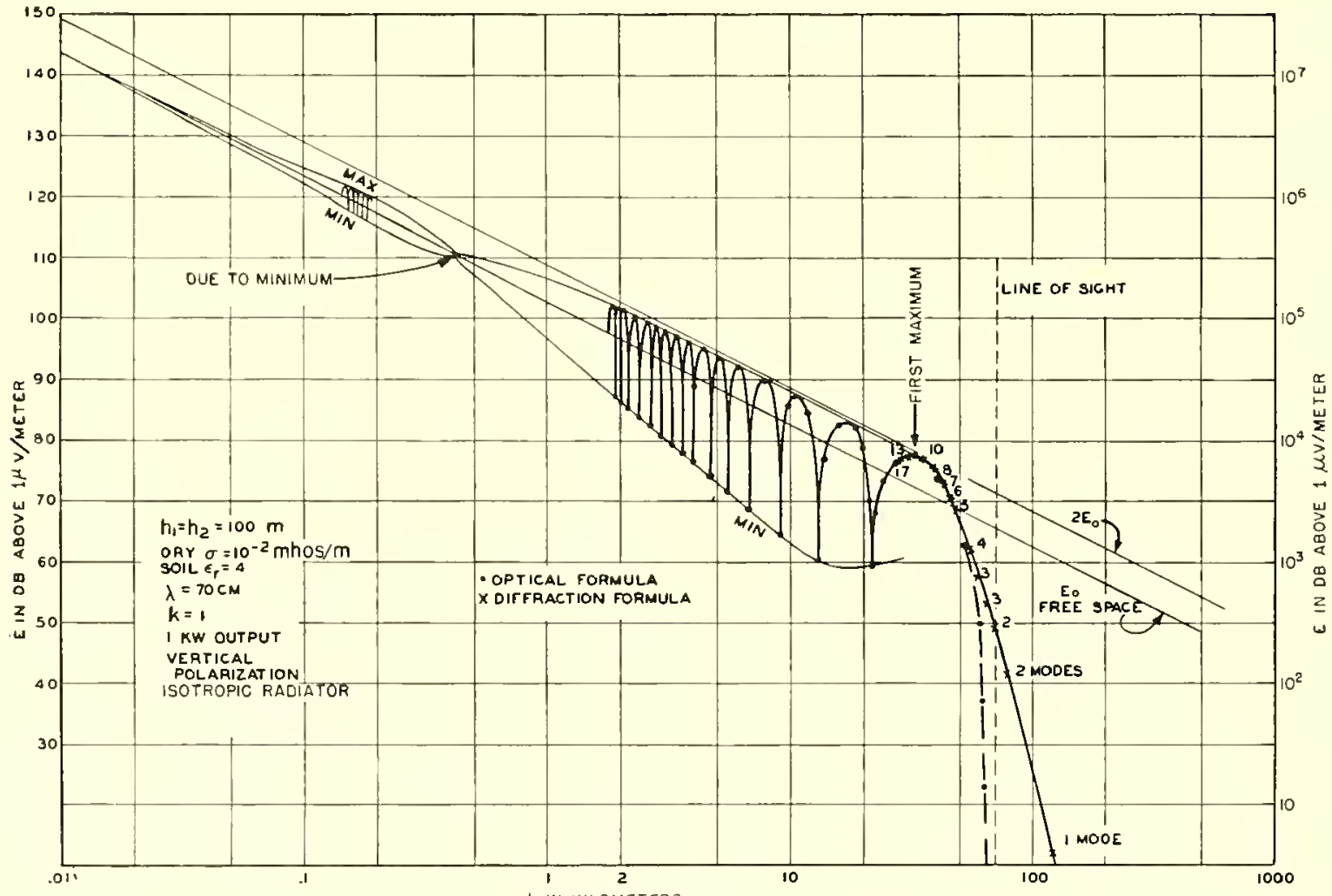

FIGURE 4. Variation of field strength with distance for propagation on vertical polarization with a wavelength of 70 chn over dry soil. The point "due to minimum" results from minimum in reflection coefficient at the pseudo-Brewster angle. 
If the earth were flat and perfectly reflecting, the envelope of the maxima of the curve in Figure 4 would coincide with the line $2 E_{0}$, twice the freespace field, corresponding to the in-phase addition of the direct and reflected waves. An envelope of the minimum points would be $E=0$, corresponding to the destructive interference of the direct and reflected waves. The curvature of the earth, resulting in increased divergence of the waves (see Section 5.2.5), and the lack of perfect reflection (see Section 5.2.4) cause the maximal and minimal envelopes to differ from $2 E_{0}$ and 0 , respectively. In the neighborhood of the first maximum in Figure 4 (i.e., when the direct ray makes small angles with the earth), the reflection coefficient tends to be unity in magnitucle for both polarizations except for the increase in divergence which results in the deviation of the maximal and minimal lines from $2 E$ and 0 , respectively. At a smaller distance, for vertical polarization, as shown in Figure 4, the deviation is caused principally by the smaller magnitude of the reflection coefficient. The virtual meeting of the maximal and minimal lines corresponds to the minimum value of the reflection coefficient at the psendo-Brewster angle. (For horizontal polarization at small distances, the envelope of maxima would virtually coincide with $2 E_{0}$ and the minima would be closer to zero. As the distance is increased, the difference between the envelopes for vertical and horizontal polarization gradually decreases.)

2. Both antennas low; $h<h_{c}$. In a discussion of the height function, it is convenient to distinguish between high and low antennas. The critical height separating the two cases for horizontal polarization or ultra-short waves is given by

$$
h_{c}=30 \lambda^{2 / 3} \text { meter's }
$$

where $\lambda$ is expressed in meters. For $\lambda=0.1$ meter, $h_{c}=6.46$ meters, and for $\lambda=10$ meters, $h_{c}=139.5$ meters. If both antennas are at elevations less than $h_{c}$, the height-gain functions $f(h)$, to a first approximation, are the same for all the modes, so that the complete solution

$$
f_{1}\left(h_{1}\right) \cdot f_{1}\left(h_{2}\right) \cdot F_{1}(d)+f_{2}\left(h_{1}\right) \cdot f_{2}\left(h_{2}\right) \cdot F_{2}(d)+++
$$

can be written in the form

$$
f\left(h_{1}\right) \cdot f\left(h_{2}\right) \cdot\left(F_{1}+F_{2}+\cdots\right),
$$

or

$$
f\left(h_{1}\right) \cdot f\left(h_{2}\right) \cdot F(d),
$$

where $f\left(h_{1}\right)$ replaces $f_{1}\left(h_{1}\right), f\left(h_{2}\right)$ replaces $f_{1}\left(h_{2}\right)$, etc., while $F(d)$ stands for the sum $F_{1}+F_{2}+\cdots$.
The distance function $F(d)$ can be calculated for particular cases. This has been done for high frequencies and is represented graphically in Section 5.7, the results being valid for low antennas for all distances in the optical as well as in the diffraction region such that $2 h_{1} h_{2}<<\lambda d$ (see Figure 3 ). The condition $2 h_{1} h_{2}<<\lambda d$ assures that the antennas are below the interference pattern.

At the ground, $f(h)=1$, so that if both antennas are close to the ground, the distance dependence is given by $F(d)$ only.

3. One or both antennas elevated; $h>h_{c}=30 \lambda^{2 / 3}$. For elevated antennas, $h>h_{c}$ and the height-gain functions of $f(h)$ vary with the modes. Consequently, it is not possible to separate the height and distance effects as in the previous paragraph.

In the optical-interference region, it is more advantageous to use the method of combining the direct and reflected wares. This is equivalent to the rigorous solution which is illustrated by the dots in Figure 4.

Simple graphical aids can be given for points well within the diffraction region where the first mode predominates. The range of usefulness of the first mode can be extended by plotting the field strength given by the first mode as a function of height (or distance) and plotting a similar curve by using the ray method as far as the lowest (or first) maximum (see Figure 7 ). Then by joining these partial curves into a smooth overall curve, a fairly good value of the field can be obtained for intermediate points.

There is a further possibility occurring with the transmitting antemna elevated, the receirer low and lying below the interference pattern, and the distance short. In this event, none of the previous methods apply. However, the reciprocity principle (see Chapter 2) can be applied to find the radio gain at the receiver by interchanging the role of receiver and transmitter. Suppose the original transmitter height is 100 meters, the original receiver height 15 meters, and the wavelength 1 meter. Now let the transmitter height be 15 meters. If the receiver height is low ( $h_{2}<30$ meters), values of the gain can be found (Section 5.7); if the receiver height is in the interference region, the gain can be found by the ray method. Now suppose a curve be drawn for these results, giving the attenuation versus receiver height. From this graph, the value of the gain factor $A$ at $h_{2}=100$ meters can be read. This value of $A$ by the principle of reciprocity is the gain factor for the original heights. 
4. Cltra-short waves in the diffraction region. Dielectric earth. For $\lambda<10$ meters $(f>30 \mathrm{mc})$ and for either polarization, land acts as a dielectric earth or absorbing earth in contradistinction to a condueting earth. Propagation orer a dielectric earth is practi-

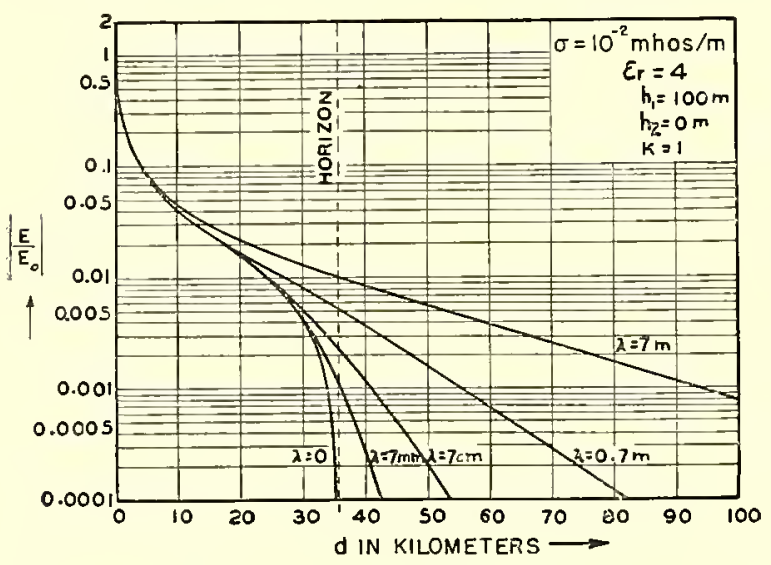

Figure 5. Field strength ratio versus distance for vertical polarization over dry soil for $h_{1}=100$ meters and $h_{9}=0$.

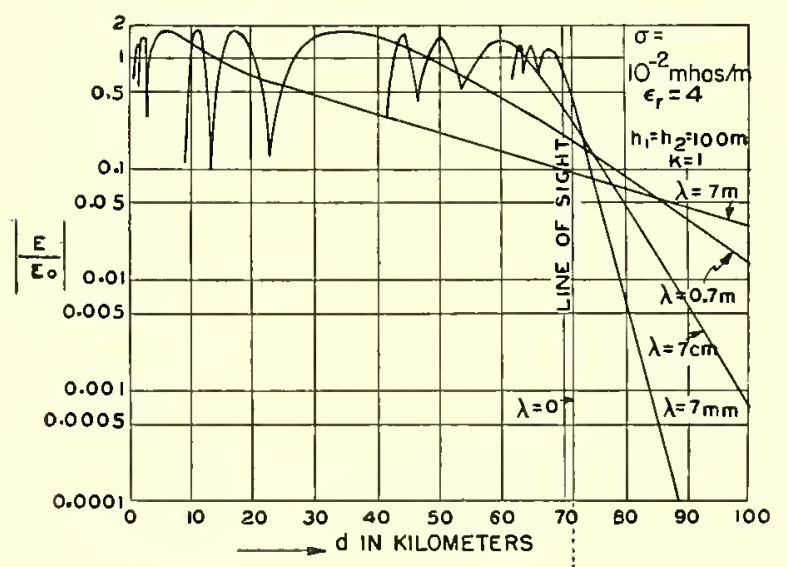

Figure 6. Field strength ratio versus distance for vertical polarization and heights $h_{1}=h_{2}=100$ meters.

cally independent of earth constants. For a given type of polarization, the chief variables affecting gain are then the heights of the antennas, their distance apart, and the wavelength. Within the diffraction region, the effect of incrcasing wavelength is to increase the field strength. This is illustrated by the curves in Figures 5 and $\mathbf{6}$. The dielectric earth is characterized by a value of $\delta>>1 . \delta$ is given by equation (193).

While sea water has i relatively high conductivity, radio wave propagation over it is the same as that over a dielectric earth in the case of horizontal polar- ization for $\lambda<10$ meters, and in the case of vertical polarization for $\lambda<1$ meter. Consequently, vertically polarized radiation of wavelength range 1 to 10 meters over sea water is given special treatment in Section 5.7.t.

In the same range, 1 to 10 meters, for vertically polarized radiation and for distances less than those given in Table 3 , propagation conditions over land atso deviate slightly from those corresponding to a dielectric earth.

5. Optical region. In the optical-interference region, the lobes for the shorter waves are more numerous, narrower, and lower, as can be seen from the oscillatory part of the field strength versus distance curves of Figure 6.

The dependence of reflection coefficients upon polarization, wavelength, and ground constants is diseussed in Seetion 4.2.

6. Horizontal versus vertical polarization. In the optical region, for rays at small grazing angles, there is not much difference between the two types of polarization. For larger grazing angles, the difference is more marked (see Section 4.2 on reflection coefficients, and see Section 5.2.4).

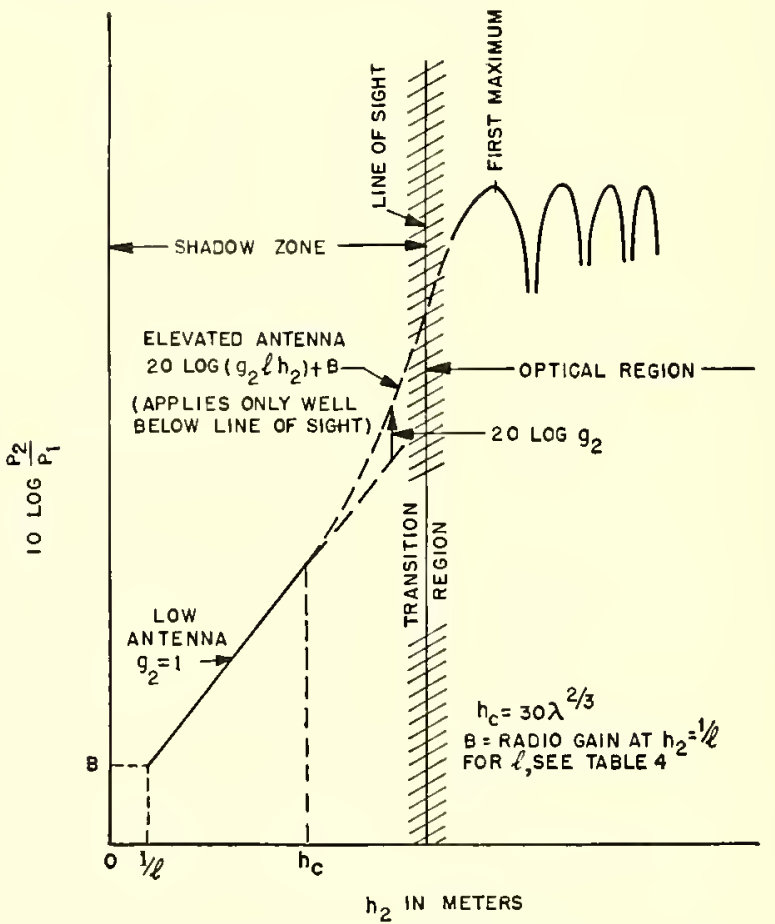

Figure 7. Gain versus height at distances beyond the radio horizon.

It has been pointed out in the previous paragraph that within the diffraction region for $\lambda<10$ meters 
and propagation over land, there is practically no difference in intensity between a horizontally and a vertically polarized radiation field. For $\lambda<1$ meter there is, similarly, no difference for propagation over sea water. When there is a difference, as for low antennas, horizontal polarization gives a smaller gain; but as the antennas are raised, the two cases approach equality (see Section 5.7.4).

\subsection{PROPAGATION FACTORS IN THE INTERFERENCE REGION}

\subsubsection{Propagation Factors}

The factors affecting gain in the region where the methods of geometrical optics may be applied are discussed in Sections 5.2 to 5.5 inclusive.

5.2 .2

\section{Spreading Effect}

From the formula of equation (1) in Chapter 2, for the field intensity components of the radiation field, it follows that for distances from the transmitter large in comparison to the wavelength, the dominant term falls off inversely as the distance from the transmitter, or

$$
E=\frac{E_{1}}{d}
$$

where $E_{1}$ is the field strength at unit distance. This means that the porrer per unit area in the radiation field varies inversely as the square of the distance. This spreading effect is the consequence of the fact that the energy of the wave is distributed over larger and larger areas as the wave progresses away from the transmitter.

\section{Interference}

When a wave travels orer a conducting surface, constructive and destructive interference occurs between the direct wave from the transmitter and the wave reflected by the surface. This is illustrated in Figure 8, which is drawn for a plane earth. If there is no energy lost in reflection, the direct and reflected waves are of equal intensity, and their resultant varies from zero to twice the free-space value, depending upon the phase difference between the two components. The reflected wave lags the direct wave by an angle $\delta+\phi$, where $\delta$ is the phase retardation caused by the greater path length

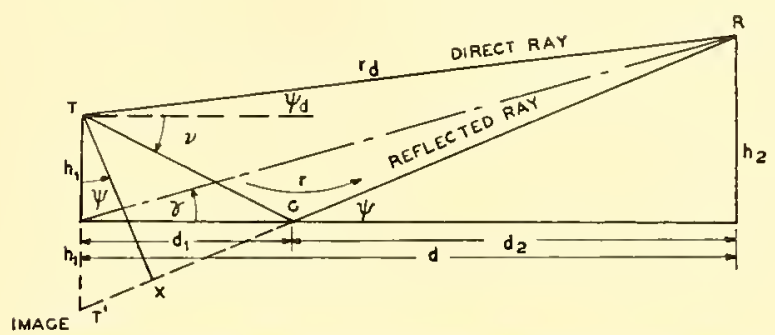

Figure S. Geometry for radio propagation over a plane earth.

traversed by the reflected wave and $\phi$ is the phase lag occurring at reflection.

Figure 9 shows the vector diagram for the case where the phase shift at reflection is $\phi=180$ degrees.

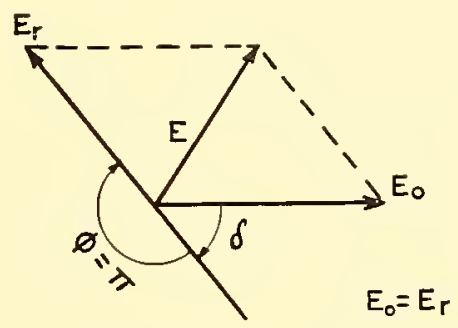

Figure 9. Tector diagram showing the addition of the direct and reflected waves for $\phi=180^{\circ}$ and $\rho=1$.

This condition holds for horizontally polarized radiation of frequency above 100 megacycles, reflected from sea water at grazing angles of less than 10 degrees. The resultant electric field is equal to

$$
\begin{aligned}
E & =\sqrt{E_{0}^{2}+E_{r}^{2}-2 E_{0} E_{r} \cos \delta} \\
& =\sqrt{\left(E_{0}-E_{r}\right)^{2}+4 E_{0} E_{r} \sin ^{2} \frac{\delta}{2}} .
\end{aligned}
$$

If the reflection is complete, as from a conductor of infinite conductivity,

$$
E_{r}=E_{0}, \quad E=2 E_{0} \sin \frac{\delta}{2} .
$$

\section{Imperfect Reflection}

In general, the strength of the reflected wave $E_{r}$ is less than that of the incident wave $E_{i}$, partly because of diffuse reflection and partly because some energy is refracted into the surface and absorbed. 
Furthermore, the phase lag usually differs from 180 degrees, depending upon the frequency and grazing angle. This is especially true for vertical polarization where the reflection coefficient is a critical function of both the grazing angle and frequency. The ratio

$$
R=\frac{E_{r}}{E_{i}}=\rho e^{-j \phi}
$$

is a complex number and defines the reflection coefficient $R$, which has an absolute value $\rho$ and a phase angle $\phi$.

In equation (27), a lagging angle is considered positive. Writing $\phi=\pi+\phi^{\prime}$, equation (27) may be expressed as

$$
R=\frac{E_{r}}{E_{i}}=\rho c^{-j\left(\pi+\phi^{\prime}\right)}=-\rho e^{-j \phi^{\prime}} .
$$

In equation (27), the lag angle $\phi$ is measured with respect to zero-degree phase shift at reflection. For horizontal polarization, $\phi$ varies from 180 degrees to 183 degrees, and from 180 degrees to 3 degrees for vertical polarization at 3,000 me over sea water. In equation (28), lag angle $\phi^{\prime}$ is measured with respect to a 180-degree phase shift (that is, from $E_{i}$ reversed), and varies from 0 degree to 3 degrees for horizontal polarization and from 0 degree to -177 degrees for vertical polarization.

The resultant field intensity is

$$
\begin{aligned}
E=E_{0}+\rho E_{0} e^{-j(\delta+\phi)} & =E_{0}+\rho E_{0} e^{-j\left(\delta+\phi^{\prime}+\pi\right)} \\
& =E_{0}\left(1-\rho e^{-j \Omega}\right),
\end{aligned}
$$

where

$$
\Omega=\delta+\phi^{\prime}=\delta+\phi-\pi .
$$

The absolute value of the received field $|E|$ is given by

or

$$
|E|=E_{0} \sqrt{\left(1-\rho e^{+j \Omega}\right)\left(1-\rho e^{-j \Omega}\right)}
$$

$$
\begin{aligned}
|E| & =E_{0} \sqrt{1+\rho^{2}-2 \rho \cos \Omega} \\
& =E_{0} \sqrt{(1-\rho)^{2}+4 \rho \sin ^{2} \frac{\Omega}{2}} .
\end{aligned}
$$

Equation (30) shows that the received field intensity has a maximum of $2 E_{0}$ when

$$
\begin{aligned}
& \rho=1, \\
& \Omega=(2 n+1) \pi .
\end{aligned}
$$

The value of $E$ is a zero when

$$
\begin{aligned}
& \rho=1, \\
& \Omega=2 n \pi .
\end{aligned}
$$

In equations (31) and (32), $n$ includes all integral values and zero. Equation (30) may be written as

$$
E=\frac{E_{1}}{d} \sqrt{(1-\rho)^{2}+4 \rho \sin ^{2} \frac{\Omega}{2}}
$$

where $E$ is the field at distance $d$ from the transmitter, and $E_{1}$ is the field strength at unit distance. From equation (33),

$$
d=\frac{E_{1}}{E} \sqrt{(1-\rho)^{2}+4 \rho \sin ^{2} \frac{\Omega}{2}} .
$$

In free space where there is no reflecting earth, $\rho=0$, and

$$
d_{0}=\frac{E_{1}}{E}
$$

where $d_{0}$ is the equivalent free-space distance from the transmitter at which the field strength $E$ would be found. Hence equation (34) may be written in the form

$$
d=d_{0} \sqrt{(1-\rho)^{2}+4 \rho \sin ^{2} \frac{\Omega}{2}} .
$$

5.2 .5

\section{Divergence}

The divergence factor $D$ is introduced to account for the decreased gain produced by the spreading

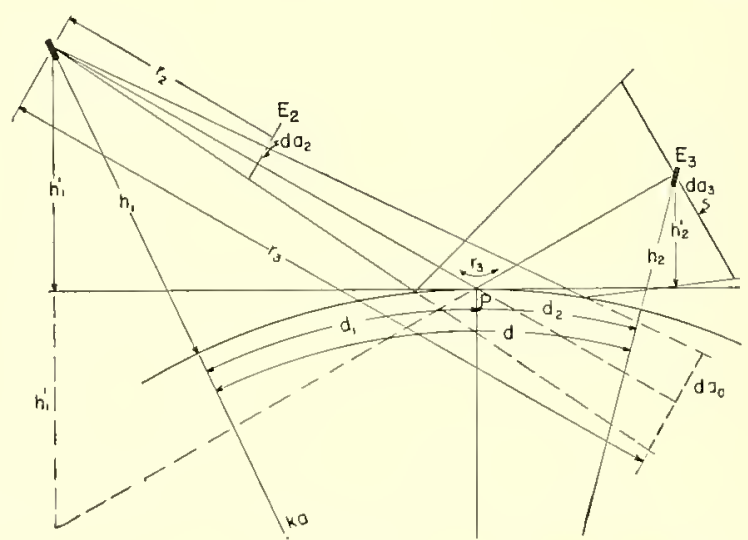

Figure 10. Increased divergence resulting from reflection by a sphere.

of a wave reflected from a spherical surface. Referring to Figure 10,

$$
\frac{E_{3}}{E_{2}}=\sqrt{\frac{d a_{2}}{d a_{3}}}=\sqrt{\frac{d a_{2}}{d a_{0}} \frac{d a_{0}}{d a_{3}}}=\frac{r_{2}}{r_{3}} \sqrt{\frac{d a_{0}}{d a_{3}}}=\frac{r_{2}}{r_{3}} D .
$$


In calculating the field intensity reflected from a spherical earth, the inverse distance attenuation factor $1 / r_{d}$ used for the direct ware must be multiplied by the divergence factor $D$, which is always less than unity.

As a result of this divergence the reflection coefficient for a spherical surface is less than that for a plane surface as given by

$$
\rho D=\rho^{\prime}
$$

where $\rho^{\prime}$ is the spherical earth reflection coefficient. Equations (30) and (36) may then be written as

$$
|E|=E_{0} \sqrt{\left(1-\rho^{\prime}\right)^{2}+4 \rho^{\prime} \sin ^{2} \frac{\Omega}{2}}
$$

and

$$
d=d_{0} \sqrt{\left(1-\rho^{\prime}\right)^{2}+4 \rho^{\prime} \sin ^{2} \frac{\Omega}{2}} .
$$

\subsubsection{Antenna Gain and Directivity}

The effects of antenna gain and directivity are expressed by means of the gain factor $G$, defined in Section 2.2.2, and the antenna pattern factors $F_{1}$ and $F_{2}$, which are the fractions of the maximum radiation amplitude in the direction of the direct and reflected rays respectively. The maximum amplitude for a transmitting antenna with gain $G_{1}$ is $E_{0}{ }^{\prime}=\sqrt{G_{1}} E_{0}$, where $E_{0}$ is the free-space field strength radiated by a doublet.

When the antemna pattern factors are taken into account, the resultant field intensity, following equation (30), is

$$
E=\sqrt{G_{1}} E_{0} \sqrt{F_{1}^{2}+F_{2}^{2} \rho^{2} D^{2}-2 F_{1} F_{2} \rho \cos \Omega} .
$$

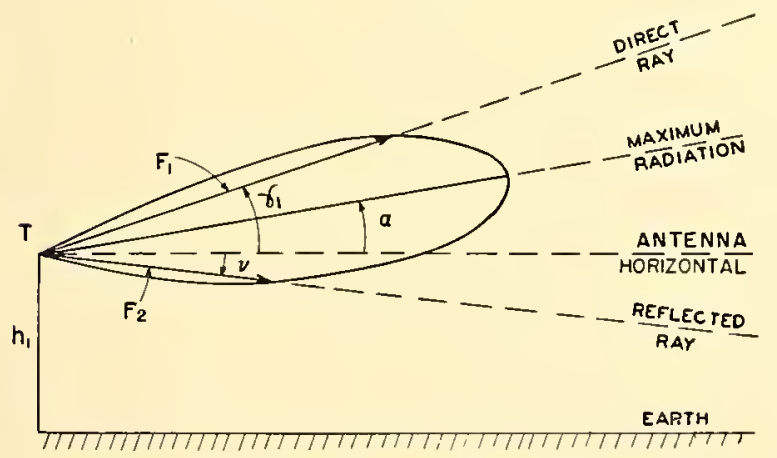

Figure 11. Antenna pattern factors $F_{1}$ and $F_{2}$.

The factors $F_{1}$ and $F_{2}$ are functions of the angles $\gamma_{1}-\alpha$ and $\nu+\alpha$ which the direct and reflected rays make with the axis of the beam. Figure 11 is a typical antenna pattern.

\section{GENERAL SOLUTION}

\subsubsection{Generalized Reflection Coefficient}

Equation (41) may be simplified by introducing a generalized coefficient which includes the effects of reflection, divergence, and directivity. The amplitude of this coefficient will be denoted by $K$ and is given by

$$
K=\frac{F_{2}}{F_{1}} \rho D .
$$

Substituting $F_{2}=F_{1} K / \rho D$ in equation (41) gives

$$
E=\sqrt{\sigma_{1}} F_{1} E_{0} \sqrt{1+K^{2}-2 K \cos \Omega}
$$

or

$$
E=\sqrt{G_{1}} F_{1} E_{0} \sqrt{(1-K)^{2}+4 K \sin ^{2} \frac{\Omega}{2}} .
$$

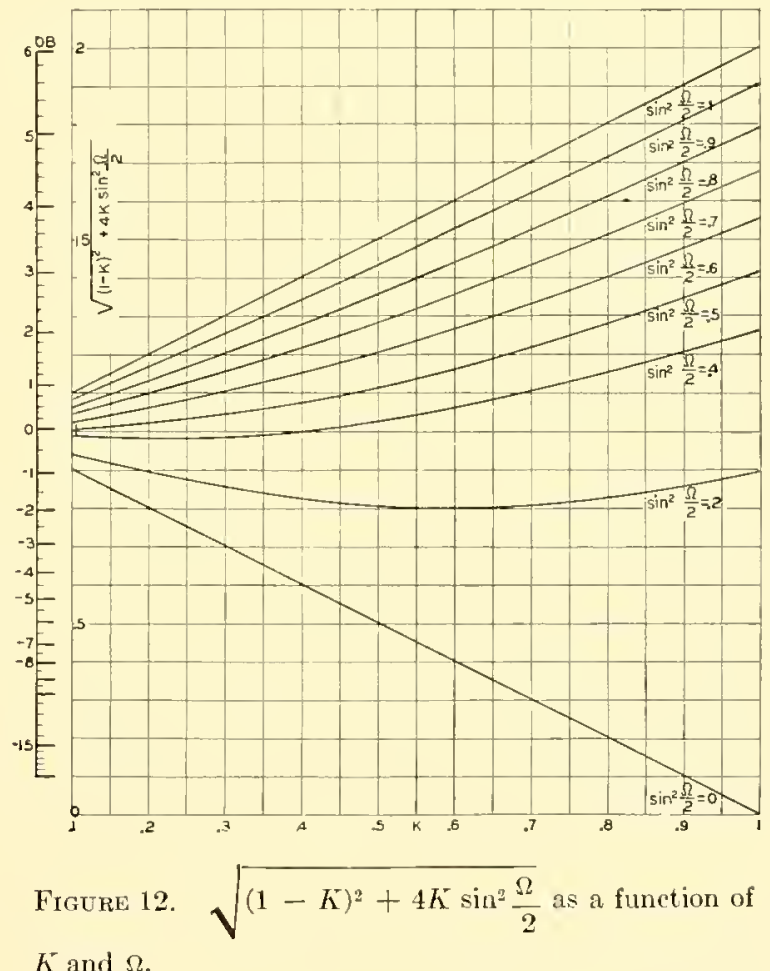

If the transmitting antenna is pointed so that the direct ray lies in the direction of maximum gaim, $F_{1}=1$, and

$$
E=\sqrt{G_{1}} E_{0} \sqrt{(1-K)^{2}+4 K \sin ^{2} \frac{\Omega}{2}}
$$

and

$$
d=\sqrt{G_{1}} d_{0} \sqrt{(1-K)^{2}+4 K \sin ^{2} \frac{\Omega}{2}}
$$


Figure 12 shows

$$
\sqrt{(1-K)^{2}+4 K \sin ^{2} \frac{\Omega}{2}}
$$

as a function of $K$ for various values of $\sin \Omega / 2$ and may be used to calculate $E$.

The value of $G_{1}$ to be used in equation (46) may be found from the antenna specifications for a given set. The free-space ficld $E_{0}$ at distance $d$ from a transmitting doublet with power output $P_{1}$ is equal to

$$
E_{0}=\frac{3 \sqrt{5} \sqrt{P_{1}}}{d}
$$

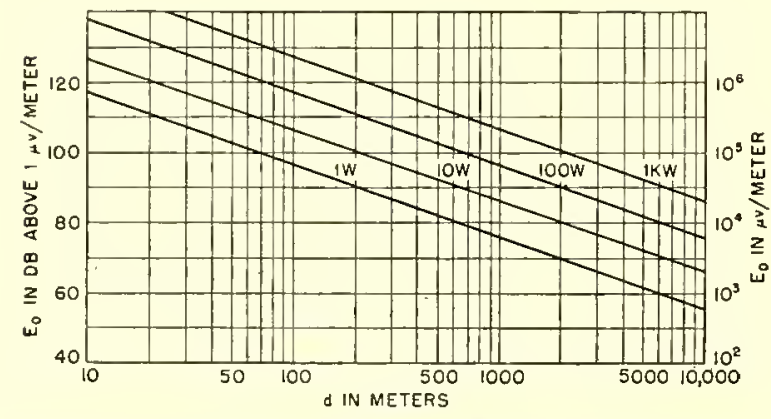

F1Gure 13. Free-space range $d$ as a function of field strength $E_{0}$ and transmitter power $P_{1}$.

Figure 13 shows $E_{0}$ in decibels above 1 mierovolt per meter as a function of $d$ for various values of transmitted power. The free-space field at distance of $d$ meters expressed in decibels above 1 microvolt per meter for $P_{1}$ watts radiated is

$$
\text { Decibels }=20 \log _{10}\left[\frac{3 \sqrt{5} \sqrt{P_{1}}}{10^{-6} d}\right] .
$$

\subsection{PLANE EARTH}

\subsubsection{Use of Plane Earth Formula}

It will be shown in Section 5.5.5 that under certain conditions ealculations based upon the assumption of a plane earth yicld satisfactory results. Calculations for propagation over a plane earth are given in this section.

5.4 .2

\section{Path Difference}

It follows from equation (29) that when $\rho=1$ and $\phi=180$ degrees, the received field depends only upon the phase lag caused by path difference. Referring to Figure 8 ,

$$
\begin{aligned}
& r_{d}=\sqrt{d^{2}+\left(h_{1}-h_{2}\right)^{2}}=d \sqrt{1+\left(\frac{h_{1}-h_{2}}{d}\right)^{2}}, \\
& =d\left[1+\frac{1}{2}\left(\frac{h_{1}-h_{2}}{d}\right)^{2}-\frac{1}{8}\left(\frac{h_{1}-h_{2}}{d}\right)^{4}+\ldots\right] \text {, } \\
& r=\sqrt{d^{2}+\left(h_{1}+h_{2}\right)^{2}}=d \sqrt{1+\left(\frac{h_{1}+h_{2}}{d}\right)^{2}}, \\
& =d\left[1+\frac{1}{2}\left(\frac{h_{1}+h_{2}}{d}\right)^{2}-\frac{1}{8}\left(\frac{h_{1}+h_{2}}{d}\right)^{4}+\ldots\right] \text {, } \\
& \Delta=r-r_{d}=d\left[\frac{2 h_{1} h_{2}}{d^{2}}-\frac{h_{1} h_{2}\left(h_{1}^{2}+h_{2}^{2}\right)}{d^{4}}+\ldots\right], \\
& =\frac{2 h_{1} h_{2}}{d}\left[1-\frac{h_{1}^{2}+h_{2}^{2}}{2 d^{2}}+\ldots\right] \text {. } \\
& \frac{h_{1}^{2}+h_{2}^{2}}{2 d^{2}}<<1, \\
& \Delta \cong \frac{2 h_{1} h_{2}}{d} \text {. }
\end{aligned}
$$

The phase lag caused by the path difference $\Delta$ is equal to

$$
\delta=\frac{2 \pi}{\lambda}\left(\frac{2 h_{1} h_{2}}{d}\right)=\frac{4 \pi h_{1} h_{2}}{\lambda d},
$$

where $\lambda$ is the wavelength of the radiation.

\subsubsection{Field Strength Equations}

When $\rho=1$ and $\phi=180^{\circ}$, equation (26) may be used. Substituting equation (53) for $\delta$ into equation (26) gives

$$
E=2 E_{0} \sin \left(\frac{2 \pi h_{1} h_{2}}{\lambda d}\right)
$$

If $\delta / 2<10^{\circ}, \sin (\delta / 2) \rightarrow \delta / 2$ and

$$
E=E_{0} \frac{4 \pi h_{1} h_{2}}{\lambda d}
$$

When $h_{1}$ or $h_{2}$ equals 0 , equation (55) indicates that the received field intensity is equal to zero, which is contrary to fact. For this case the diffraction field must therefore be calculated and included as explained in Section 5.1.7.

In the general casc $(\rho<1)$, equation (41) may be applied with $K=\left(F_{2} / F_{1}\right) \rho D$. A refinement may be added to the calculation by taking into account 
the fact that the image source (Figure 8) of the reflected wave is at a distance $r+\Delta$ from the receiver. The reflected wave is attenuated more than the direct wave, according to the free-space attenuation ratio $(r+\Delta) / r$. If this is taken into account the ordinary reflection coefficient is replaced by

$$
K^{\prime}=\frac{F_{2}}{F_{1}}\left(\frac{r}{r+\Delta}\right) \rho D .
$$

The correction is not necessary when $2 h_{1} h_{2}<<d^{2}$ [see equation (52)].

5.5

\section{SPHERICAL EARTH}

\subsubsection{Measurement of Distance}

The difference between the slant range $r_{d}$ and the distance measured along the surface of the earth and designated by $d$ in Figure 14 is usually negligible. For a transmitter height of 1,500 meters, the error in assuming $r_{d}=d$ is 0.04 per cent at a distance of $161 \mathrm{~km}$ and height of 6,900 meters, and 1 per cent at the same distance but at a height of 22,500 meters. As the transmitter height is increased, the error is increased.
In order to express the slant range $r_{d}$ in terms of the eurved distance $d$ to a higher order of accuracy, the cosine law is applied to the triangle, transmitterreceiver-earth center. This gives the equation

$$
\begin{aligned}
r_{d}^{2}= & \left(k a+h_{1}\right)^{2}+\left(k a+h_{2}\right)^{2} \\
& -2\left(k a+h_{1}\right)\left(k a+h_{2}\right) \cos \left(\frac{d}{k a}\right) .
\end{aligned}
$$

Selecting the relatively important terms of the order' $d^{2} h_{1} h_{2}$ and $d^{t}\left(h_{1}+h_{2}\right)$, as well as powers of $d$ higher than the fourth, the above equation reduces to

$$
r_{d^{2}}=d^{2}+\left(h_{2}-h_{1}\right)^{2}+\frac{d^{2}}{k a}\left(h_{1}+h_{2}-\frac{d^{2}}{12 l_{i a}}\right) .
$$

\subsubsection{Equivalent Heights}

Solving equations (13) and (14) for $h_{1}$ and $h_{2}$ gives

$$
\begin{aligned}
& h_{1}=\frac{d_{T^{2}}}{2 k a}, \\
& h_{2}=\frac{d_{R}^{2}}{2 k a} .
\end{aligned}
$$

These results may also be expressed by saying that the distance from the surface of the earth to a plane which is tangent at a distance $d_{1}$ from the transmitter is $d_{1}^{2} / 2 k a$.

$$
\begin{aligned}
& p=\frac{d_{1}}{d_{T}} \quad q=1-s=\frac{d_{2}}{d} \\
& v=\frac{d}{d_{T}} \quad u=\frac{h_{2}}{h_{1}} \\
& s=\frac{p}{v}=\frac{d_{1}}{d}=1-q
\end{aligned}
$$

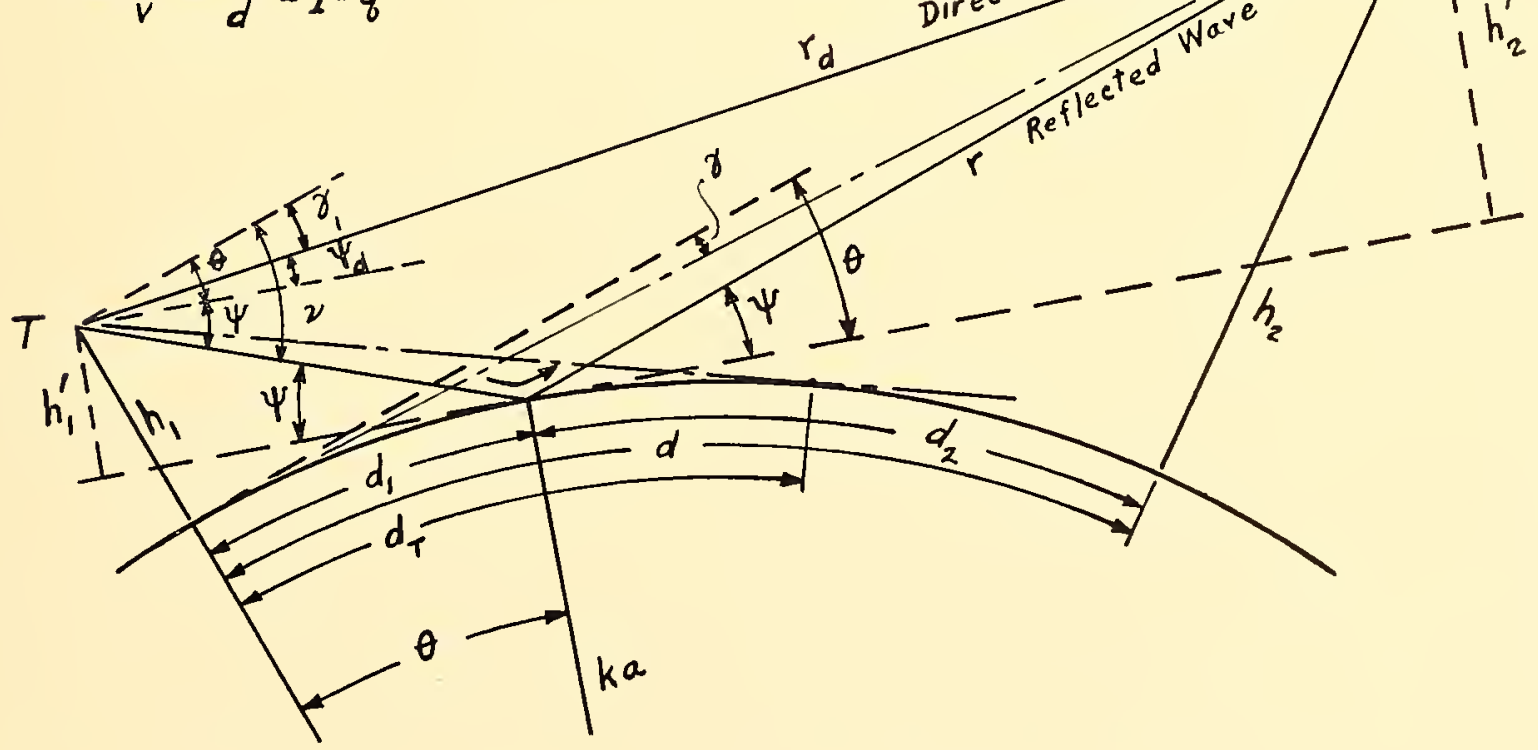

Figure 14. Geometry for radio wave propagation over a spherical earth. (Vertical dimensions greatly exaggerated.) 
Hence for a transmitter of height $h_{1}$ above the ground the height above the tangent plane at the reflection point, the so-called equivalent height is, to a first approximation,

$$
h_{1}^{\prime}=h_{1}-\frac{d_{1}{ }^{2}}{2 k a},
$$

and for the receiver the equivalent height is

$$
h_{2}{ }^{\prime}=h_{2}-\frac{d_{2}{ }^{2}}{2 k a} .
$$

The equivalent heights are shown in Figure 14, which illustrates the geometry of the spherical earth having an effective radius of $k a$.

5.5 .3

\section{Angles}

Referring to Figure 14 and remembering that the angles are greatly exaggerated in the figure, it is seen that

$$
\begin{aligned}
\tan \psi & \cong \frac{h_{1}{ }^{\prime}}{d_{1}}=\frac{h_{2}^{\prime}}{d_{2}}, \\
\tan \gamma & \cong \frac{h_{2}}{d}-\frac{d}{2 k a}, \\
\tan \psi_{d} & \cong \frac{h_{2}-h_{1}}{d}-\frac{d}{2 k a}, \\
\nu & \cong \psi+\frac{d_{1}}{k a} .
\end{aligned}
$$

Angle $\psi$ must be evaluated in order to determine the reflection coefficient. Angles $\psi_{d}$ and $\nu$ determine the antenna pattern factors $F_{1}$ and $F_{2}$, which are shown in Figure 11. The angle $\gamma$ is significant in coverage calculations and angular approximations.

\subsubsection{Determination of Reflection Point $\left(d_{1}\right)$}

Inasmuch as several equations of Seetions 5.5.2 and 5.5.3 depend upon $d_{1}$, it is necessary to be able to determine this distance when the transmitter and receiver heights are given and the distance between them is known. Let

$$
d_{1}=\frac{d}{2}(1+b)
$$

and

$$
h_{\mathrm{I}}=\frac{\left(h_{1}+h_{2}\right)}{2}(1+c)
$$

so that

and

$$
\begin{aligned}
& d_{2}=\frac{d}{2}(1-b), \\
& h_{2}=\frac{h_{1}+h_{2}}{2}(1-c),
\end{aligned}
$$

where

Where

$$
b=\frac{d_{1}-d_{2}}{d_{1}+d_{2}},
$$

and

$$
c=\frac{h_{1}-h_{2}}{h_{1}+h_{2}} \text {. }
$$

Assume $h_{1}>h_{2}$ and $d_{1}>d_{2}$, so that $b$ and $c$ will always be positive. This is always possible because of the principle of reciprocity. From equation (60),

$$
\frac{h_{1}^{\prime}}{d_{1}}=\frac{h_{2}^{\prime}}{d_{2}} \quad \text { or } \quad \frac{h_{1}}{d_{1}}-\frac{d_{1}}{2 k a}=\frac{h_{2}}{d_{2}}-\frac{d_{2}}{2 k a} .
$$

Substituting for $d_{1}$ and $d_{2}$ from equations (6t) and (66),

$$
\begin{aligned}
& \frac{h_{1}+h_{2}}{d}\left(\frac{1+c}{1+b}\right)-\frac{d(1+b)}{4 k a} \\
= & \frac{h_{1}+h_{2}}{d}\left(\frac{1-c}{1-b}\right)-\frac{d(1-b)}{4 k a} .
\end{aligned}
$$

Simplifying,

$$
\frac{h_{1}+h_{2}}{d} \cdot \frac{2(c-b)}{1-b^{2}}=\frac{b d}{2 k a} .
$$

Solving for $c$,

$$
c=b+b m\left(1-b^{2}\right)
$$

where

$$
m=\frac{d^{2}}{4 k a\left(h_{1}+h_{2}\right)}
$$

To determine $d_{1}$, equation (71) must be solved for $b$. This is a cubic equation, which is easily solved when $m$ is small in comparison to unity. However, for $m$ values eomparable to unity, or larger, it is easier to plot a series of eurres showing $c$ as a function of $m$ for assigned values of $b$ ranging from 0 to 1 . These are straight lines with a slope of $b\left(1-b^{2}\right)$ and are given in Figure 15.

The procedure for calculating $d_{1}$ when $h_{1}, h_{2}$, and $d$ are given is as follows:

1. Compute $c=\frac{h_{1}-h_{2}}{h_{1}+h_{2}}$. 
2. Compute $m=\frac{d^{2}}{4 k a\left(h_{1}+h_{2}\right)}$.

3. Read $b$ from Figure 15.

4. Calculate $d_{1}=\frac{d}{2}(1+b)$.

It should be noted that $h_{1}$ may be the height of either the transmitter or receiver. The only re-
From equation $(70)$

$$
\frac{h_{1}}{s d}-\frac{s d}{2 k a}=\frac{h_{2}}{(1-s) d}-\frac{(1-s) d}{2 k a} .
$$

Simplifying,

$$
s^{3}-\frac{3}{2} s^{2}-s\left[\frac{k a}{d^{2}}\left(h_{1}+h_{2}\right)-\frac{1}{2}\right]+\frac{k a h_{1}}{d^{2}}=0 .
$$

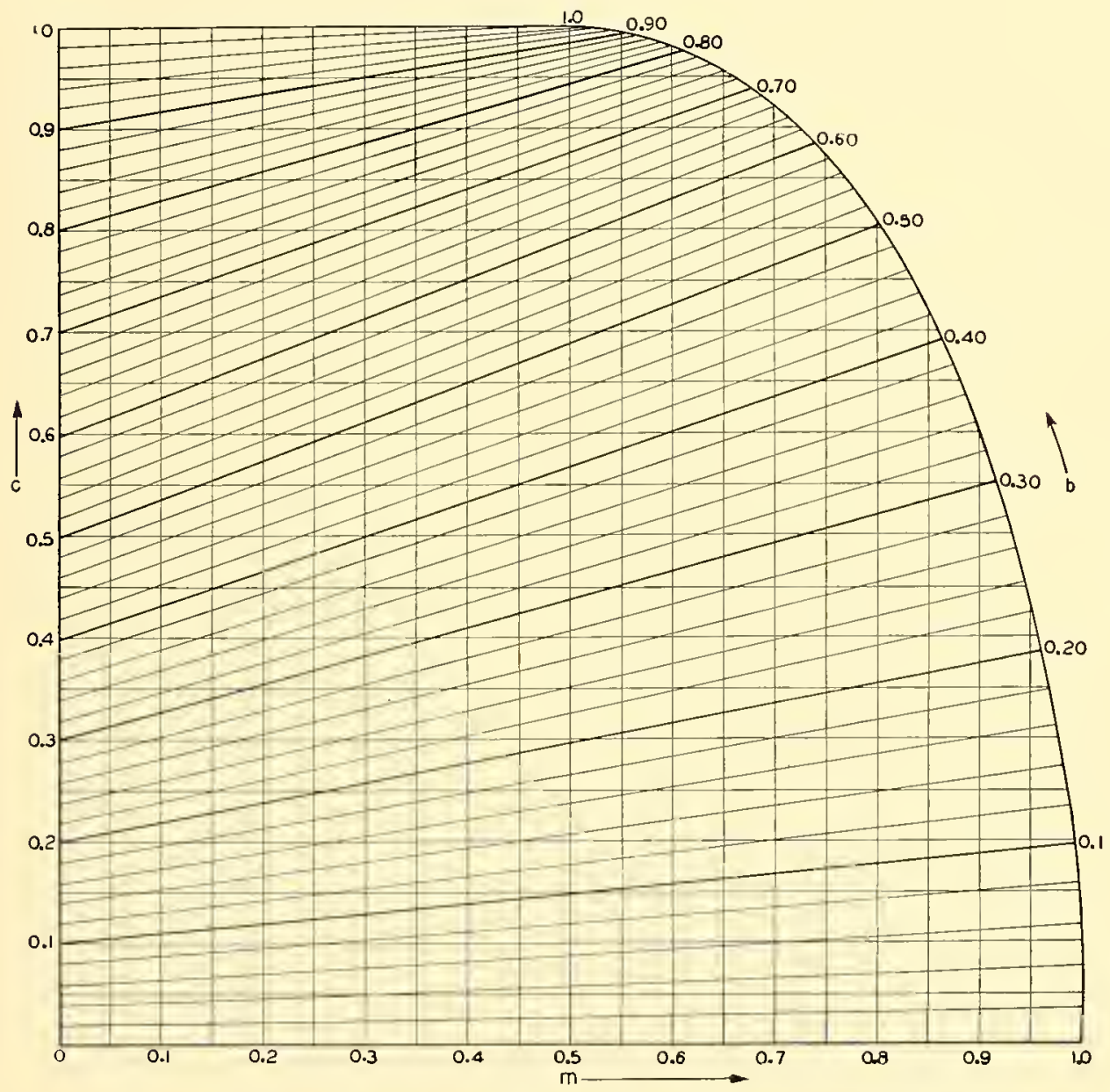

F1Gure 15. Graph for obtaining $b$ for given values of $c$ and $m$. (Marconi, Ltd.)

strictions are that $h_{1}>h_{2}$ and $d_{1}$ represents the distance from $h_{1}$.

Another method will now be given for calculating $d_{1}$. This method will be particularly useful when $d_{1} / d<<1$ and will be applied in Section 5.5.S on generalized eoordinates. Let

$$
d_{1}=s d, \quad s<1 .
$$

Hence

$$
d_{2}=(1-s) d
$$

If $s<<1$, the terms $s^{3}$ and $s^{2}$ may be neglected to a first approximation, and

$$
s=\frac{h_{1}}{\left(h_{1}+h_{2}\right)-\frac{d^{2}}{2 k a}} .
$$

If $d<<d_{L}$ (Section 5.1.5), $h_{2}$ is well above the line of sight and $s$ reduces to

$$
s=\frac{h_{1}}{h_{1}+h_{2}}=\frac{d_{1}}{d} .
$$


Equation $(76)$ is the plane earth formula.

Curves showing $s$ as a function of $h_{2} / h_{1}$ and $d / d_{T}$ are given in Figures 19 and 20. These may be used for the direct calculation of $d_{1}=s d$ within the limits of interpolation.

\section{5 .5}

\section{Path Difference}

Referring to Figure 14, the path difference $\Delta$ for a spherical earth is equal to

$$
\begin{aligned}
\Delta=r-r_{d}=\sqrt{d^{2}+} & \left(h_{2}{ }^{\prime}+h_{1}{ }^{\prime}\right)^{2} \\
& -\sqrt{d^{2}+\left(h_{2}{ }^{\prime}-h_{1}{ }^{\prime}\right)^{2}} .
\end{aligned}
$$

It is usually sufficient to expand the square roots and neglect powers and products of $h_{1}{ }^{\prime}$ and $h_{2}{ }^{\prime}$ beyond the second. This gives

$$
\Delta=r-r_{d} \cong \frac{2 h_{1}{ }^{\prime} h_{2}{ }^{\prime}}{d}
$$

which is the same as the plane earth formula when $h_{1}^{\prime}$ and $h_{2}{ }^{\prime}$ are written instead of $h_{1}$ and $h_{2}$. Equation (78) is accurate to within 1 per cent for values of $\gamma$ (the angle at the base of the transmitter) less than about 8 degrees. The error is less than 10 per cent for values of $\gamma$ less than about 24 degrees. When equation (78) is not sufficiently accurate, the following may be used:

$$
\Delta=r-r_{d}=\frac{2 h_{1}{ }^{\prime} h_{2}{ }^{\prime}}{\sqrt{d^{2}+\left(h_{1}^{\prime}\right)^{2}+\left(h_{2}\right)^{2}}},
$$

provided

$$
\frac{1}{2} \frac{\left(h_{1}{ }^{\prime}\right)^{2}+\left(h_{2}\right)^{2}}{d^{2}}<<1 .
$$

All the above equations for the path difference depend upon the distance to the reflection point $d_{1}$. However, the calculation of $d_{1}$ may be eliminated by first computing the path difference from the plane earth formula and then subtracting the correction term $\Delta\left(\Delta_{\boldsymbol{p}}\right)$. Thus

$$
\Delta=\Delta_{p}-\Delta\left(\Delta_{p}\right)
$$

where

$$
\Delta_{p}=\frac{2 h_{1} h_{2}}{d}
$$

and where

$$
\frac{5.50}{h_{1}^{3 / 2}} \Delta\left(\Delta_{p}\right)
$$

is given in Figure 16, plotted against.

$$
\frac{d}{d_{L}}=\frac{d}{\sqrt{2 k a}} \cdot \frac{1}{\sqrt{h_{1}}+\sqrt{h_{2}}} \cdot
$$

If $h_{2}<h_{1}$, interchange $h_{2}$ and $h_{1}$ on the curres and ordinate of Figure 16.

The maximum value of $\Delta\left(\Delta_{p}\right)$ is

$$
\Delta\left(\Delta_{p}\right)_{\max }=5.33 \times 10^{-4} \frac{h_{1} h_{2}}{\sqrt{h_{1}}+\sqrt{h_{2}}} .
$$

If the plane earth correction factor is negligible for the wavelength under consideration, the plane earth formula may be used throughont the whole range within the optical region, not only for the given value of $h_{2} / h_{1}$ but for all lower values of $h_{2} / h_{1}$ with the same $h_{1}$.

When $h_{2}>>h_{1}$, so that the reflection point is much closer to the transmitter than to the receiver, a good approximation to $\lrcorner$ is obtained by replacing $h_{1}{ }^{\prime}$ by $h_{1}$ and $h_{2}{ }^{\prime}$ by $h_{2}-d^{2} / 2 h a$ in equation (78).

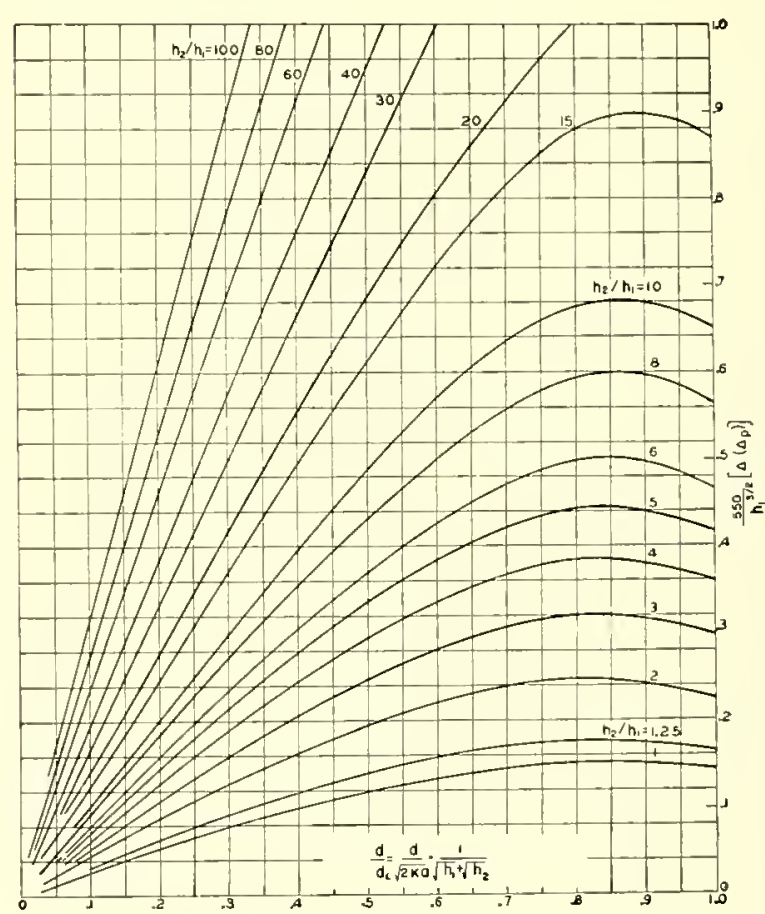
Figvre 16. Plane earth correction factor versus $\frac{d}{d L}$.
(Radiation Laboratory.)

Then

$$
\Delta \cong \frac{2 h_{1}}{d}\left(h_{2}-\frac{d^{2}}{2 k a}\right)
$$

which, to the same approximation, means that

$$
\Delta \cong 2 h_{1} \tan \gamma
$$

In general, equation (82) is an improvement over the plane earth approximation except close to the transmitter and at low heights where $h_{2}$ is not much greater than $h_{1}$. 
The dependence of path difference upon distance and height may be seen by considering the path difference parameter

$$
R=\frac{k a \Delta}{h_{1} d_{T}} .
$$

Since $h_{2}^{\prime}=h_{1}^{\prime} d_{2} / d_{1}$, it follows from equations (78) and (59) that

$$
\Delta=\frac{2 h_{1}{ }^{\prime} h_{2}{ }^{\prime}}{d}=\frac{2\left(h_{2}{ }^{\prime}\right)^{2} d_{2}}{d d_{1}}=\frac{2 d_{2}}{d} h_{1}{ }^{2} \frac{\left(1-\frac{d_{1}{ }^{2}}{2 k a h_{1}}\right)^{2}}{d_{1}} .
$$

Hence, using $d_{r^{2}}=2 k a h_{1}$.

$$
\Delta=\frac{2 h_{1}^{2}}{d_{T}}\left(\frac{d_{2}}{d}\right) \frac{\left[1-\left(d_{1} / d_{T}\right)^{2}\right]^{2}}{d_{1} / d_{T}}
$$

and

$$
\begin{aligned}
R & =\frac{d_{2}}{d} \times \frac{\left[1-\left(d_{1} / d_{T}\right)^{2}\right]^{2}}{d_{1} / d_{T}} \\
& =\left(1-\frac{d_{1} / d_{T}}{d / d_{T}}\right) \frac{\left[1-\left(d_{1} / d_{T}\right)^{2}\right]^{2}}{d_{1} / d_{T}}
\end{aligned}
$$

'The form of this expression suggests the introduction of two new dimensionless parameters

$$
p=\frac{d_{1}}{d_{T}} \text { and } v=\frac{d}{d_{T}} .
$$

In terms of these parameters, equation (85) for $R$ assumes the turm

$$
R=\left(1-\frac{p}{v}\right) \frac{\left(1-p^{2}\right)^{2}}{p},
$$

and in terms of $s$ and $v$

$$
R=(1-s) \frac{\left(1-s^{2} v^{2}\right)^{2}}{s v} .
$$

\subsubsection{Divergence Factor}

The reflection of a beam of radiation from the spherical earth increases the divergence of the beam and reduces the intensity of the reflected wave by spreading, as explained in Section 5.2.5. This is taken into account by introducing a divergence factor $D$, less than unity, which appears in the formulas as a multiplier of the plane earth reflection coefficient. Expressions for $D$ are

$$
D=\frac{1}{\sqrt{1+2 h_{1} h_{2}{ }^{\prime} / \text { kad } \tan ^{3} \psi}} .
$$

Using equation (60), $D$ becomes

$$
D=\frac{1}{\sqrt{1+2 d_{1}^{2} d_{2} / k a h_{1}^{\prime} d}} .
$$

If $d_{2} \rightarrow d$,

$$
D=\frac{1}{\sqrt{1+2 h_{1}{ }^{\prime} / k a \tan ^{2} \psi}}\left(\gamma<3^{\circ}\right)
$$

and if $\psi$ is small, so that $\tan \psi \rightarrow \psi$,

$$
D=\frac{1}{\sqrt{1+2 h_{1}^{\prime} / k a \psi^{2}}} .
$$

\subsubsection{Parameters $p$ and $q$}

Useful expressions for the divergence factor, path difference, and receiver height may be obtained by use of the dimensionless parameters,

$$
p=\frac{d_{1}}{\sqrt{2 k a h_{1}}}=\frac{d_{1}}{d_{T}}
$$

and

$$
q=\frac{d_{2}}{d}
$$

or

$$
d_{1}=(1-q) d=s d .
$$

The divergence factor may be expressed directly in terms of $p$ and $q$ by modifying equation (90) as follows:

$$
\begin{aligned}
D & =\frac{1}{\sqrt{1+2 d_{1}^{2} d_{2} / k a h_{1}{ }^{\prime} d}} \\
& =\frac{1}{\sqrt{1+\frac{4\left(d_{2} / d\right)\left(d_{1}^{2} / 2 k a h_{1}\right)}{1-\left(d_{1}{ }^{2} / 2 k a h_{1}\right)}}},
\end{aligned}
$$

where $h_{1}^{\prime}$ has been replaced by its equivalent expression, given in equation (58). The above form of $D$ shows that it can be expressed in terms of $p$ and $q$ only:

$$
D=\frac{1}{\sqrt{1+4 p^{2} q /\left(1-p^{2}\right)}} .
$$

Figure 17 shows contours of constant $D$ as a function of $p$ and $q$.

The path difference $\Delta$ may be written in terms of $p$ and $q$ by substituting into equation (78):

$$
\Delta=\frac{2 h_{1}{ }^{\prime} h_{2}{ }^{\prime}}{d}=\frac{2\left(h_{1}^{\prime}\right)^{2} d_{2}}{d d_{1}}=\frac{2 d_{2}}{d} h_{1}{ }^{2} \frac{\left(1-d_{1}{ }^{2} / 2 k a h_{1}\right)^{2}}{d_{1}} .
$$




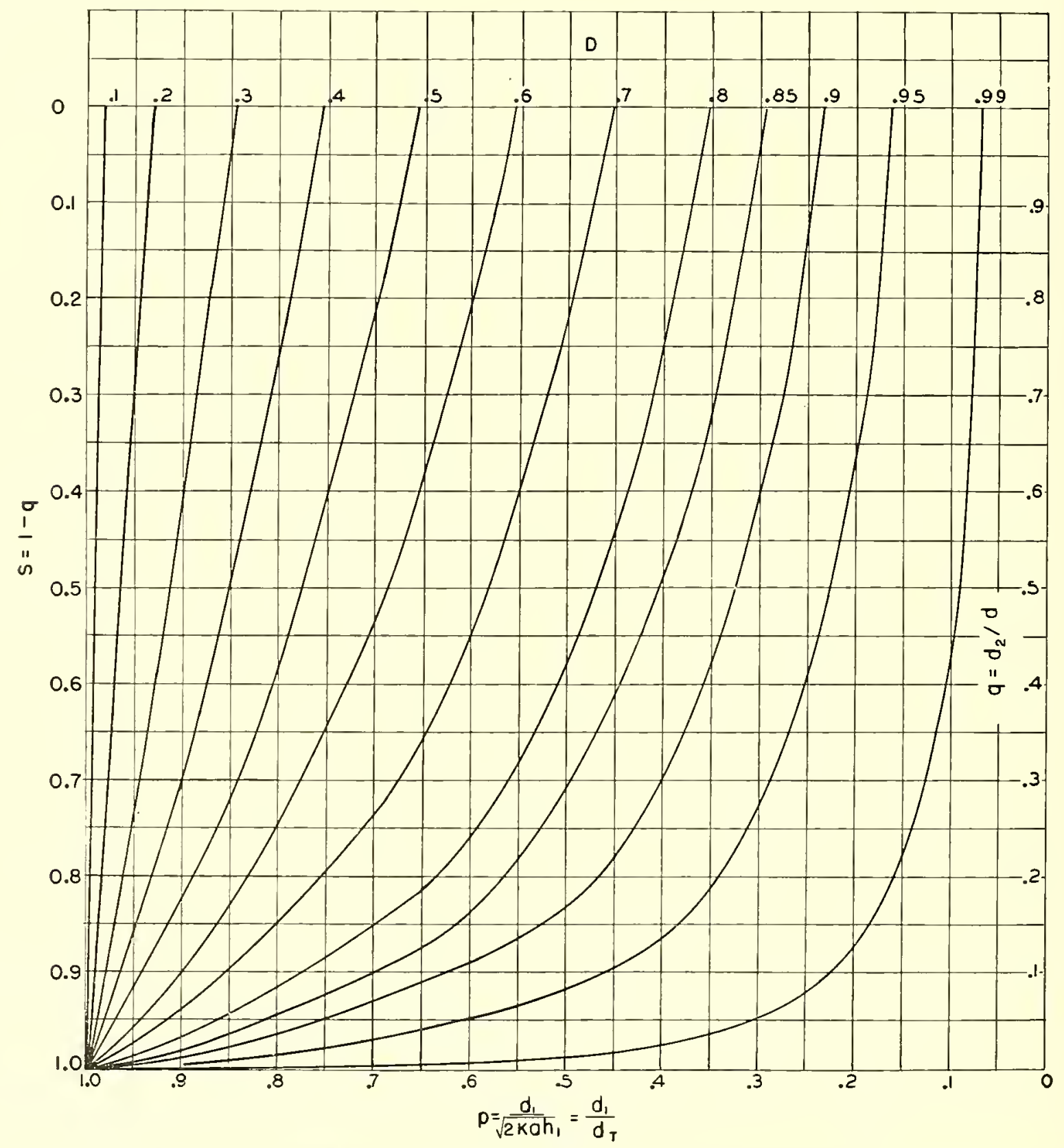

F1Gure 17. Divergence factor $D$ as a function of $p$ and $q$ or $p$ and $s$. (Radiation Laboratory.) ( $p=1$ is the line of sight where $D=0$.) Note: $S=1-q=d_{1} / d$.

Hence, on using equations (93), (94), and (95),

$$
\Delta=\frac{2 h_{1}{ }^{2}}{d_{\Gamma}} q \frac{\left(1-p^{2}\right)^{2}}{p} .
$$

Figure 18 shows $\left(1-p^{2}\right)^{2} / p$ as a function of $p$.

The reeeiver height $h_{2}$ may also be expressed in terms of $h_{1}, p$, and $q$. This will be found useful in drawing coverage diagrams in which both $h_{2}$ and $d$ are unknowns. From equation (60)

$$
\frac{h_{1}^{\prime}}{d_{1}}=\frac{h_{2}^{\prime}}{d_{2}}
$$

or

$$
h_{2}-\frac{d_{2}^{2}}{2 k a}=\frac{d_{0}}{d_{1}}\left(h_{1}-\frac{d_{1}^{2}}{2 k a}\right) .
$$


Hence it follows that

$$
h_{2}=h_{1}\left[\left(1-\frac{d_{1}^{2}}{2 k a h_{1}}\right) \frac{d_{2}}{d_{1}}+\frac{d_{2}^{2}}{2 k a h_{1}}\right] \text {. }
$$

Since

$$
\begin{aligned}
& \frac{d_{2}}{d_{1}}=\frac{d_{2}}{(1-q) d}=\frac{q}{1-q}, \\
& h_{2}=h_{1}\left(\frac{q}{1-q}\right)\left(1-p^{2}+\frac{q p^{2}}{1-q}\right) .
\end{aligned}
$$

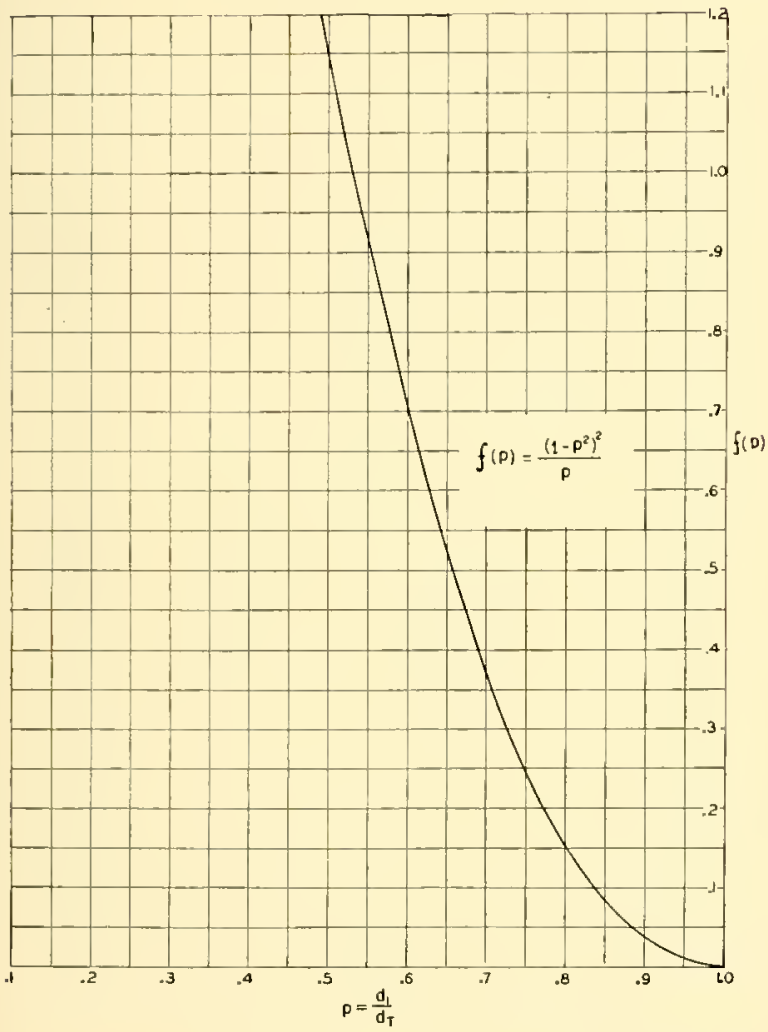

Figure 18. $\left(1-p^{2}\right)^{2} / p$ as a function of $p$. (Radiation Laboratory.)

\subsubsection{Generalized Coordinates}

The distance from the transmitter to the reflection point $d_{1}$ and the ratio $p$ may be eliminated by using the dimensionless coordinates

$$
\begin{aligned}
& u=\frac{h_{2}}{h_{1}}, \\
& v=\frac{d}{d_{T}} .
\end{aligned}
$$

The advantage of this substitution lies in the fact that the coefficients of $s=d_{1} / d$ in the cubic equation (74) may be expressed as functions of $u$ and $v$ only.
Thus,

$$
s^{3}-\frac{3}{2} s^{2}-s\left[\frac{k a}{d^{2}}\left(h_{1}+h_{2}\right)-\frac{1}{2}\right]+k a \frac{h_{1}}{d^{2}}=0,
$$

or

$s^{3}-\frac{3}{2} s^{2}-\frac{1}{2} s\left[\frac{\left(1+h_{2} / h_{1}\right)}{d^{2} /\left(2 k a h_{1}\right)}-1\right]+\frac{1}{2 d^{2} /\left(2 k a h_{1}\right)}=0$.

In terms of $u$ and $v$,

$$
s^{3}-\frac{3}{2} s^{2}-\frac{s}{2}\left(\frac{1+u}{v^{2}}-1\right)+\frac{1}{2 v^{2}}=0 .
$$

Figures 19 and 20 show contours of constant $s$ plotted in $u, v$ coordinates. The curves are parabolas.

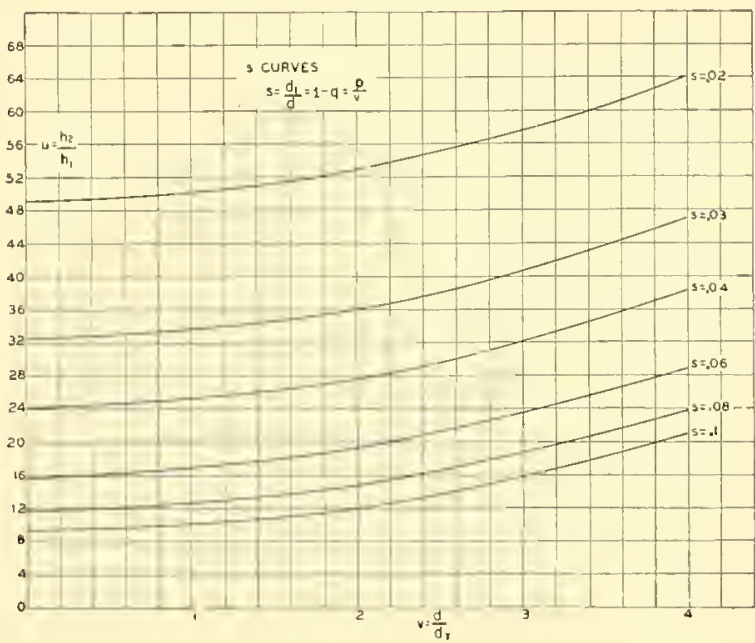

Figure 19. $s$ as a function of $u$ and $v$. (See Figure 14 for definition of lengths.)

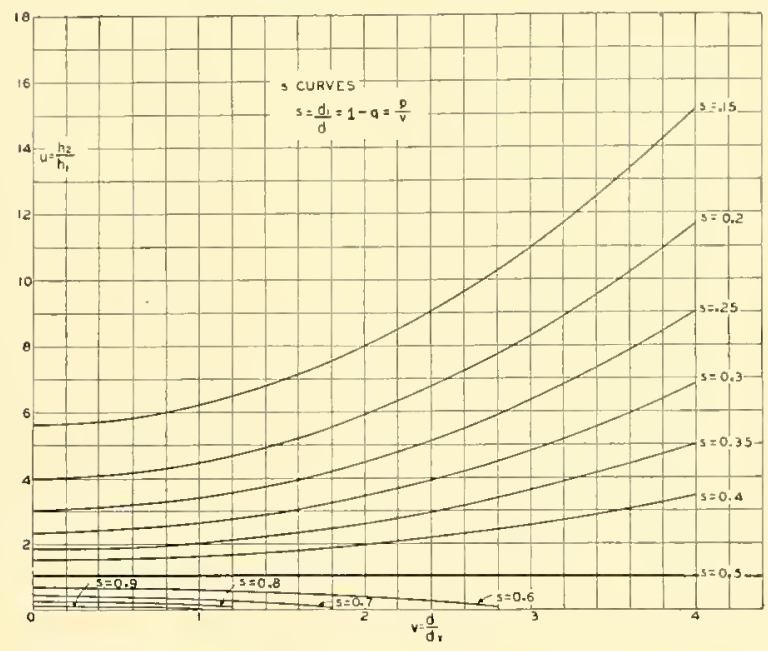

Figure 20. $s$ as a function of $u$ and $v$. (See Figure 14 for definition of lengths.) 
The divergence factor $D$ is given by

$$
D=\frac{1}{\sqrt{1+4 p^{2} q /\left(1-p^{2}\right)}},
$$

where

$$
p=s v
$$

and

$$
q=\frac{d_{2}}{d}=\frac{d-d_{1}}{d}=1-\frac{d_{1}}{d}=1-s .
$$

Since $s$ is a function of $u$ and $v$ only, it follows that $D$ may be plotted in $u, v$ coordinates. This may be accomplished by solving equation (96) with respect to $q$, which gives

$$
q=\frac{\left(1-p^{2}\right)\left(1-D^{2}\right)}{4 p^{2} D^{2}} .
$$

Equation (103) gives $s=1-q ; v=p / s$, and $u$ may be read from Figure 19 or 20. Contours of constant $D$ are shown in Figures 21, 22, and 23.

The grazing angte $\psi$ is important in calculations for vertical polarization since it determines the magnitude and phase of the reflection coefficient for a particular frequency and reflecting surface. The grazing angle $\psi$ may be expressed in terms of $s, u$, and $v$, as follows. From equation (60),

$$
\tan \psi \cong \frac{h_{1}^{\prime}}{d_{1}}=\frac{h_{1}}{d_{1}}\left(1-\frac{d_{1}^{2}}{2 k a h_{1}}\right)
$$

Hence

$$
\tan \psi=\frac{h_{1}}{d_{T}\left(s d / d_{T}\right)}\left(1-s^{2} v^{2}\right)=\frac{h_{1}}{d_{T}}\left(\frac{1}{s v}-s v\right)
$$

or

$$
\frac{\tan \psi}{\sqrt{h_{\mathrm{I}}}}=\frac{1}{\sqrt{2 k a}}\left(\frac{1}{s v}-s v\right)
$$

and for $k=4 / 3$,

$$
\frac{\tan \psi}{\sqrt{h_{1}}}=2.4 \times 10^{-4}\left(\frac{1}{s v}-s v\right) .
$$

From Figure 2t, $\psi$ may be obtained for given values of $h_{1}$ and $s v=p$.

The generalized coordinates described in this section will be found highly useful both in field strength and coverage calculations.

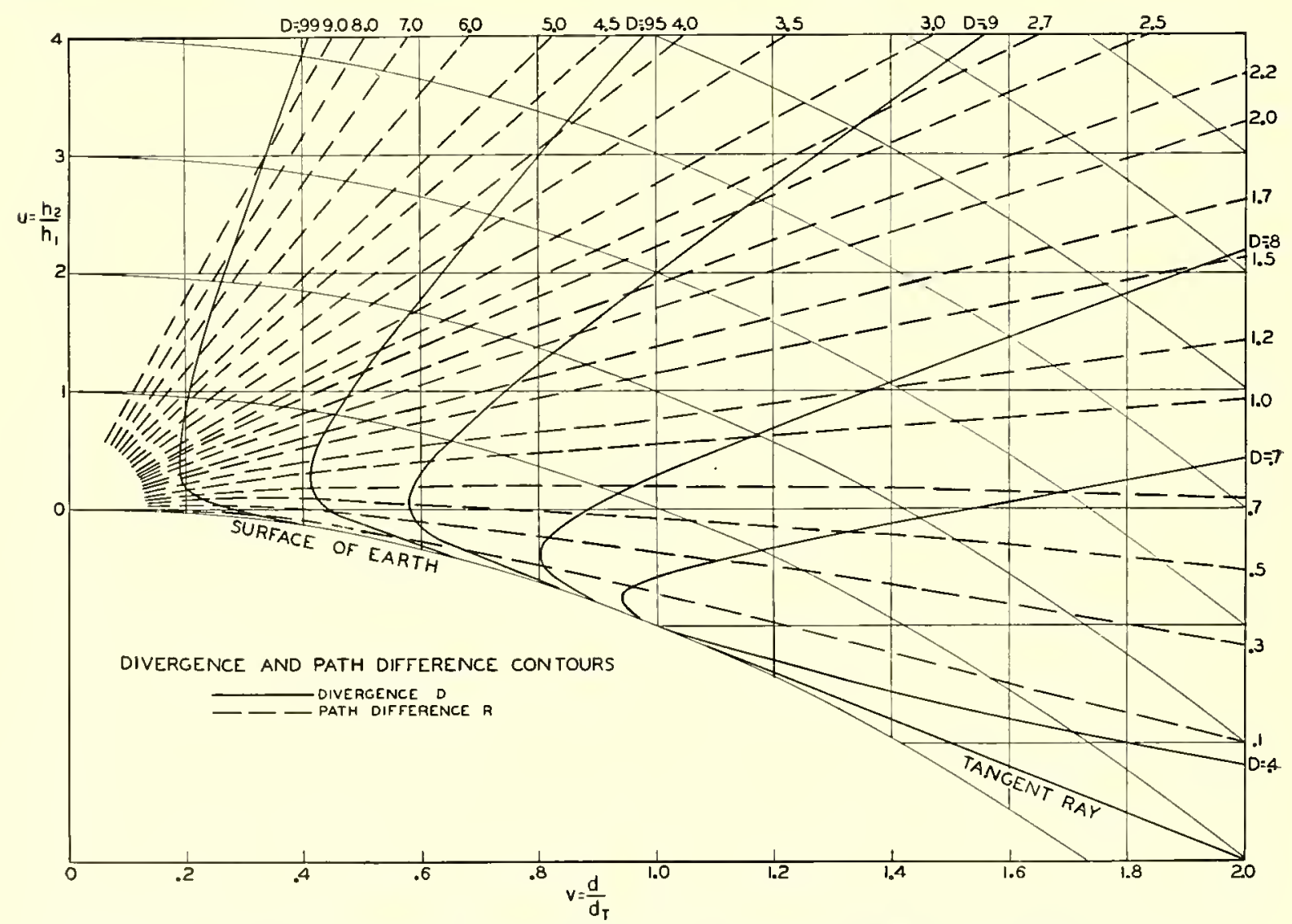

Figure 21. Contours of constant divergence factor $D$ and path difference variable $R$. (Radiation Laboratory.) 


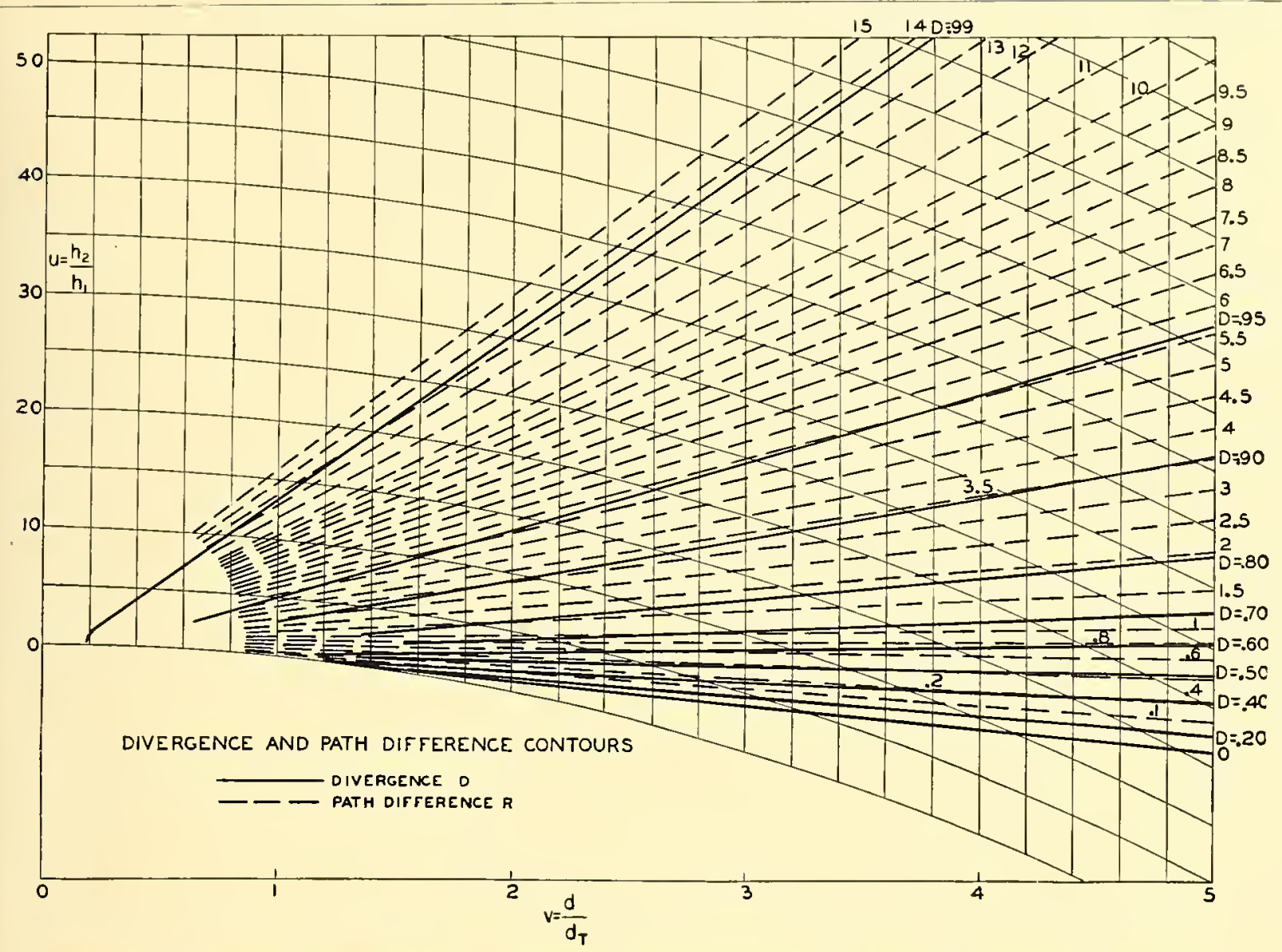

F1Gure 22. Contours of constant divergence factor $D$ and path difference variable $R$. (Radiation Laboratory.)

\subsection{ILLUSTRATIVE CALCULATIONS FOR THE OPTICAL-INTERFERENCE REGION}

5.6 .1

\section{Introduction}

The general expression for the gain factor $A$ in the interference region is obtained by combining equations (44) and (7). Then

$$
A=A_{0} F_{1} \sqrt{(1-K)^{2}+4 K \sin ^{2} \frac{\Omega}{2}} \text {. }
$$

The value of the radio gain is then given by equation (3) and the value of radar gain is given by equation (5). The value of the radical which defines the interference pattern has a range of values between 0 and 2 . The extreme values can occur only when $K=1\left(p=1, D=1, F_{2} / F_{1}=1\right)$; the value 0 (nults) is then given by $\sin ^{2}(\Omega / 2)=0$ and the value 2 (maxima) is given by $\sin ^{2}(\Omega / 2)=1$.

In general, the value of $A$ lies between the two extremes

$$
A=A_{0} F_{1}(1 \pm K)
$$

the positive sign giving a maximum and the negative giving a minimum. At any other point, the value lies between these two extremes. For range calculations (which involve maxima), the variation in $A$ is from 1 to 2 times the free-space value, according to the value of $K$, so that in practice a quick rule of thumb for range may be derised. Assume $(1+K)$ equal to 1.9 for favorable conditions (sea water, horizontal polarization, or, in the case of vertical polarization, small grazing angles) down to $(1+K)$ $=1$ or $K=0$ for propagation over rough terrain. The problem of finding the range is thus reduced to a problem for free space. In range calculations, $P_{2} / P_{1}$ is given by the ratio of minimum detectable power to power output. $A$ is then determined by equations (3) or (5), and the range is given by finding $d$ from the relation (writing $A_{0}=3 \lambda / 8 \pi d$ )

$$
A=\left(\frac{3 \lambda}{8 \pi d}\right)(1+K)
$$

More detailed calculations are presented in this 


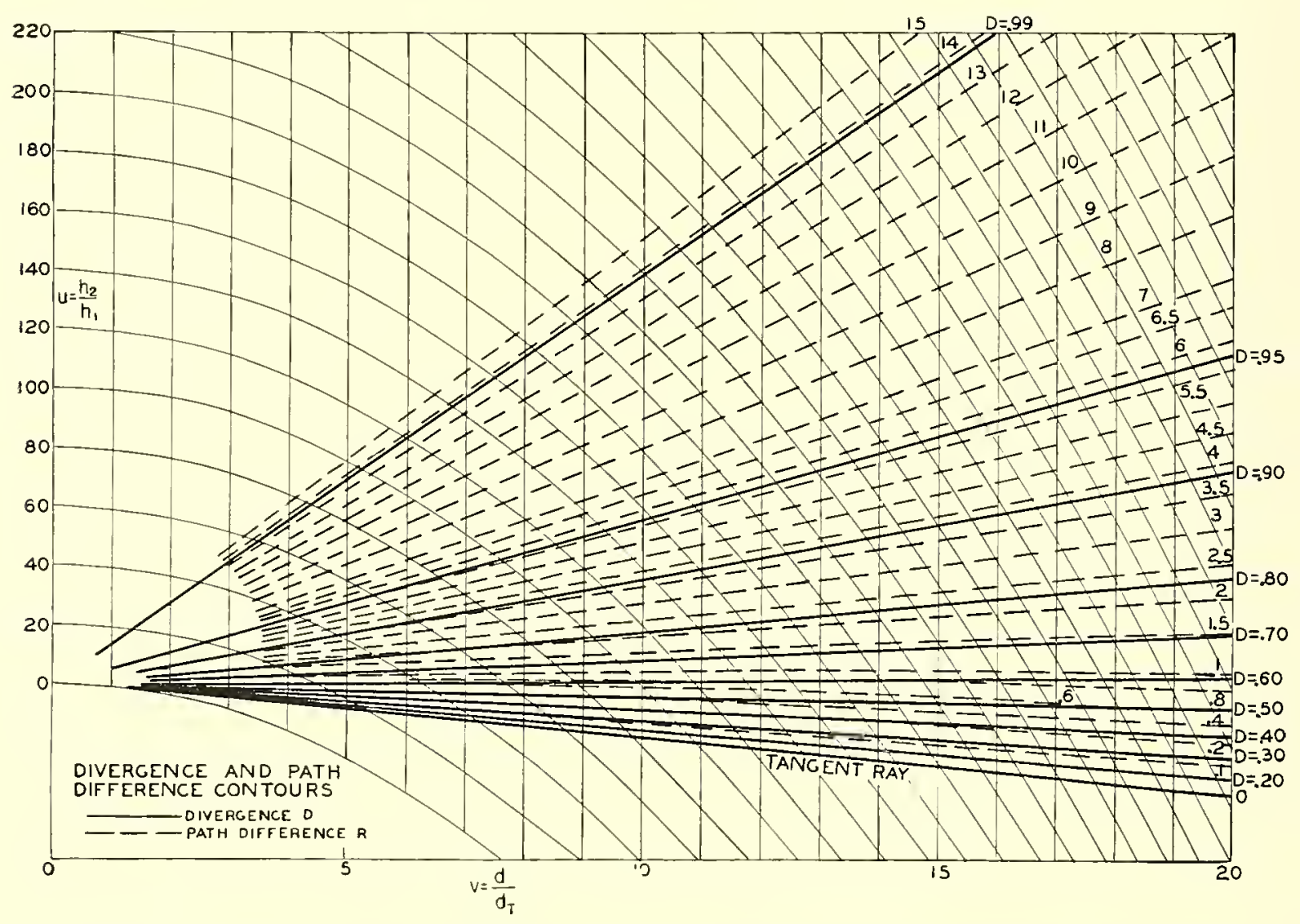

Figure 23. Contours of constant divergence factor $D$ and path difference variable $R$. (Radiation Laboratory.)

section. However, the assumption is made generally that the reflection coefficient is equal to -1 (i.e., $\rho=1$ and $\phi=180$ degrees) and that the direct and reflected rays do not differ appreciably on account of the shape of the antenna beam pattern $\left(F_{2}=F_{1}\right)$. For large distances over sea water, these assumptions are approximately realized. For most of the calculations it offer's no inherent difficulty to consider the effect of directivity or of a reflection coefficient different from - 1 but may require considerable additional calculation (see Sections 5.6.2 and 5.6.5).

For convenience, the formulas required in the calculations are recapitulated here. Putting $p=F_{1}$ $=F_{2}=1$, equation (108) takes the form

$$
A=A_{0} \sqrt{(1-D)^{2}+4 D \sin ^{2} \frac{\Omega}{2}}
$$

or, in decibels,

$20 \log A=20 \log A_{0}+10 \log \left[(1-D)^{2}+4 D \sin ^{2} \frac{\Omega}{2}\right]$.
The reflection point variable

$$
\begin{aligned}
p & =\frac{d_{1}}{d_{T}}=\frac{d_{1}}{\sqrt{2 k a h_{1}}} \\
& =\frac{d_{1}}{\left(4120 \sqrt{h_{1}}\right)} \quad(\text { when } k=4 / 3)
\end{aligned}
$$

will be used extensively. It has been found that the interference pattern is very sensitive to slight changes in $p$, so that an accuracy to the fourth significant figure is generally required.

The path difference variable $R$ is related to $p=d_{1} / d_{T}$ and $v=d / d_{T}$ by the equation

$$
R=\left(\frac{1}{p}-\frac{1}{v}\right)\left(1-p^{2}\right)^{2} .
$$

Resolved with respect to $1 / v$, this equation assumes the form

$$
\frac{1}{v}=\frac{1}{p}\left(1-\frac{R p}{\left(1-p^{2}\right)^{2}}\right)=\frac{1-q}{p}
$$


Another convenient expression for $R$ is obtained by replacing, in equation (83), the path difference $\Delta$

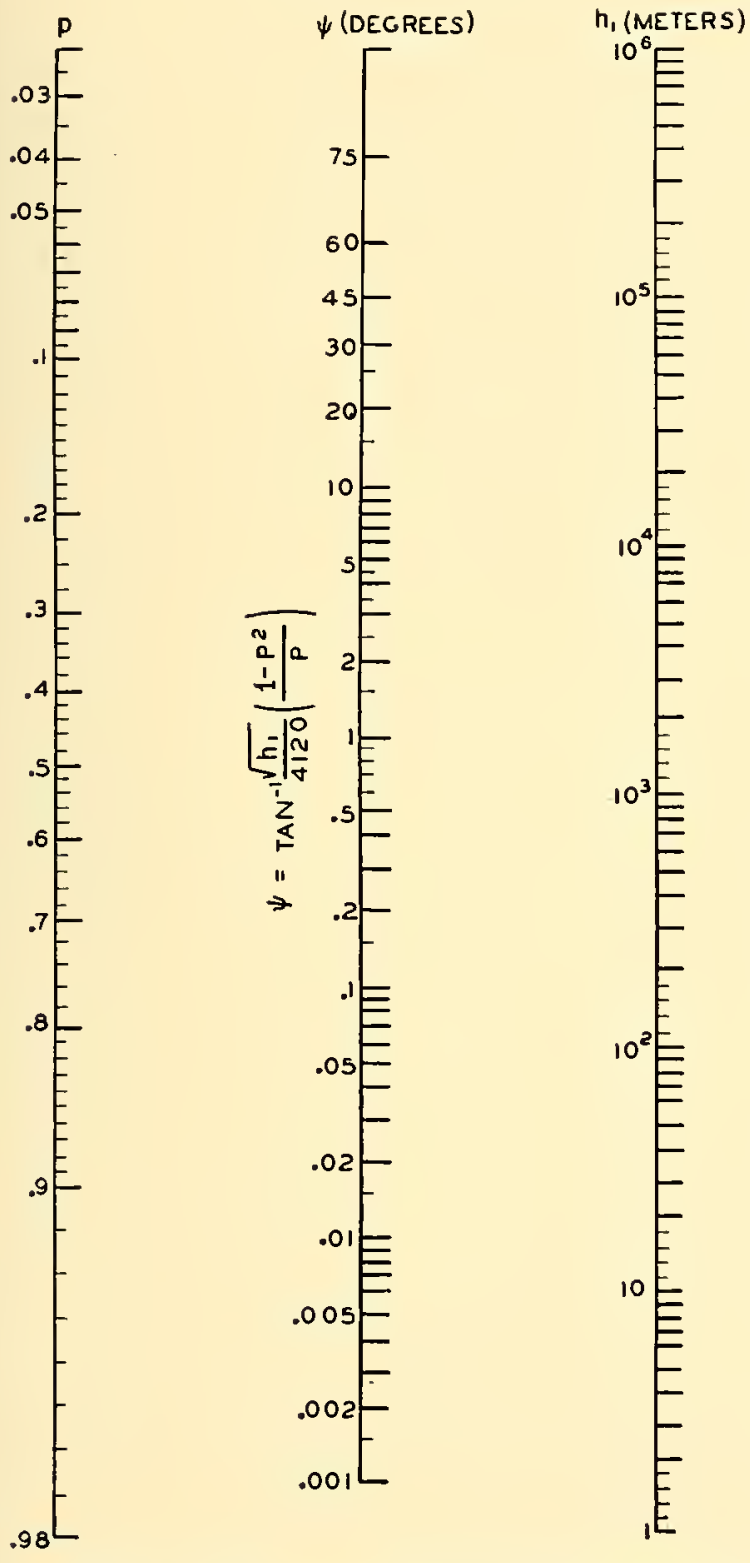

Figure 24. $\psi$ as a function of $h_{1}$ and $p=s v=d_{1} / d_{T}$. [See equation (107).] (See Figure 14 for definition of lengths.)

by $n \lambda / 2$, where $n$ (see below) may assume any positive value. Substituting $\sqrt{2 k \cdot a h_{1}}$ for $d_{T}$, equation (83) assumes the form

$$
R=n r \text {, }
$$

where

$$
r=\frac{1}{2} \sqrt{\frac{k a}{2}} \frac{\lambda}{h_{1}^{3 / 2}}
$$

or $\quad r=1030 \frac{\lambda}{h_{1}^{3 / 2}} \quad($ for $k=4 / 3)$.

A graphical representation of $r$ is given in Figure 15 in Chapter 6.

Then for a reflection coefficient of $\rho=1, \phi=180$ degrees (i.e., $\boldsymbol{\phi}^{\prime}=0$ ), equation (29) gives

$$
\Omega=\delta=\frac{2 \pi}{\lambda} \Delta=n \pi .
$$

If $r$ is fixed, a complete pattern of contour lines (along which $A$ is constant) is determined. Take as independent variables $p$ and $n$ (rather than $u$ and $v$ ). A given choice of $p$ and $n$ determines $R$ by equation (114), $\Omega$ by equation (116), $v$ by equation (113), $s$ by equation (118), u by equation (121), $D$ by equation (117), and finally $20 \log A$ by equation (110). By varying $r$, new patterns are obtained. Accordingly, $r$ may be called a pattern or chart parameter (see Section 6.8.3).

The lobes on the charts depend on $n$, in accordance with equation (116). Accordingly, for $\rho=1$, $\phi=180$ degrees, $n$ is the lobe variable. For the first (lowest) lobe, $n=0$ gives the first null, $n=1$ gives the first maximum and $n=2$ the second null. For the second lobe, $n$ varies from 2 to 4 , with a maximum at $n=3$, and so on. It should be remembered that if $n<1$, corresponding to the lower side of the lowest lobe, the value of the field (or of A) given by the optical formula is too low. A more accurate vahe can be obtained by joining the curves found in the optical and diffraction regions into a smooth overall curve.

Combining equations (102), (103), and (113) gives the divergence factor $D$.

$$
D=\left[1+\frac{4 R p^{3}}{\left(1-p^{2}\right)^{3}}\right]^{-1 / 2}=\left[1+\frac{4 R p^{2}}{1-p^{2}} \cdot \frac{d_{2}}{d}\right]^{-1 / 2} .
$$

The variable $s=d_{1} / d$ is

$$
s=p / v,
$$

and, repeating equation (101),

$$
s^{3}-\frac{3}{2} s^{2}-\frac{s}{2}\left(\frac{1+\imath}{v^{2}}-1\right)+\frac{1}{2 \vartheta^{9}}=0 .
$$

In terms of $p$, equation (119) becomes

$$
2 p^{3}-3 p^{2} v+p\left(v^{2}-u-1\right)+v=0 .
$$

Equation (120), resolved for $v$ and $u$, gires

$$
\begin{aligned}
& v=\frac{1}{2}\left[3 p-\frac{1}{p}+\sqrt{\left(\begin{array}{l}
1 \\
p
\end{array}-p\right)^{2}+4 u}\right], \\
& u=2 p^{2}-3 p v+\frac{v}{p}-1+\imath^{2} .
\end{aligned}
$$


Some useful approximations :

For $v$ large, $q \rightarrow 1$ and $R=\frac{q}{p}\left(1-p^{2}\right)^{2}$ approaches the value

$$
R \sim \frac{1}{p}-2 p
$$

which, solved for $p$, becomes

$$
p \sim \frac{-R+\sqrt{R^{2}+S}}{t} .
$$

If $R>2$,

$$
p \sim \frac{1}{R}
$$

If $R<2$

$$
p \sim \frac{3-R}{4} .
$$

For $R>3$,

$$
D \sim 1 .
$$

The calculations will be divided into four types.

Type I. The direct calculation of the radio gain (or field) when the heights and distance apart of the antennas and the wavelength are given.

Type II. The calculation of the radio gain as a function of the receiver leight $h_{2}$ when the transmitter antema height $h_{1}$, distance $d$, and wavelength $\lambda$ are given.

Type III. The calculation of the radio gain as a function of the distance $d$ when the transmitter antenna height $h_{1}$, receiver height $h_{2}$, and wavelength are given.

Type IV. The calculation of the possible positions of the receiver in space $\left(h_{2}, d\right)$ when the radio gain will have the given value for given values of the gain factor $A$, the transmitter antenna height $h_{1}$, and the wavelength $\lambda$. Special cases, such as the receiver antenna height, $h_{2}$ for given $d$, or $d$ for given $h_{2}$, can be solved by use of the curves in Type II and "Type III, in Sections 5.6.3 and 5.6.4.

This type of problem is of importance in estimating the range of a set when the minimum detectable porrer of the receiver and power output of the transmitter are known.

\subsubsection{Problem of Type I. Radio Gain for Fixed Heights and Distance}

The radio gain at a given receiver is to be found, their heights, as well as the wavelength being given.
The polarization is assumed horizontal, the effective earth's radius $t a / 3$.

The following data are assumed.

Transmitter height: $h_{1}=\mathbf{5 0}$ meters

Receiver height: $\quad h_{2}=1,500$ meters

Distance apart: $\quad d=100$ kilometers

Wavelength: $\lambda=1$ meter $(f=300 \mathrm{mc})$

Gains (over doublet): $G_{1}=G_{2}=100(20 \mathrm{db})$

\section{ONE- I'AY Transuission}

1. $d_{L}=188$ kilometers (Figure 2). $d<d_{L}$, so that the receiver is in the optical region.

2 . The $u, v$ coordinates of the receiver are

$$
\begin{aligned}
u & =\frac{h_{2}}{h_{1}}=\frac{1,500}{50}=30, \\
d_{T} & =\sqrt{2 k a h_{1}}=29,100 \text { meters } \\
v & =\frac{d}{d_{T}}=\frac{100}{29.1}=3.43 .
\end{aligned}
$$

3. Referring to Figure 19, $s(=p / v)$ is estimated to be 0.05 . Since the result is very sensitive to slight changes in $s$, it is desirable to improve the value of $s$. In Newton's method, the next approximation, using equations (101) or (119), is

$$
s^{\prime}=s-\frac{f(s)}{f^{\prime}(s)}=\frac{2 s^{3}-\frac{3}{2} s^{2}-\frac{1}{2 v^{2}}}{3 s^{2}-3 s-\frac{1}{2}\left[\frac{1+u}{v^{2}}-1\right]},
$$

$s^{\prime}=0.04794$.

The next approximation gives the same result.

4. Using the above value of $s^{\prime}$ and the relation $p=s v(v=3.43), p$ is equal to

$$
p=0.16+5 \text {. }
$$

Witl the value of $p=0.1645$, equation (117) and Figure 22 give the value

$$
D=0.95 \text {. }
$$

5. The path difference variable $R$ is obtained from equation (112) or Figures 21, 22, 23, as

$$
R=5.4 \pi \text {. }
$$

6. The number $r$, from equation (115), is 2.91 , so that the lobe number $n$ is

$$
n=\frac{R}{r}=1.88 .
$$

Hence the receiver is on the upper part of the first lobe close to a null. 
7. $\Omega=n \pi=5.9$ [see equation (116)],

$$
\begin{aligned}
\frac{\Omega}{2} & =2.95, \\
\sin ^{2} \frac{\Omega}{2} & =0.0353 .
\end{aligned}
$$

8. To use equation (110), the value of the freespace gain factor $A_{0}$ is needed. Figure 3 in Chapter 2 gives

$$
20 \log A_{0}=-118 .
$$

Substituting in equation (110),

$20 \log A=20 \log A_{0}+10 \log (0.0025+0.1342)$

$$
=-118-8.64 \cong-127
$$

By equation (3), using gains of $20 \mathrm{db}$,

$$
10 \log \frac{P_{2}}{P_{1}}=-127+40=-87 .
$$

Accordingly the radio gain is $-87 \mathrm{db}$ and the received power is given by

$$
P_{2}=P_{1} 10^{-8.7} \text {. }
$$

Suppose the receiver has a minimum detectable power of $10^{-10}$ watt, then the required minimum power output under the given conditions would be

$$
\begin{aligned}
P_{1} & =P_{2} \times 10^{8.7} \\
& =10^{-10} \times 10^{8.7}=10^{-1.3} \text { watts. }
\end{aligned}
$$

\section{RADAR}

Suppose that, instead of a receiver, there is a target at the same position with a radar cross section of $\sigma=50$ square meters. The value of $P_{2} / P_{1}$ at the radar receiver can be found from equation (5) using the value of $A$ found above and the given values of $\sigma$ and $\lambda$. If the radar uses the same antenna for transmitting and receiving, $G_{1}=G_{2}$, which in this case is 100 or $20 \mathrm{db}$, and the raclar gain

$10 \log \frac{P_{2}}{P_{1}}=20 \log G_{1}+10 \log \frac{16 \pi}{9}+10 \log \sigma$

$$
\begin{aligned}
& +40 \log A-20 \log \lambda, \\
& =40+7.5+17-254-0, \\
= & -189.5 \mathrm{db}
\end{aligned}
$$

This gives $P_{1}=10^{8.9}$ watts, which obviously is unattainable.

\section{Effect of Vertical Polarization}

The general value for $K$ in equation (108), when the reflection coefficient $\rho$ differs from -1 and $F_{2} / F_{1}=1$ is (see Section 5.3.1)

$$
K=\rho D .
$$

$\Omega$ in equation (10S) is no longer given by equation (116) but is the sum of two phase shifts, one caused by path difference, $(R / r) \pi=n \pi$, while the other is $\phi^{\prime}=\phi-\pi$, the difference between the phase of the reflection coefficient and that for perfect reflection. Hence

$$
\Omega=\frac{R}{r} \pi+\phi-\pi .
$$

The lobe variable (for imperfect reflection) is now $N$, defined by

$$
\Omega=N \pi
$$

rather than $n=R / r$. The relation between $N$ and $n$ is derivable from equation (129), giving

$$
N=n-\frac{\pi-\phi}{\pi} .
$$

The propagation is assumed to take place over sea water. The angle between the reflected wave and the earth is given by equation (107) or Figure 24, and is

$$
\Psi=0.582^{\circ} .
$$

From Figures 14 and 15 in Chapter 4,

$$
\phi=168^{\circ}, \rho=0.76 .
$$

The lobe variable $N$, in ter'ms of the old lobe variable (for $\rho=1, \phi=180^{\circ}$ ), by equation (131), is

$$
\begin{aligned}
& N=1.88-\frac{12}{180}=1.81, \\
& \frac{\Omega}{2}=163^{\circ}, \\
& \sin ^{2} \frac{\Omega}{2}=0.085 .
\end{aligned}
$$

The fact that $N<n$ signifies that the lobe for vertical polarization (other things being equal) has a greater angle of elevation than the lobe for horizontal polarization.

$$
K={ }_{\rho} D=0.76 \times 0.95=0.722
$$

The value of $10 \log \left[(1-K)^{2}+4 K \sin ^{2} \frac{\Omega}{2}\right]$

$$
=10 \log 0.322=-5 .
$$

Therefore, for vertical polarization,

$$
20 \log A=-118-5=-123,
$$

which may be compared with the value $20 \log A$ $=-127$, obtained for horizontal polarization with $\rho=1$. 


\subsubsection{Type II. Radio Gain Versus Receiver Height for Given Distance}

Radio gain versus receiver antenna height are to be found, while transmitter antenna height, warelength, and distance are given.

Suppose that a radar set has an antenna height of 30 meters, and an antenna gain of $13.5 \mathrm{db}$. Polarization is horizontal and the wavelength is 1.5 meters. Assume also a receiver with a gain of $G_{2}=1(\mathrm{or} 0 \mathrm{db})$ at a distance of $100 \mathrm{~km}$.

The following ealculations are made:

1. The variation of the radio gain $P_{2} P_{1}$, with receiver antemna height $h_{2}$ is to be found.

2. Instead of a receiver assume a target with cross section of 50 square meters. The value of the radar gain $P_{2} / P_{1}$ at the radar receiver is to be found as a function of target height $h_{2}$.

The diffraction part of the calculation is given in Section 5.7.3; the optical part in this section. The results are represented in Figure 25, the two partial curves for one-way transmission haring been combined into a smooth overall curve which makes possible the estimation of $10 \log P_{2} / P_{1}$ in the transition region near the line of sight. The radar gain varies as $40 \log A$ [equation (5)] rather than as $20 \log A$ and contains a constant shift $10 \log \left[G_{1}{ }^{2}\left(16 \pi \sigma / 9 \lambda^{2}\right)\right]$ rather than $10 \log C_{1} G_{2}$.

\section{Radio Gain: Oxe-May Transmission}

The ealculation is most readily performed by using $p=d_{1} / d_{T}$ as the independent rariable and then finding the corresponding values of $h_{2}$, the receiver height, and $A$.

1. From Figure 15 in Chapter 6 or equation (115),

$$
r=9.403 \text {. }
$$

For $n=1$, corresponding to the first maximum, $p$ is approximately $1 / r$, since $R=n r>2$. Accordingly, we begin with $p=0.1$.
2. $d_{T}=22.5 \mathrm{~km}$ (from Figure 2), and

$$
v=\frac{d}{d_{T}}=\frac{100}{22.5}=4.45 .
$$

3. From equation (112),

$$
R=9.58 \text {. }
$$

4. From equation (121),

$$
u=\frac{h_{2}}{h_{1}}=62.028
$$

and hence

$$
h_{\mathrm{g}}=1,861 \text { meters. }
$$

5. At $d=100 \mathrm{~km}$, the free-space attenuation (Figure 3 in Chapter 2) is

$$
20 \log A_{0}=-115 .
$$

6. Compute the factor

$$
\sqrt{(1-D)^{2}+4 D \sin ^{2} \frac{\Omega}{2}}
$$

From equation (117)

$$
\begin{aligned}
D & =0.980, \\
n & =\frac{R}{r}=1.019, \\
\Omega & =n \pi=(1.019) \cdot(3.1416)=3.19 \text { radians, } \\
\frac{\Omega}{2} & =1.60 \text { radians, } \\
\sin ^{2} \frac{\Omega}{2} & =0.9992 .
\end{aligned}
$$

\begin{tabular}{|c|c|c|c|c|c|c|c|c|c|}
\hline$p$ & $R$ & $s$ & $h_{2}$ & $n$ & $\frac{\Omega}{2}$ & $D$ & $\dagger$ & $\begin{array}{l}\text { Radio Cain } \\
10 \log \frac{P_{2}}{P_{1}}\end{array}$ & $\begin{array}{c}\text { Radar Gain } \\
10 \log \frac{P_{2}}{P_{1}}\end{array}$ \\
\hline 0.15 & 6.16 & 0.0337 & 1,396 & 0.655 & 1.03 & 0.96 & 5 & -96.5 & -172 \\
\hline 0.1 & $9.5 \mathrm{~S}$ & 0.0225 & 1.861 & 1.019 & 1.60 & 0.98 & 6 & -95.5 & -170 \\
\hline 0.05 & 19.68 & 0.0112 & 3,216 & 2.09 & 3.29 & 0.995 & -11 & -112.5 & -204 \\
\hline 0.04 & 24.70 & 0.00898 & 3,885 & 2.63 & 4.13 & 0.997 & 4 & -97.5 & -174 \\
\hline 0.03 & 33.05 & 0.0067 & 5,005 & 3.51 & 5.52 & 0.998 & 3 & -98.5 & -176 \\
\hline 0.02 & 49.74 & 0.0045 & 7,235 & 5.29 & 8.31 & 0.999 & 5 & -96.5 & -172 \\
\hline 0.01 & 99.76 & 0.0022 & 13,917 & 10.61 & 16.67 & 1.000 & 4 & -97.5 & -174 \\
\hline
\end{tabular}

From Figure 12,

$$
20 \log \sqrt{(1-D)^{2}+4 D \sin ^{2} \frac{\Omega}{2}}=6 \mathrm{db} .
$$

Hence

$$
\begin{aligned}
& 20 \log A=-115 \mathrm{~d} b+6 \mathrm{db}=-109 \mathrm{db}, \text { and } \\
& 10 \log \frac{P_{2}}{P_{1}}=-109 \mathrm{db}+10 \log G_{1} G_{2}=-95.5 \mathrm{db} .
\end{aligned}
$$

7. The foregoing values, together with results obtained with other values of $p$, are listed in Table 1.

TABLE $1^{*}$

* Sce also Table 5. 
The value of $P_{2} / P_{1}$ [equation (3)] is represented graphically in Figure 25.

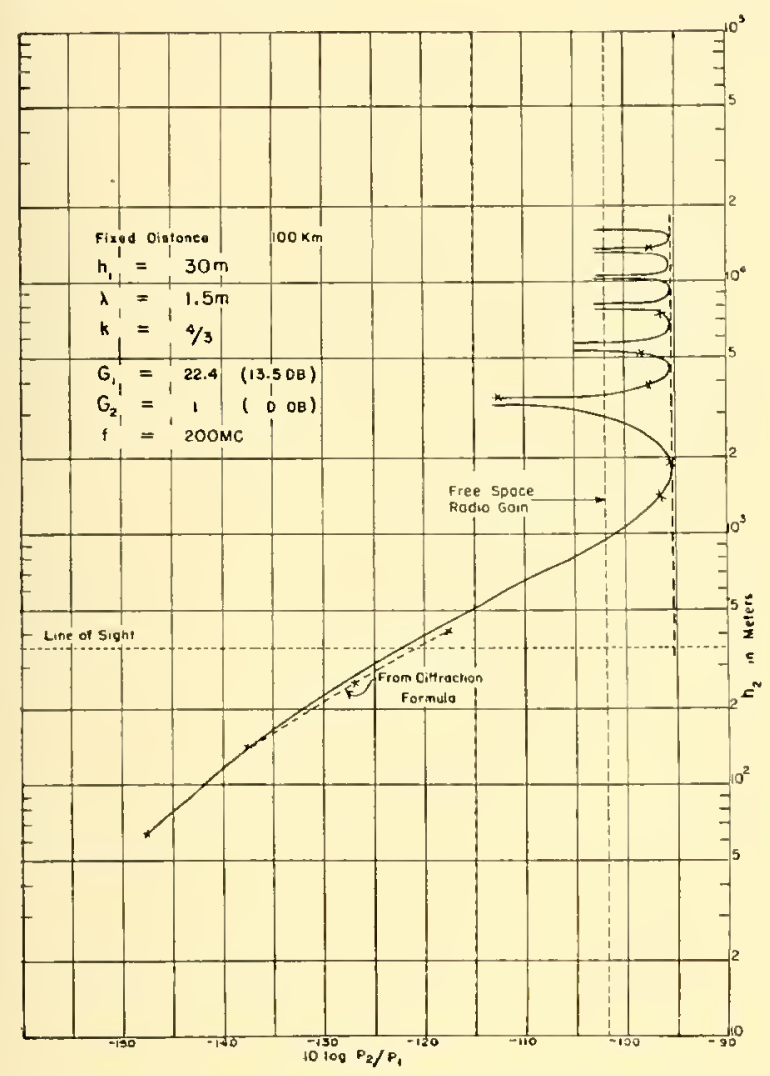

Figure 25. Radio gain in decibels versus height $h_{2}$. Horizontal polarization.

\section{Radar Galn: Two-Way Transmission}

Knowing the values of $20 \log A$, the corresponding values of $P_{2} / P_{1}$ are given by equation (5). Taking $G_{1}=G_{2}=13.5 \mathrm{db}, 16 \pi / 9=5.58, \lambda=1.5$,

$$
\begin{aligned}
10 \log \frac{P_{2}}{P_{1}} & =27+7.5+17+40 \log A-20 \log 1.5 \\
& =+40 \log A+48 .
\end{aligned}
$$

\subsubsection{Type III. Radio Gain Versus Distance for Given Antenna Heights}

A radar used over the sea has a wavelength of 1.5 meters. The transmitter is 30 meters above sea level and the target is at an altitude of 1,000 meters above the sea. The power gain of the transmitting antenna is $13.5 \mathrm{db}$, the polarization horizontal. The onc-way radio gain is to be found as a function of distance. Also, the radar gain at the radar set by echo from the target of cross section $\sigma=10$ square meters is to be calculated.
RadioGaln:One-Way Transmission (see Figure 26).

1. The number $r$ from Figure 15 in Chapter 6 or equation (115) is found to be 9.403 .

2. $u=h_{2} / h_{1}=33.3$.

3. For $r>2$ and $n=1, p$ is approximately equal to $1 / r$. Hence we start with $p=0.1$.

4. Equation (121), with $p=0.1$ and $u=33.3$, gives $v=2.76$.

5. From equation (112), $R=9.445$.

6. $n=R / r=1.005$. Hence the target is on the first (i.e., lowest) lobe. Moving in the direction of increasing distance, the target soon approaches the first maximum. To get points beyond, i.e., $n<1$, we need greater values of $p$ but it is not necessary or desirable to go below about $n=0.8$, since the curve beyond $n=0.8$ is generally in the transition region in which the curve is more easily and more accurately obtained by joining the optical and diffractive curves. The diffractive part of the calculation is given in Section 5.7. Accordingly $y_{2}$ in Table 2 , the values of $p$ are taken only slightly above $p=0.1$ (corresponding to $n=1$ ) and are diminished to find points at the nearer distances.

7. To find $A$, we need first the free-space value $A_{0}$, which is given in Figure 3 in Chapter 2 as a function of $d$. Since $d_{T}=4.12 \sqrt{30}=22.6 \mathrm{~km}$, the value of $d$ corresponding to $v=2.76$ is $d=v d_{T}=62.3 \mathrm{~km}$, and $20 \log A_{0}=-111$.

8. To find the value of

$$
10 \log \sqrt{(1-D)^{2}+4 D \sin ^{2} \frac{\Omega}{2}},
$$

we need $D$. Since $n$ is practically unity, corresponding to the first maximum, $\sin ^{2}(\Omega / 2)$ may be taken as unity. Hence the radical reduces to $1+D$. Calculation using equation (103) and Figure 17 gives $D=0.98$, and hence

$$
\begin{aligned}
1+D & =1.98, \\
20 \log (1+D) & =6 .
\end{aligned}
$$

Since the transmitting antenna gain $G_{I}$ is $13.5 \mathrm{db}$, and assuming the receiving antenna gain to be $0 \mathrm{db}$, $10 \log \left(P_{2} / P_{1}\right)$ has the value $20 \log A+13.5$ or

$$
\begin{aligned}
& 10 \log P_{2} \\
& =A_{0}^{2} \sqrt{(1-D)^{2}+4 D \sin ^{2} \frac{\Omega}{2}} G_{1} G_{2}+0.0 \mathrm{db} \\
& \underbrace{}_{-111 \mathrm{db}} \underbrace{+13.5 \mathrm{db}}_{+6 \mathrm{db}} \\
& =-105+13.5=-91.5 \mathrm{db} \text {. }
\end{aligned}
$$




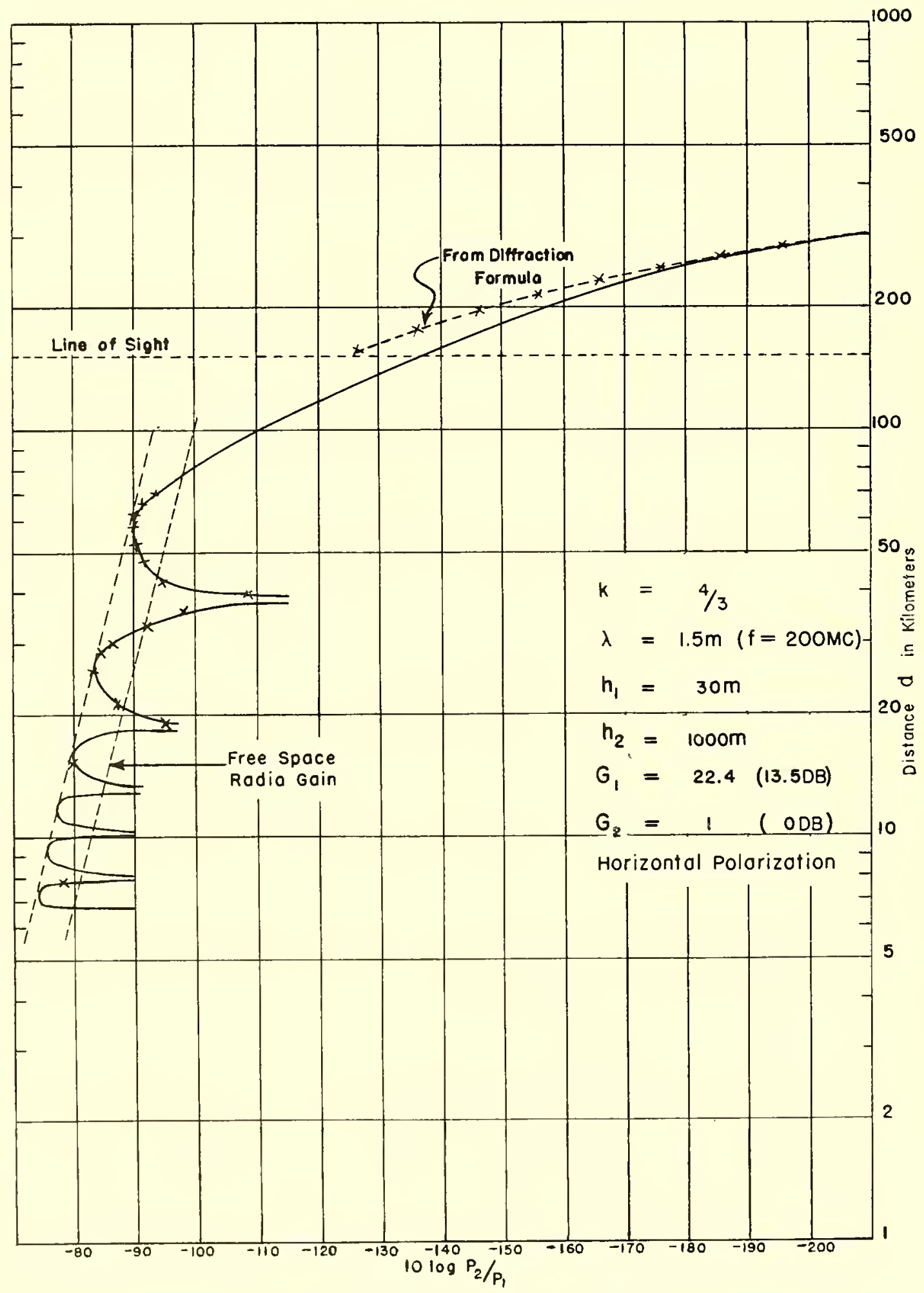

Figure 26. Radio gajn (in db) versus distance. 
TABLE 2. $10 \log G_{1}=13.5 \mathrm{db} ; 10 \log G_{2}=0.0 \mathrm{db} ; \lambda=1.5$ meters; $h_{1}=30$ meters; $h_{2}=1,000$ meters; $\sigma=10 \mathrm{~m}^{2}$.

\begin{tabular}{|c|c|c|c|c|c|c|c|c|c|c|c|}
\hline 0.11 & 2.934 & 66.75 & 8.51 & 0.91 & 0.98 & 1.43 & 6 & -111 & -105 & -92.5 & -170 \\
\hline 0.10 & 2.755 & 62.68 & 9.45 & 1.005 & 0.98 & 1.58 & 6 & -110 & -104 & -91.5 & -169 \\
\hline 0.09 & 2.561 & 58.25 & 10.55 & 1.22 & 0.98 & 1.76 & 6 & -110 & -104 & -91.5 & -169 \\
\hline 0.06 & 1.870 & 42.54 & 16.02 & 1.70 & 0.99 & 2.68 & -1 & -107 & -108 & -94.5 & -175 \\
\hline 0.055 & 1.738 & 39.22 & 17.50 & 1.86 & 0.99 & 2.92 & -7.5 & -106 & -113.5 & -100 & -186 \\
\hline 0.05 & 1.600 & 36.4 & 19.28 & 2.05 & 1.00 & 3.22 & -16 & -106 & -122 & -108.5 & -203 \\
\hline 0.045 & 1.457 & 32.89 & 21.45 & 2.28 & 1.00 & 3.58 & -1.5 & -105 & -106.5 & -93 & -172 \\
\hline 0.04 & 1.311 & 29.59 & 24.16 & 2.57 & 1.00 & $4.0 \pm$ & +3.8 & -104 & -100 & -87 & -159 \\
\hline 0.035 & 1.158 & 26.14 & 27.64 & 2.94 & 1.00 & 4.62 & 6 & -103 & -97 & -79.5 & -153 \\
\hline
\end{tabular}

* Lobe pattern factor $=20 \log \sqrt{(1-D)^{2}+4 D \sin ^{2}(\Omega / 2)}$

Repeating the process for other values of $p$, a table of $10 \log \left(P_{2} / P_{1}\right)$ versus $d$ is obtained. These points have been plotted in Figure 26, together with the points in the diffraction region obtained with the same data in Section 5.7.3. For sketching in the optical part, the value of $n$ is kept in mind, since this indicates on which lobe and where on the lobe the point lies.

\section{Radar Gain: Two-Way Transmisston}

To change from $20 \log A=-105$ to $10 \log \left(P_{2} / P_{1}\right)$ at the radar receiver, using equation (5) and $G_{1}=G_{2}$ $=13.5 \mathrm{db}$,

$10 \log \left(P_{2} / P_{1}\right)=27+7.5+10 \log \sigma-20 \log 1.5$ $+40 \log A$

$=27+7.5+10-3.5+40 \log A$,

$=41+40 \log A=-169 \mathrm{db}$.

\subsubsection{Type IV. Determination of Con- tours Along which the Gain Factor $A$ Has a Given Value, the Transmitter Height and Wavelength Being Given}

This is the so-called coverage problem which is treated in greater detail in Chapter 6 . While the usual coverage diagram is derived on the basis of one-way or communication formulas, the diagram is still useful for radar since a target will return more or less energy to the receiver according to its position on the coverage diagram. The contour gain factor $A$ is readily converted to $P_{2} / P_{1}$ for either one-way or radar by means of equations (3) and (5).

The method described here is more accurate than the graphical methods giren in Chapter 6 . It is best suited for finding maximum lobe ranges corresponding to a given radar gain. If $A$ is given, and $h_{2}$ for a given distance $d$ is wanted, a curve can be drawn as in Section 5.6.3 and then $h_{2}$ foumd for the given value of $A$.

\section{Problems}

A radar set operating over the sea has a transmitter with antenna height of 30 meters and a wavelength of 1.5 meters. As in the previous problems, a receiver with an antenna of $0 \mathrm{db}$ gain is assumed in place of a target and the gain of the transmitter is again assumed as $13.5 \mathrm{db}$. The polarization is horizontal. The gain factor $A$, for illustration, is chosen as $-130 \mathrm{db}$. Positions of the receiver are to be found at which the gain factor takes that value.

In Chapter 6, purcly graphical methods of determining the contours (lobes) are given. Here we are concerned with finding individual points on the contour. Thus, for example, $n=1$ if the tip of the lowest contour is wanted (as in range determination). Points near the tip recuire values of $n$ near 1 , such as $n=1.2$ or $n=0.9$. For the next higher lobe, the tip of the lobe corresponds to $n=3$ and so on. The nulls are at $n=0,2,4, \ldots$ However, while the optical formula gives a null at $n=0$, that is, near the line of sight, the true value of the field, or of $A$, 
is greater. To obtain the correct result, the contours for the diffraction field (Section 5.7.3) and those obtained for the optical region should be merged into smooth overall eurves.

For values of $r>3, R=n r>3$, the tip and upper part of the lowest lobe, and the higher lobes, by equation (126), have a value of $D$ close to 1 . Consequently, equation (110) reduces to

$$
A=2 A_{0} \sin \frac{\Omega}{2} \text {. }
$$

If $n$ is given, $\sin \Omega / 2$ is determined and the calculation can be performed as a free-space calculation. In terms of $d$, the equivalent free-space distance $d_{0}$, corresponding to $A$ is given by Figure 3 in Chapter 2 and is equal to

$$
d=2 d_{0} \sin \frac{\Omega}{2} .
$$

In the stated problem $r>3$, so that the frec-space calculation suffices. This is given in paragraph (1). In paragraph (2), the method including the divergence is given.

1. Frce space. In the given problem, $r=9.4$, so that the simplified calculations should be sufficient. The value of $d_{0}$ corresponding to $20 \log A=-130$ is found from Figure 3 in Chapter 2 to be $566 \mathrm{~km}$. Hence by equation (133) for $n=1$, the true distance $d$ for complete reinforcement by the reflected wave is twice as much and

$$
d=2 \cdot(566)=1,132 \mathrm{~km} .
$$

For $n=1.2, \Omega / 2=0.6 \pi$ [from equation (116)], giving

$$
\begin{aligned}
d & =2 \cdot(566) \sin (0.6 \pi), \\
& =1,076 \mathrm{~km} .
\end{aligned}
$$

To find the corresponding height $h_{2}$, a curve of the type in Section 5.6.3 is needed for $-20 \log A$ versus height. From this, the height corresponding to $20 \log A=-130$ can be read. Alternatively, the calculation given later in (2) will determine both $d$ and $h_{2}$.

If instead of $A$, either the raclio gain or radar gain is given, $A$ is first found from cquations (3) or (5).

2. Calculation including divergence, $n=1$. As in the previous problem, $r=9.4$ (from Figure $15 \mathrm{in}$ Chapter 6). A convenient value of $p$, approximately equal to $1 / r$, is selected. In this case, we take $p=0.1$. This value is then improved by applying Newton's method $p_{1}=p-\frac{f(p)}{f^{\prime}(p)}$ to the equation

$$
f(p)=\frac{1}{v_{0}}-\frac{1}{v} \sqrt{(1-D)^{2}+4 D \sin ^{2} \frac{\Omega}{2}},
$$

remembering that $D$ is a function of $p$ as given in equation (117), where $v_{0}$ is the value of $v$ which corresponds to the distance $d_{0}$ at which the given value of $A$ would occur in free space.

If $n=1$, equation (13t) reduces to

$$
\frac{1}{v_{0}^{\prime}}=\frac{1}{v}(1+D) \text {. }
$$

The correction formula corresponding to equation (134), denoting by $p_{1}$ the improved value of $p$, is

$$
p_{1}=p-\frac{v-v_{0} \sqrt{(1-D)^{2}+4 D \sin ^{2}(\Omega / 2)}}{\frac{v^{2}}{p^{2} D^{2}}+\frac{3\left(1-D^{2}\right)}{4 p D}\left[\left(1-D^{2}\right) v_{0}^{2}+v^{2}\right]\left(\frac{1+p^{2}}{1-p^{2}}\right)} .
$$

The correction formula corresponding to equation (135) $(n=1)$ is

$$
p_{1}=p-\frac{\frac{1}{v_{0}}-\frac{(1+D)}{v}}{\frac{1+D}{p^{2} D^{2}}+\frac{3 D}{2 p v}\left(\frac{1+p^{2}}{1-p^{2}}\right)\left(1-D^{2}\right)} \text {. }
$$

Taking $n=1, d_{0}$ from $A=3 \lambda / 8 \pi d_{0}$ (or Figure 3 in Chapter 2) is $566 \mathrm{~km}$, and

$$
\imath_{0}=\frac{566}{d_{T}}=\frac{566}{22.5}=25.1 .
$$

Taking $p=0.1$ as an initial value as explained above, then $\mathrm{D}$ is found to be 0.9788 [equation (117)]. Using $R=n r=9.4$ and equation (113),

$$
v=165.6 \text {. }
$$

Substituting these values in equation (135) gives

$$
p_{1}=0.10385 \text {. }
$$

Repetition of this procedure requires no change in these five figures. Accordingly, for $n=1$ and $A=-130 \mathrm{db}, p=0.10385$. The corresponding values of $D$ and $v$ are

$$
\begin{aligned}
D & =0.9789 \\
v & =49.38, d=1,110 \mathrm{~km} .
\end{aligned}
$$

In (1) $d$ was found to be $1,132 \mathrm{~km}$. Now $s=p / v$ $=0.0021$. From equation (121),

$$
\begin{aligned}
u=\frac{h_{2}}{h_{1}} & =2,89 \mathrm{~S} \\
& h_{2}=86,940 \text { meters. }
\end{aligned}
$$

For $n=1.2$, the calculation proceds as for $n=1$ except that equation (134) rather than equation (135) is used:

$$
R=11.283 \text {. }
$$


The value of $p$ by successive approximations in equation (136) is found to be

$$
\begin{aligned}
p & =0.08127 \\
v & =47.35, d=1,065 \mathrm{~km} \\
D & =0.9851 \\
s & =0.0018 t \\
\frac{h_{2}}{h_{1}} & =2,772 \\
h_{2} & =83,160 \text { meters }
\end{aligned}
$$

3. Vertical polarization, $\rho \neq 1, \phi \neq 180^{\circ}$ (sea water). The procedure given here is first to find the position of the point for a given $n$ assuming the reflection coefficient to equal -1 and then find the shift caused by the change in the reflection coefficient. For the most important case, $n=1$, the new maximum distance is given by

$$
d=d_{0}(1+\rho D),
$$

where $d_{0}$ is the free-space distance corresponding to the given value of $A . \quad \rho$ is found from the value of $\psi$, the grazing angle at the reflection point given by $p$ found above in the calculation for $\rho=1$ and $\phi=180^{\circ}$. For the new contour tip $p$ clianges, but the angle $\psi$ does not change sharply, so that $\rho$ and $\phi$ as found for the $p$ obtained by the simplified calculation is a close approximation.

For $\rho \neq 1, \phi \neq 180^{\circ}, \Omega=\delta+\phi-\pi$ [see equation (29)] consists of two parts. $\delta=\pi R / r=(\Delta / \lambda) 2 \pi$ $=n \pi$ and $\phi^{\prime}=\phi-\pi$. Writing $\Omega=N \pi, N$ the lobe number for the case $\rho \neq 1, \phi \neq 180^{\circ}$, the requircment for the new lobe tip is $N=1$; for the first maximum $\Omega=\pi=R \pi / r+\phi-\pi$ where $R$ is the path difference variable at the new lobe tip. Hence the value of $R$ at the new tip is given by

$$
R=r(2-\phi / \pi) \text {. }
$$

Using the results in (2),

$$
\begin{aligned}
p & =0.10385, \\
D & =0.9788, \\
r & =9.4, \\
d_{0} & =566,
\end{aligned}
$$

we find from Figure 24 or equation $(107), \psi=0.725^{\circ}$; from Figures 14 and 15 in Chapter $4, \rho=0.76$, $\phi=170^{\circ}$. Substituting in equation (138),

$$
d=566(1.744)=987 \mathrm{~km} \text {. }
$$

From equation (139),

Also

$$
R=(9.4)(1.050)=9.92 \text {. }
$$

$$
v=\frac{d}{d_{T}}=\frac{987}{22.5}=43.9 .
$$

The above value of $p$ can now be improved by substitution in equation (137) with $D$ replaced by $\rho D$ which gives

$$
p=0.0985 \text {. }
$$

In the calculation just made, $\rho$ has been assumed constant. This can be checked by finding $\psi$ as determined by the new value of $p$. The result is $\psi=0.766^{\circ}$ and the corresponding values are $\rho=0.74$ (as against 0.76 previously) and $\phi=169^{\circ}$ (as against $170^{\circ}$ ), which is good enough. We now find

$$
\begin{aligned}
s & =\frac{p}{v}=\frac{0.0985}{43.9}=0.00224 \\
\frac{h_{2}}{h_{1}} & =2,359 \\
h_{2} & =70,770 \text { meters. }
\end{aligned}
$$

Hence the new maximum point of the lobe is at a distance of $987 \mathrm{~km}$ and at an elevation of 70,700 meters, as compared with a distance of $1,110 \mathrm{~km}$ and height $\$ 6,900$ meters for perfect reflection.

\subsubsection{Maximum Range Versus Receiver (or Target) Height}

If the value of $A$ has been determined by using the minimum detectable power in equation (3) or (5), the corresponding contour is a curve of maximum range versus receiver height for communication or maximum range versus target height for radar. Generally, the lower part of the lowest curve $(n<1)$ is of greatest interest. If $A$ is sufficiently small (i.e., $20 \log A$ numerically large and negative), the complete contour has points below the line of sight. If the transmitter antenna is low $\left(h_{1}<30 \lambda^{2 / 3}\right.$ meters), the lower points are likewise given by the diffraction formula, discussed in Sections 5.1.7 and 5.7.3. Several such curves, the lower part of the lowest lobe corresponding to various transmitter heights for $\lambda=1$ meter and $20 \log A$ $=-130$, are given in Figure 27 .

Consider, for example, the curve for $h_{1}=2$ meters. The uppermost points of the curve correspond to the tip of the lowest lobe and were found by the procedure used in Section 5.6.5, putting $n=1$. The lowest points were found from the diffraction formula by the method of Section 5.7.3 with the aid of Figures 31 to 36 . It has been pointed out that for $n<1$, the optical interference formula is inadequate.

To locate a point between the upper extreme $(n=1)$ and the diffraction points, a curve of $A$ 


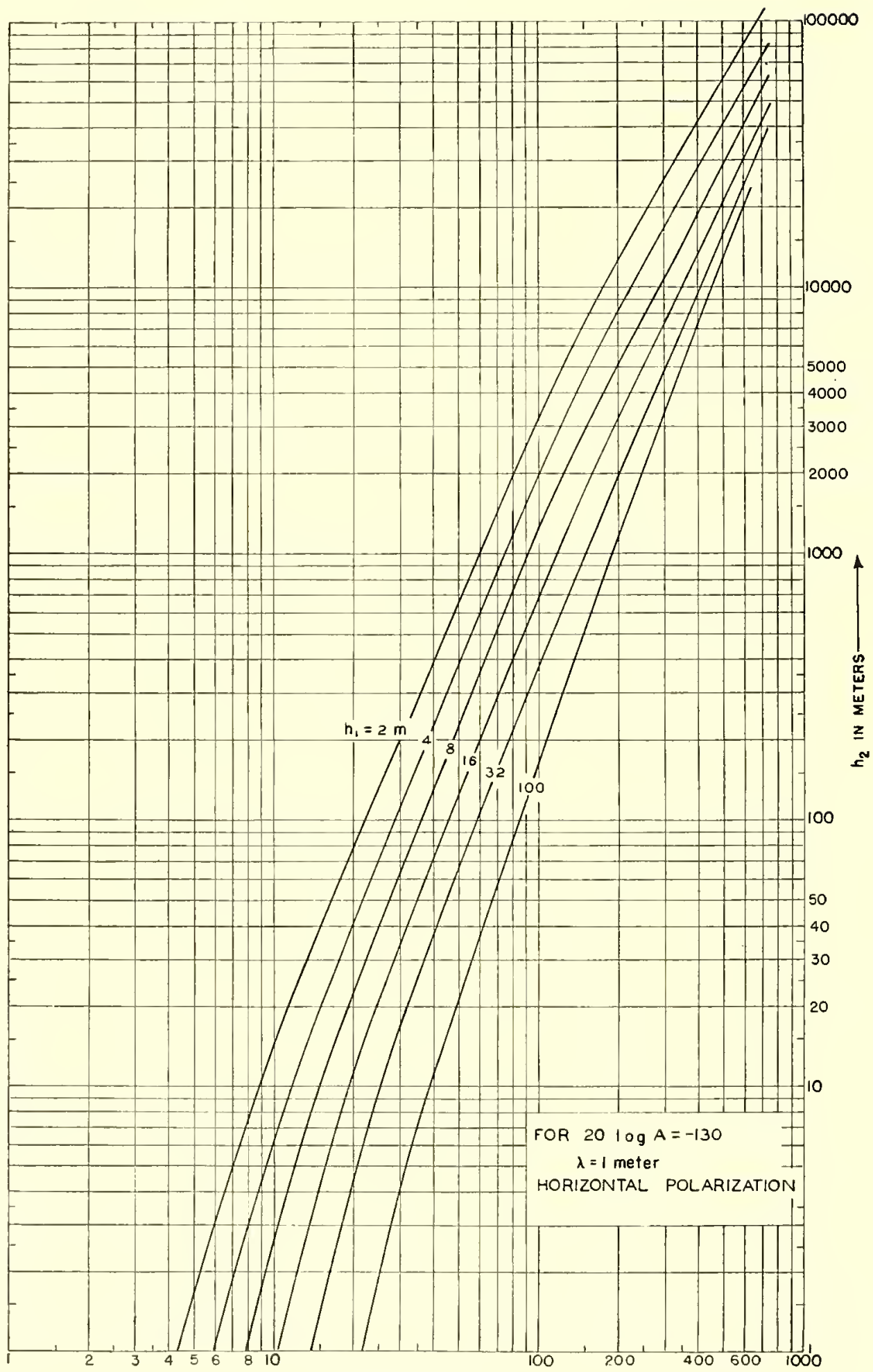

d IN KILOMETERS $\longrightarrow$

FIGURE 27. Maximum range versus receiver height $h_{2}$ for given values of transmitter height $h_{1}$. 
rersus $h_{2}$ for some distance is constructed. In Figure 28, a set of such curves is given for the distance $100 \mathrm{~km}$ and for rarious transmitter lieights. By taking the intersection of $20 \log A$ equal to -130

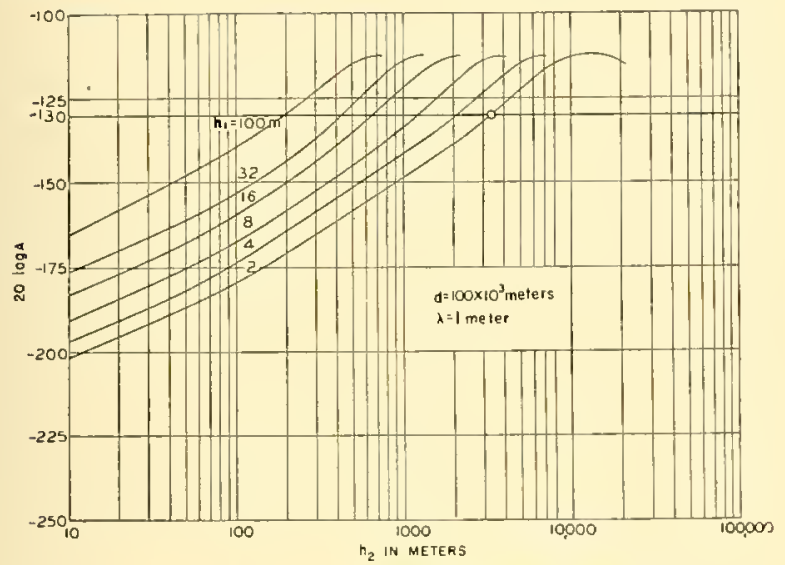

Figure 28. Radio gain versus receiver height $h_{2}$, for given values of transmitter height $h_{1}$.

with the curve $h_{1}=2$, a value of $h_{2}$ is obtained. This value $h_{2}=3,300$ and $d=100 \mathrm{~km}$ represent the coordinates of a point on the contour $h_{1}=2$ in Figure 27.

It is of some interest to observe the shortening of the lobe for $h_{1}=100$ meters on account of the divergence which is close to unity at the tips of the lobes corresponding to the low transmitter heights but drops to 0.65 at the tip of the lobe for $h_{1}=100$. Since for $h_{1}=100$, the formula for both antennas low $\left(h<30 \lambda^{2 / 3}\right)$ is not applicable, the lowest points on the curve $h_{1}=100$ in Figure 27 were obtained by applying the reciprocity principle to the curves already obtained. The distance at which $h_{2}=100$ on the $h_{1}=2$ curve is the same as that at which $h_{2}=2$ on the $h_{1}=100$ curve.

\subsection{BELOW THE INTERFERENCE REGION}

\subsubsection{Analysis of the First Mode}

Except for the numerical constants involved, the discussion for one mode applies to all the other modes. Each mode is of the form $\Phi(d) \cdot f\left(h_{1}\right) \cdot f\left(h_{2}\right)$, i.e., the product of a distance function $\Phi$ by two antenna height-gain functions $f$ (see Section 5.1.7).

\section{Distance}

$\Phi_{1}(d)$, the distance function of the first mode, can be represented as the product of two physically significant factors, one for free space and one for the earth effect. The latter may be divided into a plane earth factor and a shadow factor.

1. Free space. It has been shown in equation (18) in Chapter 2 that for doublet antenuas, with matched loar at the receiver and adjusted for maximum power transfer, the free-spare gain factor $A_{0}$ is given by

$$
A_{0}=\sqrt{\frac{P_{2}}{P_{1}}}=\frac{3 \lambda}{8 \pi d} .
$$

For other types of antennas in free space, this takes the form

$$
A_{0} \sqrt{G_{1}} \sqrt{\bar{G}_{2}}=\sqrt{\frac{P_{2}}{P_{1}}}=\frac{3 \lambda}{8 \pi l} \sqrt{i_{1}} \sqrt{i_{2}}
$$

Under actual conditions when earth and atmospheric effects are of importance, each mocke will be considered to have $A_{0}$ as one factor.

It may also be recalled that for given power $P_{2}$ delivered to the load, the corresponding electricficld strength, under matched conditions, is given by

$$
E=\frac{S_{\pi} \sqrt{5}}{\lambda} \sqrt{\frac{P_{2}}{1.2}} .
$$

Combining equations (110) and (141) gives the frec-space value of the electric ficld strength $E$, in terms of transmitted porver

$$
E=\frac{3 \sqrt{5}}{d} \sqrt{P_{1}} \sqrt{{\left(i_{1}\right.}_{1}},
$$

which is the same as equation (7) in Chapter 2 with the adclition of the transmitter gain $G_{1}$.

2. Plane earth. The earth modifies the field by absorbing and reflecting radiation. If the earth were plane and perfectly conducting, the value of the gain factor would be $21_{0}$ and the electric field $2 E_{0}$ for vertical antennas several wavelengths above the ground and at distances sufficiently large. The imperfect conductivity of the earth produces a change in the gain. Representing this effect by the factor $A_{1}$ (where $A_{1}<1$ ),

$$
A=2 A_{0} A_{1} \text {. }
$$

The plane earth factor $A_{1}$ depends on distance and on the electrical properties of the earth. Fortunately the earth constants enter the problem in an intrinsically simple way, as the main effect is taken into account by multiplying the distance $d$ by a certain factor which we shall denote by $p^{\prime}$, so that $A_{1}$ is mainly a function of $p^{\prime} d$. The new parameter $p^{\prime}$ is 
different for the two states of polarization. For vertically polarized radiation,

$$
p^{\prime}=\frac{2 \pi}{\lambda} \frac{\left|\epsilon_{c}-1\right|}{\left|\epsilon_{c}\right|^{2}},
$$

where $\epsilon_{c}$ is the complex dielectric constant $\epsilon_{\tau}-j 60 \sigma \lambda$,

$$
\text { or } \quad p^{\prime}=\frac{2 \pi}{\lambda} \frac{\sqrt{\left(\epsilon_{r}-1\right)^{2}+(60 \sigma \lambda)^{2}}}{\epsilon_{r}{ }^{2}+(60 \sigma \lambda)^{2}} \text {. }
$$

For horizontal polarization,

$p^{\prime}=\frac{2 \pi}{\lambda}\left|\epsilon_{c}-1\right|=\frac{2 \pi}{\lambda} \sqrt{\left(\epsilon_{r}-1\right)^{2}+(60 \sigma \lambda)^{2}}$.

$A_{1}$ depends also on the phase of the complex dielectric constant. The phase is determined by the parameter

$$
Q=\frac{\epsilon_{r}}{60 \sigma \lambda}
$$

For ultra-short waves, with the exception of vertically polarized waves over sea water at distances less than 50/ $p^{\prime}$ (see Seetion 5.7.4 and Figure 45) and a wavelength greater than 1 meter, $A_{1}$ is independent of $Q$ and, to a sufficient approximation, is given by

$$
A_{1}=\frac{1}{p^{\prime} d} .
$$

$A_{1}$ as a function of $p^{\prime} d$ is plotted in Figure 56 .

In the case excepted above, $A_{1}$ deriates substantially from $1 / p^{\prime} d$ (see Figure 45 ) for distances less than $50 / p^{\prime}$. Table 3 gives $50 / p^{\prime}$ as a function of $\lambda$. It appears that the deviations are immaterial for practical purposes as long as the wavelength is smaller than 3 meters, since we are usually eoncerned with ranges larger than $7 \mathrm{~km}$. It should further be mentioned that, in the above case, $A_{1}$ depends to a small degree on Q. However, the variations are less than $1 \mathrm{db}$ and may be neglected for wavelengths less than 10 meters.

TABLE 3. Sea water (vertical polarization).

\begin{tabular}{rrrrrrrrrrrr}
\hline \hline & 1 & 2 & 3 & 4 & 5 & 6 & 7 & 8 & 9 & 10 & meters \\
$\frac{50}{p^{\prime}}$ & 2 & 4 & 7 & 12 & 17 & 24 & 30 & 50 & 71 & 200 & $\mathrm{~km}$ \\
\hline
\end{tabular}

The condition that the earth may be considered plane is that the shadow faetor $F_{s}$ [see equations (149) and (207)] shall be approximately unity. For $F_{s}=0.9$, which is approximately 1 db below unity, $\eta=\zeta f(\delta)=0.4$ from Figure $58 ; f(\delta)$ in Figure $5 \bar{\gamma}$ is approximately unity, so that $\zeta=s d \cong 0.4$. Since $s$, from equation (150) for $k=4 / 3$, is given by $4.43 \times 10^{-5} \lambda^{-1 / 3}$, it follows that $d$, for the plane-earth approximation to hold, must be less than $10^{4} \lambda^{1 / 3}$,

$$
d<10^{4} \lambda^{1 / 3} \quad \text { (plane earth). }
$$

3. Curved earth. The screening effect of the earth curvature results in a further decrease in gain. Well within the diffraction region, the sliadow effect produces an exponential drop in field strength with distance, which is much greater for higher modes than it is for the first mode.

Denoting the screening or shadow factor by $F_{s}$ and the distance gain factor by $\Phi_{1}$, we have

$$
\Phi_{1}=2 A_{0} A_{1} F_{s} .
$$

For the dielectric case (sce Sections 5.1.7 and 5.7.3) $\delta>>1$ and for distances greater than $1.5 / s$, the shadow factor is

$$
F_{s}=2.507(s d)^{3 / 2} e^{-1.607(s d)},
$$

where

$$
\begin{aligned}
s & =\left[\frac{2 \pi}{\lambda} \frac{1}{(k a)^{2}}\right]^{1 / 3}, \\
& =4.43 \cdot 10^{-5} \lambda^{-1 / 3}\left(\frac{4}{3 k}\right)^{+2 / 3} .
\end{aligned}
$$

Equation (149) gives the value of the shadow factor for the first mode only. In Figure 32, the curve marked "diclectric earth" is a plot of the shadow factor evaluated by using all terms or modes. However for sd $>1.5$ only the first mode is important. Consequently, equation (149) represents this eurve accurately for all values of $s d$ larger than 1.5 .

\section{HEIGHT-GANN}

For antennas at zero height, the height-gain functions $f$ are equal to unity, so that equation (148) represents the actual value of the first mode for both intennas at zero height.

When the antennas are raised above the ground, it is convenient to distinguish between low and high antennas, the division between the two eases being given by the critical height $h_{c}=30 \mathrm{\lambda}^{2 / 3}$.

1. Low antenna; $h<h_{c}=30 \lambda^{2 / 3}$. For a low antenna, $f$ is a function of $l h$ and of $Q$, where $l$ is a quantity that depends on the complex dielectric constant and is given by

$$
l=\sqrt{\frac{2 \pi p^{\prime}}{\lambda}},
$$


in which $p^{\prime}$ is the distance coefficient given by equations (144) and (145). Let the value of $f$ for a low antenna be denoted by $H_{L}$.

The maguitude of $H_{L}$ is given by

$$
H_{L}=\sqrt{1+\frac{2 l h}{4 Q^{2}+1}+l^{2} h^{2}} .
$$

and is plotted in Figure 47 .

For height $h$ larger than $4 / l$, the two first terms under the square root may be neglected in comparison to the third term, and $H_{L}$ becomes approximately

$$
H_{L} \cong l h \text {. }
$$

In order to show more clearly when this approximation is justified, Table 4 gives values of $4 / l$ for different wavelengths and vertical polarization. For horizontal polarization, $4 / l$ is quite small.

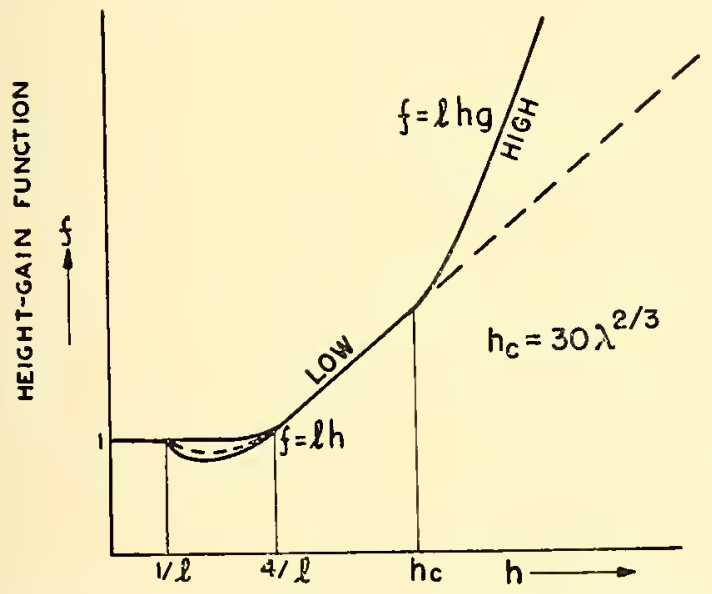

Figure 29. Height-gain as a function of height. (See Figures 7, 25, and 47.)

Inspection of Table 4 shows that, except for the case of sea water at wavelengths above 1 meter, the approximate equation (153) is good for heights above about 50 meters.

TABLE 4. Values of $4 / l$ for different wavelengths

\begin{tabular}{|c|c|c|c|c|c|c|c|c|c|c|c|}
\hline$\lambda$ in meters & 0.1 & 1 & 2 & 3 & 4 & 5 & 6 & 7 & 8 & 9 & 10 \\
\hline Sea & 0.06 & 10 & 28 & 508 & 80 & 115 & 133 & 160 & 235 & 285 & 300 \\
\hline water & & 6 & 11 & 172 & 22 & 30 & 33 & 40 & 44 & 53 & 57 \\
\hline Mloist soil & & 3 & 71 & 111 & 15 & 18 & 21 & 25 & 28 & 33 & 36 \\
\hline Fertile ground & & 3 & 5 & 81 & 10 & 13 & 16 & 17 & 20 & 25 & 27 \\
\hline Very dry ground & & 2 & 3 & 4 & 6 & 8 & 9 & 10 & 12 & 15 & 16 \\
\hline
\end{tabular}
(vertical polarization).

To a first approximation, the value of $f$ for low antennas is the same for all modes, so that the heightgain functions may be factored out, as was pointed out in Section 5.1.7. The gain factor for the case when both antennas are low can then be represented by

$$
A=2 A_{0} A_{1} F_{s}\left(H_{L}\right)_{2}\left(H_{L}\right)_{2}
$$

where $F_{s}$ is sum of the shadow factors of all the modes. $F_{s}$ has been plotted in Figure 32 . Equation (154) is also valid in the optical region, provided

$$
d>>\frac{2 h_{1} h_{2}}{\lambda} \text {. }
$$

This condition is added to insure that the point is well below the center of the lowest lobe.

2. Elevated antenna; $h>30 \lambda^{2 / 3}$. In this case the height-gain function $f$ increases exponentially. Representing the increase over $l h$ by $g$, we have

$$
f=g l h \text {. }
$$

For the dielectric case $\delta>>1$, refer to equation (193) to define $\delta$. (See Sections 5.1.6 and 5.7.3.)

$$
g=0.1356(e h)^{-0.904} 10^{0.948 \sqrt{e h}}
$$

where

$$
\begin{aligned}
e & =\left(\frac{4 \pi^{2}}{\lambda^{2} k a}\right)^{1 / 3}, \\
& =\frac{1}{60} \lambda^{-2 / 3}\left(\frac{4}{3 k}\right)^{1 / 3} .
\end{aligned}
$$

See Figures 35 and 36.

For the case of sea water, vertical polarization and wavelength in the VHF (1 to 10 meters) range, $g$ requires a correction factor $g$ ' which depends on $\lambda$. A table of $g^{\prime}$ is given with the graph of $g$ in Figure 36.

The change of $f$ with height $h$ is represented schematically in Figure 29 (see also Figures 7, 25, and 47 ).

Formula for the Dielectric Eartir. (See Sections 5.1.7 and 5.7.3.) $\delta>>1$.

The first mode has the form $\Phi_{1}(d) \cdot f\left(h_{1}\right) \cdot f\left(h_{2}\right)$. If the value of the gain is given substantially by the first mode, and substitution from equation (148) is made, it is found that

$$
A=\left[2 A_{0} A_{1} F_{s 1}\right] f\left(h_{1}\right) \cdot f\left(h_{2}\right) .
$$

For the dielectric case equation (160), using equations (140), (147), (151), and (156), becomes

$$
A=\frac{3}{2} \frac{F_{s 1}}{d^{2}}(g h)_{1}(g h)_{2}
$$

The same formula holds for sea water, vertical polarization, wavelengths in VHF range, $d>50 / p^{\prime}$, 
and $h>4 / l$, as described above, except that $F_{s}$ or $F_{s 1}$ is represented by various curves in Figure 32, according to the value of $\lambda$, and $g$ is modified slightly by a correction factor $g^{\prime}$.

Formula for Siea Water. Vtertical PolarizaTION. VHF.

Equation (160), the general formula for the first mode, in the VHF (1 to 10 meters) range, becomes

$$
A=\left[2 A_{0} A_{1} F_{s 1}^{\prime}\right]\left(H_{L} g g^{\prime}\right)_{1}\left(H_{L} g g^{\prime}\right)_{2},
$$

where $H_{L}$ is the low antenna height-gain function whose formula is giren by equation (152), and $g g^{\prime}=1$ for low antemnas. As pointed out above, if $h>4 / h$ and $d>50 / p^{\prime}$, equation (162) reduces to equation (161). $g^{\prime}$, the correction factor for $g$, is given in Figure 36. For a more cxtended discuscion, see Section 5.7.4.

\section{${ }^{5.7 .2}$ Effect of Changing the Value of $\boldsymbol{k}$}

In the optical region, the effect of a linear gradient of refractive index has been shown to be equivalent to replacing the radius of the earth $a$ by an effective radius $k a$ and then treating the atmospherc as homogeneous (see Chapter 4).

In view of the equivalence of the sum of modes to the optical formula in the optical region, it follows that the modes should be changed in the same way in the optical region, i.e., $a$ should be replaced by $k a$. These same nodes supply the solution of the wave equation in the diffraction region, so that in both regions the substitution of $k a$ for $a$ will take care of an atmosphere with a linear variation of refractive index with height for all values of $k$.

For given trunsmitter and receiver antenna heighis, $h_{1}$ and $h_{2}$, the first maximum of the ficld-streugth versus distance curve (see Figure 4) will frequently represent the limit of detection. The first maximum occurs not far from the line of sight which, for a standard atmosphere $(k=4 / 3)$, is given by

$$
d_{L}=\sqrt{2\left(\frac{4}{3}\right) a}\left(\sqrt{h_{1}}+\sqrt{h_{2}}\right) .
$$

If $4 / 3$ is changed to $k$, the more general form is

$$
d_{L}^{\prime}=\sqrt{2 k a}\left(\sqrt{h_{1}}+\sqrt{h_{2}}\right) .
$$

The first maximum will now be near

$$
d_{L}^{\prime}=\sqrt{\frac{3 k}{4}} d_{L}
$$

Suppose next that the point at distance $d_{L}{ }^{\prime}$ for the given heights was originally well within the diffractive region. The exponential part of the gain due to change of $k$ from $4 / 3$ is equal to

$$
\left(c^{-1.6078\left(l_{L}+d_{L}\right)}\right.
$$

or

$$
e^{-1.607(\sqrt{3 k / 4}-1)_{s t} l_{L}} \text {. }
$$

The above formula is obtained by combining equation (149) for $F_{s}$, and $s$ is olstained from Figure 31 $(k=4 / 3)$. This corresponding gain in decibels is

$$
\begin{aligned}
& 20 \log _{10} e-1.607(\sqrt{3 k / 4}-1)_{s t} L \\
= & 20\left[-1.607 \times .434\left(\sqrt{\frac{3 k}{4}}-1\right) s d_{L}\right], \\
= & -14 s d_{L}\left(\sqrt{\frac{3 k}{4}}-1\right) \mathrm{db},
\end{aligned}
$$

below the free-space vahue. Since a maximum is now near $d_{L}^{\prime}$ as a result of changing $4 / 3$ to $k$, i.e., the field has a value of $6 \mathrm{db}$ above the free-space value, the gain is approximately the exponential vahue

$$
-14 s d_{L}\left(\sqrt{\frac{3 k}{4}}-1\right)
$$

as a consequence of refraction giving a value $k$ greater than $4 / 3$. For $k=12$, and $s d_{L}=5$, the gain would be about $140 \mathrm{db}$ at some point near $d_{L}^{\prime}=3 d_{L}$, at heights $h_{1}$ and $h_{2}$ such that

$$
d_{L}^{\prime}=\sqrt{2 k a}\left(\sqrt{h_{1}}+\sqrt{h_{2}}\right)
$$

and such that $h_{2}$ is well within the diffraction region.

For given transmitter height $h_{1}$ and distance $d$, the effect of increasing $h$ is to lower the lowest lobe roughly by the amount by which the line-of-sight elevation at the distance $d$ is lowered in changing from $4 a / 3$ to $k a$. At this distance the height of the line of sight is $h_{L}$, and

$$
\sqrt{h_{L}}=\frac{d}{\sqrt{8 \frac{a}{3}}}-\sqrt{h_{1}}\left(\text { for } l_{i}=4 / 3\right)
$$

becomes

$$
\sqrt{h_{L}^{\prime}}=\frac{d}{\sqrt{2 k^{\prime}}}=-\sqrt{h_{1}}
$$

so that there is a downward shift of approximately

$$
h_{L}-h_{L}^{\prime}=\frac{3 d^{2}}{8 a}\left(1-\frac{4}{3 k}\right)-2 \sqrt{h_{1}} \sqrt{\frac{3}{8 a}} d\left(1-\sqrt{\frac{4}{3 k}}\right) \text {. }
$$


If $h_{1}$ is small compared with $h_{L}$ and $h_{L}{ }^{\prime}$, the result is approximately

$$
h_{L}-h_{L}{ }^{\prime}=h_{L}\left(-\frac{4}{3 k}+1\right) .
$$

Receivers situated between $h_{L}$ and $h_{L}{ }^{\prime}$ were formerly below the line of sight but are now above it (assuming $k>4 / 3$ ), with a consequent substantial gain in signal strength at some points and loss at others. The chances of detection in the region, how ever, have improved.

If both antemas are low, a change of $4 a / 3$ to $k a$ gives a field strength change such that the field where

$$
\begin{aligned}
h_{1,2}^{\prime} & =h_{1,2}\left(\frac{3 k}{4}\right)^{-1 / 3}, \\
d^{\prime} & =d\left(\frac{3 k}{4}\right)^{-2 / 3},
\end{aligned}
$$

where $k$ may now have any value. 1 itself will change to $A^{\prime}$ where

$$
A^{\prime}=A \quad\left(\frac{3 k}{4}\right)^{2 / 3} .
$$

Figure 30 illustrates the values of $(3 \mathrm{k} / 4)^{n}$ for various values of $k$ and $n$ used in equations (169), (170), and

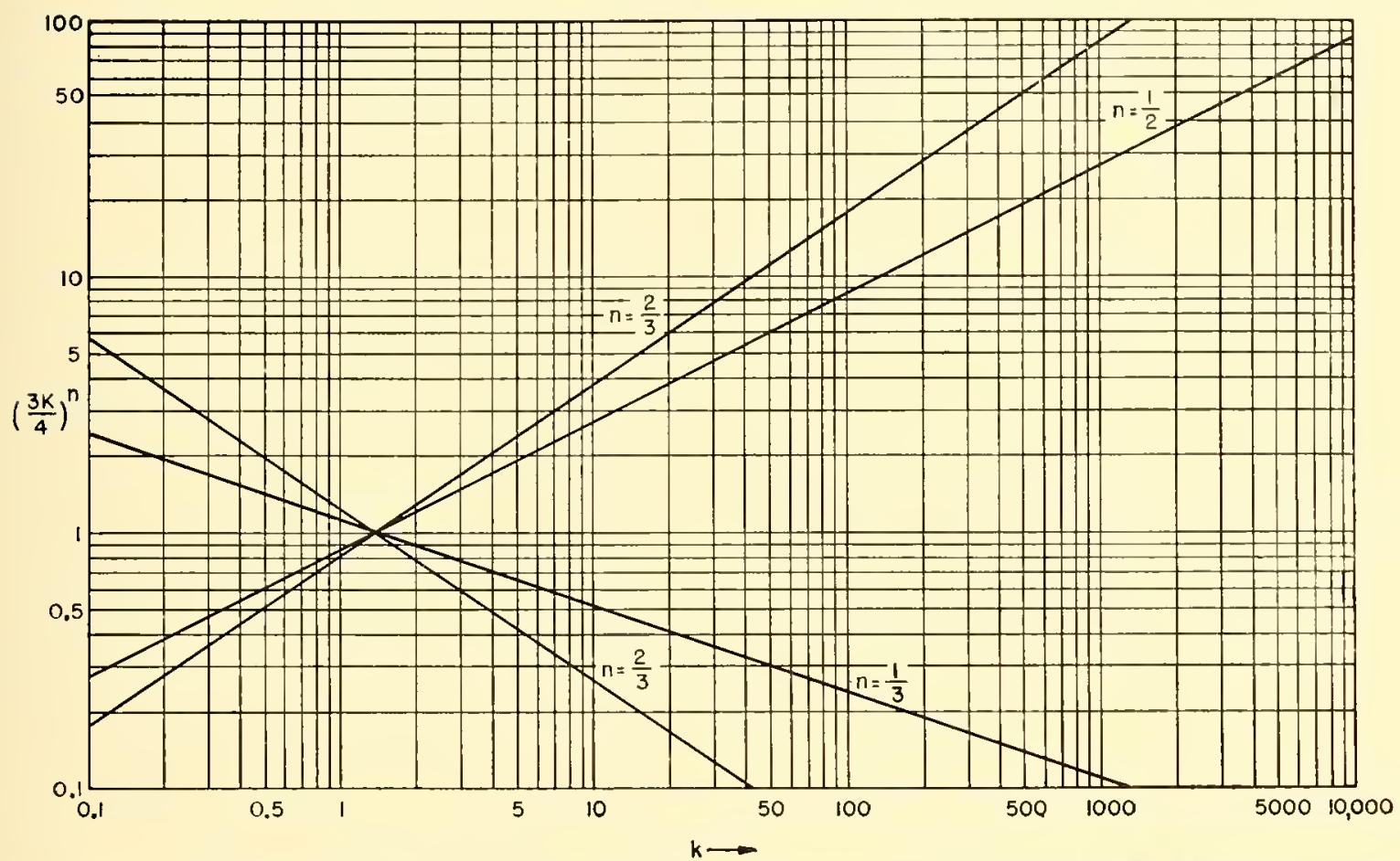

Figure 30. Values of $(3 k / 4)^{n}$. Note: Values shown between 0.1 and 0.5 of the ordinate scale are minus values.

which was formerly at a point $d$ well within the diffraction region will now be found at a distance of approximately

$$
d\left(\frac{3 k}{4}\right)^{2 / 3}
$$

except for the decrease in free-space gain $(20 / 3) \log$ $3 k / 4$, occasioned by the increase in distance. If, for instance, $k=12$, the free-space gain is equal approximately to $-7 \mathrm{db}$, and the ratio of the new distance to the old is equal to $(3 k / 4)^{2 / 3} \cong 9^{2 / 3} \cong 4$.

Nore generally, if $A / A_{0}$ is known at a point $\left(h_{\mathbf{2}}, d\right)$ for a transmitter height $h_{1}$ and for $k=4 / 3$, the same value of $A / A_{0}$ will be found at $h_{1}{ }^{\prime}, h_{2}{ }^{\prime}, d$
(17I). Figure 43 gives the same information in nomographic form.

Hence if a coverage diagram is known for $k=4 / 3$, then the same diagram can be used for $k \neq 1 / 3$ if the diagram is interpreted in terms of $h^{\prime}, d^{\prime}$, and $A^{\prime}$.

\subsubsection{Graphs for the Case of the Dielectric Earth $(\delta>>1)$}

1. Fundamentalformula for gain factor. This formula is (sce Sections 5.1.7 and 5.7.1)

$$
A=\frac{3}{2} \frac{F_{s}}{d^{2}}(g h)_{1}(g h)_{2},
$$

where $g=1$ when $h<30 \lambda^{2 / 3}$. 


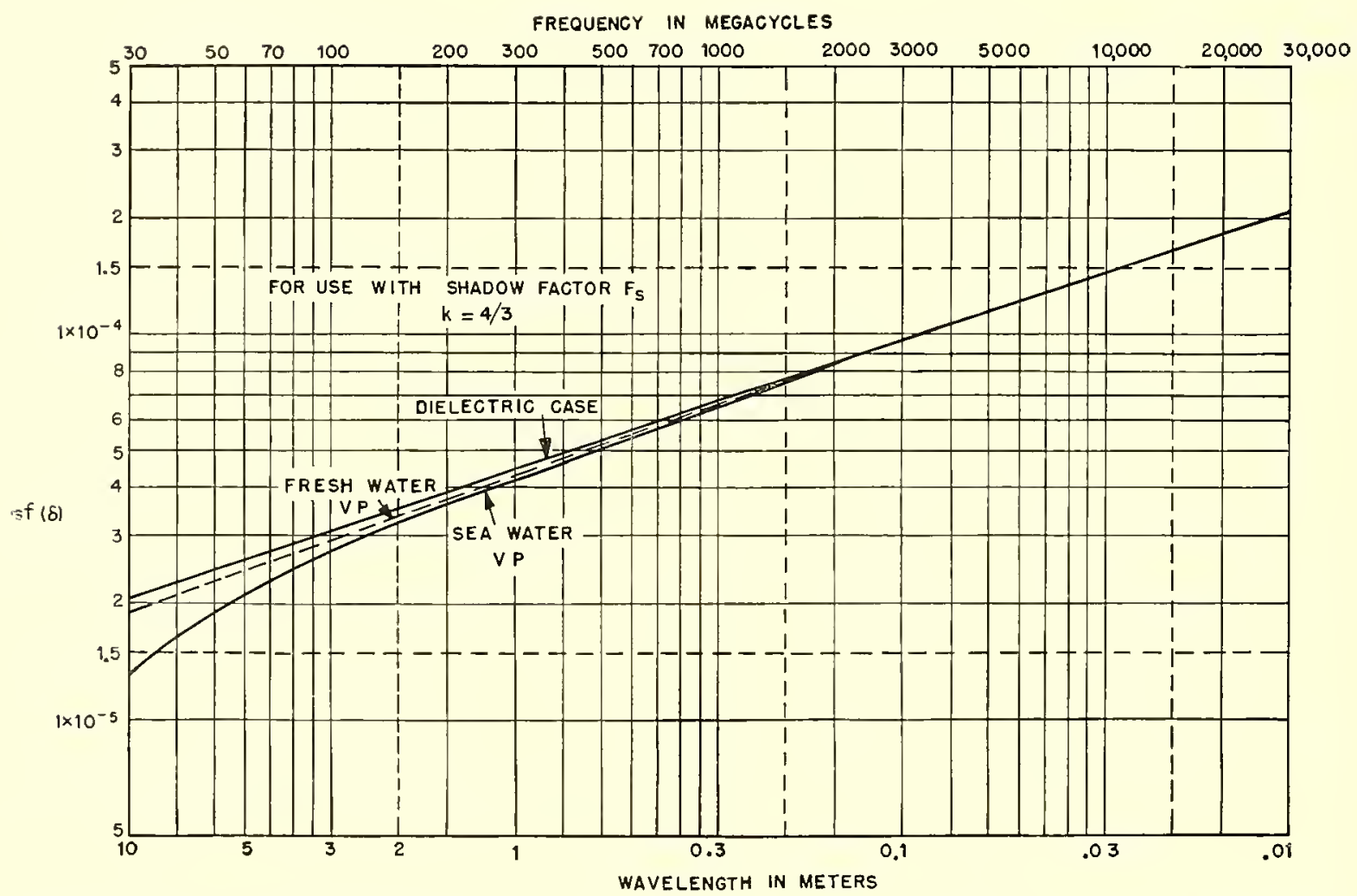

FIGURE 31. $s f(\delta)$ versus wavelength and frequency. For dielectric earth and $k=4 / 3, f(\delta)=1$, and $s=4.43 \times 10^{-5} \lambda^{-1 / 3}$. (See Figure 57 for $f(\delta)$.) 


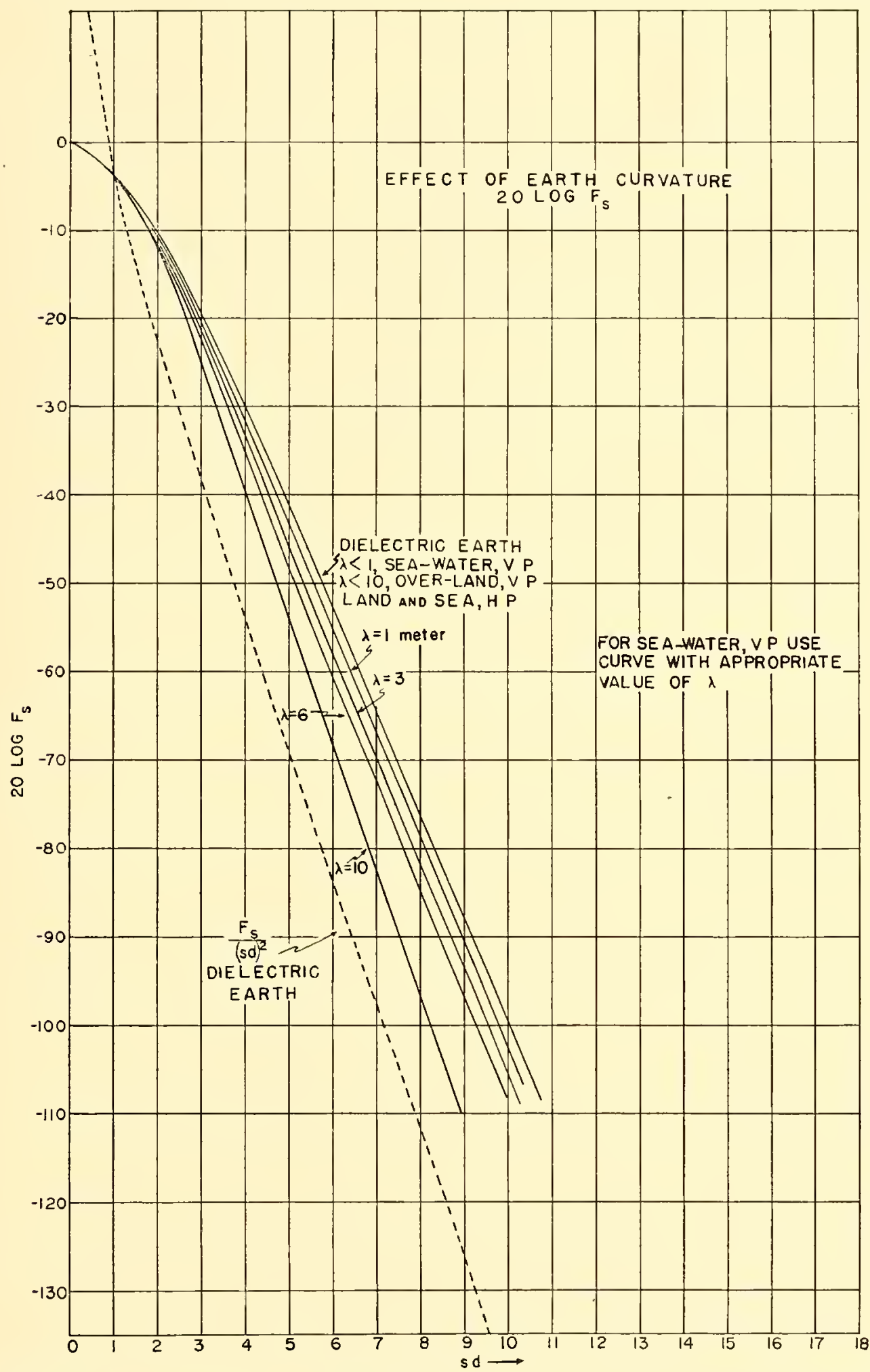

Figure 32. Shadow factor $F_{s}, 20 \log F_{s}$ (evaluating all modes), or $20 \log F_{s} /(s d)^{2}$ versus s $d$. Curve for_dielectric earth, $20 \log F_{s} /(s d)^{2}$, is the same as curve $O$ in Figure 58 . 


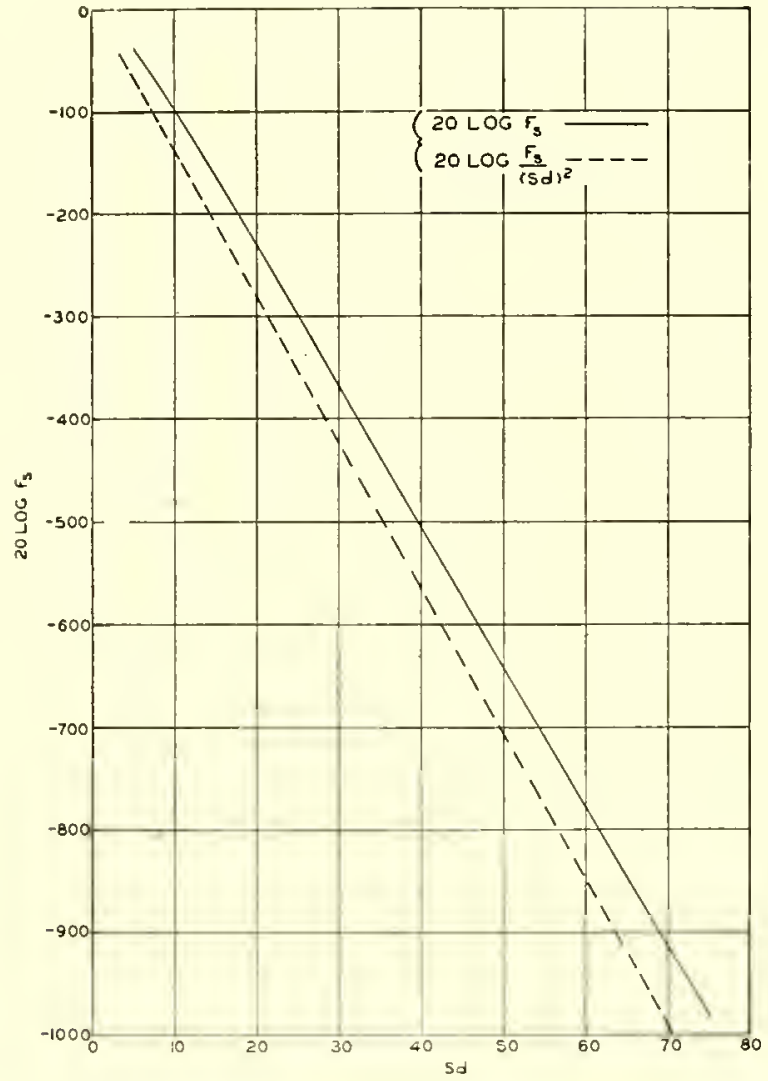

Figure 33. Shadow factor for dielectric earth.

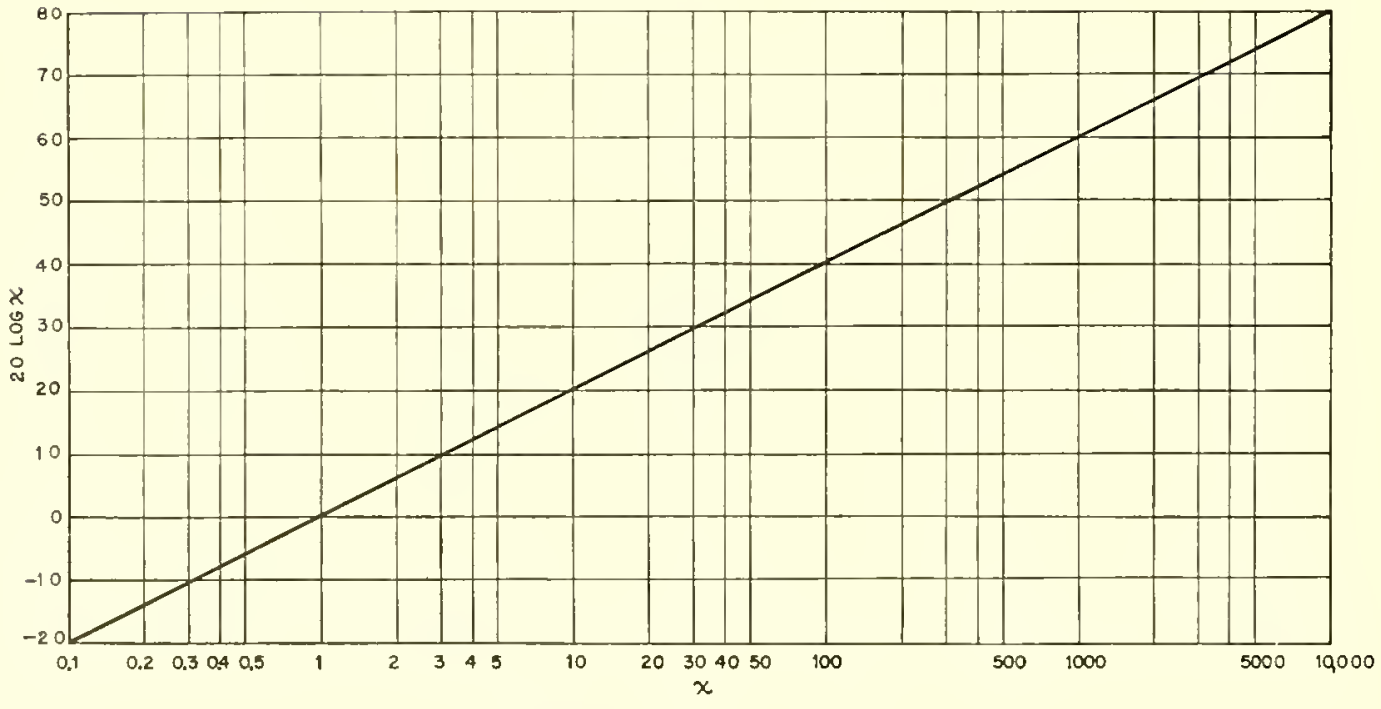

Figure $31.20 \log x$ versus $x$. 


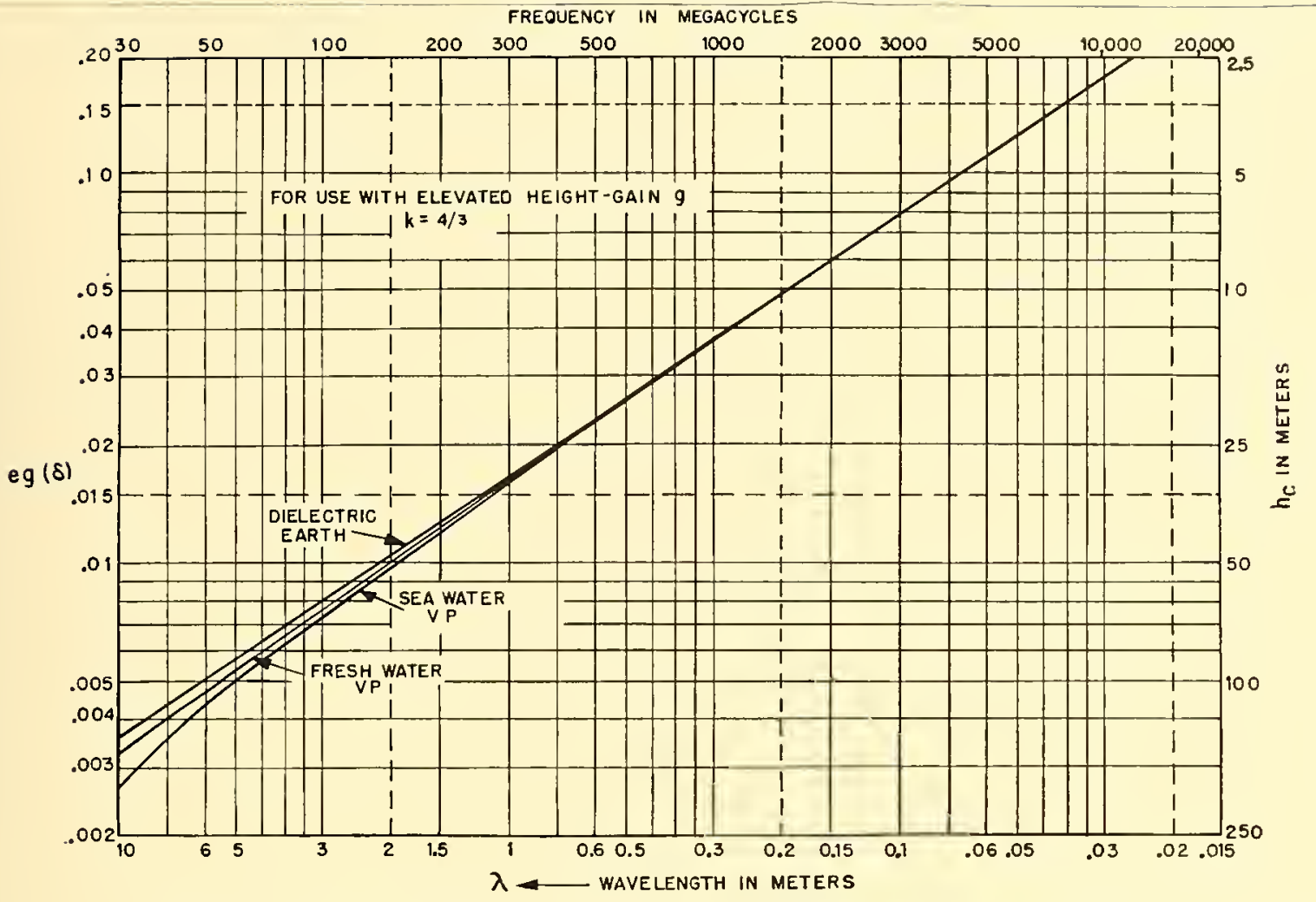

Figure 35. $e g(\delta)$ versus wavelength or frequency. For dielectric earth $e=1 / 2 h_{c}$ and $g(\delta)=1$.
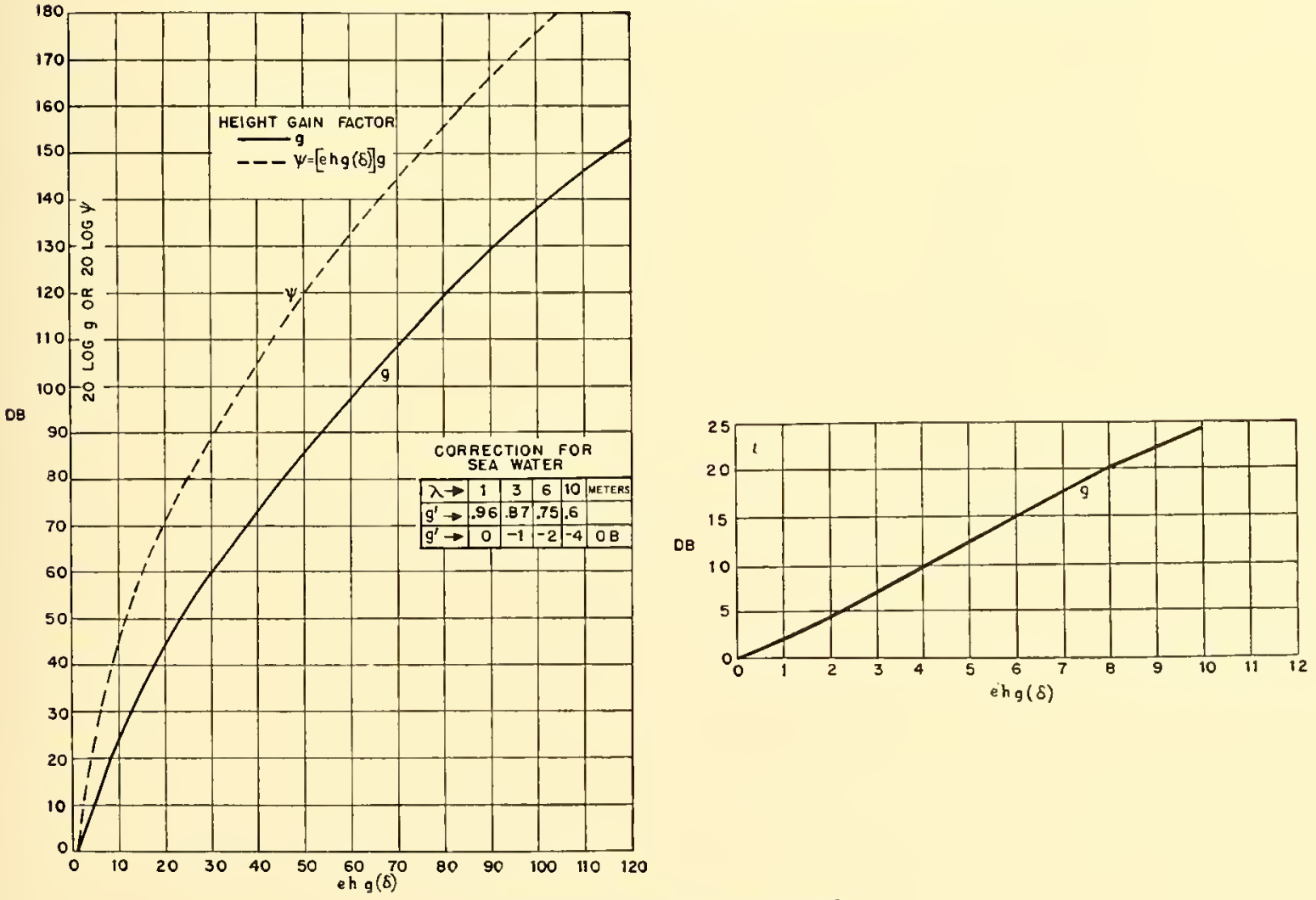

Figure $36 . g$ and $\psi$ versus $e h g(\delta)$. 

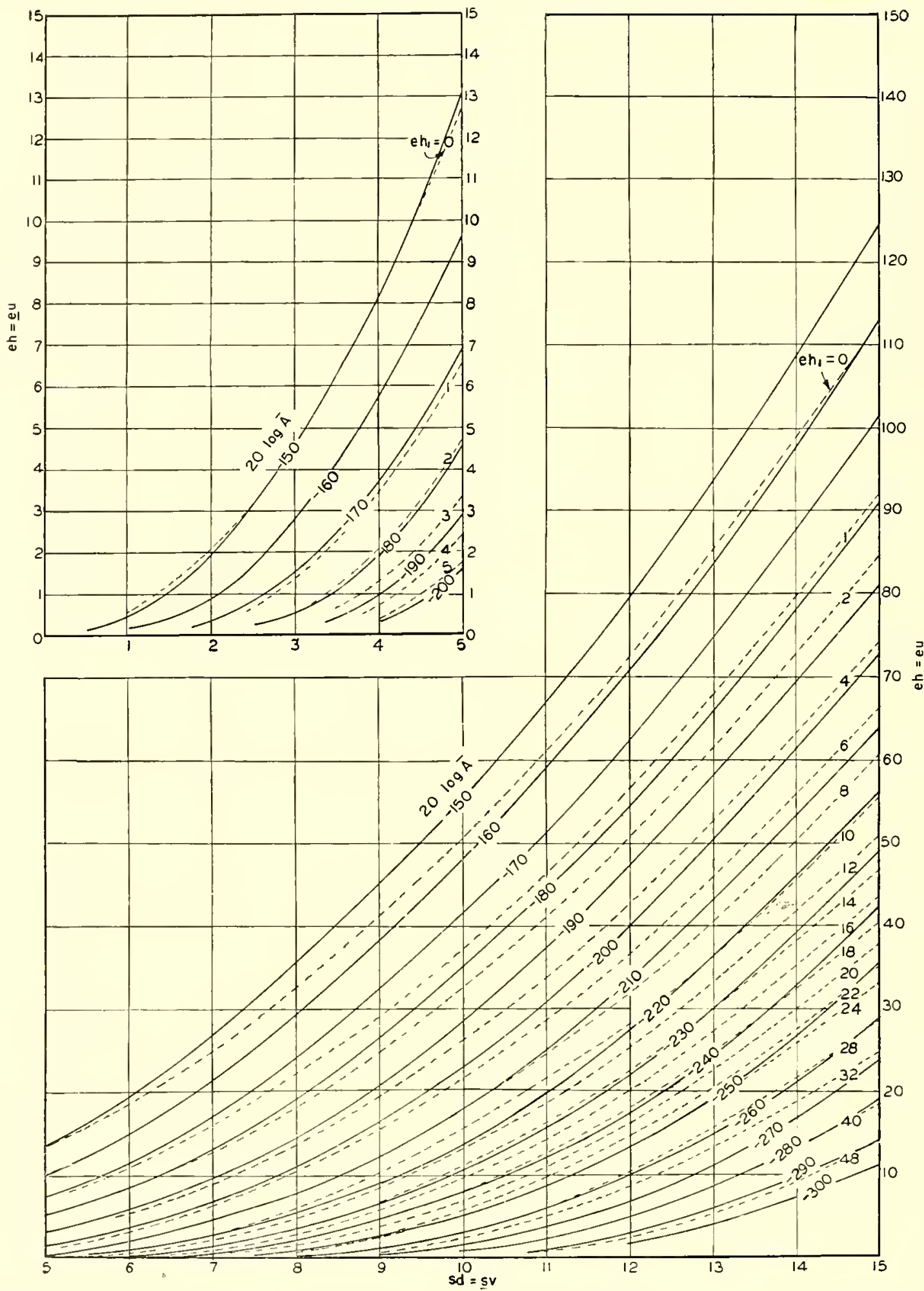

FiguRe 37. $e h_{2}=\varepsilon u$ versus $s d=\underline{s} v$. Points $e h, s d$, lying to right of dotted line corresponding to $c h_{1}$ for a given transmitter indicate that the points lie in the diffraction region of the transmitter, or $d>d_{L}$. Diffraction region dielectric earth - curve parameter: $20 \log \bar{A}=20 \log A-20 \log h_{1}-20 \log g_{1}$. 


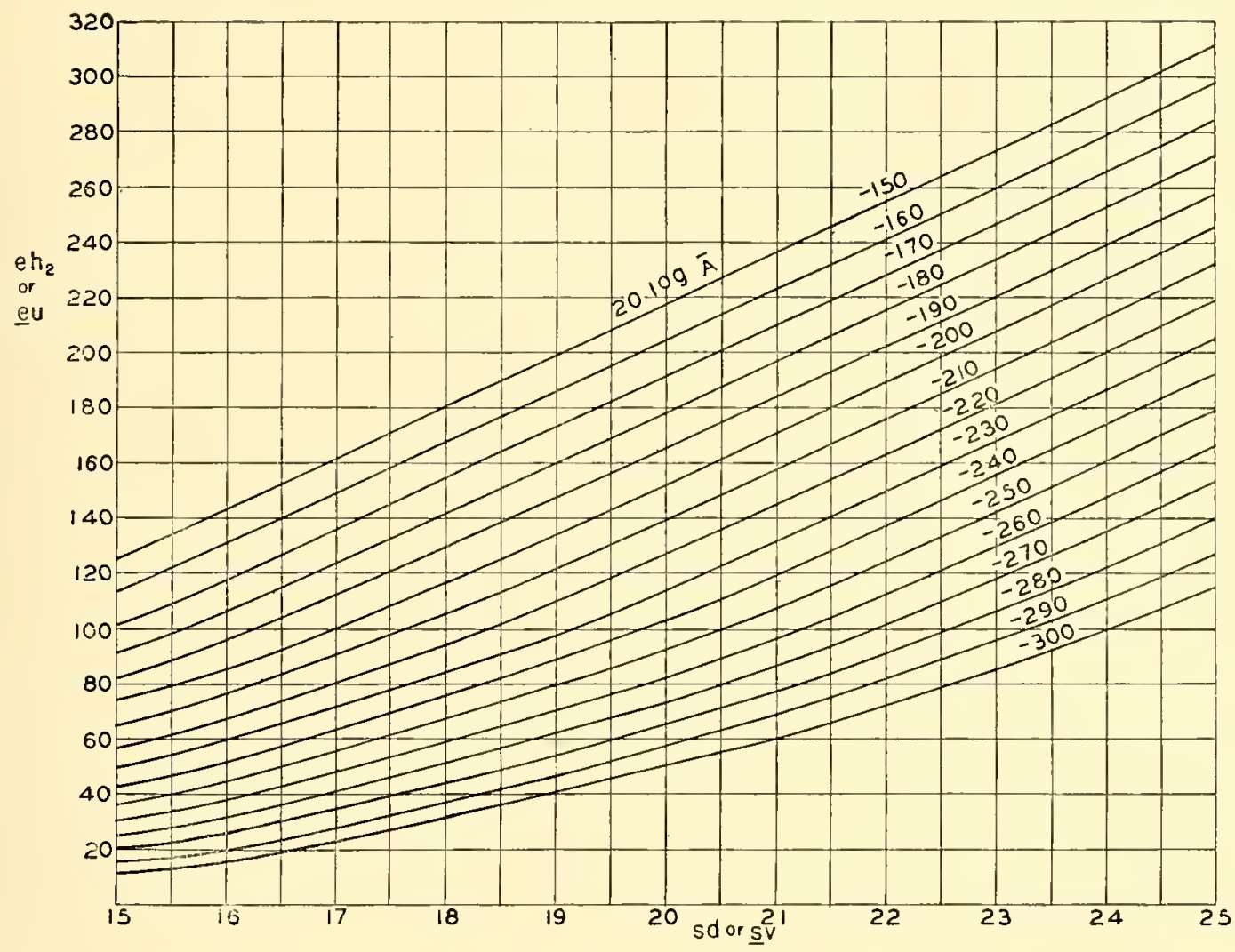

FIGURE 38. $\quad e h_{2}=\underline{e} u$ versus $s d=\underline{s} v$.

BOTH ANTENNAS LOW $\left.\quad \begin{array}{l}h_{1} \\ h_{2}\end{array}\right\}<30 \lambda^{2 / 3}$

$20 \log \bar{A}=20 \log A-20 \log n_{1}$

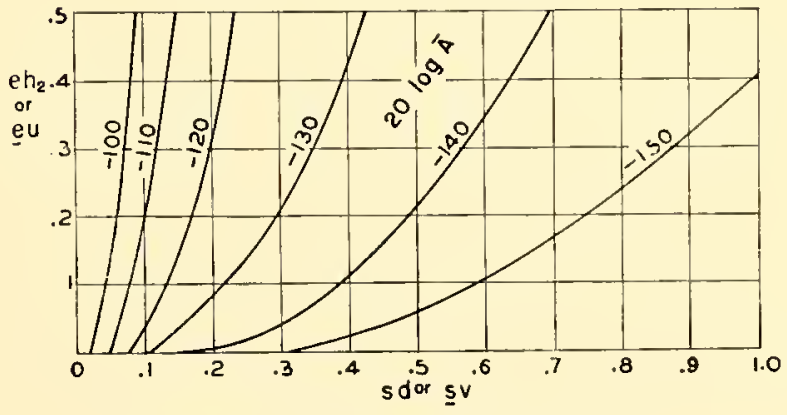

Figure 39. $\quad e h_{2}=\underline{e} u$ versus $s d=\underline{s} v$. 


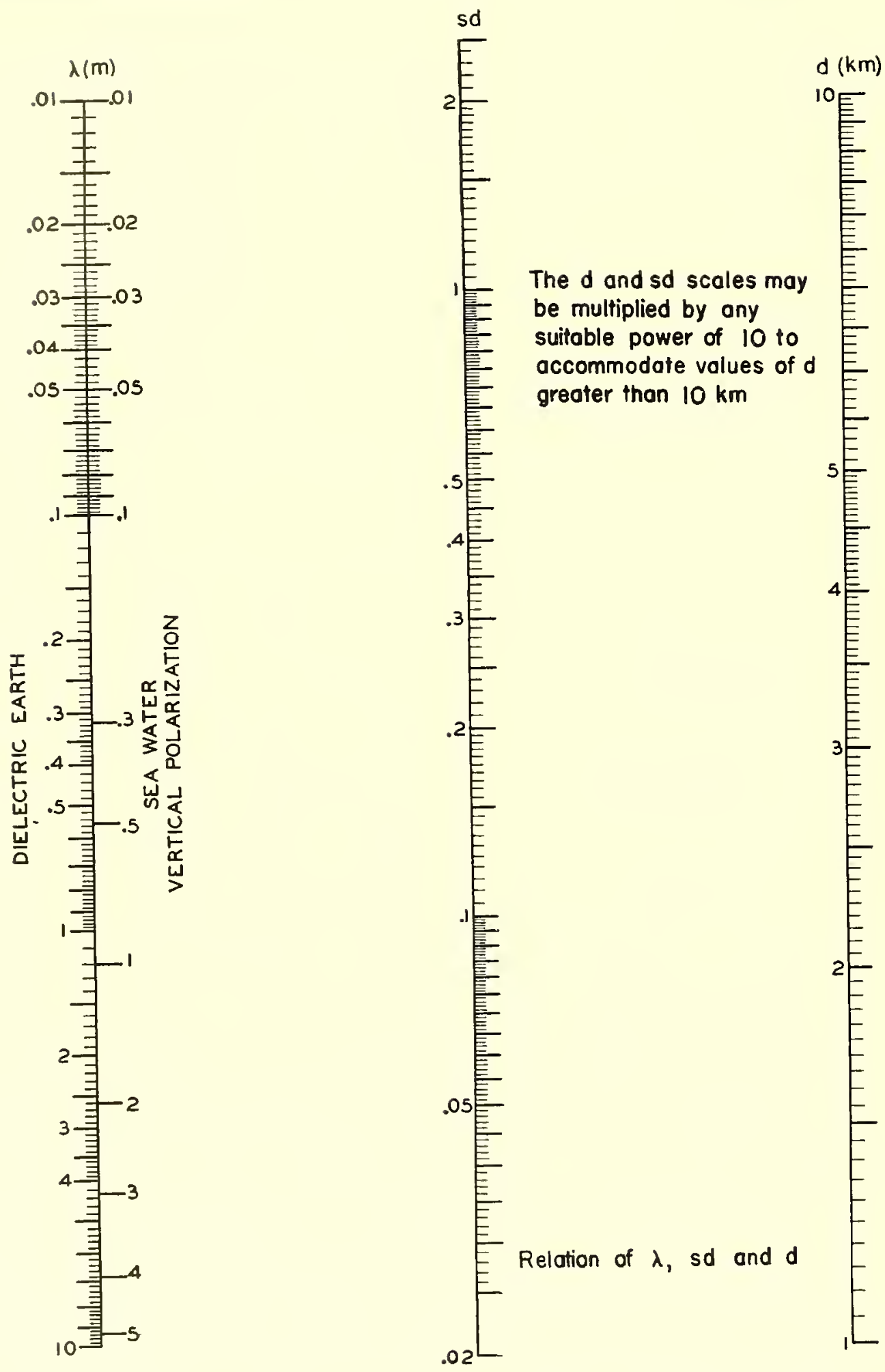

Figure 40. Relation of $\lambda, s d$ [representing $s d f(\delta)]$, and $d$. See Figure $31 . f(\delta)=1$ for $\delta>>1$. 

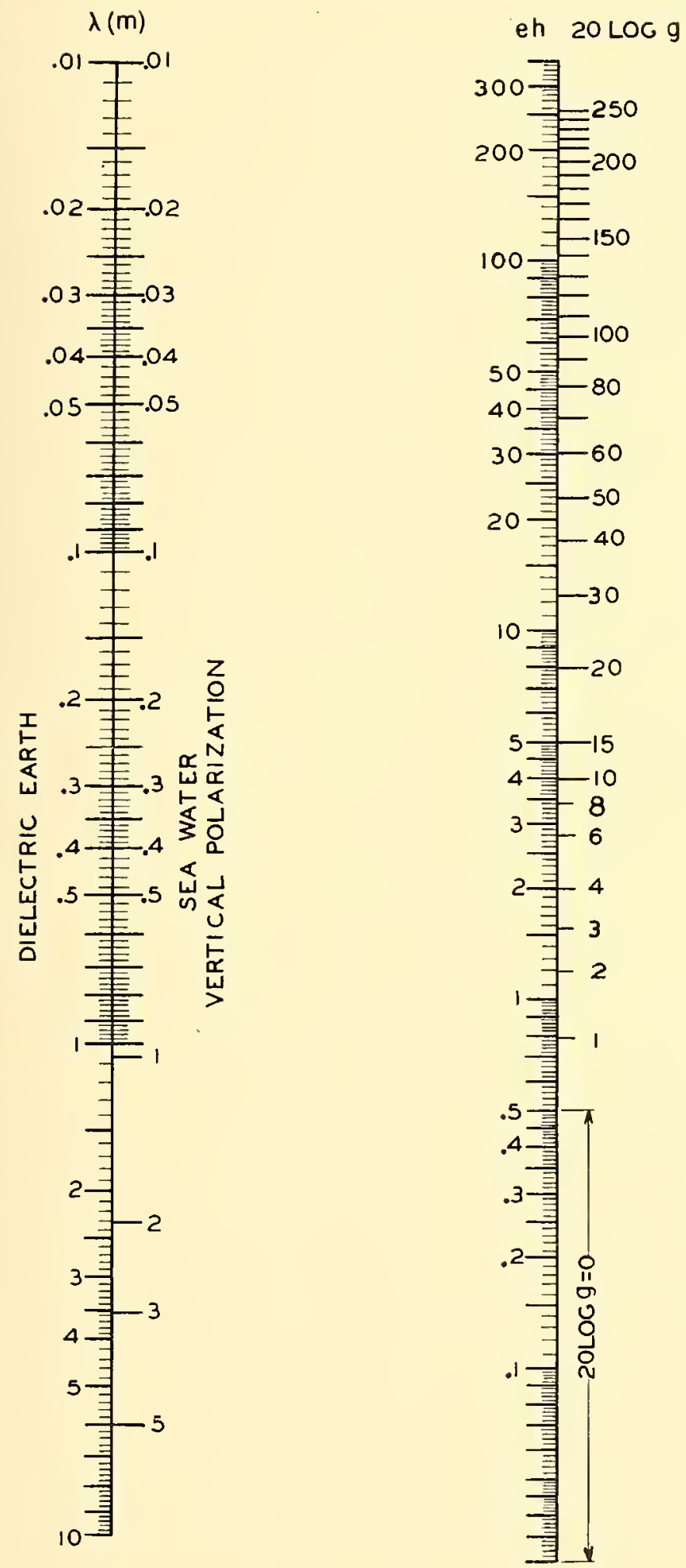

$h(m)$ zOLOG $h$

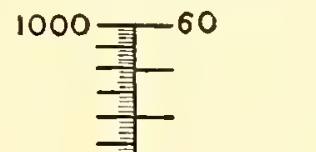


For the dielectric earth, $\delta>>1$. See equation (193). If both antennas are low $\left(h<30 \lambda^{2 / 3}\right)$ equation (172) and the accompanying figures (Figures 31 to 41 ) are valid for all distances $d$ such that

$$
d>>\frac{2 h_{1} h_{2}}{\lambda} \text {. }
$$

If one or both antennas are elevated, equation (172) is valid only well within the diffraction region of the transmitter, i.e., for

$$
d>>d_{L}
$$

The following quantities required to find $20 \log A$ are given in Figures 31 to 41 :

$$
\begin{aligned}
& s \text { as a function of } \lambda \text { is given in Figure } 31 . \\
& 20 \log F_{s} \text { versus } s d \text { in Figures } 32 \text { and } 33 . \\
& 20 \log d \text { ean be found by using Figure } 34 . \\
& e \text { as a function of } \lambda \text { by Figure } 35 \text {. } \\
& 20 \log g \text { versus } e h \text { is given by Figures } 36 \text { and } 41 \text {. }
\end{aligned}
$$

When one anterna is low, $h<30 \mathrm{\lambda}^{2 / 3}$, and the other quite elevoted, $h>1,200 \lambda^{2 / 3}$, a result valid for $h_{2}$ near the line of sight can be found from the formula and graphs in Section 5.7.5, obtained by summing several modes.

A more general method of finding the gain near the line of sight is to use equation (172) well below the line of sight to obtain a eurve of $A$ rersus $h_{2}$ and by constructing a similar curve for the optical region

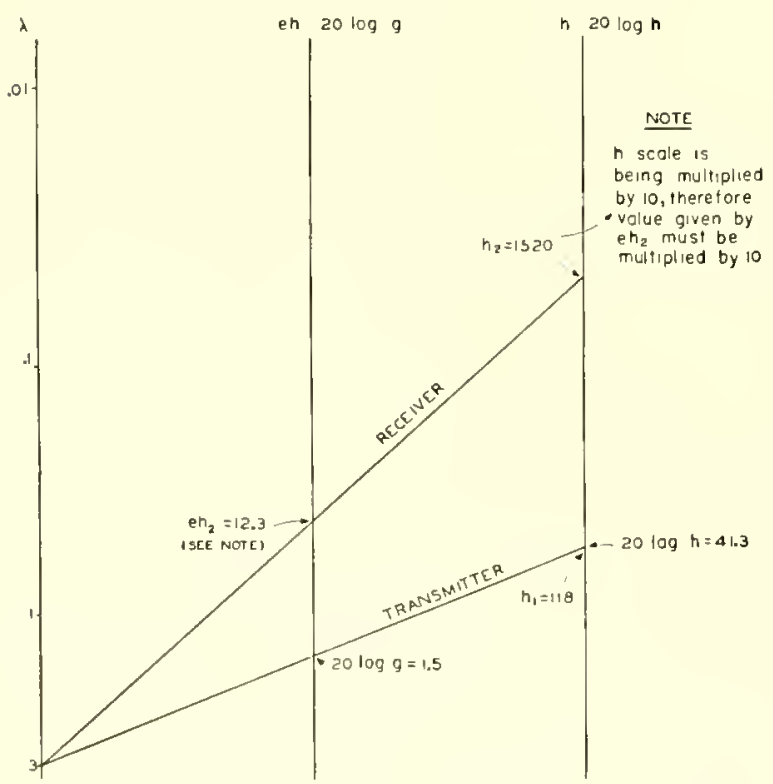

1'IGURE 42. Illustrating use of Figure 41. Note: (eh represents $\operatorname{ehg}(\delta)) ;\left[e h_{2}=12.3\right]$ represents $\left[e h_{2} g(\delta)=\right.$ 12.3]. by the method of Chapter 6, "Coverage Diagrams." $\mathrm{By}$ joining the two curves into a smooth overall curve, it is possible to estimate $A$ in the transition region near the line of sight.

For the case of short distances and receiver below the interference region, see Section 5.1.7.

2. For $h<4 / l$. Vertical polarization. A more aceurate result can be obtained by replacing the height-gain $(g h)$ by $I_{L} / l$ or in decibels by $20 \log H_{L}$ $-20 \log l . H_{L}$ is given by Figure 47 and $l$ by Figure 46 (see Table 3 ).

3. Graphieal aids (continued).

A. Definition of $\bar{A}$. Figures 31 to 36 can be combined into a form more convenient for numerical computation. In Figure 37, a curve parameter $\bar{A}$ is introduced, defined by

$$
\bar{A}=\frac{A}{h_{1} g_{1}},
$$

where $g_{1}$ is a function of $e h_{1}$. This may also be expressed in the form

$$
20 \log \bar{A}=20 \log A-20 \log h_{1} g_{1} .
$$

(For $h_{1}<4 / l, 20 \log \bar{A}=20 \log A-20 \log H_{L}+20$ $\log l$.)

Equation (172) can be written as

$$
\bar{I}=1.77 \times 10^{-\tau} \frac{F_{s}}{(s d)^{2}} \psi
$$

$\mathrm{or}^{\mathrm{N}}$

$20 \log \bar{A}=-135+20 \log \frac{F_{s}}{(s d)^{2}}+20 \log \psi$,

where

$$
\psi=\left(e h_{2}\right) g_{2},
$$

with $g_{2}$ a function of $e h_{2}$. Note that $F_{s} /(s d)^{2}$ is a function of $s d$ only and is independent of height. While $h_{1}$ usually represents the transmitter antenna height and $h_{2}$ that of the receiver, the role of $h_{1}$ and $h_{2}$ in equations (176) and (17\%) may be interchanged.

To faeilitate the use of Figure 37, three nomograms have been added (Figure 40 gives $s d$ when $\lambda$ and $d$ are given. Figure 41 gives $e h_{1}, 20 \log h$ and $20 \log g$ when $\lambda$ and $h$ are known. Figure 43 gives the modified leight $h^{\prime}$ and distance $d^{\prime}$ for given $h, d$, and $k$.) To find $s d$ for a value of $d$ which is not on the nomogram, say $120 \mathrm{~km}$, find $s d$ corresponding to a distance 100 times smaller (i.e., $1.2 \mathrm{~km}$ ) and multiply resulting sd by 100. Proceed similarly for eh.

B. Both antennas low. In the case of both antennas low, $h_{1}$ and $h_{2}<30 \lambda^{2 / 3}$, the contours $20 \log \vec{A}$ 

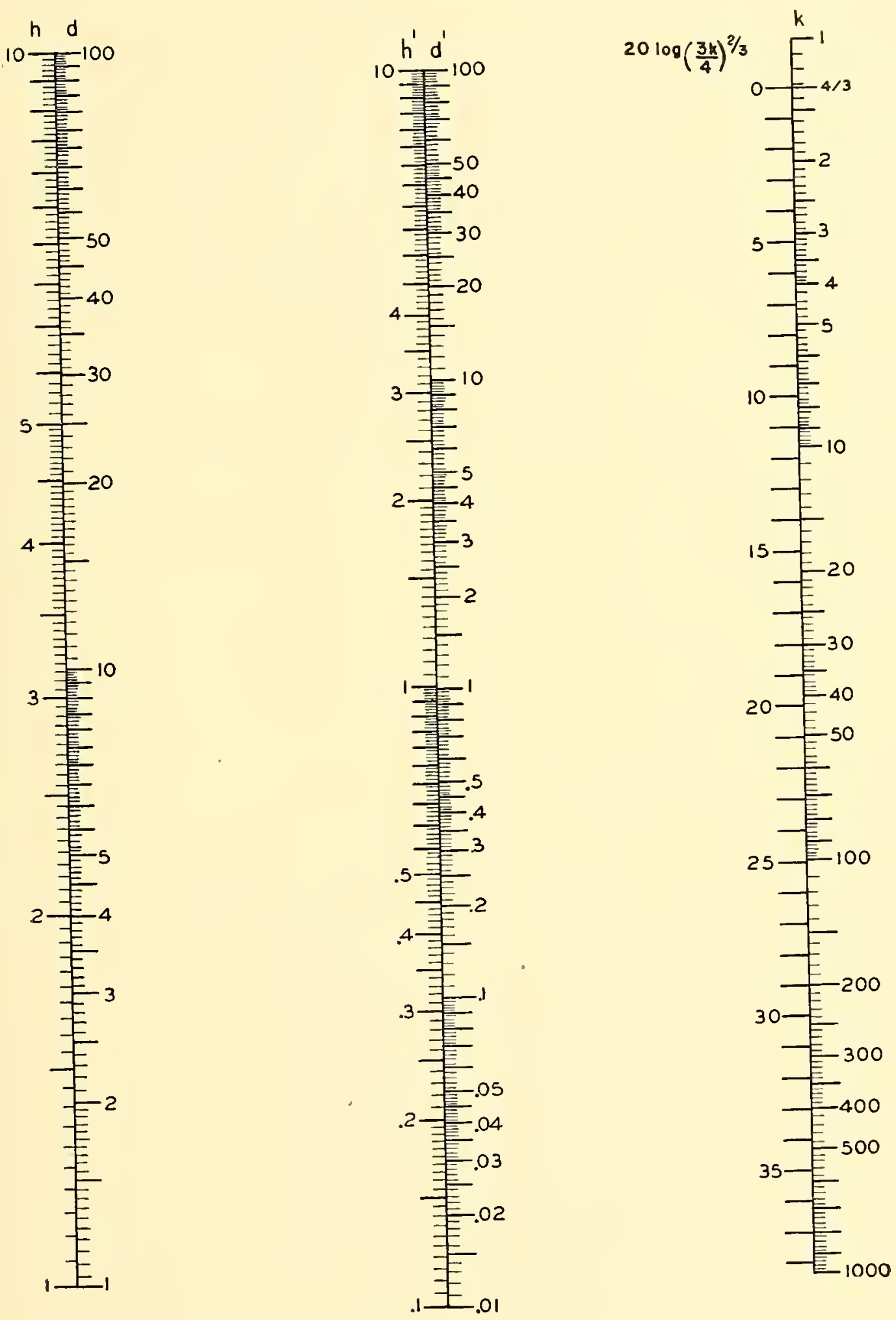

Figure 43. Relation of $h, d$ to $h^{\prime}, d^{\prime}$ as a function of $k$. 
are given by Figure 39. If both antennas are so low, say, $h_{1}$ and $h_{2}<4 / l$ (see Table 4 ) that it is desired to use $H_{L}$ for greater accuracy for vertical polarization (Figure 47), then for $20 \log \bar{A}$ we take $20 \log A$ $+20 \log l-20 \log H_{L}$ (see Section 5.7.3) and instead of $\mathrm{ch}_{2}$ in Figure 39, we use as ordinate $e H_{L 2} / l$. Then for given $s d$, Figure 39 would give the value of $e H_{L 2} / l$. If the frequency is given, $e / l$ is known (Figures 35 and 46 ), and we must find the value of tha which corresponds to a known value of $H_{L 2}$ (Figure 47) for the appropriate value of $Q=\epsilon_{r} / 60 \sigma \lambda$. From $l h_{3}, h_{2}$ is found by dividing by $l$.

If only one antenna height is less than $4 / l$, then define $20 \log \bar{A}$ as $20 \log A+20 \log l-20 \log H_{L}$ for that antenna and $h_{2}$ then refers to the other antenna.

C. Non-standard atmosphere. $k \neq 4 / 3$. The preceding graphs are all based on $k=4 / 3$. If $k \neq 4 / 3$, $h_{1}, h_{2}, d$, and $A$ should be replaced by $h_{1}{ }^{\prime}, h_{2}{ }^{\prime}, \lambda^{\prime}$, and $A^{\prime}$, where

$$
\begin{aligned}
& h^{\prime}=h\left(\frac{37}{4}\right)^{-1 / 3}, \\
& d^{\prime}=d\left(\frac{3 k}{4}\right)^{-2 / 3}, \\
& A^{\prime}=A\left(\frac{3 k}{4}\right)^{2 / 3},
\end{aligned}
$$

or

$$
20 \log A^{\prime}=20 \log A+20 \log \left(\frac{3 k}{4}\right)^{2 / 3} .
$$

The change of $h, d, A$ to the primed values can be made with the aid of Figure 43 , i.e., if $h, d$ are known, change to $h^{\prime}, d^{\prime}$, then Figure 37 will give $\bar{A}^{\prime}$, which in turn will give $A^{\prime}$, and this with the aid of Figure 43 will give $A$.

D. Change to dimensionless coordinates. In the optical region, convenient coordinates are (see Section 6.5)

$$
\begin{aligned}
& v=\frac{d}{\sqrt{27_{i} a h_{1}}}=\frac{d}{d_{T}} \\
& u=\frac{h_{2}}{h_{1}} .
\end{aligned}
$$

For these coordinates, equation (175) becomes

$$
\bar{A}=\frac{A}{h_{1} g(e)} \text {. }
$$

Writing

$$
\begin{aligned}
& \underline{e}=e h_{1}, \\
& s_{-}=s \sqrt{2 l_{i} a h_{1}},
\end{aligned}
$$

it follows that

$$
\begin{aligned}
e h_{2} & =\underline{e} u, \\
\underline{s} v & =s d,
\end{aligned}
$$

and, using equations (150) and (159),

$$
\underline{s}^{2}=2 \underline{e} .
$$

Consequently, Figure $3 \bar{\gamma}$ can be used with $\underline{s} v$ replacing $s d$, eu replacing $c h_{2}$, and $\bar{A}$ is defined in equation (179).

Caution: In using the graphs, eare must be exereised when one or both antennas are clevated to see that the receiver antenna is well within the diffraction region, i.e.,

$$
d>>d_{L} .
$$

E. Illustrative problems; diffraction formula; dielectric eurth. In Section 5.6, four types of problems were considered for the optical-interference region. The same four types are given here, for a receiver below the optical-interference region. A dielectric earth is assumed so that the figures in Scction 5.7.3 are applicable. These require supplementing by equations (3) and (5).

For one-way transmission the radio gain is

$$
10 \log \frac{P_{2}}{P_{1}}=20 \log A+10 \log \left(G_{1}\left(r_{2}\right) .\right.
$$

For two-way transmission the radar gain is

$$
\begin{aligned}
10 \log \frac{P_{2}}{P_{1}}=40 \log A & +10 \log \left(G_{1} G_{2}\right) \\
& +7.5+10 \log \sigma-20 \log \lambda .
\end{aligned}
$$

Type I. The heights and distance apart of the transmitter and receiver antennas and the wavelength are known. The radio gain is to be found.

An early-warning set has a horizontal antenna, located 118 meters above sea level. A receiver is located in an airplane 1,520 meters above sea level, at a distance of $300 \mathrm{~km}$. The wavelength is 3 meters. The gain of the radar antenna is $96 \mathrm{db}$ and its power output $100 \mathrm{kw}$. (a) The power received by the airplane receiver, assuming a gain of $10 \mathrm{db}$, is to be found. (b) The power returned to the radar by the airplane, assuming that the airplane has a radar cross section $\sigma$ of 40 square meters, is to be found.

One-uay: From Figure 2, $d_{L}=205 \mathrm{~km}$. Hence the receiver may be assumed well within the diffraction region.

From Figure 40, $s d=9.3$, with $f(\delta)=1$.

From Figure $41, c h_{2}=12.3$, with $g(\delta)=1$.

From Figure 37, $20 \log \bar{A}=-213$. 
To convert $20 \log \bar{A}$ to $20 \log A$ by equation (175), we need $20 \log h_{1}$ and $20 \log g_{1}$, which are given by Figure 41 :

$$
\begin{aligned}
& 20 \log h_{1}=41.3, \\
& 20 \log g_{1}=1.5 .
\end{aligned}
$$

Hence

$$
20 \log A=-170
$$

and by equation (182)

$$
10 \log \frac{P_{2}}{P_{1}}=-170+96+10=-6+.
$$

Since $P_{1}$ is $10^{5}$ watts,

$$
P_{2}=10^{5} \times 10^{-6.4}=10^{-1.4} w
$$

Radar: Substituting in equation (183), the radar gain is given by

$$
\begin{aligned}
10 \log \left(P_{2} / P_{1}\right) & =-310+2(96)+7.5+16-9.5, \\
& =-134 \mathrm{db}
\end{aligned}
$$

or

$$
P_{2}=P_{1} \times 10^{-13.4} \text {. }
$$

The porrer output $P_{1}$ is $10^{5}$ watts, so that the maximum receired powel

$$
P_{2}=10^{-8.4} \mathrm{r} \text {. }
$$

The minimum detectable power of the set is given as $1.6 \times 10^{-8}=10^{-7.9}$ watt, so that under the given conditions the power returned by the target would be slightly below the threshold of deteetion.

Type II. Gain versus receiver (or target) height is to be found for given distance, given wavelength. and given transmitter height: A radar has an antenna height of $h_{1}=30$ meters, a wavelength of $\lambda=1.5$ meters and a distance from a receiver (or target) of $d=100 \mathrm{~km}$. Assuming a receiver antenna gain $G_{2}=1$, the variation of $P_{2} / P_{1}$ at the reeeiver with receiver height is to be found. Also, assuming a target of cross section $\sigma=50$ sq meters, the variation of $P_{2} / P_{1}$ at the radar receiver with target height is to be found. The radar antenna has a gain of $13.5 \mathrm{db}$.

\section{One-way:}

$$
s d=3.9 \text { (from Figure } 40) .
$$

From Figure 37, for the fixed value of $s d=3.9$, we find a correspondence between values of $20 \log \bar{A}$ and $c h_{2}$, listed in Table 5 below. By means of Figure 41 or equation (159), eh is changed to $h_{2}$ and by means of equation (176), $\bar{A}$ to $A$. From Figure 41 , it is seen that $20 \log h_{1}=29.5$ and $20 \log g_{1}=0$. To change $A$ to $P_{2} / P_{1}$, the transmitter gain of $13.5 \mathrm{db}$ and the receiver gain of $0 \mathrm{db}$ must be taken into account, according to equation (182). The result is given in
Table 5. The values of $10 \log \left(P_{2} / P_{2}\right)$ are plotted in Figure 25, together with the results found with the same data in Section 5.6.3 for the optical-interference region.

TABLE $5^{*}$

\begin{tabular}{r|c|c|c|c|c}
\hline $\begin{array}{c}h_{2} \\
\text { Meters }\end{array}$ & $e h_{2}$ & $20 \log \bar{A}$ & $20 \log A$ & $\begin{array}{c}\text { Radio } \\
\text { Gain } \\
\text { in db }\end{array}$ & $\begin{array}{c}\text { Radar } \\
\text { Gain } \\
\text { in db }\end{array}$ \\
\cline { 1 - 2 } 63 & 0.8 & -190 & -160.5 & -147 & -273 \\
142 & 1.8 & -180 & -150.5 & -137 & -253 \\
259 & 3.3 & -170 & -140.5 & -127 & -233 \\
417 & 5.3 & -160 & -124.5 & -117 & -208 \\
\hline
\end{tabular}

* See also Table 1 and Figure 25.

Radar:

$$
\begin{aligned}
10 \log \frac{P_{2}}{P_{1}}= & 40 \log A+27+7.5+10 \log \sigma \\
& \quad-20 \log 1.5, \\
= & 40 \log A+27+7.5+17-3.5, \\
= & 40 \log A+48 .
\end{aligned}
$$

Type III. Gain rersus distance is to be found, with antenua heights and wavelength given: Using the same data given in Section 5.6.4, the gain as a function of distance in the diffraction region is to be found. The result has been plotted in Figure 26 . The polarization is horizontal.

$$
\begin{aligned}
& h_{1}=30 \text { meters } \quad G_{1}=22.4(13.5 \mathrm{db}) \\
& h_{2}=1000 \text { meters } \quad G_{2} \text { (one-way) }=1(0 \mathrm{db}) \\
& \lambda=1.5 \text { meters } \quad G_{2}(\text { radar })=22.4(13.5 \mathrm{db}) \\
& \sigma=10 \text { square meters }
\end{aligned}
$$

From Figure 41,

$$
e h_{2}=12.5 \text {. }
$$

Referring to Figure 37, we find a correspondence between $\bar{A}$ and $s d$. Talues of $\bar{A}$ are to be assumed. To change $s d$ to $d$, use Figure 40 . To change $\bar{A}$ to $A$, use equation (176). From Figure 41,

$$
\begin{aligned}
& 20 \log 30=29.5 \\
& 20 \log g_{1}=0, \\
& 20 \log A=20 \log A+29.5+0 .
\end{aligned}
$$

The radio gain is then given by:

One-uay:

$$
10 \log \frac{P_{2}}{P_{1}}=20 \log A+13.5+0 ;
$$

The radar gain is then given by:

Radar:

$$
\begin{aligned}
10 \log \frac{P_{2}}{P_{1}} & =40 \log A+7.5+27+10-3.5 \\
& =40 \log A+41 .
\end{aligned}
$$


These equations are evaluated in Table 6 and the one-way ralues are plotted in Figure 26.

\section{TABLE 6}

\begin{tabular}{c|c|c|c|c|c}
\hline \hline $20 \log \bar{A}$ & $s d$ & $d$ & $20 \log A$ & $\begin{array}{c}\text { Radio Gain } \\
\text { in db }\end{array}$ & $\begin{array}{c}\text { Radar Gain } \\
\text { in db }\end{array}$ \\
\hline-170 & 6.2 & 159 & -140 & -127 & -239 \\
-180 & 6.9 & 177 & -150 & -137 & -259 \\
-190 & 7.6 & 193 & -160 & -147 & -279 \\
-200 & 8.3 & 213 & -170 & -157 & -299 \\
-210 & 9.0 & 231 & -180 & -167 & -319 \\
-220 & 9.7 & 249 & -190 & -177 & -339 \\
-230 & 10.3 & 263 & -200 & -187 & -359 \\
-240 & 11.0 & 282 & -210 & -197 & -379 \\
-250 & 11.8 & 305 & -220 & -207 & -399 \\
-260 & 12.5 & 320 & -230 & -217 & -419 \\
-270 & 13.2 & 340 & -240 & -227 & -439 \\
-280 & 13.8 & 357 & -250 & -237 & -459 \\
& & & & & \\
\hline
\end{tabular}

Type IV. The determination of eontours along which the radio gain (or $A$ ) is constant (the coverage problem): A radar has a wavelength of 0.107 meter and a porrer output of $750 \mathrm{kw}$. Assume a receiver in space with a minimum detectable power of $10^{-10}$ watt. The maximum possible distance between the radar transmitter whose elevation is 100 meters and the receiver for varying heights of the receiver is to be found. The gain of the radar antenna is 10,000 (or 10 decibels), the gain of the receiver will be assumed to be 30 decibels.

For the radar problem, a target of radar cross section $\sigma=50$ square meters is assumed to take the place of the receiver. The minimum detectable power of the radar is taken as $10^{-10}$ watt; the range of the set for varying altitudes of the target will be calculated.

One-uay: The radio gain sought is the ratio of the minimum detectable power to the porrer output, or

$$
\frac{P_{2}}{P_{1}}=\frac{10^{-10}}{750 \times 10^{3}}=\frac{4}{3} \times 10^{1-16},
$$

or

$$
10 \log \left(P_{2} / P_{1}\right)=-160+1=-159 \mathrm{db} .
$$

From equation (3),

$$
\begin{aligned}
20 \log A & =-159-40-30, \\
& =-229 \mathrm{db} .
\end{aligned}
$$

From Figure 41,

$$
\begin{aligned}
& 20 \log g_{1}=18.5 \\
& 20 \log h_{1}=40 .
\end{aligned}
$$

Therefore

$$
\begin{aligned}
20 \log \bar{A} & =-229-40-18.5, \\
& =-287.5 \mathrm{db} .
\end{aligned}
$$

Referring to Figure 37 , the pairs of values of $s d$ and $e h_{2}$ along the contour $20 \log \bar{A}=-287$ are given in Table 7. By means of Figures 40 and $41, s d$ and $e h_{2}$ are changed to $d$ and $h_{g}$. The points found are to the right of the curve for $c h_{1}=7.3$, so that they correspond to points in the diffraction region.

\begin{tabular}{c|r|r|r}
\multicolumn{3}{c|}{ TABLE 7} \\
\hline \hline$d_{k m}$ & $s d$ & $e h_{3}$ & $h_{3 \text { meters }}$ \\
\cline { 1 - 2 } 118 & 11 & 1.3 & 18 \\
129 & 12 & 3.5 & 48 \\
140 & 13 & 7.0 & 96 \\
151 & 14 & 11.5 & 158 \\
161 & 15 & 16.5 & 226 \\
\hline
\end{tabular}

Radar: The value of $10 \log \left(P_{2} / P_{1}\right)$ is the same as for the one-way calculation, $-159 \mathrm{db}$. This must be changed to $20 \log A$ by equation (5),

$$
20 \log A=-141 \mathrm{db},
$$

and, as above,

$$
\begin{aligned}
20 \log \bar{A} & =-141-40-18.5 \\
& =-199.5 \mathrm{db} .
\end{aligned}
$$

Referring to Figure 37 , we see that the contonr $20 \log \bar{A}=-199.5$ is to the left of $e h_{1}=7.3$ [see caution in equation (181)]. Therefore it is not possible to get the necessary power return $P_{2}$ from the given target so long as it is below the line of sight. The desired contour would he above the line of sight. The determination of the eontour is cliscussed in Seetion 5.6.5.

5.7 .4

\section{Sea Water, VHF, Vertical Polarization}

1. Graphical Aids. Graphical aids are given in this section, which, as in Seetion 5.7.3, are valid for all practical distances when both antennas are low $\left[h<h_{c}\right.$ (see Figure 35)] and $2 h_{1} h_{2}<<\lambda d$. If one or both antennas are elevated, they will give the value of the radio gain for the first mode, which is a good approximation for the result found by summing all the modes when the receiver is well within the diffraction region, i.e., when $d>d_{L}$. [If one antenna is low $\left(h<h_{c}\right)$ and one wcll elevated $\left(h>40 h_{c}\right)$, the result found by using several modes is given in Section 5.7.5.] 
Referring to equation (162) and Section 5.7.1,

$$
A=2 A_{0} A_{1} F_{s}\left(H_{L} g g^{\prime}\right)_{1}\left(H_{L} g g^{\prime}\right)_{2},
$$

with $g g^{\prime}=1$ for $h<h_{c}$.

$h_{c}$ is given by Figure 35,

$20 \log g g^{\prime}$ is given by Figure 36,

$20 \log A_{0}$ is given by Figure 3 in Chapter 2,

$F_{s}$ is given by Figures 31,32 , and 33 .

2. Plane earth factor $A_{1}$. This has been discussed in Section 5.7.1. $A_{1}$ is a function of $p^{\prime} d ; p^{\prime}$ is given in Figure 44 and $20 \log A_{1}$ in Figure 45 . The curve shift of $A_{1}$ " ith $\lambda$ in the VHF band is less than $1 \mathrm{db}$. When $p^{\prime} d>50, A_{1}=1 / p^{\prime} d$, as in the dielectric case.

3. The low height-gain $H_{L}$ is a function of $l h$ (see Section 5.7.1). $l$ is given by Figure $46, H_{L}$ or $20 \log H_{L}$ by Figure $47 . H_{L}$ depends on the curve parameter $Q=\epsilon_{r} / 60 \sigma \lambda$ which for sea water is $\frac{1}{3 \lambda}$. Since $H_{L} \rightarrow l h$ for $l h>4,20 \log H_{L}$ can be found from Figure 34 .

4. $h_{1}, h_{2}>4 / l$ and $d>50 / p^{\prime}$. As in Section 5.7.1 [noting especially equations (151) and (153)], equation (184) reduces, when $h>4 / l$ and $d>50 / p^{\prime}$, to

$$
A=\frac{3}{2} \frac{F_{s}}{d^{2}}\left(g g^{\prime} h\right)_{1}\left(g g^{\prime} h\right)_{2}
$$

TABLE 8. Values of $4 / l$ and $50 / p^{\prime}$ for various wavelengths.

\begin{tabular}{c|c|c|c|c|c|c|c|c|c|c|c}
\hline \hline$\lambda$ & 1 & 2 & 3 & 4 & 5 & 6 & 7 & 8 & 9 & 10 & $\mathrm{~m}$ \\
\hline$\frac{4}{t}$ & 3 & 27 & 50 & 80 & 110 & 143 & 174 & 222 & 267 & 308 & $\mathrm{~m}$ \\
$\frac{50}{p^{\prime}}$ & 2 & 17 & 17 & 30 & 50 & 71 & 100 & 125 & 175 & 200 & $\mathrm{~km}$ \\
\hline
\end{tabular}

Equation (185) may be used generally, provided $\left(g g^{\prime} h\right)$ is replaced by $H_{L} / l$ when $h<4 / l$, and the right-hand member is multiplied by $A_{1} p^{\prime} d$ or, in decibels, $20 \log A_{1}+20 \log p^{\prime} d$ are added, when $d<50 / p^{\prime}$. In this formula $d<50 / p^{\prime}$ is given in meters.

5. Horizontal versus vertical polarization. It is of interest to compare the gain of vertically and horizontally polarized waves over sea water at VHF. (It has been pointed out earlier that there is no marked difference in attenuation between horizontally and vertically polarized waves for wavelengths less than one meter.) Equation (184) is valid for horizontally polarized waves also by using the appropriate $F_{s}$ curve and putting $g^{\prime}=1$ and can be made the basis of a comparison between vertical and horizontal polarization. See, for comparison, equation (160).

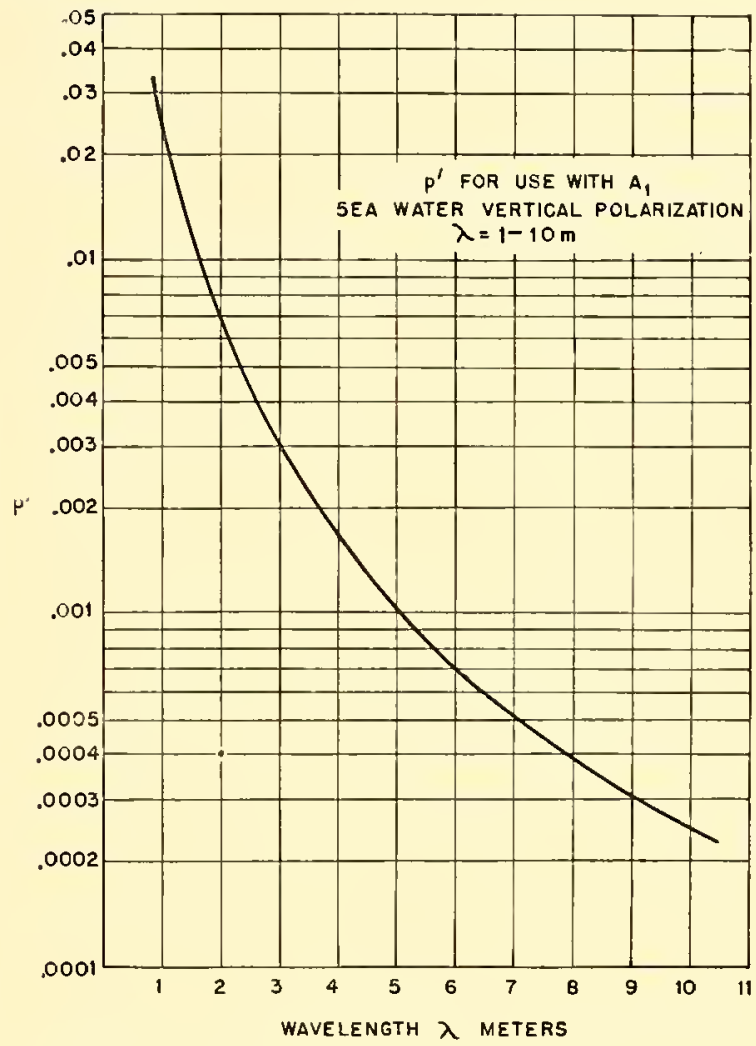

FigURE 44. $p^{\prime}$ versus $\lambda$ for sea water, vertical polarization.

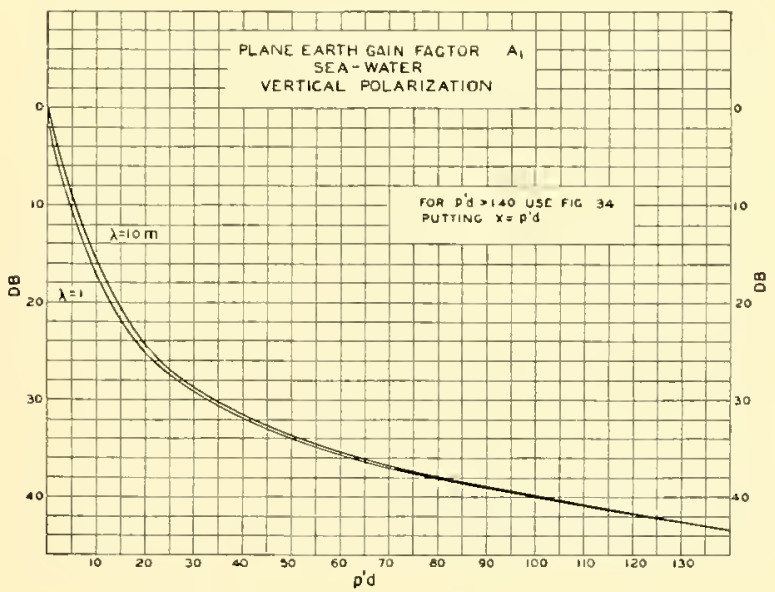

Figure 45. Plane earth factor $A_{1}$, for sea water, vertical polarization. Note: All db values are negative. 


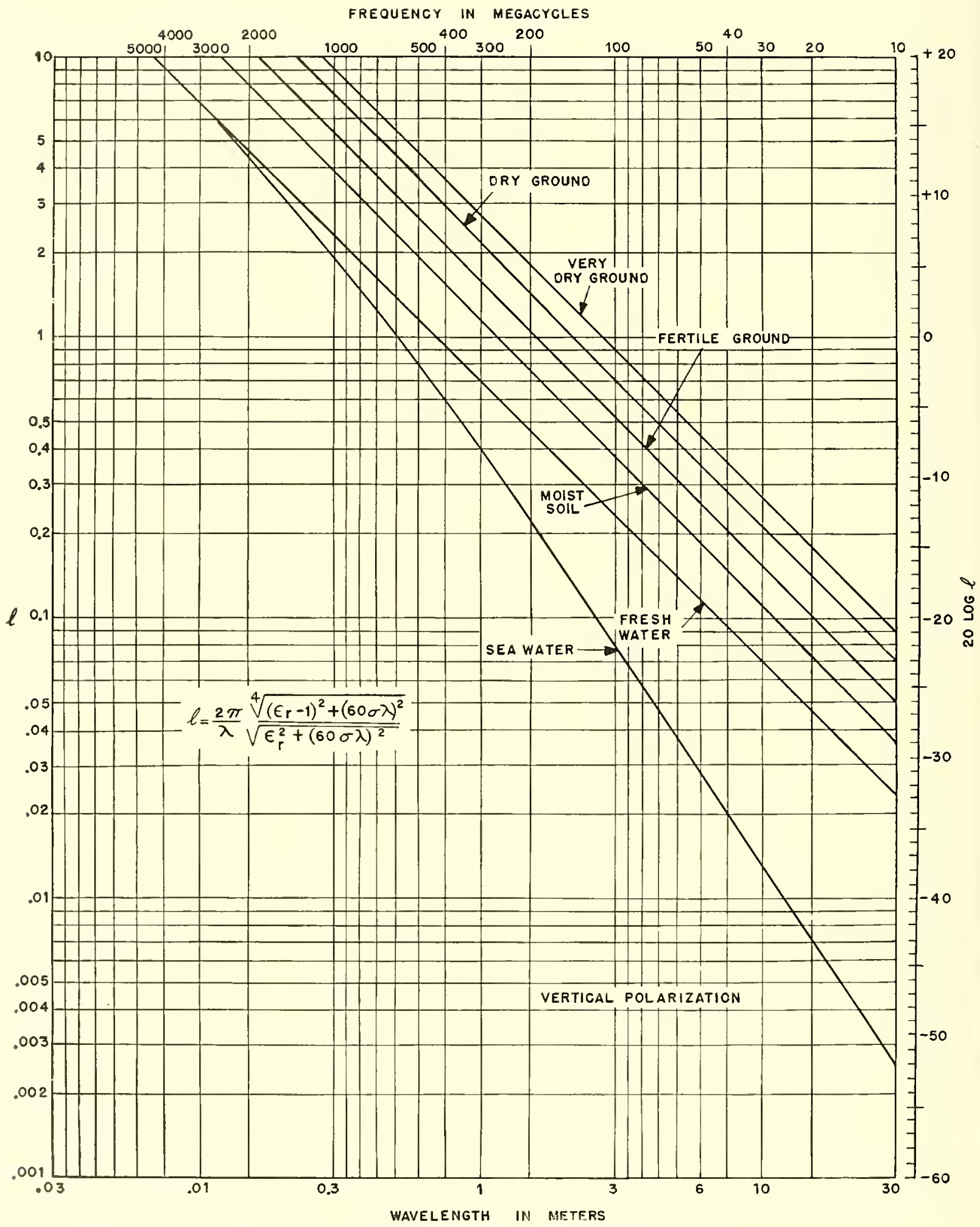

Figure 46. Parameter $l$ versus frequency for vertical polarization. 
Fol antennas at, or very close to, zero height, the gain-factor ratio depends on $A_{1} F_{s}$. While $F_{s}$ gives greater attenuation (lower gain) for horizontal than for vertical polarization, the difference between the two lics principally in the values of $A_{1}$. For $\lambda=1$ meter, the ratio is 64,000 to 1 in favor of vertical polarization. For $\lambda=10$ meters, the ratio is about $8.6 \times 10^{6}$.

However, as the antennas are raised above the ground, the strength of a horizontally polarized field increases much more rapidly than does the corresponding vertically polarized field, for a given which, as in Section 5.7.3, can be written

$$
\bar{A}=1.77 \times 10^{-7}\left[\frac{F_{s}}{(s d)^{2}}\right] A_{1} p^{\prime} d \psi
$$

or

$$
\begin{aligned}
20 \log \bar{A}=-135+ & 20 \log \frac{F_{s}}{(s d)^{2}}+20 \log A \\
& +20 \log p^{\prime} d+20 \log \psi
\end{aligned}
$$

Since $F_{s}$ has a graphical representation which depends on the ravelength, it is recessary to assume a particular value of $\lambda$; equation (18S) then becomes

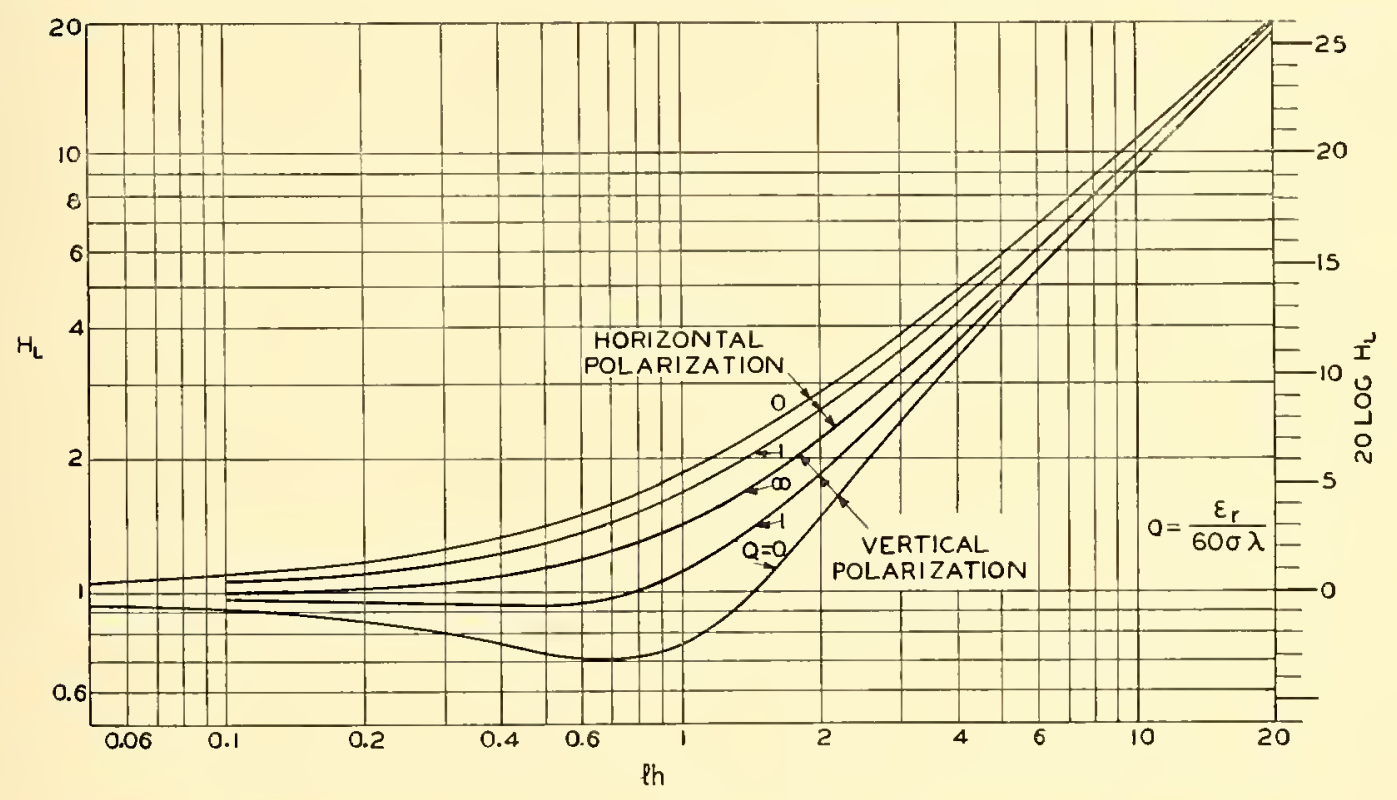

FIgURE 47. Height-gain function $H_{L}$ versus $l h$, for low antenna heights. [See equation (152.)]

wavelength up to a certain height above which the field is substantially independent of polarization. For example, above a height of 3 meters for $\lambda=1$ meter, and above 77 meters for $\lambda=10$ meters, the two fields are practically equal.

6. Parameter $\bar{A}$. As in Section 5.7 .3 , curves can be drawn in terms of the parameter $\bar{A}$ where

$$
\bar{A}= \begin{cases}\frac{A}{\left(h g g^{\prime}\right)_{1}} \quad \text { for } h_{1}>4 / l, \\ \frac{A l}{H_{L}} \quad \text { for } h_{1}<4 / l\end{cases}
$$

Equation (185), including the correction for $d<50 / p^{\prime}$, becomes

$$
A=\frac{3}{2} \frac{F_{s}}{d^{2}}\left(A_{1} p^{\prime} d\right)\left(g g^{\prime} h\right)_{1}\left(g g^{\prime} h\right)_{2}
$$

a relation between $\bar{\alpha}, d_{1}$, and $h_{2}$, and Figures $t 8,49$, and 50 for $\lambda=1,3,6$ meters are in terms of these coordinates. The height-gain function of the transmitter $g_{1}$ can be found from Figure 41 .

7. Illustrative example: Communication. A communication set used in ship-to-ship work has a wavelength of 1 meter, a receiver sensitivity of 10 microvolts with a resistance of 50 ohms across the input terminals and a transmitter power output of 100 watts. The transmitter and receiver antennas are vertical half-rrave dipoles at an elevation of 30 meters. The range is to be found.

To produce a voltage of 10 microvolts across 50 ohms, a power of

$$
P_{2}=\frac{V^{2}}{R}=\frac{100 \times 10^{-12}}{50} \text { watt }=2 \times 10^{-12} \text { watt, }
$$




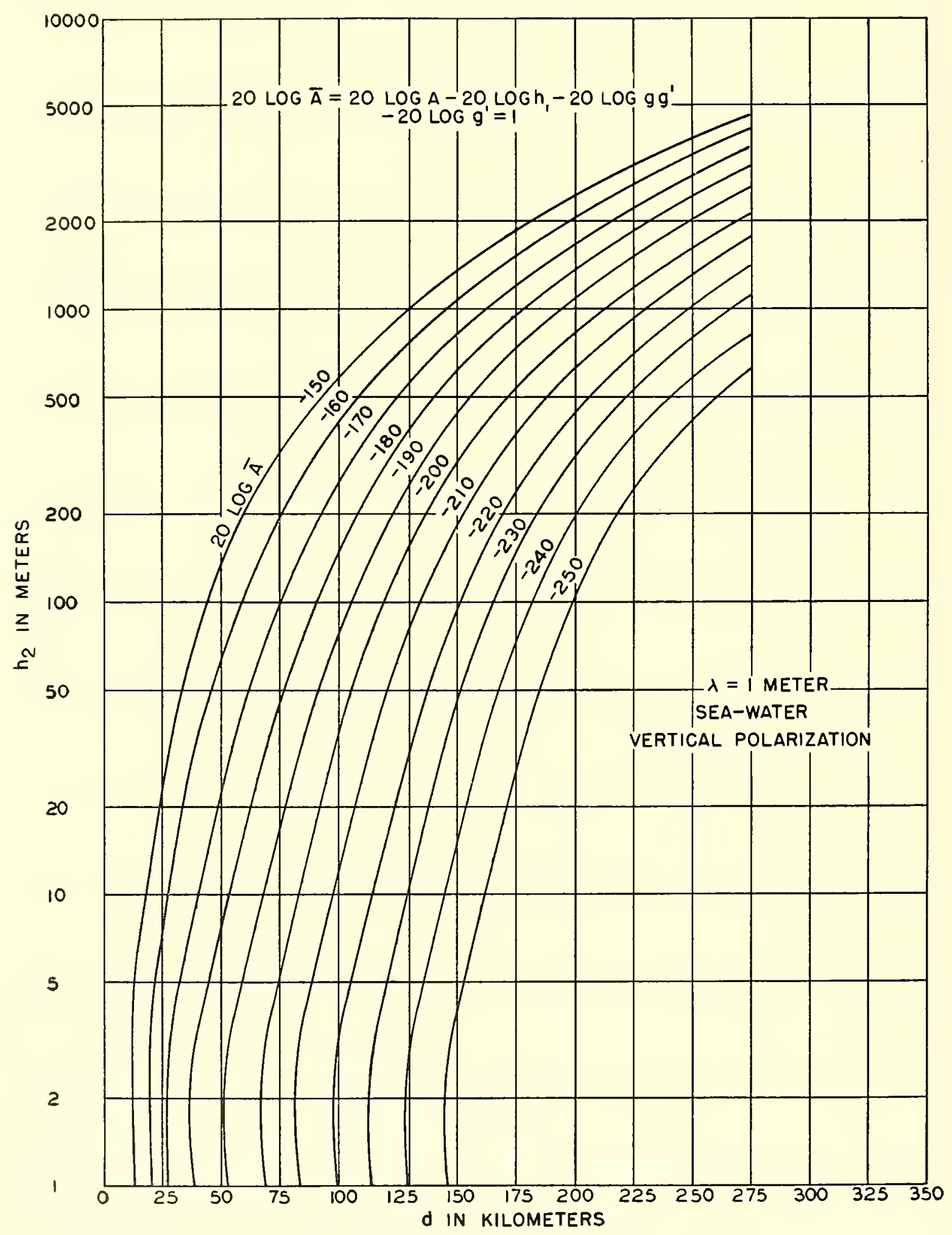

Figure 48. Maximum range for $\lambda=1$ meter, vertical polarization. [See equation (186).] 


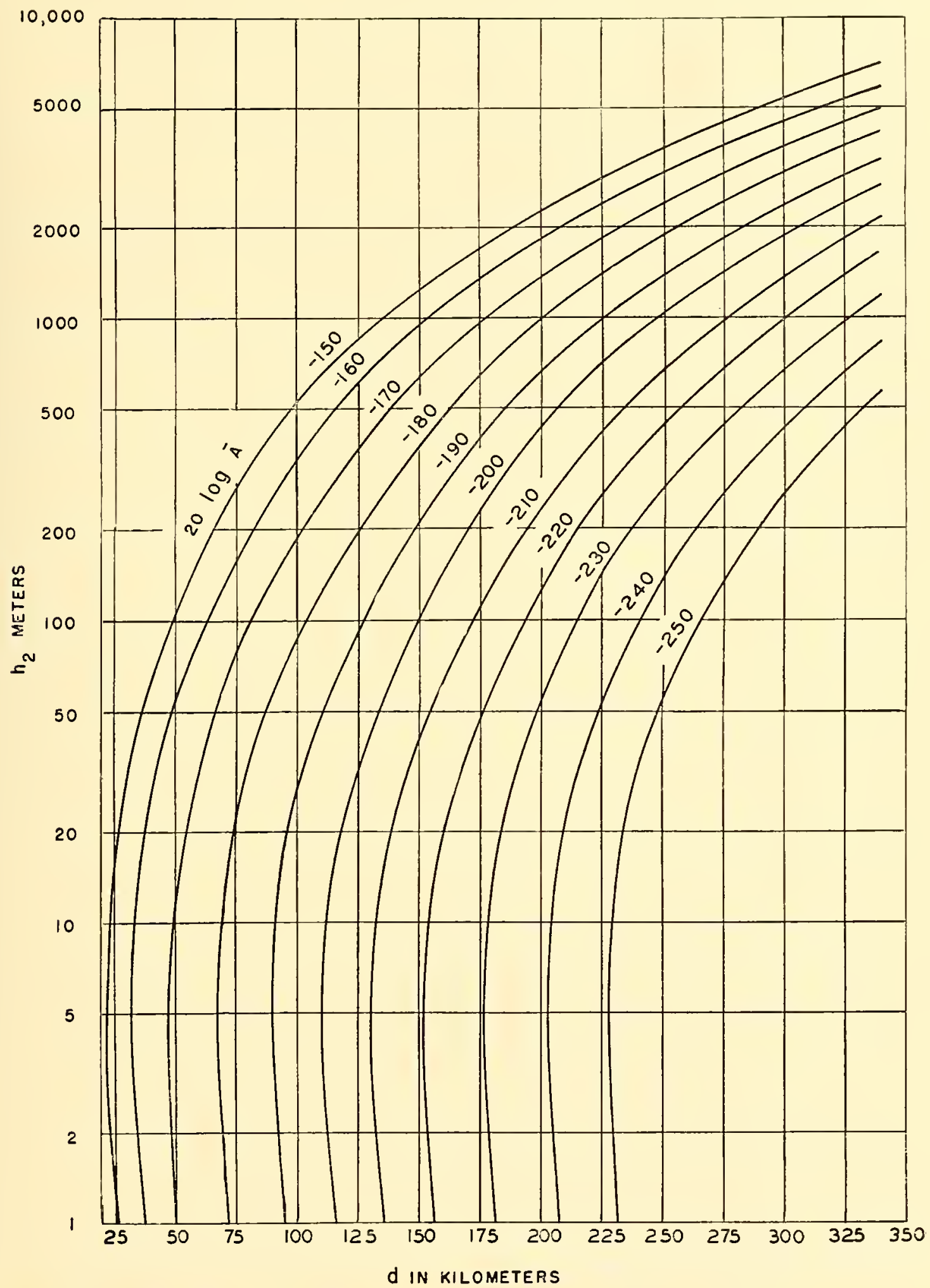

Figure 49. Naximum range for $\lambda=3$ meters (sea water vertical polarization $-20 \log g^{\prime}=1$ ). See equation (186). 


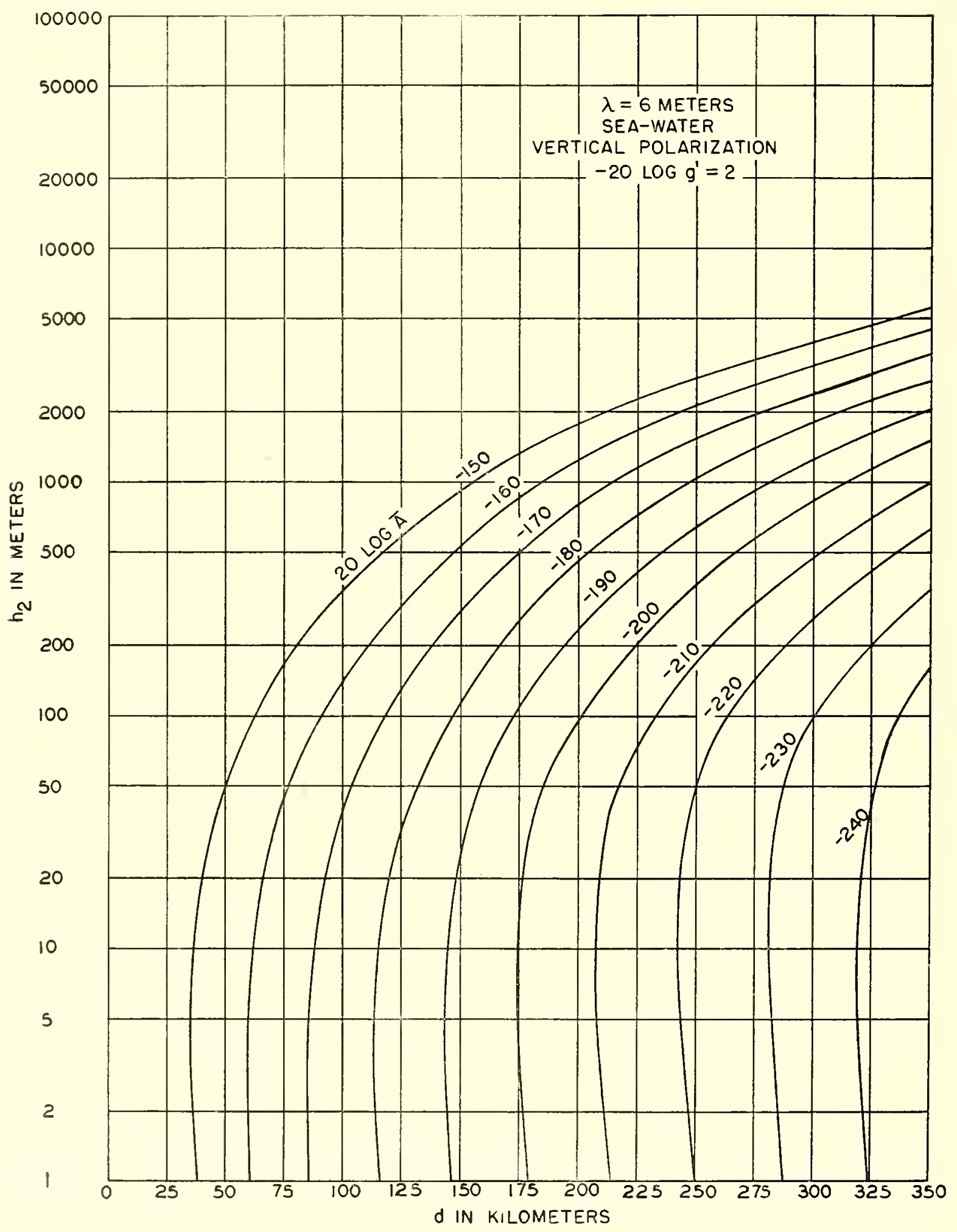

Fictere 50. Maximum range for $\lambda=6$ meters, vertical polarization. See equation (186). 
so that this is the minimum detectable power. The value of $P_{2} / P_{1}$ for the given power output of 100 watts is

$$
\frac{2 \times 10^{-12}}{100}=2 \times 10^{-14}
$$

and

$$
10 \log \frac{P_{2}}{P_{1}}=3+(-140)=-137 .
$$

This is to be changed to $20 \log A$ by equation (3). The gain of a half-wave dipole over a doublet is 1.09 (see Sections 2.2.2 and 3.2.3), so that $G_{1}=G_{2}=1.09$ ol $0.4 \mathrm{db}$,

$$
20 \log A=-137-0.8 \cong-138 \mathrm{db} .
$$

In changing from $20 \log A$ to $20 \log \bar{A}$ it must be determined which of the relations in equation (186) is required by comparing the transmitter height of 30 meters with $4 / l$. The value of $l$ as given by Figure 46 is 0.4 . Hence the vahue of $4 / l$ is 10 , which is less than 30 . Then

$$
\begin{aligned}
20 \log \bar{A} & =20 \log A-20 \log 30-20 \log g g^{\prime}, \\
& =-138-30-0, \\
& =-168 .
\end{aligned}
$$

Refering to the chart for $\lambda=1$, Figure 48 , we ind that for $h_{z}=30$ meters and $20 \log \bar{A}=-168$, the distance $d$ is 53 kilometers. This then is the maximum theoretical range between the two sets.

\subsubsection{Radio Gain Near the Line of Sight}

For $d$ much greater than $d_{L}$, the first mode is sufficient, as given in Sections 5.7.3 and 5.7.4. For $d$ nearly equal to $d_{L}$, i.e., the receiver near the line of sight, a formula [equation (190)] can be given which takes into account several modes and still permits the use of graphical aids. This formula is valid only when the elevated antenna is very ligh, i.e., $h>1,200 \lambda^{2 / 3}$ and the other antenna is low, i.e., $h<30 \lambda^{2 / 3}$. (Otherwise the transition curve near the line of sight must be sketched in graphically, as indicated by the broken portion in Figure 7 .)

Denoting by $H_{L 1}$ the height-gain of the low antenna at height $h_{1}$,

$$
A=2 A_{0} H_{L 1} M(\delta)\left[\frac{(s d)^{2}}{2 c h_{2}}\right]^{1 / 4} F_{2}(\Delta)
$$

where $h_{2}$ and $F_{2}(\Delta)$ refer to the elevated antenna.
1. $s d$ and eh are given in Figures 40 and $41 . \delta$ is given in Section 5.7.6.

2. $\Delta=\sqrt{g(\delta)}\left(s d-\sqrt{2 c h_{2}}\right)$.

3. $g(\delta)=1$, except for the VHF range, rertical polarization, orer sea water. The values are given in Table 9.

TABLE 9. Values of $\sqrt{g(\delta)}$ for VHF (sea water).

\begin{tabular}{c|cccccccccc}
$\lambda$ & 1 & 2 & 3 & 4 & 5 & 6 & 7 & 8 & 9 & 10 meters
\end{tabular}

$\checkmark g(\delta) \quad 0.980 .970 .950 .940 .930 .910900 .850 .860 .84$
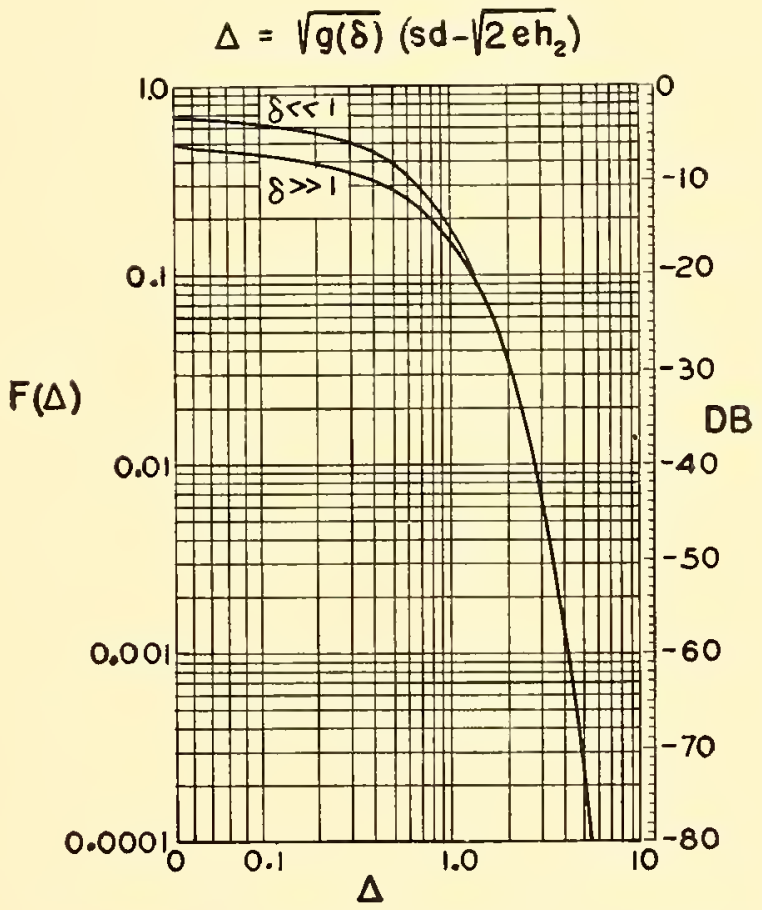

Figure 51. $F(\Delta)$ [representing $F_{2}(\Delta)$ ] versus $\Delta$ for use in equation (190).

4. $F_{2}(\Delta)$ is given by Figure 51 .

5. $M(\delta)$ for vertical polarization is given by Figure 52 as a function of $\delta$ which can be found from Figures 53 and 54 .

For horizontal polarization

$$
\begin{aligned}
M(\delta) & =\frac{1}{\sqrt{|\delta|}} \\
& =\left(\frac{\lambda}{2 \pi k a}\right)^{1 / 3} \frac{1}{\sqrt{\epsilon_{c}-1}} \\
& =\left(\frac{\lambda}{2 \pi k a}\right)^{1 / 3} \frac{1}{\sqrt[4]{\left(\epsilon_{\tau}-1\right)^{2}+(60 \sigma \lambda)^{2}}} .
\end{aligned}
$$




\subsubsection{General Solution for Vertical (or Horizontal) Dipole Over a Smooth Sphere}

1. Field strength of dipole. The vertical component of the electrical field of a rertical dipole radiating in a homogeneous atmosphere over a sphere of radius lia (or horizontal component in the cise of a horizontal dipole) is given by equation (192). The solution is ralid provided the distance between receiver and transmitter and the radius of the
For horizontal polarization,

$$
\begin{aligned}
\delta & =\left(\frac{2 \pi h a}{\lambda}\right)^{2 / 3}\left(\epsilon_{c}-1\right) \\
& =\frac{14.2 \times 10^{1}}{\lambda^{2 / 3}}\left(\epsilon_{c}-1\right) \text { for } k=\frac{4}{3} .
\end{aligned}
$$

d. $\zeta=s d$,

where

$$
s=\left(\frac{2 \pi}{\lambda k^{2} a^{2}}\right)^{1 / 3}
$$

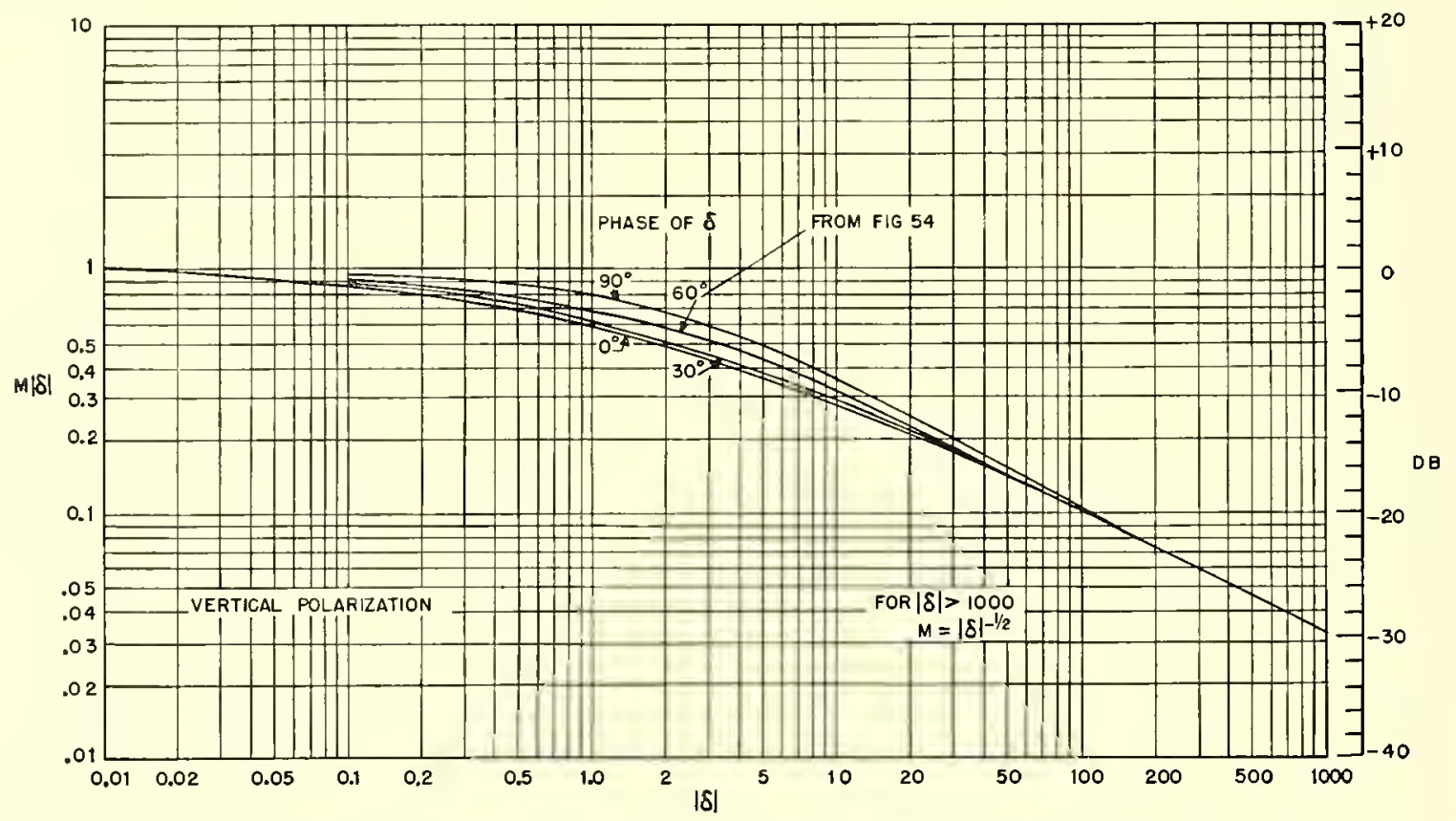

Figure 52. $M(\delta)$ versus $|\delta|$ for use in equation (190), vertical polarization.

sphere are much greater than a wavelength, conditions which are fulfilled in any practica! applieation of short waves.

$E=2 E_{0}(2 \pi \zeta)^{1 / 2}\left|\sum_{n=1}^{\infty} \frac{e^{-j \tau_{n} \zeta}}{\delta+2 \tau_{n}} f_{n}\left(h_{1}\right) \cdot f_{n}\left(h_{2}\right)\right|$.

a. $h_{1}$ and $h_{2}$ are antenna heights,

b. $E_{0}$ is the vahe of $E$ for a doublet in free space,

c. $\delta$ is the ground parameter which depends on the complex dieleetric constant $\epsilon_{c}=\epsilon_{r}-j 60 \sigma \lambda$.

For vertical polarization,

$$
\begin{aligned}
\delta & =\left(\frac{2 \pi k a}{\lambda}\right)^{2 / 3} \frac{\epsilon_{c}-1}{\epsilon_{c}{ }^{2}} \\
& =\frac{14.2 \times 10^{1}}{\lambda^{2 / 3}} \frac{\epsilon_{c}-1}{\epsilon_{c}{ }^{2}} \text { for } k=\frac{4}{3} .
\end{aligned}
$$

or

$$
s=4.43 \times 10^{-5} \lambda^{-13}\left(\frac{4}{3 k}\right)^{2 / 3} .
$$

e. $\tau_{n}$ are complex numbers which characterize the individual terms (modes) in equation (192). They are a function of $\delta$.

f. $f_{n}\left(h_{1}\right)$ and $f_{n}\left(h_{2}\right)$ are height-gain functions for the $n$th mode.

2. The complex ground parameter $\delta$. $\delta$ depends on the wavelength and the electrical constants of the ground. (The dielectrie constant is referred to air as unity.) $\delta$ is large for horizontally polarized waves, but for vertically polarized waves it may vary eonsiderably, as may be seen from Figure 53. In Figure 54 , the phase of $\delta$ is given. For wavelengths less 


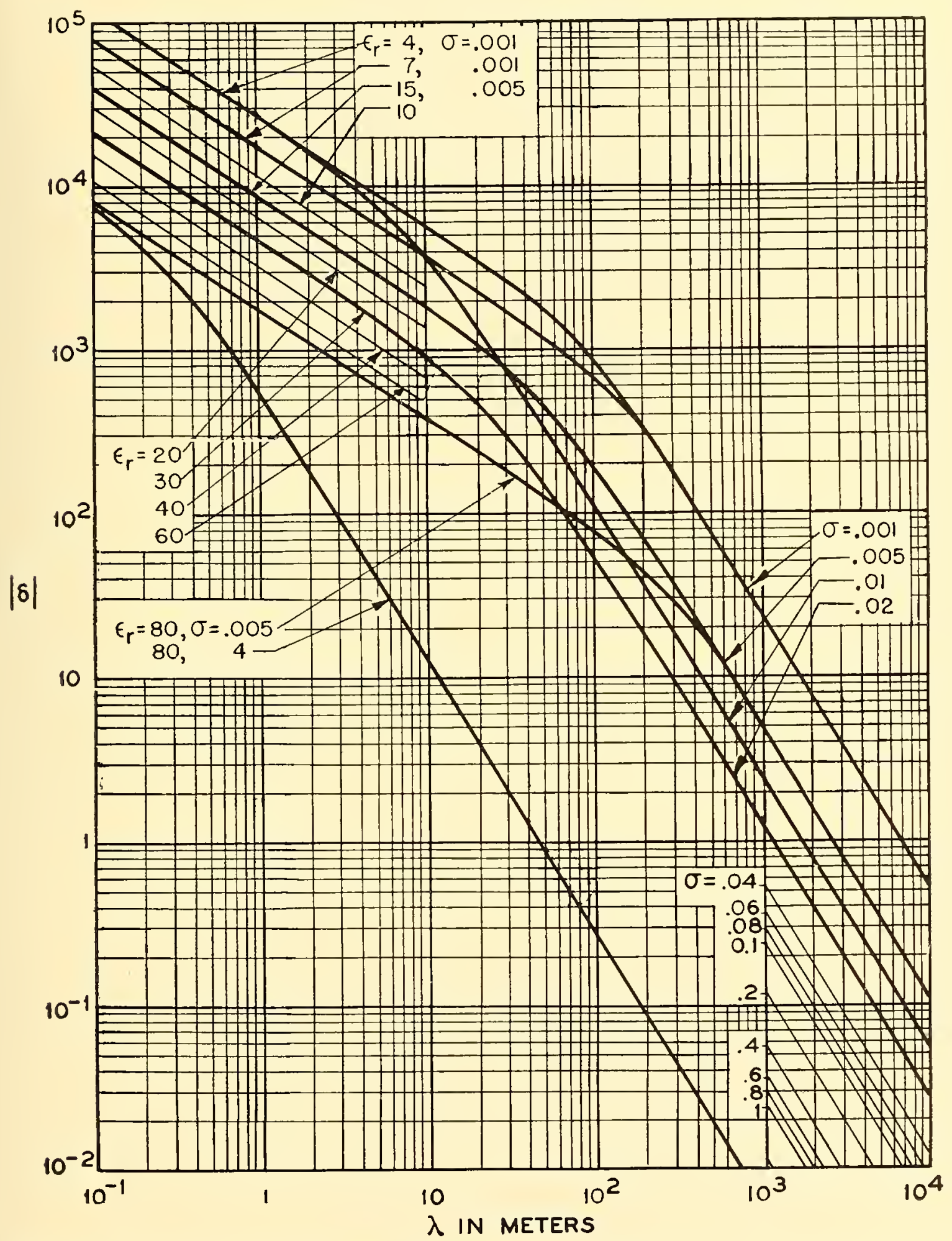

Figure 53. $|\delta|$ versus $\lambda$ for vertical polarization (see Table 10). Dielectric earth, $\delta \rightarrow \infty$; perfect conductor, $\delta \rightarrow 0$. Phase of $\delta$ is $45^{\circ}$ for intersection of two asymptotes for any particular ground. 
than 10 meters $|\delta|$, as giren by Figure 53 for rertical polarization, is large except in the VHF range orer sea water, $\epsilon_{r}=80, \sigma=4$.

The ground constants $\epsilon_{r}$ and $\sigma$ for water and various types of earth are given in Table 10. For $\lambda<\lambda_{c}$ the ground material is a diclectric earth.

TaBle 10. Ground constants.

\begin{tabular}{lll|c}
\hline & Type of ground & $\lambda_{c}=\frac{\epsilon_{r}}{60 \sigma}$ \\
\hline Sea water & $\epsilon_{r}=80$ & $\sigma=4$ mhos per meter & $0.33 \mathrm{~m}$ \\
Fresh water & $\epsilon_{r}=80$ & $\sigma=5 \times 10^{-3}$ & 267 \\
Toist soil & $\epsilon_{r}=30$ & $\sigma=0.02$ & 25 \\
Fertile ground & $\epsilon_{r}=15$ & $\sigma=5 \times 10^{-3}$ & 50 \\
Rocky ground & $\epsilon_{r}=7$ & $\sigma=10^{-3}$ & 117 \\
Drysoil & $\epsilon_{r}=4$ & $\sigma=10^{-2}$ & 6.7 \\
Tery dry soil & $\epsilon_{r}=4$ & $\sigma=10^{-3}$ & 66.7
\end{tabular}

3. The mode numbers $\tau_{n}$. These numbers are given below for the two limiting values for $\delta=0$ (infinite conductivity or $\lambda \rightarrow \infty$ ), and for $\delta=\infty$, i.c., the dielectric rarth.

\begin{tabular}{|c|c|c|}
\hline $\begin{array}{l}\text { Mode } \\
\text { No. }\end{array}$ & $\delta=0$ & $\delta=x$ \\
\hline 1 & $\tau_{1,0}=0.885 e^{-\jmath \pi / 3}$ & $\tau_{1, \infty}=1.856 e^{-j \pi / 3}$ \\
\hline 2 & $\tau_{2,0}=2.577 e^{-j \pi / 3}$ & $\tau_{2 \infty}=3.245 e^{-j \pi / 3}$ \\
\hline 3 & $\tau_{3,0}=3.824 e^{-3 \pi / 3}$ & $\tau_{3, \infty}=4.382 e^{-\jmath \pi / 3}$ \\
\hline$\geqq 4$ & $\begin{array}{l}\text { If } n \geqq 4 \\
\tau_{n, 01}=\frac{1}{2}\left[3 \pi\left(n+\frac{3}{4}\right)\right]^{2} 3^{-j \pi / 3}\end{array}$ & $\begin{array}{l}\text { If } n \geqq 4, \\
\tau_{n, \infty}=\frac{1}{2}\left[3 \pi\left(n+\frac{1}{4}\right)\right]^{2 / 3} e^{-j \pi / 3}\end{array}$ \\
\hline
\end{tabular}

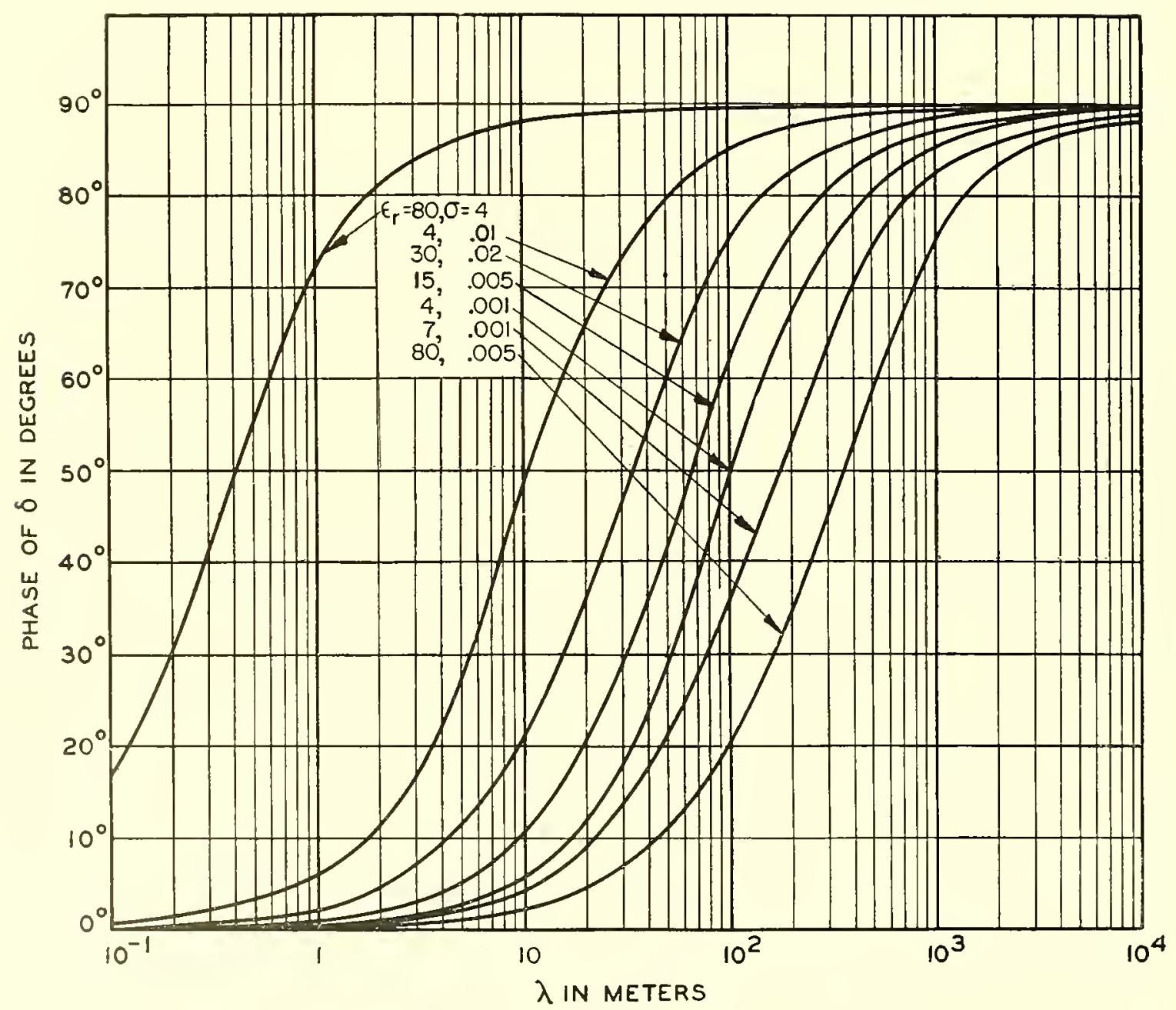

Figure 54. Phase of $\delta$ for vertical polarization. See Table 10. 
From these limiting values of $\tau$ the value of $\tau$ in general for any given $\delta$ can be found from the following two series, of which only the first is of interest in short-wave work.

$\delta$ large,

$\tau_{n}=\tau_{n, \infty}-\delta^{-1 / 2}-\frac{2}{3} \tau_{n, \infty} \delta^{-3 / 2}+\frac{1}{2} \delta^{-2}-\frac{4}{5} \tau_{n, \infty}^{2} \delta^{-3 / 2}$

$+\cdots$

$\delta$ simall,

$\tau_{n}=\tau_{n, 0}-\frac{\delta^{1 / 2}}{2 \tau_{n, 0}}=\frac{\delta}{8 \tau_{h, 0}^{3}}$

$-\frac{1}{12 \tau_{n, 0}^{2}}\left(1+\frac{3}{4 \tau_{n, 0}^{3}}\right) \delta^{3 / 2}+\cdots \cdot$

4. The height-gain functions $f(h)$.

(a) For low antennas $\left(h<30 \lambda^{2 / 3}\right)$, the height-gain functions, to the first approximation, are independent of $n$.

$f(h)=1+j\left[\frac{2 \pi h}{\lambda} \frac{\sqrt{\epsilon_{c}-1}}{\epsilon_{c}}\right]$ for vertical polarization,

(196)

$f(h)=1+j\left[\frac{2 \pi h}{\lambda} \sqrt{\epsilon_{c}-1}\right]$ for horizontal polarization

Note that the magnitudes of the bracketed quantities are equal to $l h$. The magnitude of $f$ has been denoted by $H_{L}$ and is represented in Figure 47 as a function of $t h$. The phase of the bracketed quantities in equation (196) is taken into account by using different curves with the parameter $Q=\epsilon_{r} / 60 \sigma \lambda$. For large values of $l h, H_{L} \rightarrow l h$.

(b) For elevated antennas $\left(h>>30 \lambda^{2 / 3}\right)$. The function $f_{n}$ can be represented by

$f_{n}=\frac{3}{\sqrt{2 \pi}} \frac{1}{(2 e h)^{1 / 4}} \frac{\exp \left\{+j \frac{\pi}{4}-j_{3}^{1}\left[(e h)-2 \tau_{n}\right]^{3 / 2}\right\}}{J_{1 / 3}(x)+J_{-1 / 3}(x)}$,

where, from equation (159),

$$
e=\frac{\left(4 \pi^{2}\right)^{1 / 3}}{\left(\lambda^{2} k a\right)^{1 / 3}}=\frac{\lambda^{-2 / 3}}{60}\left(\frac{4}{3 k}\right)^{1 / 3}
$$

and where the argument of the two Bessel functions is

$$
x=\frac{1}{3}\left(-2 \tau_{n}\right)^{3 / 2} e^{-j \pi}
$$

For the $n$th mode, if $(c h)>>2 \tau_{n}$, the magnitude of $f_{n}$ can be written

$$
\left|\frac{3}{\sqrt{2 \pi}} \frac{1}{(2 e h)^{1 / 4}} \frac{\exp \left(j \tau_{n} \sqrt{2 e h}\right)}{J_{1 / 3}(x)+J_{-1 / 3}(x)}\right|
$$

For large $\delta$, using the first two terms of equation (195), and writing $x_{\infty}$ for $x$ when $\tau_{n}$ is replaced by $\tau_{n, \infty}$,

$$
x=x-\left(\frac{-2 \tau_{\infty}}{\delta}\right)^{1 / 2} .
$$

Substituting this in $J_{1 / 3}(x)+J_{-1 / 3}(x)$, writing down the first two terms of the Taylor expansion, making use of the fact that the $\tau_{n_{, \infty}}$ are roots of $J_{1 / 3}(x)+$ $J_{-1 / 3}(x)=0$ and of the relation given by a property of the Bessel function,

$$
\begin{aligned}
J_{1 / 3}{ }^{\prime}(x)+J_{-1 / 3}{ }^{\prime}(x)=-1 /(3 x) & {\left[J_{1 / 3}(x)+J_{-1 / 3}(x)\right] } \\
& +J_{-2 / 3}(x)-J_{2 / 3}(x),
\end{aligned}
$$

we have

$$
\begin{aligned}
J_{1 / 3}(x)+J_{-1 / 3}(x) & \\
& \cong-\left(-\frac{2 \tau_{\infty}}{\delta}\right)^{1 / 2}\left[J_{-2 / 3}\left(x_{\infty}\right)-J_{2 / 3}\left(x_{\infty}\right)\right] .
\end{aligned}
$$

If these results are substituted into equation (192) for both antennas, the factor $\left(\delta+2 \tau_{n}\right)$ becomes $1+2 \tau_{n_{\infty}} / \delta$, which approaches unity for large $\delta$. This means that if both antennas are sufficiently slevated, short-wave propagation is practically independent of ground constants.

The value of $f$ given by cquation (199) can be written as glh so that $g$ represents the gain over $l h$. the value approached by $H_{L}$, when $l h>4$. The ralue of $g$ for $\delta \rightarrow \infty$ is represented in Figure 36 .

If $\delta$ is not very great, as in the case of vertically polarized VHF orer sea water, the effect of $\delta$ can be taken into acocunt by changing $e$ to $e g(\delta)$ and $g$ to $g g^{\prime}$. The functions $g(\delta)$ and $g^{\prime}$ are given by Figure 55 .

5. Plane earth gain factor and shadow factor. The field near the ground over a plane earth with infinite conductivity is equal to $2 E_{0}$, twice the free-space field. For an imperfectly conducting ground, the field for antemnas at zero height over a plane earth may be written

$$
E=2 E_{0} \AA_{1}
$$

$A_{1}$ is represented in Figure 56 as a function of $p^{\prime} d$, where

$p^{\prime}=\frac{2 \pi}{\lambda} \frac{|| \epsilon_{c}-1 \mid}{\left|\epsilon_{c}\right|^{2}}=\frac{2 \pi}{\lambda} \frac{\sqrt{\left(\epsilon_{r}-1\right)^{2}+(60 \sigma \lambda)^{2}}}{\epsilon_{r}^{2}+(60 \sigma \lambda)^{2}}$

for vertical polarization. For horizontal polarization.

$p^{\prime}=\frac{2 \pi}{\lambda}\left|\epsilon_{c}-1\right|=\frac{2 \pi}{\lambda} \sqrt{\left(\epsilon_{r}-1\right)^{2}+(60 \sigma \lambda)^{2}}$.

The enre parameter is $Q=\epsilon_{r} / 60 \sigma \lambda$. 
Comparing equations (202) and (203) with equations (193) and (191), we find that

$$
p^{\prime} d=|\delta| \zeta .
$$

Hence, equation (192) may be witten as

$$
E=2 E_{0}(2 \pi)^{1 / 2} \frac{\xi^{3 / 2}}{p^{\prime} d}\left|\sum \frac{e^{-j \tau} n^{\zeta}}{1+2 \tau_{n} / \delta} f_{n}\left(h_{1}\right) f_{n}\left(h_{2}\right)\right|
$$

height [i.e., $f(0)=1$ ] and equation (205) is now of the form

$$
E=2 E_{0} A_{1} F_{s}
$$

If $\delta$ is large (c.g., $\lambda$ small), $2 \tau_{n} / \delta$ in equation (207) may be neglected and $\tau_{n}$ replaced by $\tau_{n, \infty}$ so that the shadow factor is practically independent of ground constants. The shadow factor is represented graphi-

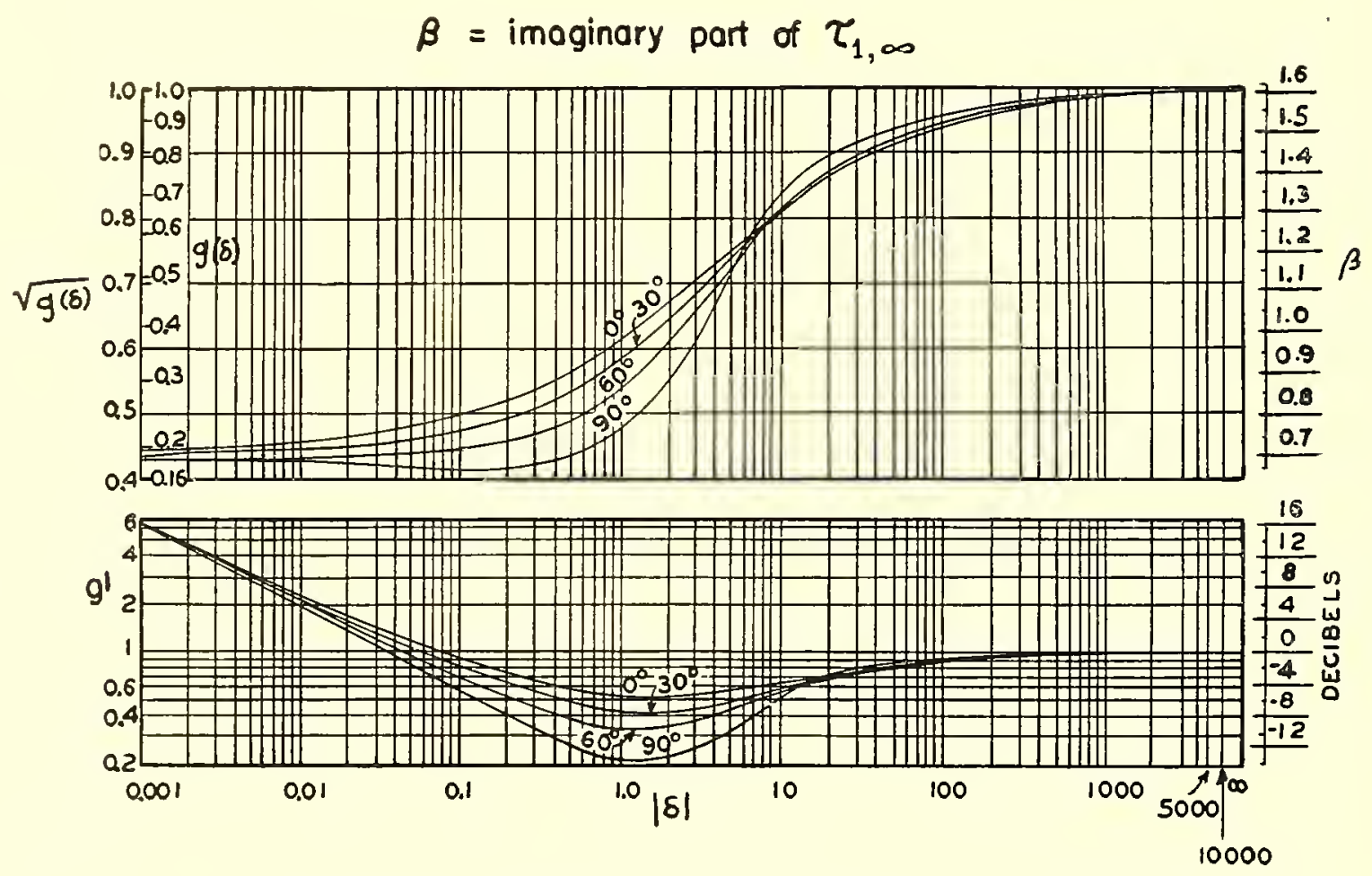

Figure 55. $\sqrt{g(\delta)}$ and $g^{\prime}$ as functions of magnitude and phase.

If $p^{\prime} d$ is large, we see from Figure 56 that

$$
A_{1} \cong \frac{1}{p^{\prime} d}
$$

so that the physical significance of $1 / p^{\prime} d$ in equation (205) becomes apparent.

The factor

$$
F_{8}=(2 \pi)^{1 / 2} \xi^{3 / 2}\left|\sum \frac{e^{-j \tau} n^{\zeta}}{1+2 \tau_{n} / \delta}\right|
$$

represents the effcet of earth curvature in increasing the attenuation over that of a plane earth at zero cally in Figures 32 and 58 . Where the factor $2 \tau_{n} / \delta$ cannot be neglected, as in the VHF range, vertical polarization over sea water, the dependence of $F_{s}$ on $\delta$ is accommodated by changing the abscissa from $\xi=s d$ to $\eta=s^{\prime} d$ where $s^{\prime}=s f(\delta)$, and by representing $F_{s}$ by a family of curres in Figure 58, whose parameter is given by dotted lines in Figure 57. $f(\delta)$ is represented also in Figure 57. For $\eta<0.4$, $F_{s}$ is less than 1 db below unity. This corresponds to a distance over which the earth may be considered plane, i.e., $d<10^{t} \lambda^{1 / 3}$, as given in Section 5.7.1. The greater the warelength, the smaller the effect of the earth's curvature. 
내,

$\left({ }^{\circ} \exists Z / 3\right) \quad$ YOLOVJ NIVO HIYVJ $3 N \forall 7 d$

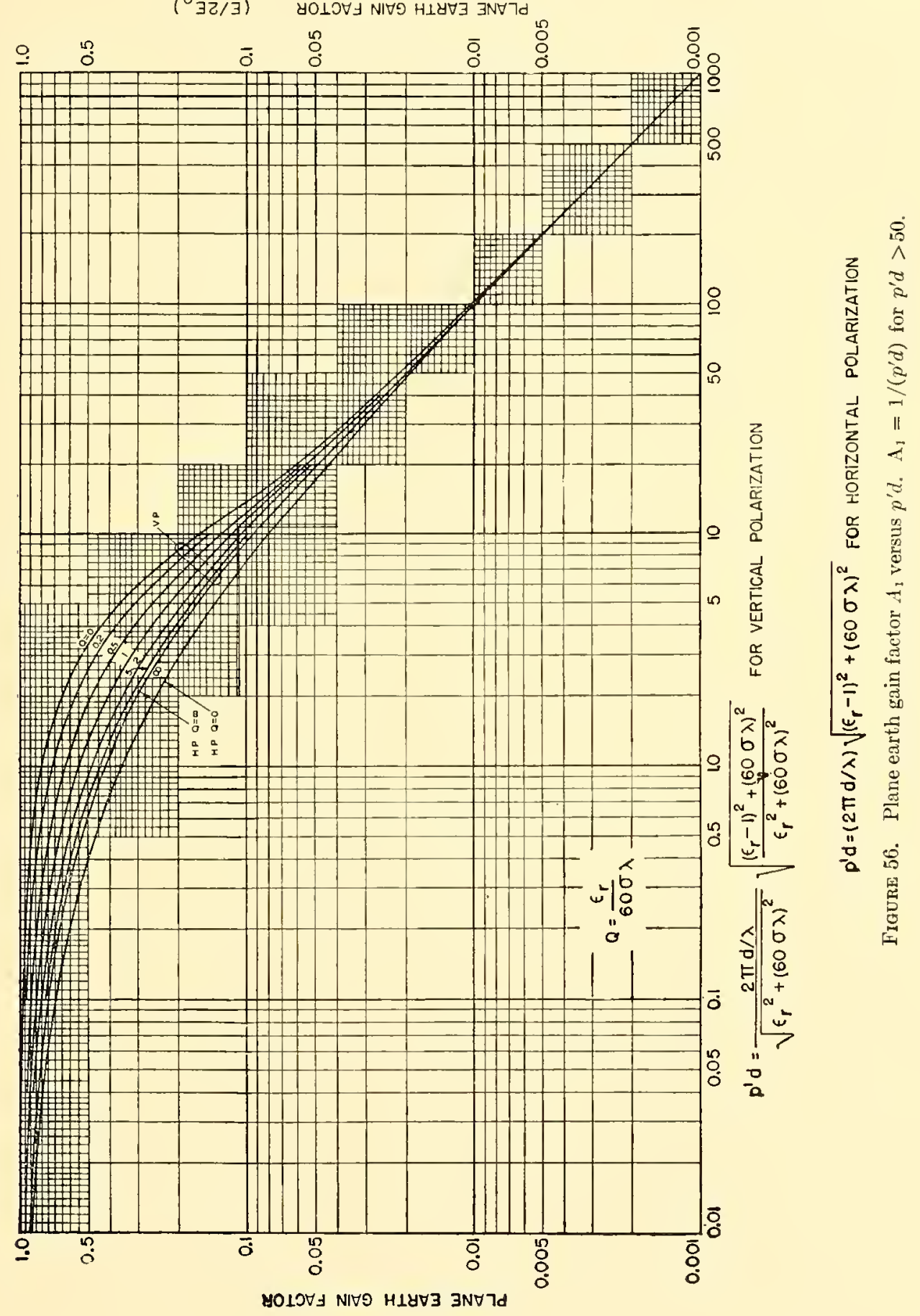




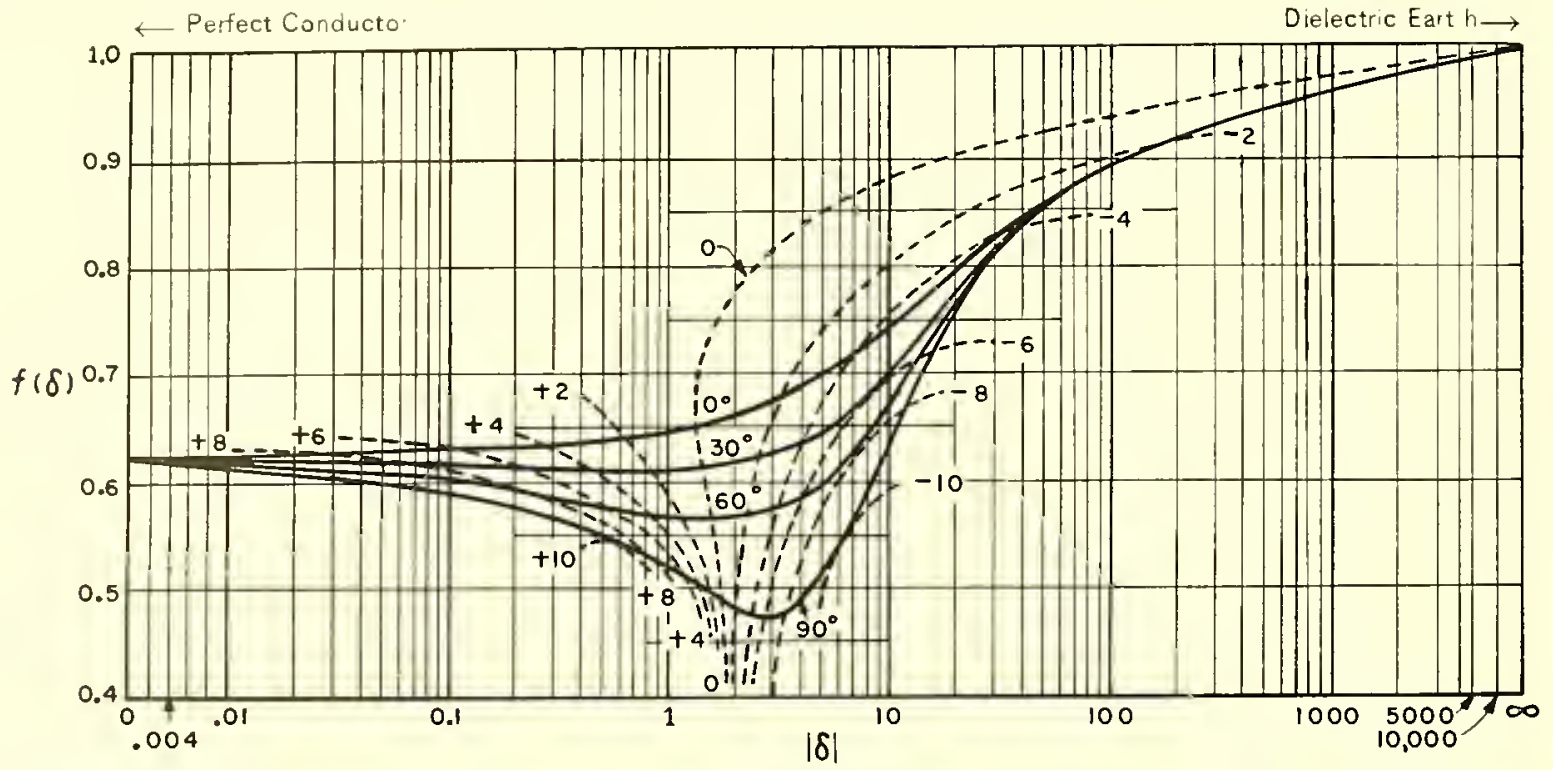

Figure 57. $f(\delta)$ versus $\delta$. Solid lines correspond to phase of $\delta$. Dotted lines indicate curve in Fimure is. For horizontal polarization $f(\delta)=1$.

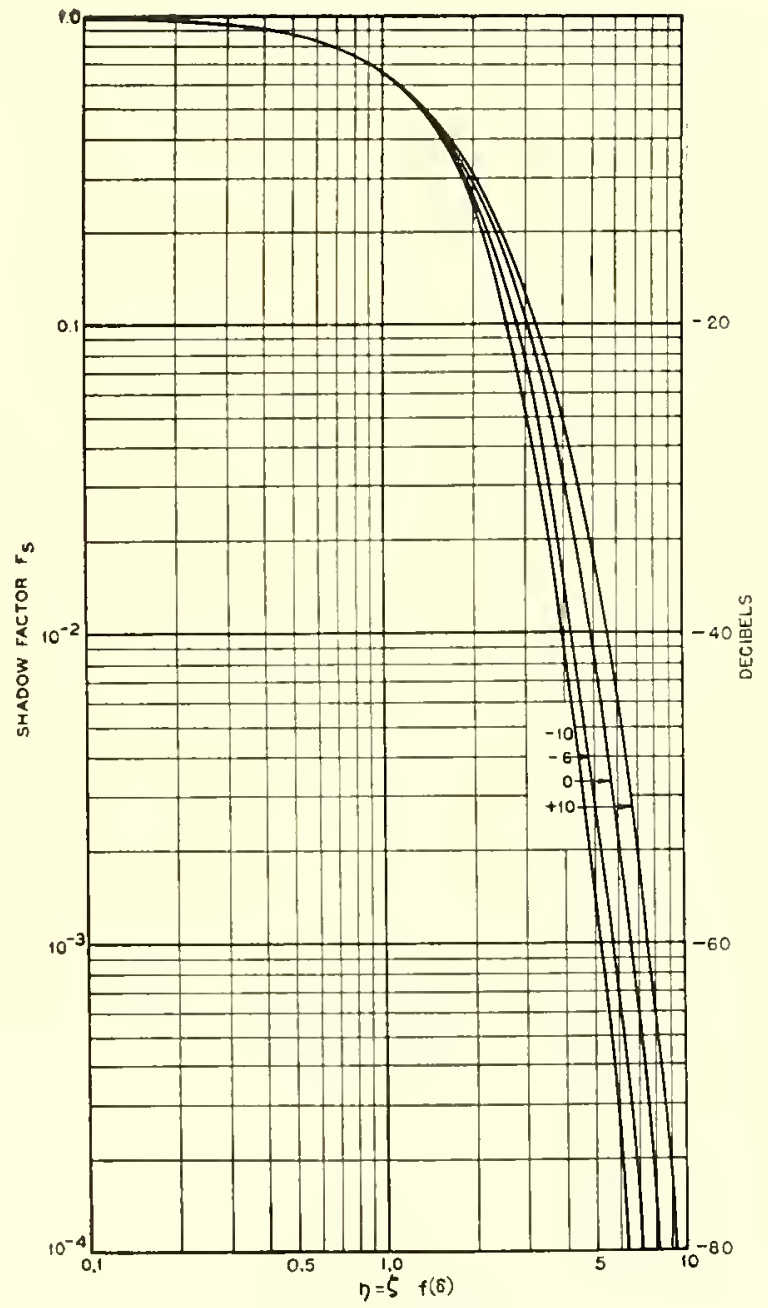

Figure 58. Shadow factor $F_{s}$ versus $\eta=\zeta f(\delta)=\operatorname{sdf}\langle\delta\rangle$. See Figures 32,33 . and 57 . Curve +10 corresponds to a perfect conductor. 
If the antennas are elevated, $F_{s}$ can still be used for the first mode for great distances where the first mode gives most of the field.

\section{7 .7 \\ Sample Calculation for Very Dry Soil}

The general solution given in Section 5.7.6 is here illustrated for the case of doublet antennas, either function of distanee $d$ for doublets at zero height. Figure 60 gives the first mode height-gain factors for transmitter and receiver heights, $h_{1}$ and $h_{2}$, respectively.

To obtain the radio gain under different conditions it is merely necessary to add the decibel gains of the transmitter and receiver antennas, the radio gain for zero height (Figure 59), the height-gain factor (Figure 60) for the transmitter, and a similar figure

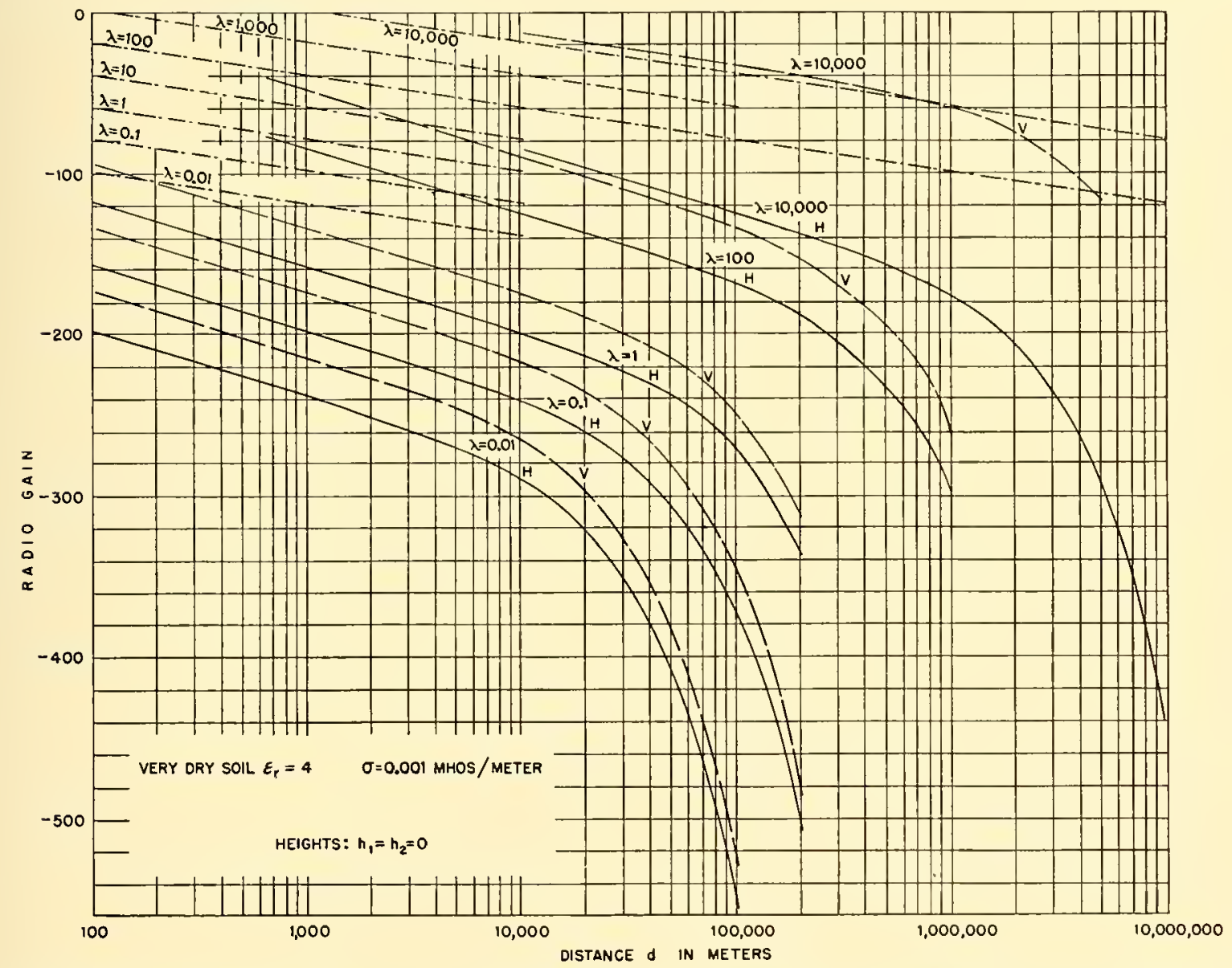

FIgure 59. Free-spare radio gain $A_{0}(--\infty)$ and radio gain $A$, in decibels, for propagation over very dry soil with doublet antennas on the ground (__ for horizontal polarization and —- for vertical polarization). Numbers on the curves give the wavelength $\lambda$. N.te: Radio gain is independent of the radiated power.

horizontally or vertically polarized, placed at various heights over an earth assumed to be rery dry soil for which the constants are $\epsilon_{r}=4$, and $\sigma=0.001$ mho/meter. The following graphs cover, in decimal steps, the frequency range of 30,000 to $0.03 \mathrm{me}$ or wavelengths $\lambda=0.01$ to 10,000 meters.

Figure 59 gives the free space radio gain $A_{0}$ and the radio gain $A$ decibels over very dry soil, as a for the receirer (Figure 60). This process, howerer, is subject to the restriction mentioned in the next paragraphs.

The addition of the factors given in the preceding paragraph is valid all the way up to the maximum of the first lobe, where the field is given by the sum of the direct and reflected rays, provided that the antennas have comparable heights. 
The radio gain ean therefore never be more than $6 \mathrm{db}$ greater than the free-space gain with the same antennas.

If, however, one antenna is low, $h<h_{c}$, and the other is very high, $h>40 h_{c}$, the method discussed four tables of computations are given. Table 11 gives the values of certain quantities, for a wide range of frequencies and for very dry soil, which are independent of polarization. Those quantities which are dependent on polarization are given in Table 12.

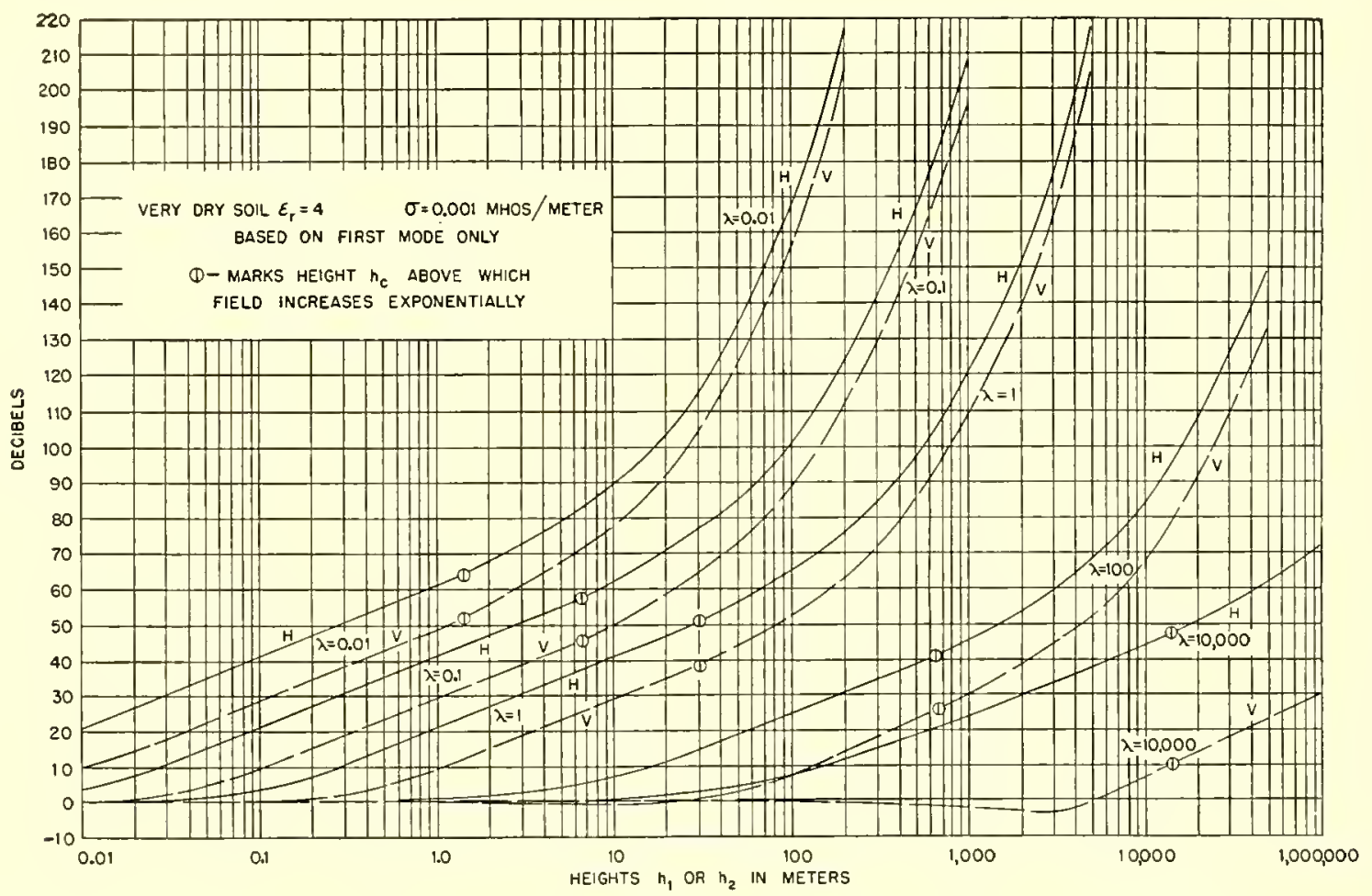

Figure 60. First mode height-gain factors for transmitter and receiver.

fails since the height-gain factors are based on the first mode only. In this event, either the methods outlined in Section 5.7.5 must be employed or the radio gain at low elevations must be connected graphically with the ralue obtained in the optical region for the first maximum.

As an aid to the computer in cheeking his results,
Table 13 gives detailed calculations for ground level radio gain for doublets for a wavelength of $\lambda=1.0$ meter, while Table 14 gives the first mode heightgain factors for the same wavelength.

Similar charts and tables may be prepared easily for transmission orer other types of earth for a similar range of frequencies. 


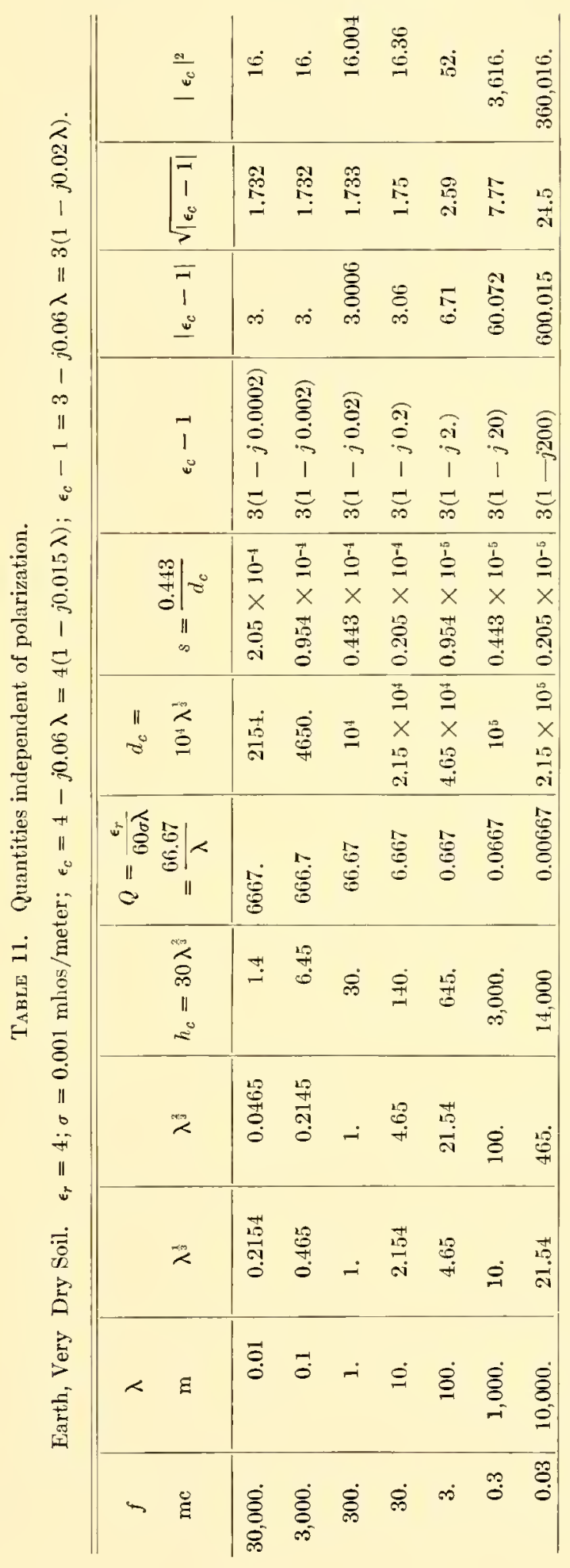




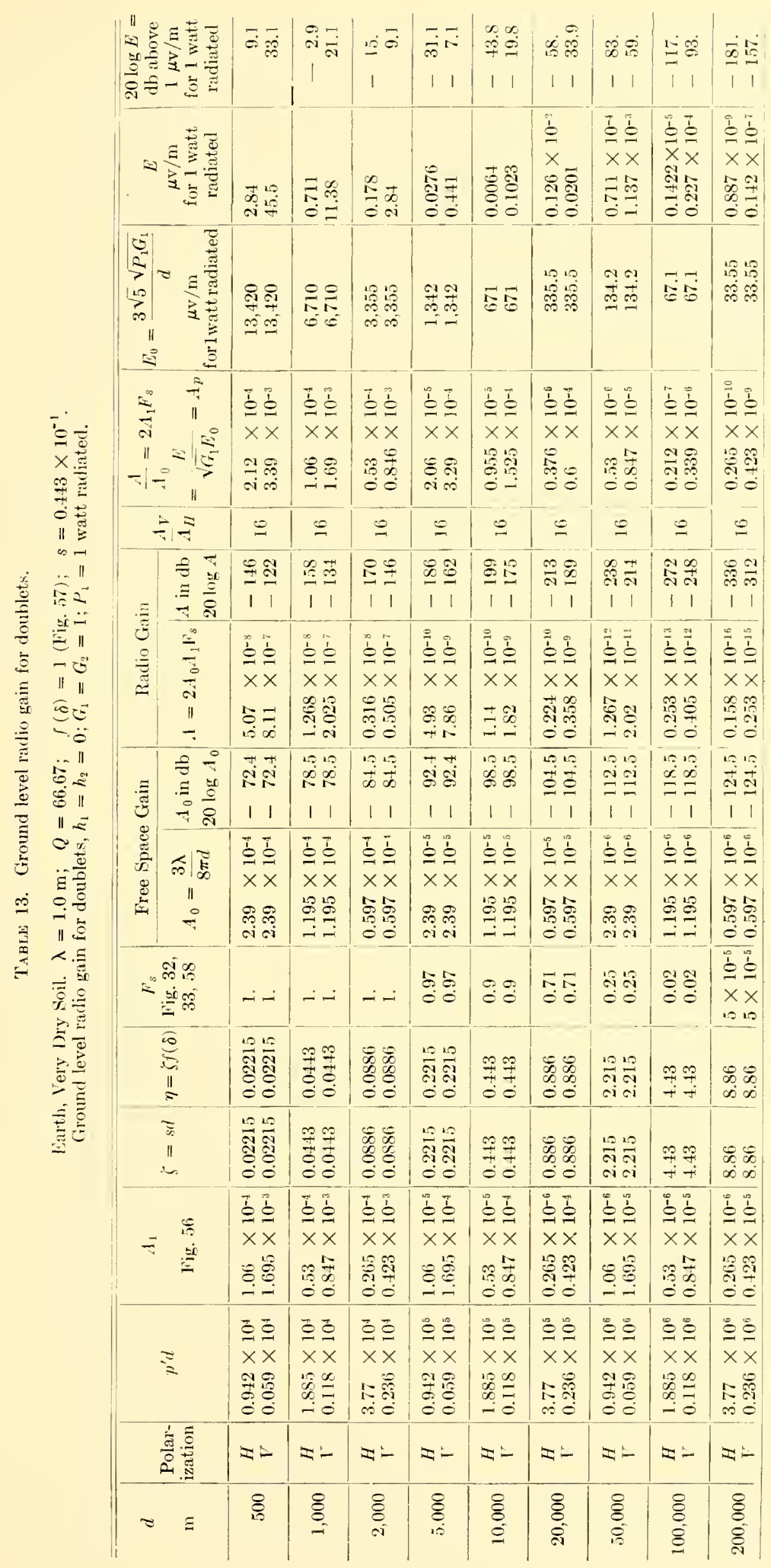


TABLE 14. Height-gain factors (first mode).

Earth, Very Dry Soil. $\lambda=1.0 \mathrm{~m} ; \delta_{H}=0.426 \times 10^{6}\left(-1.1^{\circ}\right) ; \delta_{V}=2.6 \times 10^{4}\left(0^{\circ}\right) ; l_{H}=10.86 ; l_{V}=2.72$.

First mode height-gain factors, $h_{c}=30 . \mathrm{m} ; e=\frac{1}{2 h_{c}}=0.01667 ; Q=66.67$.

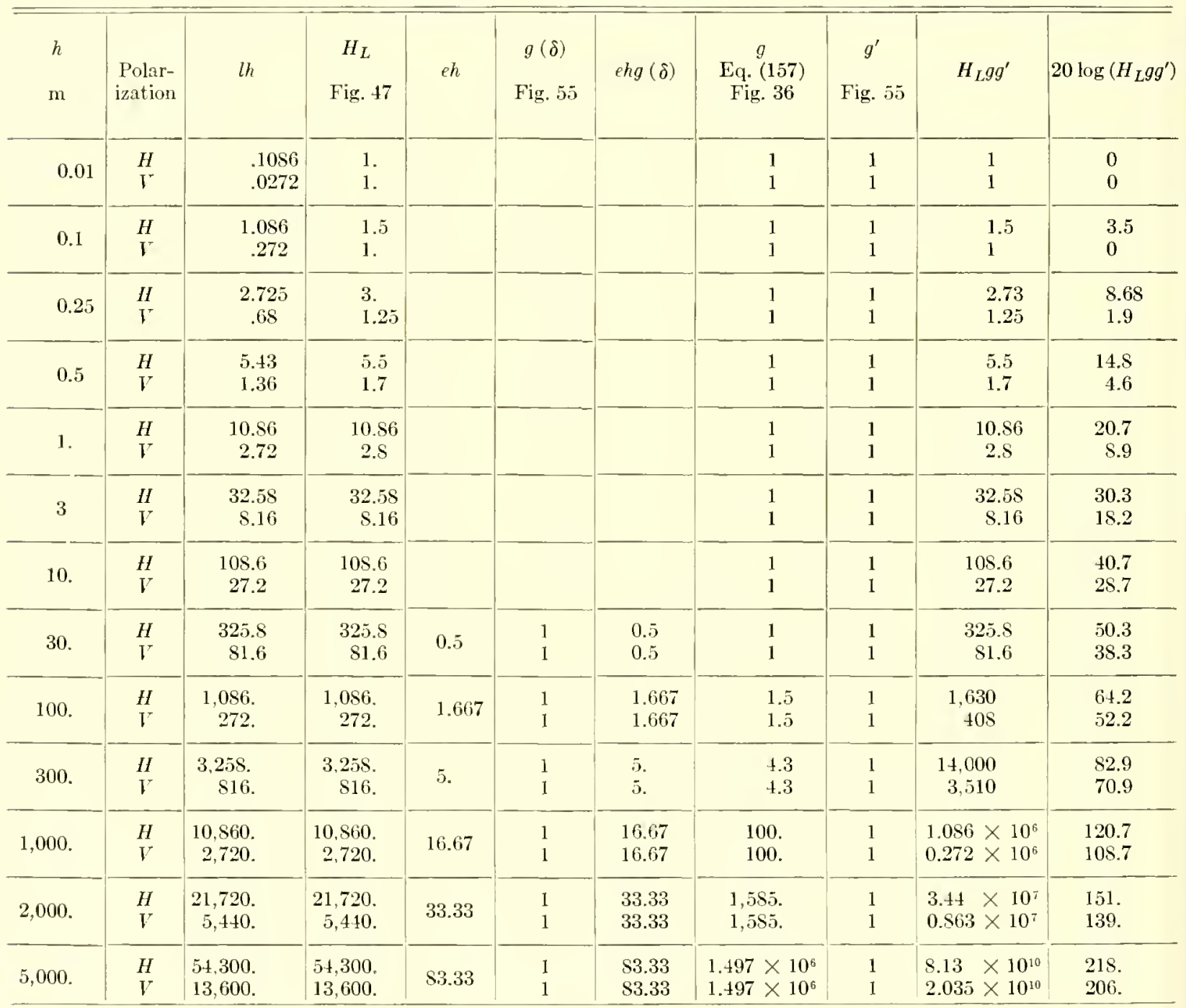




\section{Chapter 6}

\section{COVERAGE DIAGRAMS}

6.1

$\mathrm{T}$ HE LOCUs of points in space having a constant field strength is called a coverage diagram. In the optical region this is also called a lobe diagram. The construction of these diagrams is an important part of the predetermination of the performance of radar and communication sets. The basic concepts and formulas will be developed first for the case of the plane earth and then applied with necessary modifications to propagation over a spherical earth.

The method outlined in this chapter is applicable only to the lobe structure lying above the tip of the first lobe. In this region the field is given almost entirely by the vector sum of the direct and reflected waves. The lower portion of the first lobe is distorted from the regular lobe structure because, in this region, the field strength is determined in part by contributions from the diffraction terms as well as by the contributions of the direct and reflected waves.

\section{2}

6.2 .1

\section{PLANE EARTH}

\section{Field Strength}

For horizontal polarization and a reflection coefficient equal to -1 (i.e., $\rho=1, \phi=180^{\circ}$ ), the received field intensity oscillates from zero to twice the free-space value, depending on the position of the point in space, as shown in Figure 1. The position in space determines the path difference $\Delta$,

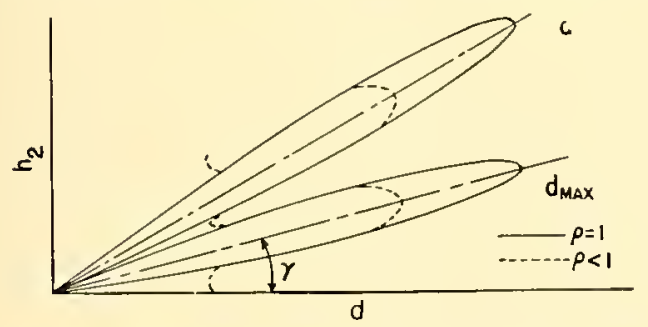

Figure 1. Coverage diagram for plane earth (heights $h_{2}$ are exaggerated relative to distance $d$ ). $n=1,3,5$ ... for the first, second, third . . . lobes. $d=d_{\max }$ $\sin (\pi n / 2)$ and $d_{\max }=2 d_{0}$.

which in turn determines the phase retardation, $\delta_{\text {lag, }}$ due to path difference. The angle $\Omega / 2$ used in calculating $E$ by equation (29) in Chapter 5 is a function of $\delta_{\operatorname{lag}}$ and $\phi$, since $\Omega=\delta_{\mathrm{lag}}+\phi^{\prime}$. The effect of a reflection coefficient $\rho$ less than unity is to reduce the length of the lobe maxima to values less than $2 d_{0}$ and to increase the minima above zero as indicated by the dotted lines of Figure 1. The angles at which the maxima and minima occur depend upon the phase shift at reflection, as will be explained in the following section.

\subsubsection{Angles of Lobe Maxima (Horizontal Polarization)}

Lobe maxima occur whenever the sum of the phase shifts caused by reflection and path difference equals an even multiple of $\pi$ radians, while lobe minima (nulls) oceur when the total phase shift is an odd multiple of $\pi$ radians. If $\rho=1$, the nulls are equal to zero.

It follows that for horizontal polarization $(\phi \cong \pi)$, maxima occur when $\delta$ equals $\pi, 3 \pi$, $5 \pi$, etc., and minima when $\delta=0,2 \pi, 4 \pi$, etc. This means that a path difference equal to an odd multiple of $\lambda / 2$ gives a lobe maximum while a path difference equal to an even multiple of $\lambda / 2$ gives a null. This holds only for horizontal polarization $(\phi=\pi)$. Applying equation (52) in Chapter 5 to the case when $d_{1}<<d_{2}$ (i.e., $d_{2} \cong d$ ),

$$
\Delta=\frac{2 h_{1} h_{2}}{d}=2 h_{1} \tan \psi \cong 2 h_{1} \tan \gamma \cong 2 h_{1} \gamma,
$$

where $\gamma$ is the angle in radians between the horizontal and the line joining the receiver or target to the base of the transmitter (see Figure 8 in Chapter 5). For equation (1) to hold, $\psi$ must be less than 0.2 . Hence for maxima,

$$
2 h_{1} \gamma=\frac{n \lambda}{2}, \quad n=\text { odd integer },
$$

and for minima,

$$
2 h_{1} \gamma=\frac{n \lambda}{2}, \quad n=\text { even integer, }
$$

or

$$
\gamma=\frac{n \lambda}{4 h_{1}}
$$


and $n$ has a range of 0 to 2 for the first lober 2 to 4 for the second lobe, ete.

The limitations of equation (2) maybe summarized ats follows:

1. The phase shift at reflection is $\pi$ radians. This assumes horizontal polarization, or $\psi \cong 0 \mathrm{for}$ vertical polarization.

2. The reflection point is (relatively) close to the transmitter.

3. The grazing angles corresponding to lobe maxima are less than 0.2 radians. In connection with limitation (2), it should be noted that the angles for which the approximation $\gamma=\psi$ holds depend upon the wavelength and transmitter height. The following table shows the minimum angle for various transmitter heights and warelengths at which the crrer in the path difference $\Delta$ introduced by this assumption is less than 1 per cent. To satisfy equatiom (2), and have an croror less than I per cent,

\begin{tabular}{|c|c|c|c|}
\hline $\begin{array}{l}\text { Intenua } \\
\text { lieight, } h_{1} \\
\text { (meter's) }\end{array}$ & $\underset{\text { Minimum }}{\gamma}$ & $\begin{array}{c}n \lambda \\
\text { Minimum } \\
\text { (meters) }\end{array}$ & $\begin{array}{l}\text { Minimum } n \text { at } \\
\lambda=3 \text { meter }\end{array}$ \\
\hline 120 & 1.44 & 12.9 & 4.3 \\
\hline 60 & 1.1 & 4.5 & 1.5 \\
\hline 30 & 0.78 & 1.6 & 0.53 \\
\hline 15 & 0.56 & 0.6 & 0.2 \\
\hline 9 & 0.4 & 0.25 & 0.08 \\
\hline
\end{tabular}

$\gamma$ should lie between the ninimum value and 0.2 radians. Table 1 shows, lor increasing transmitter anterna height, how the angle for which eruation (2) is valid within 1 per cent also incrases.

If $n$ is set equal to $2 m-1$, integral values of $m$ correspond to lobe maxima and half-odd integers to lobe minimi. The advantage of this notation is that the value of $m$ is the number of the maxima or lobe number. Thus for the fifth lobe, $m=5$. The general expression for the grazing angles corresponding to hlo maxima, for a plane earth and for horizontal polarization, is

$$
\gamma=\frac{(2 m-1)}{4 h_{1}} \lambda,
$$

whre integral values of $m$ give maxima and halfodel integers give minima.

\subsubsection{Angles of Lobe Maxima (Vertical Polarization)}

With rertically polarized radiation the reflection phater shift $\phi$ [equation (27) in Chapter 5] is less than $\pi$ radians (i. e., $\phi^{\prime}=\phi-\pi$ is negative). It follows that the path difference for lobe maxima must be greater than $\lambda / 2$ and greater than $\lambda$ for the mulls. In other words, the value of $n$ in equation (2) must be increased by $(\Delta+n)$ to compensate for the decreased phase shift of reflection, so that

$$
(\Delta n)\left(\frac{\lambda}{2}\right)\left(\frac{2 \pi}{\lambda}\right)-(\pi-\phi)=0 .
$$

Hence

$$
(\Delta n)=\frac{\phi}{\pi}-1=+\frac{\phi^{\prime}}{\pi} .
$$

l'or rertical polarization, equation (2) becomes

$$
\gamma=\frac{n+(\Delta n)}{4 h_{1}} \text {. }
$$

0.2 .4

\section{Lobe Equation}

When $\rho=1$, and $\phi=\pi, \phi^{\prime}=0$, and $\Omega=\delta$, copuation (46) iu Chapter 5 may be written as

$$
d=2 \sqrt{G_{1}} d_{0} \sin \frac{\Omega}{2}=2 \sqrt{G_{1}} d_{0} \sin \frac{\delta}{2} .
$$

Substituting

$$
\delta=\frac{2 h_{1} h_{2}}{d}\left(\frac{2 \pi}{\lambda}\right)
$$

gives

$$
d=2 \sqrt{G_{1}} d_{0} \sin \left(\frac{2 \pi h_{1} h_{2}}{\lambda d}\right)
$$

where $d_{0}$ is the free-space range which may be computed from the gain corresponding to the given coverage diagram by use of the nomogram given in Figure 3 in Chapter 2. Equation (8) may be written as

$$
d=2 \sqrt{G_{1}} d_{0} \sin \left(2 \pi \frac{h_{1}}{\lambda} \cdot \sin \gamma\right)
$$

Eruation (9) shows that for fixed values of frecspace range $d_{0}$, transmitter height $h_{1}$, and warelength, the coverage lobe may be represented by a polar sine function of the angle $\gamma$ at the base of the antenna. This assumes that the slant range measured to any point on the lobe may be considered equal to the distance $d$ measured along the surface of the earth. 
If $n$ in equation (2) is allower to assume all fractional and integral values from 0 to $2, \sin \gamma \rightarrow \gamma$ may be expressed as

$$
\sin \gamma=\gamma=\frac{n \lambda}{4 h_{1}} .
$$

Substituting this vahe into equation (9) gives

$$
d=2 \sqrt{\bar{r}_{1}} d_{0} \sin \left(\frac{n \pi}{2}\right)=2 \sqrt{G_{1}} d_{0} \sin \left(90^{\circ} n\right) .
$$

Equation (11) is useful in sketching the lobe contour. It holds only when the reflcetion coefficient equals -1 (i.e., $\rho=+1$ ) and the angle $\gamma$ is small enough so that equation (10) is valid. small mull areas. The shape of the contom is, therefore, less important and it is suffieient to find the maximum and minimum ranges and then to sketch the lobe from the polar sine formula [equation (11)]. On the other hand, the shape of the contour is of great importance when there are few lobes and the null area is large. This is illustrated in Figure 3, where there are only two complete lobes in the region of interest.

The second point to be noted is the rarying effect of divergence on the lobe number and angle. It may be seen from equation (89) in Chapter 5 that the divergence factror approaches unity as $\tan \psi$

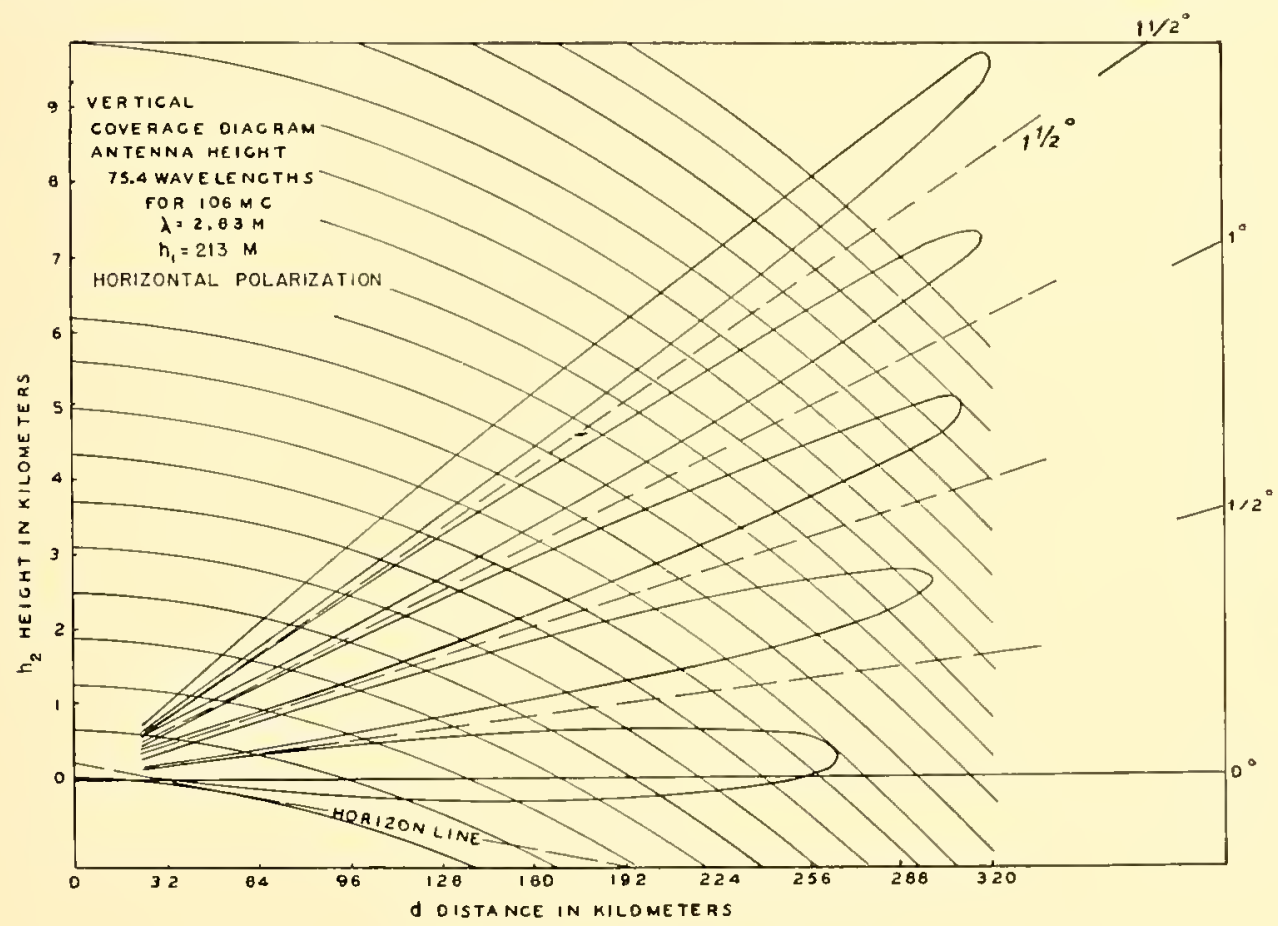

Figure 2. Vertical coverage diagram.

SHHERICAL EAR'TII

\section{Lobe Characteristics}

Figures 2 and 3 are typical vertical coverage diagrams for a smooth spherical earth. They illustrate two important points.

The first is the dependence of the number of lobes on the ratio of transmitter height to wavelength. Figures 2 and 3 show that for $h_{1}$ equal to 75.4 wavelengths, the lobes are much more closely spaced than for a transmitter height of 32.3 wavelengths.

When the number of lobes is large, there is little possibility for a target to escape detection in the increases (sec also ligure 11). 'The divergence lactor is low for sinall angles and then approaches unity rapidly. This aecounts for the reduced range of the first three lobes of Figure 2. For the larger angles, the maximum range is approximately equal to twice the free-space range. When the ratio $h_{1} / \lambda$ is small, the angle at the first lobe maxima is large, since $\gamma=n \lambda / 4 h_{1}$. In this case, the effects of divergence will be negligible except for the lower part of the first lobe, and the polar sine function derived for the ylane earth may be used.

There exists also the intermediate case where the effects of divergence may not be neglected and an 
accurate knowledge of the lobe shape is required. Three different solutions of this problem are given in Sections 6.4, 6.5, 6.6, and 6.7. They are

1. The $p-q$ method for horizontal polarization only.

2. The $u-v$ method, which may be used for both horizontal and vertical polarization.

3. Lobe-angle method which has the advantage of determining the lobe angles directly and is used for either polarization. where $D$ replaces $K$ in equation (46) in Chapter 5, since for horizontal polarization $\rho=1$ and since we are neglecting any effect due to the antenna radiation pattern. For this expression, $D$ and $\Omega$ are certain functions of the antenna heights $h_{1}$ and $h_{2}$ as well as $d$ which were considered earlier (see Sections 5.2.4 and 5.5.6). For a given transmitter, $h_{1}$, $G_{1}, d_{0}$, and $\lambda$ are given, so that for a given gain contour the only variables in equation (12) are $d$ and

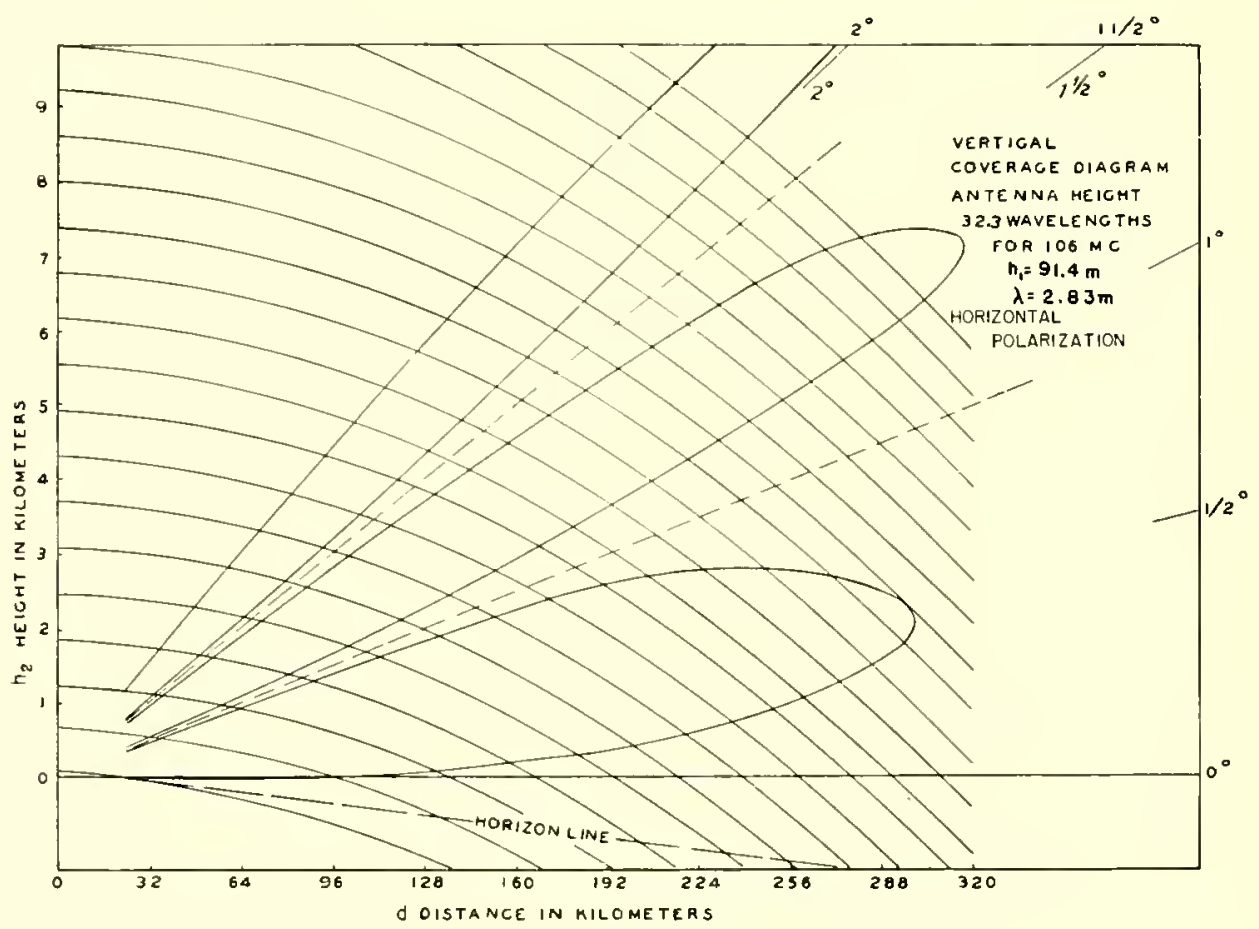

Figure 3. Vertical coverage diagram.

\section{THE $p-q$ METHOD \\ (HORIZONTAL POLARIZATION)}

6.4 .1

\section{Outline of Method}

This method consists in plotting the locus of points having a constant range $d$ and locating those points on this curve which are at such a distance from the transmitter that the phase shift caused by path difference corresponds to the required range. The range corresponding to a total phase shift $\Omega$ is given by

$$
d=\sqrt{G_{1}} d_{0} \sqrt{(1-D)^{2}+4 D \sin ^{2} \frac{\Omega}{2}},
$$

$h_{2}$. The difficulty of the problem consists in the fact that equation (12) provicles an extremely complicated relation between $h_{2}$ and $d$ which cannot be solved explicitly for either coordinate.

Under such circumstances, the natural procedure is to introduce new coordinates which make the handling of equation (12) easier. The method described in the following makes use of the variables

$$
p=\frac{d_{1}}{d_{T}} \quad \text { and } \quad q=\frac{d_{2}}{d}
$$

discussed in Sections 5.5.5 and 5.5.7, and the procedure will be called the $p-q$ method. 
It may be recalled that expressed in coordinates $p$ and $q$

$$
\begin{gathered}
d=\frac{p}{1-q} \cdot d_{l}, \\
D=\left[1+\frac{4 p^{2} q}{\left(1-p^{2}\right)}\right]^{-1 / 2},
\end{gathered}
$$

and the total phase shift, by equations $(9 \overline{7})$ and (29) in Chapter 5, is

$$
\Omega=\frac{4 \pi}{\lambda} \frac{h_{\perp}^{2}}{d_{T}} \frac{q\left(1-p^{2}\right)^{2}}{p}+\phi^{\prime} .
$$

then proceed in the following manner. To a fair approximation, we may assume the extreme range of the lobes to correspond to $\sin ^{2}(\Omega / 2)=1$, so that by equation (12) the corresponding distance $d_{\max }$ is given by

$$
d_{\max }=\sqrt{G_{1}} d_{0}(1+D) .
$$

Expressing $d_{\max }$ and $D$ by $p$ and $q$, the above equation determines the envelope of all lobe maxima. The practical way of doing this is to use a graphical representation of $D$ in $p$ and $q$ coordinates (Figure 17 in Chapter 5) and to start by selecting a particular

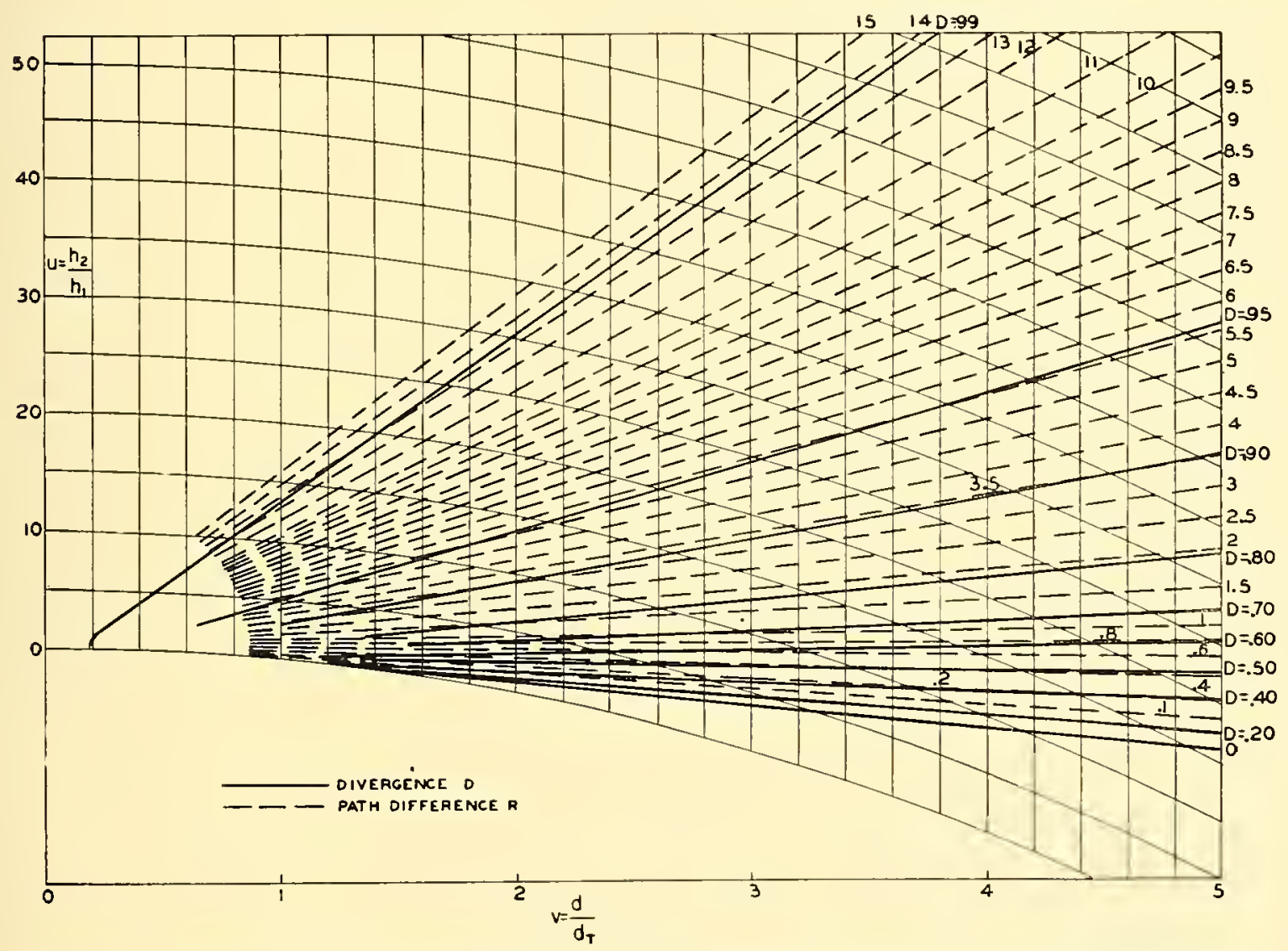

Figure 4. Curves of constant-divergence factor $D$ and path difference parameter $R$. (Radiation Laboratory.)

For horizontal polarization, $\phi \rightarrow 0$, so that for this case (which is the one under consideration) all variable quantities in equation (12) have been expressed in terms of $p$ and $q$.

\subsubsection{Construction of Range Loci}

Suppose to start with that we want to compute the position of the extreme range of a lobe. We may value of $D$, say $D=D_{1}$. Inserting this in equation (16) gives a corresponding value of $d_{\max }$, and inserting this value of $d_{\max }$ for $d$ in equation(13) determines a straight line in the $p, q$ plane, since $d_{T}=\sqrt{2 k a h_{\mathrm{I}}}$ is known. Whatever is the value of $d_{\max }$, this line passes through the point $q=1, p=0$. In order to determine the position of the line, only one more point is needed. A convenient point to choose is to take $q=0.9$ and compute the corresponding $p$ from 
$p=0.1 d_{\max } / d_{T}$. The point of intersection of this line with the seleeted $D_{1}$-contour then gives the desired $p, q$ combination that corresponds to the giren values of $d_{\max }$ and $D_{1}$.

From this pair of values $(p, q)$, the corresponding receiver height $h_{2}$ may be calculated by the relation [equation (98) in Chapter 5]

$$
h_{2}=h_{1} \frac{q}{1-q}\left[1-p^{2}+\frac{p^{2} q}{1-q}\right] .
$$

6.4 .3

\section{Construction of Path-Difference Loci}

An assumed value of $\Omega$ in equation (12) determines $\delta$, the phase shift caused by the path difference, as

$$
\delta=\Omega+2 \pi n,
$$

where $n$ assumes all integral values and zero. This value of $\delta$ determines the path difference $\Delta=r-r_{d}$,

$$
\lrcorner=\frac{\lambda \delta}{2 \pi} .
$$

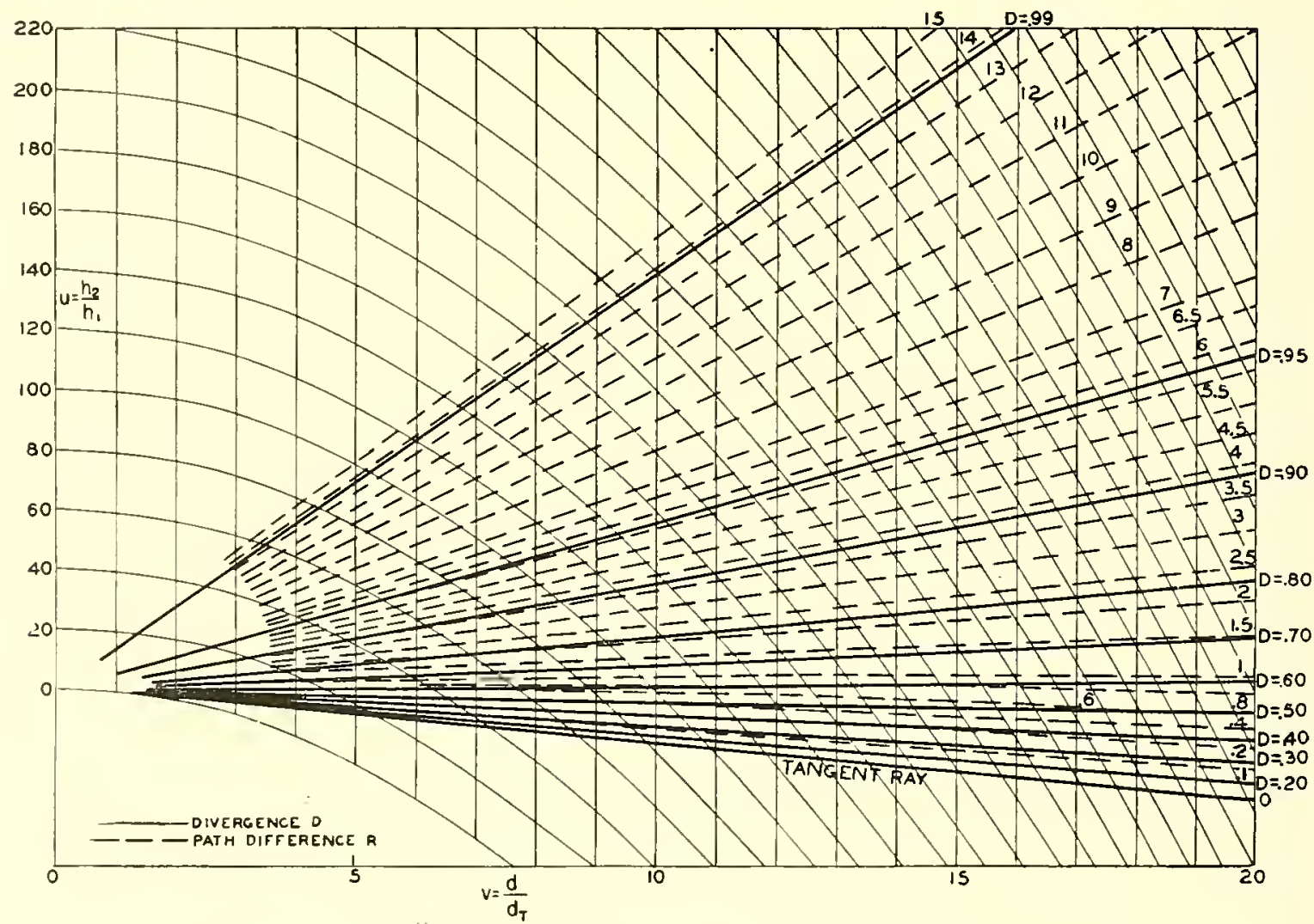

FIGURE 5. Curves of constant-divergence factor $L$ and path difference parameter $R$. (Radiation Laboratory.)

Now both coordinates of the desired point are known, and the point may be plotted. Plotting a series of different points by the same method yields a smooth curre, which is the envelope of all lobe maxima.

The locus of minima may similarly be plotted by using $\sin ^{2} \frac{\Omega}{2}=0$ and

$$
d_{\min }=\sqrt{C_{1}^{1}} d_{0}(1-D)
$$

instead of equation (16). Intermediate range curves are found by assigning nonintegral values to $m$ in the equation $\Omega=m \pi$ and substituting in equation (12).
But from equation (97) in Chapter 5

$$
د=\frac{2 h_{1}{ }^{2}}{d_{T}} q \frac{\left(1-p^{2}\right)^{2}}{p}=\frac{2 h_{1}^{2}}{d_{T}} q f(p)
$$

where $f(p)$ is given in Figure 18 in Chapter 5 .

In this calculation, $q$ may be taken as the independent variable. The assumed values of $q$ together with $\Delta$ from equation (19) determine $f(p)$ in equation $(20)$. For given values of $f(p)$, the eorresponding values of $p$ may be read from Figure 18 in Chapter 5 . The coordinates $h_{2}$ and $d$ on the path-differ- 
ence loci are found from equation (98) in Chapter 5 and equation (13) in Chapter 6 , giving

$$
h_{2}=\frac{h_{1} q}{1-q}\left(1-p^{2}+\frac{p^{2} q}{1-q}\right)
$$

(46) in Chapter 5] are constructed and their intersections with path-difference contours corresponding to the assumed values of $\Omega / 2$ determine points on the lobes.

\subsubsection{Construction of Lobes (Horizontal Polarization)}

In this method, the divergence factor $D$ is considered to be the independent variable. Dividing
Intersections of the path-difference loci with the range curves determine points on the lobes.

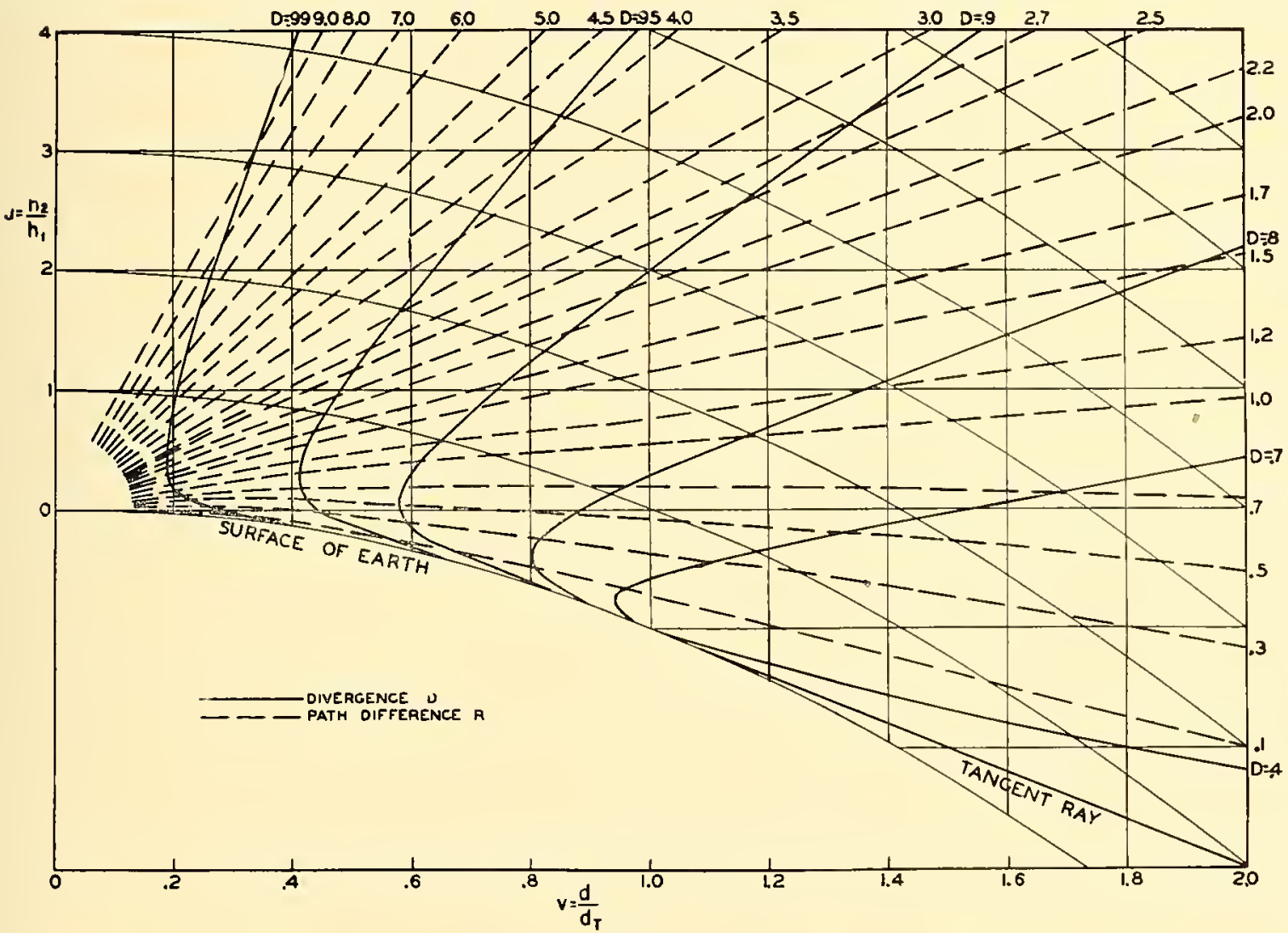

Figure 6. Curves of constant-divergence factor $D$ and path difference parameter $R$. (Radiation Laboratory.)

6.5

6.5
THE $u-v$ METHOD

\section{Outline of Method}

This method makes use of the generalized coordinates $u=h_{2} / h_{1}$ and $v=d / d_{T}$ described in Section 5.5.8. The curves of constant-divergence factor $D$ and path-difference parameter $R$ are plotted on the same shect in Figures 4,5 , and 6 . The divergence lines are shown in full and the path-difference curves are dotted. Envelopes of constant $\sin ^{2}(\Omega / 2)$ [equation both sides of equation (46) in Chapter 5 by $d_{T}$ gives

$$
\begin{aligned}
\frac{d}{d_{T}} & =\sqrt{G_{1}} \frac{d_{0}}{d_{T}} \sqrt{(1-K)^{2}+4 K \sin ^{2} \frac{\Omega}{2}} \\
& =\sqrt{G_{1}} \frac{d_{0}}{(1-K)^{2}+4 K \sin ^{2} \frac{\Omega}{2}},
\end{aligned}
$$

where

$$
\underline{d_{\mathrm{n}}}=\frac{d_{0}}{d_{T}} .
$$


If $\rho=1$, and the effect of antenna directivity is neglected, $K=D$, and

$$
v=\frac{d}{d_{T}}=\sqrt{G_{1}} \frac{d_{0}}{(1-D)^{2}+4 D \sin ^{2} \frac{\Omega}{2}} .
$$

given by Figure 12 in Chapter 5. Equation (22) then gives the value of $v$. These quantities together with $R=n r$ may conveniently be tabulated, as in Table 2. Corresponding values of $D$ and $v$ are plotted as crosses on Figure 7. The line drawn through these

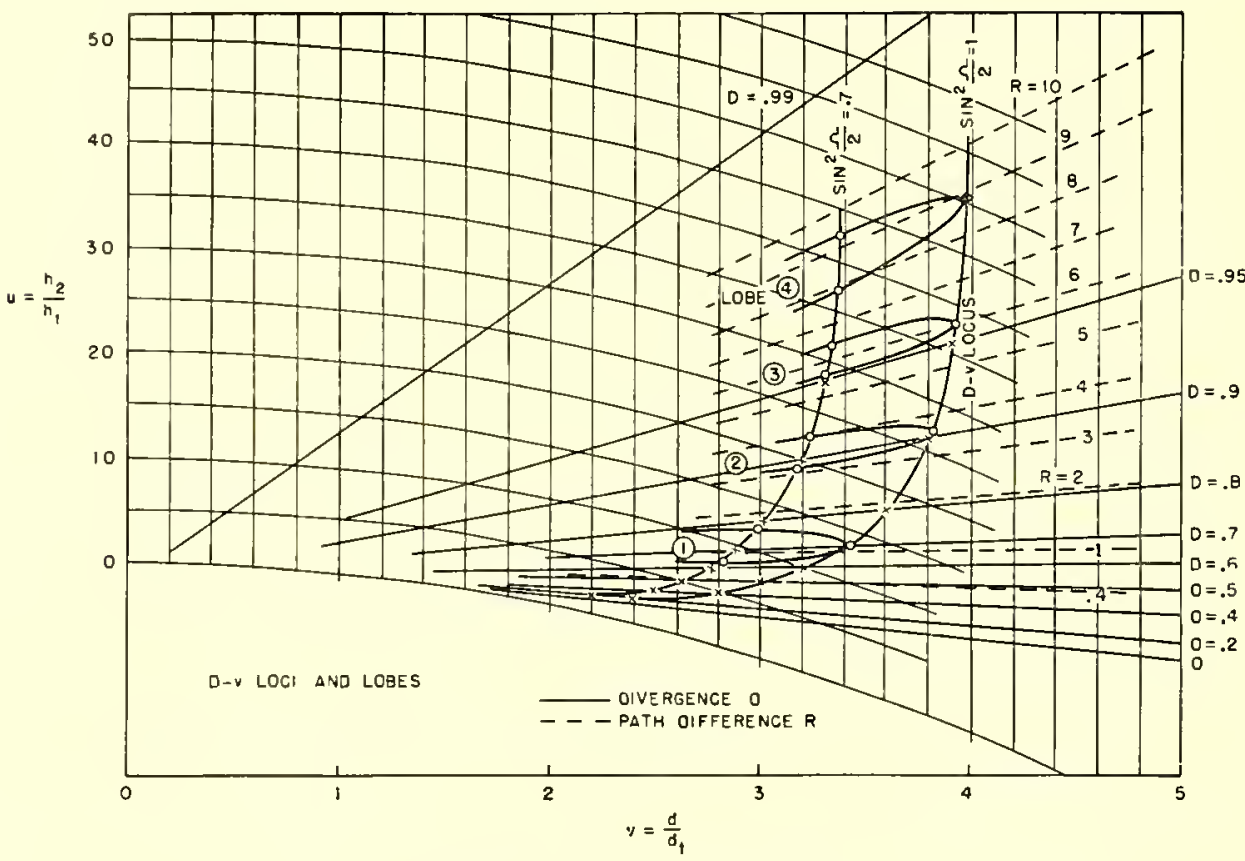

Figure 7. $D-v$ loci and lobes.

The following discussion illustrates how one contour of a coverage diagram, corresponding to a particular value of radio gain, may be plotted on Figure 4 or its equivalent, Figure 7 . The result is a curve similar to Figure 2 , but plotted in $u, v$ coordinates instead of $h_{2}$ and $d$. See also Figures 16 to 39.

For illustration, let the transmitter gain, $C_{1}=1$ and let the radio gain be such a value that $\underline{d_{0}}=d_{0} / d_{T}=2$. Further, let $\lambda=0.1$ meter and $h_{1}=20$ meters. From Figure 15 it is seen that $r=1,030 \lambda / h_{1}^{3 / 2}=1.2$. Select one of the curves for $\sin ^{2}(\Omega / 2)$ in Figure 12, Chapter 5 , say $\sin ^{2}(\Omega / 2)=1$, for which $\Omega=\pi, 3 \pi$, $5 \pi$, etc. These values correspond to tips of the lobes for which $n=1,3,5$, etc., since, for perfect reflection, $\Omega=n \pi$ by equation (116) in Chapter 5 .

Next select values of $K=D$ and note the corresponding value of the radical

$$
\sqrt{(1-K)^{2}+4 K \sin ^{2} \frac{\Omega}{2}}
$$

\begin{tabular}{|c|c|c|c|c|c|}
\hline \multirow[b]{2}{*}{$D$} & \multirow{2}{*}{$\begin{array}{l}\sqrt{(1-D)^{2}+4 D \sin ^{2} \frac{\Omega}{2}} \\
\text { (Figure 12, Chapter 5) }\end{array}$} & \multirow{2}{*}{$\begin{array}{c}v \\
\text { [equation } \\
(22)]\end{array}$} & \multicolumn{3}{|c|}{$(r=1.2)$} \\
\hline & & & $n$ & $R=n r$ & $\begin{array}{c}\text { Lobe } \\
\text { maxima }\end{array}$ \\
\hline $\begin{array}{l}0.2 \\
0.3\end{array}$ & $\begin{array}{l}1.2 \\
1.3\end{array}$ & $\begin{array}{l}2.4 \\
2.6\end{array}$ & 1 & 1.2 & 1st lobe \\
\hline $\begin{array}{l}0.4 \\
0.5\end{array}$ & $\begin{array}{l}1.4 \\
1.5\end{array}$ & $\begin{array}{l}2.8 \\
3.0\end{array}$ & 3 & 3.6 & $2 \mathrm{~d}$ lobe \\
\hline $\begin{array}{l}0.6 \\
0.7\end{array}$ & $\begin{array}{l}1.6 \\
1.7\end{array}$ & $\begin{array}{l}3.2 \\
3.4\end{array}$ & 5 & 6.0 & 3d lobe \\
\hline $\begin{array}{l}0.8 \\
0.9\end{array}$ & $\begin{array}{l}1.8 \\
1.9\end{array}$ & $\begin{array}{l}3.6 \\
3.8\end{array}$ & 7 & 8.4 & 4th lobe \\
\hline $\begin{array}{l}0.95 \\
1.0\end{array}$ & $\begin{array}{l}1.95 \\
2.0\end{array}$ & $\begin{array}{l}3.9 \\
4.0\end{array}$ & & & \\
\hline
\end{tabular}

Table 2. Values of $v$ and $R$ for $\sin ^{2}(\Omega 2)=1, \underline{d_{0}}=2$.

points is the locus of the tips of the lobes. The actual position of each lobe tip is then marked with a eircle where the corresponding ralue of $R$ crosses the loeus in Figure 7 . 
Additional lobe points are loeated by ehoosing some other value of $\sin ^{2}(\Omega / 2)$, say $\sin ^{2}(\Omega / 2)=0.7$. Each value of $\sin ^{2}(\Omega / 2)$ now gives two points on each lobe, one on the upper braneh and the other on the lower. Again choose values of $K=D$, obtain the corresponding ralues of the radical

$$
\sqrt{(1-K)^{2}+4 K \sin ^{2} \frac{\Omega}{2}}
$$

from Figure 12 in Chapter 5, and ealeulate new values for $v$. The $D-v$ values are plotted as crosses on Figure 7 and the line through them is the loeus of points for which $\sin ^{2}(\Omega / 2)=0.7$ (see Table 3$)$.

TABLe 3. Values of $v$ and $R$ for $\sin ^{2}(2 / 2)=0.7, \underline{d_{0}}=2$.

\begin{tabular}{|c|c|c|c|c|c|c|}
\hline \multirow{2}{*}{$D$} & \multirow{2}{*}{$\begin{array}{l}\sqrt{(1-D)^{2}+4 D \sin ^{2} \frac{5}{2}} \\
\text { (Figure } 12, \text { Chapter } 5 \text { ) }\end{array}$} & \multirow{2}{*}{ 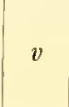 } & \multicolumn{4}{|c|}{$(r=1.2)$} \\
\hline & & & $K$ & u & $R=n r$ & Lobe \\
\hline 0.2 & 1.1 & 2.2 & 0 & 0.63 & 0.756 & 1 \\
\hline 0.3 & 1.15 & 2.3 & 0 & 1.37 & $1.6 \pm 4$ & 1 \\
\hline 0.4 & 1.22 & $2.4 t$ & 1 & 2.63 & 3.16 & 2 \\
\hline 0.5 & 1.28 & 2.56 & 1 & 3.37 & 4.04 & 2 \\
\hline 0.6 & 1.36 & 2.72 & 2 & 4.63 & 5.56 & 3 \\
\hline 0.7 & 1.43 & 2.86 & 2 & 5.37 & 6.44 & 3 \\
\hline 0.8 & 1.52 & 3.04 & 3 & 6.63 & 7.96 & 4 \\
\hline 0.9 & 1.6 & 3.2 & 3 & 7.37 & 8.81 & 4 \\
\hline 0.95 & 1.64 & $\begin{array}{l}3.28 \\
3.36\end{array}$ & & & & \\
\hline 1.0 & 1.68 & 3.36 & & & & \\
\hline
\end{tabular}

For $\sin ^{2}(\Omega / 2)=0.7, \sin (\Omega / 2)= \pm 0.836, \Omega / 2$ $=0.315 \pi$ or $0.685 \pi$. Then $\Omega=n \pi=0.63 \pi+2 k \pi$ and $1.37 \pi+2 k \pi$, in which $k$ is an integer. Then $n=0.63+2 k$ and $1.37+2 k$, and $R=n r$. Values of $n$ and $R$ are listed in Table 3. The intersections of the $R$ values and the loeus for $\sin ^{2}(\Omega / 2)=0.7$ are plotted as cireles on Figure 7 .

The entire lobe structure for one contour may be drawn by ehoosing additional vahues of $\sin ^{2}(\Omega / 2)$. A large number of contours have been ealeulated by the Radiation Laboratory and are plotted in Figures 16 to 39 .

In order to construet one contour of a coverage diagram, it remains to find the intersection between the eurves giving values of $u$ for constant $\sin ^{2}(\Omega / 2)$ and the corresponding path-difference contours. The equations relating $R$ to $\Omega / 2$ are given below. From equation (18)

$$
\delta=\Omega+2 \pi n \quad\left(\phi=180^{\circ}, \phi^{\prime}=0\right),
$$

and from equation (19)

$$
\Delta=\frac{\lambda}{\pi}\left(\frac{\delta}{2}\right)
$$

From equation (83) in Chapter 5

$$
R=\frac{k a \Delta}{h_{1} d_{T}} .
$$

An assigned value of $\Omega$ fixes two values of $\delta$ for each lobe, as explained in the previous paragraph. All vahues of $\sin ^{2}(\Omega / 2)$ other than 1 or 0 determine two intersections with the lobe. When $\sin ^{2}(\Omega / 2)=1$, the envelope of maxima is obtaincl, while $\sin ^{2}(\Omega / 2)$ $=0$ eorresponds to the envelope of minima.

By selecting several values of $\sin ^{2}(\Omega / 2)$ in Figure 12, Chapter 5, and following the method outlined above, a coverage diagram may be constructed in generalized $(u, v)$ eoordinates. The actual values of $h_{2}$ and $d$ are

$$
\begin{aligned}
h_{2} & =h_{1} u, \\
d & =d_{T} v .
\end{aligned}
$$

\subsubsection{Construction of Lobes (Vertical Polarization)}

Problems involving vertical polarization or cases where the ratio of the antenna-pattern factors $F_{2} / F_{1}$ cannot be negleeted, may be solved by successive approximation.

As a first approximation the method of Section 6.5.2 is applied to determine points $\left(h_{2}, d\right)$ on the lobe. The corresponding values of $u$ and $v$ determine $s$ in Figure 19 or Figure 20, in Chapter 5, and $\tan \psi$ may be found from Figure 24 in Chapter 5 for the given transmitter height $h_{1}$. An alternate method is to ealculate $\tan \psi$ from equations (73), (58), and (60) in Chapter 5, which are

$$
\begin{aligned}
d_{1} & =s d, \\
h_{1}{ }^{\prime} & =h_{1}-\frac{d_{1}{ }^{2}}{2 k a}, \\
\tan \psi & =\frac{h_{1}{ }^{\prime}}{d_{1}} .
\end{aligned}
$$

The angles $\psi_{d}$ and $\nu$ required to ealeulate the antenna pattern faetors $F_{1}$ and $F_{2}$ are found from equations (62) and (63) in Chapter 5,

$$
\begin{aligned}
\tan \psi_{d} & =\frac{h_{2}-h_{1}}{d}-\frac{d}{2 k a} \\
\nu & \cong \psi+\frac{d_{1}}{k a}
\end{aligned}
$$


The values of $\rho, \phi\left(\mathrm{ol}^{\prime} \phi^{\prime}\right)$ may now be read from the reflection curves in Chapter $t$.

Equation (46) in Chapter 5 may now be applied with $K=\left(F_{2} / F_{1}\right) \rho D$ where $D$ assumes the same values as in the approximate solution. Equation (21) determines the value of $d$ from which $u=d / d_{T}$ may be calculated. This value of $u=d / d_{T}$ is laid off on the original divergence contour in Figures 4, 5 , or 6 . This determines $v$. The assumption underlying this procedure is that the divergence factor is not appreciably affected by the change in coordinates caused by imperfect reflection and an unsymmetrical antenna pattern. The corrected phase difference $\delta^{\prime}$ is found from

$$
\delta^{\prime}=\Omega-\phi^{\prime}
$$

and the path difference $\Delta^{\prime}$ and parameter $R^{\prime}$ from

$$
\begin{aligned}
\Delta^{\prime} & =\frac{\lambda}{\pi}\left(\frac{\delta^{\prime}}{2}\right) \\
R^{\prime} & =\frac{k a \Delta^{\prime}}{h_{1} d_{T}} .
\end{aligned}
$$

The intersections between the path-difference contours and the distance envelope determine points on the coverage diagl an.

The above method should be applied even for horizontal polarization when the directivity of the antenna is such that $F_{2} / F_{1} \neq 1$. This follows from the concept of generalized reflection coefficients of Section 5.3.1.

\section{6 \\ LOBE-ANGLE METHOD (HORIZONTAL, POLARIZATION)}

\subsubsection{Outline of Method}

In this method the angles of lobe maxima are determined by modifying the plane earth formula, equation (2). In this equation, $h_{1}$ is replaced by $h_{1}{ }^{\prime}$, which is the equivalent height above a plane tangent to the earth's surface at the reflection point, as shown in Figure 8.

The vahe of $h_{1}{ }^{\prime}$ is given in equations (58) and (60) in Chapter 5. The maxinum and minimum distances from the transmitter base to a point on the lobe are calculated by equation (46) in Chapter 5, using a modified divergence factor to be deseribed in Section (i.6.5.
6.6 .2

\section{Basic Relations}

Referring to Figure $S$ and assuming $d_{1}<<d_{2}$, $\gamma^{\prime}<10^{\circ}$ and $\psi \cong \gamma^{\prime}$, the following relations hold.

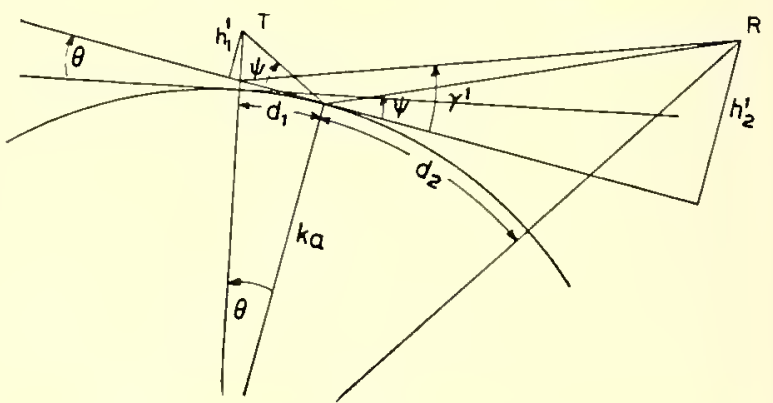

Figure 8. Lobe angles corrected for earth curvature.

$$
\begin{aligned}
\tan \gamma^{\prime} \rightarrow \gamma^{\prime} & =\frac{n \lambda}{4 h_{1}{ }^{\prime}} \\
\tan \gamma^{\prime} \rightarrow \gamma^{\prime} & \cong \frac{h_{1}{ }^{\prime}}{d_{1}}
\end{aligned}
$$

Hence

$$
\frac{h_{1}^{\prime}}{d_{1}}=\frac{n \lambda}{4 h_{1}^{\prime}}
$$

and

$$
d_{1}=\frac{\left(2 h_{\mathrm{I}}{ }^{\prime}\right)^{2}}{n \lambda},
$$

where, from equation (58) in Chapter 5 ,

$$
h_{1}{ }^{\prime}=h_{1}-\frac{d_{1}^{2}}{2 k a} \text {. }
$$

The basic equations of the lobe-angle method are

$$
\gamma^{\prime}=\frac{n \lambda}{4 h_{1}{ }^{\prime}}=\frac{n \lambda}{4\left(h_{1}-\frac{d_{1}{ }^{2}}{2 k a}\right)}
$$

and

$$
d_{1}=\frac{4\left(h_{1}-\frac{d_{1}{ }^{2}}{2 k a}\right)^{2}}{n \lambda} .
$$

\subsubsection{Reflection-Point Curves}

The elimination of $d_{1}$ from equations (32) and (33) is most convenicntly accomplished by graphical aids, which may be used in the following way.

1. From equation (33), a curve may be plotted showing $d_{1}$ as abscissa and $n$ as ordinate for a given transmitter height and wavelength. This is illus- 
trated in Figure 9 for two stations $A$ and $B$ with heights equal to 146.5 meters and 302 meters and wavelengths $\lambda=1.50$ meters and $\lambda=1.42$ meters respectively.

2. From equation (58) in Chapter 5, a second curve may be plotted with $d_{1}$ as abscissa and the equivalent height $h_{1}{ }^{\prime}$ as ordinate as shown in Figure 10. To illustrate, computed data for station $A$ are given in Table 4 , for a free-space range of $d_{0}=100 \mathrm{~km} . d$ is calculated from equations (16) and (17). where

$$
\theta=\frac{d_{1}}{k a}
$$

Hence by equation (32)

$$
\gamma=\frac{n \lambda}{4\left(h_{1}-\frac{d_{1}^{2}}{2 k_{a}}\right)}-\frac{d_{1}}{l_{i} a},
$$

TABLE 4. Data for station A of Figure 9.*

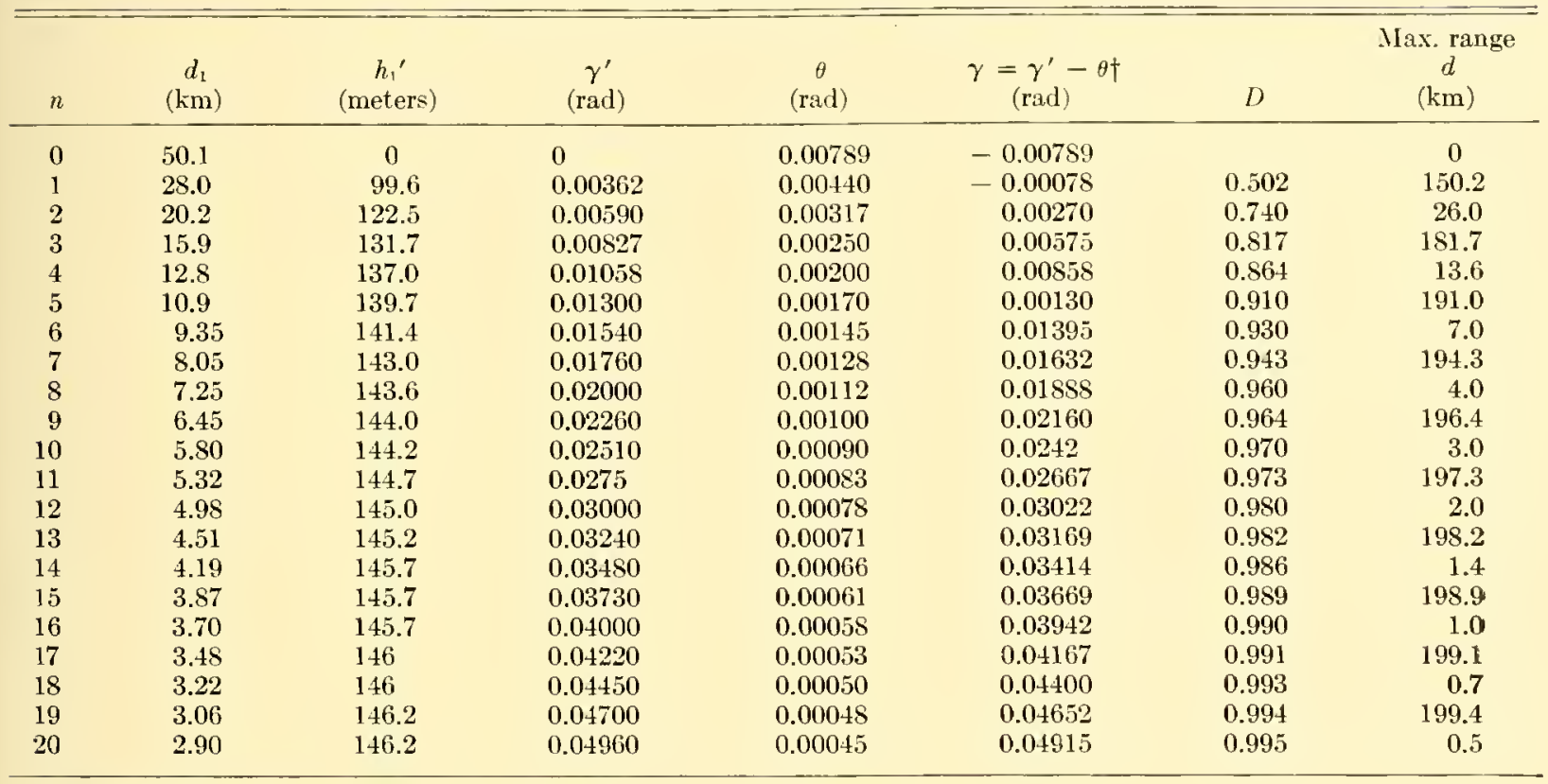

* Antenna gain and directivity factors have bees omitted from the above calculations.

t See Section 6.6.4.

3. For any $n$, including integral and fractional values, $d_{1}$ may be found from Figure 9 and the corresponding $h_{1}{ }^{\prime}$ from Figure 10. The angles $\gamma^{\prime}$ corresponding to lobe maxima may then be calculated from equation (32).

\subsubsection{Lobe Angles with Horizontal}

The angle $\gamma^{\prime}$ given by equation (32) is measured with respect to the tangent plane through the reflection point shown in Figure 8 . This plane is inclined at an angle $\theta$ with the horizontal at the base of the transmitter. The true angle $\gamma$ which the lobe-center line makes with the horizontal is

$$
\gamma=\gamma^{\prime}-\theta
$$

where odd values of $n$ give maxima and even values minima, provided the reflection phase shift is $\pi$ radians. The angle may be either positive or negative, as shown by equation (36).

\subsubsection{Use of Modified Divergence Factor}

The value of the divergence factor must be determined in order to calculate the maximum and minimum lobe lengths by equation (46) in Chapter 5 . A convenient formula for the divergence factor at the angles of lobe maxima is obtained by substituting $\gamma^{\prime}$ for $\psi$ in equation (92) in Chapter 5. The errors involved in this assumption have been given in Section 6.2.3. Substituting $\gamma^{\prime}=\psi$ in equation (92) 
in Chapter 5 yields

$$
D=\frac{1}{\sqrt{1+\frac{2 h_{1}{ }^{\prime}}{k a\left(\gamma^{\prime}\right)^{2}}}} .
$$

For lobe maxima

$$
h_{1}^{\prime}=\frac{n \lambda}{4 \gamma^{\prime}} .
$$

Hence

$$
D=\frac{1}{\sqrt{1+\frac{n \lambda}{2 k a\left(\gamma^{\prime}\right)^{3}}}} .
$$

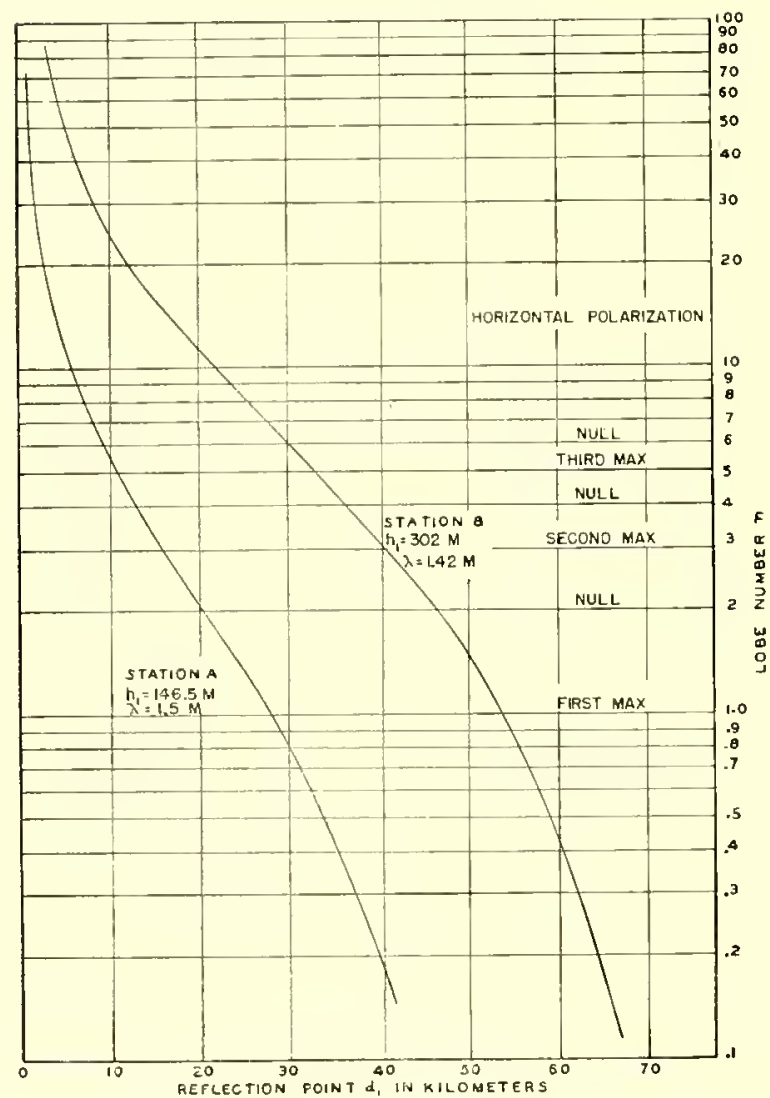

F1Gure 9. Loration of reflection point $d_{1}$ as a function of lobe number $n$.

Contours of constant $\gamma^{\prime}$ are shown in Figures 11 and 12 where $\gamma^{\prime}$ is a function of $D$ and $n \lambda$.

\subsubsection{Construction of Lobes}

For horizontal polarization, the distance $d_{\max }$ from the base of the transmitter to the lobe maximum is calculated from equation $(46)$ in Chapter 5 by substituting $K=\left(F_{2} / F_{1}\right) \rho D$ and $\sin ^{2}(\Omega / 2)=1$. For horizontal polarization $\rho=1$. Thus,

$$
d_{\max }=\sqrt{G_{1}} d_{0} \sqrt{\left[1-\frac{F_{2}}{F_{1}} D\right]^{2}+4 \frac{F_{2}}{F_{1}} D}
$$

or

$$
d_{\max }=\sqrt{G_{1}} d_{0}\left[1+\frac{F_{2}}{F_{1}} D\right]
$$

Here $F_{2}$ and $F_{1}$ are computed from the angles $\psi_{d}$ and $\nu$ given by equations (62) and (63) in Chapter 5 . The distance $d_{\min }$ from the transmitter base to the minimum

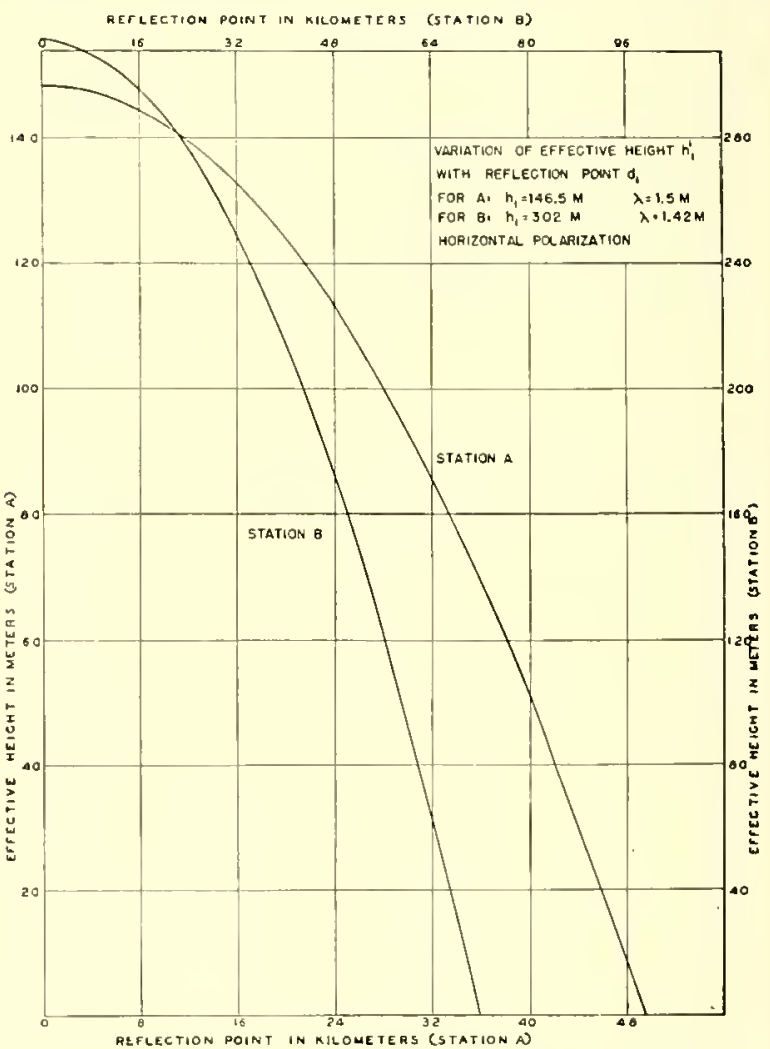

Figure 10. Variation in effective height $h_{1}^{\prime}$ with reflection point $d_{1}$.

point is obtained by substituting $\sin ^{2}(\Omega / 2)=0$ and $K=\left(F_{2} / F_{1}\right) D$ in equation (46) in Chapter 5 . Thus

$$
d_{\min }=\sqrt{G_{1}} d_{0}\left[1-\frac{F_{2}}{F_{1}} D\right] .
$$

The values of $D$ to be used in equations (40), (41), and (42) may be read directly from Figure $11 \mathrm{or}$ Figure 12, or calculated by equation (39). 
Intermediate points in the lobe may be formed by axsigning fractional values to $n$. The corresponding path-difference angle $\delta$ may be calculated in the following manner. Suppose it is desired to find intermediate points on the fourth lobe. The values of $n$ for this lobe range from $n=6$ to $n=8$, with the maximum at $n=7$. It follows that a change of as given in equation (46) in Chapter 5 , in which $K=\left(F_{2} / F_{1}\right)_{\rho} D$ and $\rho=1$. The value of $D$ may be read from Figures 11 and 12, using the assigned value of $n \lambda$ and the relation $\gamma^{\prime}=n \lambda / 4 h_{1}{ }^{\prime}$. The proper value of $d_{1}$ to be used in equation (63) in Chapter 5 to determine the antemua-pattern angle $\nu$ may be read from Figure 9 .

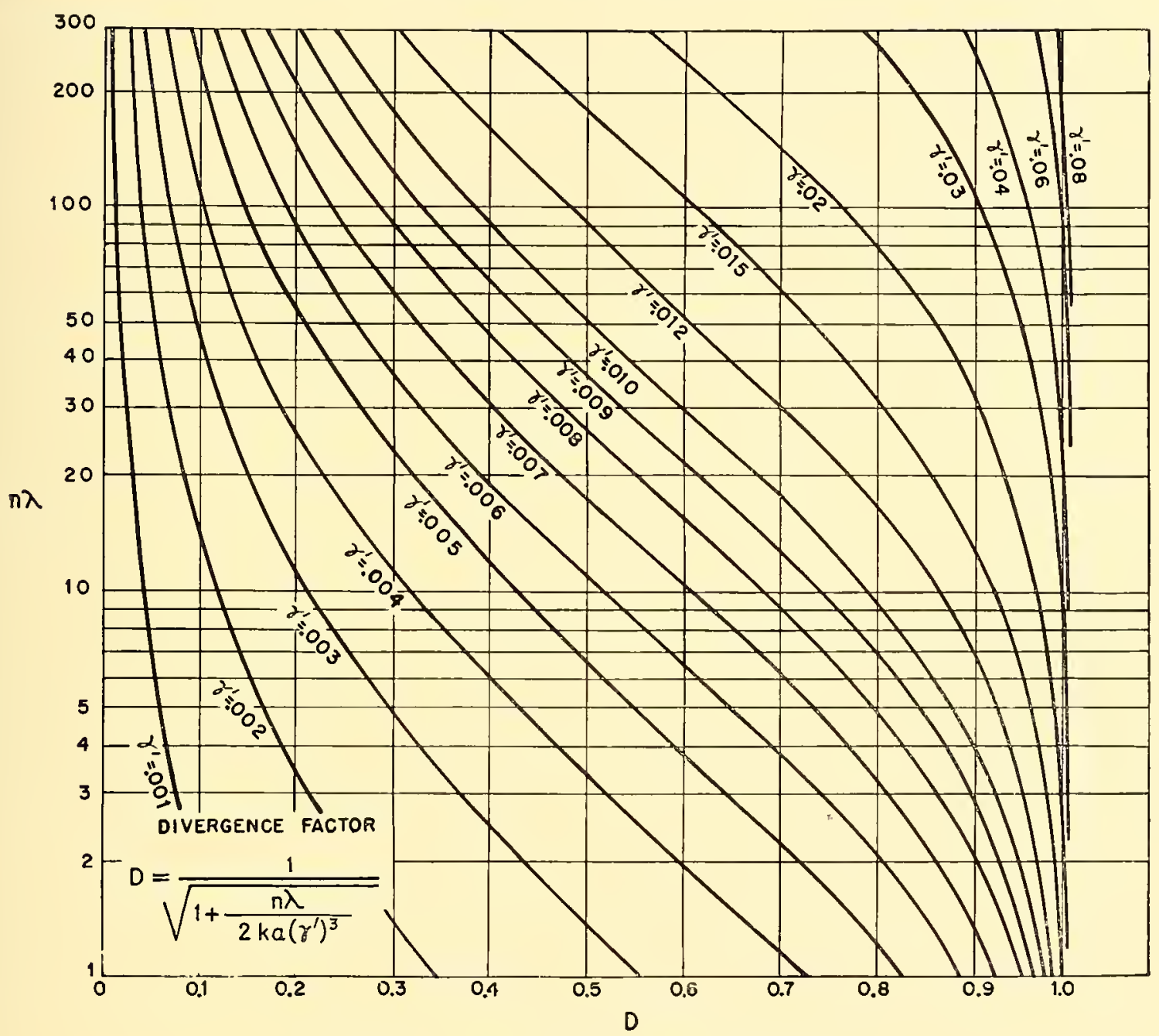

Figure 11. Contours of $\gamma^{\prime}$ as a function of $D$ and $n \lambda$.

2 in $n$ corresponds to a change of $2 \pi$ in $\delta$. Thus if $n=6.5, \delta=(0.5 / 2)(2 \pi)=\pi / 2=90^{\circ}$. For horizontal polarization $\Omega\left(=\delta+\phi^{\prime}\right)$ reduces to $\delta$, since $\phi^{\prime} \cong 0$. The distance from the transmitter base to this intermediate point in the lobe is equal to

$$
d=\sqrt{G_{1}} d_{0} \sqrt{(1-K)^{2}+4 K \sin ^{2}\left(\frac{\Omega}{2}\right)},
$$

\subsubsection{Correction for Low Angles}

The method outlined in Sections 6.6.2 to 6.6 .6 depends upon the assumption that $\gamma^{\prime} \rightarrow \psi$ or $d_{1} \rightarrow 0$. This assumption gives good lesults when $n$ is a large number but serious errors are involved for small values of $n$. The method described in this paragraph is designed to aroid this difficulty. The procedure 
consists in plotting point by point the lobe-center lines and angles of lobe maxima. The points will be locater by polar coordinates with the pole at the
Equations [(4ta to e)] are obtained by inspection of Figure 13. Equations (4tf) and (4tg) are derived as follows:

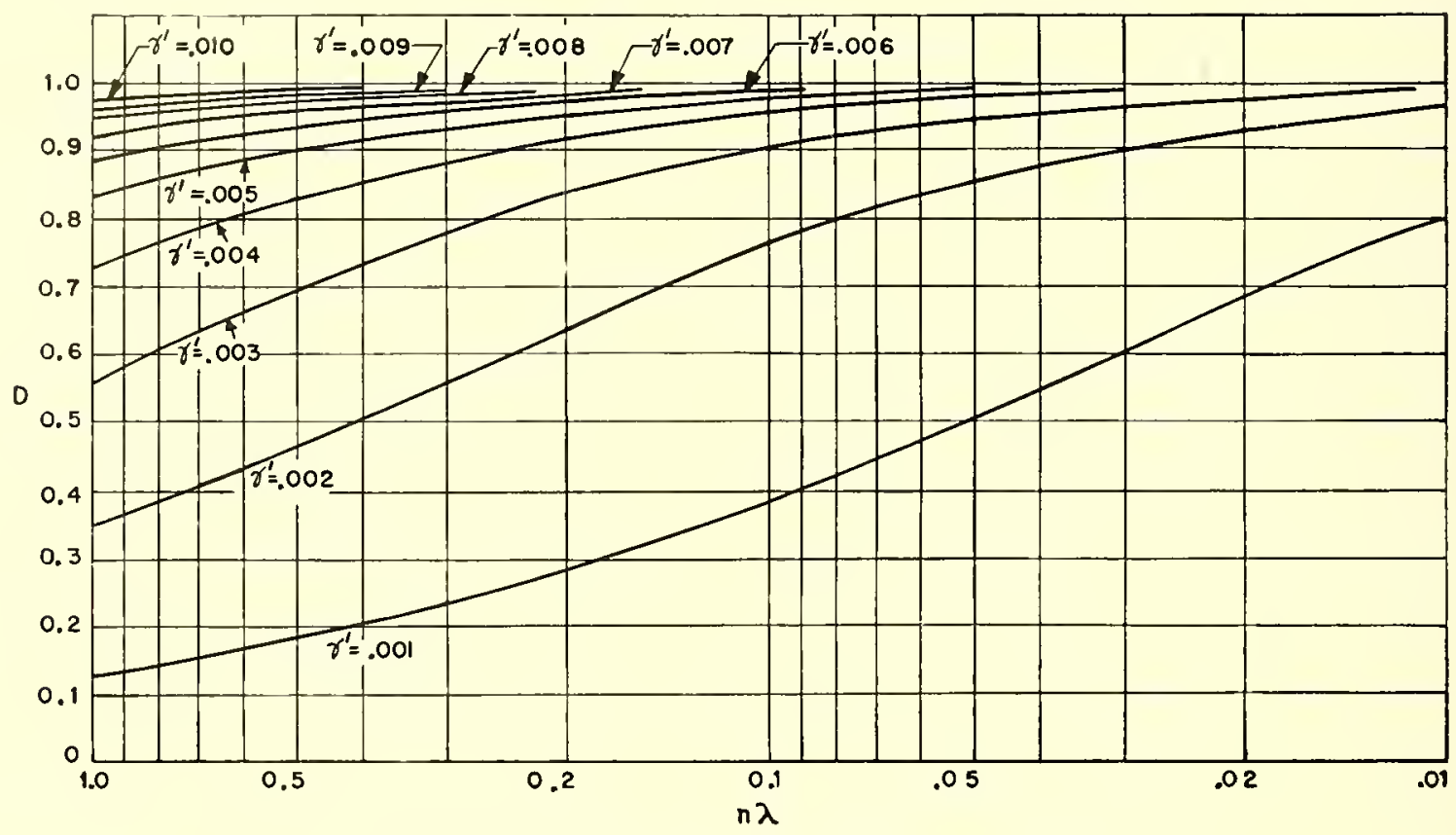

Fintre 12. Contours of $\boldsymbol{\gamma}^{\prime}$ as a function of $D$ and $n \lambda$.

transmitter. The coordinates are shown in Figure 13 as $r_{d}$ and $\gamma_{1}$.

Referring to Figure 13, the following relations hold when the angle $\psi$ is less than 10 degrees:

(a)

(b)

(c)

(e) $\quad r_{l} \rightarrow A+B=d_{1}+B$

(g)

$$
\psi=\frac{h_{1}{ }^{\prime}}{A}=\frac{h_{1}{ }^{\prime}}{d_{1}}
$$$$
\theta=\frac{d_{1}}{l_{i \prime l}}=\frac{A}{l_{i} a}
$$

$\gamma_{1}=\psi_{d}-\theta^{\circ}$

$\quad h_{1}^{\prime}=h_{1}-\frac{d_{1}^{2}}{2 l^{\prime} \prime}$

$\psi_{l l}=\left(\frac{B-d_{1}}{r_{d}}\right) \psi$

$$
B=\frac{(n \lambda / 2) d_{1}}{2 d \psi^{2}-n \lambda 2}
$$

$$
\begin{aligned}
\frac{\sin 2 \psi}{\sin \left(\psi+\psi_{d}\right)} & =\frac{r_{d}}{B} \rightarrow \frac{2 \psi}{\psi+\psi_{d}}, \\
\psi+\psi_{d} & =\frac{2 \psi B}{r_{d}}, \\
\psi_{d} & =\psi\left(\frac{2 B-r_{d}}{r_{d}}\right)=\psi\left(\frac{B-d_{1}}{r_{d}}\right) .
\end{aligned}
$$

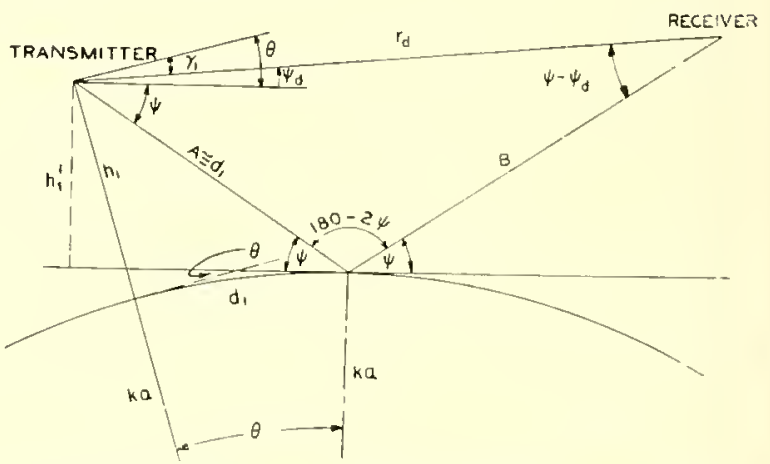

Figure 13. Geometry for radio propagation over spherical earth. See Figure 14 in Chapter 5. 
The path difference $\perp$ is given by

$$
\lrcorner=A+B-r_{d}=\frac{n \lambda}{2} \quad\left(\phi=180^{\circ}\right) .
$$

Hence

$$
A+B-\sqrt{A^{2}+B^{2}+2 A B \cos 2 \psi}=\frac{n \lambda}{2} .
$$

Squaring.

$$
B(2 A-n \lambda-2 A \cos 2 \psi)=n \lambda A-\left(\frac{n \lambda}{2}\right)^{2} .
$$

Therefore,

$$
B=\frac{\frac{n \lambda A}{2}-\frac{1}{2}\left(\frac{n \lambda}{2}\right)^{2}}{A(1-\cos 2 \psi)-\frac{n \lambda}{2}} .
$$

For small angles,

$$
\cos 2 \psi=1-\frac{(2 \psi)^{2}}{2}=1-2 \psi^{2}
$$

Hence

$$
\because=\frac{\frac{n \lambda A}{2}-\frac{1}{2}\left(\frac{n \lambda}{2}\right)^{2}}{2 \psi^{2} A-\frac{n \lambda}{2}}=\frac{n \lambda}{2} \frac{A-\frac{n \lambda}{4}}{2 A \psi^{2}-\frac{n \lambda}{2}} .
$$

Since the angle $\psi$ is of the order of a few degrees only, it is permissible to write $A=d_{1}$ in the above equation.

Neglecting further the term $n \lambda / t$ in comparison with $d_{1}$, which is permissible for short waves and small values of $n$, the above expression for $B$ reduces to equation $(44 \mathrm{~g})$.

The method of determining the locus of points having a path difference of $\lambda / 2$ (i.e., $n=1$ ) is as follows. Assume a value of $d_{1}$ and calculate the corresponding values of

$$
\begin{gathered}
h_{1}{ }^{\prime} \text { by equation }(4+\mathrm{d}), \\
\psi \text { by equation }(44 \mathrm{a}), \\
B \text { by equation }(44 \mathrm{~g}), \\
r_{d} \text { by equation }(44 \mathrm{e}), \\
\psi_{d} \text { by equation }(44 \mathrm{f}), \\
\theta \text { by equation }(44 \mathrm{~b}), \\
\gamma_{1} \text { by equation }(44 \mathrm{c}) .
\end{gathered}
$$

The assumed values of $d_{1}$ are limited to those which will give positive values of $B$ in equation ( $44 \mathrm{~g})$. Select as many values of $d_{1}$ as are necessary to plot a smooth path-difference locus. Repeat for $n=3$, etc.
6.7

This method of determining the angles of lobe maxima is of particular value in constructing the first few lobes, since the approximations of Sections 6.6.2 to 6.6 .6 may cause considerable error for low angles. These path-difference loci will intersect a vertical line drawn from the antenna to the ground below at heights equal to $n \mathrm{\lambda} / 4$. For short waves. this height is negligible for the lower lobes.

\section{LOBE-ANGLE METHOD (VERTICAL POLARIZATION)}

\subsubsection{Angles of Lobe Maxima}

The values of $n$ corresponding to the angles of lobe maxima are determined exactly as in Section 6.2.3 for the case of a plane earth. The values of $n$. in the expression $\gamma^{\prime}=n \lambda / 4 h_{1}{ }^{\prime}$ are increased above those for horizontal polarization by an amount $(\Delta n)$ to compensate for the reduced phase shift on reflection. In other words, the path difference must be greater than integral multiples of $\lambda / 2$ to compensatc for the reduced phase shift. The expression for this compensation, from equation (5), is

$$
\Delta n=\frac{\phi^{\prime}}{\pi}
$$

Hence

$$
\gamma^{\prime}=(n+\Delta n) \frac{\lambda}{4 h_{1}^{\prime}} .
$$

[See Figure 8 and equation (32).]

\subsubsection{Construction of Lobes}

As a first approximation, the angles of lobe maxima are calculated on the basis of horizontal polarization. A table is constructed giving values of $n$ and $\gamma^{\prime}$ for maxima and minima. The next approximation is to let $\psi=\gamma^{\prime}$. This assumes that $d_{1}<<d_{2}$. The values of $\phi^{\prime}$ and $\rho$ may then be found from reflection curves, and $(\Delta n)$ calculated from equation (45). The corrected values of $\gamma^{\prime}$ may be determined from equation (46). It is simpler to find $\gamma^{\prime}$ by interpolating between integral values of $n$ in the $n$ versus $\gamma^{\prime}$ table previously constructed. The values of $d_{\max }$ and $d_{\min }$ are

$$
\begin{aligned}
& d_{\max }=\sqrt{G_{1}} d_{0}(1+K), \\
& d_{\min }=\sqrt{G_{1}} d_{0}(1-K),
\end{aligned}
$$


where $K=\left(F_{2} / F_{1}\right) \rho D$. The divergence factor may be found directly from Figure 11 for the corrected values of $n$ and $\gamma^{\prime}$. It will be found that for the higher lobes, the effect of $(\Delta n)$ upon the ralue of the divergenee factor is negligible.

Table 5 shows calculations of the corrected values of $n$ and $\gamma$ when the radiation from antema $A$ of 6.6.3 and Table $t$ is vertically polarized. Transmission over sea water is assumed. Tables 6 and 7 illustrate the effects of vertical polarization on reducing the maxima and increasing the minima. of $n$ and minimum ranges. The free-space range is $100 \mathrm{~km}$, as in Section 6.6.3. The last column shows the ratio of ranges for horizontal and rertical polarization

\subsection{GENERALIZED COVERAGE DIAGRAMS (HORIZONTAL POLARIZATION) 6.8.1 Basic Parameters}

The $u-v$ method applied to generalized coordinates which was given in Section 6.5.2 may be

TABLE 5

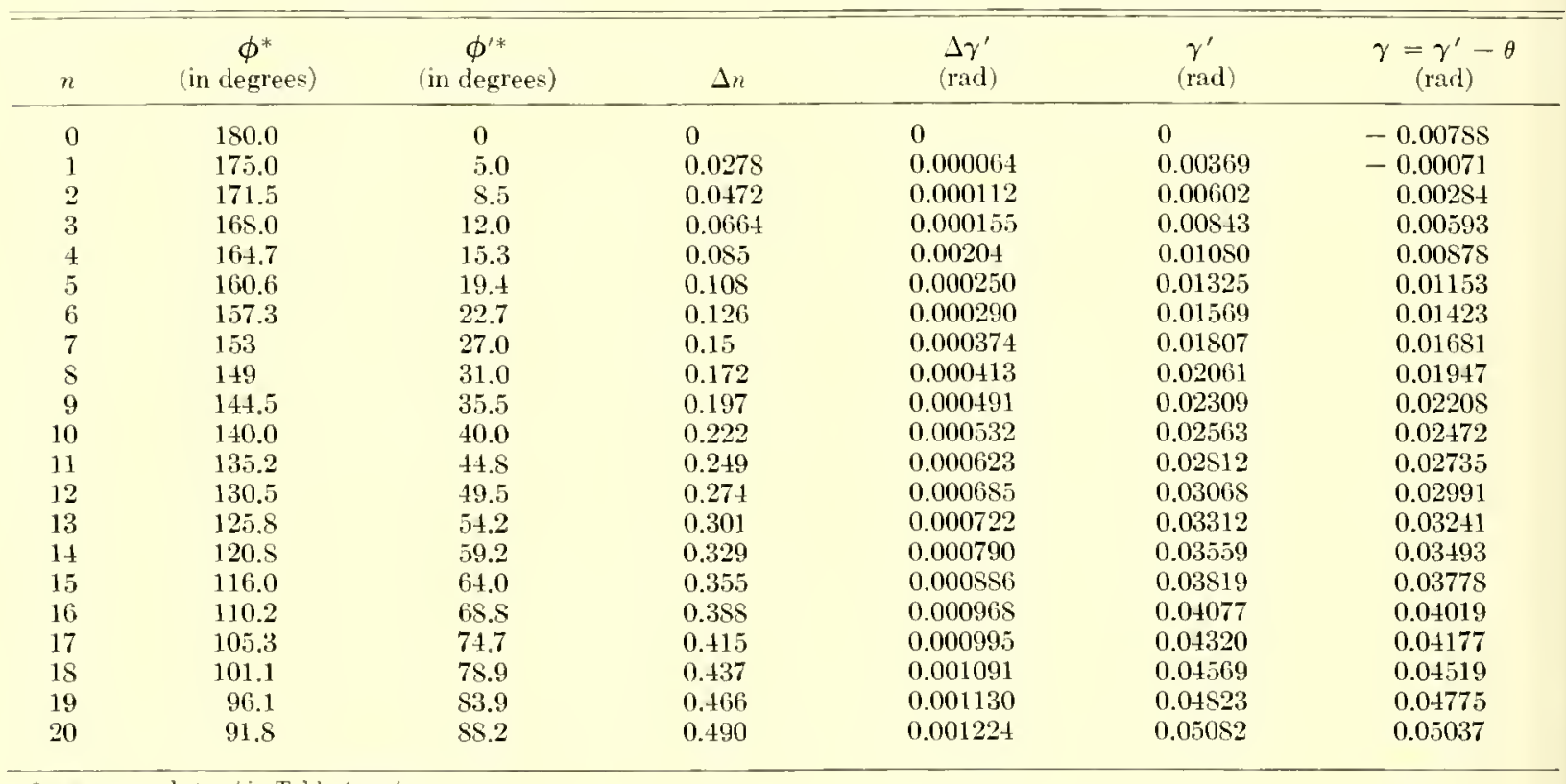

" $\phi$ corresponds to $\gamma$ in Table $4 . \quad \phi^{\prime}=\pi-\phi$.

\begin{tabular}{|c|c|c|c|c|}
\hline$n$ & $K$ & $\begin{array}{c}d_{\max }(\mathrm{HP}) \\
(\operatorname{km})\end{array}$ & $\begin{array}{c}d_{\max }(\mathrm{VP}) \\
(\mathrm{km})\end{array}$ & $\frac{d_{\max }\left(\mathrm{T}^{\top}\right)}{d_{\max }(\mathrm{HP})}$ \\
\hline 1 & 0.904 & 150.2 & 145.5 & 0.968 \\
\hline 3 & 0.765 & 181.7 & 162.5 & 0.895 \\
\hline 5 & 0.670 & 191.0 & 161.0 & $0.8 t t$ \\
\hline 7 & 0.585 & 194.3 & 155.2 & 0.80 \\
\hline 9 & 0.516 & 196.4 & 149.8 & 0.762 \\
\hline 11 & 0.458 & 197.3 & 141.6 & 0.733 \\
\hline 13 & 0.415 & 198.2 & 140.8 & 0.710 \\
\hline 15 & 0.385 & 198.9 & 138.0 & 0.695 \\
\hline 17 & 0.362 & 199.1 & 135.7 & 0.681 \\
\hline 19 & 0.360 & 199.4 & 135.8 & 0.680 \\
\hline
\end{tabular}

Tables 6 and 7 show the effect of a reflection coefficient which is less than unity upon the maximum and minimum ranges of station $\mathrm{A}$ in Section 6.6.3. The first table gives odd values of $n$ and maximum ranges; the second table gives even values

\begin{tabular}{|c|c|c|c|c|}
\hline$n$ & 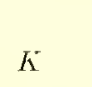 & $\begin{array}{c}d_{\min }(\mathrm{IIP}) \\
(\mathrm{km})\end{array}$ & $\begin{array}{c}d_{\min }(V P) \\
(\mathrm{km})\end{array}$ & $\frac{d_{\min }(\mathrm{VP})}{d_{\min }(\mathrm{HP})}$ \\
\hline 2 & 0.835 & 26.0 & 38.2 & 1.47 \\
\hline 4 & 0.725 & 13.6 & 37.4 & 2.75 \\
\hline 6 & 0.625 & 7.0 & 41.9 & 5.98 \\
\hline 8 & 0.548 & 4.0 & 44.8 & 11.2 \\
\hline 10 & 0.486 & 3.0 & 52.9 & 17.6 \\
\hline 12 & 0.436 & 2.0 & 57.3 & 28.6 \\
\hline 14 & 0.40 & 1.40 & 60.6 & 43.3 \\
\hline 16 & 0.375 & 1.0 & 62.9 & 62.9 \\
\hline 18 & 0.360 & 0.70 & 64.3 & 91.9 \\
\hline 20 & 0.355 & 0.50 & 64.7 & 129.4 \\
\hline
\end{tabular}

extended to all transmitter heights and wavelengths. In this method, points on the lobe are located by the intersection of the path-difference locus with the normalized distance envelope. The basic parameters are $d_{0}$ and $R$. 
In constructing a coverage diagram for a doublet transmitter, the transmitter height, the wavelength and the radio gain are known. It will be shown in Section 6.8.2 that the normalized free-space distance, $\underline{d_{0}}=d_{0} / d_{T}$, is related to the gain factor $A$ by the equation

$$
\frac{1}{\underline{d_{0}}}=\frac{8 \pi}{3 \lambda} d_{T} A
$$

The path-difference parameter $R$ has been expressed in equation (114) in Chapter 5 in terms of a heightwavelength factor $r$ which is defined by

$$
R=n r,
$$

where

$$
r=\frac{1}{2} \sqrt{\frac{k a}{2}} \frac{\lambda}{h_{1}^{3 / 2}} .
$$

The first maximum, which for horizontal polarization occurs when $\Delta=\lambda / 2$, corresponds to $n=1$, the second minimum to $n=2$, etc. Recalling the discussion in Section 6.5.2, it follows that it is possible to construct coverage diagrams in generalized coordinates with $r$ the pattern or chart parameter and $d_{0}$ the curve parameter on a chart for which $r$ is fixed.

\subsubsection{Determination of $d_{0}$}

It is possible to express $\underline{d}_{0}=d_{0} / d_{T}$ in terms of $E / E_{1}$, the ratio of the field strength $E$ corresponding to the lobe, to the free-space field $E_{1}$ at unit distance from the transmitter. Since

$$
d_{0}=\frac{E_{1}}{E},
$$

it follows that

$$
\underline{d_{0}}=\frac{d_{0}}{d_{T}}=\frac{1}{d_{T}} \frac{E_{1}}{E} .
$$

The ratio $E_{1} / E$ may be expressed in terms of the gain factor $A$ through the following relationships. By equation (16) in Chapter 2

$$
P_{1}=\frac{E^{2} d^{2}}{45} .
$$

When $d=1$, this gives

$$
P_{1}=\frac{E_{1}^{2}}{45} .
$$

For a doublet receiver with matched load and ad- justed for maximum power transfer, equation (17) in Chapter 2 gives

$$
P_{2}=\frac{E^{2}}{120 \pi} \cdot \frac{3 \lambda^{2}}{8 \pi}
$$

Hence

$$
\frac{E_{1}}{E}=\frac{3 \lambda}{8 \pi} \sqrt{\frac{P_{1}}{P_{2}}}=\frac{3 \lambda}{8 \pi A} .
$$

Substituting the value of $E_{1} / E$ from equation (54) into equation (52):

$$
\underline{d_{0}}=\frac{1}{d_{T}}\left(\frac{3 \lambda}{S \pi A}\right)
$$

and

$$
\frac{1}{\underline{d_{0}}}=d_{T}\left(\frac{8 \pi}{3}\right) \frac{\lambda}{A}=\sqrt{2 k a}\left(\frac{8 \pi}{3}\right) \frac{\lambda}{A} \sqrt{h_{1}} .
$$

Equation (56) shows that if $\log h_{1}$ is plotted against $\log A$ for fixed values of $\underline{d}_{0}$ and $\lambda$, a straight line results. These straight lines are shown in Figure 14.

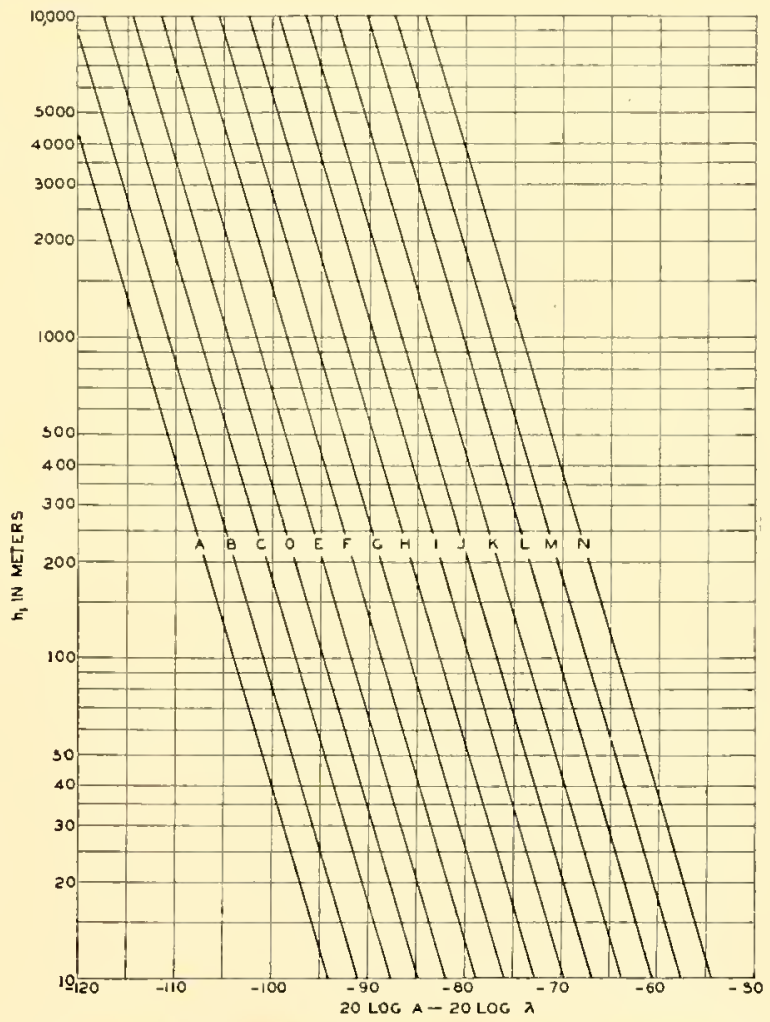

Figure 14. Values of $d_{0}$ as functions of $h_{1}$ and $20 \log A$ $-20 \log \lambda$. (See equation 56. The letters refer to coverage diagrams plotted in Figures 16 to 39 .)

If $h_{1}$ and $E / E_{1}$ or $h_{1}, \lambda$, and $A$ are known, $d_{0}$ may be calculated from equations (52) or (55). The 

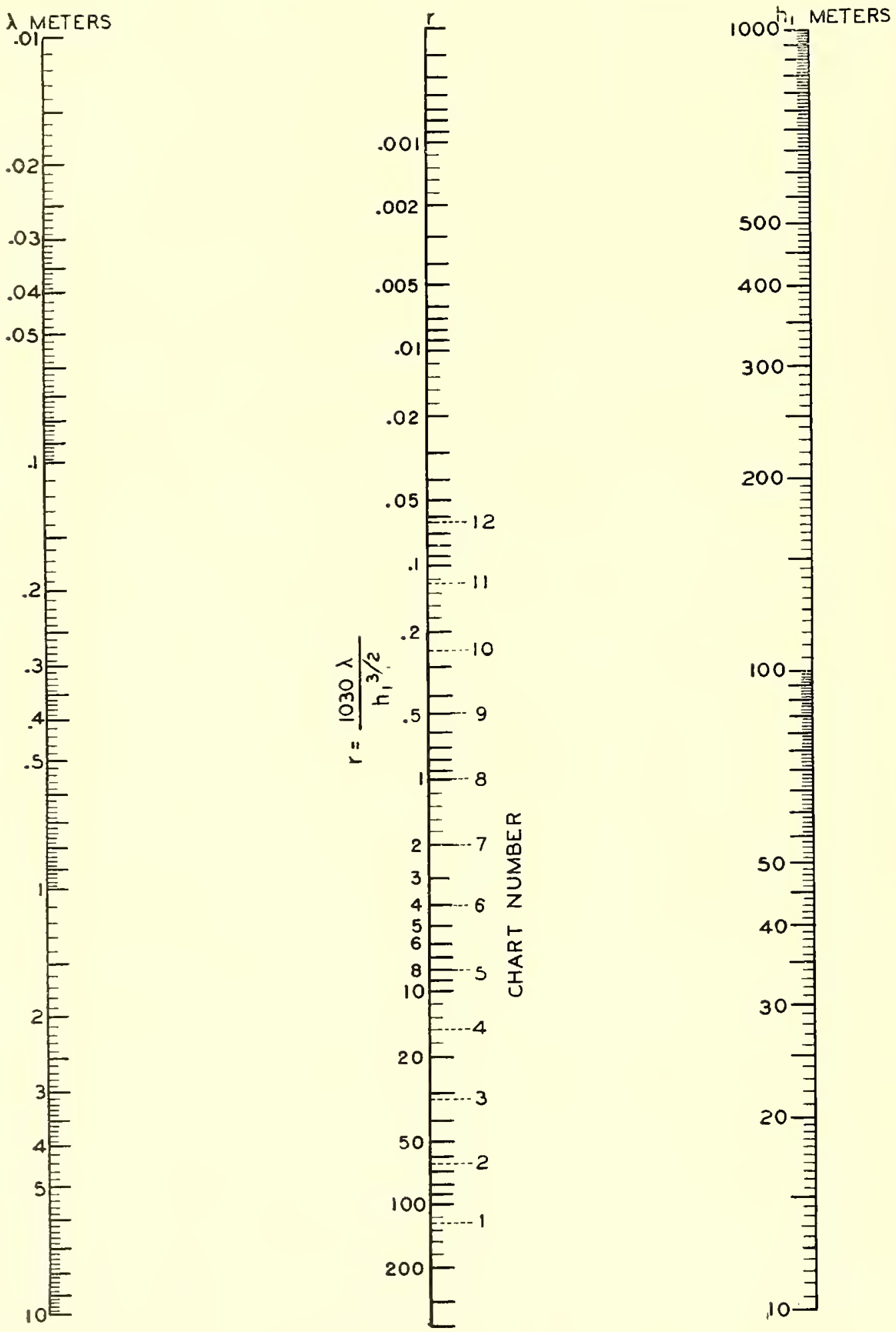

I'IGUR: 15. Chart number and $r$ as a function of $\lambda$ and $h_{1}$. (See Figures 16 and 17.) 
value of $\underline{d}_{0}$ determines the range of the lobe for specified values of $r$. In Figure 1t, the various values of $d_{0}$ used in constructing the charts arc specified as $A, B, C, \cdots, M, N$, and are shown as functions of $h_{1}$ as ordinate and $20 \log A-20 \log \lambda$ as abscissa.

\subsubsection{Determination of $r$}

Figure 15 shows $r$ for various values of transmitter height $h_{1}$ and wavelength $\lambda$ wherc

$$
\frac{1}{r}=\frac{2 h_{1} d_{T}}{\lambda k a}=\frac{h_{1}^{3 / 2} \sqrt{2 k a}}{\lambda k a}=\frac{2 h_{1}^{3 / 2}}{\lambda} \sqrt{\frac{2}{k a}} .
$$

The values of $r$ determine how the path-difference curves intersect the envelopes corresponding to assignod vahues of $\sin ^{2}(\Omega / 2)$ in the equation

$$
v=\frac{d}{d_{T}}=d_{0} \sqrt{G_{1}} \sqrt{(1-D)^{2}+4 D \sin ^{2} \frac{\Omega}{2}} .
$$

The generalized coverage charts are designated as $1,2, \cdots, 11,12$ in Figures 16 to 39, with the chart number being given by Figure 15 .

\subsubsection{Use of Charts}

Figures 16 to 39 give 24 basic charts developed by the Radiation Laboratory and presented in report 702. On each chart are complcte lobes or lobe outlines labeled $A, B, C, \cdots, M, N$. In the following description these letters are referred to as lobe letters and the numbers 1 to 12 as chart numbers. It will be noteck that each chart number is plotted to two scales, giving 24 charts in all.

The problem of constructing coverage diagrams resolves itself into finding the chart number and lobe letter corresponding to given values of gain factor $A$, transmitter height $h_{1}$, and wavclength $\lambda$. As stated in Section 6.8.1, the basic parameters of the generalized coverage diagrams are $R$ and $\underline{d_{0}}$ or $r$ and $\underline{d_{0}}$.
The value of $r$ is given in Figure 15 as a function of $\lambda$ and $h_{1}$. Figure 15 was constructed fiom equation (51) which, after the substitution of numerical values, becomes:

$$
r=\frac{1030 \lambda}{h_{1}^{3 / 2}} \quad\left(\text { for } l_{i}=\frac{4}{3}\right)
$$

'The value of $r$ determines the chart number. The lobe letter is found from the $\underline{d}_{0}$ corresponding to the given gain factor, transmitter height, and frequency. Figure 14 gives the lobe letters $A, B, C, \cdots, M, N$ as functions of $20 \log A-20 \log \lambda$ and the transmitter height. The relationship for plotting these lines is given by equation (56).

As an illustration of the use of the generalized coverage diagrams, assume $20 \log A=-83, h_{1}=$ 33 meters, and $f=200 \mathrm{mc}(\lambda=1.5$ moters $)$. If a straight line is drawn connecting $h_{1}=33$ and $\lambda=1.5$ in Figure 15, it will intersect the $r$ scale at $r=8$. Thus the chart number is 5 . Now the lobe letter to be used in chart 5 must be found. For this case, $20 \log A-20 \log \lambda=-86.48$. The coordinates $20 \log A-20 \log \lambda=-86.48$, and $h_{1}=33$, determine the lobe letter to be $E$ in Figure 14. Figure 24 shows lobe $E$ on chart 5 . The first lobe is shown completely, together with the lower half of the second lobe. It must be noted that the coordinates of thesc charts, $v=d / d_{T}$ and $u=h_{2} / h_{1}$, are dimensionless. To convert to height $h_{2}$ and range $d$, the rertical distances must be multiplied by $h_{1}$ and the horizontal distances by $d_{T}$. In this case $h_{1}=33$ meters and $d_{T}=\sqrt{2 k a h_{1}}=23.6 \mathrm{~km}$. The actual coordinates of the position of maximum range are $h_{2}=$ $375 \times 33=12.4 \mathrm{~km}$ and $d=15.4 \times 23.6=365 \mathrm{~km}$.

The charts given in Figures 16 to 39 may be used for drawing coverage diagrams where the reflection coefficient is assumed equal to -1 and when the directivity factor $F_{2} / F_{1}$ is equal to unity. Each chart may be used for values of $r$ near that for which the chart is drawn. For intermediate values, interpolation betwoen charts is necessary. Errors inherent in interpolation limit the accuracy attained. 


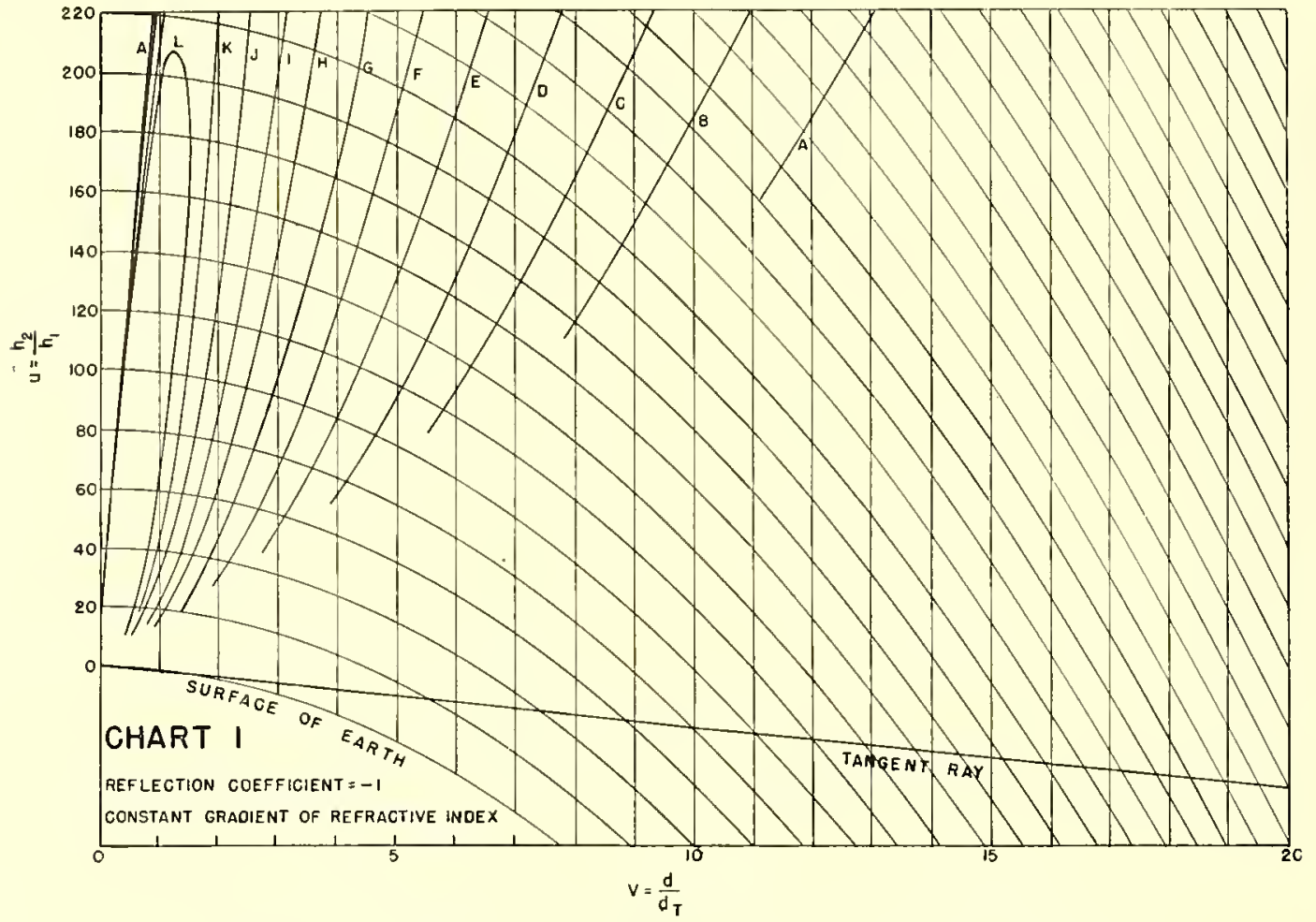

Figure 16. Generalized coverage diagram for $r=128$. (See Figure 15.)

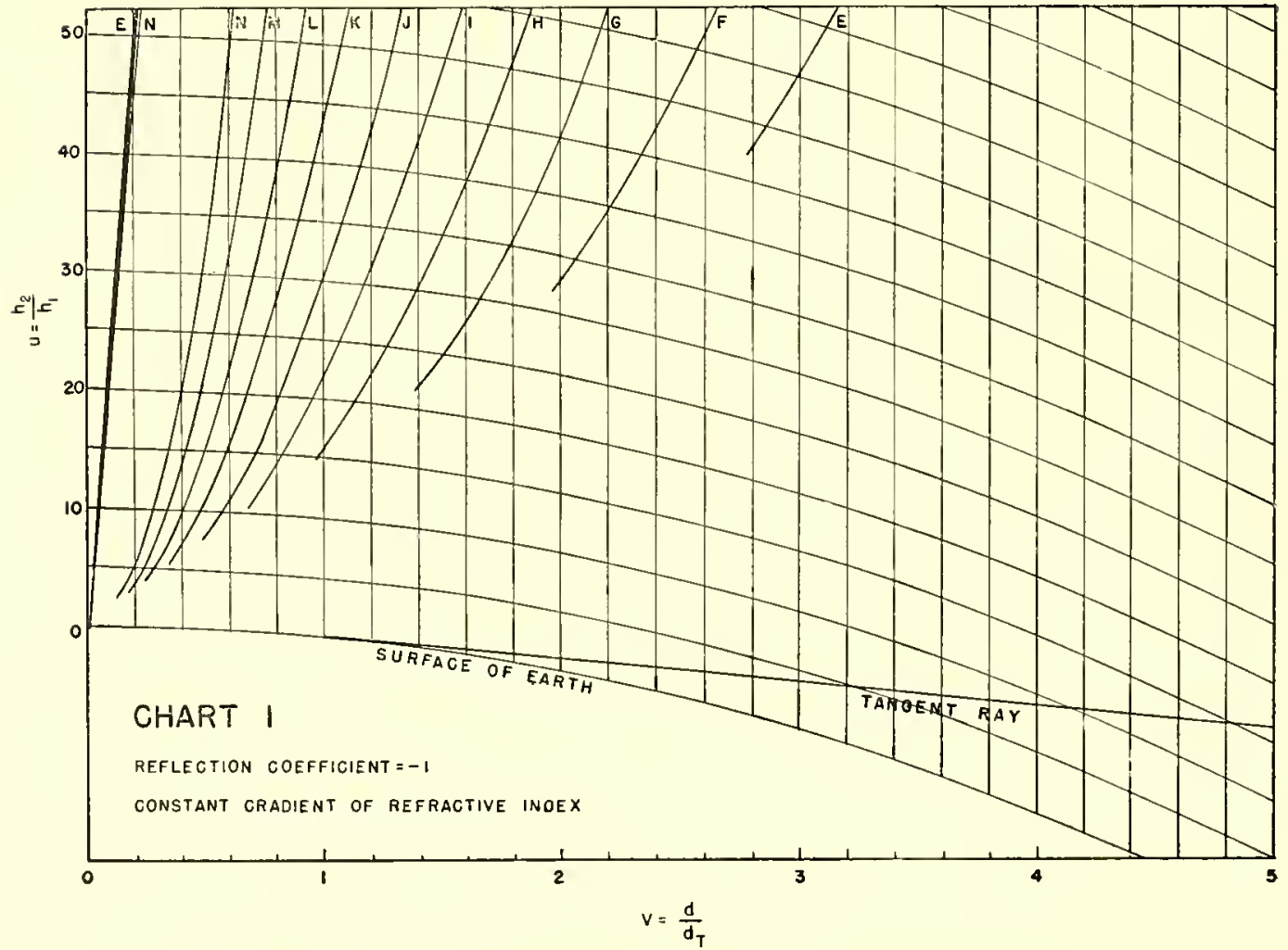

Figure 17. Generalized coverage diagram fur $r=128$ (See Figure 15.) 


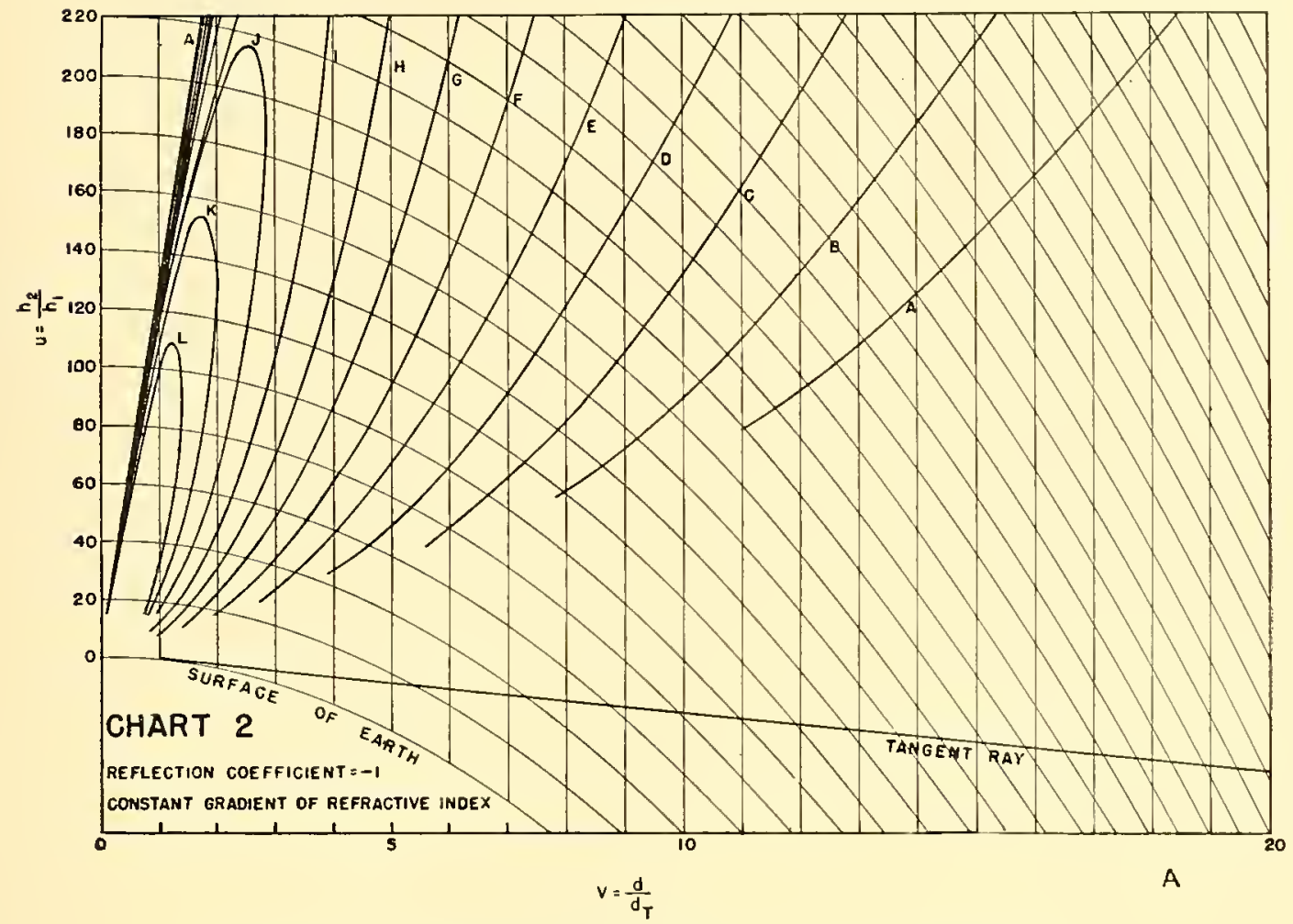

Figure 18. Generalized coverage diagram for $r=64$. (See Figure 15.)

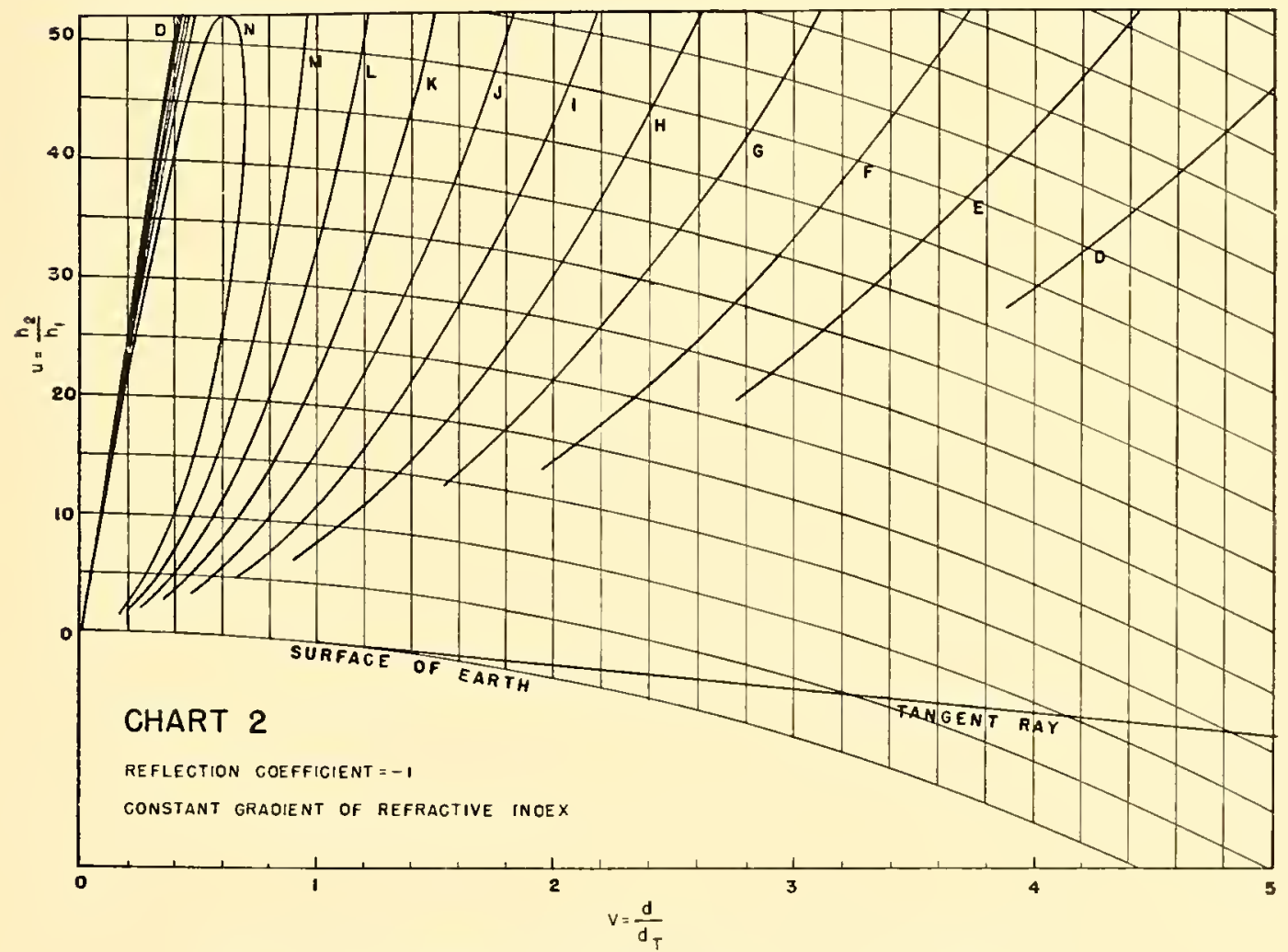

Figure 19. Generalized coverage diagram for $r=64$. (See Figure 15.) 


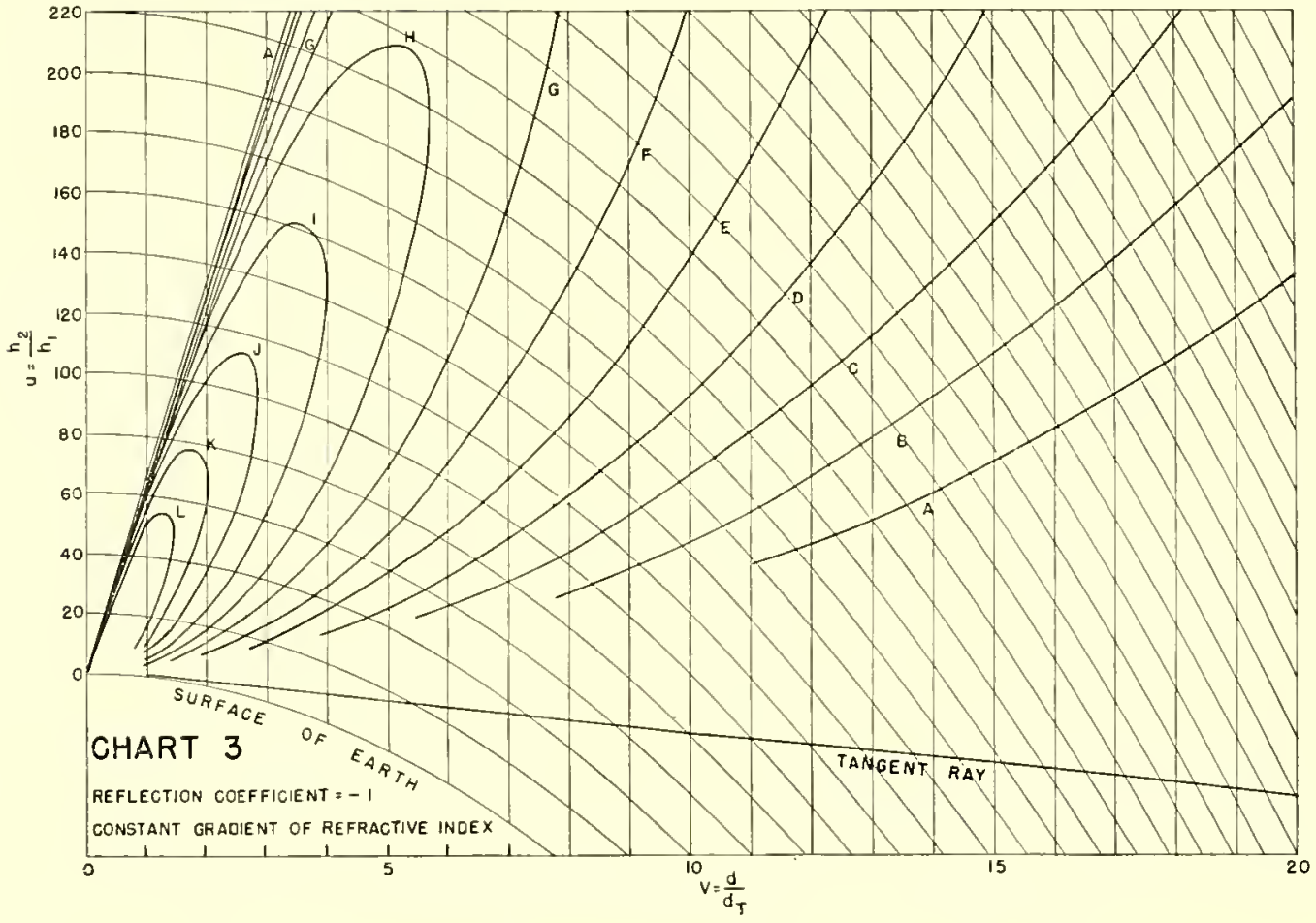

Figrre 20. Generalized coverage diagram for $r=32$. (See Figure 15.)

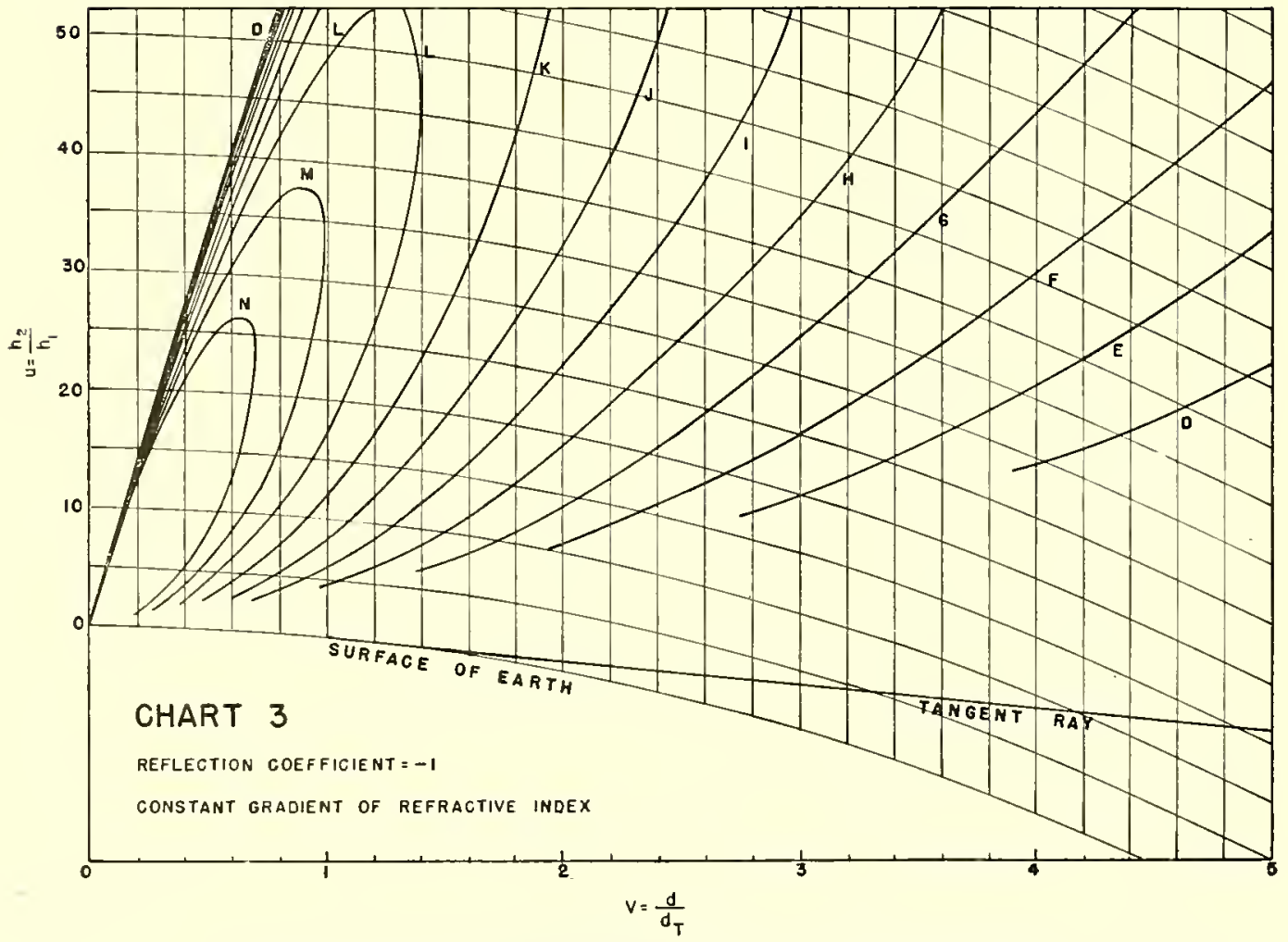

Figcre 21. Generalized coverage diagram for $r=32$. (See Figure 15.) 


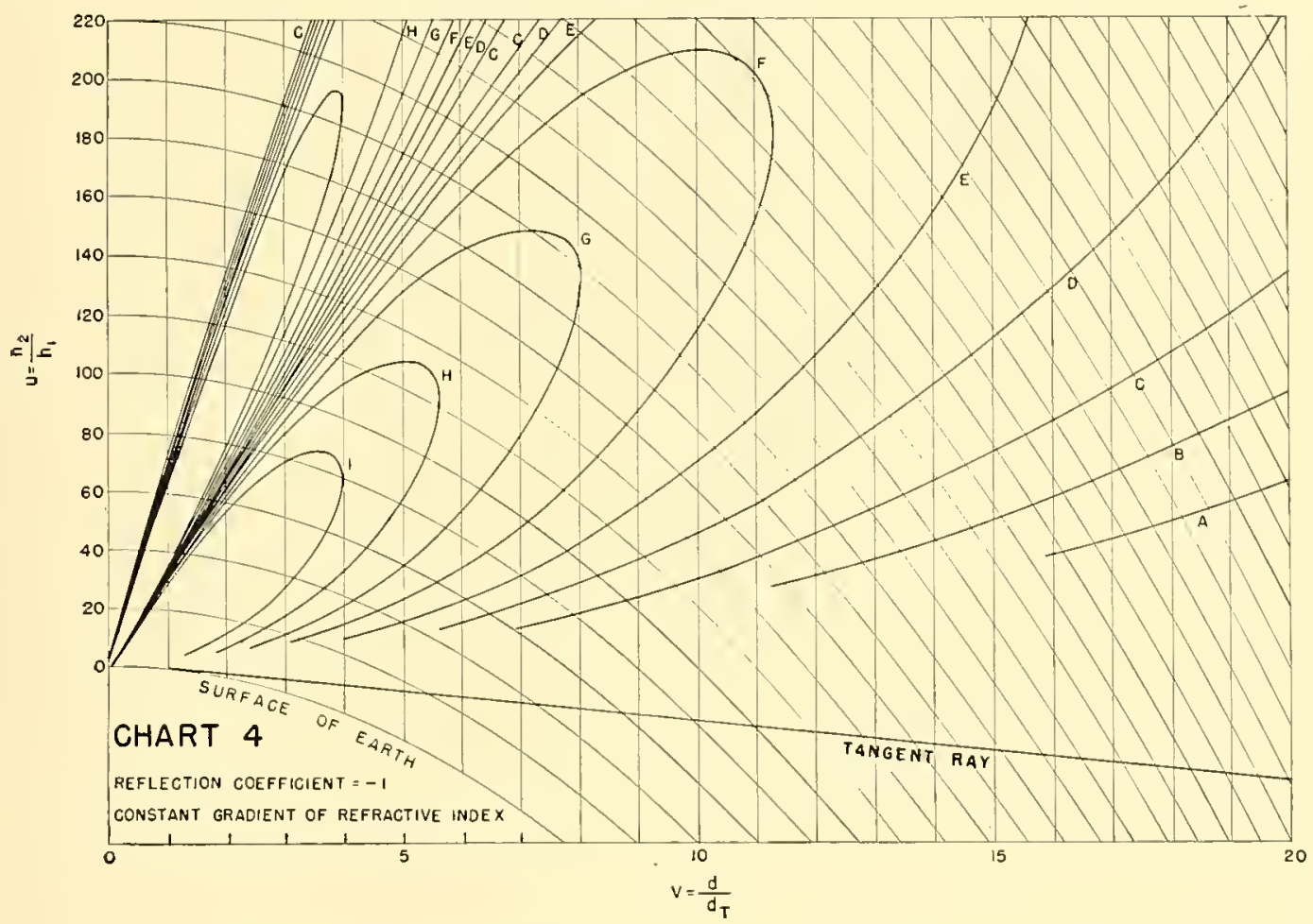

Figure 22. Generalized coverage diagram for $r=16$. (See Figure 15.)

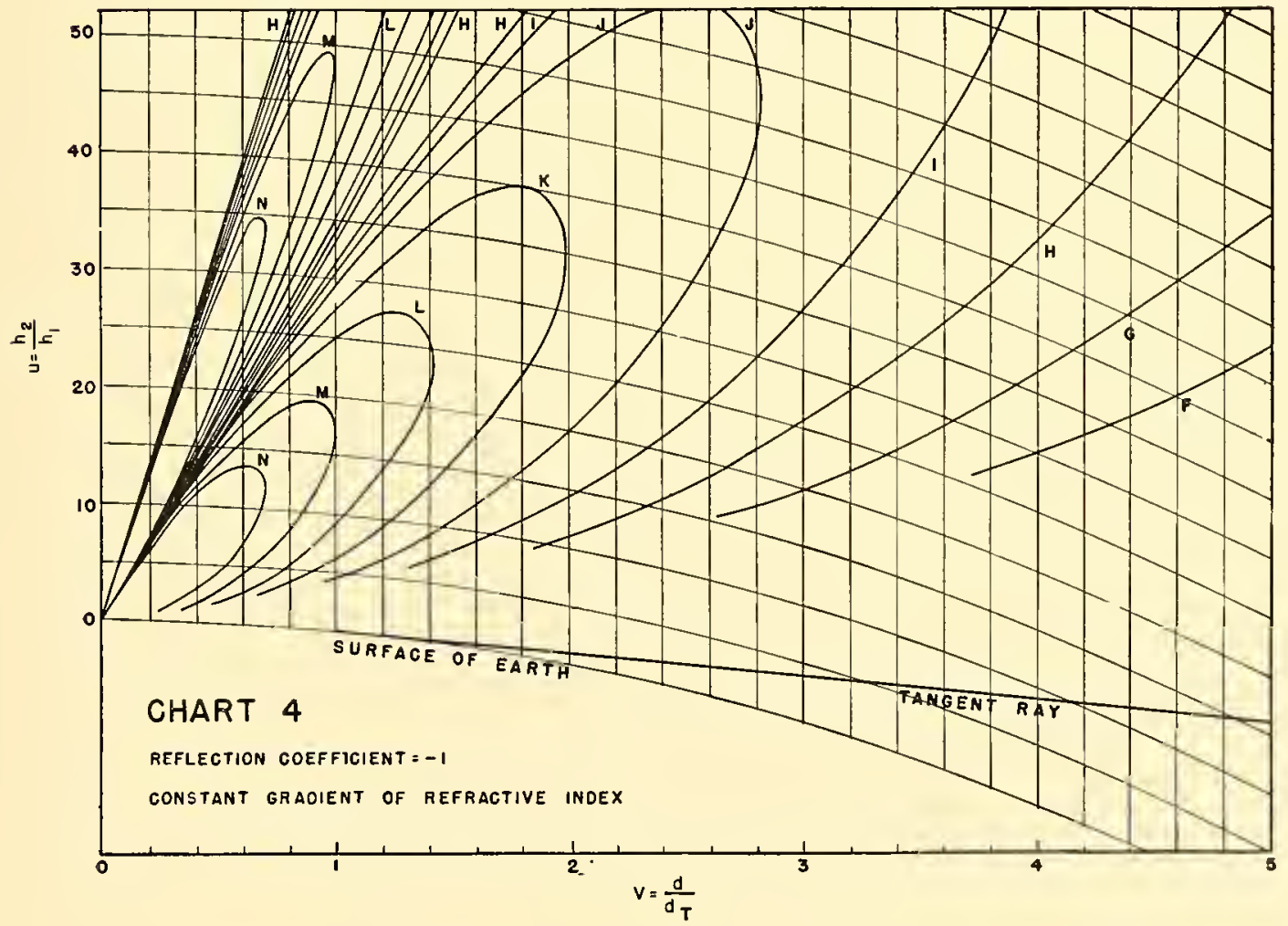

Figure 23. Generalized coverage diagram for $r=16$. (See Figure 15.) 


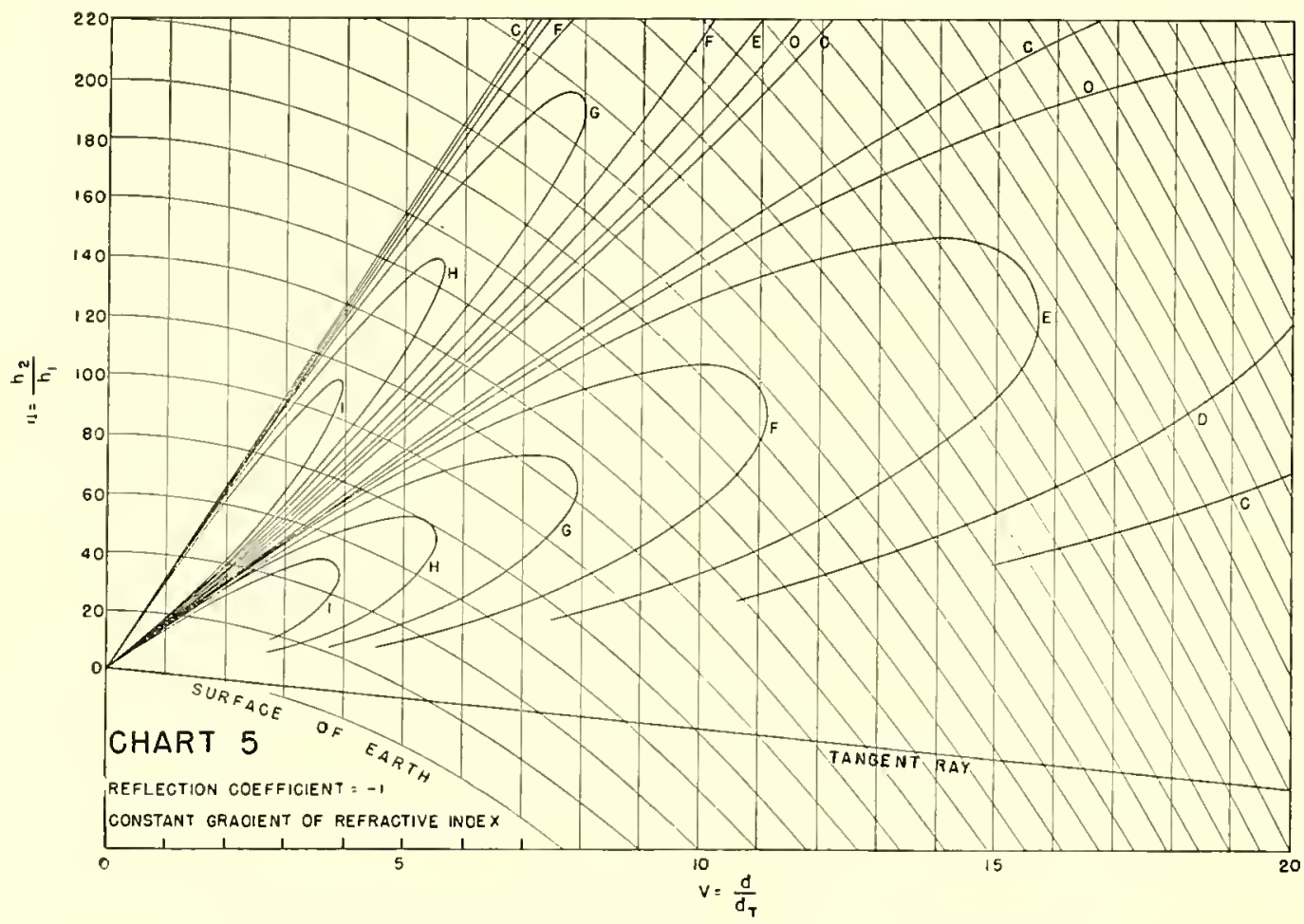

Figure 24. Generalized coverage diagram for $r=8$. (See Figure 15.)

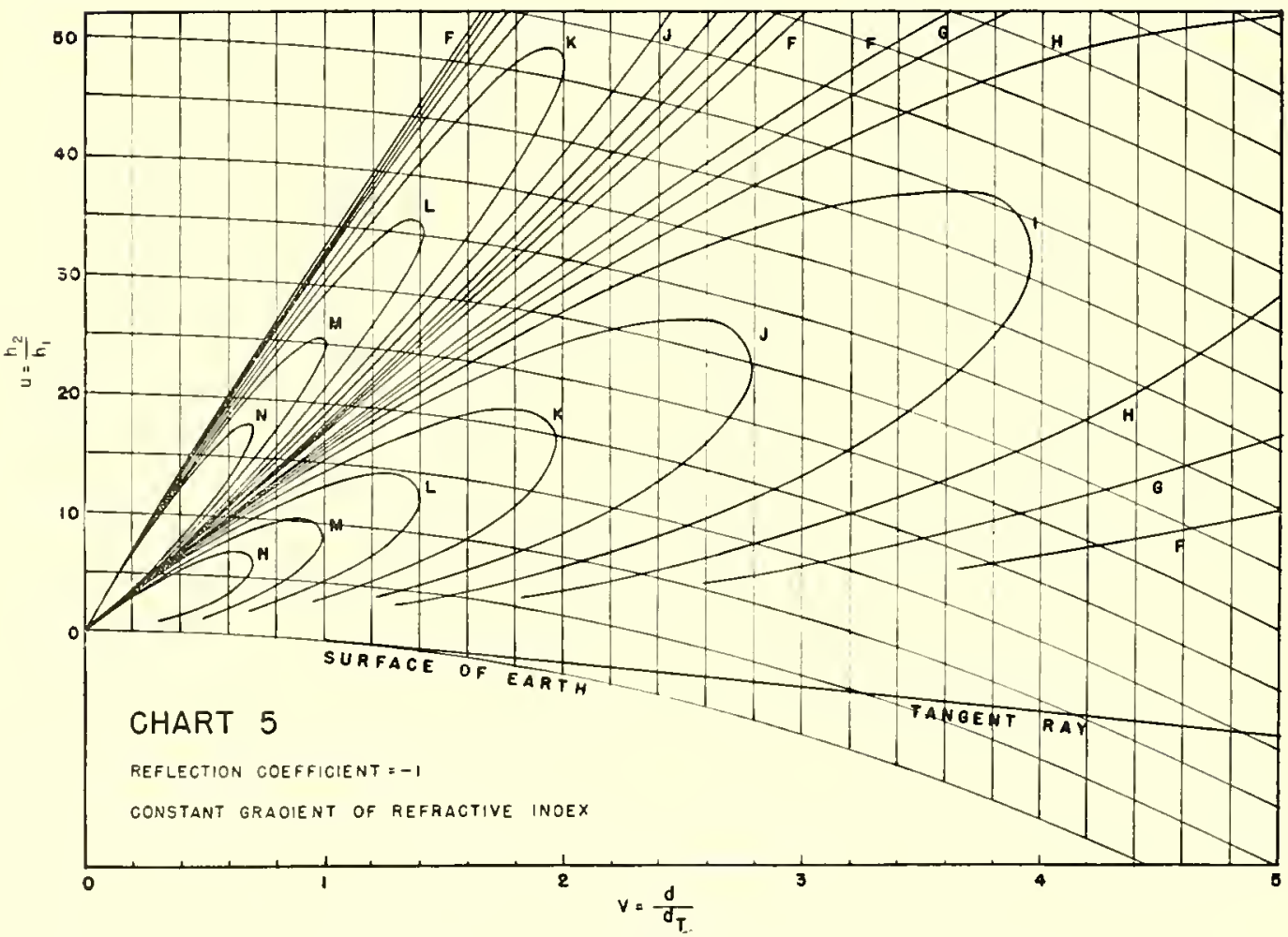

Figure 25. Generalized coverage diagram for $r=8$. (See Figure 15.) 


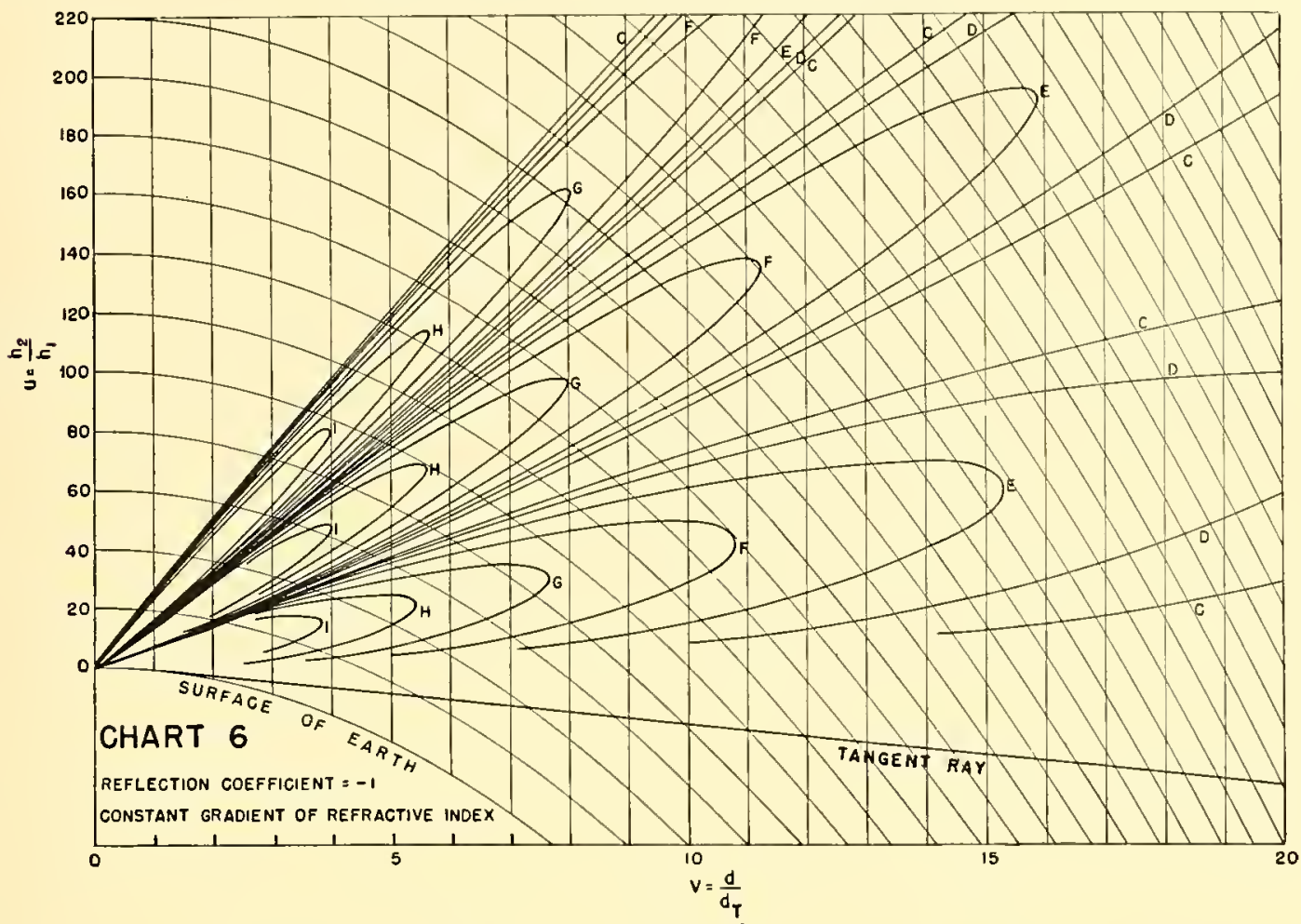

Figure 26. Generalized coverage diagram for $r=4$. (See Figure 15.)

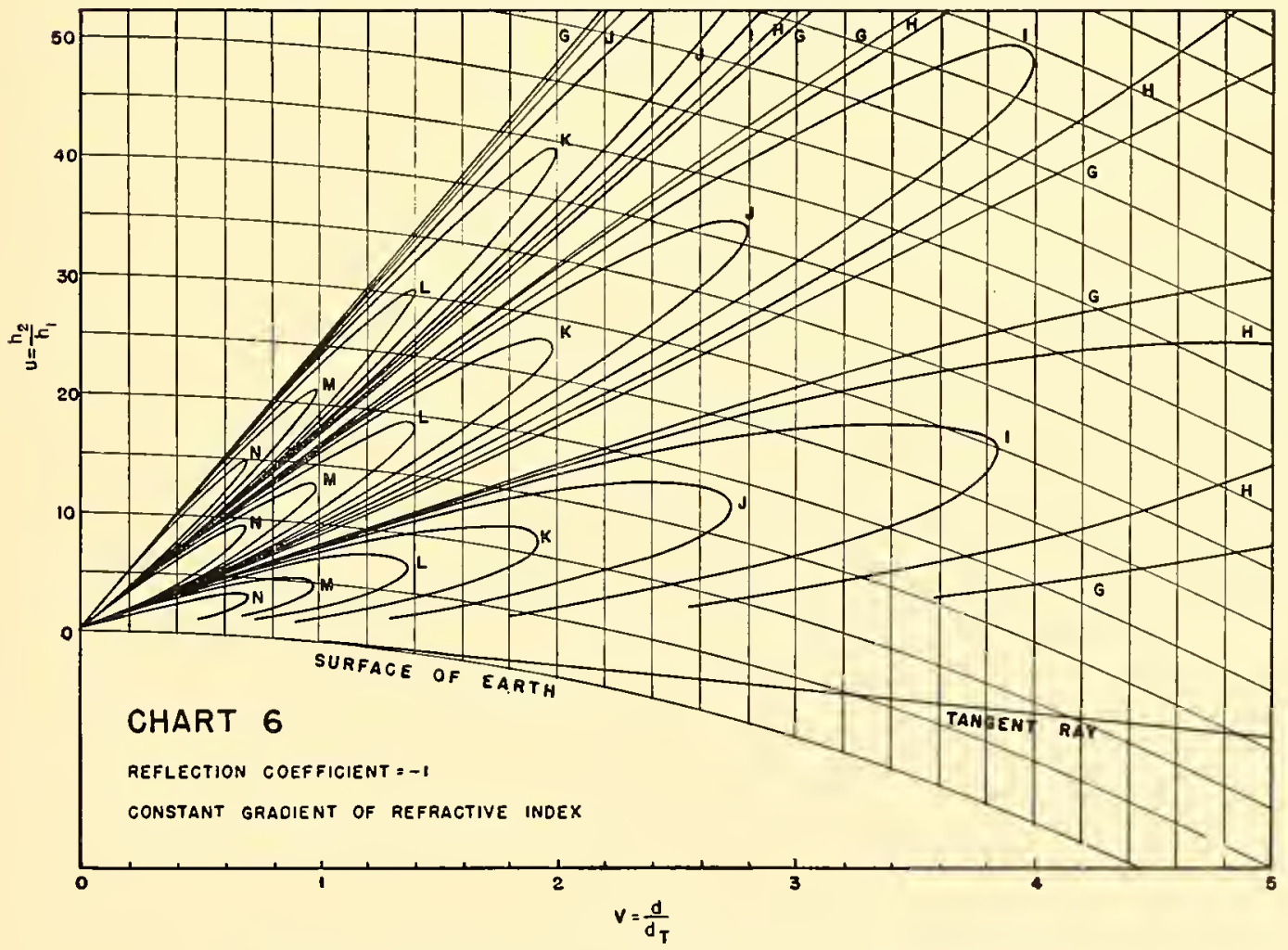

Figure 27. Generalized coverage diagram for $r=4$. (See Figure 15.) 


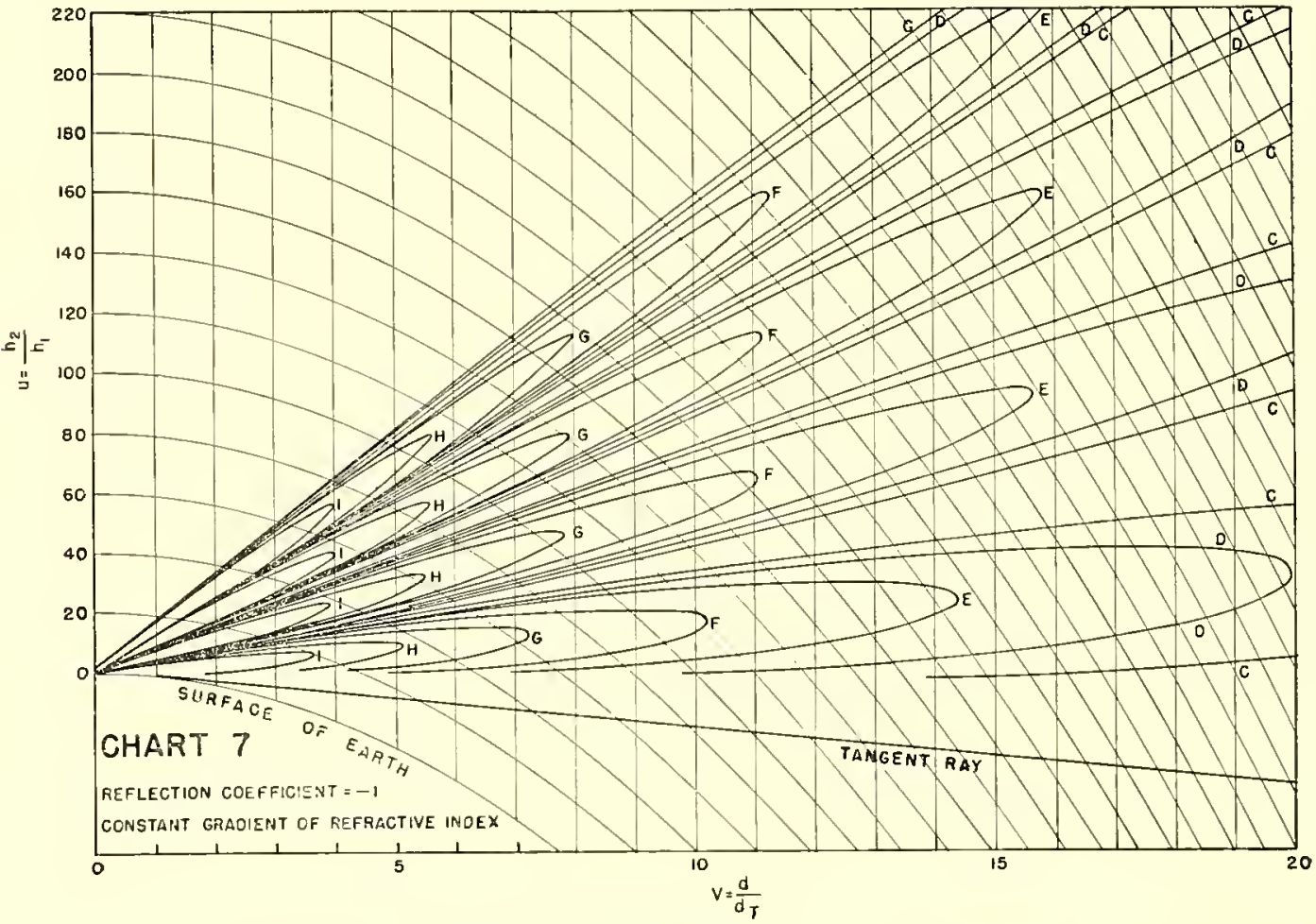

Figure 28. Generalized coverage diagram for $r=2$. (See Figure 15.)

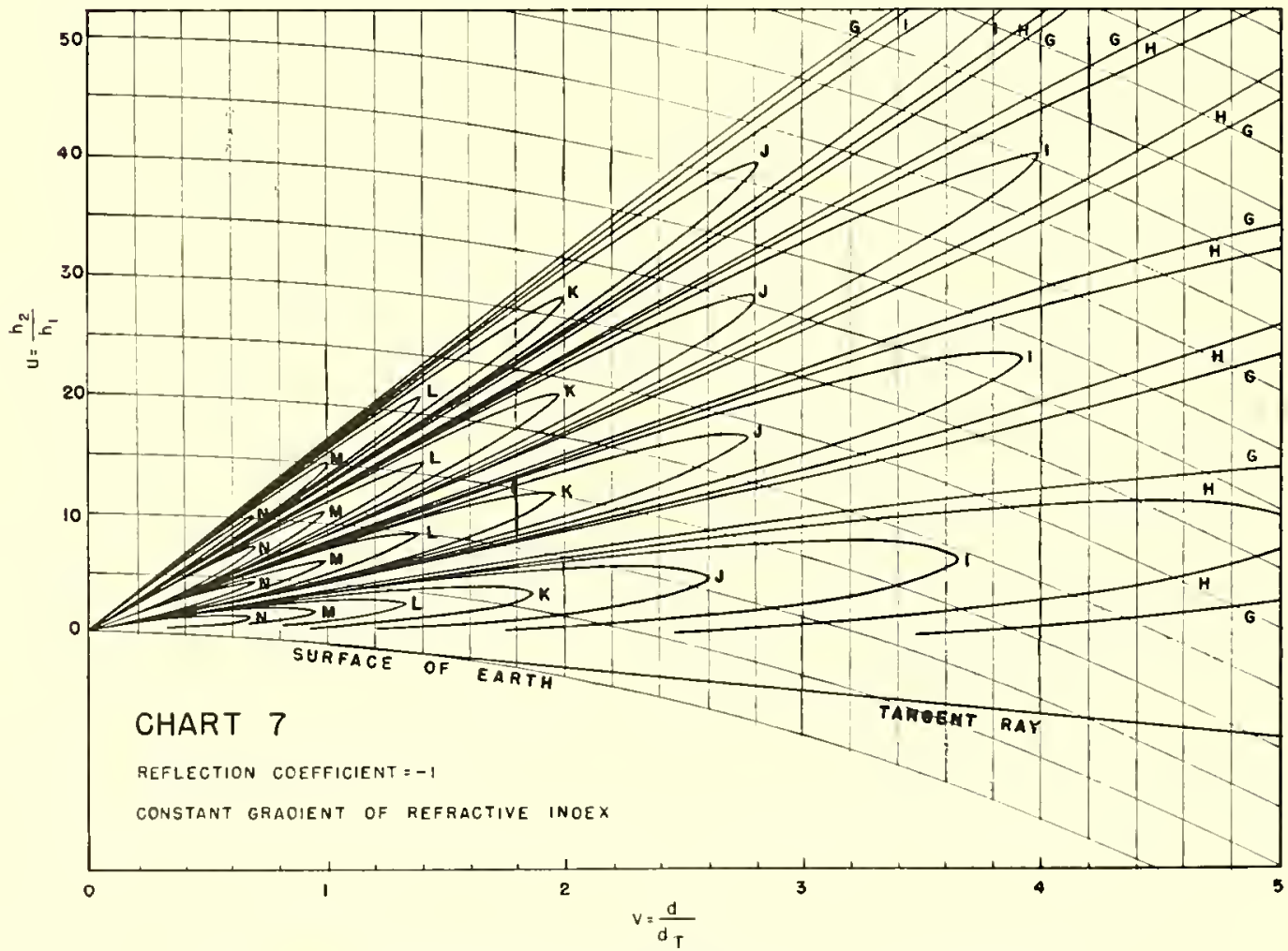

Figure 29. Generalized coverage diagram for $r=2$. (See Figure 15.) 


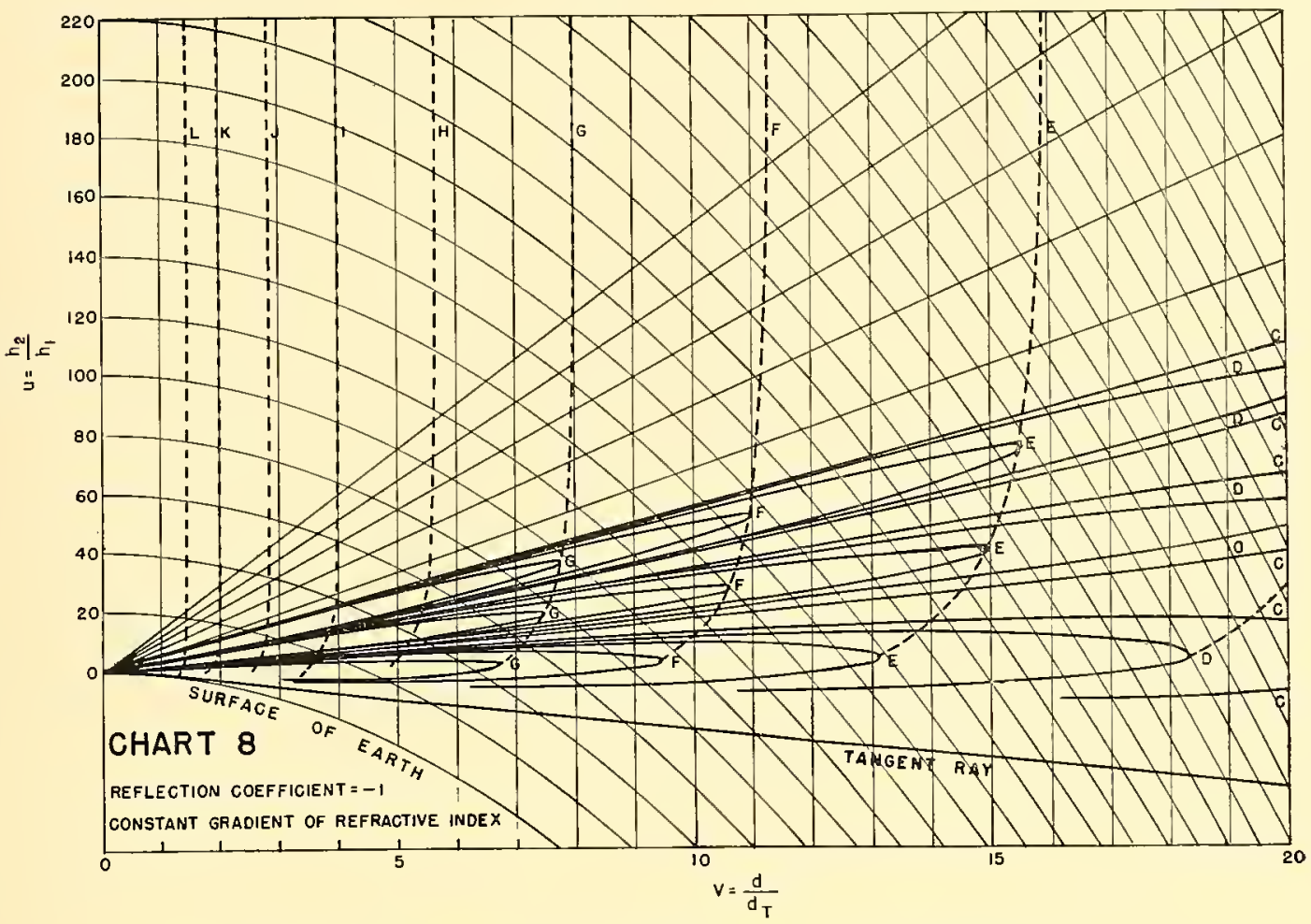

Figure 30. Generalized coverage diagram for $r=1$. (See Figure 15.)

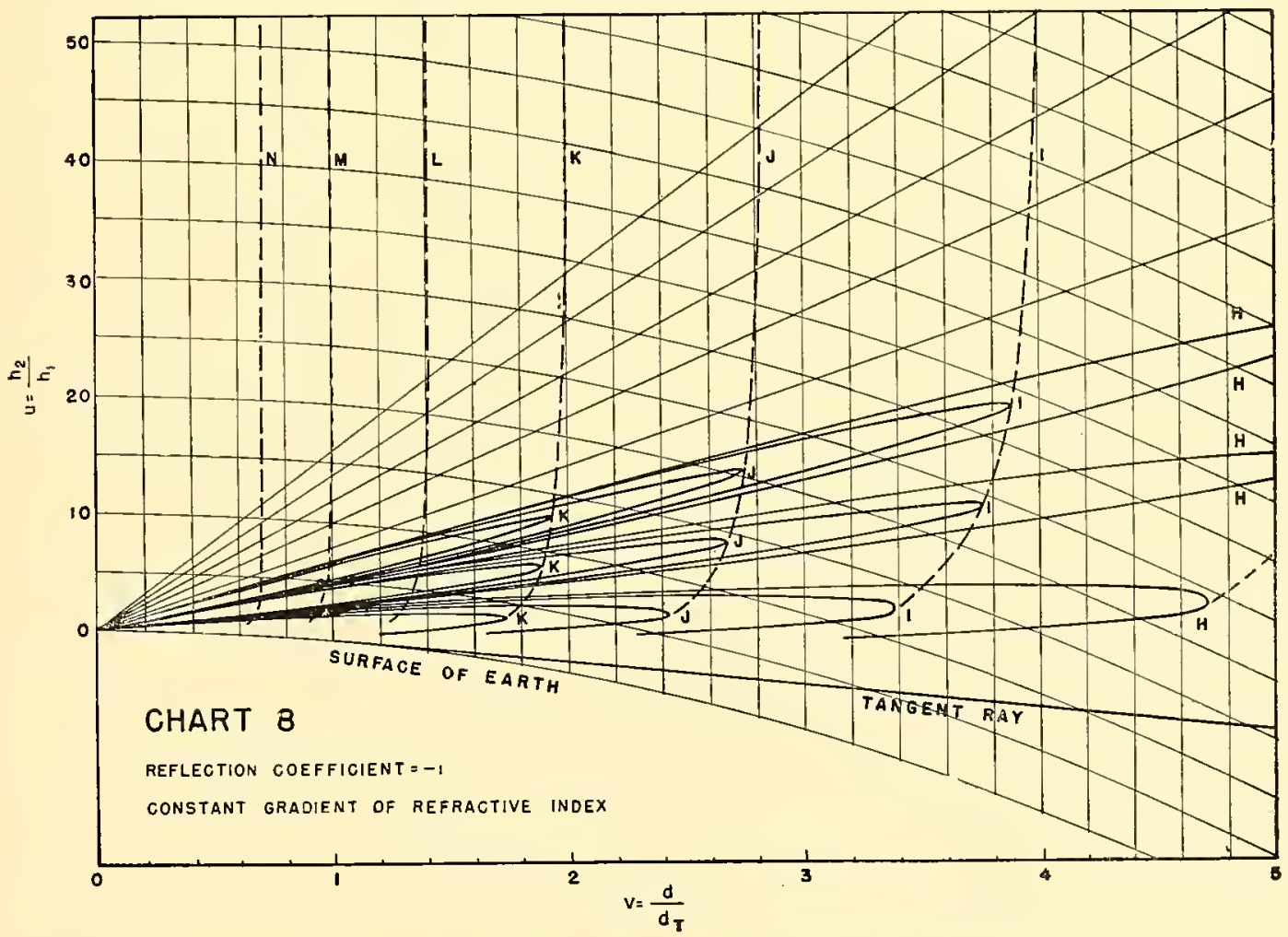

FigURE 31. Generalized coverage diagram for $r=1$. (See Figure 15.) 


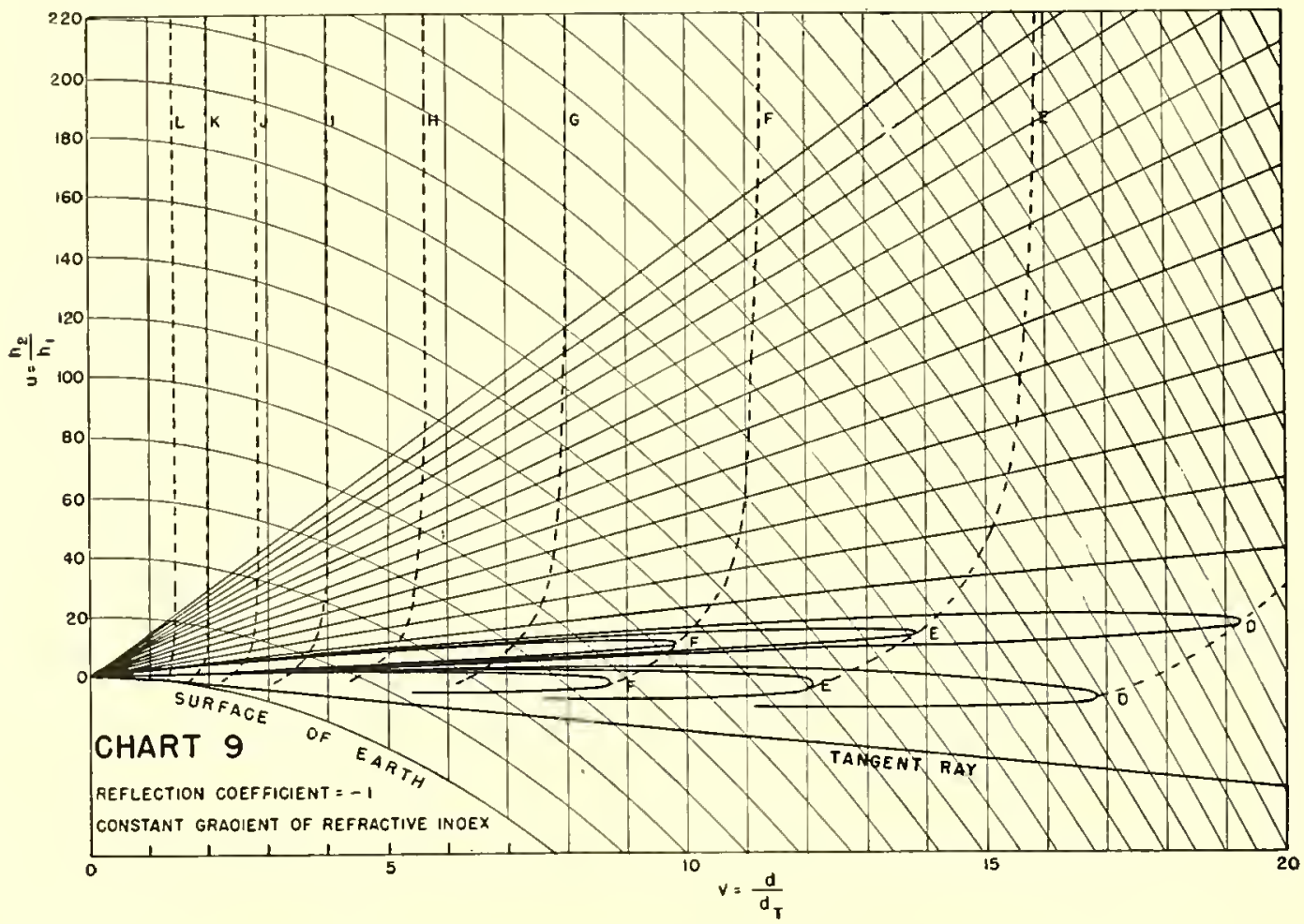

Figure 32. Generalized coverage diagram for $r=1 / 2$. (See Figure 15.)

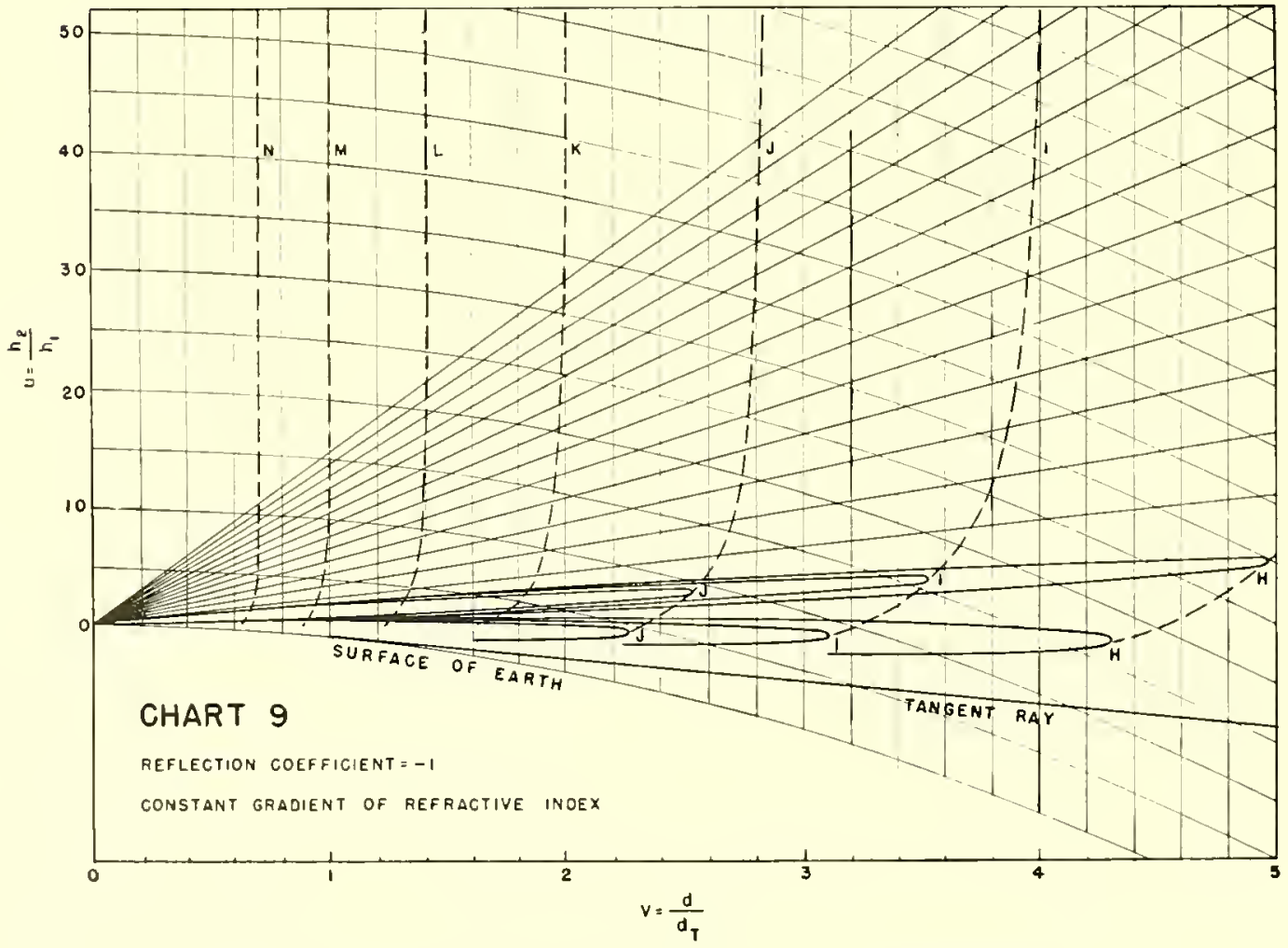

Figure 33. Generalized coverage diagram for $r=1 / 2$. (See Figure 15.) 


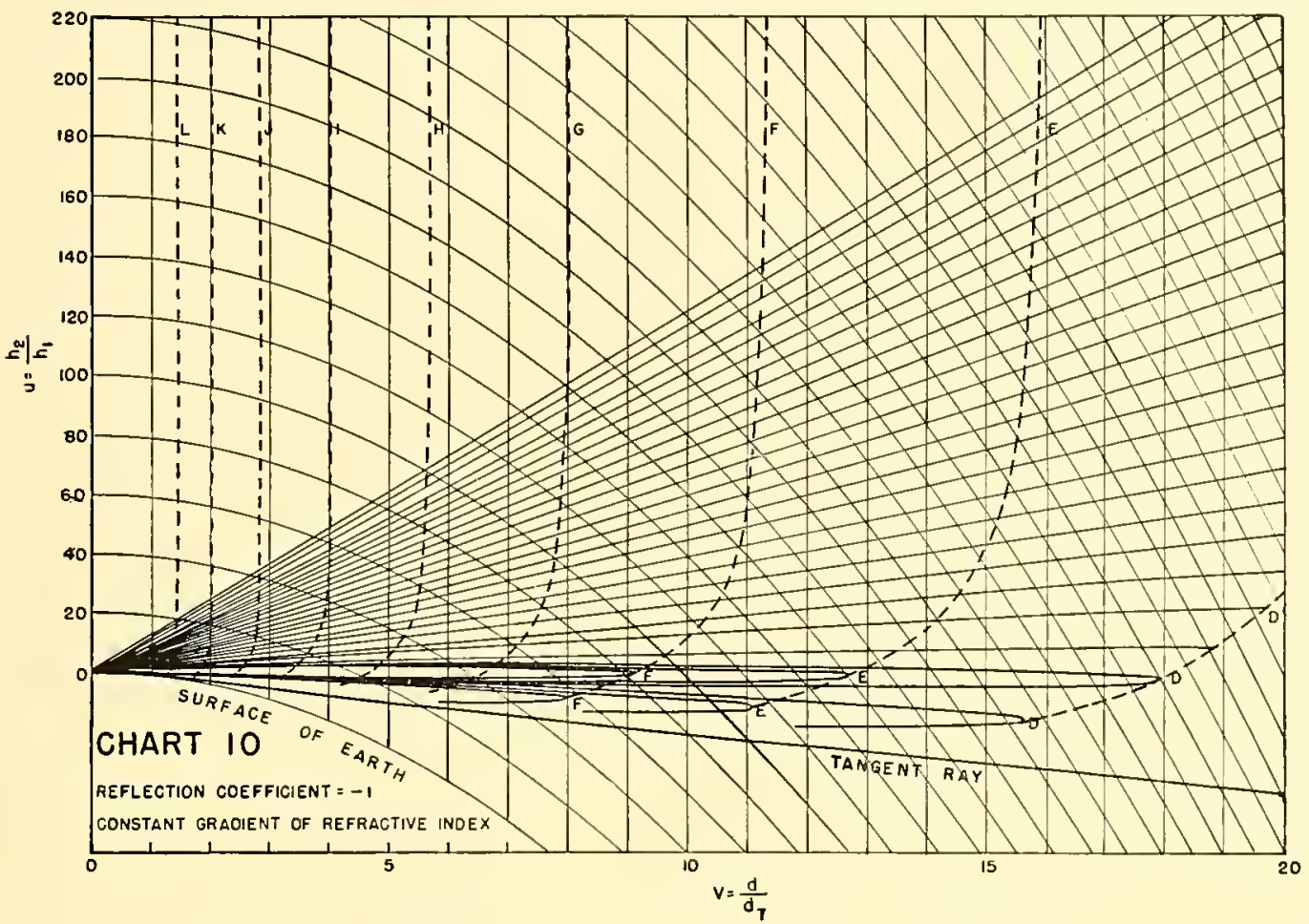

Figure 34. Generalized coverage diagram for $r=1 / 4$. (See Figure 15.)

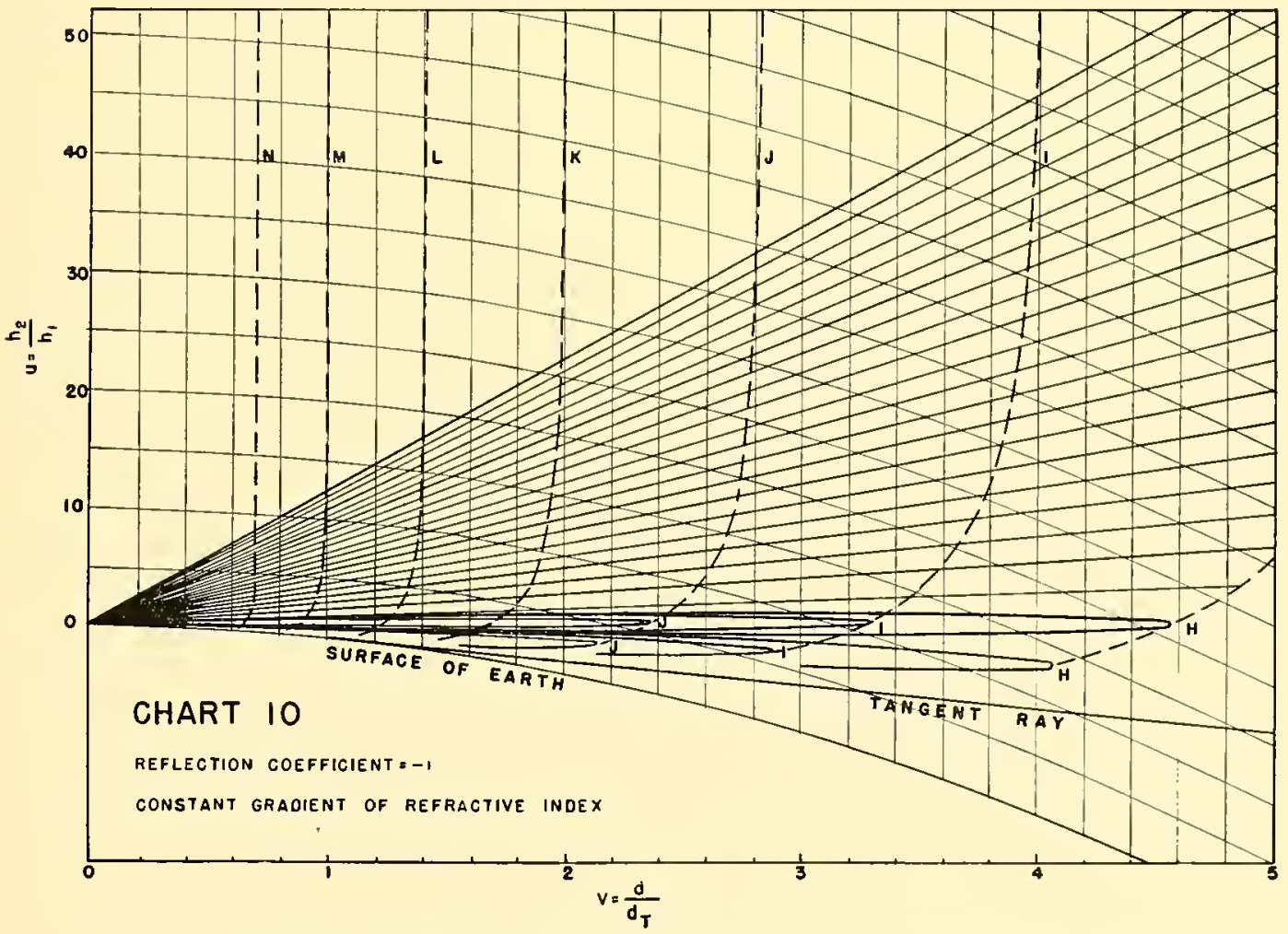

Figure 35. Generalized coverage diagram for $r=1 / 4$. (See Figure 15.) 


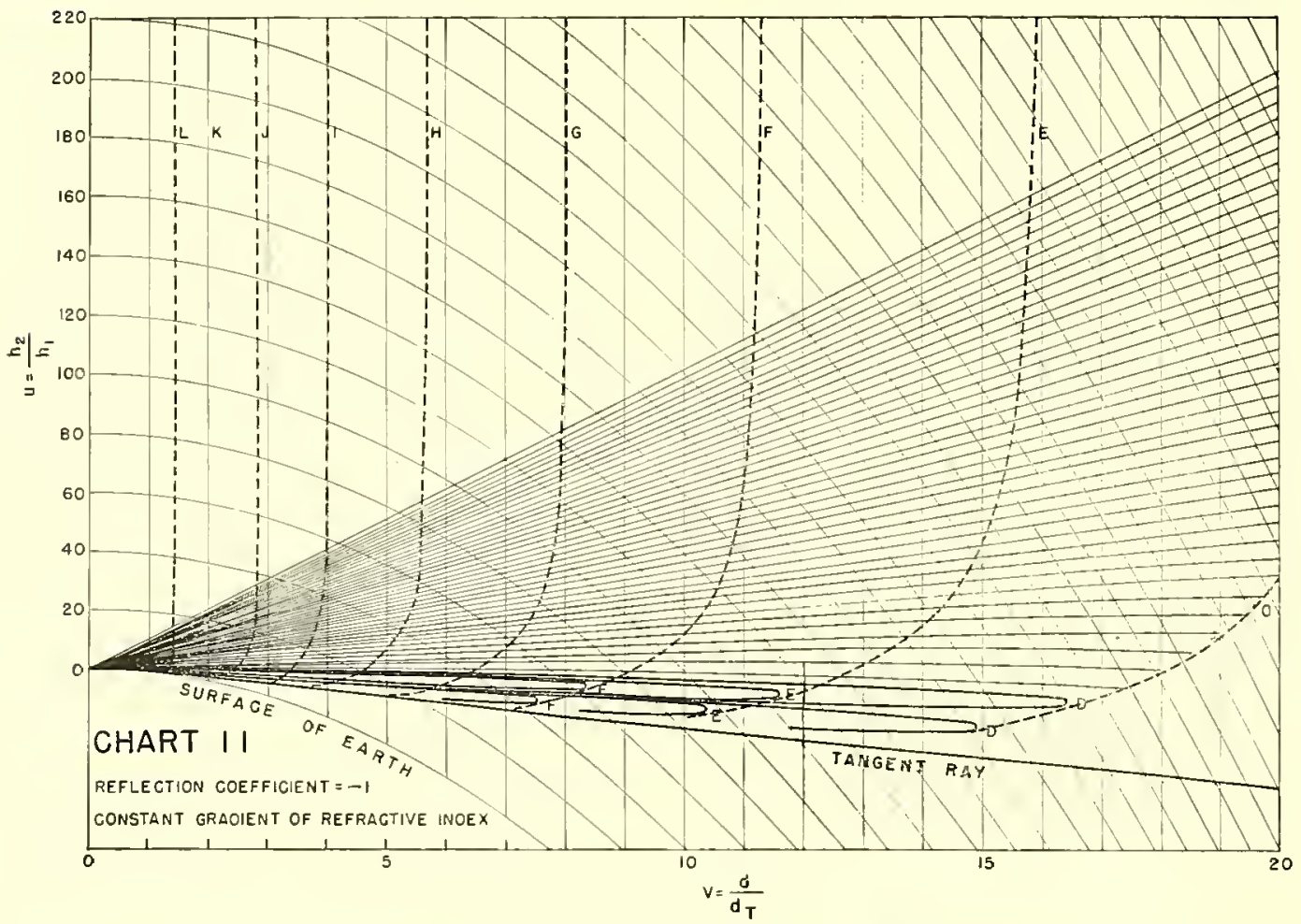

Figtre 36. Generalized coverage diagram for $r=1_{\mathrm{b}}$. (See Figure 15.)

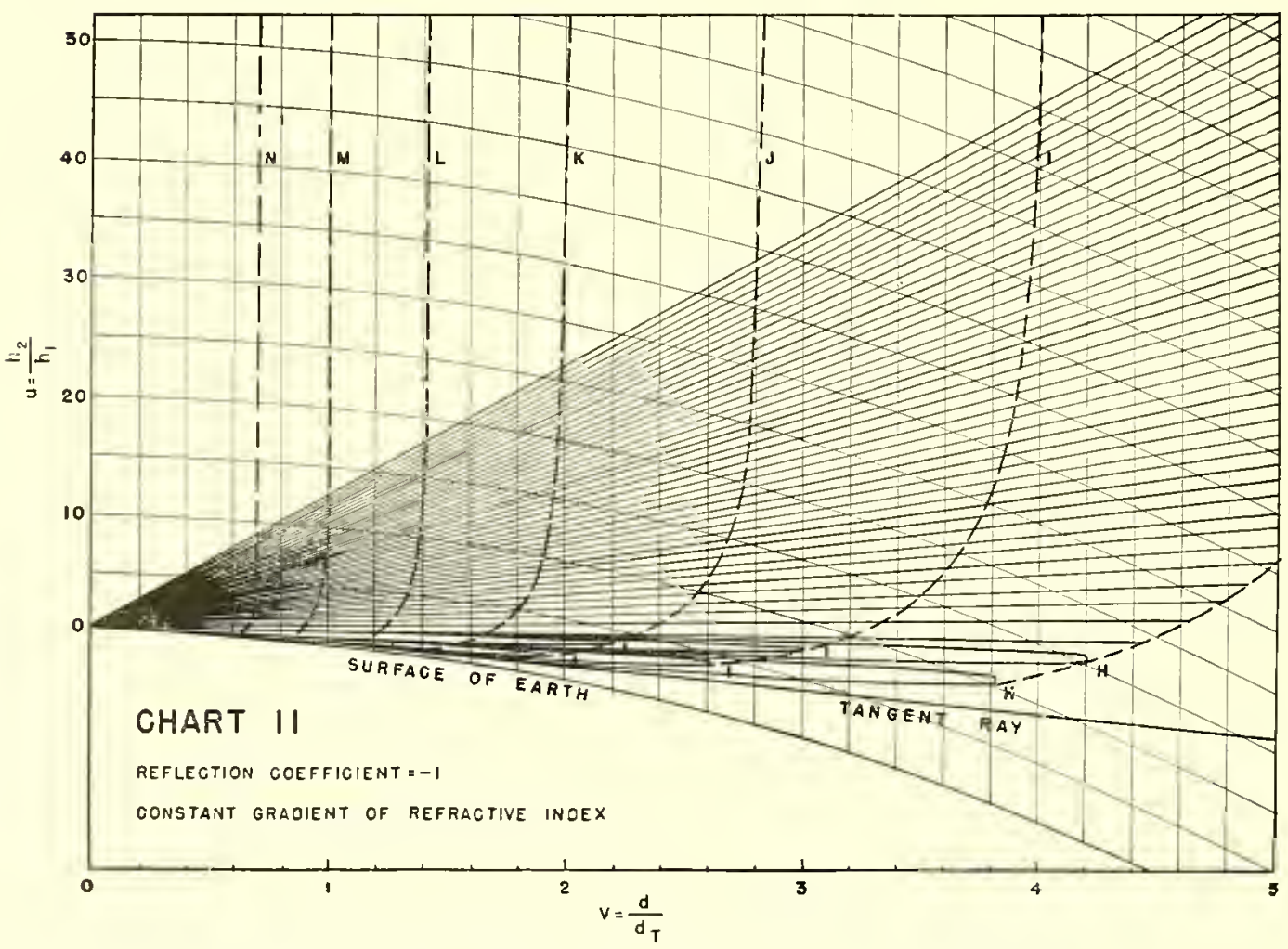

Figure 37. Generalized coverage diagram for $r=1 / \%$. (See Figure 15.) 


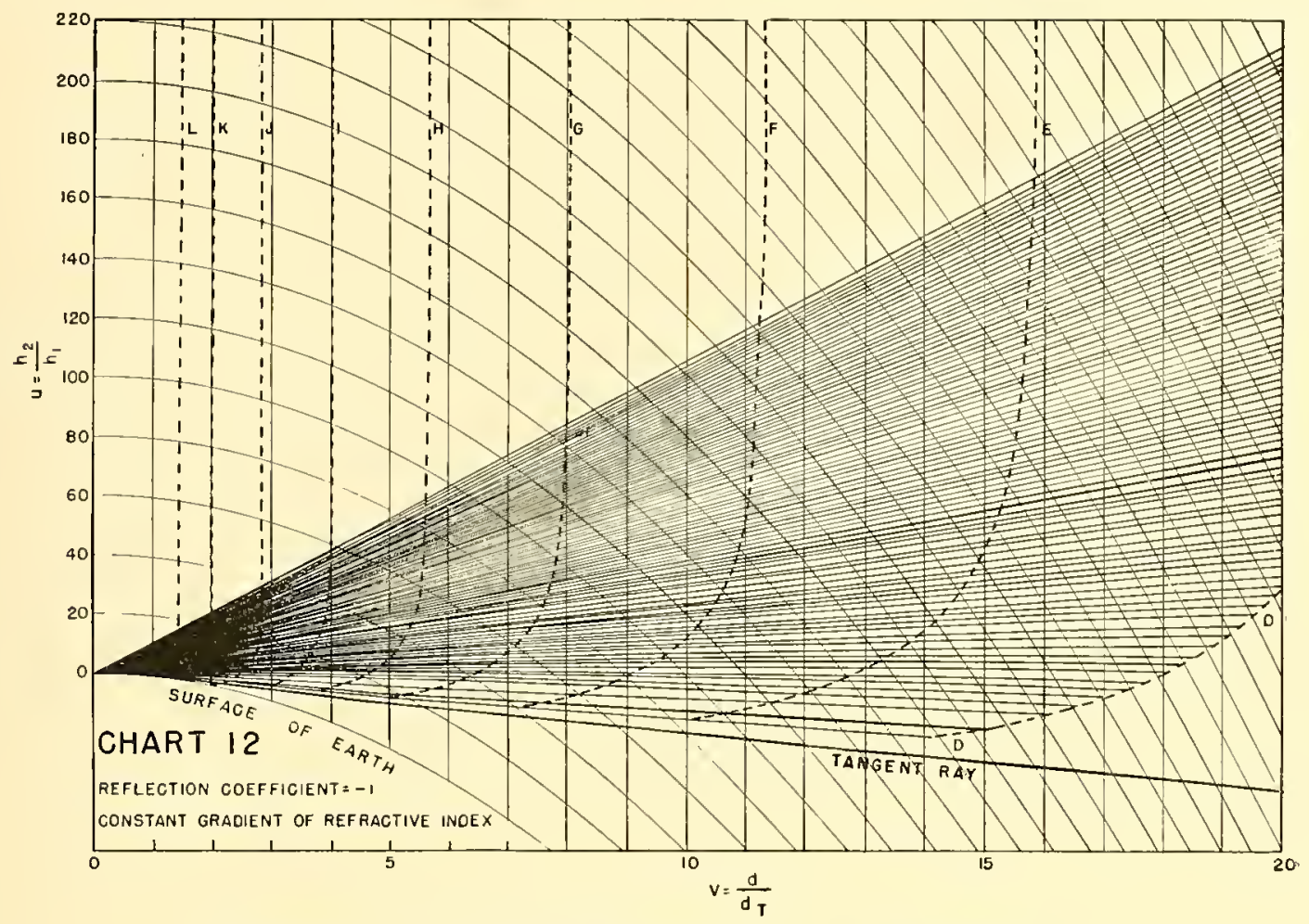

Figure 38. Generalized coverage diagram for $r=1 / 16$. (See Figure 15.)

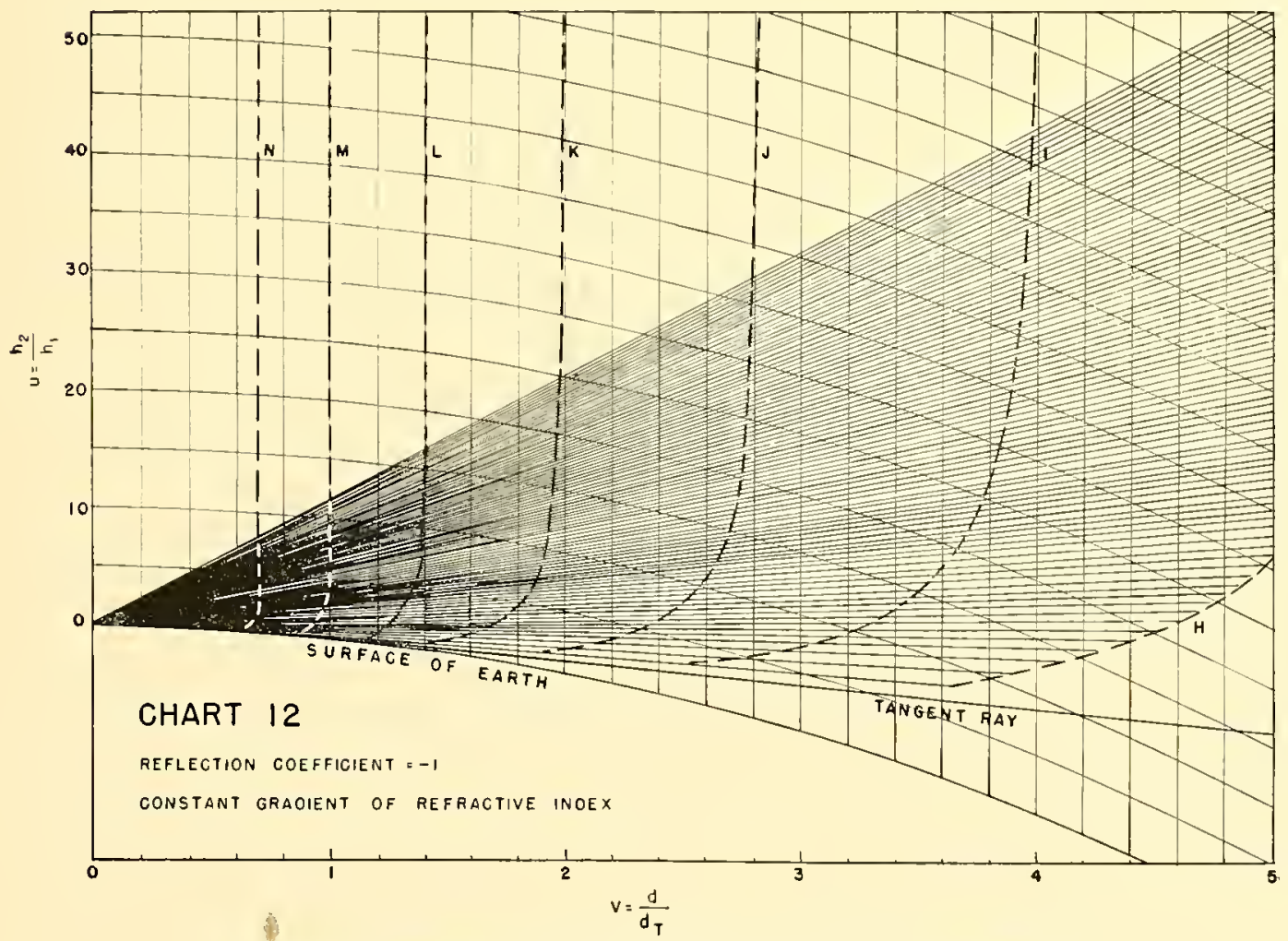

Figure 39. Generalized coverage diagram for $r=1 / 16$. (See Figure 15.) 


\section{Chapter 7}

\section{PROPAGATION ASPECTS OF EQUIPMENT OPERATION}

$\mathrm{F}$ OR A STANDARD atmosphere and with the basic assumptions set forth in Section 5.1.4, the relation between the factors affecting the power of a set and the gain factor $A$ is given in equations (3) and $(5)$ in Chapter 5 . The problem of computing $A$ depends on the set in the sense that some sets are designed to operate in free space, others with the aid of reflection from the sea, as in low-angle and surface coverage.

The characteristics of a set as given in the manufacturer's description or in Tables 3, 4, and 5 at the end of this chapter may not represent the true values for a set in field use. Expected set performance, such as maximum range and corerage, can be calculated on the basis of the set's rated characteristics. Such performance can be termed "normal." If a set is behaving abnomally, it may be that it is not functioning nost efficiently. Unfortunately, the problem is complicated by the possible presence of atmospheric ducts and by the rariability and difficulty of finding accurately the radar cross sections of aireraft and ships.

Ducts are especially important for low antennas in surface search. In the case of communication sets, the most important item of information from a propagation standpoint is the maximum range. In the case of radal, not only is knowledge of maximum range wanted but also the ability to estimate the size and type of the target.

\subsubsection{The Performance Figure and Efficieney}

The maximum range of a set depends on the peak power output $P_{p}$ of the transmitter, the minimum detectable power $P_{\text {min }}$ (see Sections 2.3.1 to 2.3.5) of the receiver, and the antenna gains $G_{1}$ and $G_{2}$. These can be grouped to give a performance figure. For communication, this figure is $\left(P_{p} / P_{\min }\right) G_{1} G_{2}$. For radar, the gains $G_{1}$ and $G_{2}$ are generally equal. The performance figure is then $\left(P_{p} / P_{\min }\right) G^{2}$. The ratio of the actual performance figure to the maximum possible value, or the difference in decibels, gives the efficiency of the set. In field use, it is generally impossible to measure the working performance figure with any precision and methods for obtaining a rough measure must be employed.

\subsubsection{Effect of Reflection}

It has been pointed out in Chapters 5 and 6 that reflection may increase the maximum range of a radar up to twice the free-space value. This aid
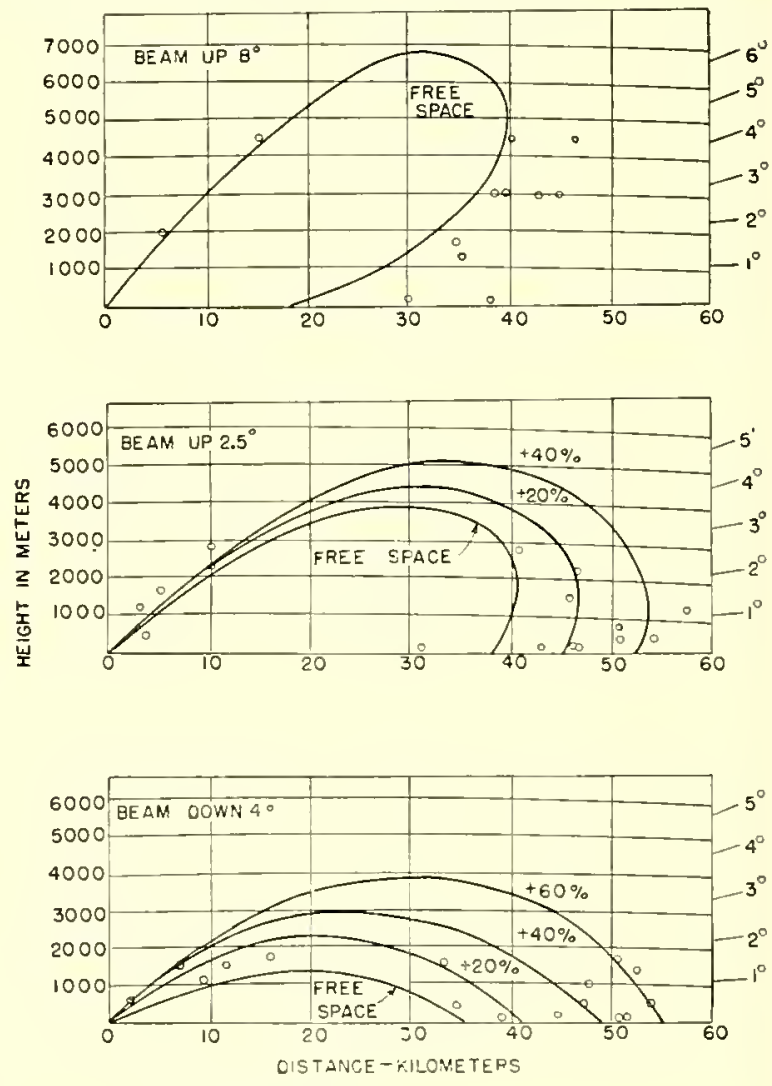

- Experimentar Points

Figure 1. Effect of beam tilt on coverage for a radar.

the early detection of aireraft at low angles. However, the minima which occur in the resulting interference pattern prevent the continuous tracking of an airship coming in. 
This effect can be counteracted in screral ways. One way is to employ microwaves whose interference lobes are narrow and close together. Vertical polarization is another means of filling in the nulls while gaining in maximum lange at low angles. Another device is to tilt the antenna beam upward so that some radiation (but substantially less than half) falls upon the sea. The result is a gain in lowangle coverage while the high-angle coverage is that of free space, without minima.

The effect of various percentages of specular reflection in comparison with the free-space pattem is shown in Figure 1 for various beam tilts of a radar with a comparatively narrow beamwidth (11 degrees between half-power points). The experimental data, shown by small circles, illustrate the increase in detection range at 0 degree while at 5 degrees elevation angle there is little gain over free space.

The roll of a ship, by varying the beam tilt, results in a shift in coverage, as can be seen from Figure 1.

\subsection{4}

\section{Signal-to-Noise Ratio}

The visibility of a signal on a scope depends on its relation to the noise. In early work with radar, the maximum range was defined by a ratio of signal voltage $S$ to noise voltage $N$ of unity, i.e.,

$$
\frac{S}{N}=1
$$

However, as pointed out in Section 2.3.5, the minimum detectable signal is greater than $N$. Since the pip on a scope includes noise, equation (1) is equivalent to

$$
\frac{S+N}{N}=2
$$

On an $\mathrm{A}$ scope, this relation signifies that the height of the signal is twice that of the noise grass.

Since the visual signal in a set functioning properly varies linearly with the signal voltage, the size of targets can be estimated by means of the size of the visual signal. The ratio $S / N$ gives a means of measuring a signal in terms of the noise. To change $(S+N) / N$ to $S / N$, the value of $(S+N) / N$ is expressed with unity as denominator. For example, if $(S+N) / N$ is estimater to be $8 / 2$ from the scope, the equivalent fraction is $4 / 1$. The value of $S / N$ is $(4-1) / 1=3 / 1$.
The relation of receiver power, $P_{2}$, to noise power, $N P$, and the signal-to-noise ratio, $S / N$, is given by

$$
10 \log \frac{P_{2}}{N} \frac{2}{P}=20 \log \frac{S}{N} .
$$

\subsubsection{Calibration of an A Scope}

On an A scope, the ratio $(S+N) / N$ can be estimated roughly by eye. To improve upon this, a calibration is employed. One method is to mark the A scope to facilitate the reading of heights. Another method goes beyond this and calibrates the gain control. A turn of $5 \mathrm{db}$ is equivalent to a raito of 1.8/1. A datum line $1 \mathrm{~cm}$ above the tine line and

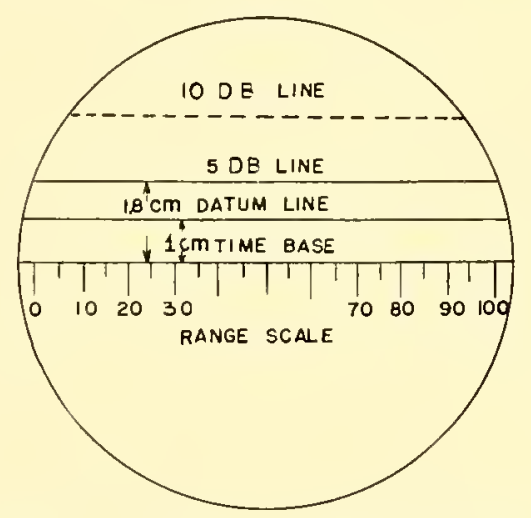

Figure 2. Method of calibrating an A scope.

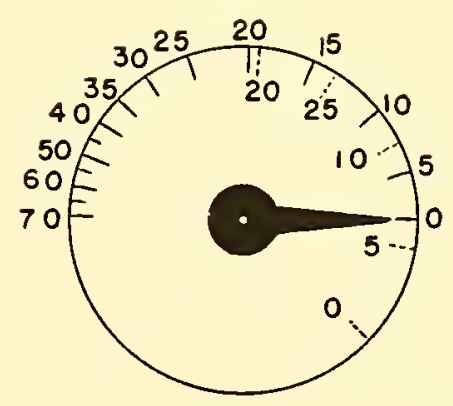

Figure 3. Calibration of gain control of an A scope. (Dotted lines are uncorrected calibration.)

another at $1.8 \mathrm{~cm}$ are drawn on the $\mathrm{A}$ scope. The noise is brought up to the datum line by means of the gain control. The position is marked 0 on the gain control (see Figure 2). A steady signal is found (permanent echo, large boat, or signal generator) which produces a signal height of $1.8 \mathrm{~cm}$ above the time line. The gain control is then turned until the signal is reduced to the datum line. The position 
on the gain control is marked $5 \mathrm{db}$ (see dotted lines in Figure 3).

Teeping the 5-db pasition on the gain control, another signal is obtained which comes up to 1.8-cm line. The gain control is turned until the signal is brought down to the datum line. The new setting is marked $10 \mathrm{db}$. This is repeated until markings up to about $70 \mathrm{db}$ are obtained.

This calibration of $(S+N) / N$ must be corrected to $S / N$ (Figure 3) which can be done by means of Table 1. For $25 \mathrm{cb}$ and above, the values of $(S+N) / N$ can be taken as equal to $S / N$. Any signal voltage can then be measured in decibels above the noise voltage $(N)$ by turning the gain control until the signal height is $1 \mathrm{~cm}$. By equation (3) this is also the signal power received, in decibels, above the noise power which is discussed in Sections 2.3.1 and 2.3.2.

Table 1. Correction of $(S+N) / N$ to $S / N$.

\begin{tabular}{cc}
\hline $\begin{array}{c}\text { Corrected } \\
\left(\frac{S}{Y}\right) \mathrm{db}\end{array}$ & $\begin{array}{c}\text { Uncorrected } \\
\left(\frac{S+N}{N}\right) \mathrm{db}\end{array}$ \\
\hline 0 & 6 \\
5 & 9 \\
10 & 12.5 \\
15 & 16.5 \\
20 & 21 \\
\hline
\end{tabular}

In the calibration just described, the pip on the scope is supposed to be proportional to the received signal strength. In a set functioning normally, this is justified, but occasionally defects in the set may destroy the linearity. The existence of a linear relation can be tested by means of a signal generator.

\subsection{FREE SPACE - HIGH-ANGLE COVERAGE}

\subsubsection{Maximum Range Formulas}

In free space, the gain factor $A$ has the ralue $3 \lambda / S \pi d$ (for maximum power transfer between doublets). Equations (3) and (5) in Chapter 5 then take the simple forms:

One-way, radio gain:

$$
\frac{P_{2}}{P_{1}}=G_{1} G_{2}\left(\frac{3 \lambda}{8 \pi d}\right)^{2}
$$

Two-way, radar gain:

$$
\frac{P_{2}}{P_{1}}=G^{2} \cdot \frac{16 \pi \sigma}{9 \lambda^{2}} \cdot\left(\frac{3 \lambda}{8 \pi d}\right)^{4} .
$$

If $P_{2}$ is replaced by the minimum detectable power of the receiver, $P_{\min }$, and $P_{1}$ by the peak power $P_{p}$ of the transmitter, the maximum range is given by:

Onc-way,

$$
d_{\max }=\frac{3}{8 \pi} \sqrt{\frac{P_{p}}{P_{\min }} G_{1} G_{2} \lambda^{2}},
$$

Two-way,

$$
d_{\max }=\sqrt[4]{\frac{P_{p}}{P_{\min }} G^{2} \lambda^{2} \frac{9 \sigma}{256 \pi^{3}}} .
$$

\subsubsection{Deviation from Maximum of Beam}

For an antenna whose direction is fixed, the equations in Section 7.2.1 apply only to points on the axis of the beam. Denoting by $f(\tau)$ the ratio of gain in a direction at an angle $\tau$ from the axis of the beam to the gain at the axis and by $2 \tau_{0}$ the beam width between half-power points, then

$$
f(\tau)=\exp -0.692\left(\tau / \tau_{0}\right)^{2} .
$$

Accordingly, for points off the axis, $G$ must be multiplied by $f(\tau)$ before substitution in the formulas of Section 7.2.1.

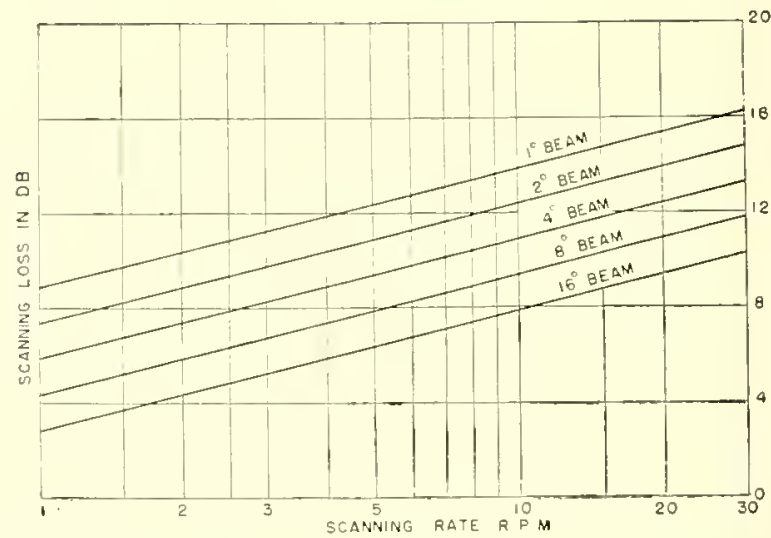

Figure t. Scanning loss as a function of scanning speed and beamwidth.

\subsubsection{Performance Figure}

Equations (6) and (7) for $d_{\max }$ depend on the performance figure defined in Section 7.1.2. The quantities which appear in the performance figure can be measured. The one which offers most difficulty is $P_{\text {min }}$, the minimum detectable power, which has been discussed in Section 2.3. In Tables 3 and 4 at 
the end of this chapter, noise figures and bandwidths of various sets are given. An important correction to $P_{\text {mi. },}$ as determined from the noise figure and bandwidth is the scanning loss. This loss for various scanning speeds and beamwidths is represented in Figure 4. Another source of loss is deviation of the product of bandwidth $B$ (mc) and the pulse width $t$ (microseconds) from the optimum value of 1.2 . The losses for various values of the product are tabulated in Table 2.

TABLE 2. Joss resulting from band- and pulse widths.

\begin{tabular}{rc}
\hline \hline$B t^{*}$ & Loss $(\mathrm{db})$ \\
\hline 0.1 & 5.0 \\
0.3 & 1.5 \\
0.7 & 0.5 \\
1.2 & 0.0 \\
2.5 & 0.8 \\
5.0 & 3.0 \\
10.0 & 5.0 \\
20.0 & 8.0 \\
\hline
\end{tabular}

${ }^{*} B=\mathrm{i}-\mathrm{f}$ bandwidth (me); $t=$ pulse width (mieroseconds).

A field measure of the performance figure of a radar can be determined by the use of a target of known radar cross section, such as a silvered balloon and equation (7). A check on variability of performance can be made by finding the maximum range on a plane (using a constant aspect, such as nose or tail).

\subsubsection{Radar Cross Section}

An important but troublesome factor in calculating $d_{\max }$ of a radar from a knowledge of the performance figure is $\sigma$, the radar cross section (see Section 2.4.1 and Chapter 9). The value of $\sigma$ can be found by:

1. Laboratory measurement of the factors which constitute the performance figure and field determination of the maximum range $d_{\max }$. The value of $\sigma$ is then given by equation ( 7 ).

2. Measuring the signal returned by the target at a convenient distance on a calibrated $A$ scope or by direct comparison with a pulsed signal generator. In this method neither $P_{\min }$ nor $d_{\max }$ enter.

The equations involving' $\sigma$ assume a point target. Since an airplane intercepts a small solid angle over which the beam strength varies little, the assumption of a point target is adequate for aireraft.
7.3

7.3 .1

\section{LOW-ANGLE AND SURFACE COVERAGE}

\section{Maximum Range}

Since the gain factor $A$ for this case is more complicated than for free space, the relation between $d_{\max }$ and the performance figure cannot be given in general by a simple expression, as can be scen from equations (172) and (18t) in Chapter 5. For ranges such that the shadow factor $F_{s} \sim 1$, i.e., distances $d$ less than $10^{4} \lambda^{1 / 3}, \lambda$, and $d$ in meters, and both antennas low, or antenna and target low $\left(h_{1}, h_{2}<\right.$ $30 \lambda^{2 / 3}$ ), the form of $A$ is simplified so that a simple relation can be given for $d_{\max }$. Otherwise, the methods of Sections 5.6, 5.7.3, and 5.7.4 must be employed, especially of Sections 5.6.5 and 5.7.3.

\subsubsection{Ducts and Set Performance}

It has been found from field tests that atmospheric ducts are likely to be found close to the surface of the sea. The consequent increase in range may mask subnormal set performance. If the antenna is tilted upward so that no radiation reaches the earth, then the free-space discussion of Section 7.2 applies to the field determination or check of the performance figure. Otherwise ficld testing, when conditions are normal, can be accomplished by the use of permanent echoes or the use of a ship of known target cross section (see Section 7.3.7).

\section{${ }^{7.3 .3}$ Low Heights and Plane Earth Ranges}

For these conditions, the following relations must hold:

$$
\left(h_{1,2}<30 \lambda^{2}{ }^{3}, d<10^{4} \lambda^{1 / 3}\right),
$$

where $h, d$ and $\lambda$ are in meters (see Section 5.7.1).

In the dielectric case (see Section 5.7.3), generally applicable to radar, the value of $A$ [equation (172) in Chapter 5] for $F_{s}=1$ and $g=1$, becomes

$$
A=\frac{3}{2} \frac{h_{1} h_{2}}{d^{2}} .
$$

Since $A=A_{0} A_{p}$ and $A_{0}=3 \lambda / s \pi d$, the preceding equation is equivalent to a path-gain factor value of

$$
A_{p}=4 \pi \frac{h_{1} h_{2}}{\lambda d} .
$$

[See equation (55) in Chapter 5.] Instead of equations (4) and (5), we now have [from equations (3) and (5) in Chapter 5] for a dielectric earth, 
One-way, radio gain:

$$
\frac{P_{2}}{P_{1}}=\frac{9}{4} G_{1} G_{2} \frac{h_{1}^{2} h_{2}^{2}}{d^{4}}
$$

Two-way, radar gain:

$$
\frac{P_{2}}{P_{1}}=9 \pi G^{2} \frac{h_{1}{ }^{4} h_{2}{ }^{4} \sigma}{\lambda^{2} d^{8}} .
$$

Hence for maximum range, replacing $P_{1}$ by $P_{p}$, and $P_{2}$ by $P_{\min }$,

One-uay,

$$
d_{\max }=\sqrt[4]{\frac{9}{4} h_{1}^{2} h_{2}^{2}\left(\frac{P_{p}}{P_{\min }} G_{1} G_{2}\right)} .
$$

Two-way,

$$
d_{\max }=\sqrt[8]{\frac{9 \pi \sigma h_{1}{ }^{4} h_{2}{ }^{4}}{\lambda^{2}}\left(\frac{P_{p} r^{2}}{P_{\min }}\right)} .
$$

The maximum range now depends on the antenna heights and on the target height.

\subsubsection{Radar Cross Section of Surface Craft}

Effective Height of Targets. Since the field varies considerably with height for a low target, the scattering by even simple geometrical objects requires integration. The formulas for radar in Section 7.3.3 apply to a point target with radar cross section $\sigma$ at an effective height $h_{\text {eff }}$.

In the case of a cylinder of radius $a$ and length $H$, the radar cross section in a uniform field is $\sigma=2 \pi a H^{2} / \lambda$. Under operating conditions the field is not uniform but a feasible approximation may be obtained by using the preceding value of $\sigma$ and a value of $h_{2}$ equal to the average vahe of the target height.

The radar cross section of a ship is a complicated problem which is not discussed here.

\section{3 .5 \\ Maximum Range Versus Height Curves}

By the method of Section 5.6.6 (see also Sections 6.5.2 and 6.8.4), maximum range versus height curves can be constructed for various values of $A$. These are of importance chiefly for low-angle aircraft coverage. If the performance figure, $\left(P_{p} / P_{\min }\right) G^{2}$, is known, equation (5), in Chapter 5 , defines the relation between $\sigma$ and $A$ so that $\sigma$ can be taken as the curve parameter instead of $A$. Equation (5), Chapter 5 , can be written

$20 \log A=-5 \log \frac{P_{p}}{P_{\min }} G^{2}-5 \log \sigma-5 \log \frac{16 \pi}{9 \lambda^{2}}$.

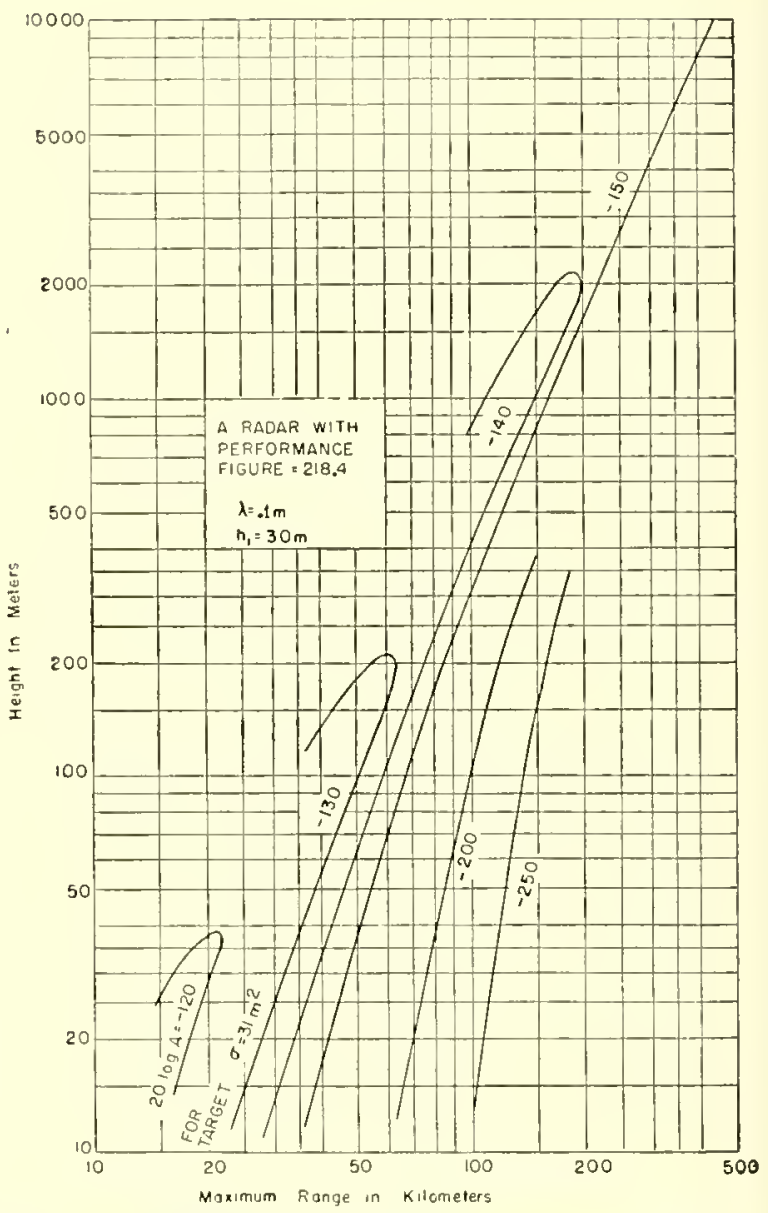

Figure 5. Maximum range velsus height curves for various targets.

A set of curves for a 10-cm radar is given in Figure 5 for a transmitter height of 30 meters. The curve corresponding to $\sigma=31$ square meters is indicated. The corresponding value of $20 \log A=-130$ was found from equation (15), using the value of $218.4 \mathrm{db}$ as the performance figure.

For a ship with an effective height of 18 meters, Figure 5 gives the value of $20 \log A$ at various distances. With the aid of equation (5), in Chapter 5, the power $P_{2}$ returned to the radar by the ship at these distances can be found, using the following 


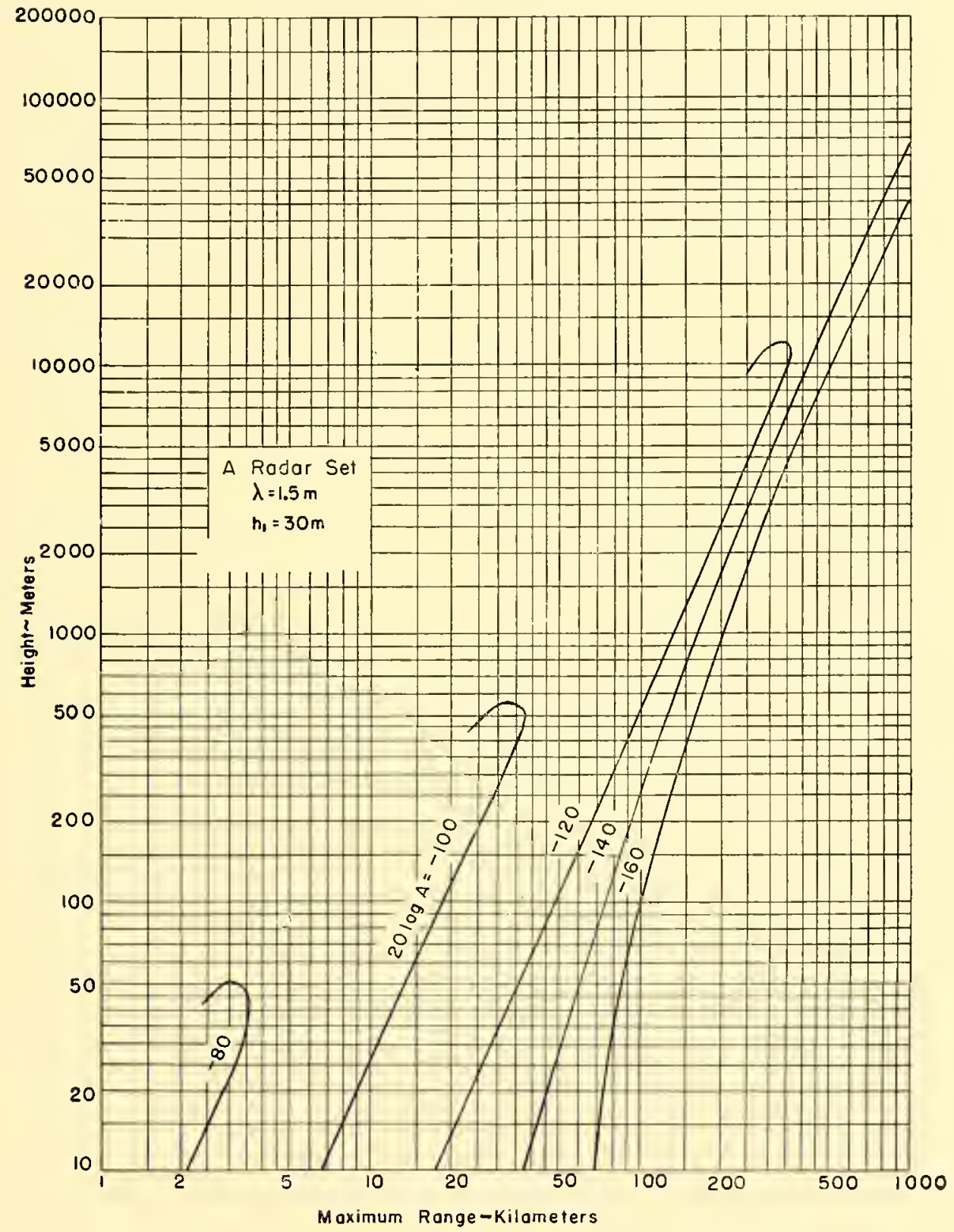

Figure 6. Maximum range versus height curves for various targets. 
data: $P_{1}=P_{p}=60 \mathrm{kw}, G=22.5 \mathrm{db}$, and $\sigma=7,440$ square meters.

In Figure 6, the maximum range versus distance curves for a set are presented. This set has a beamwidth of 60 degrees so that it is effectire for tracking aireraft coming in toward the set (increasing angle of elevation). The near coverage then approximates the free-space coverage which is dependent on the angle of inclination of the antenna.

In Figure 7, curves are presented for a 3 -cm set.

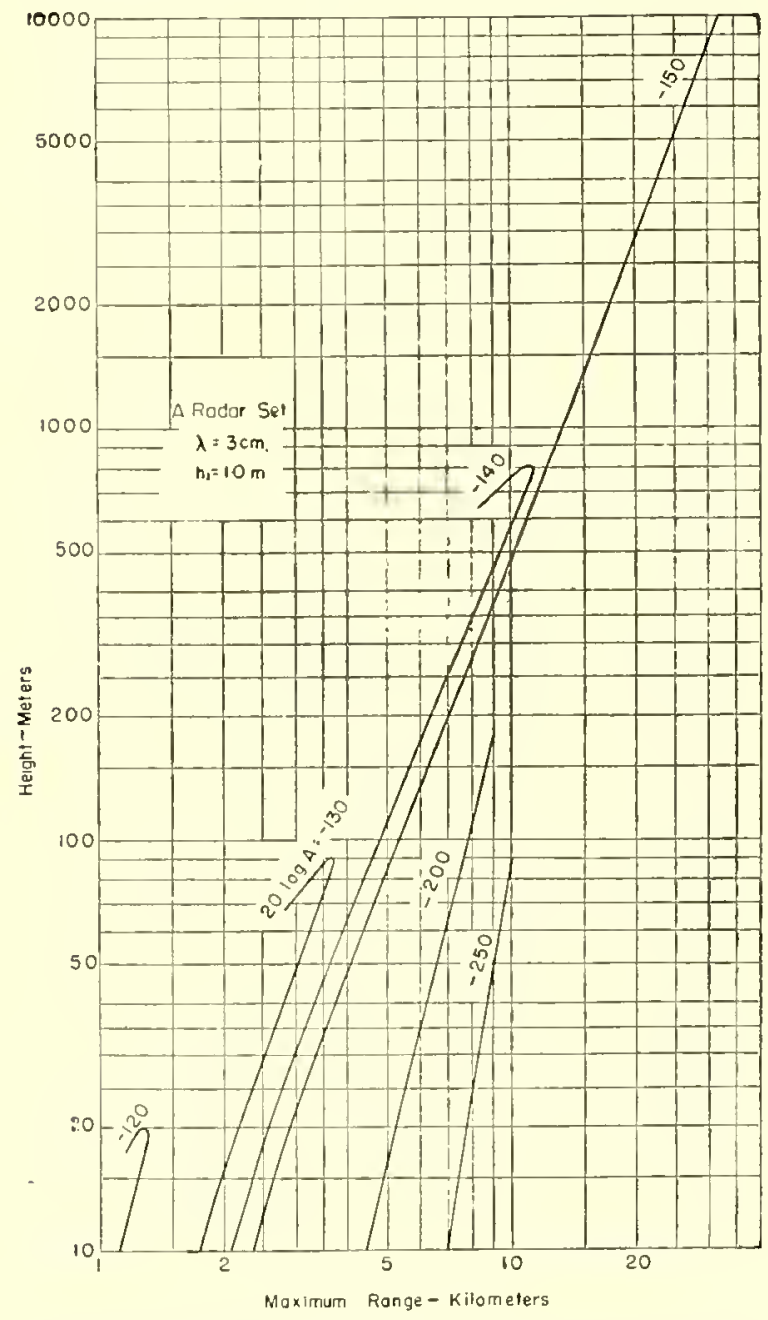

Figure 7. Maximum range versus height curves for various targets.

\subsubsection{Estimating Ship Size}

The fact that the strength of a signal received from a target depends on the size of the target can be utilized to estimate ship size. If signal strength versus distance curves are obtained for surface craft of various sizes, then by plotting readings from an unknown vessel, its size may be estimated. A field procedure for obtaining these curves is to use a number of surface craft of assorted sizes and follow them on a radar with a calibrated A scope, recording signal strength versus distance. If a number of such runs are taken during periods judged to be standard and the results averaged, a fairly reliable chart should result.

The curves can be obtained by calculation using measured power output and antenna gain and empirically determined values of $\sigma$ in equation (5) in Chapter 5. The shape of such a curve for a "point" target is given in Figure 20 in Chapter 5. The radar curve can be obtained from this curve by changing the ordinate back to $20 \log A$ [equation (3) in Chapter 5] and then using equation (5) in Chapter 5.

Since an actual surface target is generally large and at short distances intercepts a considerable part of the energy, the oscillatory part of the point curve of Figure 26 in Chapter 5 is smoothed out. The value of $A$ for short distances is sometimes taken as $6 A_{0}$, or $15 \mathrm{db}$ above the free-space ralue.

In Figure 8, an example is given of curves computed for various ship sizes. Most of the plotted points fall in the 200- to 600-ton region. However, the precaution of a performance eheek is necessary in order to use this method most effectively (see Section 7.3.7). Since shape plays an important part in radar cross section, a ship of unusual shape may give a signal outside its class.

\subsubsection{Performance Check}

Since the performance of a set varies, before a chart described in Section 7.3.6 is used to detect unknown ships, it should be checked to determine whether set performance is normal. The curves can be used for this check by using a known ship. Such a check is shown in Figure 9 for two different days. On one day, the points for the ship fell in the correct region. On the other day, they fell too low by about $20 \mathrm{db}$. Accordingly, on the latter day the curves should have been used with an ordinate correction of $-20 \mathrm{db}$.

Other types of performance check may be used, such as permanent echoes, free-space range of aircraft, or a signal generator. 


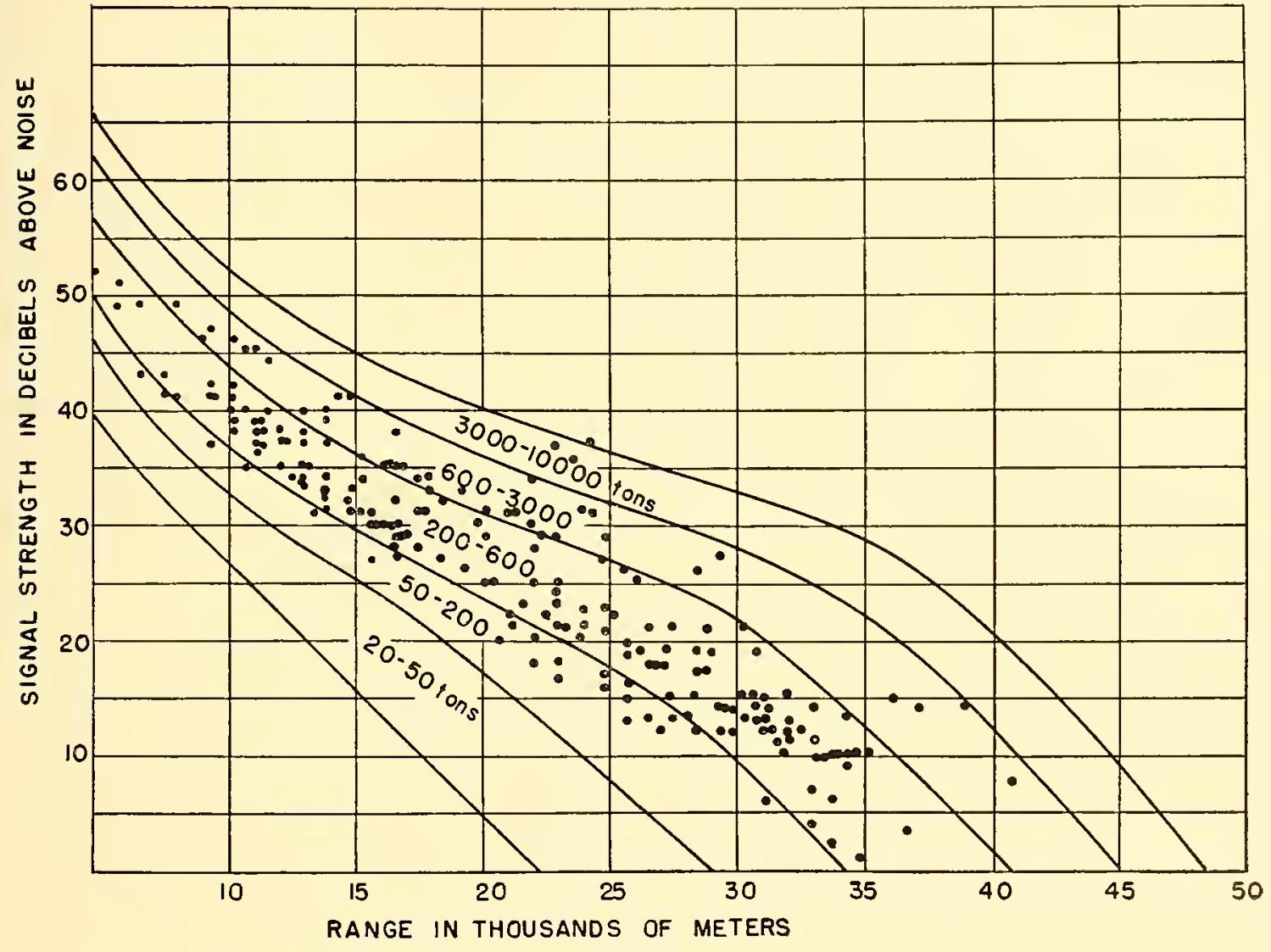

Figure 8. Estimation of ship sizes. (From Coast Artillery Experimental Establishment, England.) 


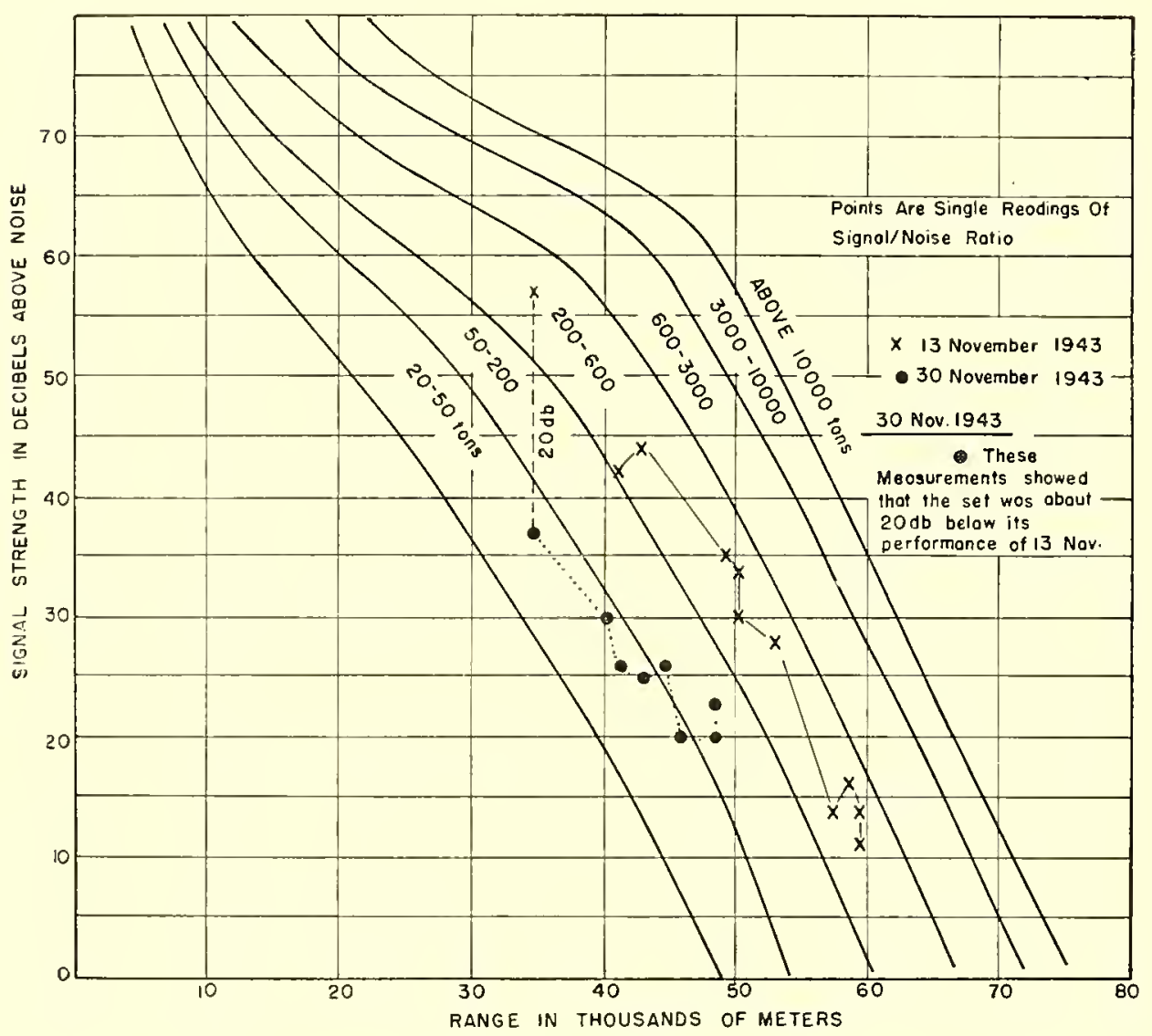

Figure 9. Range in thousands of meters. (From Coast Artillery Experimental Establishment, England.) 
DATA ON COMMUNICATION

AND RADAR EQUIPMENT

The tables given in this section are not intended to be exhaustive. These are the best available data but should be used with caution, since specification changes may change rated characteristics of sets.

Table 3. Communication equipment.

\begin{tabular}{|c|c|c|c|c|c|c|}
\hline \multirow{3}{*}{$\begin{array}{c}\text { Communication } \\
\text { equipment }\end{array}$} & \multirow{3}{*}{$\begin{array}{l}\text { Frequency } \\
\text { (mc) }\end{array}$} & \multirow{3}{*}{$\begin{array}{l}\text { Power } \\
\text { output } \\
\text { (watts) }\end{array}$} & \multirow{3}{*}{$\begin{array}{l}\text { Receiver } \\
\text { sensitivity }\end{array}$} & \multicolumn{2}{|c|}{ Antenna } & \multirow[b]{2}{*}{ Beamwidth } \\
\hline & & & & Polarization & Gain & \\
\hline & & & & & $(\mathrm{db})$ & Horizontal \\
\hline SCR-300 & 44 & 0.5 & $2.5 \mu \mathrm{V}$ & $V$ & & Whip \\
\hline SCR-522 & 125 & 6 & $4 \mu_{\mathrm{V}}$ & $V$ & & Whip \\
\hline $\mathrm{AN} / \mathrm{TRC}-8$ & 240 & 12 & $\begin{array}{l}30 \mu_{v} \\
\text { (4 channel) }\end{array}$ & $H$ & 7 & \\
\hline AN/TRC-5 & 1,400 & 400 & $N F^{*} 12 \mathrm{db}$ & $H$ & 14 & \\
\hline AN /TRC-6 & 4,600 & 2 & $\mathrm{NF}^{*} 19 \mathrm{db}$ & $H$ & 33 & \\
\hline
\end{tabular}

* Noise figure.

TABLE 4. Radars.

\begin{tabular}{|c|c|c|c|c|c|c|c|c|}
\hline \multirow[b]{3}{*}{ Radars } & \multirow{3}{*}{$\begin{array}{l}\text { Frequency } \\
\text { (mc) }\end{array}$} & \multirow{3}{*}{$\begin{array}{c}\text { Power } \\
\text { output } \\
\text { (kw) }\end{array}$} & \multicolumn{2}{|c|}{$\begin{array}{c}\text { Receiver } \\
\text { sensitivity }\end{array}$} & \multirow{2}{*}{\multicolumn{2}{|c|}{ Antenna }} & \multirow{2}{*}{\multicolumn{2}{|c|}{ Beamwidth }} \\
\hline & & & Noise figure & Bandwidth & & & & \\
\hline & & & $(\mathrm{db})$ & (mc) & Polarization & Gain (db) & Horizontal & Vertical \\
\hline SCR-271DA & 106 & 100 & 6 & 1 & $H$ & 19.8 & $11^{\circ}$ & $12^{\circ}$ \\
\hline AN/TPS-3 & 600 & 200 & 11 & 1.8 & $H$ & 23.4 & $12^{\circ}$ & $11^{\circ}$ \\
\hline SCR-584 & 3,000 & 300 & 15 & 1.7 & $H$ & 30.8 & $7^{\circ}$ & $7^{\circ}$ \\
\hline $\mathrm{SC}-4$ & 200 & 200 & 6 & 0.5 & $H$ & 13.5 & $20^{\circ}$ & $60^{\circ}$ \\
\hline $\mathrm{SQ}$ & 2,500 & 1 & 13 & 2 & V & 20 & $8^{\circ}$ & $15^{\circ}$ \\
\hline SG-1 & 3,000 & 50 & 18 & 1 & $H$ & 28 & $6^{\circ}$ & $15^{\circ}$ \\
\hline ASB & 515 & 10 & 15 & 1.4 & $H$ & 30 & $60^{\circ}$ & \\
\hline
\end{tabular}

TABLe 5. IFF or beacons.

\begin{tabular}{lccccc}
\hline \hline & $\begin{array}{c}\text { Frequency } \\
\text { (mc) }\end{array}$ & $\begin{array}{c}\text { Power } \\
\text { output }\end{array}$ & $\begin{array}{c}\text { Antenna } \\
\text { Pensitivity }\end{array}$ & $\begin{array}{c}\text { Polarization } \\
\text { Gain } \\
(\mathrm{db})\end{array}$ \\
\hline AN/TPX-4 & 170 & $0.5 \mathrm{~km}$ & $15 \mu_{\mathrm{V}}$ & $V$ & 6 \\
AN/UPN-1,2 & 3,000 & $50 \mathrm{kw}$ & $0.05 \mu_{\mathrm{V}}$ & $H$ & 6 \\
AN/UPN-3,4 & 9,320 & $300 \mathrm{kw}$ & $0.02 \mu_{\mathrm{V}}$ & $H$ & 12 \\
\hline
\end{tabular}




\section{Chapter 8}

\section{DIFFRACTION BY TERRAIN}

$\mathrm{T}^{\prime \prime}$ HE EFFECTs of diffraction around natural obstacles of complicated shape are difficult to analyze. Theory offers two lines of approach to diffraction problems, both based on the substitution of contours of simple shape in place of the natural obstacle.

The first and oldest method, known as the FresnelKirchhoff method, is an approximate procedure for calculating the diffraction by a flat screen. It yields comparatively simple formulas for the diffracter field; the present chapter is concerned with a presentation of this method.

The sceond method is based on the fact that the wave equation can be solved for obstacles of very simple geometrical shape, especially cylinders and spheres. If the curvature of a hill is fairly constant, so that its shape can be approximated by a cylinder or sphere, the field behind the hill can be obtained by the use of this method.

Observations on diffraction by obstacles in the short wave and microwave region are very sparse. It is, therefore, not possible to present a consistent body of results that could be utilized in radio practice. It seems, however, rather certain from the observations that when the shape of the obstacle approaches one of the special shapes dealt with by the theory, the latter gives a fair account of the facts. Such cases will not be found too frequently in practice. The hope is nevertheless justified that the right order of magnitude is obtamed by a judicious application of the theory. The main application is in the lower frequency band $(30$ to $200 \mathrm{mc})$; for higher frequencies, the diffracted field is relatively unimportant.

\subsubsection{The Fresnel Diffraction Theory}

The Fresnel-Kirchhoff approximate theory was originally developed to account for the diffraction of beams of light when cut off by diaphragms, slits, and similar optical devices. In applying this theory to the propagation of radio waves over the earth, only one basic problem is usually encountered, namely that of diffraction around a straight edge. In the present section, the general method of handling this problem and obtaining numerical results is given. On applying the method to actual cases certain accessory problems arise which will be dealt with in Sections 8.3 and 8.4. The most important of these complications is caused by ground reflection.

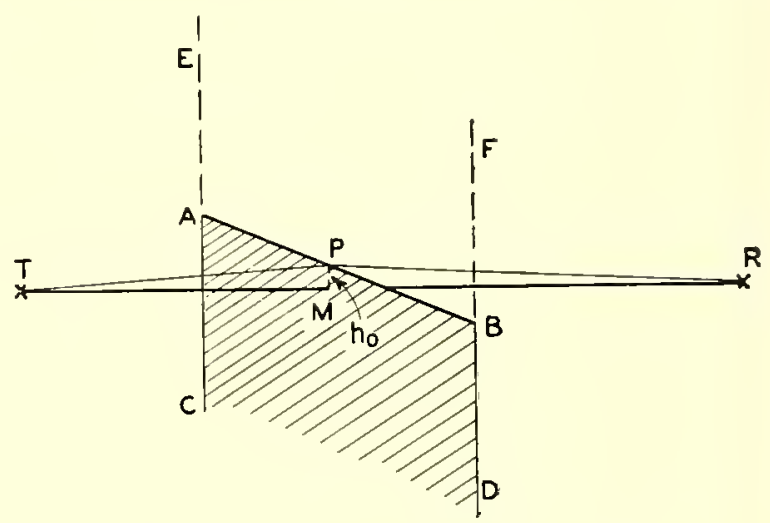

Figure 1. Diffraction around straight edge.

In Figure 1, the area $C A P B D$ forms an opaque screen bounded by a straight edge $B P A$. The width $A B$ of the screen is assumed infinite in the mathematical theory, but is here shown finite for simplicity. The line connecting the transmitter $T$ to the receiver $R$ intersects the plane of the opaque screen in the point $M$ whose distance from the edge is $P M=h_{0}$. The shortest unobstructed path of the radiation is $T P R$.

In a purely geometrical theory, the point $R$ would be in the shadow of the screen and would receive no radiation. If the ware nature of radiation is taken into account, it is found that an electromagnetic field is generated in the shadow of the screen; the waves are bent around the obstacle.

The mathematical derivation of the diffraction formulas will not be given here as it is rather intricate; however, the problem is discussed in Section 8.2. The discussion here is limited to a qualitative visualization of the mechanies of diffraction (Section 8.1.3); the final formulas used for computa- 
tions will then be written down at once (Sections 8.1.4 to 8.1.6).

\subsubsection{Mechanism of Diffraction}

The physical idea underlying the Fresnel-Iirehhoff diffraction theory may be presented as follows. At points visible from $T$ the field, to a first approximation, is equal to the free-space field $E_{0}$. This applies in particular to all points of the plane ECDF containing the sereen. The receiver $R$ receives radiation from the open part $E A P B F$ of the plane, while there is no radiation incident upon $R$ from the opaque surface $C A P B D$. In order to eompute the field at $R$, it is assumed that in the open part of the plane the field is $E_{0}$ while on the opaque sereen the field vanishes. Such a field distribution may be realized physically by assuming that there is a conducting sheet in the open region $E A P B F$ with suitably ehosen oscillating charges or eurrents such that the field $E_{0}$ is produeed on the side of the sheet facing the receiver. The total radiation received at $R$ from such a current-sheet will be equivalent to the radiation from $T$ bent around the diffracting sereen. The fictitious sheet $E A P B F$ forms a system of secondary sourees of radiation whose effeet is equivalent to that of the primary source for all points on the far side of the plane ECDF (side of the receiver), but not on the near side (side of the transmitter).

It is evident that most of the radiation received at $R$ comes from the area near the point $P$ above the line $A P B$. The relative importanee of contributions of areas more or less removed from $P$ is discussed in Section 8.2.

When the primary source at $T$ is replaced by a distribution of secondary sourees in the plane of the screen, an essential approximation is made. It is assumed that there are no secondary sourees in the opaque region $C A P B D$. In reality, the screen is a physical body and, whether it is a conduetor or a dielectric, there is an electromagnetic field in its surface layers, especially near the edge $A P B$, and this field makes a eontribution to the radiation received at $R$. In the approximate theory, it is assumed that the field on the surface of the opaque sereen is negligible. In the terminology of opties, this implies that the screen is black; in radio terminology, it means that the surface of the screen is rough (Section 8.3.2).

\subsubsection{The Straight Edge Formula}

The physical picture just described can be put into mathematical language. When the rather intrieate derivations are earried through, a relatively simple formula results.

The symbols and designations used are illustrated in Figme 2. In aceordance with praetice it is assumed that the line $T R$ is nearly horizontal. The trace of the opaque screen on a rertical plane through $T$ and $R$ is assumed perpendicular to the line $T R$

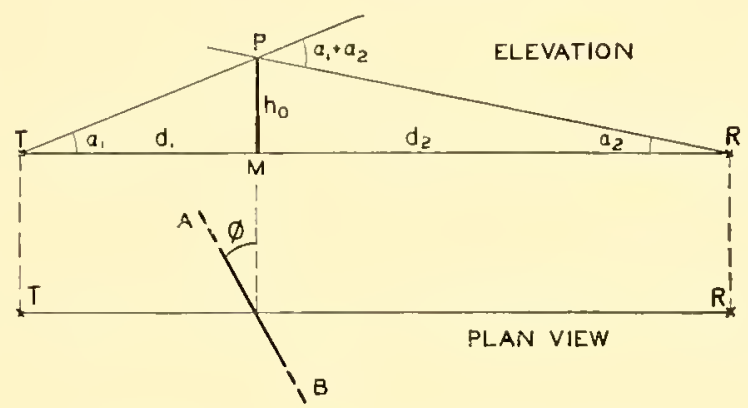

Figure 2. Diffraction around straight edge.

(upper part of Figure 2). The trace of the screen oll a horizontal plane may, however, make an angle $\phi$ with the line $T R$ (lower part of Figure 2).

In view of the approximate nature of the theory explained in the preceding paragraph, the following eonditions must be fulfilled in order to obtain reliable results:

$$
d_{1}, d_{2}>>h_{0}>>\lambda
$$

That is, the distances from the transmitter and reeeiver to the obstacle must be large compared to the height of the latter above the line $T R$, and this height must be large compared to the wavelength. The second of these conditions is likely to be fulfilled in the short-wave and microwave bands, and the first will be fulfilled when the angles of elevation $\alpha_{1}$ and $\alpha_{2}$ of the rays, drawn from the transmitter and receiver to the edge, are small.

A second condition for the ralidity of the diffraetion formula refers to the horizontal extension of the screell. The formulas are derived for a screen of infinite horizontal extent, but in practice it will usually suffice if the horizontal extension of the screen is large compared to the height $h_{0}$. 
If these conditions are fulfilled, the field at the where receiver is given by

$$
E=E_{0} \frac{e^{j \pi / 4}}{\sqrt{2}} \int_{-\infty}^{0} e^{-j \pi v^{2} / 2} d v,
$$

where $E_{0}$ is the free-space field at the receiver in absence of the screen and

$$
v= \pm h_{0} \sqrt{\frac{2}{\lambda}\left(\frac{1}{d_{1}}+\frac{1}{d_{2}}\right)}= \pm \sqrt{\frac{2 h_{0}}{\lambda}\left(\alpha_{1}+\alpha_{2}\right)} .
$$

In the last formula, use is made of the fact that $\alpha_{1}$ and $\alpha_{2}$ are small angles 'so that approximately $\alpha_{1}=h_{0} / d_{1}$ and $\alpha_{2}=h_{0} / d_{2}$.

\subsubsection{The Fresnel Integrals}

An integral of the type appearing in equation (2) is known as Fresnel's integral; its properties will now be briefly discussed and numerical data given. The standard Fresnel integral is usually defined as

$$
C(v)-j S(v)=\int_{0}^{v} e^{(-\pi / 2) v} d v^{\prime}
$$

$$
\begin{aligned}
& C(v)=\int_{0}^{v} \cos \left(\frac{\pi}{2} v^{2}\right) d v, \\
& S(v)=\int_{0}^{v} \sin \left(\frac{\pi}{2} v^{2}\right) d v .
\end{aligned}
$$

If this function is plotted in the complex plane, with $C$ and $S$ as abscissa and ordinate, respectively, for all values of $v$, a curve is obtained that is known as Cornu's spiral (Figure 3). $C-j S$ is represented, in magnitude and phase, by a vector from the origin to a point on this spiral.

It may be shown that the length of arc along the spiral, measured from the origin, is equal to $v$. In the graph, values of $v$, counted positive in the first quadrant and negative in the third quadrant, are indicated along the spiral. As $v$ approaches infinity, the spiral winds an infinity of times around two points lying at the distance $1 / \sqrt{2}$ from the origin on a 45-degree line. $C$ and $S$ for the end points are

$$
C( \pm \infty)= \pm \frac{1}{2}, \quad S( \pm \infty)= \pm \frac{1}{2}
$$

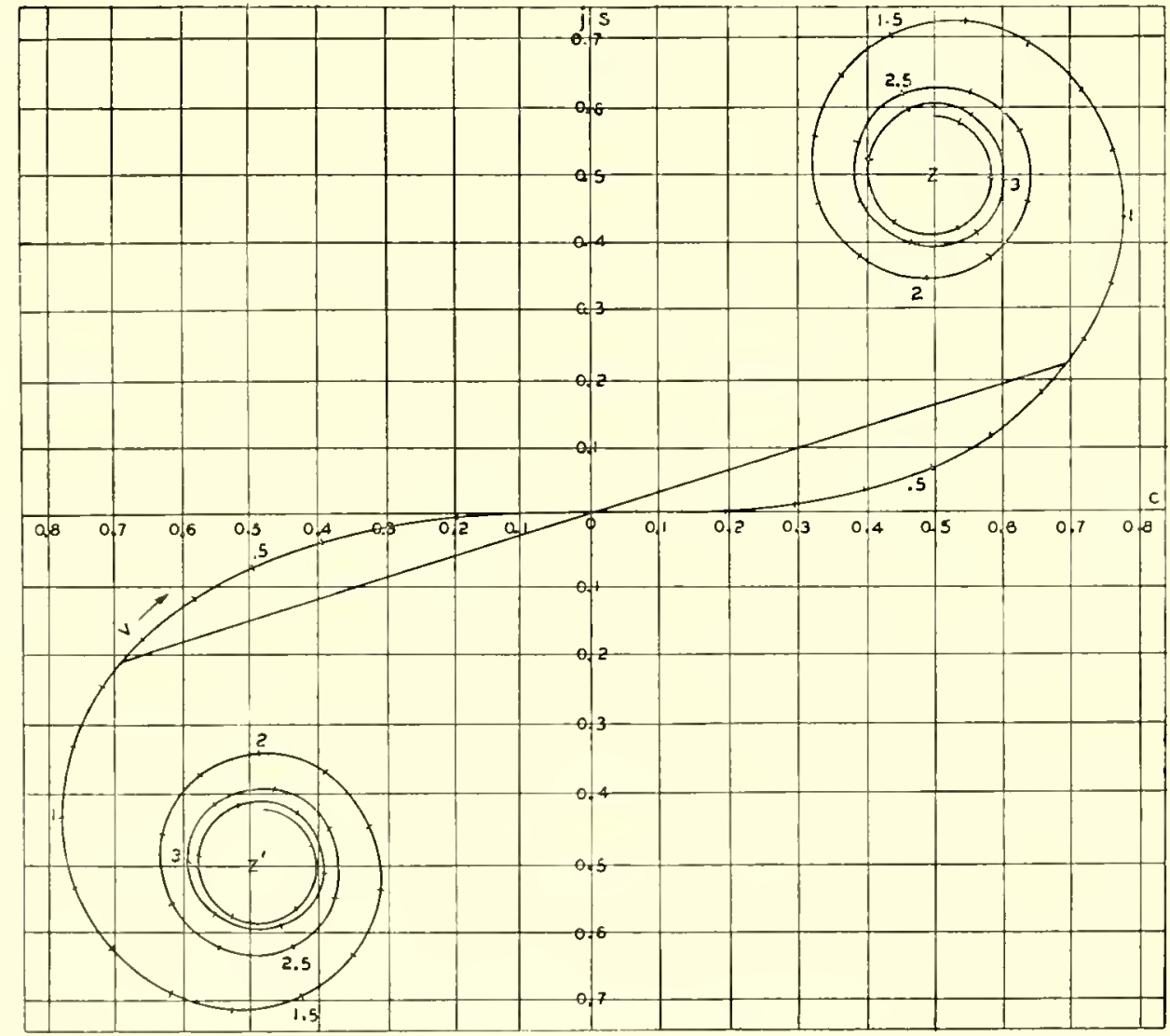

Figure 3. Cornu spiral. 


\subsubsection{Application to Straight Edge}

Since

$$
\frac{e^{j \pi / 4}}{\sqrt{2}}=\frac{1+j}{2}
$$

equation (2) may be rewritten, on using equations (4) and (5), as

$$
E=E_{0} \frac{1+j}{2}\left[\frac{1}{2}+C(v)-\frac{j}{2}-j S(v)\right] .
$$

It will be noticed that the quantities

$$
\frac{1}{2}+C, j\left(\frac{1}{2}+S\right)
$$

have a simple geometrical meaning. They are the real and imaginary components, respectively, of a vector drawn from the lorrer point of convergence (point $-1 / 2,-j / 2$ ) of the Cornu spiral to a point on this spiral. The bracket in equation (6) is equal to this vector in magnitude but with opposite phase.

The Fresnel formulas and Cornu spiral as given above will assist the reader in establishing the relations of our equations with the classical theory of diffraction as found in all textbooks on the subject. For practical purposes the field behind a diffracting straight edge given by equation (6) will be denoted by

$$
\frac{E}{E_{0}}=z e^{-j \zeta}
$$

In Figure 4, the modulus $z$ is plotted as a function of $v$. In Figure 5, the phase lag $\zeta$ is plotted in a similar. way. (With the above choice of the sign, $\zeta$ is positive in the shadow.)

The variable $v$ is given by equation (3). On account of the square root, there is an ambiguity in sign. Closer inspection shows that $v$ must be taken positive when the receiver is in the illuminated region, above the line of sight; $v$ must be taken negative when the receiver is in the shadow zone.

When $v$ tends to $-\infty$, the line $A P B$ (Figure 1) moves far upwards relative to the line $T R$; the receifver lies deep in the shadow and $E$ approaches zero by equation (6). When $v$ tends to $+\infty$, the line $A P B$ moves far downward, and the screen ceases to orm an obstruction, $E$ approaches $E_{0}$. At the line of sight (when the point $P$ in Figure 1 coincides with $M), v=F(v)=0$ and $E=E_{0} / 2$. Clearly, the effects of diffraction are not confined to the shadow region but extend considerably into the illuminated zone. If the recciver is sufficiently deep in the shadow, about $v>-1$, the following approximate formula holds:

$$
z=\left|\frac{E}{E_{0}}\right|=\frac{0.225}{v} .
$$

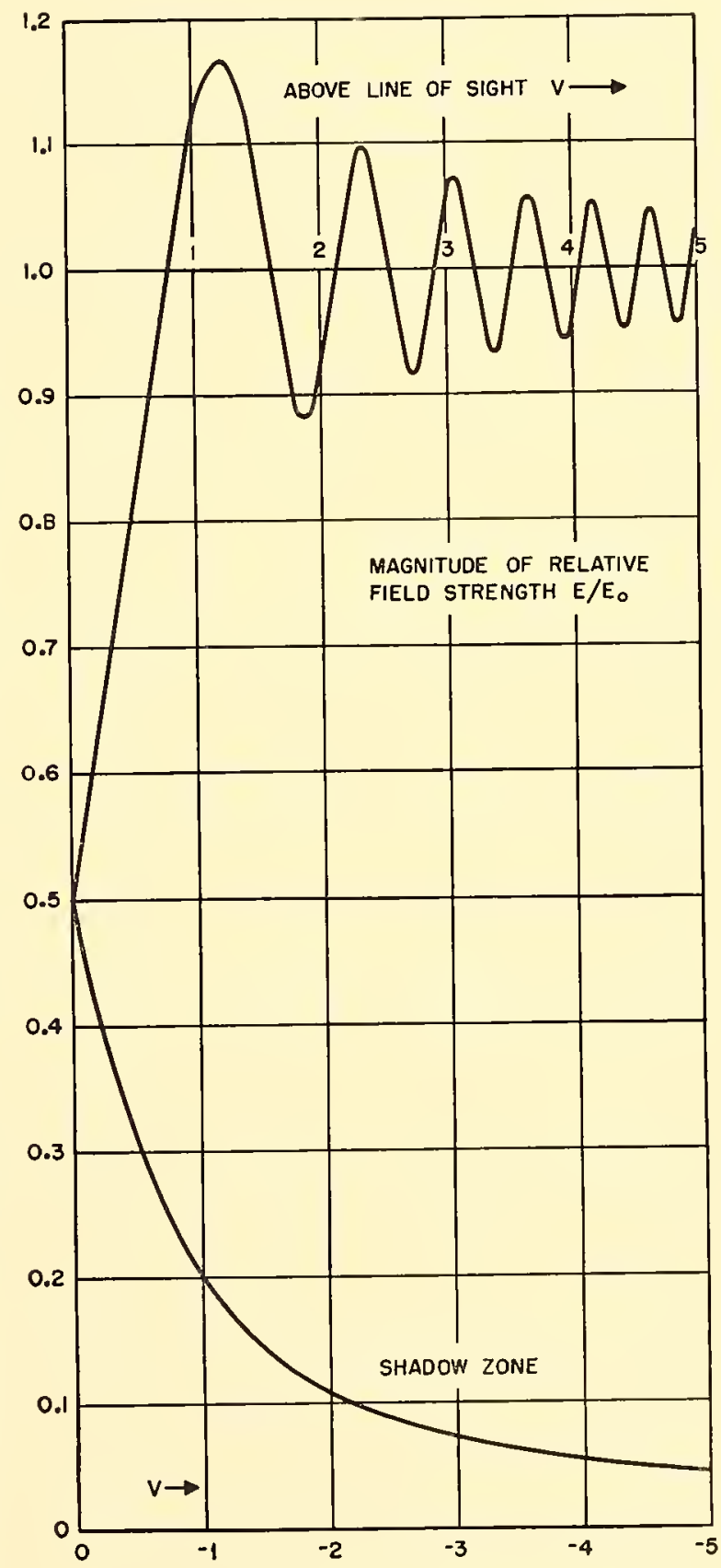

FIgure 4. Magnitude of relative field strength $E / E_{0}$ versus $v$ 


\subsubsection{Polarization. Large Angles}

It may be noticed that in the preceding equations no reference is made to the state of polarization of the diffraction field. The results of the approximate Fresnel-Kirchhoff theory are independent of the state of polarization in agreement with observation.
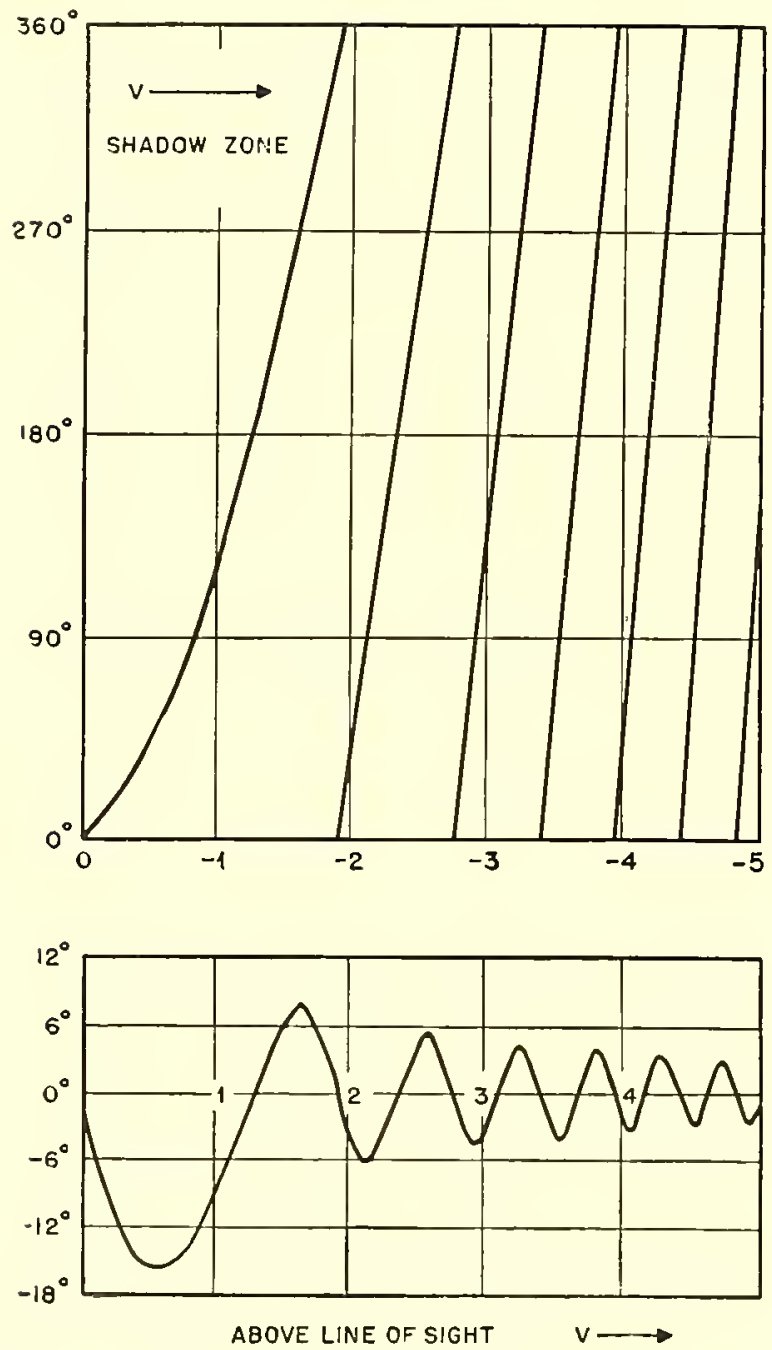

Figure 5. Phase lag (ordinate) of relative field strength $\left(E / E_{0}\right)$ versus $v$ (abscissa).

If the angles of diffraction ( $\alpha_{1}$ and $\alpha_{2}$, Figure 2) become large, larger than a fert degrees, for instance, the approximate theory no longer applies. The deviations from the Fresnel formulas then go in opposite directions for the two states of polarization. If the electric rector is parallel to the diffracting cdge, the field in the shadow at large angles is slightly diminished as compared with that given by the Fresnel formulas; if the electric field is perpendicular to the diffracting edge, the diffracted field in the shadow at large angles can become appreciably larger than the calculated one and, in the case of very large angles, the excess may reach the magnitude of, say, 6 to $15 \mathrm{db}$. In the region above the line of sight, the sign of the polarization effect is reversed (slight increase for polarization parallel to the edge, appreciable decrease for polarization perpendicular to the edge).

These effects are entirely analogous to those that are observed when the currents induced in the surface of the obstacle cannot be neglected (Section 8.1.3), and they have the same physical origin.

\subsection{DIGRESSION ON FRESNEL'S THEORY}

8.2.1

\section{Fresnel Zones}

The concept of the Fresnel zone has played an important role in the development of diffraction theory. As it is frequently referred to in papers on the subject, it may be useful to digress briefly on it. Fresnel's original construction is based on the conception that any small element of space in the path of a wave may be considered as the source of a secondary wavelet, and that the radiation field can

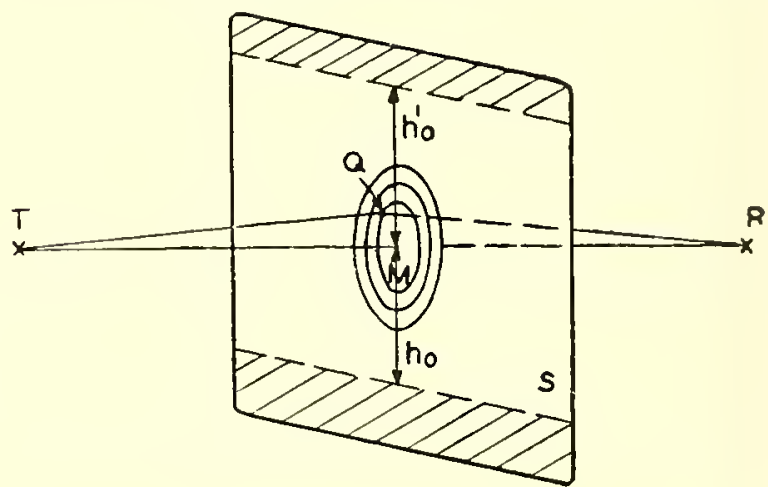

Figure 6. Relations of Fresnel zones and diffracting slots.

be built up by the superposition of all these wavelets (Huyghens' principle). In particular, consider the field produced by the transmittel in the open part of the plane containing the diffracting screen (EAPBF in Figure 1) and let each element of this plane be the souree of a secondary wavelet. This may be achieved by distributing a suitable ficti- 
tious system of oscillating currents (or a system of elementary doublets of proper strength) over the surface of the plane. The field at the receiver is then the superposition of all the fields produced by the wavelets.

Now let (Figure 6) $S$ be a plane perpendicular to the line $T R$ and let $M$ be the point in which the line $T R$ intersects the plane $S$. Let $Q$ be a point on the plane $S$ such that the difference in path between $T Q R$ and $T M R$ is just $\lambda / 2$. The locus of these points is a circle about $M$. Similarly we can construct other circles so that the corresponding path differences are integral multiples of $\lambda / 2$. The area within the first circle is ealled the first Fresncl zone, the subsequent ring-shaped areas are called the second, third, etc., Fresnel zones. The secondary wavelets originating in the first, third, fifth, ete., Fresnel zones are in phase with each other and reinforce each other by constructive interference at $R$, while the secondary wavclets originating in the second, fourth, etc., zoncs are in phase with each other but out of phase with the former group and tend to cancel the field produced by this group.

Hence if the plane $S$ is opaque except for a round hole centered on $M$, the intensity of the radiation field at $R$ will depend on the number of Fresnel zones that fall inside the hole. If we start out with a very small hole and progressively increase its size, there will be a maximum of intensity at $R$ (nearly twice the free-space field $E_{0}$ ) when the hole just comprises the first Fresnel zone. If the size of the hole is further increased, the destructive interference of the second zone comes into play, decreasing the intensity, and a minimum (very nearly zero) is reached when the hole contains just the first tro zones. On continued increase of the hole size, further maxima and minima appear. The amplitude of these oscillations decreases very gradually until eventually the field at $R$ approaches the free-space value.

\section{Diffraction by a Slot}

The preceding considerations indicate that only a comparatively small area of an opening, of the order of one Fresnel zone, is required to produce an illumination that is comparable in order of magnitude to the free-space field. It is also seen that the simple geometrical construction of the Fresnel zones is more suitable when dealing with the diffraction by round openings than with screens bounded by straightedges. Qualitatively, however, the conditions are similar.

As an example, consider the case of a slot bounded by parallel edges at distances $h_{0}$ and $h_{0}{ }^{\prime}$ from the point of intersection $M$ between the plane of the slot and the direction from the observer to the distant light source (see Figure 6). The diffracted field $E$ will obviously be equal to the free-space field $E_{0}$ if the slot is infunitely wide on both sides of $M$, which corresponds to a vector joining the two foci of the Cormu spiral. However, there is an infinite number of other finite openings of the slot which also will give the free-space field. Suppose, for instance, that $h_{0}=h_{0}{ }^{\prime}$ in Figure 6 and that the slot width is gradually increased from zero. A glance at the Cornu spiral (Figure 3) shows that when $v=0.75$ and $v^{\prime}=-0.75$, the vector representing the diffraction field is approximately equal to the free-space field. This width represents, for a slot, the analogue of the first Fresnel zone for a circular opening.

\section{S.3 DIFFRACTION HY HILLS}

\subsubsection{Introduction}

The formula for diffraction by a straight edge may be applied in radio practice to determine the diffraction field behind a ridge. The ridge need not be perpendienlar to the transmission path, but the condition given in equation (1) must be approximately fulfilled. The distance from transmitter and receiver to the ridge should be large compared to the height of the latter above the straight line $T R$; and that height should be large compared to the wavelength.

Moreover, as pointed out in Scction 8.1.3, the diffraction formula applies in principle only to the case where the effeet of the currents induced on the surface of the ridge upon the ficld at the receiver can be neglected. This is the case (1) when the ridge has the sliape of a steep and narrow knife-edge protruding from the surrounding countryside; or (2) when the surface of the ridge is rough (see Section 8.3.2). Experience shows that so long as the profile of the ridge is reasonably compact and its surface reasonably rough, the diffraction formula will give the magnitude of the field behind the ridge to within a few decibels. 
If the ground near the transmitter or receiver is smooth, howerer, it becomes necessary to take ground reflection into account. This may be done by introducing an image transmitter and receiver. The field is then the sum of four components whose relative plase must be calculated (see Figure 11).

Earth curvature will be neglected throughout the present section.

\subsubsection{Criterion for Roughness}

It is difficult to establish a quantitative criterion for the roughness of a surface. From the viewpoint of radiation theory, the effect of a rough surface is to seatter incident radiation diffusely in all directions with no preference for the direction of regular reflection, whereas a smooth surface will reflect the incident radiation according to Snell's law. In radio work, the effect of diffuse reflection is to weaken the radiation scattered in the direction of the receiver so much that its intensity may be neglected compared to the direct ray. A moderately rough surface will give a coefficient of reflection intermediate between zero and unity. A surface will be optically smoother as the incident radiation approaches grazing, and even surfaces that are comparatively rough geometrically may then give partial reflection.

A rule taken flom optics and known there as Rayleigh's criterion has been used successfully in radio practice. Assume that the roughness is produced by numerous small elevations above a level surface and let $I$ be the typical height of such an eleration. The difference in path between a ray

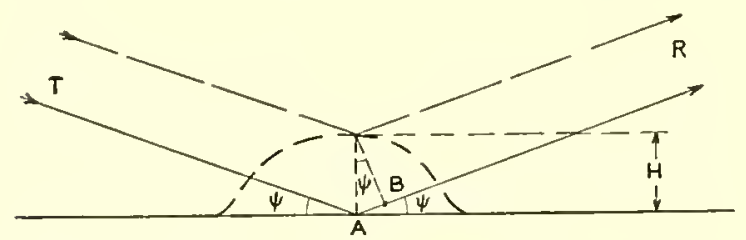

Figure 7. Geometry for Rayleigh's criterion for rough ground.

reflected from the ground and a ray from the top of the eleration is $2 A B$ in Figure 7 , which is equal to $2 I I \sin \psi$ or $2 H \psi$ approximately for small angles $\psi$. The difference in phase between the two rays is $2 H \psi(2 \pi / \lambda)$. The criterion now requires that the surface be considered as rough when this phase difference exceeds 45 degrees, or $\pi / 4$ radians.
Hence the critical value of $H$ is given by

$$
\frac{4 \pi H \psi}{\lambda}=\frac{\pi}{4}, \text { or } H=\frac{\lambda}{16 \psi},
$$

with $\psi$ in radians and

$$
H=\frac{3.6 \lambda}{\psi},
$$

with $\psi$ in degrees. The surface is considered smooth or rough according to whether $H$ is smaller or larger than this value.

Sometimes it is convenient to refer to the field pattern that would be present over a reflecting surface. This is done by introducing a new variable, the lobe number

$$
n=\frac{4 h_{1} \psi}{\lambda}
$$

( $h_{1}$ transmitter height above the ground), where $n=1,3,5$, ete., correspond to the angle of the first, second, ete., maxima in the lobe pattern and $n=0$, 2 , 4 , etc., to the nulls of the lobe pattern. Introducing $n$ into equation (8), the criterion assumes the form

$$
H=\frac{h_{1}}{4 n} .
$$

Although the criterion is approximate and gives no more than an order of magnitude estimate, it is rather surprisingly well fulfilled in radio practice. Experience has shown that when the differences in level which constitute roughness are of the order indicated by these equations, the reflection coefficient is reduced to a small fraction (about one-fifth) of the value calculated for an ideal surface.

\subsubsection{Diffraction by a Straight Ridge}

Assume that the ground intervening between the transmitter and receiver is everywhere rough, so

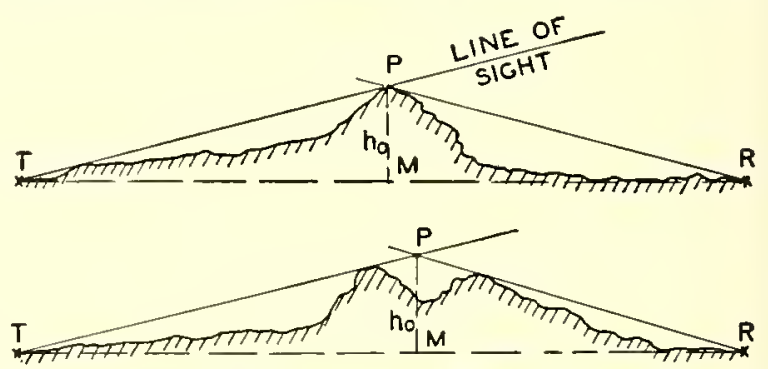

Figure 8. Diffraction by a straight ridge.

that all ground reflection may be neglected. For the sake of computation, the ridge is replaced by a vertical screen of height $h_{0}$ above the line $T R$. The 
top of the ridge forms the diffracting edge ( $P$ in Figure 8). If the profile of the ridge is somewhat more complicated, the effective diffracting edge might be a purely mathematical line, as shown in the lower part of the figure. The height $h_{0}$ is conveniently determined from a profile of the transmission path obtained from a topographic map. If the heights $h_{1}, h_{2}$, and $h$ of transmitter, receiver, and obstacie, respectively, above a given reference level such as sea level are given, we have

$$
h_{0}=\frac{d_{1} h_{2}+d_{2} h_{1}}{d_{1}+d_{2}}-h,
$$

where the signs have been chosen so that $h_{0}$ is negative when the receiver is in the shadow of the ridge and positive when it is in the illuminated region. Now, by equation (3),

$$
v=h_{0} \sqrt{\frac{2}{\lambda}\left(\frac{1}{d_{1}}+\frac{1}{d}\right)}=\sqrt{\frac{2 h_{0}}{\lambda}\left(\alpha_{1}+\alpha_{2}\right)} .
$$

In these equations, give the angles $\alpha_{1}$ and $\alpha_{2}$ the same sign as $h_{0}$ and give $v$ the same sign that $h_{0}$ has in equation (12).

The ratio of the field to the free-space field at the leceiver is now given by $\left|E / E_{0}\right|=z(v)$, defined by equation (7) and plotted in Figure 4. In Figure 9, this ratio is given in decibels as a function of the quantity $x=-h_{0} / \sqrt{\lambda d}$ (all lengths in meters). The successive curves in Figure 9 correspond to different values of the ratio $d_{1} / d_{2}$ or $d_{2} / d_{1}$ (choose whichever one is the smaller). Only the field below the line of sight is shown.

\subsubsection{Field Near the Line of Sight}

The fact that just above the line of sight the field increases above its free-space value may sometimes be usech to obtain a favorable site (Figure 10). The maximum value of the field is about 1.17 times the free-space vahue (Figure 4 ), equivalent to $1.36 \mathrm{db}$. On the other hand, there are advantages in avoiding a line $T R$ that is too close to grazing the top of an intervening obstacke, as this will substantially reduce the signal. At the line of sight, the signal is $6 \mathrm{db}$ below free space. In order to get approximately the free-space value of the field, the crest of the obstacle should be sufficiently below the line $T R$ so that $v>0.8$ where $v$ is given by equation (13), $h_{0}$ being the clearance between the line $T R$ and the obstacle. In cases where the heights and distances are not quite certain, it is therefore preferable to select a higher and definitely unobstructed site rather than to try to utilize the small gain that might possibly be had from the diffraction field.

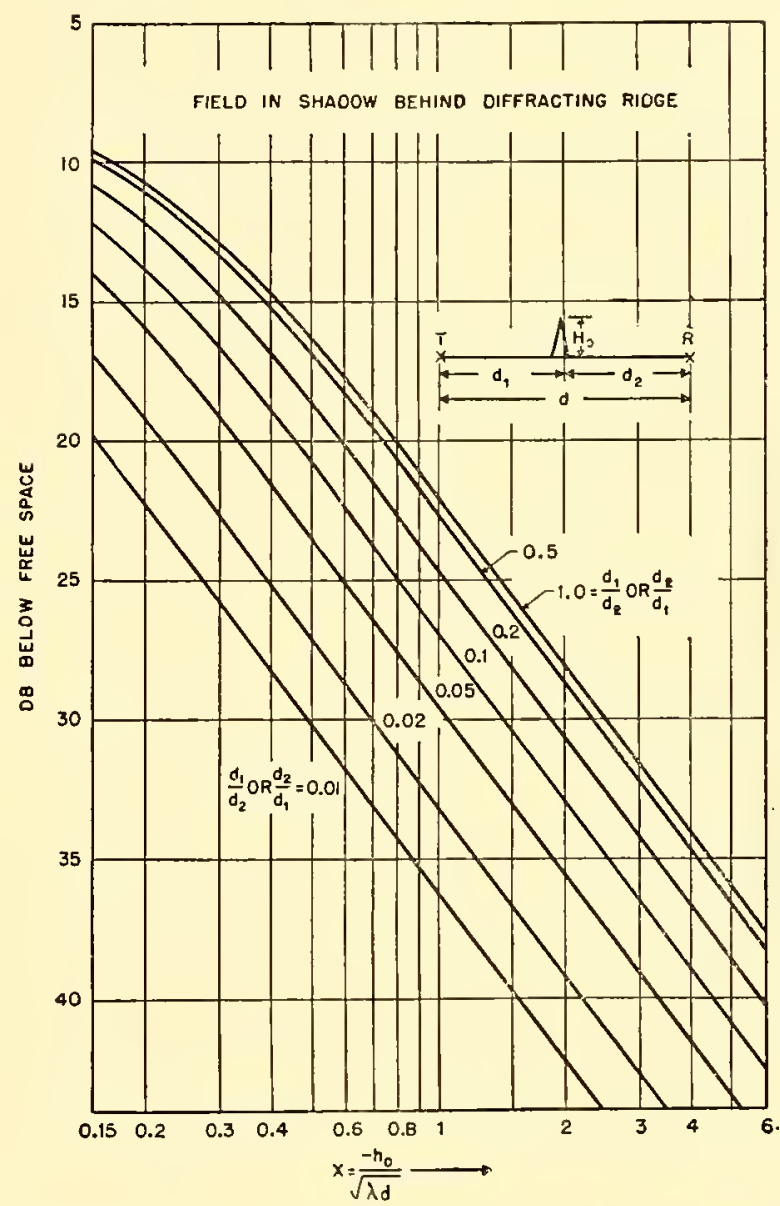

Figure 9 . Field in shadow behind a diffracting ridge.

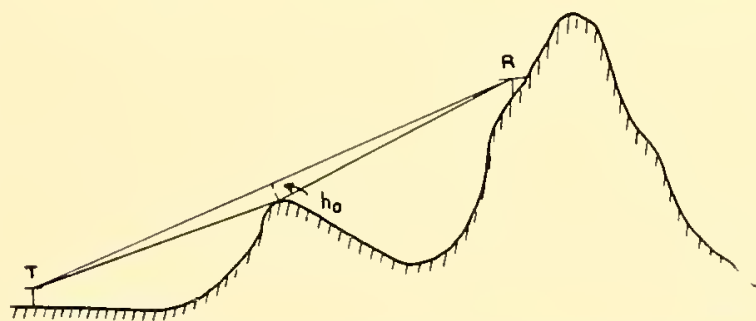

Figure 10. Diffraction field above the diffracting edge.

\subsubsection{Diffraction with Reflecting Ground}

When the ground near the transmitter or receiver is smooth and reflecting, the diffraction problem. becomes very complicated. It can be solved by the- 
method of images on assuming that the radiation reflected on the transmitter side of the obstacle issues from an image transmitter and that the radiation reflected on the receiver side is incident upon

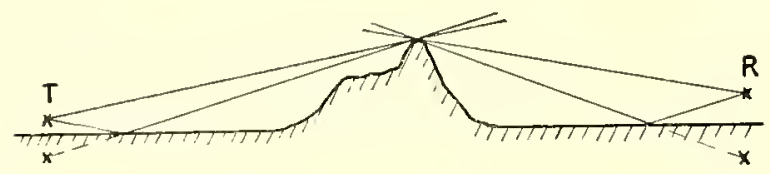

FIgURE 11. Diffraction of both direct and reflected rays.

an image receiver (Figure 11). The total field at the receiver may be written

$$
E=E_{1}-E_{2}-E_{3}+E_{4}
$$

where each term on the right-hand side is of the form of equation (6), $E_{1}$ corresponding to the direct radiation, $E_{2}$ to the radiation from the image transmitter to the receiver, $E_{3}$ to the radiation from the transmitter to the image receirer, and $E_{4}$ to the radiation from one image to the other. These four terms differ in the value of $v$ assigned to each of them; the effective height $h_{0}$ computed by equation (12) and the path lengths being different in each case.

\subsubsection{Example}

Assume that from a topographic map the profile shown in Figure 12 has been drawn. The horizontal scale is in kilometers and the vertical scale in meters above sea level. From this profile, combined with inspection of the terrain, it has been found that the ground is so rough that the reflected rays may be

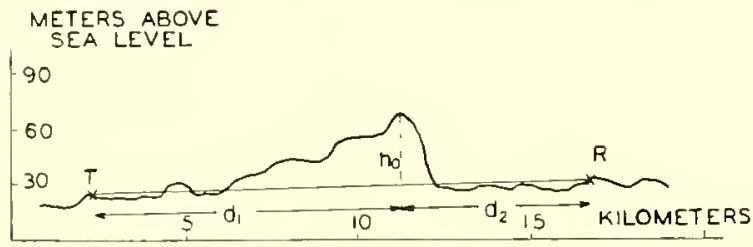

Figure 12. Assumed profile.

disregarded. The heights above sea level of transmitter, receiver, and obstacle, are respectively $h_{1}=24$ meters, $h_{2}=33$ meters, $h=69$ meters. Since $d_{1}=9,000$ meters, $d_{2}=5,400$ meters, $d=$ 14,400 meters, we find from equation (12) that $h_{0}=-39$ meters. Assume a wavelength of 1 meter :

$$
x=\frac{-h_{0}}{\sqrt{\lambda d}}=0.325 \text { with } \frac{d_{2}}{d_{1}}=0.6 .
$$

From Figure 9, the diffracted field is found to be $14 \mathrm{db}$ below the free-space field at the same distance.

8.4

8.4 .1

\section{DIFFRACTION BY COASTS}

\section{Introduction}

Diffraction occurring at coast lines is significant for coverage problems of coastal radars. It becomes particularly important when the sets are used for height-finding purposes where an accurate knowledge of the lobe angle and possible deformation of the lobes is required.

The diffraction might be due either to the fact that the radar is sited on a cliff or to the sudden change in surface properties. Reflection from rough ground is diffuse, so that there is no interference between direct and reflected rays when the reflection point lies on this type of terrain, but interference does occur when the reflection point lies on the sea surface from which regular reflection is obtained. A situation commonly occurring is that of a search radar sited on rough terrain a few miles inland from the coast. Here coastal diffraction may result in an appreciable deformation (shortening or lengthening) of the lobes.

More generally, diffraction occurs with level ground whenever there is a change, especially a sudden change, of ground properties along the transmission path. The formulas developed for coastline diffraction may equally be applied to the case where rough ground suddenly changes into smooth, reflecting ground. Similarly, the effect of patches of smooth ground in rough surroundings, such as a lake in wooded country and, vice rersa, rough patches in smooth terrain, may be treated by means of the Fresnel-Kirchhoff theory. Here, attention will be confined to the case of a straight boundary, applying the diffraction theory developed in Section S.1.

\subsubsection{Level Site Near Coast}

Assume a transmitter sited on rough ground near a coast. If diffraction were disregarded, the coverage pattern would appear as follows. When the reflection point is on the land, the reflected ray is diffusely seattered and its field at the receiver is negligible. Again, if the reflection point falls on the sea, the reflected ray will be present and will interfere with 
the direet ray with its full or nearly its full intensity. The ray leaving the transmitter at an angle $\psi_{0}$ (Figure 13), sueh that its refleeted counterpart undergoes refleetion light at the shore line, divides the coverage diagram into two parts. For angles of

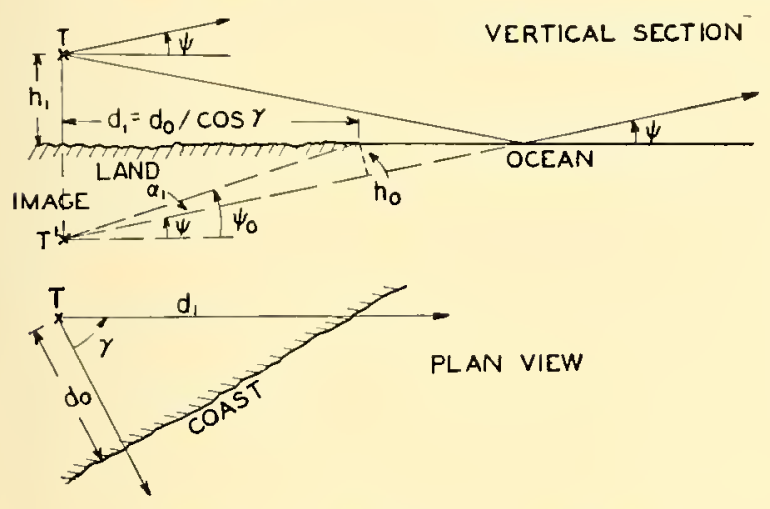

Figure 13. Diffraction by a coast line.

elevation larger than $\psi=\psi_{0}$ the field will be essentially the free-space field; for angles of elevation less than $\psi=\psi_{0}$ the familiar lobe pattern, for eomplete refleetion, will appear with maxima equal to twice the frec-space field. When diffraction by the coast line is taken into account, the discontinuity expressed by this rough picture is replaced by a smooth transition of the field from one region to the other.

The land surface may be considered as an opaque sereen for the image transmitter from which the refleeted rays seem to eome (Figure 13). This problem is somewhat clifferent from the diffraetion problem treated previously since the trace of the screen in the vertieal plane through $T^{\prime \prime}$ and $R$ is no longer perpendicular to the line $T^{\prime} R$ as it was, for instance, in Figure 2, upper part. In the present ease, the effective height $h_{0}$ of the diffraeting edge for any given ray is the perpendieular projection from the eoast line upon this ray, as shown in Figure 13. The slant distance of the eoast from the transmitter is $d_{1}$. Assuming that the receiver (target) is far clistant, a eondition usually fulfilled in radar praetice, $d_{2}>>d_{1}$ and the angle $\psi$ between the direet ray and the horizontal will be equal to the angle between the image ray and the horizontal. Then approximately, since the angles are small,

$$
h_{0}=d_{1} \alpha_{1}=d_{1}\left(\psi_{0}-\psi\right),
$$

where $d_{1}$ and $\alpha_{1}$ have the signifieance given them in Section 8.1.4. Here the signs have again been chosen so that $h_{0}$ is negative when the receiver (target) is in the shadow of the screen with regard to the image transmitter.

The distance from the transmitter to the diffracting coast depends on the azimuth (Figure 13). Therefore, with the designations of the figure,

$$
d_{1}=\frac{d_{0}}{\cos \gamma} \text {. }
$$

\subsubsection{Equation for Field Strength}

The expression for the diffraeted field of the image transmitter is given by the straightedge formula, equation (6), with $v$ given by equation (3). Since $1 / d_{2}$ is assumed negligibly small compared to $1 / d_{1}$, we find, on using equation (14),

$$
v=\left(\psi_{0}-\psi\right) \sqrt{\frac{2 d_{1}}{\lambda}} .
$$

This may be further simplified by introducing (as in Section 8.3.2) a new variable, the lobe number

$$
n=\frac{4 h_{1} \psi}{\lambda} .
$$

( $h_{1}=$ transmitter height). This quantity is equal to $1,3,5 \cdots$ at the interference maxima and equal to $0,2,4, \cdots$ at the interference minima but is here taken as a continuous rariable, defined for any value of $\psi$. In partieular for $\psi=\psi_{0}$ we put $n=n_{0}$. Sinee $\psi_{0}=h_{1} / d_{1}$, we have by equation (15)

$$
n_{0}=\frac{4 h_{1} \psi_{0}}{\lambda}=\frac{4 h_{1}^{2} \operatorname{eos} \gamma}{\lambda d_{0}} .
$$

Equation (16) may now be written

$$
v=\frac{n_{0}-n}{\sqrt{2 n_{0}}} .
$$

The cliffraction formula will again be written, in the form of equation ( 7 ), as

$$
\frac{E}{E_{0}}=z e^{-j \zeta}
$$

where $z$ and $\zeta$ are the functions of $v$ shown in Figures 4 and 5 .

The total field obtained by the interferenee of the direct and reflected ray is

$$
E=E_{0}\left(1-z e^{-j \pi n-j \xi}\right),
$$

where the negative sign in front of the second term in parentheses aecounts for the 180-degree phase shift at refleetion, and the phase lag $\pi n$ eorresponds 
to the path difference between the reflected and direct rays.

The absolute value of the field is

$$
\left|\frac{E}{E_{0}}\right|=\sqrt{(1-z)^{2}+4 z \sin 1 / 2(\pi n+\zeta)} .
$$

Figure 12 in Chapter 5 may be used for the numerical evaluation of this equation.

The formula can readily be generalized to the case where the reflected ray is weakened by (1) a reflection coefficient, $R$, different from unity, and (2) the effect of the earth's curvature expressed by the divergence factor, $D$, (Chapter 5). If, moreover, the phase lag at reflection is not $\pi$ but $\pi+\phi^{\prime}$, the equation becomes

$$
\left|\frac{E}{E_{0}}\right|=\sqrt{(1-2 R D)^{2}+4 z R D \sin ^{2} 1 / 2\left(\pi n+\phi^{\prime}+\zeta\right)} .
$$

\section{4 .4}

\section{Example}

Assume the following conditions. A radar set of $200 \mathrm{me}(\lambda=1.5$ meters $)$ is sited at a height $h_{1}=15.3$ meters (about 50 feet) and at a distance to a straight shore line of $d_{0}=195$ meters (about 0.12 mile). The ground between the radar and the seashore is level but can be considered as rough for practically any angle of elevation, on applying the criterion of Section 8.3.2. The coverage diagram will first be determined in the azimuth perpendicular to the coast line, where $d_{1}=d_{0}$, or $\cos \gamma=1$. Then by equation (18), $n_{0}=3.20$. With this value of $n_{0}$ the variable $v$ is determined by equation (19). We shall confine ourselves to integral values of $n$, that is, to those angles which, in the presence of simple reflecting ground, correspond to lobe minima and maxima. Having obtained $v$, one then determines $z$ and 5 from Figures 4 and 5 . The field in terms of the free-space field is then obtained from equation (21), either by direct computation or by means of Figure 12 in Chapter 5. The numerical data for the first five lobes are summarized in Table 1. The last eolumn of this table contains the values of $E / E_{0}$ which would be obtained if the magnitude of the reflection coefficient were assumed to be zero over land and unity over the sea and if diffractions were neglected.

The same calculations are carried out for an azimuth inclined by an angle $\gamma=45^{\circ}$ with respect to the coast line. Then, from equation (18), $n_{0}=4.5$. The results are given in Table 2 .
It is seen from these data that the lobes near the critical ray (ray whose reflection point is at the coast line) undergo very considerable deformation. The

\begin{tabular}{|c|c|c|c|c|c|}
\hline$n$ & $v$ & $z$ & $\begin{array}{c}5 \\
\text { (degrees) }\end{array}$ & $\begin{array}{l}\left|E E_{0}\right| \text { with } \\
\text { diffraction }\end{array}$ & $\begin{array}{c}\left|E / E_{0}\right| \text { without } \\
\text { diffraction }\end{array}$ \\
\hline 0 & 1.27 & 1.17 & 0 & 0.17 & 0 \\
\hline 1 & 0.87 & 1.05 & -12 & 2.05 & 2 \\
\hline 2 & 0.48 & 0.80 & -15 & 0.75 & 0 \\
\hline 3 & 0.08 & 0.54 & -4 & 1.54 & 2 \\
\hline 4 & -0.32 & 0.36 & 24 & 0.69 & 1 \\
\hline 5 & -0.71 & 0.26 & 11 & 1.26 & 1 \\
\hline 6 & -1.11 & 0.19 & 145 & 1.16 & 1 \\
\hline 7 & -1.51 & 0.14 & 242 & 0.94 & 1 \\
\hline 8 & -1.90 & 0.12 & 5 & 0.88 & 1 \\
\hline 9 & -2.30 & 0.10 & 158 & 0.92 & 1 \\
\hline 10 & -2.70 & 0.08 & 339 & 0.94 & 1 \\
\hline
\end{tabular}

TABLE $1 . \quad\left(\gamma=0^{\circ}\right)$.

TABLE 2. $\quad\left(\gamma=45^{\circ}\right)$

\begin{tabular}{llllll}
\hline & & & & $\left|E / E_{0}\right|$ with & $\left|E / E_{0}\right|$ without \\
diffraction
\end{tabular}

\begin{tabular}{rrrrrl}
\hline 0 & 1.50 & 1.07 & 6 & 0.10 & 0 \\
1 & 1.17 & 1.17 & -3 & 2.17 & 2 \\
2 & 0.83 & 1.03 & -13 & 0.21 & 0 \\
3 & 0.50 & 0.82 & -15 & 1.80 & 2 \\
4 & 0.17 & 0.59 & -8 & 0.42 & 0 \\
5 & -0.17 & 0.42 & 11 & 1.42 & 1 \\
6 & -0.50 & 0.31 & 42 & 0.80 & 1 \\
7 & -0.83 & 0.23 & 90 & 0.91 & 1 \\
8 & -1.17 & 0.18 & 157 & 0.85 & 1 \\
9 & -1.50 & 0.14 & 240 & 0.99 & 1 \\
10 & -1.83 & 0.12 & 338 & 1.12 & 1 \\
\hline
\end{tabular}

coverage pattern corresponding to Table 1 is shown graphically in Figure $1 t$.

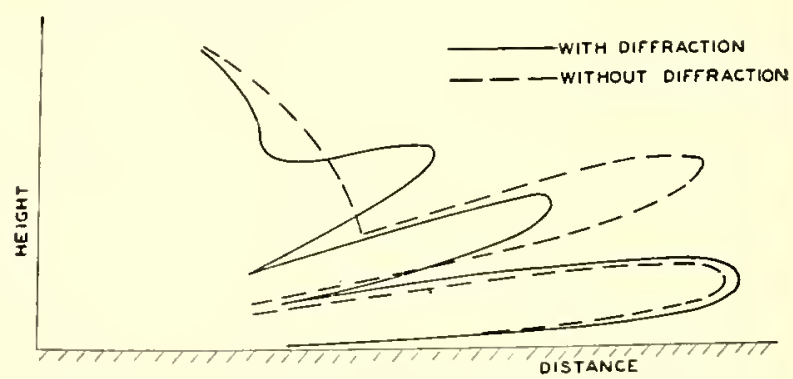

Figure 14. Coverage diagram (relative field strength). (leights exaggerated 3.5 to 1 .)

In the problem considered here, the angles of elevation are comparatively large (for $n=1$, $\psi=1^{\circ} 2 t^{\prime}$ ). If the effects of diffraction occur at 
lower angles, the divergence factor $D$ must be taken into account (see Chapter 5). This is done by computing $D$ for the angles desired and replacing $z$ by $z D$ in equation (21).

\subsubsection{Cliff Site}

If the radar is sited on a cliff and if the land intervening between the radar and the reflecting plane (ocean) is rough, the equations of Section 8.4.3 apply. We shall now consider the case where the radar is sited at some distance from the cliff edge and where the ground between the radar site and the cliff edge is reflecting. There are then two reflecting planes, the lower of which might be the ocean, or

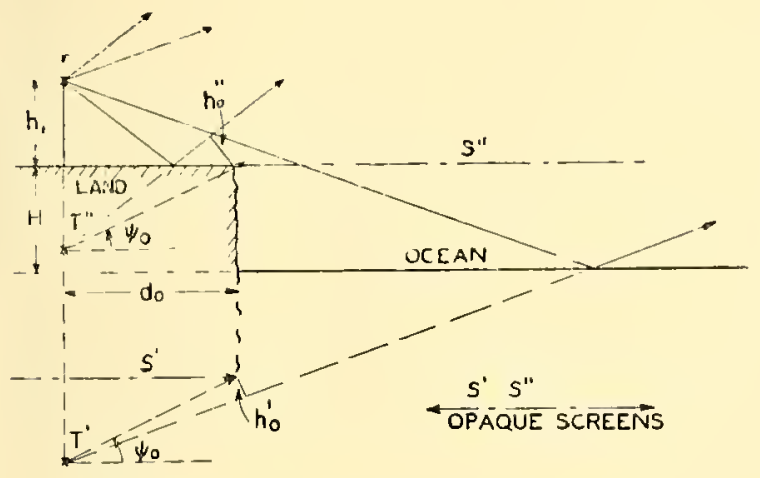

Figure 15. Diffraction from a cliff site.

might be a reflecting land surface. In Figure 15, this surface has been designated as ocean. The upper plane is at a height $H$ above the lower plane and the transmitter at a height $h_{1}$ above the upper plane. Assume that the azimuth chosen is perpendicular to the direction of the cliff edge; the distance of the radar to the cliff edge is $d_{0}$. For any other azimuth (angle $\gamma$ in Figure 13), replace $d_{0}$ by $d_{0} / \cos \gamma$ in the following equations.

Two images are shown in Figure 15 and two fictitious opaque screens, one corresponding to each image. The corresponding variables are distinguished by single and double primes. The lobe numbers are given by

$$
\begin{aligned}
& n^{\prime \prime}=\frac{4 h_{1} \psi}{\lambda} \\
& n^{\prime}=\frac{4\left(H+h_{1}\right) \psi}{\lambda}=\frac{n^{\prime \prime}\left(H+h_{1}\right)}{h_{1}} .
\end{aligned}
$$

The critical angle, $\psi_{0}=h_{1} / d_{0}$, is the same for both image transmitters. Thus

$$
\begin{aligned}
& n_{0}{ }^{\prime \prime}=\frac{4 h_{1}^{2}}{\lambda d_{0}}, \\
& n_{0}{ }^{\prime}=\frac{4\left(H+h_{1}\right) h_{1}}{\lambda d_{0}}=\frac{n_{0}{ }^{\prime \prime}\left(H+h_{1}\right)}{h_{1}}
\end{aligned}
$$

Further

$$
\begin{aligned}
v^{\prime} & =\frac{n_{0}^{\prime}-n^{\prime}}{\sqrt{2 n_{0}^{\prime}}}, \\
v^{\prime \prime} & =\frac{n^{\prime \prime}-n_{0}^{\prime \prime}}{\sqrt{2 n_{0}{ }^{\prime \prime}}}=-v^{\prime} \frac{1}{1+H / h_{1}} .
\end{aligned}
$$

Again, the field is given by

$$
E=E_{0}\left(1-z^{\prime} e^{-j \pi n^{\prime}-j \zeta^{\prime}}-z^{\prime \prime} e^{-j \pi n^{\prime \prime}-j \zeta^{\prime \prime}}\right) .
$$

The expression for the field strength [equation (23)] is in a form where all the quantities involved may be evaluated for any given height of transmitter, height of the cliff, and any wavelength by using graphs and tables given in earlier paragraphs. 


\section{Chapter 9}

\section{TARGETS}

9.1 .1

SCATTERING PARAMETERS

\section{Radar Cross Section}

$I^{\top}$ $\checkmark$ DETERMINING the coverage to be expected of radar systems, it is important to know what fraction of the power incident upon a target will be retumed to the receiver. A parameter involving the dimensions and orientation of the target, and usually also the warelength, and which measures the proportion of power retumed, is called a scattering parameter.

The most generally used of these parameters is the radar cross section introduced in Section 2.4.1. It is denoted by $\sigma$ and is defined by

$$
\sigma=4 \pi d^{2} \frac{W_{r}}{W_{i}}
$$

where $\mathrm{H}_{r}^{+}$is the scattered power per unit area at the receiver and $W_{i}$ is the incident power per unit area at the target. In terms of $\sigma$, the radar gain is

$$
\frac{P_{2}}{P_{1}}=G_{1} G_{2} \frac{\sigma}{4 \pi d^{2}}\left(\frac{3 \lambda}{8 \pi d}\right)^{2} A_{p^{4}} .
$$

This equation may also be written in the form

$$
\frac{P_{2}}{P_{1}}=G_{1} G_{2} \cdot \frac{16 \pi \sigma}{9 \lambda^{2}} A^{4},
$$

where

$$
A=\left(\frac{3 \lambda}{8 \pi d}\right) A_{p}=A_{0} A_{p}
$$

is the gain factor introduced earlier (see Section 5.1), $A_{0}$ is the free-space gain factor and $A_{p}$ is the pathgain factor.

\subsubsection{Target Gain}

Another scattering parameter is $G_{R}$, the target gain, cliseussed in Section 2.4.2. It is the gain of the target in the direction of the receiver relative to a shorted (dummy) doublet antenna. The target gain is comnected with $\sigma$ by the retation

$$
\sigma_{R}=\frac{\sqrt{4 \pi \sigma}}{3 \lambda} .
$$

The corresponding radar gain is

$$
\frac{P_{2}}{P_{1}}=4 G_{1} G_{2} C_{R^{2}} A^{4}
$$

The factor 4 is due to the calculation of $G_{R}$ relative to a shorted doublet rather than to a matched load doublet. If the calculation of $G_{R}$ were made relative to the matched load doublet, the factor 1 would be replaced by 1.

\subsubsection{Echo Constant}

The ccho constant, denoted by $K$, is defined by

$$
K=\frac{W_{r}}{W_{i}}\left(\frac{d}{\lambda}\right)^{2}
$$

and is related to $\sigma$ by

$$
K=\frac{\sigma}{4 \pi \lambda^{2}} .
$$

The corresponding power ratio is

$$
\frac{P_{2}}{P_{1}}=K G_{1} G_{2}\left(\frac{8 \pi}{3}\right)^{2} A^{4} \text {. }
$$

Except for the factor $1 / 4 \pi, K$ is just $\sigma$ measured in square wavelengths.

\subsubsection{Equivalent Plate Area}

A plate of area $S$ placed normal to the direction of propagation has a radar cross section given by

$$
\sigma=4 \pi \frac{S^{2}}{\lambda^{2}}
$$

provided the linear dimensions of the plate are large compared with $\lambda$. Any target may be supposed to scatter (in the direction of the radar) an amount of energy cqual to the amount that a plate of area $S$ would seatter in this direction. This area $S$ is called the equivalcnt plate area of the target. The corresponding radar gain is

$$
\frac{P_{2}}{P_{1}}=C_{r_{1}} r_{2}\left(\frac{8 \pi S}{3 \lambda^{2}}\right)^{2} A^{4}
$$


9.1 .5

\section{Seattering Coefficient or Characteristic Length}

This parameter has also been called the radar length of the target. The definition is

$$
L=d \frac{E_{r}}{E_{i}},
$$

where $E_{r}=$ field strength at the reeeiver,

$E_{i}=$ field strength incident on target.

It is evident that

$$
L^{2}=\frac{\sigma}{4 \pi}
$$

connects $L$ with radar eross section. The radar gain becomes

$$
\frac{P_{2}}{P_{1}}=G_{1} C_{2}\left(\frac{8 \pi L}{3 \lambda}\right)^{2} A^{4}
$$

\section{2 \\ RADAR CROSS SECTION OF SIMPLE FORMS \\ 9.2 .1}

where

$$
\begin{aligned}
& a=\text { radius, } \\
& L=\text { length }(L>>\lambda) .
\end{aligned}
$$

This formula assumes that the direction of incidence is normal to the cylindrical surface. If the eylinder is tilted so that there is a small angle $\theta$ between the normal to the cylinder and the direction of incidence, the result is

$$
\sigma=\frac{2 \pi a L^{2}}{\lambda} \cdot\left[\frac{\sin \frac{2 \pi L \theta}{\lambda}}{\frac{2 \pi L \theta}{\lambda}}\right]^{2} .
$$

This result holds for small angles of tilt $\theta$ such that

$$
\sin \theta \cong \theta \text {. }
$$

9.2 .3

\section{Plates}

A flat plate of area $S$ with all dimensions large compared with $\lambda$ and oriented so that the normal to the plate is in the direction of incidenee, has a radar cross section given by

$$
\sigma=4 \pi \frac{S^{2}}{\lambda^{2}},
$$

The radar cross section of any large curved conducting surface having principal radii of curvature $\rho_{1}$ and $\rho_{2}$ at the reflection point is given by

$$
\sigma=\pi \rho_{1} \rho_{2} \text {. }
$$

This formula applies if the surface is sufficiently large and sufficiently curved to contain many Fresnel zones. For a sphere of radius $a$, where $a>>\lambda$,

$$
\sigma=\pi a^{2} .
$$

Thus, in the case of a large conducting sphere, the radar cross section is equal to the geometrical cross section and is independent of wavelength.

The result for small spheres $(a<<\lambda)$ is

$$
\sigma=144 \pi^{5} \frac{a^{6}}{\lambda^{4}} .
$$

There is no simple formula for the radar cross section in the region $a \sim \lambda$.

\section{Cylinders}

The radar cross section of a cylinder whose length is large compared with the wavelength is

$$
\sigma=\frac{2 \pi a L^{2}}{\lambda}
$$

regardless of shape.

For a circular plate (a disk) of radius $a$, whose normal is at an angle $\theta$ with the direction of incidence,

$$
\sigma=\pi a^{2}\left[\cot \theta \cdot J_{1}\left(\frac{4 \pi a}{\lambda} \sin \theta\right)\right]^{2},
$$

where $J_{1}$ is the first-order Bessel function. The maximum value is at $\theta=0$, where

$$
\sigma=\frac{4 \pi^{3} a^{4}}{\lambda^{2}} .
$$

This agrees with equation (18), since at normal incidence $S=\pi a^{2}$.

The peculiar feature of equation (19) is that the maximum at $\theta=0$ is very sharp. For example, if $\lambda / a=1 / 10, \sigma$ is only $1 / 10$ of its maximum value when $\theta=1.25^{\circ}$.

The average value of $\sigma$ over all orientations is

$$
\sigma=\frac{1}{2} \pi a^{2} .
$$

This result is independent of wavelength and suggests that a large number of flat plates oriented at random will have a cross section independent of $\lambda$, or that a fer surfaces of rapidly ehanging orientation may have this property. 


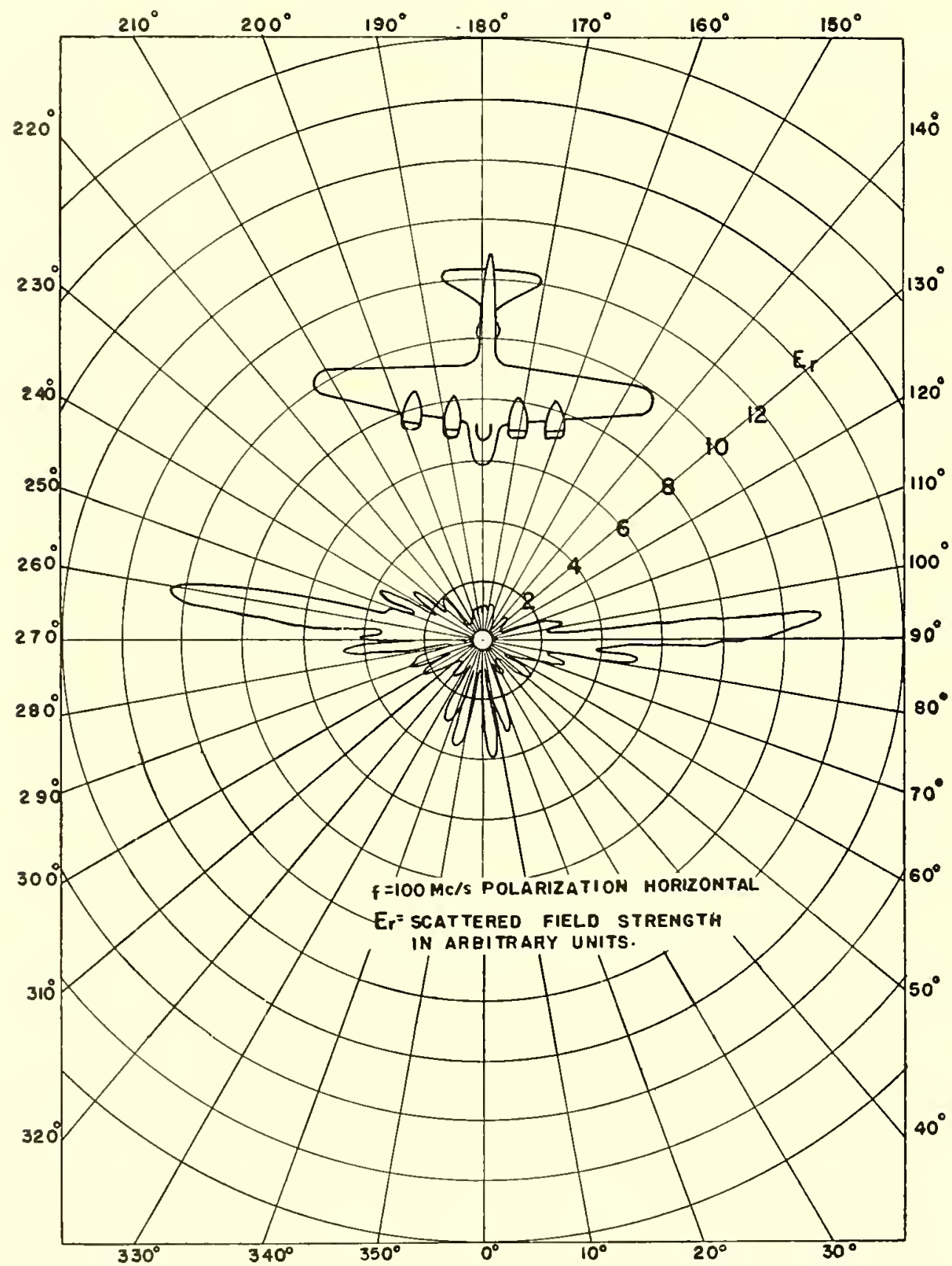

Figure 1. Aspect diagrams of B-17E, 5 degrees above horizon. 
The results for a rectangular plate are practically the same as for a disk. If the dimensions of the plate are $b$ and $c, \pi a^{2}$ is replaced by $b c$ in equation (20) and equation (21); equation (19) is replaced by

$$
\begin{aligned}
& \sigma=\frac{4 \pi b^{2} c^{2}}{\lambda^{2}}\left[\cos \theta \frac{\sin \left(\frac{2 \pi b}{\lambda} \sin \theta \cdot \cos \phi\right)}{\frac{2 \pi b}{\lambda} \sin \theta \cdot \cos \phi}\right. \\
& \left.\frac{\sin \left(\frac{2 \pi c}{\lambda} \sin \theta \cdot \sin \phi\right)}{\frac{2 \pi c}{\lambda} \sin \theta \cdot \cos \phi}\right]^{2}
\end{aligned}
$$

where the sides $b$ and $c$ are parallel to the $x$ and $y$ axes, and $\sin \theta \cos \phi$, $\sin \theta \sin \phi$, and $\cos \theta$ are the direction cosines of the direction of incidence relative to the plate normal.

These results hold when the linear dimensions of the target are large compared with the wavelength. If the linear dimensions are small compared with the warelength, a plate of area $S$, oriented so that the normal to the plate is in the direction of incidence, has a radar cross section given by

$$
\sigma=\frac{32 \pi^{2}}{3} \frac{S^{3}}{\lambda^{4}}
$$

9.2 .4

\section{Corner Reflectors}

The corner formed by three mutually perpendicular conducting planes forms what is called a corner reflector. The faces may be triangular or square or have other shapes, depending on how the planes are bounded. A line drawn to the corner making equal angles with the three edges is called the axis of symmetry.

Reflection from a comer reflector may be analyzed by the methods of geometrical optics, provided the linear dimensions of the reflector are large compared with the wavelength. A ray which is reflected from all three surfaces is said to be triply reflected. Triply reflected rays always return to the radar and make the only large contribution to the radar cross section. The radar cross section of a corner reflector' is

$$
\sigma=\frac{4 \pi S^{2}}{\lambda^{2}},
$$

where $S$ is the cross section of the triply reflected beam. $S$ is a function of the shape of the faces of the corner reflector and of the angle of incidence of the radiation.

For a triangular corner reflector, $\sigma$ is given approximately by

$$
\sigma=\frac{4 \pi L^{4}}{3 \lambda^{2}}\left(1-0.00076 \theta^{2}\right),
$$

where $L=$ length of edge of reflector,

$\theta=$ angle between direction of incidence and the axis of symmetry in degrees $\left(\theta<26^{\circ}\right)$.

As a function of $\theta, \sigma$ has a broad, flat maximum. Consequently, the return to the radar receiver from such a target is not sensitive to the precise orientation of the axis of symmetry.

9.3

9.3 .1

\section{AIRCRAFT}

\section{Variation with Aspect}

Diagrams showing the dependence of $\sigma$ on orientation indicate very large and irregular fluctuations. Radar cross section $\sigma$ can change from values of nearly 1,000 square meters to a few square meters as a result of a change of aspect of a few degrees. These instantaneous values of the raclar cross section are dependent on wavelength, polarization, details of plane design, areas of specular reflection, propeller rotation, etc. Reflection patterus have been neasured for a few simplified models by laboratory means (see Figure 1 as an example). It would be difficult to calculate instantaneous values of $\sigma$ by theoretical methods.

In practice, however, an airplane is in motion and is affected by air currents. These factors cause the airplane, in a short interval of time, to present many widely different instantaneous values of $\sigma$ to the radar, so that the signal actually seen on the scope by the observer is in effect a time average, where the most violent fluctuations of instantaneous values of $\sigma$ have been smoothed out.

\subsubsection{Measurement of $\sigma$}

The radar equation for free space, equation (45), in Chapter 2, may be used for the computation of average values of $\sigma$ from observed instantaneous values, provided conditions are such that ground reflections are unimportant. The received power $P_{2}$ is determined by matching the signal from the plane with the measured signal from a signal generator.

The procedure followed in work at the Radiation Laboratory is to measure the maximum value of $P_{2}$ 
for each of a series of 3 -second intervals. I plot is made of $P_{2}$ against range $d$ on $\log \log$ eoordinates. As might have been antieipated from equation (45), in Chapter 2, it is found that a line with a constant slope of -4 passes through the average of the 3 second interval maximum points, although the individual points fluctuate widely. The value of $\sigma$ corresponding to this line is calculated.

The resulting value of $\sigma$ still cannot be called an average value because the maximum value of $\sigma$ has been used for each point. Consequently these values of $\sigma$, substituted into equation (45) in Chapter 2 , eannot be expeeted to give the arerage ralue of $P_{2}$, or to give observed maximum ranges. However, it is found that if the values of $\sigma$ thus computed are reduced 40 per cent, they give correet results.

These empirical cross sections are relatively independent of wavelength. This result may be interpreted to mean that a plane in motion behaves more or less like a collection of speeularly reflecting surfaces oriented at random, as equation (21) indieates.

Attempts have been made to develop formulas giving operational cross sections as a function of some large feature of plane design, such as wing span or length of fuselage, but these attempt: have not been suceessful. 


\section{Chapter 10}

\section{SITING}

Citisf ReFers to the selection and utilization of $\boldsymbol{N}$ local terrain features which affect propagation and the performance of equipment. From a preliminary analysis, the general location, type of equipment, and height may be determined. The specific sites arailable may, however, profoundly alter performance in sereral ways. Careful analysis and tests may then be necessary to determine the best use of the facilities at hand and for an understanding of the limitations due to the terrain.

\subsubsection{Siting Requirements}

With communication equipment, the siting problems are principally concerned with visibility and, in wooded areas, absorption by vegetation. When siting direction-finding equipment, it is important to realize that reflections from mountains or other irregularities may cause serious angular errors which should be avoided by proper choice of the location. Both direction-finding and radar equipment require orientation.

Radar siting requirements are rather different and depend on whether ground reflection is of importance or not. The siting of radars operating mainly on the direct ray is relatively easy and is principally concerned with permanent echoes and visibility. The most exacting site requirements are presented by the VHF early warning and height-finding radars, which to a large extent depend on ground reflection for successful operation. The siting problem then requires the consideration of terrain effects such as limited reflection areas, cliff edges, obstacles, etc., which involve diffaction problems of considerable complexity. Recommendations for specific sets are given in instruction manuals furnished with the equipment.

10.2

10.2 .1

Radar and direction-finding systems, which may cover a large area and involve many services, use a grid for plotting purposes. The grid location, height, and orientation of each station must be known with reasonable accuracy. Topographic maps of a scale of one or two miles to the inch and contour intervals of not more than 100 feet, preferably 20 feet, should be secured. These may be supplemented by aerial photographs and surveys.

10.2 .2

\section{Profiles}

In a complicated terrain, it is usually necessary to have profiles on several azimuths to determine the effective height above the reflecting surface. The accuracy required decreases with the distance from the transmitter. In most cases sufficient detail is not available on maps, so that a personal inspection of the terrain should be made to become familial with the nature of the soil and degree of roughness. Special attention should be given to ridges, flat areas, bodies of water, distance to the shore, hills to the rear, obstacles in the operating area and at the boundaries.

10.2 .3

\section{Orientation}

Where long distances and directive beams are involved, fairly accurate orientation of the order of one-half degree is required. Care must be taken when using compasses because of local attractions or inadequate information on declinations. Observations on Polaris give the greatest precision but this star is not always visible and it is often inconvenient to use a transit at night. Cantion must be used in aligning on permanent echoes, as they may be difficult to identify. In general, several methods should be used to obtain independent checks.

Solar azimuths, correct to the nearest quarter of a degree, may be determined from the date time to the nearest minute, and the latitude and longitude to the nearest degree. Two methods will be given for obtaining the azimuth of the sun: (1) by calculation, (2) from tables. A third method gives true south only. 
The azimuth of the sum may be calculated from the formula,

$$
\tan \beta=-\frac{\sin (\mathrm{HA})}{\cos \phi \tan \delta-\sin \phi \cos (\mathrm{HA})},
$$

where $\beta=$ bearing of the sun. The bearing is east or west of south when $\phi-\delta$ is positive. The bearing is east or west of north when $\phi-\delta$ is negative. The bearing is east in the moruing ( $\beta$ will be negative) and west in the afternoon ( $\beta$ will be positive).

H. = lour angle of the sun. During the molning hours when the hour angle is greater than 12 hours, its value should be subtracted from 24 hours for use in the formula.

$\phi=$ latitude of the place of observation.

$\delta=$ declination of the sun at the time of observation. The signs of $\phi$ and $\delta$ are important and each is positive when north of the equator and negative when south.

The hour angle $\mathrm{HA}$ is the local apparent time (LAT) minus 12 hours. To convert the observed time into LAT, the civil time at Greenwich (GCT) must be found and combined with the equation of time to correct for the apparent irregular motion of the sun. This gives Greenwich apparent time GAT, which is converted to LAT, by allowing for the longitude. The equation of time and the declination of the sun are plotted for 1945 in Figure 1. The annual change is small and these eurves may be used for most orientations without regard to the year. Standard time meridians are given every 15 degrees east or west of Greenwich, each zone corresponding to one hour. Care should be used to take daylight saring or other changes from standard into account correctly.

The ealculations may be illustrated from the following data: date, 16 March; time, 1345 hours PWT; latitude, $40^{\circ}$ north; longitude, $118^{\circ}$ west. The HA is computed first.

\begin{tabular}{|c|c|}
\hline $\begin{array}{l}\text { Observed time (PVIT) } \\
\text { Zone difference }\end{array}$ & $\begin{array}{r}13 \mathrm{hr} \quad 45 \mathrm{~min} \\
+\quad 7 \mathrm{hr}\end{array}$ \\
\hline $\begin{array}{l}\text { Greenwich civil time } \\
\text { Equation of time (Figure 1) }\end{array}$ & $\begin{array}{r}20 \mathrm{hr} \\
\quad 45 \mathrm{~min} \\
-9 \mathrm{~min} \\
\end{array}$ \\
\hline $\begin{array}{l}\text { Greenwich apparent time } \\
\text { Longitude difference (for } 118^{\circ} \mathrm{W} \text { ) }\end{array}$ & $\begin{array}{r}20 \mathrm{hr} 36 \mathrm{~min} \\
-\quad 7 \mathrm{hr} \quad 52 \mathrm{~min} \\
\end{array}$ \\
\hline $\begin{array}{l}\text { Local apparent time (LAT) } \\
\text { LAT }-12 \text { hours }=\mathrm{HA}\end{array}$ & $\begin{aligned} & 12 \mathrm{hr} \\
&-12 \mathrm{hr} \\
&\end{aligned}$ \\
\hline $\begin{array}{l}\text { Hour angle of sun } \\
\text { HA in are } \\
\text { Latitude } \phi \\
\text { Declination of sun } \hat{o} \text { (Figure } 1 \text { ) }\end{array}$ & $\begin{array}{l}+0 \mathrm{hr} \quad 44 \mathrm{~min} \\
+11^{\circ} \\
+40^{\circ} \\
-2^{\circ}\end{array}$ \\
\hline
\end{tabular}

Substituting in equation (1),

$$
\begin{aligned}
\tan \beta & =-\frac{\sin 11^{\circ}}{\cos 40^{\circ} \cdot \tan \left(-2^{\circ}\right)-\sin 40^{\circ} \cdot \cos 11^{\circ}} \\
\beta & =16^{\circ} 10^{\prime}
\end{aligned}
$$

Since $\phi-\delta$ is positive, $\beta$ is the bearing from the south. The bearing is west of south, since HA is positive (p.m.). The azimuth of the sun is $180^{\circ}+16^{\circ} 10^{\prime}=196^{\circ} 10^{\prime}{ }^{\mathrm{a}}$

The equal altitude method is less convenient but requires no calculation. This method consists in measuring the horizontal angles between the sun and a mark taken when the sun is at the same altitude on both sides of the meridian of the observer. The bisector of the horizontal angle between the two equal altitude positions of the sun during the observations is very close to true south, and the azimuth of the mark may be determined.

\subsection{GEOMETRICAL LIMITS OF VISIBILITY}

10.3 .1

\section{Horizon Formula}

It is assumed throughout that the earth radius is ka (see Section 4.1). Whenever numerical examples are given, the standard value, $k=4 / 3$, is used. The alternate method of accounting for refraction given in Section 4.1 .5 may also be used in connection with the following equations if $k \neq 4 / 3$.

When a horizontal ray, tangential to the earth, is drawn, the earth slopes away (Figure 2) at the rate of

$$
h=\frac{d^{2}}{2 k a} .
$$

Hence the horizon distance $d_{T}$ for a transmitter at a height $h$ above level ground is equal to

$$
d_{T}=\sqrt{2 k a \bar{h}} .
$$

Numerically, when all the lengths are in meters

$$
d_{T}=4,120 \sqrt{h} \text { for } k=\frac{4}{3} .
$$

With $h$ in feet and $d_{T}$ in statute miles, by a curious numerical coincidence,

$$
d_{T}=\sqrt{2 h} \quad \text { for } k=\frac{4}{3} .
$$

\footnotetext{
a This result could have been obtained directly from Azimuth of the Sun, Ho71, C.S. Xaval Department, Hydrographic Office. The equation of time may be obtained from a current copy of The American Nautical Almanac, I.S. Naval Observatory. Washington. D.C
} 
When both terminals of a path are clevated above the ground (Figure 3), the horizon distance is

$$
d_{L}=\sqrt{2 k a}\left(\sqrt{h_{1}}+\sqrt{h_{2}}\right)
$$

10.3 .2

As a first case, consider a smooth earth and two teminals at the ground. The earth itself forms an

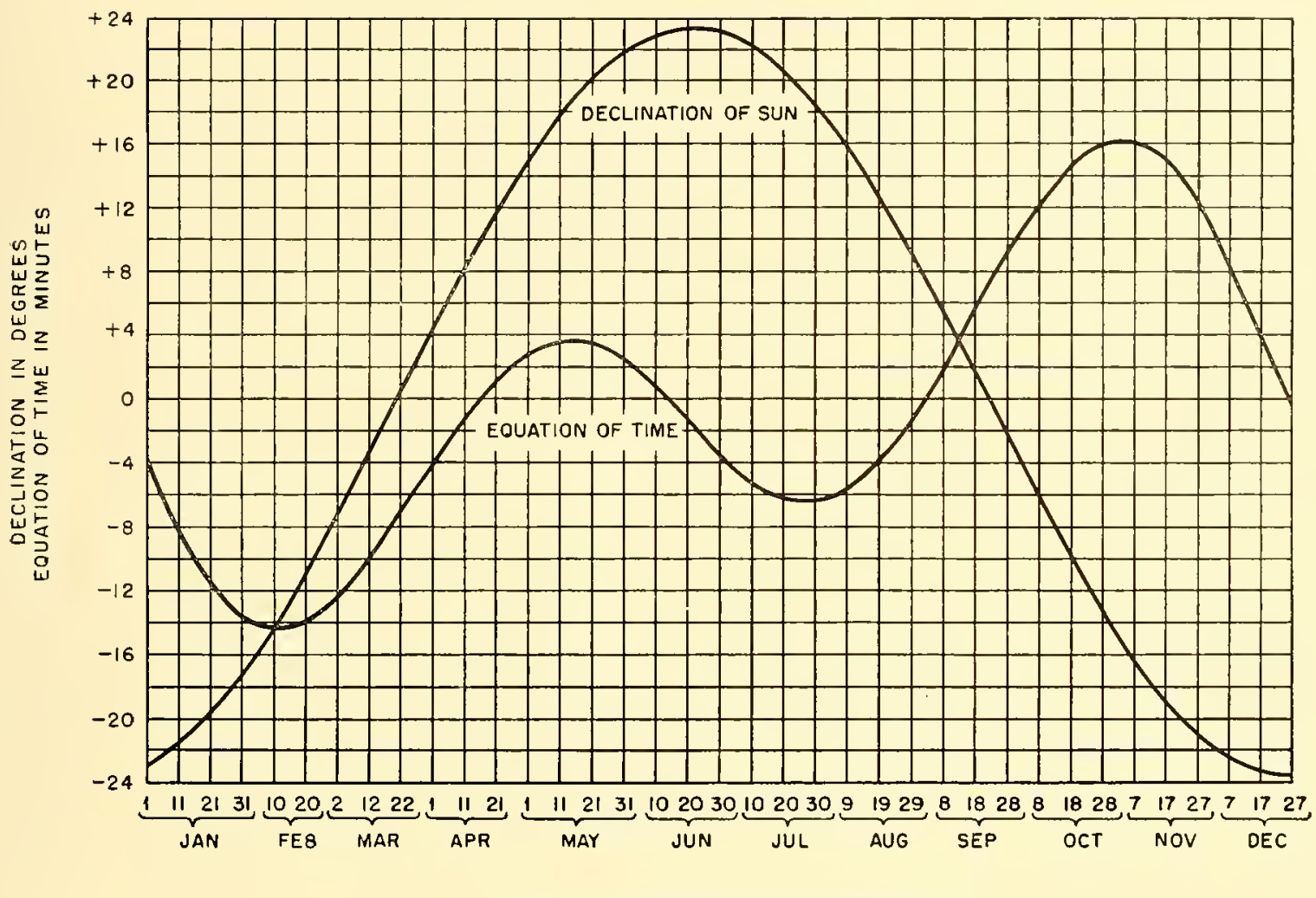

Figure 1. Calculation of solar azimuth.

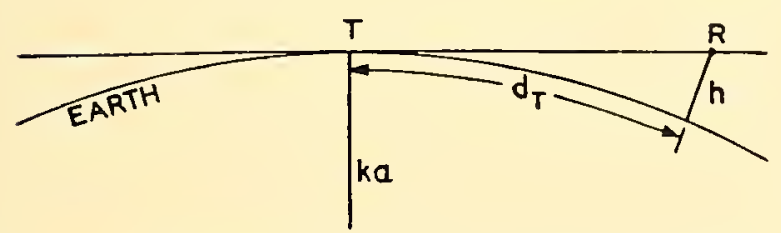

Figure 2. Geometry for lrorizon distance for zero height transmittes'.

where again $\sqrt{2 / a}=4.120$ in the metric system. If $d$ is in statute miles and $h$ in feet,

$$
d_{L}=\sqrt{2 h_{1}}+\sqrt{2 h_{2}} \text { for } k=\frac{4}{3} .
$$

The relation between $h_{1}, h_{2}$ and $d_{L}$ is graphically presented in Chapter 5 in the form of a nomogram, Figure 2.

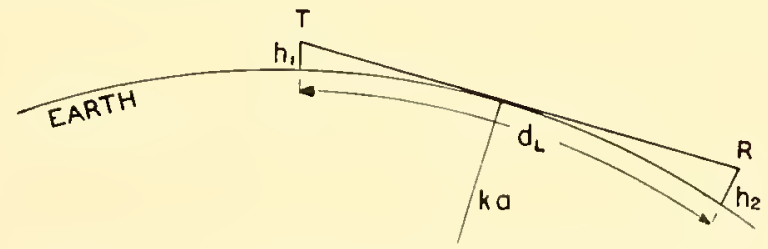

Figure 3. Geometyy for horizon distance with elevated transmitter.

obstacle which reaches its maximum height $h_{m}$ in the middle of the path (Figure 4). By equation (2)

$$
h_{m}=\frac{\left(\frac{d}{2}\right)^{2}}{2 k a}=\frac{d^{2}}{8 k a} .
$$

A point $P$ on the ground at distances $d^{\prime}$ and $d^{\prime \prime}$ from the two terminals (see Figure 4 ) has an elevation 
above the straiglt line comnecting the terminals given by

$$
h=\frac{\left(\frac{d^{\prime}+d^{\prime \prime}}{2}\right)^{2}}{2 k a}-\frac{\left(\frac{d^{\prime}-d^{\prime \prime}}{2}\right)^{2}}{2 k a}
$$

or, after a simple reduetion,

$$
h=\frac{d^{\prime} d^{\prime \prime}}{2 k a}=5.9 \cdot 10^{\prime \prime} d^{\prime} d^{\prime \prime},
$$

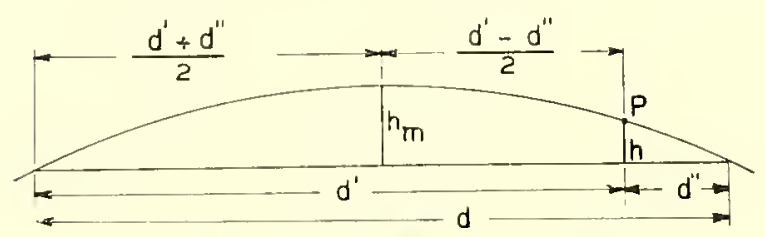

Figure 4. Height of earth as an obstacle.

where $h, d^{\prime}$, and $d^{\prime \prime}$ are given in meters. If $h$ is in feet and $d^{\prime}$ in statute miles,

$$
h=\frac{d^{\prime} d^{\prime \prime}}{2} \text { for } k=t / 3 .
$$

Secondly, assume that the terminals are elevated (Figure 5). The elevation of the straight line connecting the terminals, for a flat earth, is equal to

$$
h=\frac{d^{\prime} h_{2}-d^{\prime \prime} h_{1}}{d^{\prime}-d^{\prime \prime}}
$$

where $d^{\prime}, d^{\prime \prime}$ are again the distances to the terminals, and $h_{1}, h_{2}$ are the corresponding elevations.

In order to account for the effeet of the earth's curvature, Figure 5 may be considered as a plane earth diagram on which a lay will appear curved, the deviation from a straight line being downward and given by equation (10). This is indicated by the dashed line in Figure 5.

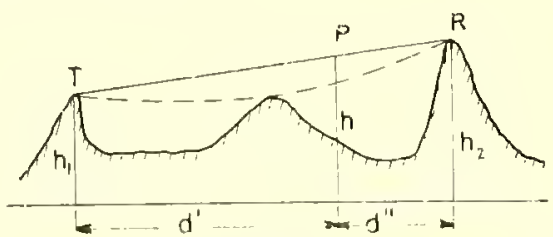

FIGURE 5. Height for elevaterl terminals.

Hence, the total height above the theoretical ground is

$$
h=\frac{d^{\prime} h_{2}-d^{\prime \prime} h_{1}}{d^{\prime}-d^{\prime \prime}}-\frac{d^{\prime} d^{\prime \prime}}{2 k a} .
$$

When the heights are expressed in feet and the distances in miles, the first term remains unaltered, while the second term again becomes $d^{\prime} d^{\prime \prime} / 2$ for $l_{i}=4 / 3$.

Equation (13) is used to decide whether and by low much an obstacle sueh as a hill will obstruct a given transmission path.

\subsubsection{Extended Obstacle}

When the obstaele is of appreciable horizontal extension, it may not possess a single peak to which equation (13) can be applied without ambiguity. The case of twin mountains is show $n$ in Figure 6 for straight rays (earth's radius kin).

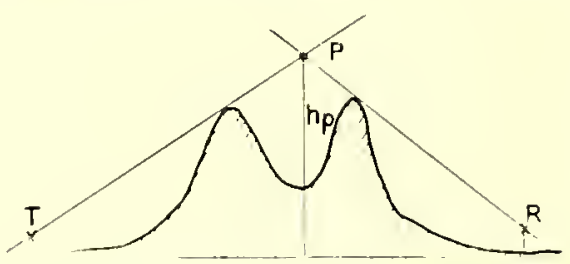

FIGURE 6. Height of equivalent diffracting edge.

The optical peak $P$ of the obstacle for radio or radar transmission is the point from which both terminals are just visible. For a given profile, the limiting rays to the terminals may be found by trial and error by applying equation (13) to those points of the profile whieh are most likely to represent limiting elevations. In the theory of diffraction given in Chapter 8, $P$ marks the position of the equivalent diffracting edge.

\subsubsection{Degree of Shielding}

As a measure of the degree of shielding, the angle between the two limiting rays drawn from the terminals to the (actual or erquivalent) peak of the obstacle of height $h_{p}$ may be used.

Since all angles considered are small, the sine or tangent of the angle may be replaced by the angle in radians. Consider first the ray going from the first terminal to $P$ (Figure $\overline{7}$ ). The angle of the ray with the horizontal at the terminal is

$$
\alpha_{1}=\frac{h_{p}-h_{1}}{d^{\prime}}-\frac{d^{\prime}}{2 k a}
$$

and its angle with the horizontal at $P$ is

$$
\beta_{1}=\alpha_{1}+\frac{d^{\prime}}{k a}=\frac{h_{p}-h_{1}}{d^{\prime}}+\frac{d^{\prime}}{2 l^{\prime} a} .
$$

The angle of the ray going from the second terminal to $P$ is determined correspondingly. 
The angle between the two rays is then equal to

$$
\beta_{1}+\beta_{2}=d\left(\frac{1}{2 k a}+\frac{h_{p}}{d^{\prime} d^{\prime \prime}}-\frac{h_{1}}{d^{\prime}}-\frac{h_{2}}{d^{\prime \prime}}\right),
$$

where $d=d^{\prime}+d^{\prime \prime}$, and $(k a)^{-1}=1.18 \cdot 10^{-7}$ (meter $)^{-1}$. When $d$ is measured in miles and $h$ in leet, equation (16) becomes

$$
\beta_{1}+\beta_{2}=1.89 \cdot 10^{-4} d\left[\frac{h_{p}}{d^{\prime} d^{\prime \prime}}+1-\frac{h_{1}}{d^{\prime}}-\frac{h_{2}}{d^{\prime \prime}}\right]
$$

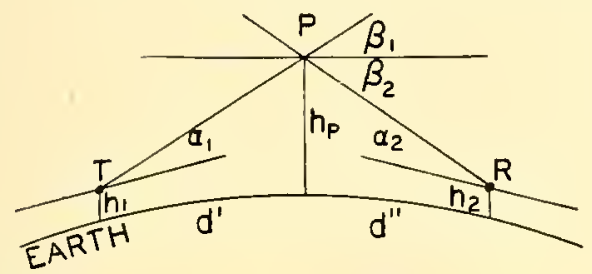

Figtre 7. Shielding between transmitter and receiver.

10.4

10.4.1

\section{PERMANENT ECHOES}

\section{Introduction}

Permanent echoes are caused by reflections from terrain features such as mountains or even smooth surfaces near the antenna (ground clutter). With radars, the indicator is obscured by the strong echoes from hills and the minimum detection range is increased by ground clutter. With direction finclers, erroneous indications are caused by the spurious reflections. Permanent echoes are among the principal problems involved in siting, as many otherwise excellent sites are rendered wor thless by excessive fixed echoes. Several methods are available for determining the suitability of sites in this regard without actual field tests.

A number of factors combine to make permanent echoes more troublesome than might be expected.

1. Hills and land surfaces are so much greater in extent than the target which the equipment is designed to detect that strong echoes may be obtained from distances where an ordinary target would give an echo far below normal detection levels.

2 . The low elevation of the land surfaces places them in regions most subject to nonstandard propagation effects where extreme ranges and large responses are frequently obtained.

3. Side lobes of the horizontal pattern of the antenna cause permanent echoes to appear at several other azimuths in addition to that of the main lobe. t. Strong permanent cchoes from mountains to the rear may be caused by back radiation from the antenna. The low intensity of the back radiation may be compensated by the size of the mountains. such echoes are especially harmful as they obscure the operating sector.

5. Objects appear wider becanse of the antemna beamwidth and of greater extent in range as a resutt of the pulse width.

6. Diffraction over intervening ridges nay be sufficient to nullify their sereening action so that. objects behind a ridge are visible.

7. The use of a permanent echo as a standard target may be very misleading. I decrease in performance that seriously affects echoes from small targets may not have any noticeable effect on the response from large targets. An echo used for a standard target should be weak and near by.

\subsubsection{Permanent Echo Diagrams}

The permanent echoes associated with a radar station may be plotted on a polar chart and their extent, location, and strengtl represented. Such diagrams should be prepared for each unit of a radar system, using a standard procedure for taking and presenting the data.

Permanent ccho data should be taken under arerage conditions with the gain set at some standard level. At intervals of azimuth such as 5 de grees, the ranges of the permanent echoes are recorded. These data are then plotted on a polar chart and the points are connceted to indicate obscured areas. The skill and judgment of the operator are important lactors. In most cases the amplitudes of the echoes ate so far abore that of ordinary target echoes that the actual amplitudes need not be noted.

In Figure 8 is shown an observed permanent echo diagram for a VHF radar. This was selected for purposes of illustration rather than as an example of a good site. The mountains to the north are unwhielded and cause extensive echoes. The large echo at 200 degrees is due to a mountainous island 260 miles away and appears only during times when propagation is nonstandard.

Care must be exercised in identifying the canse of an echo. Antenna side lobes cause spurious echoes and distant echoes may come in on the second $o$ third sweep on the scope after the main pulse. These latter echoes may be checked by changing the pulse repetition rate and observing the shift of the echo. 
Permanent echo diagrams are useful for:

1. Indicating blind areas in a station's coverage.

2. Assigning the operating area of a station.

3. Checking the range and azimuth accuracy.

4. Checking the performance.

5. Estimating nonstandard propagation.

6. Planning test flights. hills are sercened by a local obstruction. This local echo at, say, three miles, is combined with the main pulse or ground return and the distant echo is weakened or eliminated entirely.

Shielding causes a loss of corerage, which in operating regions may be more serious than the permanent echoes. Rear areas which are not scanned

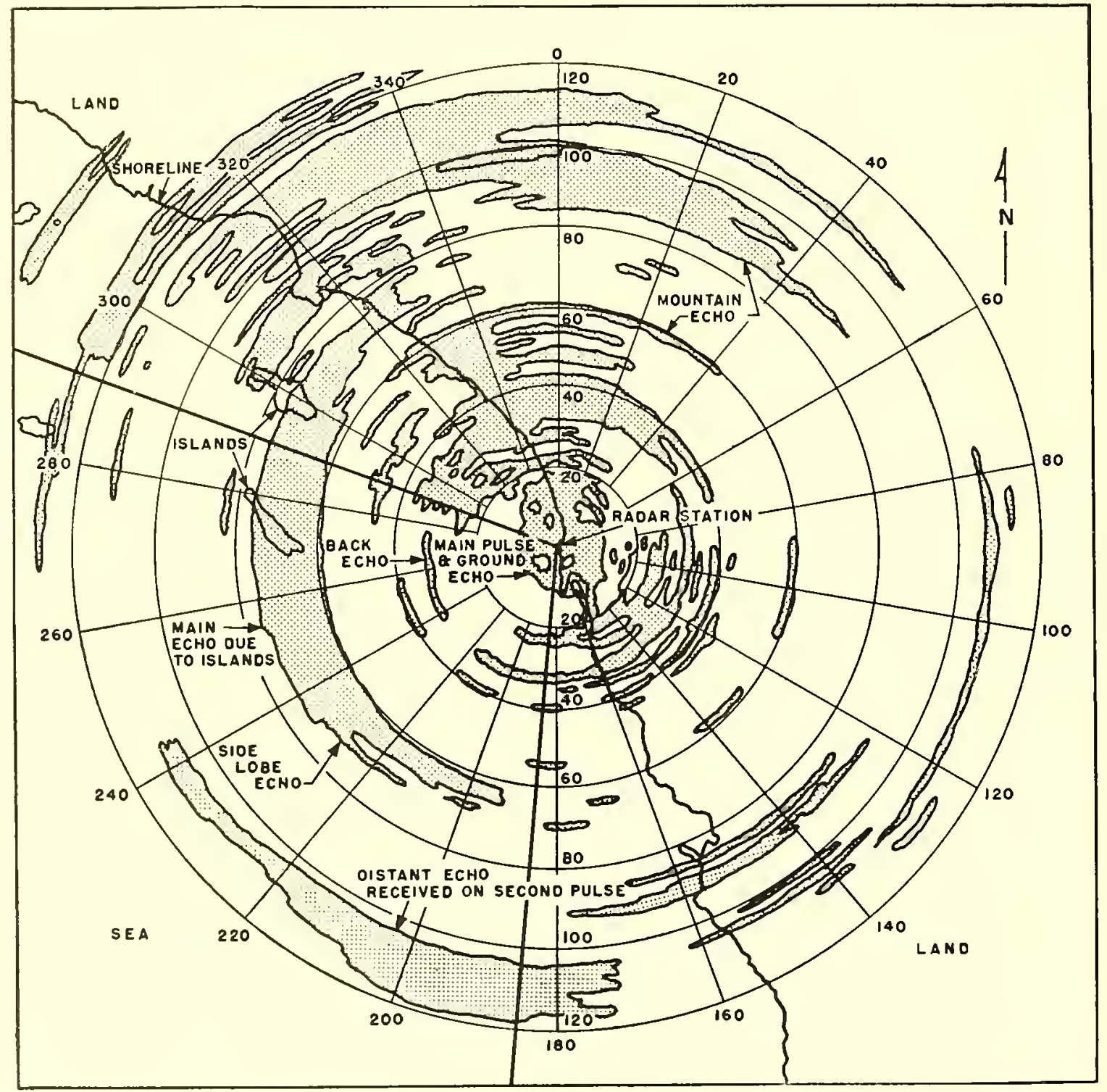

Figure 8. A typical permanent echo diagram for a VHF radar.

10.4 .3

Shielding

The principal device in the field lor the control of permanent echoes is shielding. This means that the antenna must be sited in such a way that distant should be well shielded so that back and side echoes do not interfere with targets in important tactical regions. Operation over such shielded sectors would be limited to high targets. 


\subsubsection{Prediction of Permanent Echoes}

Permanent echoes may be determined by several methods: (1) tests with the radar at the site; (2) radar planning device [RPD]; (3) supersonic method; and (4) profile method.

The feasibility of moving the radar to the site to determine the permanent echoes is dependent on the portability, accessibility, etc. Echoes obtained with one type of equipment may be very different from those of another type of radar with a different antema directivity, frequency, and range.

The RPD technique requires construction of a relief model of the terrain considered. A small light source is used to simulate the radar transmitter and the echoes are plotted as a result of a study of the areas illuminated. This method is useful for short ranges and microwaves where the diffraction and side and back lobe radiation are small. Construction of a fairly difficult relief model may take a crew of specially trained men several days to a week, as the model should be accurate. Once completed, all possible sites or aspects from a plane or ship may be readily examined. Models of enemy areas may be used to predict the coverage of possible enemy sites and evasive action may be planned. The RPD is well suited for training and briefing of air personnel.

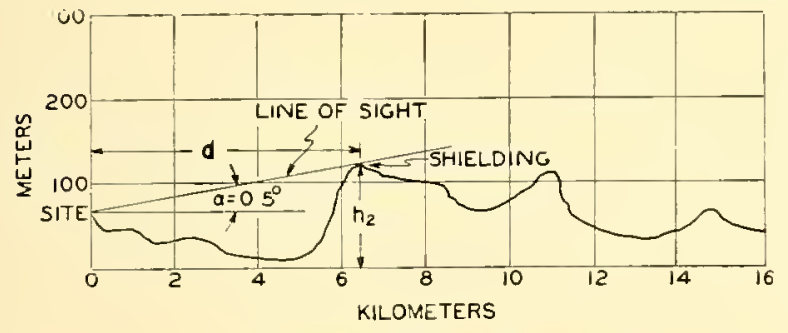

Figure 9. Typical profile.

Kits are provided containing the light source, supports, etc. Photographic and darkroom facilities are also required.

The supersonic method uses a relief model under water. Supersonic gear is used to send out pulses which are reflected like radar pulses and an echo is picked up and presented on a plan position indicator [PPI] scope. Photos may be taken of the scope or it may be used directly to train operators and for briefing. Considerable equipment is required, but the construction of the models is comparatively simple.

The profile method involves a study of topo- graphical maps and plotting of the echoes according to their visibility and the amount of diffraction. A fairly difficult site may be handled in perhaps eight man-hours. This method is adapted to long-range, low-frequency radars where diffraction and side and back lobe radiation are important. On microwave equipment, prediction of permanent echoes is simpler and the profile method may be worked out in a few hours.

\subsubsection{Prediction by the Profile Method}

The discussion here refers chiefly to VHF (1 to $10 \mathrm{~m}$ ) radars in a mountainous terrain, but the methods have general application. The principal requirements are topographic maps of the surrounding area with a scale of one or two miles to the inch and a contour interval of 20 feet, although intervals up to 100 feet may be used. Regional aeronautical maps with a scale of about 1 inch to 16 miles and 1,000-ft contours are suitable for checking distant echoes.

From the maps, profiles are prepared for various azimuths about the radar station. The first mile or so should be plotted accurately; at greater distances, the critical points, such as hills and breaks, should leceive the most attention. On each profile is drawn the tangent line from the center of the antenna to the point on the profile which determines the shielding, as in Figule 9. The angular elevation $\alpha$ of this line of sight is marked on the diagram. If $\alpha$ is negative, the profile should be checked out to the radar horizon to obtain the correct shielding angle. On a plane earth diagram, the line of sight is actually curved, but for distances up to 10 miles it may be taken as straight with small error. The height difference, with $\alpha$ in radians, is then equal to

$$
h_{2}-h_{1}=d \tan \alpha .
$$

When the distance is larger so that earth curvature has to be taken into account, to the above expression for the height difference $h_{2}-h_{1}$ must then be added the amount by which the earth is sloping eway over the distance $d$. This amount is $d^{2} / 2 k a$, and the complete expression for $h_{2}-h_{\mathrm{I}}$ becomes

$$
h_{2}-h_{1}=d \tan \alpha+\frac{d^{2}}{2 k a} .
$$

For easier handling of this equation, a set of curves may be drawn where $h_{2}-h_{1}$ is plotted against $d$ for various constant values of $\alpha$. These curves may then be used to determine the height of the 
shielded region at any range. Thus all other mountains along the profile in Figure 9 might be checked for visibility by comparing the height of the mountain with the ralue of $h_{2}-h_{1}$ read from the curve $\alpha=0.5^{\circ}$. Any desired allowance for diffraction may be made by using a different curve such as $\alpha=0$. When the shicld consists of several ridges lined. From an examination of the map, the azimuths at which profiles should be prepared are determined. This will normally be about every 10 degrees. Where the shielding is obviously good, the interval may be 20 degrees, but where the terrain is questionable, such as in a region of low hills, the profiles should be taken at 5-degree intervals.

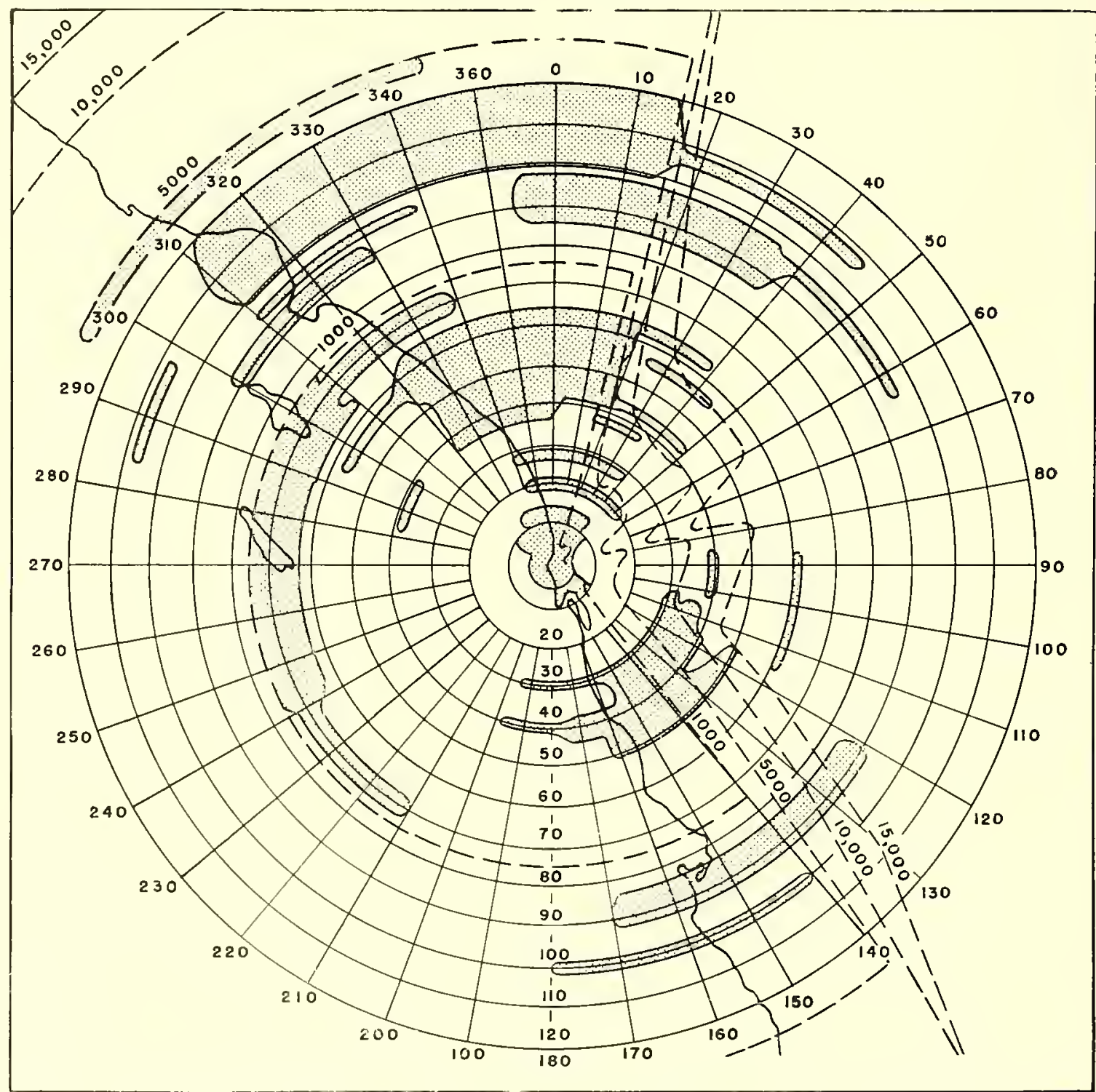

Figure 10. Theoretical permanent echo diagram.

(close together, an equivalent shicld should be used. This is derived by enclosing the ridges in a triangle, whose apex is taken as the shield (see Figure 6).

The general procedure to be followed in preparing a prediction of permanent cehoes will now be out-
An overlay of the region is then prepared, showing important geographical features and a polar-grid system. On this chart is drawn the coverage contour lines (broken lines in Figure 10). These lines represent the limits of the heights of the shielded regions. 
Targets or mountains below and beyoud the eoverage eontours will not be risible except by diffraction. These eontours may be drawn for scveral heights. Where they are close together, the shielding is good but the coverage is poor. Where the lines are widely separated, as toward the sea, there is little or no shielding except that due to earth eurvature. With the coverage contour diagram superimposed on a map, the peaks exposed to radiation may be noted.

The extent of the echoes due to these peaks depends, besides the size of the peak, on the horizontal radiation pattern, the pulse width, and the power and sensitivity of the radar. It should be noted that the half-power beamwidth is only a rough measure of the width of an echo and some greater angle between the half-power points and the nulls will usually be obtained for the echoes.

The extension of the echo in range will be at least as great as the pulse width in miles as represented on the seope. This is about 0.1 mile per microseeond of pulse width. Aetual echoes are thicker than this, since all the exposed hill sends back echoes.

After a careful inspection of the profiles, taking into aecount the rarious factors mentioned above, the echoes are sketched in on the ehart. In doing this, judgment and experience are important factors, but the following rules may be used as a guide.

1. Shade in a cirele for the main pulse several miles wide, depending on the pulse width and local return.

2. Check each profile in turn and for each peak or hillside in front of the shielding ridge or mountain plot an echo for the main and all side lobes of the antenna.

3. A series of sharp hills within the shielding part of the terrain should be plotted as a single large echo.

4. The inner edge of an echo should be at the same range as the hill.

5. Peaks beyond the shield may be in the diffraction region and the relative strength of the echo may be estimated from a diffraetion eurve.

6 . In general, the echo strength varies as the inverse square of the distance and is roughly proportional to the target area.

7. Where there is any doulbt, the echo should be plotted.

Experience is an essential factor in permanent echo prediction, regardless of the method used. The methods described here have been used successfully in many areas and are capable of aceuracy adequate for most purposes.

\subsection{EFFECT OF TREES, JUNGLE, ETC.}

10.5 .1

\section{The Effect of Trees}

Trees form very effective obstacles for' high-frequeney radio wares. A single tree may eause a drop in signal strength of several deeibels. The attenuation is less for horizontal polarization than for rertieal polarization for frequencies below 300 to 500 megacyeles. For higher frequencies, the polarization is not an important factor. With the transmitting antenna sited in a moderately wooded area, representative ralues for the losses are given in Table 1.

TABLE 1. Decrease in gain for transmitting antenna situated in a moderately wooded area.

\begin{tabular}{clc}
\hline Frequency & $\begin{array}{c}\text { Horizontal } \\
\text { polarization }\end{array}$ & $\begin{array}{c}\text { Vertical } \\
\text { polarization }\end{array}$ \\
\hline $30 \mathrm{me}$ & Negligible & $2-3 \mathrm{db}$ \\
$100 \mathrm{me}$ & $1-2 \mathrm{db}$ & $5-10 \mathrm{db}$
\end{tabular}

When both antennas are in the woods these losses should be doubled. Measurements at 200 me for transmission through a grove of trees 100 feet wide show losses of $21 \mathrm{db}$ for rertical polarization and $6 \mathrm{db}$ for horizontal polarization.

When the antennas are in elearings, so that each is more than 200 or 300 feet from the edge of the woods, the decrease in gain is small. With rertieal polarization, there may be large and rapid variations of field intensity within a small area, due to reflections from near-by trees.

\subsubsection{The Effect of Jungles}

In jungles or heavy undergrowth, an exponential absorption is to be expeeted. Tests made of transmission through heavy jungles, such as are found in Panama or in New Guinea, show that the limit of transmission for ordinary field sets is 1 mile. An inerease of power of several hundred fold is needed for a range of 2 miles. The decreases in gain encountered are of the order of 50 to $60 \mathrm{db}$ per mile.

If the antennas are elevated above the jungle or loeated in clearings, the effect of the jungle may be minimized. Antennas should be 10 or more feet away from trees to aroid a change in antenna impedance.

The best solution is sky-wave transmission even for distanees as short as 1 mile. Due eonsideration should be given to the selection of optimum fre- 
quencies based on ionosphere predictions. For distances up to 100 or 200 miles, a half-wave horizontal wire antenna should be used and the frequency range is about 2 to $8 \mathrm{mc}$. The decrease in gain for the short path up through the trees is negligible at these frequencies.

\section{${ }^{10.5 .3}$ The Effect of Trees and Obstacles on Microwaves}

At $10 \mathrm{~cm}$, the absorption is so great with most objects that the diffracted energy is the principal portion transmitted. Only windows, light wooden walls, or branches of leafless trees show less than
$10 \mathrm{db}$ loss. Opaque objects include:

1. Rows of trees in leaf if more than two in depth.

2. Screens of leafless trees if so dense that the skyline is invisible through them.

3. Trunks of trees.

4. Walls of masonry.

5. Any but the lightest wooden buildings, especially if there are partitions.

Losses of a brick wall may be increased from $12 \mathrm{db}$ to $46 \mathrm{db}$ by wetting. In computing diffraction over treetops, the diffracting edge may be taken to be 5 feet or so less in height. In a $1.25-\mathrm{cm}$ test, the transmission loss through two medium-sized bare trees increased $18 \mathrm{db}$ after leaves appeared. 


\section{GLOSSARY}

a. 1) Radius of the earth

2) Radius of scattering plate, sphere, or cylinder

A. Gain factor $=A_{0} A \rho$

A. Gain factor for doublet antennas in free space, adjusted for maximum transfer of power $=$ $3 \lambda .5 \pi d$

$A_{1}$. Plane-earth factor

Ap. Path-gain factor

$\bar{A}$. Gain-factor curve parameter

b. $\quad b=\frac{d_{1}-d_{2}}{d_{1}+d_{3}}$

B. Bandwidth

c. 1) Velocity of light in free space

2) $c=\frac{h_{1}-h_{2}}{h_{1}+h_{2}}$

$C, C(v)$. Real part of Fresnel's integral

d. Distance from center of transmitting antema to a point in space measured along the surface of the earth

$d_{0}$. Free-space distance for field of strength $E$

$d_{0}$. Normalized free-space distance $=\frac{d_{0}}{d T}$

$d_{1}, d_{2}$. Distance from transmitter, receiver to reflecting point measured along the earth's surface

$d_{T,} d_{R}$. Distance from transmitter, receiver to the radio horizon measured along the earth's surface

$d_{L}$. Line of sight distance measured along the earth's surface $=d_{T}+d_{R}$

$d_{\max }$ Maximum radar range

D. 1) Divergence factor for spherical earth

2) Aperture of reflector

e. I) Water-vapor pressure

2) Coefficient for height-gain function

E. Electric-field strength

$E_{0}$. Naximum free-space field strength of a doublet transmitter at distance $d$

$E_{1}$. Radiation field strength at one meter from transmitter

f. 1) Frequeney

2) Focal length of paraboloid reflector

$f_{c}$. Cutoff frequency of a wave guide

$f(h)$. Height-gain function

$f_{n}$. Height-gain function for the $n^{\text {th }}$ mode

$F_{1}, F_{2}$. Fraction of maximum radiation field strength in the direction of direct, reflected rays

$F_{n}$. Noise figure

$F_{s .} \quad$ Shadow factor for the first mode

$F_{s}{ }^{\prime}$. Sum of shadow factors for all modes

1) Receiver gain

2) Exponential factor of height-gain function for elevated antennas

$g^{\prime}$. Correction to $g(2)$

$G_{1}$. Transmitting antenna gain

$G_{2}$. Receiving antenna gain
$G_{R}$. Radar gain of a target

$h$. Height above ground

$h_{1}, h_{2 *} \quad$ Height of transmitter, receiver above ground

$h_{1}{ }^{\prime}, h_{2}{ }^{\prime}$. Height of transmitter, receivel above tangent plane at point of reflection

$h_{c}$. Critical height distinguishing high and low antennas located in diffraction region $=30 \lambda^{2 / 3}$

$h_{0 .} \quad$ Virtual height of obstructing screen

H. 1) Magnetic field strength

2) Height of a reflecting or diffracting obstruction

$H L$. Height-gain function for low antennas

IIA. Hour angle of the sun

I. RMS current

$I_{i}$. Input current to antenna or circuit

j. $\quad \sqrt{-1}$

k. I) Boltzinann's constant

2) Factor multiplying earth's radius to account for atmospheric refraction

K. 1) Amplitude of generalized reflection coefficient

2) Echo constant of a target

l. 1) Length of a doublet

2) Height coefficient to mclude effect of earth's eonstants and wavelength

L. 1) Effective length of a doublet

2) Characteristic length or scattering coefficient of a target

3) Radar length of a target

$m$. I) Ratio of radius of curvature of a ray to the radius of the earth $=\rho, \alpha$

2) $m=\frac{d^{2}}{4 k a\left(h_{1}+h_{2}\right)}$

M. Modified index of refraction

n. 1) Index of refraction

2) Number of elements in an antenna array

$n^{\prime}, n^{\prime \prime} \quad$ Lobe numbers

$N$. Lobe variable for imperfect reflection

NF. Noise figure

p. 1) Total pressure of the atmosphere

2) Dimensionless parameter $=d_{1} / d T$

$p^{\prime}$. Distance coefficient to include earth constants

$P$. Power

$P_{1}$. Power output of a transmitting doublet

$P_{2}$. Power delivered by a receiving doublet to a matched load

$P_{\text {min. }} \quad$ Minimum power detectable by a receiver

$P_{n .} \quad$ Noise power

$P_{r}$. Power received by load circuit of receiving antenna

$P_{s}$. $\quad$ Scattered power

q. Dimensionless parameter $=d_{\mathbf{2}} / d$

Q. Parameter determining phase of beam reflected by the earth $=\frac{\epsilon_{r}}{60 \sigma \lambda}$ 
r. 1) Distance from center of antenna to a point in space (usually replaced by $d$ in applications)

2) Height wavelength factor

3) Pattern or chart parameter

4) Path length of reflected ray

$r_{d}$. Path length of direct ray

$R$. 1) Resistance

2) Plane-earth reflection coefficient $=\rho e^{-j \phi}$

3) Path-difference parameter $=(k a \Delta) /\left(h_{1} d T\right)$

$R_{a}$. Resistive component of antenna impedance

$R H . \quad$ Relative humidity in per cent

$R_{l}$. Resistive component of load impedance

Rr. Radiation resistance of an antenna

s. 1) Spacing between dipoles in an antenna array

2) Coeffieient of distance for shadow factor

3) Dimensionless condinate $=d_{1} / d$

S. 1) Scattering cross section

2) Area

$S, S(v)$. Imaginary part of Fresnel's integral

t. 1) Time

2) Pulse width

3) Degrees centigrade

T. Absolute temperature

$u$. Dimensionless coordinate $=h_{2} h_{1}$

$v$. 1) Veloeity of wave propagation $=c_{\text {in }}$

2) Algument of Fresnel's integral

3) Dimensionless parmeter $=d / d_{T}$

r. Voltage

$V_{n}$. Noise voltage

IV. Power per unit area

$W_{i}$. Ineilent power per mit area

$I_{r}$. Scattered power per unit area at the receiver

z. Amplitude of ratio of diffracted field to free-space field

Z. Impelance

$Z_{a}$. Antenna impelance

$Z_{l}$. Load impedance

$\alpha$. 1) Attenuation constant, real part of propagation constant $\gamma$

2) Angle made by ray with the horizontal

$\alpha_{1}, \alpha_{2}$. Angle of elevation of diffracting edge as seen from transmitter, receiver

$\beta$ 1) Bearing of the sun

2) Pliase constant, imaginary part of propagation constant $\gamma$

$\beta_{1}, \beta_{2}$. Angle between ray from transmitter, receiver, and horizontal at diffracting edge

$\gamma$. 1) Propagation constant $=\alpha+j \beta$

2) Angle between horizontal at base of transmitter and line joining transmitter base to receiver

$\gamma_{1}$. $\delta$.

1) Declination of the sun

2) Angle of phase retardation due to path-length difference between direct and reflected rays

3) Ground parameter depending on complex dielectrir constant

$\Delta . \quad$ Path-length difference hetween chirect and reflected rays $=r-r_{d}$

$\Delta \rho . \quad=\left(2 h_{1} h_{2}\right) / d$

$\Delta\left(\Delta_{p}\right)$. Correction factor for $\Delta \rho$

3f. Bandwidth

$\eta$ Variable used in diffraction region

$\epsilon_{0}$. Dielectric constant of free spar'e

$$
=8.854 \times 10^{-12} \cong \frac{1}{36 \pi} 10^{-9}
$$

$\epsilon_{c}$.

$\epsilon_{i}$.

Complex dicleetrie constant $=\epsilon_{r}-j \epsilon_{i}$

$\epsilon r . \quad$ Real part of dielectric constant

5. 1) Phase-angle lag clue to diffraction

2) Dimensionless distance variable for curved-earth diffraction

o. 1) Angle between horizontal at transmitter base and lorizontal at point of reflection

2) Angle of tilt of seattering cylinder

$\lambda$. Wavelength

$\mu_{0}$. Permeability of free space $=4 \pi 10^{-7}$

$\mu_{r}$ Permeability relative to free space

$\nu$

p.

Angle between reflected ray and horizontal at transmitter

1) Raclins of curvature

2) Amplitude of reflection coefficient

1) Conductivity

2) Radar cross section

$\tau$.

1) Complex mode numbers

2) Half beam-width angle

$\phi$ 1) Plase angle of reflection coefficient

2) Latitude

$\phi^{\prime} . \quad=\phi-\pi=$ phase angle of reflection coeflieient relative to a perfect reflector

$\Phi$. Distance function for the first mode

$\psi$ 1) Phase-angle difference between currents in dipole-antenna array

2) Angle between direet or reflected ray and the horizontal at the reflection point

3) Height variable in the diffraction region

$\psi_{d}$. Angle between direct ray and horizontal at reflection point

w. Angular velocity $=2 \pi f$

$\Omega$. Total phase lag between direct and reflected rays $=\phi^{\prime}+\delta=\phi-\pi+\delta$ 


\section{OSRD APPOINTEES}

COMMITTTEE ON PROPAGATION

\section{Chairman}

Chas. R. Burrows

\section{Members}

H. H. Beverage

T. J. Carroll

J. H. Dellinger
Mirtin Katzin

D. F. Tierr

J. A. Stratton

\section{Consultants}

S. S. ATTWOOD

C. E. Buell

J. A. Stratton

Technical Aides

A. F. Murray

R. J. HEARON

S. IV. Thomas 
CONTRACT NUNBERS, CONTRACTORS, AND SUBJECT OF CONTRACTS

Contract No. Contractor Subject

OEMIsr-I207 Columbia University New York City, New Tork

OEXIS1-728

OEMSr-I 497

OEXIsr-1496

OEXIsr-I502
State College of Washington Pullman, Washington

Humble Oil and Refining Co. Houston, Texas

University of Texas Austin, Texas

Jam Ilandy Organization, Ine. Detroit, Michigan
Correlation, analysis, and integration of data on radio and radar propagation.

Develop meteorological equipment and conduct meteorological soundings in the Southwest Pacific and correlate it with radio-propagation data.

Development and construction of microwave fieldstrength measuring sets.

Devclopment of equipment for, and making measurements of, time and space deviations in radio-wave propagation.

Preparation of a General Ontline of Training Material and the preparation of manuals, films, and other training aids for use in instructing technical and other personnel in radio-weather and radio propagation. 
The Committee on Propagation dicl all of its work under Project Control SOS-9, which was originally set up through the request of the Combined Chiefs of Staff following recommendations submitted by the Combined Meteorological Committee [CMIC] (1): that the Committee on Propagation of the National Defense Research Committee be requested to act as a coordinating agency for all meteorological information associated with short-wave propagation, (2) that the Committee on Propagation be requested to forward periodically to the CMC a list of all reports and papers dealing with the meteorological aspects on short wave propagation which have been received or transmitted by that Committee.

Later the Combined Meteorological Committee in its thirty-seventh meeting on Tuesday, February 22, 194t, agreed that the NDRC Committee on Propagation be recognized as the supervising committee on all basic research being done in the United States on the related problems of radar propagation and weather, in addition it shall be the recognized channel whereby international exchange of papers of the two related sciences will be effected.

The Joint Communications Board [JCB] therefore approved the following policy, which was concurred in by NDRC and by the Joint Meteorologieal Committee:

1. The NDRC Propagation Committee and its associated working groups will initiate and exercise techmical supervision over such tests and investigations as they deem necessary to ascertain the nature of the above mentioned propagation anomalies in the VHF, UHF, and SHF bands, to devise the most practicable methods to determine the occurrence and characteristics of these anomalies from appropriate meteorological forecasts, with a view to improving the interim solutions offered by the Joint Wave Propagation Committee of the JCB.

2. The Army and Navy will furnish by direct coordination between them the basic staff guidance for such tests and investigations. They will accomplish this by determining $(a)$ the specific forms in which basic prediction data shall be presented, and $(b)$ the method of use required for operational forecast of propagation anomalies in the VHF, UHF, and SHF bands.

3. When the NDRC requires the cooperation of the operating units of the Army and Navy in conducting such tests and investigations as it deems necessary and this cooperation is of such an extent and nature that it cannot be furnished by informal coordination, it will be requested through the Joint Wave Propagation Committee of the JCB. Such requests will be initiated by the NDRC representative on the Wave Propagation Committee and recomnended to the JCB by the Joint Wave Propagation Conmittee for consideration.

4. The Joint Wave Propagation Committee will be responsible for devising and funnishing immediately interim operational forecasting guides based upon information already available.

On April 3, the Coorlinator of Researeh and Development requested that the Army Project SOS-9 be made a joint Army-Navy project. Project No. AN-I6 was assigned to this.

On May 23, 1944, the Chief Signal Officer requested that under Project AN-16 the following work be inaugurated:

Project AC 230.04, "Wave Propagation Study of Line-of-Sight Communication and Navigation." 
i 


\section{INDEX}

The subject indexes of all STR volumes are combined in a mister index printed in a separate volume. For aceess to the index volume consult the Army or Navy Ageney listed on the reverse of the half-titlo page.

A scope, calibration, $161-162$

lireraft targets, rudar cross section measurement, $185-186$

Intenna, general characteristics beam width, 22

characteristics in transmission, 6

cliameter, 29-31

effective length, 13

function, 22

horns, 14

impedance of nearby eonductors, $2+$

pattern factors in ground reflection, 23

radiation patterns, 22-23

radiation resistance, 24

Intenna arrays, 33-39

binomial, 38-39

broadside, $3 \pm-38$

colinear, $34-38$

dipole, basic types, 34

multidimensional, 38

principle, 33-34

ring, 39

two-dipole side-by-sicle, $34-35$

unidircetional, 38

Intenna gain

ealculation, $16-17$

definition, 16

description, 22

jungle locations, effect on gain, 195 196

propagation factor in interference region, 69

wood location, effect on gain, 195196

Intennat types, 22-33, 39-43

directive antennas, 22-23

multiple half-wave, $2 \overline{7}-28$

parabolic reflector antennas, 42-43

parasitic autemas, $39-12$

resonant antennas, 23

standing-wave antennas, 23, 25-32

traveling-wave antennas, 23-24, 3233

Atmospheric ducts

effect on set performance, 1633

formation, 4

types, 4

Itmospheric stratification, effect on refraction, $50-52$

Attenuation, definition, 5

Attenuation factors, rarlio gain calculation, 61

Beacons, performance characteristies, 169
Beam wilth, antemis, 22

Binomial arrays, antenna, 35-39

Brewster angle of reflection, define $1,5-1$

Broadside arrays, antenna, 34-38

one-dimensional array, 35-3s

side-by-side array, 34

unidirectional array, 39

Calibration, A scope, 161-162

Clarendon Laboratory at Oxford, eonductivity of sea water, 55

Coastal diffraction, transmission, 178181

eliff site, 181

field strength, 179-181

level site near coast, 178-179

Colinear arrays, antenna, $3 t-38$

one-dimensional array, 38

two half-wave dipole array, 35

unidirectional array, 38

Columbia University Wave Propagation Group, 1

Communication equipnent, performance characteristics, 16 ?

Conductivity, soil, 56-57

Cophased dipole antenna, 28-2?

Corner-reflector antenna, 42

Cornu's spiral, diffraction theory, 172173,175

Coverage diagrams, generalized coordinates, $1 \pm \neq-159$

basic parameters, $1 \pm 1-1+5$

normalized free-space distance, 145 147

path-difference parametcr, 1-15, 147

use of charts, $14 \bar{\gamma}-159$

Coverage diagrams, nethods of construction, 132-144

lobe-angle method, 135-1 14

P-Q method, 132-135

U-V method, 135-138

Coverage diagrams, plane carth, 129131

field strength, 129

horizontal polarization, 129-130

vertical polarization, 130-131

Coverage diagrams, spherical estrth, 131-132

Curvature of earth

calculation of radio gain below interference region, 92

geometrical relationships, 62

Curvature of radio waves

curvature relationships, $47-48$

diffraction by cartl's curvature, $5 \mathrm{~s}$

rays in standard atmosphere, 3-4
Definition of propagation, 1

I) ielectrie constant, soil, 56-57

Dielectrie constant, water, 5t-5.5

Dielectric earth

graphical ealculations, radio gain, 95-108

radiation field characteristics, 65-67

sca water as dielectric earth, 60 ;

Diffraction

by terrain, 170-181

coastal diffraetion, 178-181

Cormu's spiral, 172-173, 175

definition, 58

earth's curvature liffraction, 58

Fresnel diffraction theory, 170-175

hill diffraction, 175-17s

raindrop diffraction, 59

reflecting ground, $177-178$

slot diffraction, 175

summary, 58

target diffraction, 58-59

transmission factor, 7

Diffraction regions, radio gain calculation, 62-63

Dimensionless coordinates, radio gain ealculations, $77-7 \mathrm{~S}$

Dimensionless parameters, radio gain calculation, 75-77

Dipole arrays, basic types, 34

Direetive antenuas, 22-23

Divergence factor

ground reflection, 57

lobe length determination, 139-140

propagation in interference region, $68-69$

spherical earth radio gain ealeulations, 75

transmission characteristic, 7

Doublet antemnas, radio gain, 5

Ducts, atmospheric

effect on set performance, 163

formation, 4

types, 4

Earth conductivity, 61

Echo constant, radar coverage measurement, 182

Effective length, antenna, 13

Electric doublet antenna in free space, $12-15$

radiation, 12-13

received power, 13-14

scattered power, 13-1t

transmission, $1 \frac{1}{t}-15$

End-fire arrays, antenna, 34 
Equipment performanor, propagation aspeets, 160-169

I scope calibration, 16il-102

communications and ratiar erpuipment data, 169

free space high-ingle coverage, $162-$ 163

low-angle and surface coverage, $163-$ 169

pelformance figure, me"surement of efficiency, 160

reflection (ffeet, 160-16)

signal-to-noise ratio, 161

Equivalent height, radio gain calculation, 71

Fidel strength, dofinition, 5

Free space distance, uomalizer, $115-$ 147

Free space ficld, (I) finition, 5

Free space gain factor

below intruference recrion, !)

lefinition, 5

equation, 60

Free space ralios gatin, defintion and formula, 15

Fresnel diffraction thany, 170-175

Fresisel integrals, 172-173

Fresnel zones, $174-175$

mechanism of eliffraction, 171

polarization, 174

slot diffraction, 175

straight edge formula, 171-172

Fresnel formulas, reflection, 54

Fresnel-Kirelioff optical theory

see Fresnel diffrietion theory

Generalized reftection cocfficient, 69-70

Grazing angle corresponcliug to lobe maxima, 129-130

Grazing angle in radin gain calculattions, 78

Ground reflection, 52-58

analysis of problem, 52-53

conductivity of soil, $56 j-57$

diclectric constant of soil, $56-57$

dielectric constunt of water, 5t-55

divergence factor', 57

Fresnel formulas, 54

irregularity of ground, $57-55$

overland transmission, 56

plane reflecting surface, 53

Half-wave antennas, 25

Half-wave dipole, antema, 25-29)

eomparison of alternate and cophased half-rrave dipoles, 28

cophased dipoles, 28-29

folded dipole, 27 gaill, 26

impedance, 26

quarter-wave diprole, modification, $26-27$

radiation ficle, $25-26$

radiation resistance, 26

IIill diffraction, 175-178

critcrion, roughness of surface, 176

field rear the line of sight, $17 t$

reflecting ground diffraction, 177-178

straight ridge diffrnetion, 176-17

Horizontal polarization

angles of lob $x^{2}$ maxima, 12!)-130

radio gain curves, 8-10

Horns, antenna us( 44

IIuyghens principle, rudio wave diffraction, 7

Impeciance, half-wave dipole antcuna, 26

Index of refruction, defintion, 3

Induction ficld, refinition, 12

Integral hall-wave antennas, 27-28

Interferencer, propagation factor in radio gain calculation, 67

Isotropic radiator, hypothetical antemin, 16

Jungtes, obstaches fo radio wave propagation, $1955-190$

Lime of sight, definition, 62

Iinear antennus, diameter lemgllss, 2931

Linear antennas, types, 24-25

Lobe maxima and minima, definition, 129

Lobe-angle mothou, coverago diagram construction, 135-144

basic equation, 138

correction for low angles, 141-143

lobe angles with horizontal, 139

lobe construction, $140-1+1$

modified divergence fuctor, 139-140

reflection point eurves, 138-139

vertical polarization, 143-141

Maximum range, 162-16t;

low-angle ancralt coveratage, antenna heights and tarecet lieights, 163164

low-angle areraft coverage, lueight rurves versus maximum range, 161

low-angle and surfare range coverage, 163

one-way communication, 162

radar, 162

Mlicrowave beacons, ling arrays, 39

Moist standard atmosphere, propagation, 3-4
Multidimensional arrays, antenna, 38 Multiple half-wave long antennas, 2728

Noise figure of ratio receiver, 17-19 definition, 18

measurement, 18-19

Tonstandard atmosphere, propagation, 4

Operator loss, radar reception, 19

Optical region, raliation field characteristics, 66

Optical region, radio gain calculation, $62-63$

Parabolic reflectors for antemus, 42-43

Parasitic antennas, 39-42

corner reflectror, 42

half-wave dipole and parasite, 39-41

multiple parasites, Fagi antenna, 41

reflecting sereens, 41-12

Path difference

loci construction, enverage diagrams, 134-135

parameters, 145, $14 \pi$

path difference variable equation, s0-81

plane earth, 70

spherical earth, $71-75$

Path gain factor, definition, 5

Performance figure, measurement of set efficiency, 102-163

Permanent celsoes, prediction, 193-195 profile method, 193-195

radar test at site, 193

RPD (radar planning (levice), 193

supersonic nethorl, 1 ! 3

Permanent cehoes, sitc selection factor's, 191-195

permanent echo diagrams, 191-192

shielding, coho control, 193

Plane earth

calculation of radio gain below interference region, 9l-92

coverage diagrams, 129-131

path difference, 70

Polarization

angles of lobe maxima, horizontal polarization, 129-130

angles of lobe maxima, vertical polarization, $130-131$

Fresnel diffraction theory, 174

horizontal versus vertical, optical region, 60

horizontal versus verlical, radio gain calculations, 10!

radio gain, vertical polarization effect, 83

radio gain curves, horizontal polarization, 8-10

radio wave diffaction, 174 
radio waves, $52-53$

transmission factor, 6

Power transmission, 15-17

antenna gain and polarization, 16-17

radio gain, 15-16

reciprocity principle, 17

P'Q method of coverage diagram construction, 132-13.5

path-difference loci construction, 134-135

range Ioci construction, 133-134

Profile method, ceho determination, 193-195

Propagation, assumption of standard comditions, 61-62

Propagation, atmospheric consider:tlions, 2-4

moist standard atmosphere, 3--t

nonstandard atmosphere, 1

standard atmosphere, 3

Propagation, hasic relationships, 12-2I

electric doublet in free space, 12-15

power transmission, 15-17

radar cross section, 19-20

radar gain, 20-21

receiver sensitivity, 17-19

Jropagation, definition, 1

Propagation, general charucteristics, 1-11

atmospheric considerations, 2-4

basie problems, 2

radiation field characteristics, $7-8$

radio gain, 4-5, $8-10$

transmission factors, $6-7$

units and symbols used in propagation study, 11

Propagation aspects of low-angle and surface coverage performance, 163-169

duets, effect on set perfornance, 163

low heights, effect on ranges, 163-164

maximum range, 163

maxinum range velsus height eurves 164-166

performance check before operation, 166

radar cross section of surface craft, 164

ship size estimation, 160

Propagation below interference region, radio gain calculation, 91-128

eurved earth calculations, 92

general problem an:lysis, 91-95

graphical solution, dielectric earth calculations, 95-108

plane earth calculations, 91-92

radio gain near line of sight, 115

sample calculations for general solution, 116-12s

sea water calculations, $108-115$
Jropagation in interforence region, radio gain calculation, $66-91$

antenna gain and directivity, 69

livergence, 68-69

factors affecting radiu gain, $67-69$

general solution, $69-70$

imperfert reflection, $67-68$

interference, 6.

plane earth calculations, 70-71

sample calculations, $79-91$

spherical earth calculations, $71-78$

spreading effect, 67

Quarter-wave dipole antenna, 26-27

Ratrlar cross scotion

aircraft target, 185-180

characteristic length $\mathrm{h}, 20$

maximum range calculations, 163

scattering cross section, 19-20

scattering parameters, radar coverage measurement, 182

surface eraft, 164

Radar cross section, simple forms, 183 $\mathbf{1 8 5}$

circular plate, 183-185

corner reflector, 185

eylinders, 183

flat plates, 183

rectangular plate, 18.5

spheres, $1 \mathrm{S3}$

laadar gain, 5, 20-21

Radar plaming device for ccho determination (RPD), 193

Radar receivers, sensitivity, 19

operator loss, 19

performance characteristics, 169

seanning loss, 19

sweep-specd loss, 19

liadar siting

see Siting, terrain selection and utilization

Radar targets, 182-186

aireraft, cross section measurement, $185-186$

radar eross section of simple forms, 183-185

scattering parameters, coverage neasurement, 182-183

Radiation

antenna radiation putterns, 22-23

antenna radiation resistance, 24

electric doublet antenna, 12-13

induction field, 12

reciprocity principle with reception, 17

lesistance, 13

lesistance, half-wave dipole, 20

Radiation field

electric doublet antenna, 12-13

gencral nature, $7-8$

half-wave dipole antenna, 25-26
Radiation field in standard atmosphere, radio gain ealeulations, $63-67$

field variation, $63-65$

bigh antenna calculitions, 65

low antenna caleulations, 65

ultra short waves in fliffraction region, 65-67

Radio gain

basic equation, 15

cialculation, 60-128

Hefined, $4-5,60-61$

doublet antennas in free space, 5

rarlio gain cur've's, $8-10$

Radio gain calculations, helow interference region, 91-12S

analysis of first mode, 91-94

effect of linear variation of refractive index of atmosphere, $94-95$

general solution for dipole over a smooth spliere, 116-122

graphical aids for sea water, v-li-f, vertical polarization, 10S-115

graphs for the case of the dielectric carth, 95-108

radio gain near the line, 115

sample ealculation for very dry soil 123-128

Radio gain caleulations, general considerations, 60-63

altenuation factors, 61

curved-earth geometrical relationships, 62

definition, 60-61

optical and diffraction regions, 62-63

standard propagation conditions assumed, 61-62

Radio gain calculations, in interforence legion, $67-71$

gencral solution, $69-70$

plane earth, 70-71

propagation factors, 67-69

Radio gain calculations, in standard atmosphere, 63-67

Radio gain calculations, optical-interference region, 79-91

coverage problem, $87-89$

for fixed heights and distance, 82-84

maxinum range vs. receiver hejght, 89-91

radio gain vs. distance for given antenna heights, 85-87

radio gain vs, receiver leight for given distance, $84-85$

Radio gain calculations, spherical earth, 71-7s

angle determination, 71

dimensionless coordinates, $77-78$

dimensionless parameters, $75-77$

distance measurement, 71

divergence factor, 75

equivalent height, 71 
grazing angle, 78

path difference, $74-75$

reflection point determination, $71-74$

Raindrop diffraction, 59

Range loci construction, 133-134

Rayleigh criterion, radiation theory application, 176

Receiver sensitivity, 17-19

definition, 18

noise figure, 17-19

radar receivers, 19

thermal noise, 17

Reciprocity principle, reception and radiation, 17

Reflection

Brewster angle, 54

effect on equipment performance, $160-161$

ground reflection, 52-5s

imperfect reflection, interfernce region, $6 \overline{7}-68$

reflection coefficient, $53,69-70$

transmission factors, $6-\bar{t}$

Reflection point curves, coverage diagram construction, 138-139

Reflection point determination, $71-\overline{7}$

Reflection point variable, 80

Refraction, $45-52$

atmospheric stratification, $50-52$

curvature relationships, $47-48$

definition, 3

graphical representation, 46-17

Snell's law, 45-46

standard refraction, 45

transmission factor, 6

liefractive index, 45-52

computation, $48-50$

function of temperature and height, $49-50$

function of temperature and relative humidity, 51

modified refractive inlex, 40

standard atmosphere, 6 !

Resonant antennas, 23

Rhombic antenna, traveling wave antenna, 32-33

Ring arrays, antonna, 39

RPD (radar planning device) for echo determination, 193

Scanning loss, radar reception, 19

Seattered power, radio wave reception, 13-14

Scattering parameters, radar coverage measurement, 182-183 echo constant, 182

equivalent plate area, 183

radar cross section, 182

seattering coefficient, radar lenth of target, 183

target gain, 182

Seat water

dielectric earth behavior, 66-6is

infuence on radio wave transmission, $54-55$

radio gain ealculation, 108-115

Sectoral horn, antenna, 4

Shadow zone, $T$

Shielding, permanent, echo control device, 193

Ship roll, effect on radar coverige, $160-$ 161

Ship size estimater? by strength of returned radar signal, 160

Side-by-side array, two-dipole antenua, $34-35$

Signal-to-noise ratio, radar equipnent performance, 161

Siting, terrain selection and utilization, $187-196$

geometrical limits of visibility, 188191

permanent echoes, $191-195$

radar, overlind transmission, 50

requirements of siting, 187

trees and jungles, 195-1!kj

Siting topography, 187-188

maps, 187

orientation, $18 \overline{-}-18 \mathrm{~S}$

profiles, 187

Slot diffraction, 175

Snell's law of refraction, $45-46$

soil as radio wave conductor', 56-57

Solar azimuths, calculation metlouls. $187-188$

spherical earth

coverage diagrams, $131-132$

path difference, $7 \$-75$

radio gain calculation; see Radio gain calculations, spherical earth

spreading effect, plopagation factor in interference region, 67

Standard atmosphere, relnetive index, 61

Standard dry atmosphere, properties, 3

Standard refraction, definition, 45

Standing-wave antennas, 24-32

half-wave dipole, $25-26,25-2\})$

linear antennas, 24-25, 2!)-31 multiple half-wave long antennas, $27-28$

$\mathrm{V}$ antemnas, 31-32

Supersonic method of echo determination, 193

Surface claft, extimation of size by strength of returned radar signal, 160

Surface craft, ralar closs section, 164

Sweep-speed loss, radar reception, 19

Symbols adopted for frequency ranges, 11

Target liffraction, 5S-59

Torrain diffraction, 170-181

Terrain selertion

see Siting, terrain selection and utilization

Thermal noise in radio receiver's, 17

Time computations neeessary for radar site selection, 188

Topographic maps and profiles

prediction of echoes, 193-195

- siting selection use, 187

Transmission, $6-\bar{\tau}, 45-59$

antenna characteristios, 6

diffraction, $\bar{\tau}, 5 \$-59$

divergence, 7

ground reflection, 52-58

polarization, 6

reflection, 6

refraction, $6,45-52$

Traveling-wave antennas, 23-24, 32-33

Trues as obstacles for high frequency raclio wave, 195-190

Troposphere, propagation role, 2

Ulta-short waves in diffraction region $65-67$

Cnidilectional arrays, antenna, 38

Units used in propagation study, 11

U-Y methoul of coverage tiagram construction, 135-13s

$\checkmark$ antennas, $31-32$

Vertical polarization

angles of lobe maxima, 130-131

effect on radio gain, $\$ 3$

Visibility, geometrical limits, 188-191

degree of shielding, 190-191

horizon distance of transmitter, $188-189$

obstacle beight, 189-190

Tagi antenna, multiple parasite, 41 




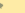




\section{.}


